\title{
Investigating the anti-cocaine effects of novel kappa opioid receptor agonists: Behavioural and cellular actions of Salvinorin A analogues
}

by

\section{Amy Wan Mun Ewald}

A thesis submitted to Victoria University of Wellington in fulfilment of the requirements for the degree of Doctor of Philosophy

Victoria University of Wellington 


\section{Abstract}

Acute kappa opioid receptor (KOPr) activation by traditional agonists produces antiaddiction properties, but side effects such as sedation and depression prevent their clinical use. The novel KOPr agonist salvinorin A (Sal A), isolated from the plant Salvia divinorum, is a potent and selective $\mathrm{KOPr}$ agonist with a unique non-nitrogenous structure. Sal A possesses anti-addiction effects with less side effects than traditional KOPr agonists, but its short duration of action limits its therapeutic usefulness. To test the hypothesis that longer acting structural analogues of Sal A may yield a new class of therapeutics, the anti-cocaine effects of Sal A analogues such as 16-bromosalvinorin A (16-brSal A), ethoxymethyl ether salvinorin B (EOM Sal B), and methoxymethyl ether salvinorin B (MOM Sal B) were evaluated. 16-brSal A $(1.0 \mathrm{mg} / \mathrm{kg})$ displayed a longer duration of action in mice compared to Sal $A$, evidenced using the tail flick test $(p<0.05)$. Both 16-brSal A and EOM Sal B produced dose-dependent decreases in cocaine-induced reinstatement of drug seeking $(p<0.05)$. On the other hand, 16-brSal A $(1.0 \mathrm{mg} / \mathrm{kg})$ but not MOM Sal B $(0.3 \mathrm{mg} / \mathrm{kg})$ attenuated cocaine-induced hyperactivity $(p<0.05)$, although both compounds showed no sedative effects in the locomotor activity test in rats. This indicates the superior behavioural anti-cocaine profile of 16 -brSal $A$ at its minimum effective dose. These three compounds, together with another analogue that also decreased cocaineinduced drug seeking, $\beta$-tetrahydropyran salvinorin B ( $\beta$-THP Sal B), were screened for typical KOPr-mediated side effects using the minimal effective doses that attenuated drug seeking. MOM Sal B but not EOM Sal B $(0.1 \mathrm{mg} / \mathrm{kg}), \beta$-THP Sal B $(1.0 \mathrm{mg} / \mathrm{kg})$, or $16-\mathrm{brSal}$ A produced depressive-like effects in the forced swim test (FST) in rats $(p<0.05)$. However, EOM Sal B displayed a reduction in swimming time coupled with an increase in climbing duration in the FST $(p<0.05)$. On the other hand, $\beta$-THP Sal B $(p<0.001$, between $30-45$ $\mathrm{min}$ ) and EOM Sal B ( $p<0.05$, between $15-30 \mathrm{~min}$ ) significantly increased sucrose intake in the rat sucrose self-administration model at different time intervals. 16-brSal A, however, produced no significant changes in natural reward intake measured by sucrose self-administration. The improved behavioural profile of 16-brSal A extended to a lack of anxiogenic effects. No significant anxiety-like behaviour was seen in the light dark or elevated plus maze, although aversion was observed in the conditioned place aversion paradigm $(p<0.05)$. The low incidence of adverse effects of 16 -brSal A compared to other 
Sal A analogues in behavioural models prompted additional cellular studies of this KOPr agonist. As the anti-cocaine effects of KOPr agonists have been attributed to their ability to modulate dopamine (DA) levels, 16-brSal A was examined for its ability to regulate dopamine transporter (DAT) function. DAT function was determined in vitro by determining uptake of a fluorescent substrate, ASP ${ }^{+}$, in HEK-293 cells expressing YFP-DAT and myc-KOPr. Ex vivo studies were also conducted by measuring DA uptake in isolated, minced rat dorsal striatum and nucleus accumbens using rotating disk electrode voltammetry. 16-brSal A significantly increased DAT function in both the in vitro (10 $\mu \mathrm{M})$ and ex vivo $(500 \mathrm{nM})$ models $(\mathrm{p}<0.05)$, an effect that was dependent on extracellular regulated kinase $1 / 2$ (ERK1/2). Since late phase ERK1/2 and p38 kinase activation have been attributed to negative KOPr behavioural responses, the effects of 16 -brSal $A$ on these pathways were also examined. Western blotting studies revealed that 16 -brSal A selectively activated only the early $(5-15 \mathrm{~min})$ but not late phase $(120-180 \mathrm{~min})$ ERK1/2 pathway in HEK-293 cells as well as rat dorsal striatum, prefrontal cortex, and nucleus accumbens ( $p<0.05)$. 16-brSal A also produced no significant activation of p38 kinase in the dorsal striatum or prefrontal cortex of rats, although significant phosphorylation was seen in the nucleus accumbens $(p<0.05)$. The ability of 16 -brSal A to produce desired behavioural anti-addiction effects with fewer adverse effects, matched with its regulation of KOPr signalling pathways, suggests that it may possibly be a functionally selective agonist that preferentially activates the G-protein signalling pathway at the KOPr. Since understanding the potential use of novel KOPr agonists in different phases of the addiction cycle is crucial to ensure effective administration of therapies, Sal A and 16-brSal A were tested in rats self-administering cocaine on the long access (Sal A) and progressive ratio (Sal A and 16-brSal A) schedules. Although no differences in cocaine responding were seen with KOPr agonist treatment in either paradigms, a higher dose or concurrent infusions of KOPr agonist with cocaine may improve the responses observed. Overall, the novel KOPr agonist, 16-brSal A has excellent potential as a pharmacotherapy due to its anti-cocaine effects and minimal adverse side effect profile. This is the first study to examine in detail the behavioural and cellular actions of 16-brSal A, and supports previous reports of Sal A-derived KOPr agonists as prospective therapeutics for cocaine abuse. 


\section{Acknowledgements}

This has been a long and crazy journey, one which I could not have completed without the help and support of many people who deserve to be acknowledged.

My supervisors Dr Bronwyn Kivell and Professor John Miller, thank you for your advice, guidance, moral support, and keeping me on track the past three years. I appreciate you making time for me and answering all my queries despite your busy schedules. To the academics who always had a good word of advice and support: Dr Katie Brennan, Professor David Harper, and Professor Susan Schenk, thank you for being so helpful and welcoming.

To my mentor and lab mate Peter Bosch who taught me everything I needed to know, thank you so much for your patience and putting up with my endless questions. I am extremely grateful for your suggestions, advice, and unwavering support. My fellow lab mates, honorary lab mates, and volunteers who have provided great (and crazy) research ideas, alcohol when needed, coffee runs, and a shoulder to cry on: Kirsty, Miles, Fraser, Tori, Leigh, Amy S., Aimee, David, Kelly, Varun, Mohan, Anna, Caroline, and Adam, thank you for your help! I appreciate the time you spent drying rats, cleaning dishes, autoclaving rubbish, and helping me work out Inkscape and R when I couldn't. Those who put up with me in Psychology: Sarah, Dane, Jeremy, Bridget, Sophie, Alanna, and Hannah, thanks for always providing a fun working environment. My office mates: Edi, Pop, Cong, Kimberley, Olivia, and Megan, thank you for always being so supportive and encouraging.

The technicians and facility managers, I could not have completed any experiments without you: Neville, Craig, Shaun, Sushila, Chris, Ange, Joyce, Richard, Peter, and Mike, thank you for putting up with my numerous requests and fixing things when they didn't work. To the front office staff who always had an encouraging word: Lesley, Mary, Sandra, Patricia, Paul, and Mark, thank you for helping with the official paperwork and administration side of things. 
My family who listened to my rants, lauded my successes, and prayed for me when things were hard: dad, mum, Allan, and granny, thank you for your unwavering love, support, and guidance. My parents in law, thank you for being so supportive and understanding. To my husband Rod, I will be eternally grateful to you for putting up with my crazy hours, working just as many hours to feed us both, cooking all our meals, and never complaining; may it last many more years to come. Finally to God, without Whom none of this would have been possible. 


\section{Table of Contents}

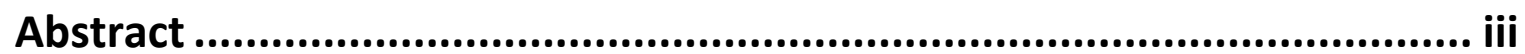

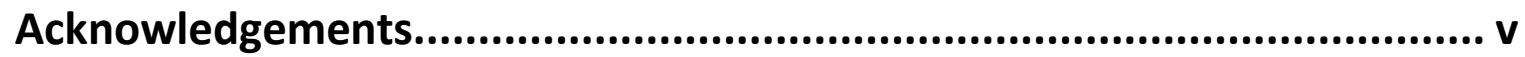

Table of Contents...................................................................................... vii

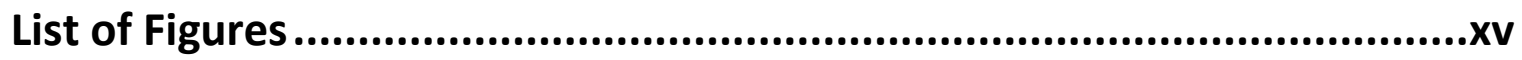

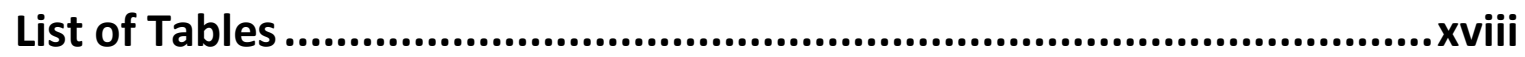

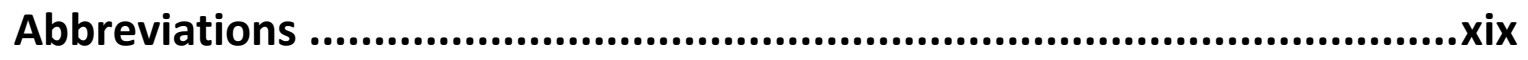

Chapter 1: Introduction ........................................................................... 1

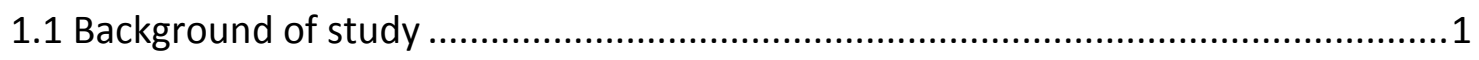

1.1.1 Drug addiction: definition and statistics .................................................

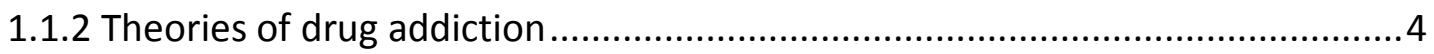

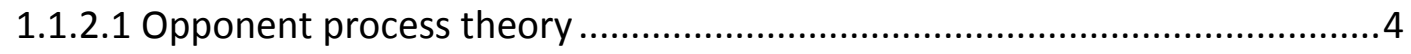

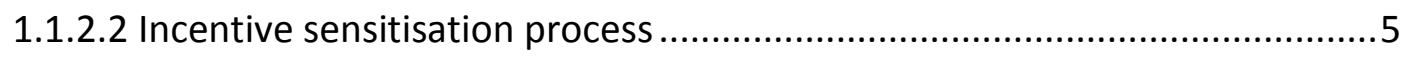

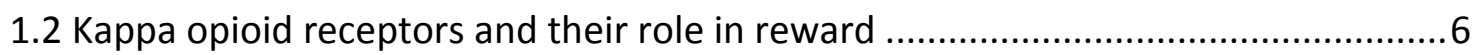

1.2.1 The brain natural reward pathway .......................................................... 6

1.2.2 The dynorphin/kappa opioid receptor system ........................................11

1.2.3 Regulation of the reward pathway by the dynorphin/KOPr system ................12

1.3 Role of the dynorphin/kappa opioid system in cocaine abuse ............................. 12

1.3.1 Effects of cocaine on the dopaminergic reward system ..............................12

1.3.2 Changes to DAT and KOPr systems in response to cocaine ...........................15

1.3.3 Current pharmacotherapies for cocaine ....................................................17

1.4 KOPr agonists as potential anti-cocaine pharmacotherapies................................19

1.4.1 Anti-cocaine and adverse effects of traditional KOPr agonists .....................19

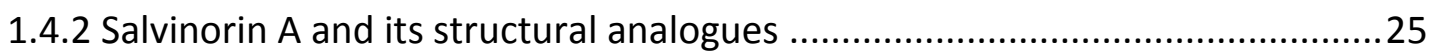




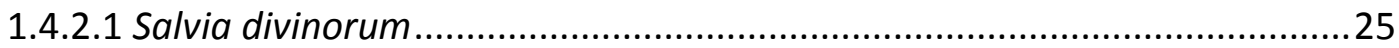

1.4.2.2 Salvinorin A and its synthetic derivatives ..............................................25

1.4.3 Anti-cocaine effects of Sal A and its analogues .............................................30

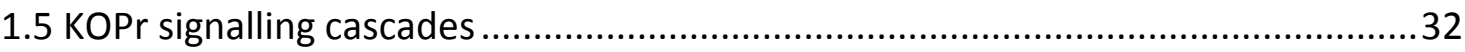

1.5.1 Extracellular signal-regulated kinase 1 and 2 (ERK1/2) ...............................34

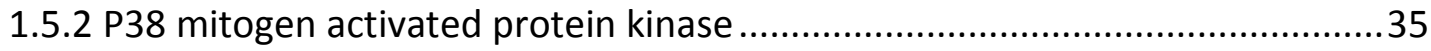

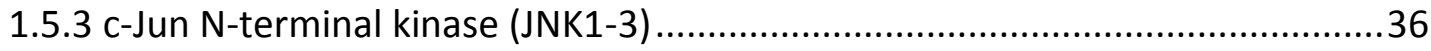

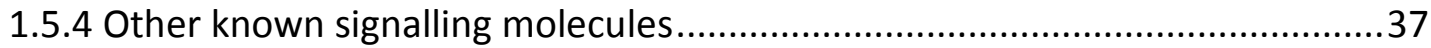

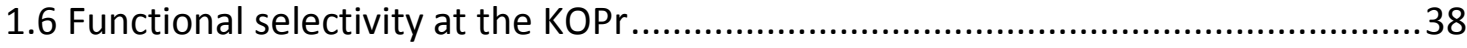

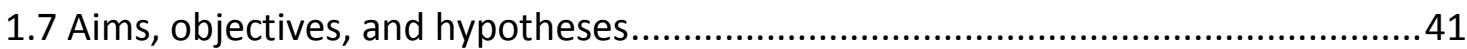

Chapter 2: Materials and methods........................................................ 43

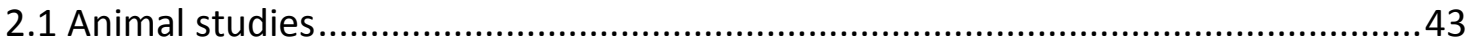

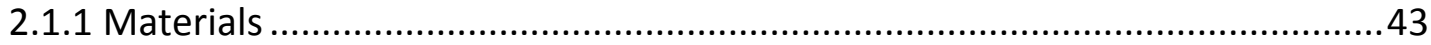

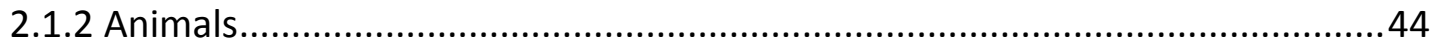

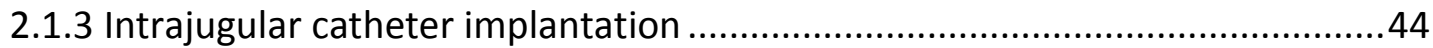

2.1.4 Cocaine self-administration training...........................................................46

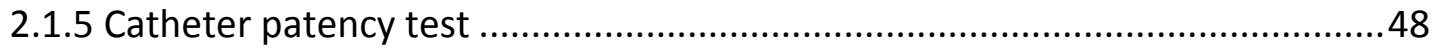

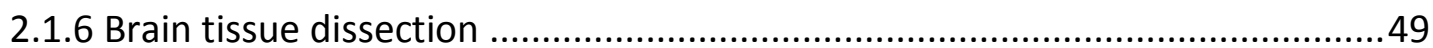

2.1.7 Rotating disk electrode voltammetry …....................................................50

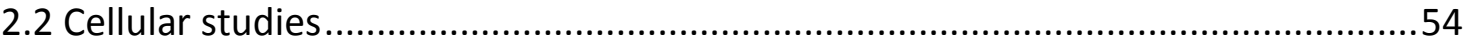

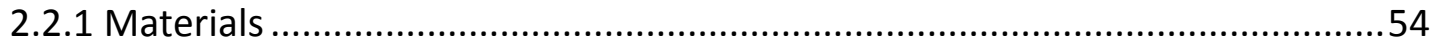

2.2.2 Cell line: Human embryonic kidney 293 (HEK-293) cells ..................................54

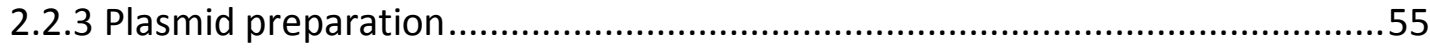

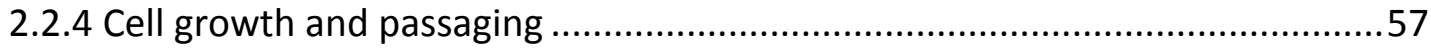

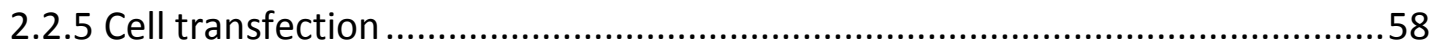

2.2.6 trans-4-[4-(dimethylamino)styryl]-1-methylpyridinium $\left(\mathrm{ASP}^{+}\right)$uptake............58 
2.2.7 Cell surface biotinylation .59

2.2.8 Protein concentration assay .60

2.2.9 SDS polyacrylamide gel electrophoresis and Western blotting .61

2.3 Statistical analysis

Chapter 3: Investigating the preclinical anti-cocaine effects of novel Sal A analogues 65

3.1 Introduction .65

3.1.1 Modelling craving and relapse behaviours: Reinstatement of drug seeking ...65

3.1.2 Modelling cocaine-induced hyperactivity. .67

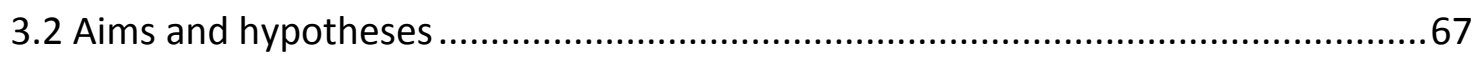

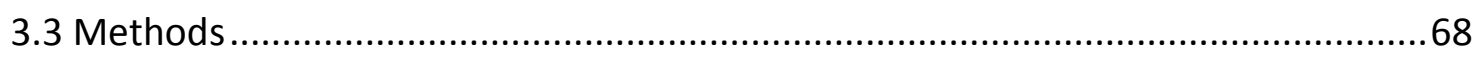

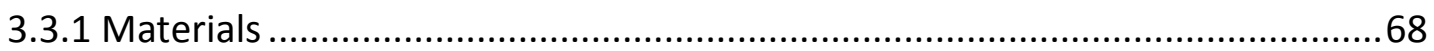

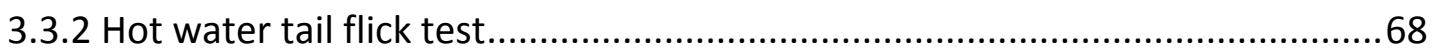

3.3.3 Cocaine prime-induced reinstatement of drug seeking ................................69

3.3.4 Cocaine-induced locomotor hyperactivity ..................................................69

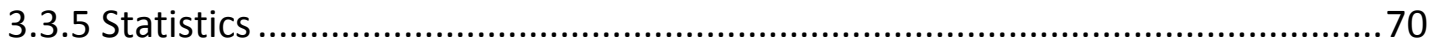

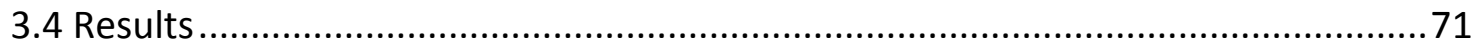

3.4.1 Onset and duration of action of 16-brSal A in vivo .......................................... 71

3.4.2 Effect of 16-brSal A and EOM Sal B on cocaine-induced drug seeking ............73

3.4.3 Effect of 16-brSal A and MOM Sal B on cocaine-induced hyperactivity ..........79

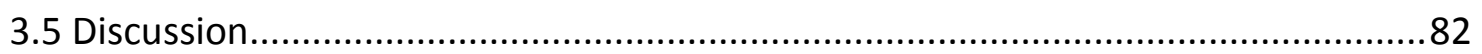

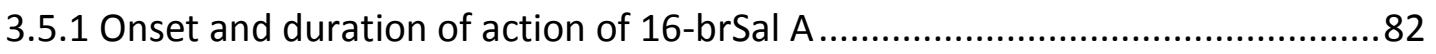

3.5.2 Cocaine self-administration and cocaine-induced reinstatement ..................83

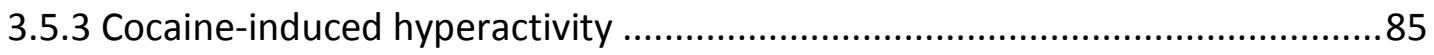

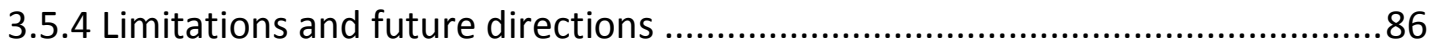

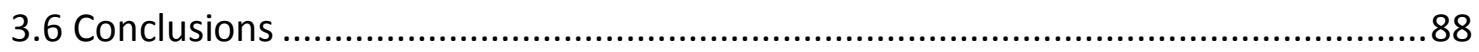


Chapter 4: Investigating the side effects of novel Sal A analogues in

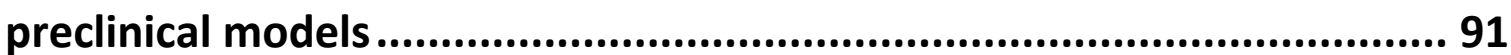

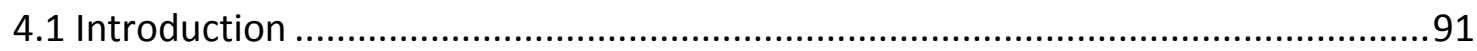

4.1.1 Effect of KOPr agonists on sedation ......................................................... 91

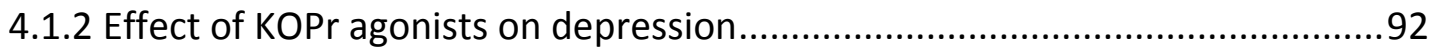

4.1.3 Effect of KOPr agonists on modulation of natural reward .............................93

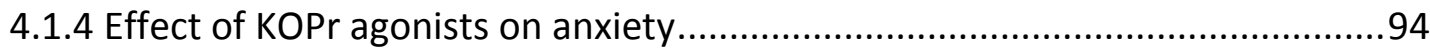

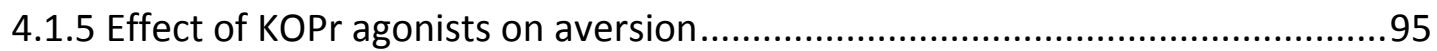

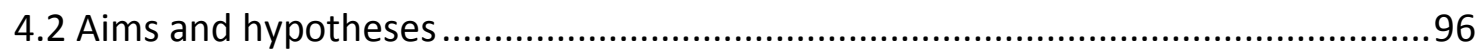

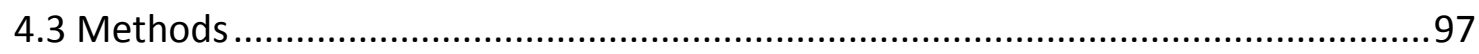

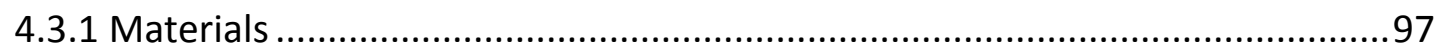

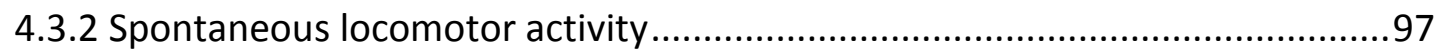

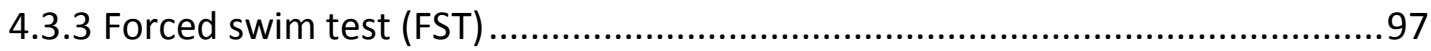

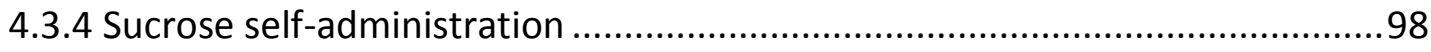

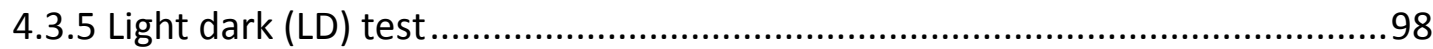

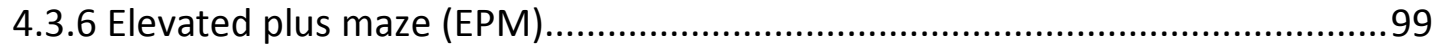

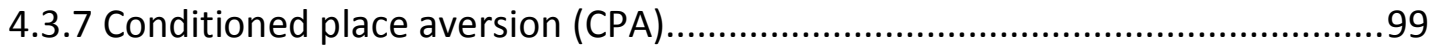

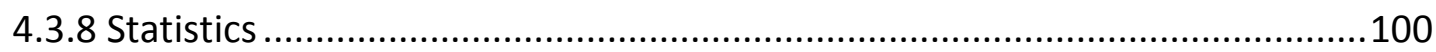

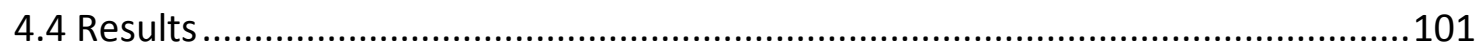

4.4.1 Effect of 16-brSal A and MOM Sal B on sedation ........................................101

4.4.2 Effect of 16-brSal A, EOM Sal B, MOM Sal B, and $\beta$-THP Sal B on depression104

4.4.3 Effect of 16-brSal A, EOM Sal B, and $\beta$-THP Sal B on sucrose self-administration 106

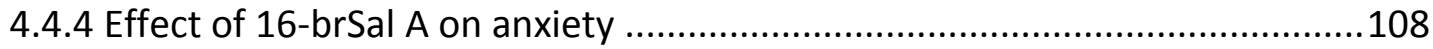

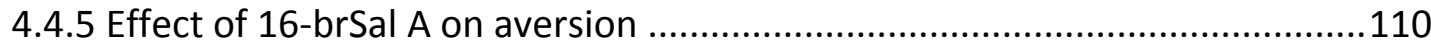

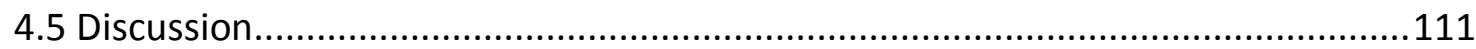


4.5.1 Sedative effects

4.5.2 Depressive effects

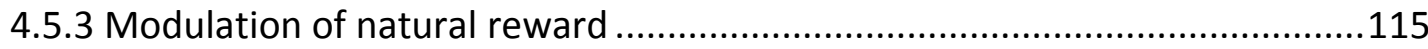

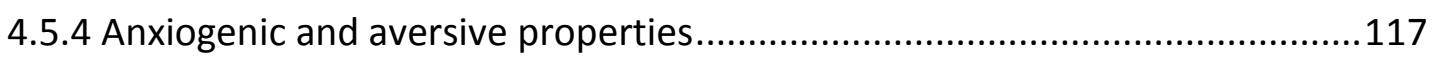

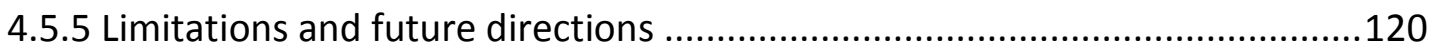

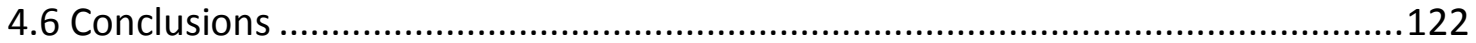

Chapter 5: Investigating the cellular mechanisms of 16-brSal A in rat brain tissue and HEK-293 cells ................................................................... 127

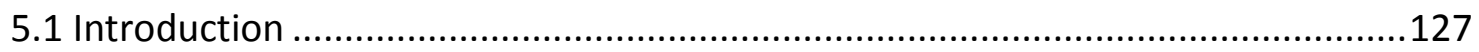

5.1.1 KOPr modulation of DAT function and expression ....................................127

5.1.2 KOPr-mediated ERK1/2 and p38 MAPK activation .....................................128

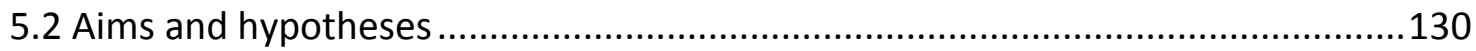

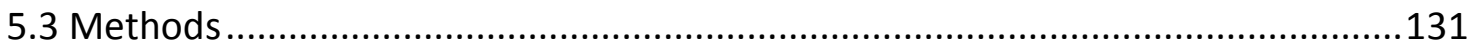

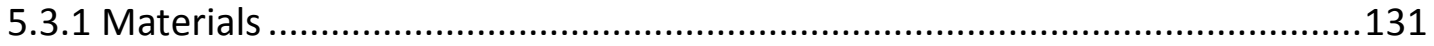

5.3.2 Immuno-labelling of myc-rKOPr for visualisation........................................131

5.3.3 trans-4-[4-(dimethylamino)styryl]-1-methylpyridinium (ASP ${ }^{+}$) uptake...........132

5.3.4 Rotating disk electrode voltammetry (RDEV) ..........................................133

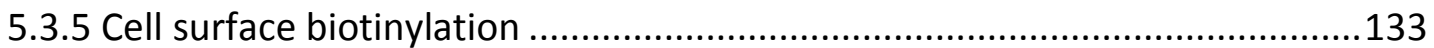

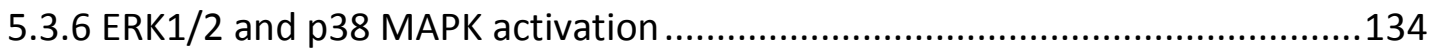

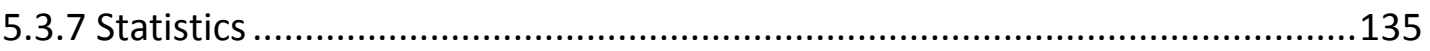

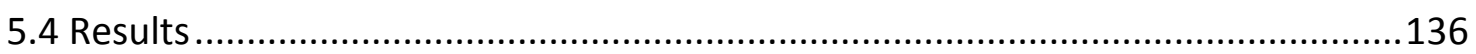

5.4.1 Validation of YFP-hDAT and myc-rKOPr co-transfection in HEK-293 cells .....136

5.4.2 Validation of ASP ${ }^{+}$uptake in KOPr and DAT co-expressing HEK-293 cells......138

5.4.3 Modulation of DAT in HEK-293 cells following acute administration with 16brSal A .141 
5.4.4 Modulation of DAT in the rat dStr and NAc following acute treatment with 16brSal A

5.4.5 Effect of ERK1/2 inhibition on the 16-brSal A-evoked increase in DAT function 150

5.4.6 Effect of 16-brSal A on ERK1/2 activation 152

5.4.7 Effect of 16-brSal A treatment on p38 MAPK activation .156

5.5 Discussion. 158

5.5.1 Validation of YFP-hDAT and myc-rKOPr expression in HEK-293 cells for ASP ${ }^{+}$ uptake testing ..... 158

5.5.2 16-brSal A modulates DAT function in a KOPr-dependent manner without altering DAT cell surface expression 159

5.5.3 16-brSal A regulates DAT by activating the early, but not late phase of ERK1/2

5.5.5 Limitations and future directions 166

5.6 Conclusions 168

Chapter 6: Investigating the preclinical effects of novel kappa opioid receptor agonists using long access and progressive ratio selfadministration paradigms .173

6.1 Introduction 173

6.1.1 Extended or long access model 174

6.1.2 Progressive ratio model 176

6.2 Aims and hypotheses. 177

6.3 Methods 178

6.3.1 Materials 178

6.3.2 Long access cocaine self-administration 178

6.3.3 Progressive ratio self-administration 178 
6.4.1 Validation of the extended access cocaine self-administration paradigm.....180

6.4.2 Effect of Sal A on extended access cocaine self-administration in rats .182

6.4.3 Determination of differences between drug naïve and KOPr agonist pre-treated rats 185

6.4.4 Effect of novel and traditional KOPr agonists on cocaine self-administration using a progressive ratio schedule of reinforcement .188

6.5 Discussion .190

6.5.1 Extended access cocaine self-administration .190

6.5.2 Progressive ratio schedule of reinforcement .193

6.5.3 Limitations and future directions 196

6.6 Conclusions .198

Chapter 7: Final Discussion. 199

7.1 Significance of study .199

7.2 Key findings 199

7.2.1 16-brSal A has a longer duration of action in vivo and similar anti-cocaine profile as Sal $\mathrm{A}$ 201

7.2.2 16-brSal A has fewer side effects compared to traditional KOPr agonists, Sal A, or other Sal A analogues. .201

7.2.3 16-brSal A modulates DAT function in a different manner than traditional KOPr agonists 202

7.2.4 Sal A and 16-brSal A do not decrease LgA self-administration or motivation to self-administer cocaine. 203

7.3 Limitations and future directions .203

7.4 Final conclusions .209

Chapter 8: Supplementary information 211 
Appendix 2.1: Determination of ideal protein loading concentration

Appendix 2.2: Protein band size quantification

Appendix 3.1: Counterbalanced treatment order for the reinstatement study 216

Appendix 3.2: Number of days required to reach extinction criteria

Appendix 3.3: Comparison between vehicle groups in the cocaine-induced hyperactivity study. 218

Appendix 4.1: Counterbalanced treatment order for the sucrose self-administration study 219

Appendix 4.2: Comparison of vehicle groups in the locomotor activity study 220 Appendix 4.3: Comparison of vehicle-treated rats of different groups in the FST study

Appendix 5.1: Non-specific uptake in $\mathrm{ASP}^{+}$uptake kinetics by non-transfected cells 222 Appendix 5.2: Representative Western blot membranes

Appendix 6.1: Counterbalanced treatment order for the LgA and PR self-administration study. .225

Appendix 6.2: Comparison of acquisition periods in all self-administration studies ..227 Appendix 6.3: Comparison of baselines throughout the PR study 228 


\section{List of Figures}

Figure 1.1: Usage prevalence of commonly abused drugs .............................................

Figure 1.2: Co-localisation of DAT and KOPr in the natural reward pathway .................... 8

Figure 1.3: Synthesis of DA and chemical structures of DA and cocaine .........................10

Figure 1.4: Physiological and cocaine-affected dopamine signalling ............................... 14

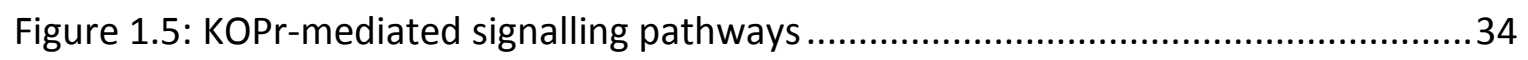

Figure 2.1: Schematic of an intrajugular catheter ...................................................... 45

Figure 2.2: Coordinates for rat brain dissection ....................................................... 49

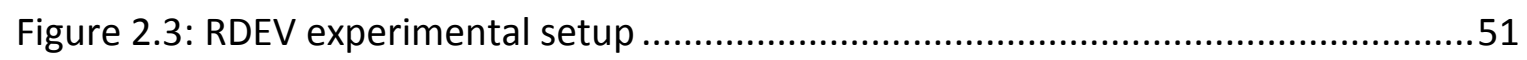

Figure 3.1: Outline for cocaine-induced reinstatement of drug seeking .........................70

Figure 3.2: 16-brSal A has a longer duration of action in vivo than Sal A......................... 72

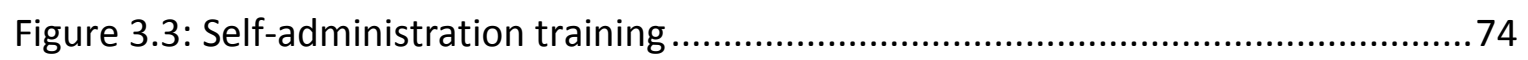

Figure 3.4: 16-brSal A and EOM Sal B decrease cocaine-primed reinstatement of cocaine

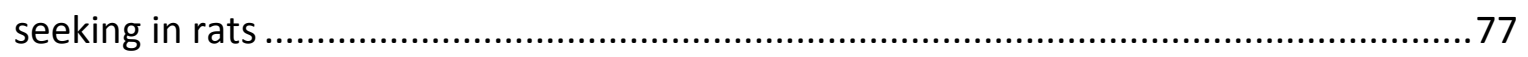

Figure 3.5: 16-brSal A reduces cocaine-induced locomotor hyperactivity........................80

Figure 3.6: MOM Sal B does not alter cocaine-induced locomotor hyperactivity .............81

Figure 4.1: 16-brSal A and MOM Sal B do not alter spontaneous locomotor activity in rats 103

Figure 4.2: MOM Sal B increases occurrences of immobility in the rat FST ...................105

Figure 4.3: U50,488, EOM Sal B, and $\beta$-THP Sal B significantly alters responding for sucrose in rats 107

Figure 4.4: 16-brSal A produces no significant anxiogenic effects in rat anxiety models

Figure 4.5: Sal A and 16-brSal A produce conditioned place aversion in rats .................110

Figure 5.1: Validation of YFP-hDAT and myc-rKOPr co-expression in HEK-293 cells ......137

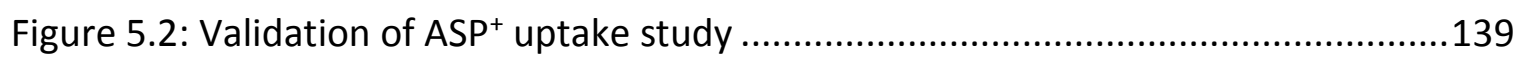

Figure 5.3: Time course of $\mathrm{ASP}^{+}$uptake in YFP-hDAT and myc-rKOPr co-transfected HEK293 cells 140

Figure 5.4: 16-brSal A increases $\mathrm{ASP}^{+}$uptake in YFP-hDAT and myc-rKOPr co-expressing HEK-293 cells in a KOPr-dependent manner .142 
Figure 5.5: 16-brSal A increases the Vmax and $\mathrm{Km}$ of $\mathrm{ASP}^{+}$uptake by DAT in YFP-hDAT and

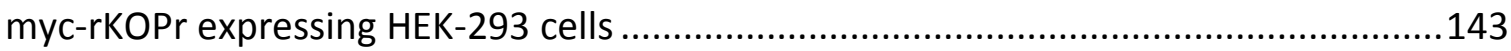

Figure 5.6: 16-brSal A increases DA uptake in the rat dStr .......................................146

Figure 5.7: 16-brSal A increases DA uptake in the rat NAc.........................................147

Figure 5.8: The selective KOPr antagonist nor-BNI inhibits 16-brSal A-mediated increase of DA uptake in the dStr and NAC

Figure 5.9: 16-brSal A increases DAT function in an ERK1/2-dependent manner but does not alter DAT cell surface expression

Figure 5.10: 16-brSal A selectively activates the early but not late phase of ERK1/2 in HEK293 cells transiently expressing YFP-hDAT and myc-rKOPr in a KOPr-dependent manner

Figure 5.11: 16-brSal A modulates ERK1/2 phosphorylation in the rat dStr, NAc, and PFC 155

Figure 5.12: 16-brSal A modulates $\mathrm{p} 38$ phosphorylation the rat NAc. 157

Figure 6.1: Self-administration training 181

Figure 6.2: Sal A does not alter responding for cocaine in an extended access model of self-administration. 184

Figure 6.3: Mesyl Sal B and U50,488 pre-treatment increases cocaine self-administration acquisition time in rats 186

Figure 6.4: Pre-treatment with KOPr agonist does not alter the rate of active or inactive lever responding in rats 187

Figure 6.5: U69,593 decreases motivation to self-administer cocaine in rats using a PR schedule of reinforcement 189

Figure 8.1: The density of protein bands is dependent on protein concentration 214

Figure 8.2: Determination of protein band size 215 Figure 8.3: Number of days required for extinction decreased with progression of the study. 217

Figure 8.4: Number of total ambulatory counts produced by vehicle-treated rats in the hyperlocomotion study. 218

Figure 8.5: Number of total ambulatory counts produced by vehicle-treated rats in the open field locomotor activity study. 220 
Figure 8.6: Time spent climbing, swimming, and immobile for vehicle-treated rats in all

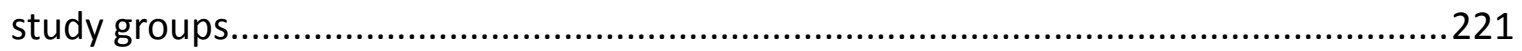

Figure 8.7: Increase of background autofluorescence in $\mathrm{ASP}^{+}$uptake kinetics..............223

Figure 8.8: Representative Western blot membranes ...............................................224

Figure 8.9: Self-administration acquisition period of drug naïve and KOPr pre-treated rats 227

Figure 8.10: Baseline number of infusions received by rats prior to PR test sessions ....228 


\section{List of Tables}

Table 1.1: Structures of U69,593, U50,488, Sal A and their pharmacology at the KOPr ..24

Table 1.2: Structures and pharmacology of Sal A and its C-2 analogues at KOPr. 28

Table 1.3: Chemical structures and pharmacology of Sal A and its C-16 analogues at KOPr

Table 2.1: Coordinates and weight of dissected brain regions

Table 2.2: Plasmid DNA vector and resistance information 57

Table 2.3: Antibodies used for Western blotting and their conditions ..... 63

Table 3.1: Acute effects of U69,593, U50,488, Sal A, and Sal A analogues on anti-cocaine behavioural models 89

Table 4.1: Acute effects of U69,593, U50,488, Sal A, and Sal A analogues on KOPrmediated adverse effects

Table 5.1: 16-brSal A increases Vmax and $\mathrm{Km}$ of ASP+ uptake in YFP-hDAT and myc-rKOPr co-expressing HEK-293 cells.

Table 5.2: 16-brSal A increases Vmax but not Km of DA uptake in the rat dStr and NAc 148

Table 5.3: The 16-brSal A-evoked increase of DA uptake in the rat dStr and NAc is inhibited with prior addition of nor-BNI

Table 5.4: Acute effects of U69,593, U50,488, Sal A, and Sal A analogues on the ERK1/2 and p38 MAPK signalling pathways 170

Table 5.5: Acute effects of U69,593, U50,488, Sal A, and Sal A analogues on DAT modulation

Table 7.1: Summary of behavioural anti-cocaine and side effects of Sal A derivatives ..200

Table 7.2: Summary of cellular effects produced by 16-brSal A 200

Table 8.1: Treatment design used in cocaine-induced reinstatement of drug seeking ..216

Table 8.2: Treatment order for rats in the sucrose reinforcement study 219

Table 8.3: Treatment order for rats in the LgA self-administration study 225

Table 8.4: Treatment order for rats in the PR study. 226 


\section{Abbreviations}

\begin{tabular}{|c|c|}
\hline 16-brSal A & 16-bromosalvinorin A \\
\hline $5-\mathrm{HT}$ & serotonin \\
\hline $6^{\prime}-\mathrm{GNTI}$ & 6'-guanidinonaltrindole \\
\hline Akt & protein kinase B \\
\hline AMPA & $\alpha$-amino-3-hydroxy-5-methyl-4-isoxazolepropionic acid \\
\hline ANOVA & analysis of variance \\
\hline $\mathrm{ASP}^{+}$ & 4-(4-(dimethylamino)-styryl)-N-methylpyridinium \\
\hline CAMP & cyclic adenosine monophosphate \\
\hline $\mathrm{CHO}$ & Chinese hamster ovary \\
\hline COMT & catechol-O-methyltransferase \\
\hline cos7 & African green monkey fibroblast \\
\hline CPA & conditioned place aversion \\
\hline $\mathrm{CPP}$ & conditioned place preference \\
\hline CREB & CAMP response element binding protein \\
\hline CTA & conditioned taste aversion \\
\hline DA & dopamine \\
\hline DAT & dopamine transporter \\
\hline DIC & differential interference contrast \\
\hline DMEM & Dulbecco's Modified Eagle's Medium \\
\hline DMSO & dimethyl sulfoxide \\
\hline DOPr & delta opioid receptor \\
\hline dStr & dorsal striatum \\
\hline EOM Sal B & ethoxymethyl ether Sal B \\
\hline EPM & elevated plus maze \\
\hline ERK1/2 & extracellular signal-regulated kinase 1 and 2 \\
\hline Ethy Sal A & 16-ethynylsalvinorin A \\
\hline FCS & foetal calf serum \\
\hline FDA & Food and Drug Administration \\
\hline FR & fixed ratio \\
\hline FST & forced swim test \\
\hline
\end{tabular}




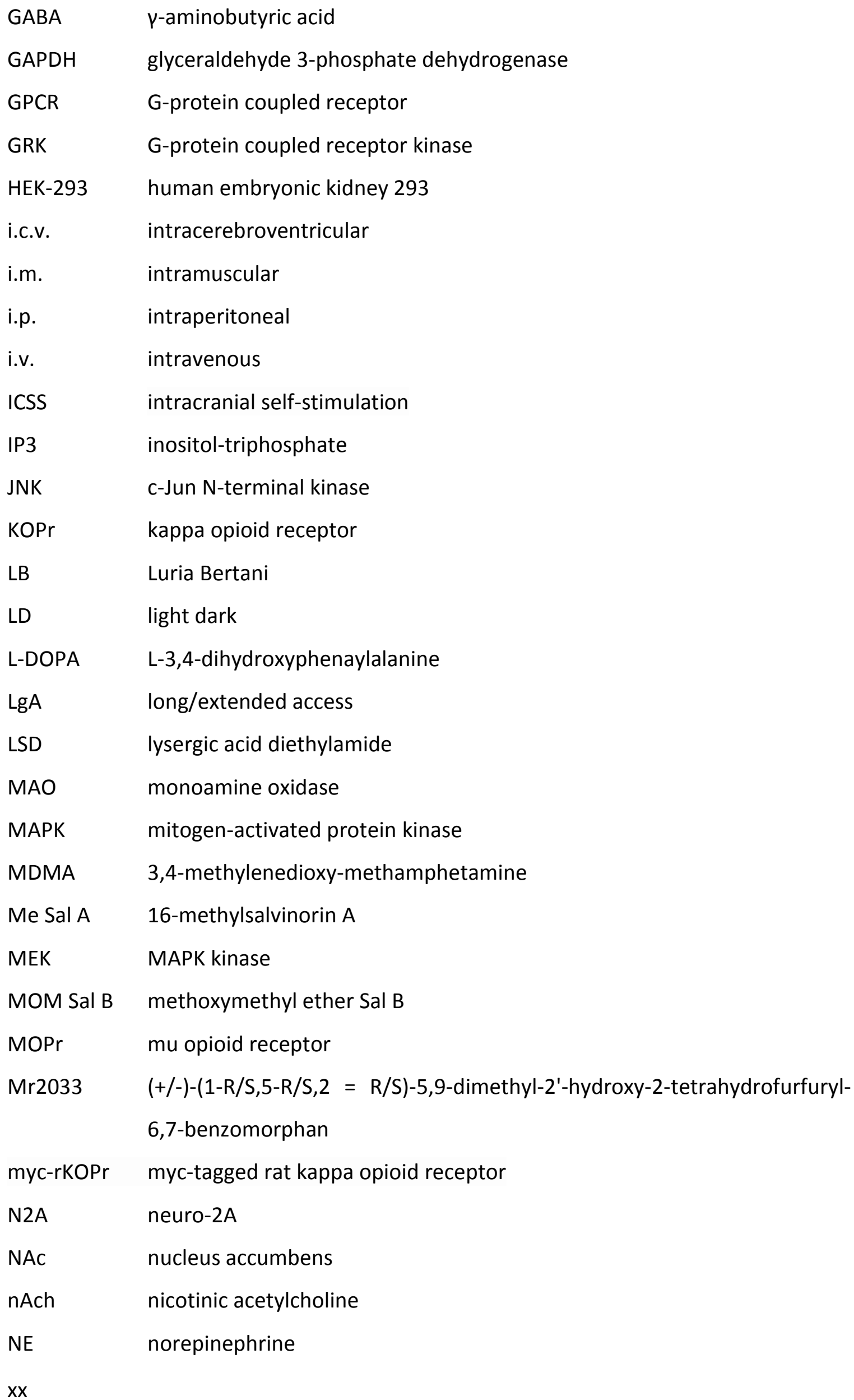




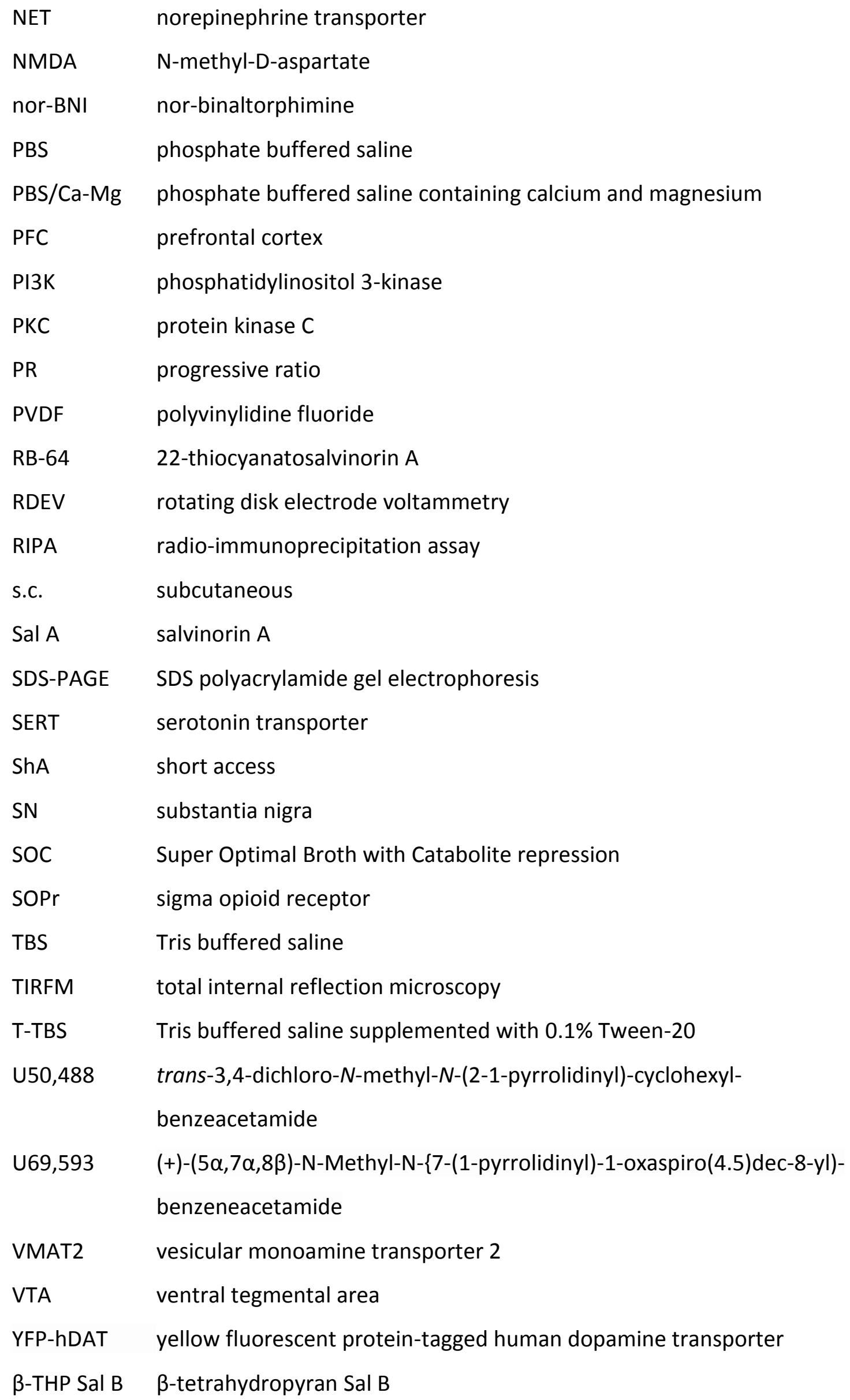




\section{Chapter 1: Introduction}

\subsection{Background of study}

Drug addiction is a compulsive disease that results in long-lasting changes in brain neurocircuitry and neurochemistry. Despite vast efforts, researchers in the addiction field have not yet developed a successful Food and Drug Administration (FDA) approved pharmacotherapy for psychostimulant abuse, of which New Zealanders are among the highest users in the world (Ministry of Health, 2010; Degenhardt and Hall, 2012). Agonists of the kappa opioid receptor (KOPr) have previously shown anti-addiction and cocaine antagonistic actions, effects attributed to their modulation of dopamine (DA) levels in the brain (Glick et al., 1998; Schenk et al., 1999; Morani et al., 2009; Morani et al., 2012). Unfortunately, traditional KOPr agonists present side effects such as sedation, aversion, anxiety, and depression that have prevented their translation into clinical use. Salvinorin A (Sal A), a structurally novel KOPr agonist has been identified as a potential therapeutic target due to its anti-cocaine effects and paucity of side effects, although its short half-life limits its therapeutic use. Synthetic analogues of Sal A with improved pharmacokinetic profiles show huge promise for the development of anti-cocaine pharmacotherapies. Research is currently ongoing to determine the behavioural and cellular effects of multiple analogues of Sal A. This PhD thesis adds to that growing knowledge and will aid in development of a Sal A analogue that may be useful as an anti-cocaine pharmacotherapy.

\subsubsection{Drug addiction: definition and statistics}

Drug addiction is a devastating disease defined as a chronic, relapsing brain disorder whereby an individual prioritises drug consumption despite negative personal and social consequences (Koob and Kreek, 2007). The most recent edition of the Diagnostic and Statistical Manual of Mental Disorders (DSM-V) classified 'substance use disorder' as a progressive condition that can be measured based on severity, in which a mild diagnosis requires at least two to three symptoms, whereas a severe diagnosis requires six or more symptoms out of 11 (American Psychiatric Association, 2013). Repeated use of drugs can cause transition to dependence and addiction (Koob et al., 2004). Addiction is a phenomenon defined by three symptoms: compulsive drug taking, loss of control over drug intake, and development of a negative emotional state (Koob and Le Moal, 2008). In 
comparison to occasional drug users, addicted individuals show intense drug cravings and experience a high risk of relapse even after a long abstinence period (Nestler, 2001).

The popularity of different drugs of abuse differ based on their geographic availability. The prevalence of use for 20 of the most popular drugs of abuse in Europe, USA, and the Oceania region as well as in New Zealand alone is shown in Figure 1.1 (Global Drug Survey, 2014). The legal depressant, alcohol, is the most commonly used drug worldwide $(90.8 \%)$ and in New Zealand (91\%). Globally, tobacco (56.7\%) and cannabis (48.2\%) use are highly prevalent, whereas caffeinated energy drinks (46.2\%) and cannabis (35.3\%) are more popular in New Zealand. Psychostimulants such as 3,4-methylenedioxymethamphetamine (MDMA or otherwise known as 'ecstasy'), cocaine, amphetamines, and methylphenidate (Ritalin) are also among the popular drugs abused (Global Drug Survey, 2014).

Addiction to drugs has severe repercussions because it not only affects the individual personally but also poses a massive burden to society. The resultant increase in crime, increased healthcare costs, unemployment, and loss of productivity are estimated to cost \$181 billion in the USA (National Institute On Drug Abuse, 2008) and \$6 billion in New Zealand (Slack et al., 2009) each year, making treatments for addiction highly sought after. Every dollar spent on treatment is estimated to save four to seven dollars as a result of drug related crimes alone (National Institute On Drug Abuse, 2008). Research in the field of addiction has also advanced rapidly due to increasing awareness that addiction is a brain disorder caused by biological and environmental factors, rather than just a moral failure (Leshner, 1997). 


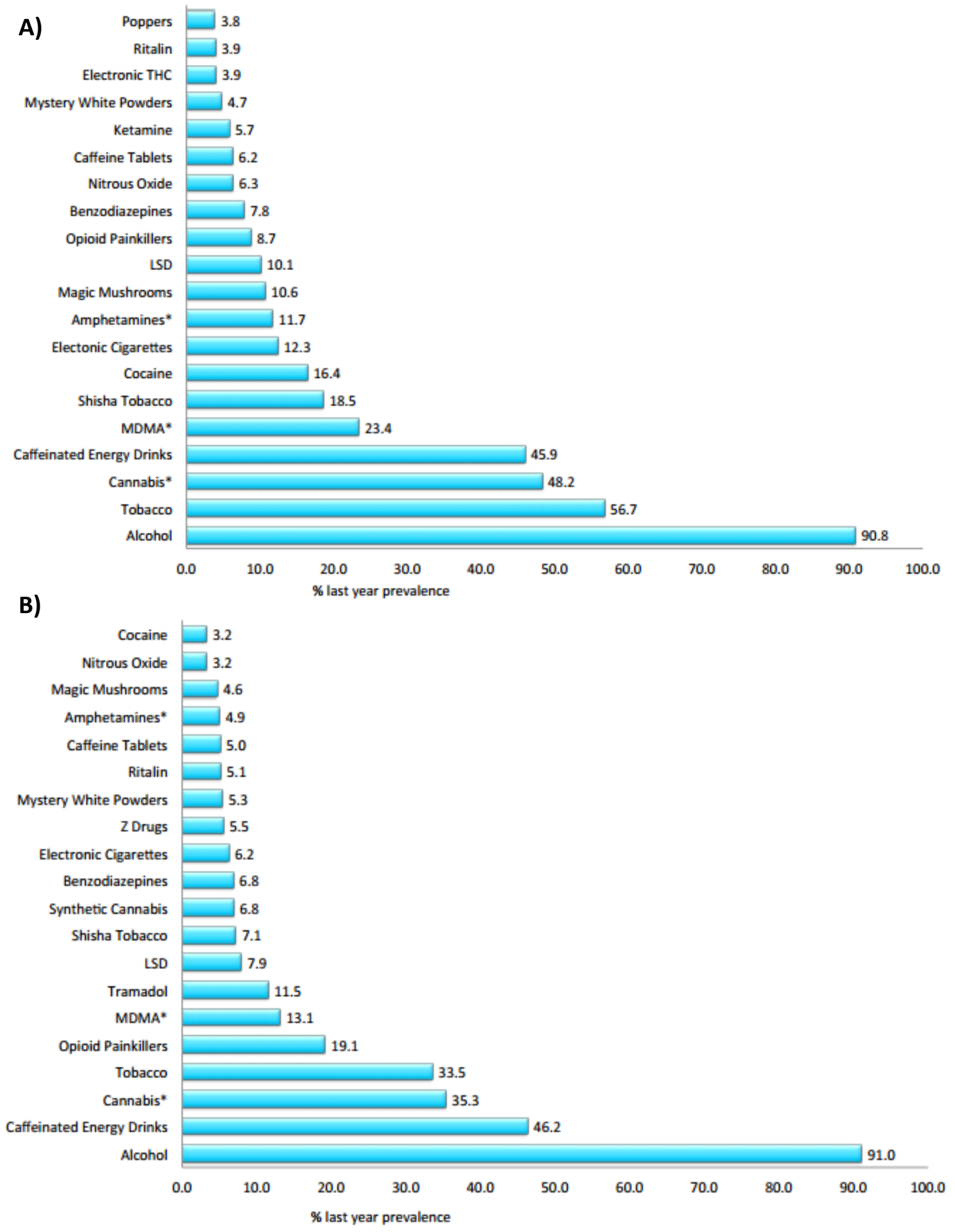

\section{Figure 1.1: Usage prevalence of commonly abused drugs}

The top 20 most commonly abused drugs in A) Europe, America, and the Oceania combined ( $\mathrm{n}=$ 78,819 people) and $B$ ) New Zealand ( $n=5,731$ people); graphed by \% prevalence of drug use in 2014. Subjects were self-nominated and between $20-40$ years of age. Poppers: alkyl nitrates inhaled for recreation; Shisha tobacco: tobacco concoction smoked using a water pipe; $Z$ drugs: non-benzodiazepines with similar effects to benzodiazepines. ${ }^{*} D e n o t e s$ all types and preparations. Figures reproduced with permission of Dr Adam R Winstock, Global Drug Survey GDS2014 ${ }^{\circ}$. 


\subsubsection{Theories of drug addiction}

Due to the complex nature of addiction, there is currently no universal theory that completely explains its development and maintenance. The susceptibility of an individual to develop drug addiction is dependent on his/her life choices as well as genetic and environmental factors. Although many theories have been suggested (refer to West (2001) for a full review), two predominant theories of addiction form the basis of most studies, and only these two will be briefly discussed here: 1) the opponent process theory (Koob and Le Moal, 2008) and 2) the incentive sensitisation theory (Robinson and Berridge, 1993).

\subsubsection{Opponent process theory}

The opponent process theory states that alterations in the brain have opposing systems that act to restore homeostasis. This opposing process was first described by Himmelsbach (1941), who suggested that acute effects produced by morphine are naturally antagonised by the brain in order to maintain neurochemical equilibrium (Himmelsbach, 1941). This long-lasting antagonism is strengthened with repeated use, whereby initial drug taking causes intense rewarding effects and have a minimal opposing impact (Solomon and Corbit, 1974). However, repeated drug use causes the opposing effect to intensify, whereby each subsequent event increases the magnitude of the opposing effect and decreases the hedonic effects. This vicious cycle of events continues until a point where drug intake is required solely to prevent the resultant negative effects, producing craving for a drug from which users derive no pleasure (Solomon and Corbit, 1974).

Over the years, this model has been adapted to our modern understanding of physiological systems. Chronic drug use creates repeated deviations from homeostasis, causing alterations in homeostatic set points (termed 'allostasis') (Koob and Le Moal, 1997). This leads to a negative affective state that is critical to the development of the addiction. The addiction cycle is made up of three stages: preoccupation/anticipation, binge/intoxication, and withdrawal/negative effect (Koob and Le Moal, 1997; Koob and Le Moal, 2001). The preoccupation/anticipation stage is characterised by a persistent desire for the drug, whereas drugs are taken in higher doses in the binge/intoxication 
stage to satisfy physical desires. These two stages are driven by positive reinforcement and mediated by dopaminergic systems in the brain (refer to section 1.2.1). However, as drug intake increases, drug users progress to the withdrawal/negative effect phase, in which drugs are used to alleviate aversive symptoms (Koob and Le Moal, 2001).

This opponent process theory described above is supported by research that shows longterm alterations in the brain of chronic drug users. Cocaine abusers, for example, reported lower levels of DA D2 receptors (Volkow et al., 1993; Volkow et al., 1999; Martinez et al., 2004) and lower glucose metabolism in the frontal regions of the brain that persist for up to four months after abstinence (Volkow et al., 1992). Chronic administration of cocaine also produced a long-term depression of D2 receptors in monkeys (Nader et al., 2006), reduction of excitatory transmission in the nucleus accumbens (NAC) of mice (Thomas et al., 2001), and alterations in the regulation of opioid receptors in rats (section 1.3.2). These long-lasting changes in the brain can be explained by processes that contribute to neural plasticity such as changes in gene transcription (reviewed in Nestler (2001)). Although the opponent process theory does not fully explain the phenomena of relapse in abstinent users (refer to section 1.1.2.2), studies that support this theory highlight the importance of identifying brain circuits affected by drug use in order to better understand the disorder.

\subsubsection{Incentive sensitisation process}

The other theory of addiction, the incentive sensitisation theory, states that drug addiction develops due to gradual and persistent neuroadaptations caused by repeated drug use. The dopaminergic system is said to attribute 'incentive salience' - a motivational attribute developed by association between the stimuli and hedonic effects - to natural rewards such as food and sex in a bid to encourage behaviours that are necessary for survival. In normal circumstances, pleasure accompanies and is required to assign incentive salience (Berridge and Robinson, 1998). However, drugs of abuse hijack this system and directly trigger incentive salience. Over a period of repeated drug use, the system becomes hypersensitive (sensitised) to drugs and drug-associated stimuli, transforming drug wanting into craving (Robinson and Berridge, 1993; Berridge and Robinson, 1995; Berridge and Robinson, 1998; Robinson and Berridge, 2001; Robinson et 
al., 2013). To this effect, the incentive sensitisation theory dissociates the act of drug 'wanting' (motivation to seek out drug) from 'liking' (hedonic impact of drug). Although these two processes often occur simultaneously, Robinson and Berridge postulate that drug wanting can occur independently of neural systems that mediate drug liking, but that only drug wanting is affected by sensitisation (Berridge and Robinson, 1995; Berridge, 1996). Upon sensitisation of neural systems associated with incentive salience, drug users may increasingly want the drug while liking it less and less; explaining the intensity at which addicts pursue drugs in the face of adverse consequences despite diminished hedonic effects. Environmental cues such as odour and drug-related stimuli that act as sensory reinforcers also influence sensitisation and can be powerful triggers to sensitised behaviour, resulting in high relapse rates in abstinent users (Robinson and Berridge, 2001).

Previous reports have provided evidence in support of this theory. Hobbs and colleagues discovered that 'wanting' and 'liking' could be dissociated in human participants using alcohol (Hobbs et al., 2005), whereas methamphetamine users displayed high rates of relapse up to five years post-abstinence/treatment (Brecht and Herbeck, 2014). In further support of this theory, preclinical studies have also shown that cocaine-dependent rats have high relapse rates up to six months after withdrawal (Lu et al., 2004). As these studies show that sensitisation of neural circuits persist long after withdrawal effects subside, the incentive sensitisation theory explains relapse more effectively than the withdrawal/negative effect phase of the opponent process theory.

\subsection{Kappa opioid receptors and their role in reward}

\subsubsection{The brain natural reward pathway}

Reward is intrinsically associated with the mesolimbic, mesocortical, and nigrostriatal dopaminergic pathways, collectively referred to as the natural reward pathway (Figure 1.2A). Due to neuronal overlap of the mesolimbic and mesocortical pathways, the whole system is generally regarded as one and referred to as the mesocorticolimbic system (Wise, 2009). The mesocorticolimbic system is responsible for hedonic effects (mesolimbic) and motivation (mesocortical) (Wise, 2009), whereas the nigrostriatal pathway is considered part of the basal ganglia motor loop and is functionally involved in 
motor control (Wise, 2009). The mesocorticolimbic reward pathway is implicated as being an important link in the neurocircuitry of reward (Fibiger and Phillips, 1988; Wise, 2009), regulating reinforcement of behaviours that are advantageous to the survival of a species such as eating, drinking, and sex (Day and Carelli, 2007).

DA is the major neurotransmitter underlying hedonic sensations in the reward pathway. It is synthesised by tyrosine hydroxylase from tyrosine to form L-3,4dihydroxyphenaylalanine (L-DOPA), which is then converted by DOPA decarboxylase to DA (Figure 1.3A) (Elsworth and Roth, 1997). DA is translocated into synaptic vesicles via vesicular monoamine transporter 2 (VMAT2), an integral membrane protein that transports monoamines from the cytosol into vesicles where DA storage occurs (Chen et al., 2010). These storage vesicles are primed for DA release by exocytosis upon arrival of an action potential to the neuronal synapse. Released DA binds to either postsynaptic receptors to propagate the signal or presynaptic autoreceptors to regulate its release (Figure 1.4).

Physiological actions of DA are mediated by five types of G-protein coupled receptors (GPCR) for DA, D1 to D5 (Missale et al., 1998). Classified as either D1-like (D1 and D5) or D2-like (D2, D3, and D4), D1-like receptors are linked to $\mathrm{G}_{\mathrm{s}}$, activate adenylyl cyclase, and increase cyclic adenosine monophosphate (CAMP) levels; whereas, D2-like receptors are linked to $\mathrm{G}_{\mathrm{i}} / \mathrm{G}_{\mathrm{o}}$, inhibit adenylyl cyclase, and activate $\mathrm{K}^{+}$channels (reviewed in Missale et al. (1998)). Unlike D1-like receptors which are primarily found on postsynaptic terminals, D2 and D3 receptors are expressed both pre- and postsynaptically and can act as DA autoreceptors to regulate DA neurotransmission (refer to Beaulieu and Gainetdinov (2011) for a review). Action of DA is terminated by the dopamine transporter (DAT) which transports DA back into presynaptic neurons (Figure 1.4) where DA is either recycled or degraded by monoamine oxidase (MAO) or catechol-O-methyltransferase (COMT) (Elsworth and Roth, 1997). DA levels in the synapse are regulated by trafficking and activation of DAT on the presynaptic neuron. DAT is regulated through phosphorylation, ubiquitination (Chen et al., 2010), glycosylation (Li et al., 2004), membrane insertion or internalisation (Eriksen et al., 2010a), and protein-protein interactions (Torres, 2006; Eriksen et al., 2010b). 
DAT is expressed on axons, dendrites, and cell soma on the extrasynaptic plasma membrane, but not in the synapse; indicating that DA released at the synapse diffuses out of the cleft to bind to DAT (Nirenberg et al., 1996; Nirenberg et al., 1997a; Nirenberg et al., 1997b). DAT is found on dopaminergic neurons in the ventral tegmental area (VTA), substantia nigra (SN) (Ciliax et al., 1995), dorsal striatum (dStr), and nucleus accumbens (NAc; Figure 1.2B) (Boja and Kuhar, 1989). Its peri-synaptic location is ideal since DAT functions as the primary mechanism by which DA is cleared from the synapse (Torres, 2006), although studies have shown that DA uptake can also take place via the norepinephrine transporter (NET) (Carboni et al., 1990) and serotonin transporter (SERT) (Larsen et al., 2011). DAT also co-localises with KOPr in the VTA, prefrontal cortex (PFC), dStr, amygdala, and NAc (Ciliax et al., 1995; Simonin et al., 1995; Ciliax et al., 1999) at the terminals, varicosities and axons (Figure 1.2B) (Nirenberg et al., 1997a; Svingos et al., 2001). A study conducted by Svingos et al. (2001) showed that KOPr and DAT appose each other at DA nerve terminals, suggesting a role for $\mathrm{KOPr}$ in regulating DAT and subsequently, DA levels (Svingos et al., 2001).

\section{Figure 1.2: Co-localisation of DAT and KOPr in the natural reward pathway}

A) The three dopaminergic pathways involved in natural reward and addiction: the mesolimbic, mesocortical, and nigrostriatal pathways. Dopaminergic neurons projecting from the VTA to the NAc make up the mesolimbic pathway, whereas neurons projecting from the VTA to the PFC make up the mesocortical pathway. The mesolimbic and mesocortical pathways are usually referred to as the mesocorticolimbic pathway. The nigrostriatal pathway has dopaminergic neurons with cell bodies in the SN which project to the dStr. B) KOPr is enriched in the PFC, Olf, dStr, Amg, NAc, Hyp, Thal, and VTA, whereas DAT is found in the PFC, dStr, NAc, Amg, VTA, and SN (Ciliax et al., 1995; Simonin et al., 1995; Ciliax et al., 1999). 
A)

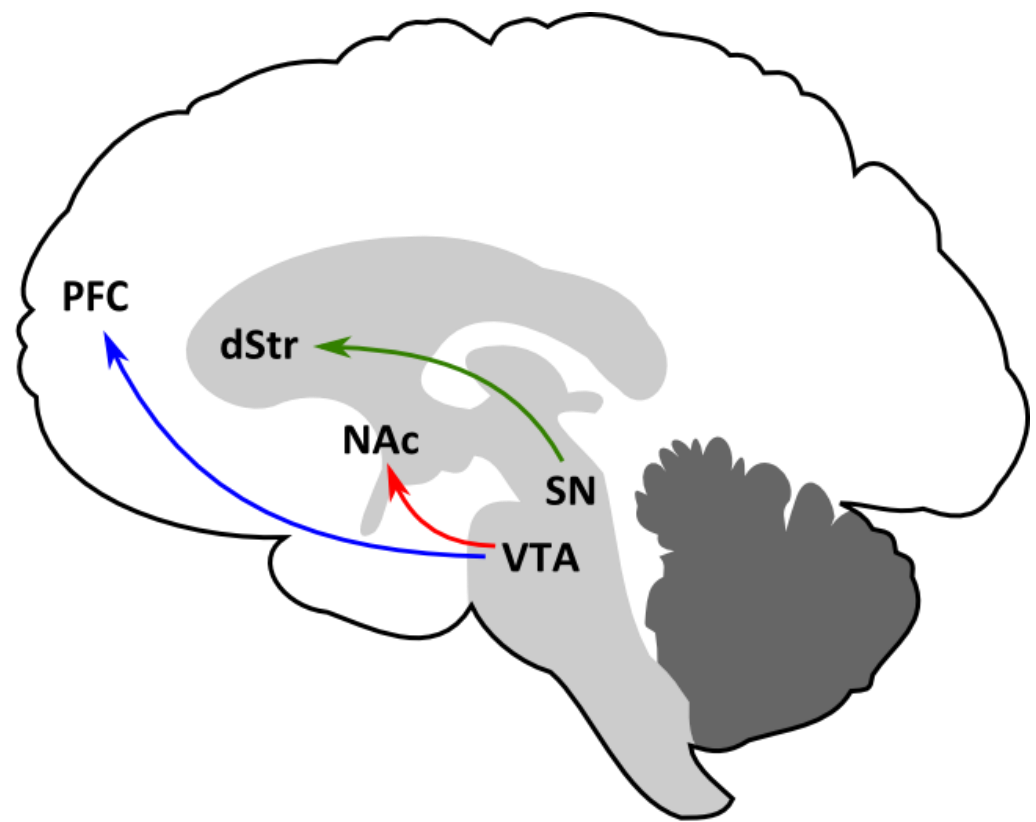

VTA $=$ Ventral tegmental area

_ Mesolimbic pathway

- Mesocortical pathway Nigrostriatal pathway
PFC = Prefrontal cortex

SN = Substantia nigra

$\mathbf{d S t r}=$ Dorsal striatum

NAc $=$ Nucleus accumbens

B)

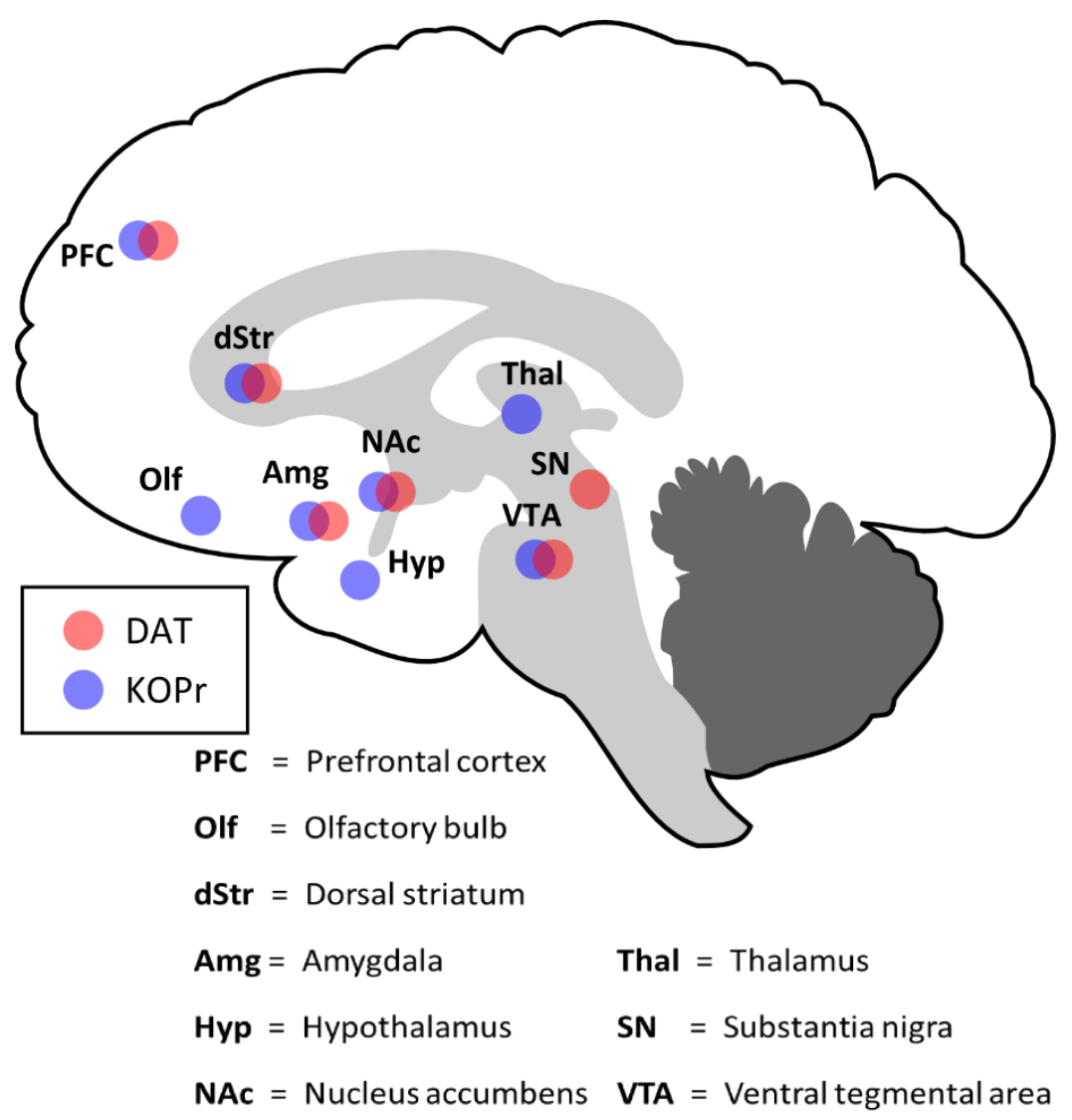


A)<smiles>CN(C)OCCN</smiles><smiles>CC(C)(C)O[Na]</smiles>

DA<smiles>NCCc1ccc(O)c(O)c1</smiles>

B) Cocaine

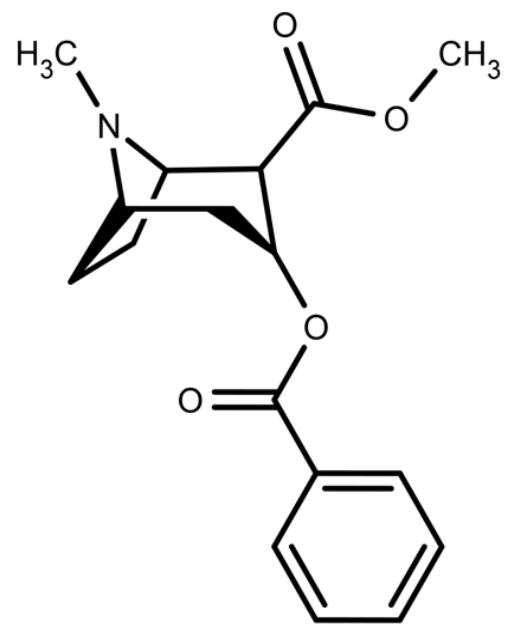

Figure 1.3: Synthesis of DA and chemical structures of DA and cocaine

A) Synthesis of DA from tyrosine. Tyrosine is first converted to L-DOPA by the rate limiting enzyme tyrosine hydroxylase, and L-DOPA is then converted by DOPA-decarboxylase to DA. B) Cocaine is a psychostimulant that exerts its effects via DAT. L-DOPA, L-3,4-dihydroxyphenaylalanine; DA, dopamine. 


\subsubsection{The dynorphin/kappa opioid receptor system}

The KOPr is a 7 transmembrane, pertussis toxin sensitive GPCR located throughout the nervous system (Avidorreiss et al., 1995; Butelman et al., 2012). The KOPr is a 380 amino acid protein (Zhu et al., 1995) encoded by the OPRK1 gene (Butelman et al., 2012) located at position q11-12 on chromosome 8 (Simonin et al., 1995). There are 3 known subtypes of $\mathrm{KOPr}(\mathrm{KOPr} 1, \mathrm{KOPr}$, and KOPr3) of which only one variant, KOPr1, has been cloned to date (Simonin et al., 1995). The KOPr is part of the opioid receptor family and is closely related to the mu opioid receptor (MOPr) and delta opioid receptor (DOPr).

KOPr is widely distributed throughout the brain, with the exception of the internal globus pallidus, where no KOPr mRNA has been detected (Figure 1.2B) (Simonin et al., 1995). $\mathrm{KOPr}$ is found in the telencephalon, particularly in the various neocortical areas: amygdala, olfactory bulb, basal ganglia, external globus pallidus, and the hippocampus. KOPr mRNA is also observed in the thalamus, hypothalamus, mammillary bodies, VTA, locus coeruleus, and cerebellar cortex. (Simonin et al., 1995). KOPr is found both pre- and postsynaptically (Svingos et al., 2001) on dopaminergic, glutamatergic, and $\gamma$ aminobutyric acid (GABAergic) neurons (Shippenberg et al., 2001; Shippenberg et al., 2007; Chartoff et al., 2008).

The endogenous ligand for KOPr, dynorphin, is a class of opioid peptide that is a posttranslational product of the PDYN gene. There are several types of dynorphin: dynorphin A, dynorphin B, big dynorphin (a 32 amino acid protein consisting of both dynorphin $A$ and $B$ that is generated due to incomplete cleaving), and $\alpha / \beta$-neo-endorphin, all of which are generated from cleavage of prodynorphin by proprotein convertase 2 (Marinova et al., 2005; Nyberg and Hallberg, 2007). Dynorphins can be found throughout the central nervous system (Watson et al., 1982) with high levels in the hypothalamus, dStr, pallidus, and SN (Gramsch et al., 1982). Moderate levels of dynorphin are found in the hippocampus, pons, medulla, amygdala, periaqueductal gray matter, and the area postrema (Gramsch et al., 1982). The most active form of dynorphin, dynorphin A (1-17), preferentially activates KOPr, although it has some affinity for MOPr and DOPr (Chavkin et al., 1982; Merg et al., 2006). 


\subsubsection{Regulation of the reward pathway by the dynorphin/KOPr system}

Activation of the dynorphin/KOPr system is intrinsically associated with the dopaminergic pathway due to its opposing actions on reward. KOPr activation produces negative behavioural effects such as stress, aversion, anxiety, hypothermia, increased submissive behaviour, sedation, and depression (Shippenberg et al., 2007; Knoll and Carlezon Jr, 2010; Wee and Koob, 2010). This 'anti-reward' role of the KOPr system is supported by the fact that stressful, anxiogenic, and depressive effects are negated by administration of KOPr antagonists (Mague et al., 2003; McLaughlin et al., 2003; Shirayama et al., 2004; Carr et al., 2009; Carr and Lucki, 2010; Chartoff et al., 2012). The negative effects of KOPr activation are attributed to decreased DA neurotransmission (Di Chiara and Imperato, 1988b; Wee and Koob, 2010). In fact, KOPr activation reduces both basal DA neurotransmission (Di Chiara and Imperato, 1988b; Spanagel et al., 1992; Margolis et al., 2003; Carlezon et al., 2006; Ebner et al., 2010) as well as drug-evoked increases of DA release in the reward pathway (Maisonneuve et al., 1994; Xi et al., 1998; Zhang et al., 2004b; Zhang et al., 2004a), suggesting that KOPr-associated therapies can prevent abnormalities produced by drug-induced alterations in DA signalling. Further evidence of the importance of the KOPr system in regulating DA neurotransmission is its contribution to the hypodopaminergic state reported in cocaine addiction (for a recent review, see Trifilieff and Martinez (2013)). Taken together, these studies suggest that the KOPr system could be a relevant target for anti-addiction pharmacotherapy development.

\subsection{Role of the dynorphin/kappa opioid system in cocaine abuse}

\subsubsection{Effects of cocaine on the dopaminergic reward system}

Cocaine is an illegal yet popular recreational drug used to elicit euphoria, mood elevation, as well as increased alertness and energy. Cocaine presents negative effects such as tachycardia, hypertension, nausea, anxiety, irritability, and confusion (Kloner et al., 1992; Dackis et al., 2003). Although moderate doses of cocaine improve memory consolidation and enhance learning (Rkieh et al., 2014), chronic cocaine intake causes cognitive decline, deficits in executive function, and memory impairment (Ardila et al., 1991; Mittenberg and Motta, 1993). 
Actions of cocaine have been associated with the dopaminergic system since the late 1970s when Roberts et al. (1977) found that dopaminergic lesions in the NAc disrupt rewarding effects of cocaine (Roberts et al., 1977). The discovery that SCH 23390, a DA receptor antagonist, increased cocaine self-administration only served to reinforce the connection between the dopaminergic system and cocaine (Koob et al., 1987). Cocaine exerts its effects by inhibiting DAT and preventing reuptake of DA into the presynaptic neuron, resulting in elevated DA levels in the synapse (Figure 1.4). Cocaine interacts with similar amino acids on DAT as DA, but does not bind in the exact same place (Huang et al., 2009). There have been conflicting reports as to whether cocaine binds to DAT via a competitive (Wu et al., 2001) or non-competitive mechanism (Povlock and Schenk, 1997), differences which are proposed to be due to different experimental techniques and design. Cellular studies have shown that cocaine mobilises DAT to the cell surface (Daws et al., 2002), an effect observed in human embryonic kidney 293 (HEK-293) and neuro-2A (N2A) mouse neuroblastoma cells but not African green monkey fibroblast (COS7) cells (Zhang et al., 1998; Daws et al., 2002). Recently however, a new 'inverse agonist' mechanism proposed that cocaine possesses dual actions, whereby it not only inhibits DA uptake but also reverses the direction of DAT transport, explaining the reason DA reuptake inhibitors with similar potency to cocaine, i.e. bupropion, do not produce the same euphoric effects (refer to Heal et al. (2014) for a recent review).

Initial work conducted by Rocha et al. (1998) in DAT knock-out mice showed that these mice were still able to self-administer cocaine, but a later study showed that cocaine is only self-administrated reliably in the presence of fully functional DAT (Thomsen et al., 2009). However, although DAT and dopaminergic systems are heavily implicated in the hedonic effects of cocaine, it is imprudent to assign sole responsibility to the DAT system. Cocaine is also effective at SERT and NET (Hall et al., 2004), with NET knock-out mice showing a higher rate of cocaine self-administration, indicating a decrease in reinforcing potency of cocaine (Rocha, 2003). In contrast, other studies showed that SERT (Sora et al., 1998) and NET (Xu et al., 2000) knock-out mice display enhanced cocaine reward, suggesting that the blockade of SERT and NET by cocaine usually causes aversive or anhedonic effects (Hall et al., 2002). In addition, DAT/SERT double knock-out but not DAT knock-out mice failed to produce cocaine place preference (Sora et al., 2001). Taken 
together, these studies are evidence that the effects of cocaine are not solely due to its actions on DAT, but rather a complex interaction between several systems.

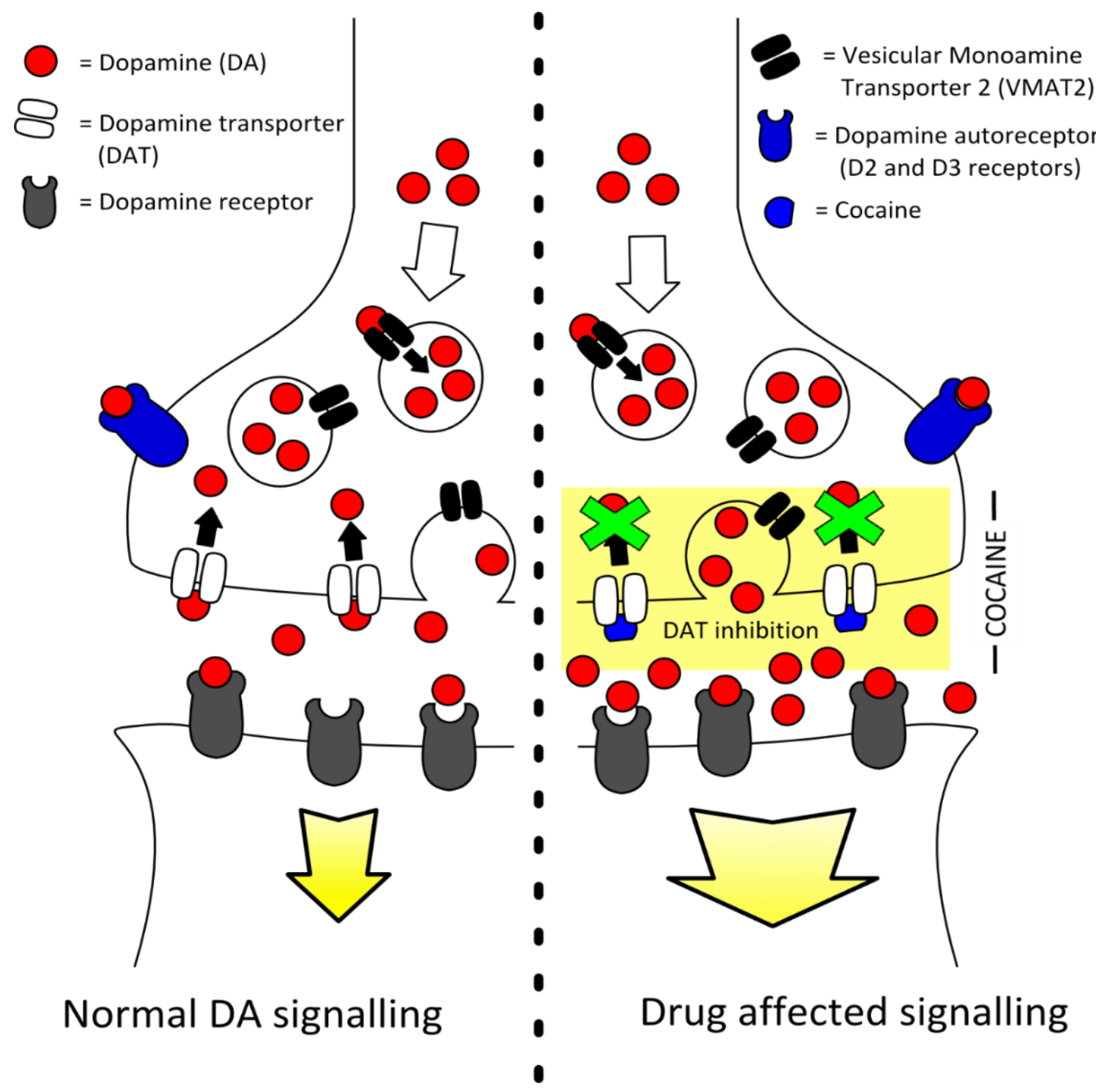

Figure 1.4: Physiological and cocaine-affected dopamine signalling

Arrival of an action potential causes influx of $\mathrm{Ca}^{2+}$, resulting in migration and fusion of synaptic vesicles to the plasma membrane. The synaptic vesicles release DA into the synaptic cleft, whereby DA binds to postsynaptic DA receptors to propagate the signal. DA also binds to presynaptic DA autoreceptors (D2 and D3 receptors) to regulate its release. DAT functions as the main mechanism by which DA is cleared from the synapse. DA diffuses out of the synaptic cleft and is transported back into the presynaptic neuron by DAT for degradation or repackaging into synaptic vesicles for reuse. The psychostimulant, cocaine, acts as a direct DAT blocker and prevents reuptake of DA into the presynaptic neuron, causing an increase of DA in the synapse. 


\subsubsection{Changes to DAT and KOPr systems in response to cocaine}

Chronic cocaine use is associated with neurophysiological dopaminergic changes such as reduced DA D2 receptor availability (Volkow et al., 1993; Volkow et al., 1999; Martinez et al., 2004), decreased glucose metabolism (Volkow et al., 1993), decreased cerebral blood flow in the PFC (Volkow et al., 1988), and enlargement of the dStr (Jacobsen et al., 2001). Chronic administration of cocaine also caused a long-term depression of DA D2 receptors in monkeys (Nader et al., 2006) and reduction of excitatory transmission in the NAc of mice (Thomas et al., 2001). Studies carried out in primates demonstrated a decrease in striatal DAT during short-term (5 days) cocaine administration, but an increase in striatal DAT with long-term (3.3 months and 1.5 years) administration (Letchworth et al., 2001). This increase in DAT level and function, also seen in humans, is proposed to be a compensatory effect of long-term cocaine abuse (Mash et al., 2002).

Continued and repeated drug use also upregulates the KOPr system (Wee and Koob, 2010), a change that causes adverse effects during withdrawal (Hurd and Herkenham, 1993). Cocaine overdose victims display elevated KOPr levels in the NAc, amygdala, and other corticolimbic areas (Mash and Staley, 1999). In the laboratory, repeated cocaine injections (45 mg/kg/day for 14 days) increased the KOPr density in the rostral cingulate cortex, caudal olfactory tubercle, dStr, and VTA of male rats (Unterwald et al., 1994). A later study with $50 \mathrm{mg} / \mathrm{kg} /$ day for 14 days also produced an increase in KOPr density in the dStr, NAc shell, claustrum, and endopiriform nucleus of rats (Collins et al., 2002). In contrast, a study which administered $60 \mathrm{mg} / \mathrm{kg} /$ day of cocaine for 5 days showed a downregulation of KOPr in the dStr and NAc, accompanied by an elevation in prodynorphin mRNA levels (Turchan et al., 1998). These contrasting effects are likely due to differences in duration of continuous cocaine exposure, whereby the elevation in KOPr density that occurs at a later time point is believed to compensate for the earlier decrease. Frequency of cocaine doses also play a major role in cocaine-induced alterations, as rats administered three separate cocaine injections daily showed significant increases in KOPr density in the cingulate cortex, NAc, and dStr after 14 days, whereas rats given only one or two injections daily showed small, insignificant changes despite the total daily dose (30 $\mathrm{mg} / \mathrm{kg}$ ) being identical (Unterwald et al., 2001). 
Upregulation of both KOPr and DAT in chronic cocaine users suggests that both systems act synergistically to regulate DA levels, with KOPr influencing DAT levels and function (Thompson et al., 2000; Kivell et al., 2004). In support of this, in vivo studies showed that subcutaneous (s.c.) administration of the traditional KOPr agonist, U69,593 (0.32 mg/kg), over 5 days decreased striatal DAT density in rats (Collins et al., 2001b). The regulation of DAT by KOPr agonists is opposite to that of cocaine. Ex vivo studies showed that repeated U69,593 treatment $(0-0.32 \mathrm{mg} / \mathrm{kg}$, s.c.) produced a dose-dependent decrease in DA uptake via DAT in the NAc of rats (Thompson et al., 2000), whereas chronic cocaine administration produced the opposite effect on DAT level and function (Letchworth et al., 2001; Mash et al., 2002). On the other hand, acute administration of cocaine prevented reuptake of DA by binding to DAT (Kuhar et al., 1991), whereas acute administration of KOPr agonists increased DA uptake by increasing DAT function (Kivell et al., 2014). In further support of the close association between KOPr and DAT, studies investigating coexpression of KOPr and DAT found that KOPr and DAT form interacting complexes in striatal synaptosomes, and this interaction was enhanced upon KOPr agonist administration (Kivell et al., 2014).

The cocaine opposing actions of $\mathrm{KOPr}$ make $\mathrm{KOPr}$ agonists attractive anti-cocaine compounds that may prevent cocaine-induced alterations in the dopaminergic system by means of DAT regulation (Chefer et al., 2000; Chefer et al., 2005). Administration of U69,593 (0.32 mg/ $\mathrm{kg}$, s.c.) in combination with cocaine for 5 days had no significant effect on DAT density in the dStr of male Sprague-Dawley rats, suggesting that alterations in DAT levels following U69,593 treatment directly counteracts those of cocaine (Collins et al., 2001a). Intracranial perfusion of U69,593 (1 $\mathrm{\mu g} / \mathrm{side})$ into both sides of the NAc prior to cocaine injections (20 mg/kg, intraperitoneal, i.p.) for 3 days also prevented alterations in DA neurotransmission that occur during abstinence in rats (Chefer et al., 1999). Further support for the role of KOPr in preventing cocaine-stimulated modifications are studies that show inhibition of cocaine-induced behavioural effects such as hyperactivity (Chefer et al., 2000; Collins et al., 2001b; Chartoff et al., 2008) and sensitisation (Heidbreder et al., 1993; Shippenberg et al., 1996; Morani et al., 2012) when KOPr agonists were administered prior to cocaine. 


\subsubsection{Current pharmacotherapies for cocaine}

The main focus in addiction studies have been the development of cocaine replacement therapies to reduce drug abuse and alleviate severe withdrawal symptoms. DA reuptake inhibitors such as cocaethylene (Baker et al., 2007) and methylphenidate (Ritalin) (Grabowski et al., 1997; Somoza et al., 2004; Levin et al., 2007) were explored as potential cocaine substitutes. However, cocaethylene was found to be associated with neurological and cardiac toxicity (Farooq et al., 2009), whereas methylphenidate only produced a decline in cocaine use in adult abusers with attention deficit hyperactivity disorder (Levin et al., 2007). The adverse physiological and psychological side effects that accompany direct actions on DAT have also prevented development of DA reuptake inhibitors as therapies (Ferragud et al., 2009). Therefore, therapies targeting other systems such as GABA pathways (i.e. using topiramate and modafinil) and glial modulators (i.e. ibudilast) have also been investigated. However, studies for ibudilast are still undergoing clinical trials (Johnson et al., 2014), whereas modafinil has shown mixed results with concurrent drug abuse (Anderson et al., 2009) and success only when low subject numbers were used (Dackis et al., 2003). Work exploring potential psychostimulant pharmacotherapies are too numerous to discuss in detail but have been reviewed extensively over the years (for reviews, refer to Shearer et al. (2002); Xi and Gardner (2008); Graves et al. (2012); Mariani and Levin (2012); Pierce et al. (2012); Rush and Stoops (2012); Brensilver et al. (2013); Kivell et al. (2013); Bailey and Husbands (2014); Beardsley and Hauser (2014); Noble et al. (2015); Shorter et al. (2015)).

One particular drug candidate that is closely related to the present study is ibogaine, an alkaloid extracted from the plant Tabemanthe iboga which grows in West Central Africa. Ibogaine has an interesting mechanism of action due to its interaction with multiple neurotransmitter systems. Ibogaine exerts its actions via the $\mathrm{N}$-methyl-D-aspartate (NMDA) receptor (Glick et al., 1997; Leal et al., 2003), KOPr (Glick et al., 1997), MOPr (Codd, 1995), sigma opioid receptor (SOPr) (Mach et al., 1995), nicotinic acetylcholine (nAch) receptor (Mah et al., 1998), SERT, and DAT (Bulling et al., 2012) (refer to Sershen et al. (1997); Alper (2001) for a full review). Perfusion of low doses $\left(10^{-6} \mathrm{M}\right.$ to $\left.10^{-4} \mathrm{M}\right)$ of ibogaine into the Str of rats attenuated synaptic DA levels, an effect attributed to KOPr activation, whereas higher doses of ibogaine $\left(5 \times 10^{-4} \mathrm{M}\right.$ to $\left.10^{-3} \mathrm{M}\right)$ increased extracellular 
DA levels by interacting with DAT (Reid et al., 1996). A study conducted on 75 subjects with various drug problems, including alcohol, cannabis, crack cocaine, and cocaine, found that doses of ibogaine ranging from $7.5-20 \mathrm{mg} / \mathrm{kg}$ increased drug abstinence in users (Schenberg et al., 2014). The opioid and stimulant anti-addiction effects of ibogaine were attributed to its actions on the KOPr and NMDA receptors, the reduction of nicotine preference to its actions on the nAch receptor, and the adverse effects of ibogaine on the SOPr (Glick et al., 1997). The efficacy of ibogaine may be due to its metabolism to the active metabolite noribogaine, which has a long half-life (28 to 49 hours) in humans (Glue et al., 2015). In fact, a study recently identified noribogaine (the long-acting metabolite of ibogaine) as a G-protein biased KOPr agonist (Maillet et al., 2015) (refer to section 1.6 for details on G-protein biased agonists), suggesting that the anti-addiction effects of ibogaine and subsequently noribogaine, may be in part due to their actions at the KOPr. However, ibogaine is classed as a Schedule I drug in the USA due to its hallucinogenic effects (US Department of Justice, 2011). In addition, ibogaine use has been associated with fatalities related to cardiotoxicity (reviewed in Koenig and Hilber (2015)), necessitating the need for development of safer structural analogues (Mash et al., 1998; Glick and Maisonneuve, 2000; Mash et al., 2000; Passarella et al., 2003; Passarella et al., 2006).

The use of ibogaine, which is metabolised to the KOPr agonist noribogaine, in antiaddiction studies suggests the possibility of a link between the KOPr and the anti-addiction effects of ibogaine. Like ibogaine, acute administration of KOPr agonists have inhibitory effects on cocaine by decreasing DA release and increasing DAT-mediated DA uptake (Thompson et al., 2000; Chefer et al., 2005). As the opposing action of the KOPr system on the behavioural and cellular effects of cocaine is paramount to its anti-cocaine properties, research into therapeutic approaches targeting the KOPr system holds great promise in field of cocaine abuse and addiction. 


\subsection{KOPr agonists as potential anti-cocaine pharmacotherapies}

\subsubsection{Anti-cocaine and adverse effects of traditional KOPr agonists}

Unlike MOPr agonists, KOPr agonists have good potential as analgesic agents (Kivell and Prisinzano, 2010) as well as anti-addiction pharmacotherapies (Kivell et al., 2013) due to their low abuse potential. Acute administration of KOPr agonists reduce cocaine selfadministration in rats (Glick et al., 1995; Glick et al., 1998; Schenk et al., 1999; Schenk et al., 2001) and rhesus monkeys (Negus et al., 1997; Mello and Negus, 1998; Mello and Negus, 2000), morphine self-administration in rats (Glick et al., 1995) and mice (Kuzmin et al., 1997), ethanol self-administration in rats (Lindholm et al., 2001; HendersonRedmond and Czachowski, 2014), and nicotine self-administration in rats (Ismayilova and Shoaib, 2010). Administration of KOPr agonists also decrease the rewarding properties of ethanol in rats (Sandi et al., 1988; Logrip et al., 2009), block morphine CPP in mice (Masahiko et al., 1993), and prevent sensitisation to cocaine (Shippenberg et al., 1996), effects that are likely explained by the modulation of dopaminergic neurons (Margolis et al., 2003; Margolis et al., 2006).

There are a few classes of KOPr agonists that have been studied extensively for their therapeutic effects: 1) arylacetamides such as trans-3,4-dichloro- $N$-methyl- $N$-(2-1pyrrolidinyl)-cyclohexyl-benzeacetamide $\quad(U 50,488), \quad(+)-(5 \alpha, 7 \alpha, 8 \beta)-N-m e t h y l-N-\{7-(1-$ pyrrolidinyl)-1-oxaspiro(4.5)dec-8-yl)-benzeneacetamide (U69,593), enadoline, spiradoline, and a cyclohexyl benzeneacetamide derivative, PD117302; 2) benzomorphans such as ethylketocyclazocine, bremazocine, (+/-)-(1-R/S,5-R/S,2 = R/S)5,9-dimethyl-2'-hydroxy-2-tetrahydrofurfuryl-6,7-benzomorphan (Mr2033), and cyclazocine; and 3) neoclerodane diterpenes (Sal A and its derivatives, Table 1.2 and 1.3). The arylacetamides have greater selectivity for KOPr than MOPr and DOPr, whereas the benzomorphans have a much lower selectivity for KOPr than MOPr and DOPr. This makes the benzomorphans more likely to produce agonist or antagonist effects at other opioid receptors as well as the KOPr (Mello and Negus, 2000). For this reason, the two main selective and potent synthetic KOPr agonists extensively reported on in the literature are the arylacetamides $U 50,488$ and $U 69,593$ (Table 1.1). In one study, a single injection of $\mathrm{U} 50,488(2.5-10 \mathrm{mg} / \mathrm{kg}$, i.p.) led to the extinction of drug taking after one day in cocaine 
and morphine self-administering rats (Glick et al., 1995). The suppression seen with the higher dose of U50,488 (10 mg/kg, i.p.) lasted up to two days, with a third of the rats showing attenuated intake for up to six days following treatment. Effects of U50,488 were reversed by prior administration of the KOPr antagonist nor-binaltorphimine (nor-BNI, 10 $\mathrm{mg} / \mathrm{kg}$, s.c.), indicating that the decrease observed in cocaine and morphine selfadministration were KOPr-mediated (Glick et al., 1995). Nor-BNI is a highly selective antagonist of the KOPr and has long-lasting effects (up to 28 days) (Endoh et al., 1992; Horan et al., 1992). Similarly, a later study reported that U50,488 (30 mg/kg, i.p.) reduced cocaine-induced reinstatement of drug seeking in rats (Morani et al., 2009). The antireward behaviour of U50,488 corresponded with a microdialysis study by Maisonneuve et al. (1994), in which U50,488 (10 mg/kg, i.p.) produced a 50\% attenuation of cocaineevoked DA levels in the NAc. Administration of U50,488 on its own also caused a moderate decrease in DA levels (Maisonneuve et al., 1994).

Similar to its sister compound, U69,593 $(0.32 \mathrm{mg} / \mathrm{kg}$, s.c.) also decreased cocaine selfadministration (Schenk et al., 1999; Schenk et al., 2001) and attenuated cocaine-induced reinstatement of drug seeking in rats (Schenk et al., 2000; Morani et al., 2009). Acute treatment of rats with $U 69,593(0.32 \mathrm{mg} / \mathrm{kg}$, s.c.) showed that $U 69,593$ increased DATmediated DA uptake in the NAc, an effect that was also inhibited by prior administration of nor-BNI (10 mg/kg, s.c.) (Thompson et al., 2000). Interestingly, the authors noted that U69,593 produced biphasic effects in opposition to cocaine. An acute administration of U69,593 increased DA uptake, whereas chronic administration of U69,593 decreased DA uptake; observations that were in direct contrast to the effects of cocaine. This opposing effect of KOPr agonists on cocaine-elicited changes in DA neurotransmission (Thompson et al., 2000) and the anti-reward behavioural effects strongly indicate that KOPr agonists can act as potential cocaine antagonists.

However, the KOPr system is also a known mediator of stress (Land et al., 2008; Bruchas et al., 2010) and is linked to the negative emotional state that leads to compulsivity in the pursuit of drugs (Koob and Kreek, 2007; Koob, 2008; Koob, 2015). Although somewhat paradoxical, KOPr agonists can also potentiate the rewarding effects of cocaine (McLaughlin et al., 2006; Ehrich et al., 2014) and cause stress-induced relapse (McLaughlin 
et al., 2006). In that regard, acute administration of KOPr agonists mimicked application of stressors such as the forced swim stress to reinstate cocaine-induced CPP (McLaughlin et al., 2006). In fact, administration of U50,488 (5 mg/kg, i.p.) potentiated cocaine CPP when administered 60 min prior to cocaine, an effect the authors hypothesised to be a consequence of KOPr-mediated dysphoria (Ehrich et al., 2014). In contrast, KOPr antagonists have been successfully used to combat stress-related drug use. Antagonism of KOPr prevented stress-induced reinstatement of CPP (Land et al., 2009), stress-induced cocaine seeking (Beardsley et al., 2005), and stress-induced potentiation of cocaine CPP (McLaughlin et al., 2003). Nor-BNI did not alter cocaine self-administration behaviour in rats (Glick et al., 1995) or rhesus monkeys (Negus et al., 1997; Hutsell et al., 2015), but decreased the intake of a threshold dose of cocaine ( $30 \mu \mathrm{g} /$ infusion) in drug naïve rats (Kuzmin et al., 1998). Nor-BNI, however, had no effect on cocaine intake when cocaine was offered at higher doses (60 $\mu \mathrm{g}$ /infusion), indicating that although nor-BNI may decrease sensitivity to cocaine, possibly because of its 'rewarding' effects through KOPr antagonism, it does not completely prevent cocaine intake (Kuzmin et al., 1998). Nor-BNI (10 mg/kg, i.p.) also inhibited stress-induced immobility in mice (McLaughlin et al., 2003), whereas another selective KOPr antagonist, JDTic (10 - $30 \mathrm{mg} / \mathrm{kg}$, intragastric gavage), significantly attenuated stress-induced reinstatement to cocaine (Beardsley et al., 2005). KOPr knock-out mice displayed no aversion to U50,488 (Land et al., 2009) and were insensitive to stress-induced reinstatement of cocaine CPP (Redila and Chavkin, 2008), further confirming the role of KOPr in modulating stress and aversive effects. Taken together, these studies advocate the use of KOPr agonists at the binge/intoxication phase of the addiction cycle (reviewed in Shippenberg (2009); Wee and Koob (2010); Kivell et al. (2013); Picetti et al. (2013)) and KOPr antagonists in the later withdrawal stage of the addiction cycle (Chavkin, 2011; Picetti et al., 2013). However, as this study focuses on KOPr agonists and their role as potential pharmacotherapies for cocaine abuse, discussion of the therapeutic potential of KOPr antagonists is beyond the scope of this study (refer to Carroll and Carlezon (2013) for a review). Nonetheless, it is notable that work using nor$\mathrm{BNI}$ suggests that the interfering effects of KOPr agonists on drug self-administration are due to direct regulation of the reward pathway rather than an increase in aversive effects (Mitchell et al., 2005). The signalling pathways activated in drug- vs. stress-induced 
reinstatement also differ (Kreibich and Blendy, 2004), indicating that KOPr may modulate the anti-cocaine and stress pathways in a different manner.

In addition to their modulation of stress pathways, use of traditional KOPr agonists have been limited due to mixed reports of side effects, with some agonists presenting severe reactions and others indicating only moderate to mild effects. In clinical trials for opioid dependence, cyclazocine-induced dysphoria, insomnia, irritability, and hallucinations have halted the progress of cyclazocine (Glick et al., 1998). Another study found a dosedependent increase in dysphoria and psychotomimesis when 30 healthy male subjects were given several isomers of the $\mathrm{KOPr}$ agonist $\mathrm{Mr} 2033(1.9 \mu \mathrm{g} / \mathrm{kg}$ to $3.8 \mu \mathrm{g} / \mathrm{kg}$, intravenous, i.v.). Although the experience of each participant differed, most subjects reported various complaints such as weakness, vertigo, dizziness, anxiety, sweating, and with the higher doses, a distortion of space and time perception (Pfeiffer et al., 1986). Enadoline, another selective and specific KOPr agonist, produced adverse effects such as fatigue, sensory distortions, and dizziness at $25 \mu \mathrm{g} / \mathrm{kg}$ and above in healthy volunteers. Interestingly, enadoline produced slightly unpleasant but tolerable effects at $20-80$ $\mu \mathrm{g} / \mathrm{kg}$ in subjects with a history of drug abuse, effects that the authors suggested may be due to tolerance derived from their past opioid use (Walsh et al., 2001). However, adverse effects such as sedation, confusion, dizziness, visual distortions, and psychotomimetic effects were observed when two of the participants with a history of drug abuse were exposed to a $160 \mu \mathrm{g} / 70 \mathrm{~kg}$ intramuscular (i.m.) injection of enadoline (Walsh et al., 2001).

The adverse effects seen after administration of KOPr agonists in humans are mirrored in preclinical models. Although i.v. infusions of ethylketocyclazocine $(0.01 \mathrm{mg} / \mathrm{kg} / \mathrm{h}$ and $0.032 \mathrm{mg} / \mathrm{kg} / \mathrm{h})$, bremazocine $(0.0032 \mathrm{mg} / \mathrm{kg} / \mathrm{h})$, enadoline $(0.001 \mathrm{mg} / \mathrm{kg} / \mathrm{h}$ and 0.0032 $\mathrm{mg} / \mathrm{kg} / \mathrm{h}), \operatorname{Mr} 2033(0.01 \mathrm{mg} / \mathrm{kg} / \mathrm{h}$ and $0.032 \mathrm{mg} / \mathrm{kg} / \mathrm{h})$, and U50,488 (0.1 mg/kg/h) decreased cocaine self-administration in rhesus monkeys, administration of enadoline and $U 50,488$ resulted in emesis, whereas ethylketocyclazocine, bremazocine, U50,488, enadoline, and Mr2033 produced a simultaneous decrease in responding for food at the higher doses (Mello and Negus, 2000). The concurrent decline in food intake suggests that the KOPr-mediated decrease of cocaine self-administration may be due to a general decrease in responsiveness (possibly due to sedative effects) or modulation of natural 
reward, a result of non-specific anti-reward effects. Administration of U50,488 ( $\geq 2.5$ $\mathrm{mg} / \mathrm{kg}$, s.c.) also produced a diminished performance in the rotarod test in rats due to a loss of muscle tone (Gallantine and Meert, 2008), suggesting that the anti-cocaine effects displayed may be due to non-specific effects on activity. Traditional KOPr agonists also produce depressive-like effects. Rats given i.p. administration of U69,593 (0.25 and 0.5 $\mathrm{mg} / \mathrm{kg}$ ) showed an increase in intracranial self-stimulation (ICSS) thresholds (Todtenkopf et al., 2004), a measure of depression. U50,488 also increased ICSS thresholds in mice (3.0, $5.5 \mathrm{mg} / \mathrm{kg}$, i.p.) (DiNieri et al., 2009) and the time mice spent immobile in the forced swim test (FST), another measure of depression (Zhang et al., 2015). The numerous side effects typical of KOPr agonists have been the greatest challenge for the clinical progression of these compounds. However, recent discovery of Sal A, a structurally novel KOPr agonist that produces fewer side effects compared to classic agonists (Braida et al., 2008; Braida et al., 2009; Morani et al., 2009; Morani et al., 2012) has reinstated interest in $\mathrm{KOPr}$ agonists as potential therapeutic agents. The following sections will give an account of the chemical, pharmacological, and behavioural differences between Sal A (and its analogues) and traditional KOPr agonists, plus the rationale for investigating the anti-cocaine effects of Sal A-derived agonists. 


\begin{tabular}{|c|c|c|c|}
\hline Compound name & U69,593 & U50,488 & Sal A \\
\hline \multicolumn{4}{|l|}{ Chemical structure } \\
\hline $\begin{array}{l}\text { Binding affinity }(\mathrm{Ki}) \text { at the } \mathrm{KOPr}(1) \\
\text { Using rKOPr and }\left[{ }^{3} \mathrm{H}\right] \cup 69,593 \text { as the radioligand }\end{array}$ & $\mathrm{Ki}=2.5 \pm 0.3$ & $\mathrm{Ki}=0.42 \pm 0.22$ & $\mathrm{Ki}=0.28 \pm 0.22$ \\
\hline $\begin{array}{l}\text { Potency }\left(\mathrm{EC}_{50}\right) \text { and binding efficiency }\left(\mathrm{E}_{\max }\right) \text { at } \\
\text { the KOPr }{ }^{(2)} \\
\text { Using }\left[{ }^{35} \mathrm{~S}\right] \mathrm{GTP}-\mathrm{\gamma}-\mathrm{S} \text { functional assay in N2A cells } \\
\text { expressing hKOPr }\end{array}$ & $\begin{array}{c}\mathrm{EC}_{50}=94 \\
\mathrm{E}_{\max }=108 \pm 6.6\end{array}$ & $\begin{array}{c}\mathrm{EC}_{50}=30 \\
\mathrm{E}_{\max }=101 \pm 4.2\end{array}$ & $\begin{array}{c}\mathrm{EC}_{50}=23 \\
\mathrm{E}_{\max }=103 \pm 2.9\end{array}$ \\
\hline $\begin{array}{l}\text { Potency and binding efficiency at the KOPr } \\
\text { Measured by intracellular } \mathrm{Ca}^{2+} \text { mobilisation in } \\
\text { HEK-293 cells co-expressing hKOPr and Gqi5 or } \\
\text { G16 protein. } E_{\max } \text { relative to U69,593 }\end{array}$ & $\begin{array}{l}\mathrm{EC}_{50}=13 \\
\mathrm{E}_{\max }=100\end{array}$ & $\begin{array}{c}E C_{50}=24 \\
E_{\max }=102 \pm 4^{*}\end{array}$ & $\begin{array}{c}\mathrm{EC}_{50}=7 \\
\mathrm{E}_{\max }=104 \pm 7 *\end{array}$ \\
\hline $\begin{array}{l}\text { Potency, binding affinity, and binding efficiency } \\
\text { at the KOPr }{ }^{(4)} \\
\text { Using }\left[{ }^{35} \mathrm{~S}\right] \mathrm{GTP}-\gamma-\mathrm{S} \text { functional assay in Chinese } \\
\text { hamster ovary (CHO) cells expressing hKOPr. } \\
\text { (U50,488 as a full agonist at the KOPr) }\end{array}$ & - & $\begin{array}{c}E C_{50}=2.2 \pm 0.3 \\
E_{\max }=100 \\
K i=11.0 \pm 0.2\end{array}$ & $\begin{array}{c}\mathrm{EC}_{50}=4.6 \pm 1.2 \\
\mathrm{E}_{\max }=110 \pm 12 \\
\mathrm{Ki}=7.9 \pm 0.8\end{array}$ \\
\hline
\end{tabular}
1) Beguin et al. (2008)
2) DiMattio et al. (2015)
3) Chavkin et al. (2004)

\section{Table 1.1: Structures of U69,593, U50,488, Sal A and their pharmacology at the KOPr}

4) Wang et al. (2005)

Values presented as the mean or mean $\pm \mathrm{SEM}\left({ }^{*}\right.$ mean $\pm \mathrm{SD}$ ); $\mathrm{Ki}$ and $\mathrm{EC}_{50}$ in $\mathrm{nM}, \mathrm{E}_{\max }$ in \%. 


\subsubsection{Salvinorin A and its structural analogues}

\subsubsection{Salvia divinorum}

Salvia divinorum, also known as diviner's sage, is a perennial herb of the Labiatae (mint) family traditionally used by the Mazatecs, an indigenous Mexican population in northeast Oaxaca, Mexico (Valdés III et al., 1983; Roth et al., 2002). S. divinorum is one of several spiritual hallucinogens used by the Mazatecs in their divination and healing rituals. It is also used as a remedy to cure diarrhoea, headaches, rheumatism, a semi-magical ailment panzón de barrego (swollen belly) caused by the curse of an evil shaman, and given to the ill, elderly, and dying as palliative care (Valdés III et al., 1983; Prisinzano, 2005). Leaves of S. divinorum can either be chewed, smoked, or crushed to prepare an infusion (Siebert, 1994).

S. divinorum was first described botanically in 1962 (Epling and Jativa-M, 1962). It has since gained popularity as a recreational drug among young adults due to its hallucinogenic properties, its legality in many countries, as well as its lack of adverse health effects. A randomised double-blind placebo trial in $\mathbf{3 0}$ healthy Salvia smokers showed no significant adverse events (Addy, 2012), although there has been one report of psychosis (Przekop and Lee, 2009). A more recent study on 30 healthy individuals who self-administered S. divinorum by combustion and inhalation using a metal smoking pipe reported a very rapid onset of action ( $0-5 \mathrm{~s}$ ) as well as an intense and unique sensory experience (Addy et al., 2015). However, despite its hallucinogenic effects, the study reported a low potential for abuse (Addy et al., 2015). S. divinorum is classed as 'relatively safe' (Listos et al., 2011), non-toxic (Mowry et al., 2003), and studies conducted on rodents have indicated a low potential for overdose (Mowry et al., 2003; Prisinzano, 2005). In fact, S. divinorum has been reported to produce anti-depressant effects and can be prescribed as a medicinal herb for depression (Hanes, 2001) in Finland, Iceland, Norway and Estonia (Siebert, 2014).

\subsubsection{Salvinorin A and its synthetic derivatives}

The active ingredient of Salvia divinorum is Sal A, a neoclerodane diterpene (Siebert, 1994). There has been substantial interest in Sal A in the scientific community due to its 
unique structure and pharmacological effects. Unlike the classical hallucinogen Iysergic acid diethylamide (LSD), Sal A does not exert its activity on the serotonin (5- $\mathrm{HT}_{2 \mathrm{~A}}$ ) receptor but instead binds with high selectivity and potency to the KOPr (Roth et al., 2002). Sal A is the first known naturally occurring KOPr agonist with a non-nitrogenous structure (Roth et al., 2002; Prisinzano, 2005), a remarkable find because it lacks an amino group, a functional group long considered essential for opioid receptor binding and efficacy (Rees and Hunter, 1990). In support of this, the structure of Sal A differs greatly from classic KOPr agonists such as U50,488 and U69,593 (Table 1.1). Despite the differences in structure, several studies have shown that Sal A is still an agonist at KOPr, with similar efficacy to U69,593 and U50,488 (Table 1.1) (Chavkin et al., 2004).

Sal $A$ is most efficient when smoked in a manner similar to freebase cocaine, i.e. using a specially designed glass pipe, with an effective dose of $200-500 \mu g$ (Perez-Reyes et al., 1982; Valdes, 1994). Studies have shown that Sal A has a short duration of action in vivo, with effects peaking at two min and disappearing after 20 min (Johnson et al., 2011). When administered sublingually, the effects of Sal A appeared within 10 min and lasted for approximately $60 \mathrm{~min}$ (Siebert, 1994). However, a more recent study reported a lack of effect after sublingual administration of Sal A (Mendelson et al., 2011), which may have been due to degradation of Sal A in the gastrointestinal system (Siebert, 1994). Studies in preclinical models including rats (elimination half-life of $75 \mathrm{~min}$ and brain half-life of 36 $\min ($ Teksin et al., 2009)) and rhesus monkeys (elimination half-life of $56.6 \pm 24.8 \mathrm{~min}$ (Schmidt et al., 2005)) show that the rapid pharmacokinetics of Sal A are similar regardless of species. In fact, positron emission tomography studies in female baboons show that Sal A had an extremely rapid brain uptake and elimination, with peak levels achieved by $40 \mathrm{~s}$ and a clearance half-life of eight min (Hooker et al., 2008). The fast elimination of Sal A from the brain has limited its clinical use, and efforts to develop derivatives that possess similar therapeutic characteristics but a longer half-life and fewer side effects have been ongoing.

Numerous analogues of Sal A have been synthesised in recent years, many of which have been derived in the laboratory of our collaborator at the University of Kansas, Professor Thomas Prisinzano (Table 1.2 and 1.3). Reduction of the Sal A C-1 ketone moiety reduced 
its binding affinity to the KOPr, whereas the C-2 group of Sal A is said to be critical for binding and function at the KOPr (Prevatt-Smith et al., 2011). Although modifications at C-2 were not well-tolerated, several derivatives synthesised by modifying the C-2 group (namely methoxymethyl ether Sal B (MOM Sal B) and ethoxymethyl ether Sal B, (EOM Sal B)) have reported increased binding affinity and potency at KOPr (Table 1.2) (Peet and Baker, 2011). Other KOPr agonists derived from C-2 modification of Sal A such as Mesyl Sal B and $\beta$-tetrahydropyran Sal B ( $\beta$-THP Sal B) displayed similar binding and efficacy to KOPr as Sal A (Prevatt-Smith et al., 2011) (refer to Table 1.2 for details and Cunningham et al. (2011) for a review of structural modifications).

On the other hand, studies of the C-12 furan ring of Sal A have shown that although it is not essential for biological activity (Cunningham et al., 2011), its complete absence results in a massive decline in affinity for KOPr compared to Sal A (Simpson et al., 2007). As published reports have concentrated on replacing the C-12 group with other cyclic structures, substitution or introduction of other specific groups to the C-12 ring of Sal A may provide a better understanding of the interaction with the KOPr (Table 1.3) (Riley et al., 2014). One compound with this modification is the main compound investigated throughout this thesis, 16-bromosalvinorin A (16-brSal A), generated by addition of a bromine group at the $\mathrm{C}-16$ position on the $\mathrm{C}-12$ furan ring. 16-brSal $\mathrm{A}$ has been found to produce similar efficacy and efficiency at KOPr as Sal A (Simpson et al., 2007; Riley et al., 2014) (Table 1.3). 


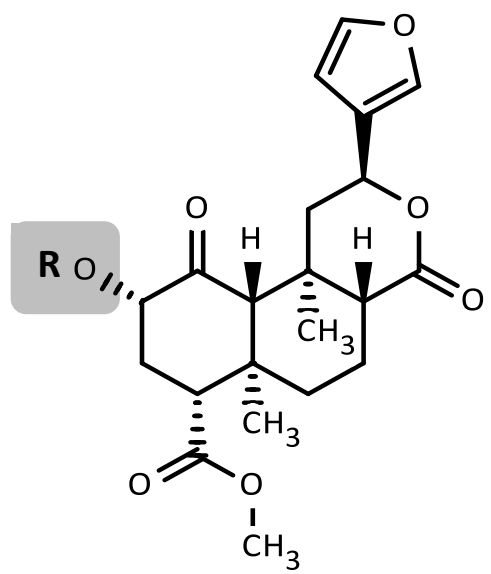

\begin{tabular}{|c|c|c|c|c|c|}
\hline Compound & Sal A & MOM Sal B & EOM Sal B & $\beta$-THP Sal B & Mesyl Sal B \\
\hline$-\mathbf{R}$ & $\prod_{0}^{\mathrm{H}_{3} \mathrm{C}}$ & $\mathrm{H}_{3} \mathrm{C}^{-}$ & $\mathrm{H}_{3} \mathrm{C}$ & & $\begin{array}{c}\mathrm{O}^{\prime \prime} \\
\mathrm{H}_{3} \mathrm{C}-\mathrm{S}^{\prime \prime} \\
\mathrm{O}\end{array}$ \\
\hline $\begin{array}{l}\text { Potency at } \\
\text { KOPr, EC } 50 \\
\text { (nM) }\end{array}$ & $40 \pm 10^{(1,2,3)}$ & $6 \pm 1^{(2)}$ & $0.65 \pm 0.17^{(2)}$ & $60 \pm 6^{(2)}$ & $30 \pm 5^{(3)}$ \\
\hline $\begin{array}{l}\text { Binding } \\
\text { affinity at } \\
\text { KOPr, Ki } \\
\text { (nM) }\end{array}$ & $\begin{array}{l}7.4 \pm 0.7^{(1,2)} \\
* 1.9 \pm 0.2^{(3)}\end{array}$ & $1.9 \pm 0.2^{(2)}$ & $3.13 \pm 0.40^{(2)}$ & $6.21 \pm 0.40^{(2)}$ & $* 2.3 \pm 0.1$ \\
\hline $\begin{array}{l}\text { Binding } \\
\text { efficiency } \\
\text { at KOPr, } \\
E_{\max }(\%)\end{array}$ & $120 \pm 2^{(1,2,3)}$ & $118 \pm 2^{(2)}$ & $127 \pm 5^{(2)}$ & $109 \pm 3^{(2)}$ & $112 \pm 4^{(3)}$ \\
\hline
\end{tabular}

1) Lozama et al. (2011)

2) Prevatt-Smith et al. (2011)

3) Harding et al. (2005)

\section{Table 1.2: Structures and pharmacology of Sal A and its C-2 analogues at KOPr}

$\mathrm{EC}_{50}$ and $\mathrm{E}_{\max }$ values obtained using the [ $\left.{ }^{35} \mathrm{~S}\right] \mathrm{GTP}-\mathrm{\gamma}-\mathrm{S}$ functional assay in Chinese hamster ovary $(\mathrm{CHO})$ cells stably expressing human KOPr; Ki values were obtained using [ $\left.{ }^{3} \mathrm{H}\right] \mathrm{U} 69,593$ as a radioligand (*or using $\left[{ }^{125} \mathrm{I}\right] \mathrm{IOXY}$ as radioligand). $\mathrm{EC}_{50}=$ effective dose that produces $50 \%$ of the maximal response, $\mathrm{E}_{\max }=\%$ at which compound stimulates [ $\left.{ }^{35} \mathrm{~S}\right] \mathrm{GTP}-\mathrm{\gamma}-\mathrm{S}$ binding compared to $\mathrm{U} 50,488$ (500 nM). Values presented as mean \pm SD. 


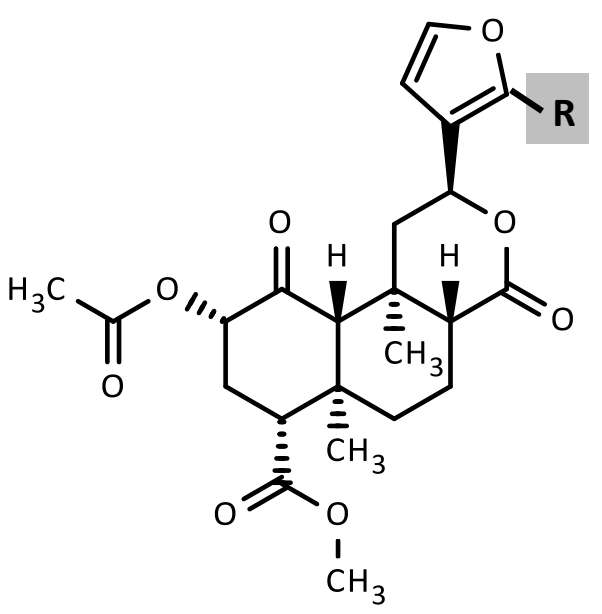

\begin{tabular}{|l|c|c|c|c|}
\hline Compound & Sal A & 16-brSal A & $\begin{array}{c}\text { 16-methyl Sal A } \\
\text { (Me Sal A) }\end{array}$ & $\begin{array}{c}\text { 16-ethynyl Sal A } \\
\text { (Ethy Sal A) }\end{array}$ \\
\hline $\begin{array}{l}\text { Potency at } \\
\text { KOPr, EC } 50 \\
\text { (nM) }\end{array}$ & $\begin{array}{c}0.030 \pm 0.004^{(1)} \\
2.1 \pm 0.6^{(2)}\end{array}$ & $\begin{array}{c}0.040 \pm 0.010^{(1)} \\
2.4 \pm 0.2^{(2)}\end{array}$ & $0.41 \pm 0.15^{(1)}$ & $0.019 \pm 0.004^{(1)}$ \\
\hline $\begin{array}{l}\text { Binding } \\
\text { affinity at } \\
\text { KOPr, Ki } \\
\text { (nM) }\end{array}$ & $2.5 \pm 0.6^{(2)}$ & $2.9 \pm 0.3^{(2)}$ & - & \\
\hline $\begin{array}{l}\text { Binding } \\
\text { efficiency } \\
\text { at KOPr, } \\
\text { Emax }_{\text {(\%) }}\end{array}$ & $105^{(2)}$ & $108 \pm 5^{(2)}$ & & \\
\hline
\end{tabular}

1) Riley et al. (2014)

2) Béguin et al. (2009)

Table 1.3: Chemical structures and pharmacology of Sal A and its C-16 analogues at KOPr

(1) $\mathrm{EC}_{50}$ values obtained by measuring inhibition of forskolin-induced cAMP accumulation in $\mathrm{CHO}$ cells stably expressing $\mathrm{KOPr}$, with all compounds reporting a KOPr $E_{\max }$ of $100 \%$. (2) $E_{50}$ values based on ability to regulate $\left.{ }^{35} \mathrm{~S}\right] \mathrm{GTP}-\mathrm{\gamma}-\mathrm{S}$ binding to membranes of $\mathrm{CHO}-\mathrm{hKOPR}$ cells. $\mathrm{E}_{\max }$ defined as \% of maximal response produced by $\mathrm{U} 50,488$ in parallel experiments. Ki values obtained by inhibiting $\left[{ }^{3} \mathrm{H}\right]$ diprenorphine binding to $\mathrm{CHO}$ cells stably expressing hKOPr. U50,488 values: $\mathrm{Ki}=$ $1.6 \pm 0.6 \mathrm{nM} ; \mathrm{EC}_{50}=3.2 \pm 0.7 \mathrm{nM}$. Values presented as mean $\pm \mathrm{SEM}$. 


\subsubsection{Anti-cocaine effects of Sal A and its analogues}

Despite its hallucinogenic properties, Sal A did not substitute for discriminative effects of LSD or ketamine (Butelman et al., 2004; Killinger et al., 2010). Instead, Sal A showed similar effects to the classical KOPr agonists U50,488 and U69,593 in spite of its structural difference. Research conducted in rats (Willmore-Fordham et al., 2007; Baker et al., 2009) and rhesus monkeys (Butelman et al., 2004) showed that animals trained to discriminate for U69,593 and U50,488 also recognised Sal A in the same way. A later study conducted by Peet and Baker (2011) showed that the Sal A analogues MOM Sal B $(0.003-0.10 \mathrm{mg} / \mathrm{kg}$, i.p.) and EOM Sal B $(0.003-0.10 \mathrm{mg} / \mathrm{kg}$, i.p.) substituted fully for Sal A $(0.5-2.0 \mathrm{mg} / \mathrm{kg}$, i.p.) when tested using a food-reinforced drug discrimination procedure (Peet and Baker, 2011), suggesting that Sal A analogues also exert their discriminative stimulus effects at KOPr.

Sal A displayed similar effects to classic KOPr agonists in numerous behavioural and pharmacological tests. Like U50,488 (30 mg/kg, i.p.) and U69,593 (0.32 mg/kg, s.c.), Sal A (0.3 $\mathrm{mg} / \mathrm{kg}$, i.p.) showed anti-cocaine effects by decreasing cocaine-induced reinstatement of drug seeking (Morani et al., 2009). Sal A also decreased cocaine selfadministration in rhesus monkeys (Freeman et al., 2014) and prevented cocaine-induced behavioural sensitisation (Morani et al., 2012) in a manner comparable to traditional KOPr agonists (Heidbreder et al., 1993; Heidbreder et al., 1995; Shippenberg et al., 1996; Shippenberg et al., 1998; Chefer et al., 1999). An acute administration of Sal A (2 mg/kg, i.p.) decreased cocaine-induced locomotion (Chartoff et al., 2008), as have U69,593 and U50,488 (Crawford et al., 1995; Vanderschuren et al., 2000). The anti-cocaine effects of Sal $A$ are attributed to its regulation of DA neurotransmission, specifically a decrease in extracellular DA levels in the NAc, similar to the actions of U69,593 and U50,488 (Di Chiara and Imperato, 1988a; Spanagel et al., 1992; Zhang et al., 2005).

Despite its similarities to traditional KOPr agonists, recent studies have highlighted several differences that make Sal A a unique potential therapy for various stress, mood, and reward disorders. Although acute activation of the KOPr by traditional agonists produce anti-cocaine effects, their application has consistently been associated with multiple side effects (refer to section 1.4.1 for further details). Sal A, however, possesses a less 
consistent adverse effect profile than classic agonists. Previous studies have shown that Sal A has both pro- and anti-depressive actions (Carlezon et al., 2006; Braida et al., 2009) in the rat FST, with an increase in immobility time being a preclinical measure of depression. Similarly, Sal A has been reported to produce both preference (Braida et al., 2008) and aversion (Sufka et al., 2014) in rats. These discrepancies can be attributed to the dose administered, as Sal A only displays typical KOPr-mediated effects such as a decrease in sucrose intake at high $(2.0 \mathrm{mg} / \mathrm{kg}$ ) but not low $(0.25 \mathrm{mg} / \mathrm{kg})$ doses (Ebner et al., 2010). Since presence of these bimodal effects advocate use of Sal A at lower doses to prevent the negative responses, it is very promising to note that anti-cocaine effects of Sal A are present below the threshold dose whereby KOPr-mediated adverse effects occur (Morani et al., 2009; Morani et al., 2012). Although Sal A still produced depressive-like effects at the minimal dose that decreased cocaine-induced drug seeking $(0.3 \mathrm{mg} / \mathrm{kg})$ (Morani et al., 2012), fewer adverse effects were observed.

Synthetic derivatives of Sal A, MOM Sal B $(0.3 \mathrm{mg} / \mathrm{kg}$, i.p.), Mesyl Sal B $(0.3$ and $1.0 \mathrm{mg} / \mathrm{kg}$, i.p.), $\beta$-THP Sal B (1.0 mg/kg, i.p.), and 16-ethynyl Sal A (Ethy Sal A, 0.1 and $0.3 \mathrm{mg} / \mathrm{kg}$, i.p.) have all demonstrated similar anti-cocaine properties to Sal A by attenuating cocaineinduced reinstatement of drug seeking (Prevatt-Smith et al., 2011; Morani et al., 2013; Simonson et al., 2015). In addition, MOM Sal B (0.3 mg/kg, i.p.) (Morani et al., 2013) and Mesyl Sal B (0.3 mg/kg, i.p.) (Simonson et al., 2015) showed no sedation at the minimal dose that attenuated cocaine seeking. Mesyl Sal B and Ethy Sal A also showed no significant effect on modulation of sucrose intake at their anti-cocaine doses (Kivell Lab, unpublished data), in direct contrast to $\mathrm{U} 50,488$, which produced a reduction in both sucrose intake and sucrose seeking at 5 and $10 \mathrm{mg} / \mathrm{kg}$ (Henderson-Redmond and Czachowski, 2014). However, it is important to note that MOM Sal B (0.3 mg/kg, i.p.), displayed a decrease in sucrose intake similar to U50,488 (Morani et al., 2013). It is also noteworthy that although a study examining the cellular effects of Sal A found that Sal A increases DAT function and expression (Kivell et al., 2014), the Sal A analogue Mesyl Sal B increased DAT function but not cell surface expression (Simonson et al., 2015). These studies indicate that despite the structural and behavioural similarities of Sal A and its analogues, the behavioural and cellular effects of Sal A analogues may differ from Sal A, necessitating further investigation of these compounds. 
Taken together, the large body of work presented above is evidence that Sal A and its analogues share similar anti-cocaine profiles with classic KOPr agonists, but present fewer KOPr-mediated adverse effects. The unique structural and behavioural differences between Sal A (and its derivatives) and traditional KOPr agonists indicate that Sal A and its analogues represent a new class of KOPr-based compounds. Recent discussion of functional selectivity - whereby activation of a receptor by functionally selective ligands can result in differential activation of signal transduction pathways associated to the receptor - suggests that it may be possible to separate desired physiological effects from adverse side effects, highlighting a new area of investigation that can be exploited for therapeutic advantage (refer to section 1.6 for more details). Since the KOPr, being a GPCR, is capable of functionally selective signalling, the following sections will highlight the different KOPr-mediated signalling pathways, the prospects of functionally selective agonists as therapies, and potential for the novel KOPr agonist Sal A to yield novel functionally selective ligands.

\subsection{KOPr signalling cascades}

Although it is established that KOPr agonists produce both anti-cocaine and negative behavioural effects, the cellular mechanisms underlying each of these behaviours are not fully known. KOPr activation produces different signalling responses depending on the type of ligand administered (agonist or antagonist), length of exposure, dose administered, and route of administration. A brief summary of the known cellular effects of KOPr activation as well as the commonly activated signalling pathways is shown in Figure 1.5 (for a full review, refer to Bruchas and Chavkin (2010)).

Acute binding of KOPr agonists to the receptor leads to dissociation of the Gai and G $\beta \gamma$ subunits of the G-protein (Figure 1.5). The Gai subunit inhibits CAMP production by inhibiting adenylyl cyclase (Taussig et al., 1993). Although the downstream consequences of CAMP inhibition are not fully understood, it has been suggested that this decrease opposes the effects of increased cAMP by stimulatory GPCRs (Bruchas and Chavkin, 2010). The $G \beta \gamma$ subunit binds to $G$-protein-gated inwardly rectifying potassium channels $\left(K_{i r} 3\right)$ and $\mathrm{L}, \mathrm{N}$, and $\mathrm{P} / \mathrm{Q}$ type calcium ion channels, inhibiting influx of $\mathrm{Ca}^{2+}$ and enhancing $\mathrm{K}^{+}$ efflux (Rusin et al., 1997). KOPr activation may also mobilise intracellular calcium pools via 
the inositol-triphosphate (IP3) pathway (Bruchas and Chavkin, 2010). Together, these actions cause hyperpolarisation of the neuron and result in decreased neuron excitability. On the other hand, chronic KOPr activation increases CAMP by upregulating adenylyl cyclase and inducing receptor phosphorylation. KOPr phosphorylation uncouples the receptor from its signal transduction networks, which in turn internalises the receptor, resulting in desensitisation of the receptor and reduced KOPr agonist binding (Figure 1.5) (Li et al., 1999; Liu-Chen, 2004; Bruchas and Chavkin, 2010). These actions of chronic KOPr activation associated with opioid tolerance are mediated by GPCR kinase (GRK) and $\beta$ arrestin (Li et al., 1999).

Binding of an agonist to KOPr also activates many signal transduction cascades, namely the mitogen-activated protein kinase (MAPK) pathways. MAPKs are a family of serine/threonine protein kinases that regulate various fundamental cellular responses such as proliferation, differentiation, gene expression, mitosis, apoptosis, embryogenesis, stress, transcription factor regulation, and protein-protein interactions (Pearson et al., 2001; Raman et al., 2007). The signalling of MAPK pathways is dependent on three essential protein kinases that sequentially phosphorylate and activate their downstream counterparts: MAPK kinase kinase kinase that activates MAPK kinase kinase that in turn activates MAPK kinase (MEK). There are several well studied MAPKs - extracellular signalregulated kinase 1 and 2 (ERK1/2), p38 kinase, and c-Jun N-terminal kinase (JNK1-3) - all of which are activated by the KOPr. JNK and early phase ERK1/2 signalling occurs with acute KOPr activation, whereas chronic activation of the KOPr leads to GRK3-dependent phosphorylation of the receptor and $\beta$-arrestin recruitment. $\beta$-arrestin recruitment in turn activates late phase ERK1/2 and p38 signalling (Figure 1.5) (Bruchas and Chavkin, 2010). 
$\beta$-arrestin-independent

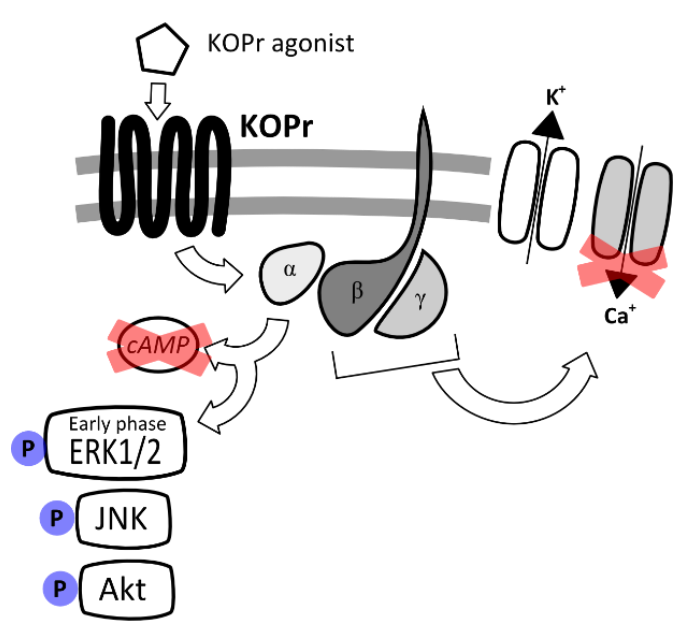

$\beta$-arrestin-dependent

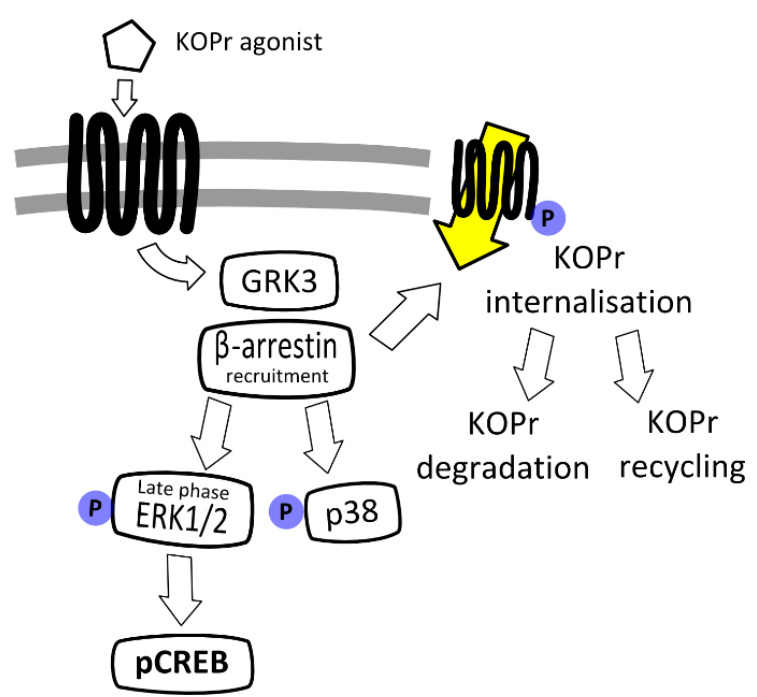

\section{Figure 1.5: KOPr-mediated signalling pathways}

Activation of the KOPr results in activation of several different signalling pathways (Figure modified from Bruchas and Chavkin (2010)). Arrows refer to activation, red crosses refer to downregulation or inhibition, $\mathrm{P}$ in purple circles represent phosphorylation. $\alpha, G$-protein $\alpha$ i subunit; $\beta \gamma, G$-protein $\beta \gamma$ subunit; $C A M P$, cyclic adenosine monophosphate; ERK1/2, extracellular signal-regulated kinase 1 and 2; JNK, c-Jun N-terminal kinase; Akt, protein kinase B; GRK3, G-protein couple receptor kinase 3; p38 MAPK, p38 mitogen activated protein kinase; $P C R E B$, phospho-cAMP response element binding protein.

\subsubsection{Extracellular signal-regulated kinase 1 and 2 (ERK1/2)}

ERK1/2 is the most well characterised downstream signalling pathway activated by KOPr to date (Bruchas and Chavkin, 2010). ERK1 and ERK2 have $84 \%$ primary amino acid sequence homology (Lefloch et al., 2008) and are expressed in most mammalian tissue. Studies of several brain regions such as the superficial cortex and medulla have shown that ERK2 is 2 to 13 times more abundant than ERK1 (Lefloch et al., 2008). Invalidation of the erk2 gene is lethal in mice while invalidation of the erk1 gene is not (Lefloch et al., 2008), suggesting a predominant and compensatory role for ERK2 that can possibly be attributed to its abundance.

ERK1/2 is activated by MAPK/ERK Kinase1/2 (MEK1/2). KOPr agonists' activation of ERK1/2 is hypothesised to be biphasic, with an early phase after $5-10 \mathrm{~min}$ of treatment (Belcheva et al., 2005) and a late phase around two hours after treatment (McLennan et al., 2008). The early phase of activation occurs via the GBy subunit and is dependent on 
phosphatidylinositol 3-kinase (PI3K), protein kinase $\mathrm{C}$ zeta (PKCZ), and $\mathrm{Ca}^{2+}$ mobilisation (Belcheva et al., 2005), whereas the late phase activation requires recruitment of $\beta$ arrestin (McLennan et al., 2008). Both KOPr (Kivell et al., 2014) and DA D2 receptors (Bolan et al., 2007) regulate DAT via the ERK1/2 pathway, whereby KOPr activation leads to ERK1/2 activation (Belcheva et al., 2005; McLennan et al., 2008), and activation of ERK1/2 leads to increased DAT function and cell surface expression (Moron et al., 2003; Bolan et al., 2007). This ERK1/2-dependent regulation of DAT is hypothesised to be one way in which KOPr-mediated anti-cocaine effects occur (Simonson et al., 2015). In direct contrast to KOPr agonists, ERK1/2 inhibitors cause a concentration and time-dependent decrease in DA uptake due to decreased DAT surface expression (Moron et al., 2003), further indicating the importance of this signalling pathway in the dopaminergic pathway of reward.

\subsubsection{P38 mitogen activated protein kinase}

P38 signalling is associated with the negative behavioural effects of KOPr activation. P38 is a class of MAPK that is responsive to stressful stimuli such as heat, osmotic shock, and UV irradiation. There are four identified p38 MAPKs: $\alpha, \beta, \gamma$, and $\delta$; with the $\alpha$-isoform being the most abundant in most cells (Roux and Blenis, 2004). The p38 family is important for cell survival, differentiation, apoptosis, cytokine production, inflammatory responses and stress modulation (Mittelstadt et al., 2005; Whitmarsh, 2010). P38 MAPK also plays an important role in chronic pain responses. For instance, KOPr-induced p38 activation in astrocytes is responsible for cellular proliferation following nerve damage (Xu et al., 2007). Increases in p38 MAPK activation were not observed in astrocytes cultured from KOPr knock-out mice or mice pre-treated with nor-BNI, indicating the role of the KOPr system in modulating p38 (Xu et al., 2007). In addition, in vivo studies have shown that inhibition of p38 MAPK prevented KOPr-induced CPA and attenuated stress-mediated immobility during the FST, whereas repeated swim stress increased both KOPr and p38 MAPK activation, effects not seen in KOPr knock-out mice or mice pre-treated with nor-BNI (Bruchas et al., 2007a).

The mechanism by which p38 mediates KOPr-dependent effects is not completely clear although it is known that p38 MAPK phosphorylates sodium channels ( $\mathrm{Na}_{v} \mathrm{1.8}$ ) (Hudmon 
et al., 2008). Activation of the KOPr leads to phosphorylation of inwardly rectifying potassium channels $\left(\mathrm{K}_{\mathrm{ir}} 3.1\right)$ and produces channel inhibition via a GRK3, p38 MAPK, and Src kinase-dependent mechanism (Clayton et al., 2009). Since the reduced inward $\mathrm{K}^{+}$ current increased neuronal excitability, Clayton and colleagues suggested that this mechanism may be responsible for KOPr-mediated behaviours, a hypothesis supported by the fact that the increase in potassium channel phosphorylation was not observed in KOPr or GRK3 knock-out mice. (Clayton et al., 2009). KOPr-dependent activation of p38 MAPK in transfected cell models, primary astrocyte cultures, and neuronal cultures required phosphorylation of KOPr at serine 369 by GRK3 and $\beta$-arrestin recruitment (Bruchas et al., 2006), unlike the biphasic actions of ERK1/2 which have both $\beta$-arrestindependent and independent phases (McLennan et al., 2008). A potential target of KOPrmediated p38 MAPK activation is the $5-\mathrm{HT}$ system, since p38 MAPK activation in the dorsal raphe nucleus, a serotonergic nucleus, mediates dysphoria in mice (Land et al., 2009). However, as possible downstream signalling substrates of p38 MAPK activation are numerous, further studies are required to determine the exact KOPr-mediated mechanism of action.

\subsection{3 c-Jun N-terminal kinase (JNK1-3)}

The JNK pathway is associated with stress, inflammation, pain, and apoptosis (Leppa and Bohmann, 1999). The JNK MAPK family consists of JNK1, JNK2 and JNK3, with differential splicing generating 10 isoforms of the protein (Waetzig and Herdegen, 2005). JNK1 and 2 are expressed in almost all cells, while JNK3 is found mainly in the brain (Bode and Dong, 2007). There have been few studies carried out on JNK activation by KOPr, but the few that have been conducted show that KOPr activates JNK. This activation is dependent on Src and focal adhesion kinase, but independent of PI3K (Kam et al., 2004a; Kam et al., 2004b). KOPr agonist-induced phosphorylation of JNK is mediated by activation of the pertussis toxin-sensitive Gai subunit (Kam et al., 2004b), whereas KOPr antagonist activation of JNK occurs in a Src kinase and pertussis toxin-insensitive manner (Bruchas et al., 2007b), suggesting that KOPr antagonist-mediated JNK activation may occur via a different pathway than the KOPr agonist signalling pathways (Bruchas and Chavkin, 2010). In further support of this, JNK phosphorylation by KOPr agonists was insensitive to norBNI (Bruchas et al., 2007b). However, the long-lasting effects of KOPr antagonists are 
attributed to JNK activation, as inhibition of JNK prevented the sustained effects of norBNI on KOPr agonist-mediated analgesia (Bruchas and Chavkin, 2010). The authors therefore postulated that long-lasting KOPr antagonists, such as nor-BNI, inactivate $\mathrm{KOPr}$ signalling by forming an association between a JNK-modulated regulator (a hypothetical substrate that prevents $G$ protein activation) and the KOPr (Bruchas et al., 2007b). The differences that exist between JNK activation by different KOPr ligands remains unclear, although it has been hypothesised that these differences are possible examples of functional selectivity (refer to section 1.6) (Bruchas and Chavkin, 2010).

\subsubsection{Other known signalling molecules}

The behavioural effects of KOPr agonists are also modulated by less studied downstream signalling pathways. One of these is the G-protein-mediated activation of protein kinase B (otherwise known as Akt). Due to its dependence on G-protein, Akt activation is pertussis toxin-sensitive (Schmid et al., 2013) and PI3K-dependent (Chen et al., 2006), but independent of $\beta$-arrestin recruitment (Schmid et al., 2013). Suppression or attenuation of Akt is said to regulate the discriminative stimulus of KOPr agonists (Mori et al., 2013), whereas stimulation of Akt mediates KOPr-modulated attenuation of hyperalgesia (Cunha et al., 2012).

Another further downstream signalling pathway crucial to KOPr-mediated effects is activation of CAMP response element binding protein (CREB), a transcription factor that regulates dynorphin gene expression (Kreibich and Blendy, 2004; Carlezon et al., 2005). Activation of CREB occurs downstream of the ERK1/2 signalling pathway (Bruchas et al., 2008), but is independent of GRK3 (Bruchas et al., 2008) and p38 MAPK (Bruchas et al., 2007a). However, upregulation of CREB in the NAc has similar behavioural effects to p38 MAPK in that it modulates KOPr-mediated depression (Pliakas et al., 2001; Mague et al., 2003). In support of this, the repeated swim stress paradigm that produces stress-induced dynorphin release (McLaughlin et al., 2003) also caused CREB phosphorylation in the mouse NAc (Bruchas et al., 2008). Another study conducted by Kreibich and Blendy (2004) showed that CREB was necessary for stress-induced but not cocaine-induced reinstatement of CPP, further validating the role of CREB in regulating stress and depression (Kreibich and Blendy, 2004). 


\subsection{Functional selectivity at the KOPr}

Activation of the KOPr initiates activation of many signalling cascades, each responsible for different KOPr-mediated behavioural responses. Since logical reasoning suggests that KOPr-based agonists which only activate the therapeutic signalling pathways (i.e. early phase ERK1/2 activation) and not the pathways associated with adverse effects (i.e. late phase ERK1/2, p38 kinase, or CREB) should yield therapeutic compounds that have anticocaine effects without the associated adverse effects, it is important to identify and correlate the KOPr-mediated signalling pathways to their respective behavioural effects. To that end, recent work has focused on KOPr agonists that possess few adverse effects in the hope that they may possibly be 'functionally selective' agonists. The idea of functional selectivity, also known as 'biased agonism' or 'collateral efficacy', was introduced as a new idea of receptor activation as a result of greater understanding of the GPCR and enhanced pharmacological approaches (Kenakin, 2007; Urban et al., 2007; Violin and Lefkowitz, 2007; Pineyro, 2009; White et al., 2015).

For many years, the receptor signalling process was thought of as a linear phenomenon, whereby all receptor-mediated activities take place upon activation of the receptor. A bound ligand would either initiate a full, or partial response by activating signalling cascades (agonist) or inhibit receptor activation and not produce any biological activity (antagonist). However, the concept of functional selectivity suggests that since GPCRs are proteins that have many effectors and can exist in numerous conformations (Bruchas and Chavkin, 2010), these different conformations can direct the receptor towards one of many reactions upon activation: 1) receptor occupancy and internalisation (without activation), 2) receptor occupancy followed by G-protein activation and receptor phosphorylation or desensitisation, 3) receptor occupancy and G-protein activation, or 4) receptor occupancy and G-protein independent MAPK activation through $\beta$-arrestin (reviewed in Kenakin (2007)). The knowledge that selective ligands can yield a fraction of the possible biological responses rather than all of them makes GPCRs and exploration of novel biased GPCR substrates an exciting endeavour with interesting potential therapeutic prospects. Although discussion of functionally selective ligands at various GPCRs are beyond the scope of this study (refer to Shonberg et al. (2014) and Violin et al. 
(2014) for recent reviews), the present project will focus on the KOPr (a GPCR) as a potential target that can be exploited for functional selectivity.

It has been hypothesised that the desirable therapeutic effects associated with KOPr activation such as antinociception and anti-addiction, are due to G-protein mediated signalling events, whereas negative side effects of KOPr agonists result from $\beta$-arrestin mediated signalling events (Bruchas and Chavkin, 2010; Kivell et al., 2013). Thus, development of KOPr agonists that are biased towards G-protein signalling, and away from $\beta$-arrestin recruitment will likely be desirable for developing therapeutics with fewer KOPr-mediated side effects. One such example of a functionally selective agonist is $6^{\prime}-$ guanidinonaltrindole (6'-GNTI), a KOPr agonist derived from the selective DOPr antagonist naltrindole. Recent in vitro reports have shown that 6'-GNTI partially activates G-protein signalling without recruiting $\beta$-arrestin (Rives et al., 2012; Schmid et al., 2013). Behaviourally, 6'-GNTI displayed huge potential as an analgesic agent, whereby an intrathecal administration of $6^{\prime}$-GNTI $(0.1-1 \mathrm{nmol} /$ mouse) produced analgesic effects measured by tail flick assay in male mice (Waldhoer et al., 2005). Intraplantar injection of 6'GNTI (1 $\mu \mathrm{g})$ also significantly increased paw withdrawal latencies of rats in a prostaglandin $\mathrm{E}_{2}$-induced thermal allodynia test, a model of inflammatory pain (Berg et al., 2012). The lack of reported side effects of $6^{\prime}$-GNTI suggests that functionally selective KOPr-based analgesic agents may produce minimal KOPr-mediated adverse effects.

In studies involving Sal A and its analogues, the improved behavioural adverse effect profile of these compounds has prompted several studies identifying differences in KOPrmediated signalling compared to classic agonists. In vitro studies have shown that Sal A is 40 times less potent at internalising the KOPr compared to U50,488, despite their similar potencies and efficacies of action at the KOPr (Wang et al., 2005). Since KOPr phosphorylation, internalisation, and down-regulation is linked to receptor desensitisation (Li et al., 1999; Li et al., 2000; Liu-Chen, 2004) and in vivo responses of a drug such as behavioural tolerance (McLaughlin et al., 2004), the reduced ability of Sal A to internalise KOPr suggests that Sal A may produce less tolerance compared to traditional KOPr agonists. Another interesting report showed differences in ERK signalling between U69,593 and the Sal A analogue, MOM Sal B. While U69,593 induced both early and late 
phase ERK1/2 activation in immortalised rat astrocytes, MOM Sal B only induced phosphorylation of early phase ERK1/2 (McLennan et al., 2008). Since late phase ERK1/2 activation is linked to $\beta$-arrestin recruitment, which in turn leads to downstream responses such as activation of CREB and p38 MAPK, both of which are implicated in dysphoria and depression (Pliakas et al., 2001; Carr and Mague, 2008), differences in ERK1/2 signalling of this sort may correlate to the different cellular mechanisms underlying their different behavioural effects.

Taken together, these studies suggest that Sal A has the potential to yield functionally selective agonists at the KOPr. In support of this, behavioural studies have shown that a novel MOPr agonist derived from Sal $A$, herkinorin, does not recruit $\beta$-arrestin or internalise MOPr (Groer et al., 2007). Behaviourally, in vivo studies have shown that an intraplantar injection of herkinorin ( 1 and $10 \mathrm{mg} / \mathrm{kg}$ ) into the hind paw of male SpragueDawley rats dose-dependently decreased the flinching response induced by formalin, with minimal tolerance. In fact, rats that were tolerant to morphine still displayed antinociceptive effects when herkinorin was administered (Lamb et al., 2012). Another Sal Aderived functionally selective KOPr agonist, 22-thiocyanatosalvinorin A (RB-64, $3 \mathrm{mg} / \mathrm{kg}$, s.c.) reported analgesic effects in mice, measured using a hindpaw hotplate assay. In accordance with its biased agonism at KOPr, RB-64 did not produce motor incoordination (evidenced by a lack of change in rotarod performance), sedation, or increase ICSS thresholds in mice (a measure of anhedonia) (White et al., 2015). However, to the best of our knowledge, there are currently no behavioural reports of anti-cocaine studies using G-protein biased KOPr agonists to date. Nonetheless, it is exciting to note that Sal Aderived agonists have showcased therapeutic analgesic effects with fewer KOPr-mediated adverse effects. These studies are evidence that G-protein biased KOPr agonists derived from Sal A may yield novel therapeutics that produce anti-cocaine actions at KOPr with little tolerance or side effects, making them compounds of huge promise in the field of anti-addiction therapy development. 


\subsection{Aims, objectives, and hypotheses}

Given the importance of the KOPr system in cocaine abuse, this research aims to investigate the behavioural and cellular effects of novel KOPr agonists. The effect of acute administration of novel KOPr agonists on drug seeking behaviour will first be evaluated using the cocaine-induced reinstatement paradigm. The present study also aims to investigate another aspect of cocaine-induced behaviour by determining the effects of acute KOPr agonists on cocaine-induced hyperactivity. Based on previous reports featuring KOPr agonists, it was hypothesised that novel Sal A agonists would show attenuation of cocaine seeking as well as hyperactivity due to their cocaine antagonistic actions. Another aim of this study is to explore the typical KOPr-mediated adverse effects of novel Sal A-derived agonists. Because past research has demonstrated that Sal A has fewer side effects than traditional KOPr agonists, it is hypothesised that Sal A derivatives would also show fewer adverse effects.

Previous work has shown that acute KOPr activation produces anti-cocaine effects by opposing the actions of cocaine in dopaminergic neurons (Maisonneuve et al., 1994; Zhang et al., 2004b; Zhang et al., 2004a), and that this cocaine antagonistic action occurs partially via an increase in DAT function and expression (Thompson et al., 2000; Kivell et al., 2014). Therefore, in vitro and ex vivo studies will be conducted to determine the effects of a promising Sal A derivative on regulation of DAT. Subsequent studies on KOPrmediated signalling pathways activated by this lead KOPr agonist will also be conducted, with the aim of determining the mechanism by which this compound exerts its behavioural effects. It is hypothesised that novel KOPr agonists with fewer KOPr-mediated adverse effects have the potential to be functionally selective agonists or at least, yield new functionally selective compounds at the KOPr. Given that behavioural characterisation of potential therapeutics are important to determine the usefulness of anti-cocaine pharmacotherapies at different stages of the addiction cycle, two short studies investigating promising novel KOPr agonists for their ability attenuate cocaine selfadministration in an extended access and progressive ratio paradigm will also be conducted. Findings from this study will reveal the potential therapeutic ability of novel Sal A-derived KOPr agonists. 


\section{Chapter 2: Materials and methods}

\subsection{Animal studies}

\subsubsection{Materials}

Ketamine, xylazine, carprofen (Carprieve), vetadine, lacrilube (Allergan), terramycin pinkeye powder (Pfizer), sodium lactate (Hartmann's solution, Baxter, Viaflex), penicillin G sodium, heparin sodium, pentobarbital and supramid suture wire (\#SD20800, Serag Wiessner) were purchased from Provet (Palmerston North, NZ). Ethanol was obtained from PureScience (Wellington, NZ). Cocaine-HCl (BDG Synthesis, Wellington, NZ) was dissolved in physiological $(0.9 \%)$ saline containing heparin sodium $(3 \mathrm{U} / \mathrm{mL})$ for i.v. infusions. For i.p. injections, cocaine- $\mathrm{HCl}$ was dissolved in physiological saline without heparin. U50,488, U69,593, and nor-BNI were obtained from Sigma-Aldrich (Auckland, NZ). Sal A, 16-brSal A, EOM Sal B, MOM Sal B, and $\beta$-THP Sal B were generous gifts of Dr Thomas Prisinzano.

Animal feed (Diet 86) was acquired from Sharpes Stock Feeds (Carterton, NZ), whereas the bedding (Kay Pine, code DK250) was purchased from Masterpet (Wellington, NZ). All animal housing equipment was obtained from Able Scientific (Perth, Australia).

Surgery equipment: silastic tubing (\#C-P96115-02, internal diameter: 0.020 inches, outer diameter: 0.037 inches, Cole-Parmer) and tygon tubing (\#AAC02346-CP, internal diameter: 0.020 inches, outer diameter: 0.092 inches, Cole-Parmer) were acquired from Thermo Fisher Scientific (Auckland, NZ). Silicon (Selleys), cotton buds, and superglue (Bostik) were obtained from the Warehouse (Wellington, NZ). Gauze swabs (\#NZNZ26611505, 8 ply, 7.5 x $7.5 \mathrm{~cm}$ ) were purchased from Global Science (Auckland, NZ). The large screws (\#CS00395) were supplied by Coastal Fasteners (Wellington, NZ), whereas small jeweller's screws (\#MCPF40210) were purchased from Eyeline Optical (Wellington, NZ). Dental acrylic (Ostron 100 powder and liquid, GC dental products) were obtained from Henry Schein Shalfoon (Auckland, NZ). All syringes and needles (PrecisionGlide ${ }^{\mathrm{TM}}$ ) were acquired from Becton Dickinson (Auckland, NZ), whereas the single use microfilters (\# 16534-K, Minisart ${ }^{\circledR}, 0.2 \mu \mathrm{m}$ ) were purchased from MicroNZ. 


\subsubsection{Animals}

Adult male B6-SJL protein tyrosine phosphatase receptor type $\mathrm{c}$ allele a $\left(\mathrm{ptprc}^{\mathrm{a}}\right)$ mice (23 $-26 \mathrm{~g}$ ) for use in tail flick assays were obtained from the Malaghan Institute of Medical Research (Wellington, NZ) and housed within the School of Biological Sciences, Victoria University of Wellington animal facility. Male Sprague-Dawley rats (Rattus norvegicus) were used for all other behavioural and ex vivo experiments. Rats were housed individually (self-administration studies), two per cage (sucrose reward studies), or three per cage (all other behavioural experiments) within the animal facilities of the School of Psychology or School of Biological Sciences at Victoria University of Wellington. The housing area was temperature $\left(19-21^{\circ} \mathrm{C}\right)$ and humidity $(55 \%)$ controlled, and lights were set to a $12 \mathrm{~h}$ light: $12 \mathrm{~h}$ dark cycle with lights on at 07:00.

All animals (apart from rats undergoing sucrose self-administration) had ad libitum access to food and water except during experimental periods. Food access in sucrose reward test subjects was restricted to maintain their weights at $85 \%$ of their initial body weight, with each rat given at least $12 \mathrm{~g}$ of food per day to prevent extreme weight loss. For all behavioural tests, animals were handled two to three days before testing in order to prevent handling stress. Experiments were carried out during the light cycle and were approved by the Victoria University of Wellington Animal Ethics Committee.

\subsubsection{Intrajugular catheter implantation}

Catheters were prepared 2 days prior to surgeries according to Figure 2.1. The catheter metalpiece was made by removing a $22 \mathrm{G}$ needle from its hub and blunting it at both ends using a rotating flat disc (Dremel rotating tool kit, Bunnings, New Zealand). The metalpiece was cleaned by rinsing it with $70 \%$ ethanol several times. One end of the metalpiece was attached to a length of silastic tubing and the resultant catheter flushed with $70 \%$ ethanol several times.

Once the catheter was dry, it was coated with a thin layer of silicon at the metalpiecetubing connection point to about $3 \mathrm{~cm}$ from the end of the silastic tubing. The silicon was left to dry overnight. The next day, a second coat of silicon was applied and a small silicon 
sphere that served as a point of attachment between the catheter and the jugular vein was created and allowed to dry overnight.

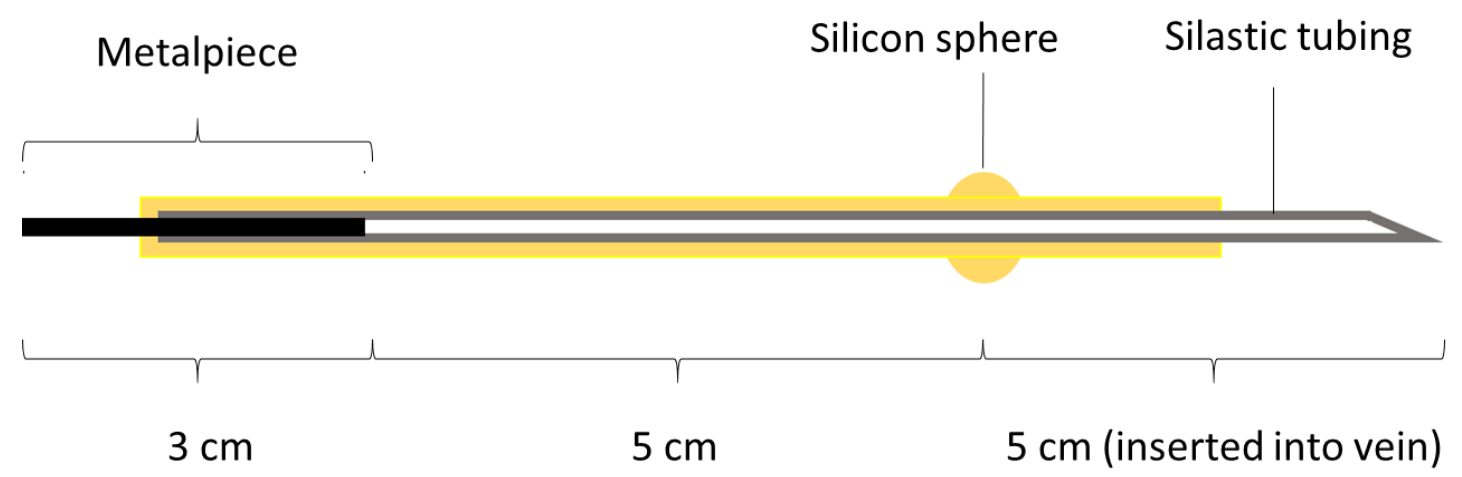

\section{Figure 2.1: Schematic of an intrajugular catheter}

Catheters were made 2 days prior to surgery in order to ensure that the two coats of applied silicon and silicon sphere were completely dry. Catheters were made up to $13 \mathrm{~cm}$ in total length, with the vein end to the silicon sphere measuring approximately $5 \mathrm{~cm}$. The vein end of the tubing was cut at an angle to allow for easier insertion into the jugular vein.

For self-administration experiments, Sprague-Dawley rats weighing between $300-350 \mathrm{~g}$ were used. On the day of surgery, rats were anaesthetised with a mixture of ketamine and xylazine $(90 \mathrm{mg} / \mathrm{kg}$ and $9 \mathrm{mg} / \mathrm{kg}$ respectively, i.p.). After $10-15 \mathrm{~min}$, rats were subjected to a rear paw pinch and surgical procedures commenced in the absence of a reflex. The top of the head and right portion of the chest was shaved with animal clippers, and swabbed with a gauze saturated with vetadine and $70 \%$ ethanol in order to prevent infection. Lacrilube was applied on the rats' eyes to prevent them from drying out during surgery. Carprofen was then injected s.c. on the right flank for pain relief.

To insert the catheter into the right jugular vein, an incision was made on the right ventral side of the rats' chest region using a scalpel. The jugular vein was isolated by teasing the tissue apart with tweezers and a ligature secured at at the rostral end of the vein with supramid wire. A small incision was made in the vein and the silastic catheter inserted into the vein. The metalpiece of the catheter was connected to a syringe filled with heparinised saline $(3 \mathrm{U} / \mathrm{mL})$ and the patency of the intravenous line verified by pulling back on the plunger to draw blood. The catheter was anchored in place using supramid suture wire 
and the exposed chest region sealed with superglue. A mid-sagittal incision was made on the top of the rats' head and the catheter passed subcutaneously under the skin from the chest region to the exposed scalp. The surface of the exposed scalp was swabbed with terramycin-dusted gauze to scrape all the tissue away. To ensure proper adhesion of the headpiece to the skull, four holes were drilled using a manual hand drill with a $1.0 \mathrm{~mm}$ drill bit (Evacut twist, Bunnings, New Zealand) and a jeweller's screw embedded into each quadrant of the skull using a flat head screwdriver. The metalpiece was bent to 90 degrees, slotted between screws, and held in place with superglue. The screws and metalpiece were then fastened in place using dental acrylic, a quick-drying paste produced by mixing Ostron 100 powder and liquid. This mixture was placed on the exposed portion of the skull to seal the incision and anchor the metalpiece. A large screw was placed upside down on the rapidly drying mixture and left to set. A small piece of close-ended tubing was placed on the exposed portion of the metalpiece to prevent clot formation. Terramycin was sprinkled at the back of the headpiece and the sealed portion of the chest area. The rats were then administered $6 \mathrm{~mL}$ of warm sodium lactate to each flank (s.c.) to replenish fluids lost during surgery before being returned to their home cage.

Rats were given carprofen $(5 \mathrm{mg} / \mathrm{kg}$, s.c.) daily for two days post-surgery for pain relief. Their catheters were infused daily with $0.2 \mathrm{~mL}$ of sterile saline containing heparin (30 $\mathrm{U} / \mathrm{mL}$ ) and penicillin $\mathrm{G}$ potassium $(250,000 \mathrm{U} / \mathrm{mL})$ to prevent clot formation. Rats were weighed and monitored daily for signs of discomfort, and given five to seven days to recover before self-administration training.

\subsubsection{Cocaine self-administration training}

Rats were trained in standard operant chambers (Med Associates, ENV-001, St Albans, Vermont, USA) in the School of Biological Sciences, Victoria University of Wellington animal facility or in the School of Psychology animal facility at Victoria University of Wellington. Prior to all sessions, the self-administration chambers were prepared by flushing the tygon tubing with contents of their respective syringes (cocaine $\mathrm{HCl}$ or heparinised saline) to dispel the air. The syringe was attached to a mechanical infusion pump (Razel, Model A with 1 RPM motor equipped with a $20 \mathrm{~mL}$ syringe). The catheter of 
each rat was flushed with heparin-penicillin solution, and rats were placed into operant chambers. The tygon tubing that was surrounded by a wire sheath (C313CS, Plastics One, Australia) was attached to the metalpiece of each rat. The wire sheath was securely anchored to the screw on the rat's headpiece. Each wire sheath was connected to a swivel, which allowed for free movement throughout experimental sessions.

The self-administration chambers contained 2 levers: one connected to an infusion pump (active lever) and one which was not (inactive lever). Upon depression of the active lever, rats received a $12 \mathrm{~s}, 0.1 \mathrm{~mL}$ infusion of cocaine $-\mathrm{HCl}$ solution $(0.5 \mathrm{mg} / \mathrm{kg} /$ infusion) accompanied by a light stimulus. Depression of the inactive lever had no programmed consequences. Rats were observed at the midpoint of each session (end of the first hour for $2 \mathrm{~h}$ sessions and end of the third hour for $6 \mathrm{~h}$ sessions) to ensure that the infusion tubing was still connected to the catheter. Syringes were also inspected to ensure that they contained sufficient solution for the rest of the session. Syringes that held less than $5 \mathrm{~mL}$ of cocaine- $\mathrm{HCl}$ at the midpoint of a $6 \mathrm{~h}$ self-administration session were replaced by temporarily removing the rat from the chamber, replacing the syringe with one containing $20 \mathrm{~mL}$ of cocaine- $\mathrm{HCl}$, and reconnecting the tygon tubing to the headpiece of the rat to resume the session. After each self-administration cycle, catheters were flushed and metalpieces plugged before rats were returned to their home cages.

Rats were initially trained on a fixed ratio (FR) 1 schedule of reinforcement, whereby one active lever press was required for an infusion of cocaine- $\mathrm{HCl}$. Once they acquired selfadministration behaviour (acquisition defined as at least 20 infusions during a $2 \mathrm{~h}$ session with the active:inactive ratio of more than 2:1), rats were required to perform $\geq 20$ active lever presses per 2 hour session with active: inactive ratio of $\geq 2: 1$ for 3 consecutive days. Following this, they progressed to FR2 in which 2 active lever presses were required for a single infusion. Rats were kept on this schedule until they received at least 20 infusions per $2 \mathrm{~h}$ session for 3 consecutive days, with the ratio of the active:inactive lever of more than $2: 1$. They then moved on to the FR5 schedule, whereby 5 lever presses were required for a single infusion. Rats on $2 \mathrm{~h}$ sessions were run in daily sessions from 08:00 to 10:00 and 10:30 to 12:30, 6 days a week. They were trained for 2 weeks to establish a baseline value (less than $20 \%$ variation in active lever presses). Rats on $6 \mathrm{~h}$ sessions were trained 
using $2 \mathrm{~h}$ sessions for at least 5 days before progressing to $6 \mathrm{~h}$ sessions, where they were run daily from 08:00 to 14:00, 5 days a week. Rats were trained for 4 weeks to establish a baseline value before testing began.

For the PR paradigm, rats that acquired self-administration were trained in $2 \mathrm{~h}$ sessions on FR1 for three days, followed by three days on FR2, and 10 days on FR5 before progressing to the $P R$ schedule. The response requirements for $P R$ sessions were increased according to the following equation:

\section{$5 e^{(0.2 \times \text { Infusion\#) }}-5$ (rounded to the nearest integer),}

resulting in the following ratios: $1,2,4,6,9,12,15,20,25,32,40,50,62$, 77 , etc. (Richardson and Roberts, 1996). Each PR session continued until 60 min elapsed without a drug infusion. The breakpoint was defined as the final ratio completed during the session or the number of infusions received for the session. All active and inactive lever responses were recorded using Med Associates software (MED-PC IV, version 4.2).

\subsubsection{Catheter patency test}

Catheters were tested every week to ensure their patency. The catheter was connected to a $1 \mathrm{~mL}$ syringe filled with heparin-penicillin solution. A $0.1 \mathrm{~mL}$ volume of solution was flushed into the rat, and the plunger of the syringe drawn back by $0.2 \mathrm{~mL}$. The presence of blood signified patency of the catheter, whereby a further $0.2 \mathrm{~mL}$ of heparin-penicillin was delivered before returning the rat to its home cage. In the absence of blood, the rat was infused with $0.15 \mathrm{~mL}$ of $50 \mathrm{mg} / \mathrm{mL}$ pentobarbital and observed for immediate loss of righting reflex. Rats which displayed no visible effects to the infusion were declared to have failed the patency test. These rats were subjected to another surgical catheter implantation on the left jugular vein the day after catheter patency testing. Since rats were able to receive a maximum of 2 intrajugular catheters, patency failure of both resulted in a rat being removed from the study. 


\subsubsection{Brain tissue dissection}

Rats were sacrificed via $\mathrm{CO}_{2}$ asphyxiation and decapitated. The brain was rapidly removed within 1-2 min and the brain regions of interest hand-dissected on an ice-cold petri dish using a brain matrix (Alto, AgnTho's AB, Sweden). Specific regions were identified using stereotaxic coordinates in Paxinos and Watson (2005). Three major brain regions were used in this study: the dStr, NAc, and PFC. Table 2.1 and Figure 2.2 shows the regions of interest and their coordinates.

\begin{tabular}{|c|c|c|}
\hline Brain regions & $\begin{array}{c}\text { Interaural } \\
\text { coordinates }\end{array}$ & $\begin{array}{c}\text { Tissue weight } \\
\text { (mg) }\end{array}$ \\
\hline $\begin{array}{c}\text { Dorsal striatum (dStr) } \\
\text { Both sides pooled for Western blotting }\end{array}$ & +9 to +11 & $23.8 \pm 1.3$ \\
\hline $\begin{array}{c}\text { Dorsal striatum (dStr) } \\
\text { Single side for RDEV }\end{array}$ & +9 to +11 & $11.7 \pm 0.5$ \\
\hline Nucleus accumbens (NAc) & +9 to +11 & $10.5 \pm 0.3$ \\
\hline Prefrontal cortex (PFC) & +12 to +14 & $19.1 \pm 0.7$ \\
\hline
\end{tabular}

Values presented as mean \pm SEM. RDEV, rotating disk electrode voltammetry.

Table 2.1: Coordinates and weight of dissected brain regions

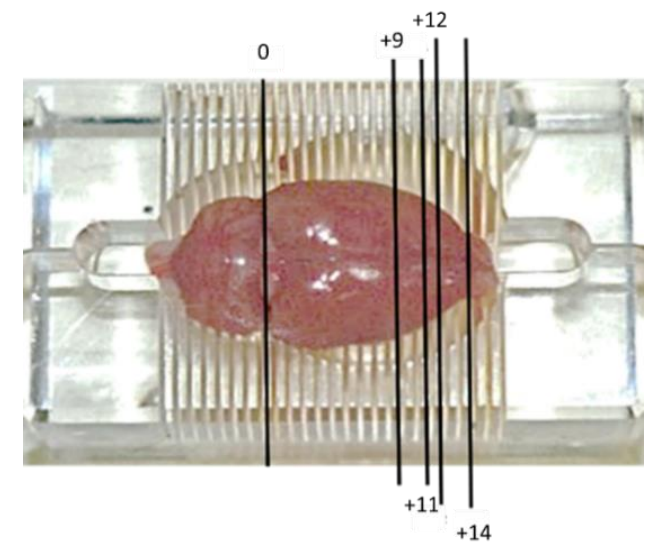

Figure 2.2: Coordinates for rat brain dissection

Adult rat brains were positioned in a brain block on ice. The dStr and NAc were removed from a 2 $\mathrm{mm}$ section between interaural coordinates +9 and +11 , whereas the PFC was removed from interaural coordinates +12 to +14 . All coordinates were obtained from the stereotaxic atlas of Paxinos and Watson (2005). dStr, dorsal striatum; NAc, nucleus accumbens; PFC, prefrontal cortex. 


\subsubsection{Rotating disk electrode voltammetry}

Rotating disk electrode voltammetry (RDEV) is a useful electrochemical technique that measures concentrations of readily oxidised neurotransmitters in cell and tissue suspensions (Earles and Schenk, 1998). It is a non-destructive technique (Schenk et al., 2005) that has been used to measure real time DA (Earles and Schenk, 1998; Jones et al., 2012), 5-HT (Hagan et al., 2010), and norepinephrine (NE) (Burnette et al., 1996) uptake and release within physiologically relevant concentrations (Earles and Schenk, 1998). The present study used RDEV to determine DAT function since the RDEV response time of approximately $20 \mathrm{~ms}$ is sufficient to kinetically resolve DAT function in cell suspensions, tissue, and synaptosome preparations (Schenk et al., 2005).

The RDEV setup consists of a heated $37^{\circ} \mathrm{C}$ chamber into which a rotating glassy carbon electrode is placed (Pine Instruments, special order AFMDO3GC, Pennsylvania, USA), a reference $\mathrm{Ag} / \mathrm{AgCl}$ reference electrode, an auxiliary Pt electrode, an eDAQ potentiostat EA161 (eDAQ, NSW, Australia), and a recording software (Chart, eDAQ, NSW, Australia) (Earles and Schenk, 1998) (Figure 2.3). A potential relative to the $\mathrm{Ag} / \mathrm{AgCl}$ reference electrode sufficient to oxidise the analyte of interest is applied to the rotating carbon electrode and the resulting change in current is measured. The potentiostat maintains a set potential of the rotating carbon electrode relative to the reference $\mathrm{Ag} / \mathrm{AgCl}$ electrode by adjusting the current of the auxiliary Pt electrode. Current from the potentiostat is fed into an oscilloscope and analysed using the Chart software. The constant angular rotation of the carbon electrode causes added DA to approach the rotating carbon electrode perpendicularly, oxidise at the surface to its quinone counterpart, releasing two electrons and generating a current. The release of electrons during oxidation can be measured according to the Levich equation (Earles and Schenk, 1998):

$$
i_{L}=0.62 n \mathrm{FAD}^{2 / 3} \mathrm{v}^{-1 / 6} \omega^{1 / 2} \mathrm{C}
$$

where $i_{L}$ is the limiting current in $\mathrm{mA}, n$ is the number of electrons transferred per mole of analyte, $\mathrm{F}$ is Faraday's constant $(96,485$ coulombs/equivalent of electrons), $\mathrm{A}$ is the area of the electrode in $\mathrm{cm}^{2}, \mathrm{D}$ is the diffusion coefficient in $\mathrm{cm}^{2} / \mathrm{s}, \mathrm{v}$ is the kinematic viscosity of the solution in $\mathrm{cm}^{2} / \mathrm{s}, \omega$ is the angular velocity of rotation in radians/s (where $\omega=2 \pi \mathrm{N}$ and $\mathrm{N}$ is in rotations/s) and $\mathrm{C}$ is concentration in $\mathrm{mM}$. 
Uptake of DA into cells or tissue in the chamber leads to a decrease of free DA, a decrease in oxidation, and a resultant decrease in current.

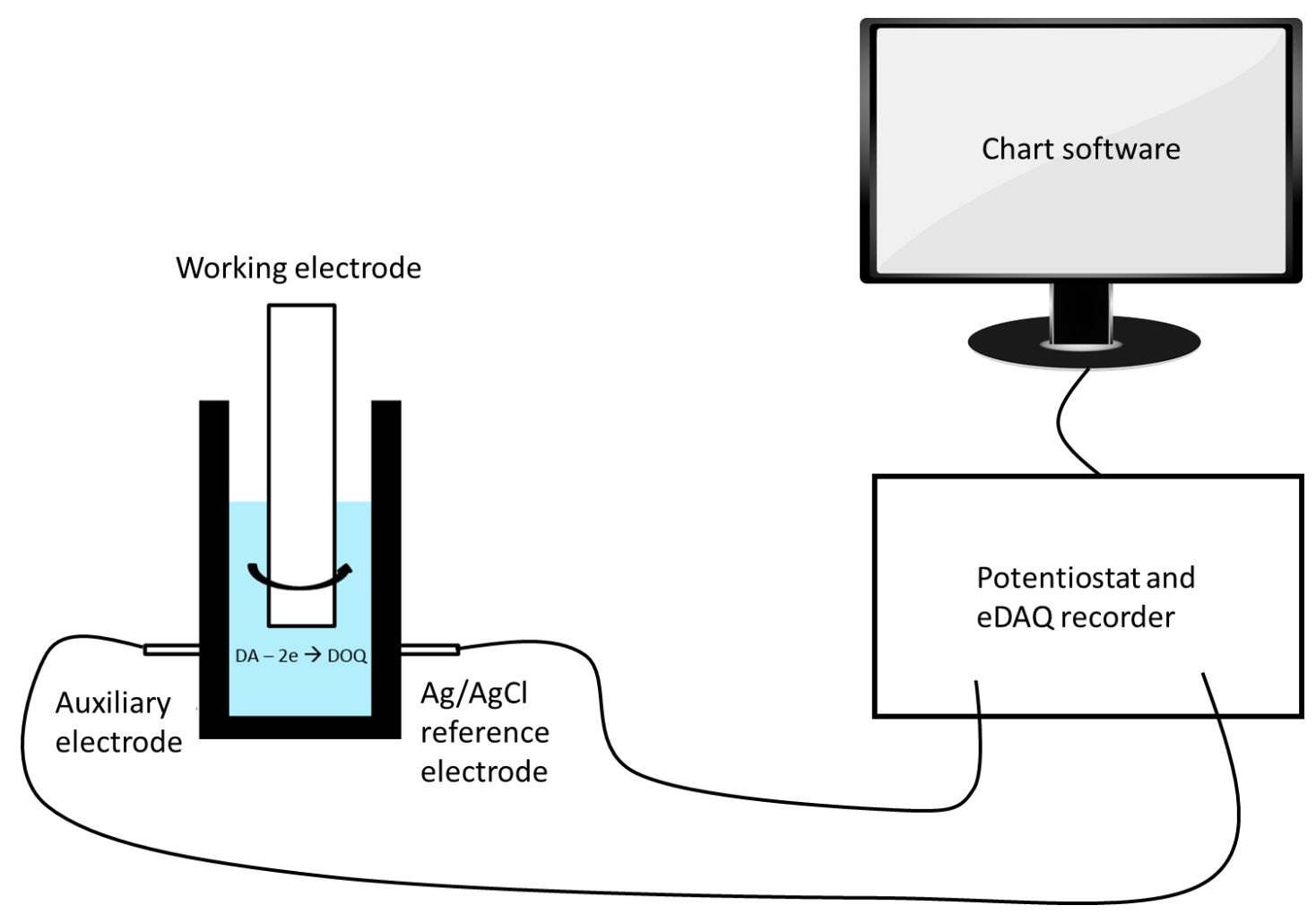

\section{Figure 2.3: RDEV experimental setup}

Minced rat brain tissue was placed into the chamber and the glassy carbon electrode rotating at $2000 \mathrm{rpm}$ lowered into the same chamber. A $+450 \mathrm{mV}$ potential was applied relative to the $\mathrm{Ag} / \mathrm{AgCl}$ electrode to oxidise DA to DA quinone. Electrons released from this reaction flow to the surface of the electrode where they are measured as a current by the eDAQ recorder. Uptake of DA into cells via DAT decreases the number of available electrons and measurable current, providing a linear estimate of DA concentration in the chamber. $D A$, dopamine; $D O Q, D A$ quinone.

There are two different methods of RDEV that can be used to determine the kinetic values of monoamine transporters such as DAT: the zero trans and low to infinite trans model (Povlock and Schenk, 1997). In the present study, the zero trans model was conducted by adding $2 \mu \mathrm{M}$ of DA ( $4 \mu \mathrm{L}$, Sigma-Aldrich) to minced rat brain tissue from a single brain region. On the other hand, the low to infinite trans model was conducted by adding increasing DA concentrations ( 0.5 to $4 \mu \mathrm{M}$ of $\mathrm{DA}, 4 \mu \mathrm{L}$ ) to tissue suspension from a single brain region, allowing current readings to return to baseline between each addition. The zero trans model is useful as it allows quick data collection, whereas the low to infinite 
trans model is suitable for observing transporter kinetics (Vmax and $\mathrm{Km}$ values) as it allows within-sample analysis and reduces the number of animals needed in this study. The low to infinite trans model has previously produced comparable results to single addition studies (Povlock and Schenk, 1997).

Each day prior to commencement of an RDEV experiment, a standard concentration response curve was generated by adding increasing concentrations of DA ( $4 \mu \mathrm{L}$ of 0.5 to $2.0 \mu \mathrm{M}$ ) to $296 \mu \mathrm{L}$ of KREBS buffer in the RDEV chamber (kindly donated by J. B. Justice Jr.,). A linear regression was calculated from the increase in current (nA) after each addition. Data from each DA concentration were plotted in a graph to produce a linear curve. The equation generated from the curve was used to convert data collected from tissue samples into $\mu \mathrm{M}$ values (as in Meiergerd and Schenk (1995); Chefer and Shippenberg (2002)).

Brain sections were dissected according to methods outlined in section 2.1.6. Samples that were not used immediately were either kept on ice for up to 20 min or incubated in pre-warmed $\left(37^{\circ} \mathrm{C}\right)$, carbogen $\left(95 \% \mathrm{O}_{2}, 5 \% \mathrm{CO}_{2}\right)$ aerated KREBS buffer to maintain tissue viability. Brain tissue was minced with an ice-cold razor blade in $300 \mu \mathrm{L}$ of KREBS and subsequently transferred into microcentrifuge tubes. The tissue was washed eight times in pre-warmed, carbogen-aerated KREBS buffer, while allowing tissue to settle between each wash. After the washes, the tissue suspension was added to the RDEV chamber to a volume of $294 \mu \mathrm{L}$. The rotating glassy carbon electrode was lowered into the chamber and rotated at $2000 \mathrm{rpm}$ with an MSR rotator (Pine Instruments). A potential of $+450 \mathrm{mV}$ relative to the $\mathrm{Ag} / \mathrm{AgCl}$ reference electrode was applied to the electrode by the potentiostat.

Once the current measurement of the suspension reached a stable baseline, DA was added to the suspension. For single additions, $4 \mu \mathrm{L}$ of $\mathrm{DA}(2 \mu \mathrm{M})$ was added and the resultant current measured for $30 \mathrm{~s}$. For multiple additions of DA, the low to infinite trans model described Povlock and Schenk (1997) was adapted. Increasing concentrations of DA were added, with the current output allowed to reach baseline between each addition. The resulting current was recorded by an eDAQ recorder 210 (eDAQ, NSW, Australia) and 
saved using the Chart software. The rotating electrode was cleaned with milliQ water on a Buehler Microcloth (Buehler, IL, USA) for $30 \mathrm{~s}$ after each use. At the end of each study, the rotating electrodes were polished with $0.05 \mu \mathrm{m}$ diameter aluminium slurry (Buehler, IL, USA) followed by milliQ water on a Buehler Microcloth in order to prevent fouling of the electrode and measurement inconsistencies.

For all DA uptake studies, approximately $10 \mathrm{~s}$ of uptake data was collected $1 \mathrm{~s}$ after addition of DA. The change in current measurements were recorded in Microsoft Excel 2013 and a linear regression calculated. Correlations with an $R^{2}$ value of lower than 0.95 were excluded from the study. The slope of the regression was then normalised to the standard concentration curve obtained at the beginning of each respective experiment and weight of rat brain tissue to obtain DA uptake values. For kinetic studies, DA uptake values (expressed as pmol/s/g) were fitted to the Michaelis-Menten curve with a one-site binding analysis, and values for Vmax (the maximum velocity of transport) and $\mathrm{Km}$ (the half-maximal velocity of uptake) were generated for each individual rat. 


\subsection{Cellular studies}

\subsubsection{Materials}

Sterile tissue culture flasks (Falcon ${ }^{\circledR}$ ), $35 \mathrm{~mm}$ plastic bottom petri dishes (Falcon ${ }^{\circledR}$ ), and disposable $10 \mathrm{~mL}$ serological pipettes (CELLSTAR ${ }^{\circledR}$ ) were purchased from Becton Dickinson and RayLab (Auckland, NZ). Glass bottom plates (\#FD35-100) were obtained from Coherent Scientific (SA, Australia). Dulbecco's Modified Eagle's Medium (DMEM), Lglutamine, penstrep antibiotic (Penicillin G sodium $5000 \mathrm{U} / \mathrm{mL}$ and streptomycin sulphate $5000 \mathrm{U} / \mathrm{mL}$ in $0.85 \%$ saline), MAX Efficiency ${ }^{\circledR} \mathrm{DH} 5 \alpha^{\mathrm{TM}}$ competent Escherichia coli cells (Invitrogen), Opti-MEM (Invitrogen), Super Optimal Broth with Catabolite repression (SOC) media, and Lipofectamine 2000 were purchased from Life Technologies (Auckland, NZ). Foetal calf serum (FCS) was obtained from ICP biologicals (Auckland, NZ).

The plasmid purification kit (Qiagen HiSpeed Maxi Kit) was purchased from Qiagen, Biostrategy (Auckland, NZ). The Bradford protein assay reagent, SDS polyacrylamide gel electrophoresis (SDS-PAGE) apparatus, and Western blotting equipment were obtained from Bio-Rad (Auckland, NZ). Immobilon-FL polyvinylidine fluoride (PVDF) low background fluorescence transfer membrane $(0.2 \mu \mathrm{M}$ pore size, Millipore Corporation) was purchased from Thermo Fisher Scientific, Auckland, NZ.

\subsubsection{Cell line: Human embryonic kidney 293 (HEK-293) cells}

HEK-293 cells are adherent immortalised cells that were originally produced by transformation of normal human embryonic kidney cells with sheared adenovirus 5 DNA (Graham et al., 1977). Cells were purchased from American Type Culture Collection (ATCC, Manassas, VA, USA) and grown in a humidified, temperature controlled $\left(37^{\circ} \mathrm{C}\right)$ incubator (Bioline, NSW, Australia) with constant flow of $5 \% \mathrm{CO}_{2}$ in air. Cells from passage numbers 5 to 30 were cultured in growth media consisting of DMEM supplemented with $10 \%$ FCS, $200 \mu \mathrm{M}$ L-glutamine, and 1\% penstrep antibiotic until they were plated for experimental use. To maintain frozen cell stocks, aliquots of cells in log growth phase ( $1 \times 10^{6}$ cells $\left./ \mathrm{mL}\right)$ were suspended in growth media containing $10 \%$ dimethyl sulfoxide (DMSO), placed in a cryovial inside a 'Mr Frosty' freezing container (Thermo Fisher Scientific) at $-80^{\circ} \mathrm{C}$ overnight to ensure a cooling rate of approximately $-1^{\circ} \mathrm{C} / \mathrm{min}$. The next day, cryovials were transferred into a liquid nitrogen dewer for long-term storage. 


\subsubsection{Plasmid preparation}

MAX Efficiency ${ }^{\circledR} \mathrm{DH} 5 \alpha^{\mathrm{TM}}$ Competent E. coli cells were allowed to thaw slowly on wet ice. Cells were mixed gently with a pipette tip and $50 \mu \mathrm{L}$ of cells were aliquoted into pre-chilled microcentrifuge tubes ( $50 \mu \mathrm{L}$ aliquot for each transformation). To transform competent cells, approximately $10 \mathrm{ng}$ of plasmid DNA (Table 2.2) was added to the cells. The tubes were tapped gently to ensure adequate mixing and incubated on ice for $30 \mathrm{~min}$. The tube without plasmid DNA was designated the negative control. Tubes were then heat shocked at $42^{\circ} \mathrm{C}$ for exactly $30 \mathrm{~s}$ in a water bath, following which they were returned to ice for 2 min. A $900 \mu \mathrm{L}$ volume of prewarmed $\left(37^{\circ} \mathrm{C}\right) \mathrm{SOC}$ media was added to each of the tubes and the tubes were shaken at $225 \mathrm{rpm}$ in a $37^{\circ} \mathrm{C}$ incubator (Bioline, NSW, Australia) for 1 h. A $100 \mu \mathrm{L}$ aliquot of each culture was then spread onto a Luria Bertani (LB) plate containing $100 \mu \mathrm{g} / \mathrm{mL}$ ampicillin to create a lawn culture. The plates were covered and allowed to dry, after which they were sealed with parafilm and incubated overnight at $37^{\circ} \mathrm{C}$ in a humid incubator.

If the control plate showed no growth after incubation, a single colony from the transformed lawn culture was taken and streaked onto LB agar plates containing ampicillin. These plates were incubated overnight at $37^{\circ} \mathrm{C}$. The next day, a single colony was removed from the plate using a sterile pipette tip and added to $2 \mathrm{~mL}$ of LB broth containing $100 \mu \mathrm{g} / \mathrm{mL}$ ampicillin. The culture was incubated in a shaking, humid $37^{\circ} \mathrm{C}$ incubator for $8 \mathrm{~h}$ at $300 \mathrm{rpm}$. A $200 \mu \mathrm{L}$ aliquot of the bacterial culture was then removed and added to $200 \mathrm{~mL}$ of LB broth containing $100 \mu \mathrm{g} / \mathrm{mL}$ ampicillin in a sterile conical flask, and then incubated for a further $12-16 \mathrm{~h}$ in the same incubator.

Plasmid DNA was then extracted from this bacterial culture according to the Qiagen protocol. Briefly, the culture was first removed from the shaking incubator and immediately placed on ice to halt further growth. The suspension was centrifuged at 6000 g for $15 \mathrm{~min}$ in a prechilled $\left(4^{\circ} \mathrm{C}\right)$ Sorvall Evolution RC centrifuge (Kendro Laboratory Products, Bath, UK). The supernatant was then discarded and the bacterial pellet resuspended in $10 \mathrm{~mL}$ of Buffer P1 (resuspension buffer) containing RNase A. A $10 \mathrm{~mL}$ volume of buffer P2 (lysis buffer) was added to lyse the cells, and the mixture mixed thoroughly by inverting the tube $4-6$ times. After 5 min incubation at room temperature, 
$10 \mathrm{~mL}$ of chilled buffer P3 (neutralisation buffer) was added to the cell lysate and the solution mixed thoroughly. The lysate was then transferred into the barrel of the QLA filter Maxi Cartridge and incubated at room temperature for $10 \mathrm{~min}$. During this time, a HiSpeed Maxi tip was equilibrated by applying $10 \mathrm{~mL}$ of buffer QBT (equilibration buffer) and allowing the column to empty via gravity.

After $10 \mathrm{~min}$, the lysate was filtered into the equilibrated tip, trapping DNA in the tip. The tip was washed with $60 \mathrm{~mL}$ of buffer QC (wash buffer) and the DNA eluted with $15 \mathrm{~mL}$ of pre-warmed $\left(50^{\circ} \mathrm{C}\right)$ buffer QF (elution buffer). DNA was then precipitated by adding 10.5 $\mathrm{mL}$ of molecular grade isopropanol ( $\geq 99.5 \%$, Sigma-Aldrich) to the eluate and incubating it for $5 \mathrm{~min}$ at room temperature. The mixture was transferred into a $30 \mathrm{~mL}$ syringe, attached to a QLA precipitator Maxi Module, and filtered into a waste container using constant pressure. The precipitator was washed by filtering $2 \mathrm{~mL}$ of $70 \%$ ethanol through it at constant pressure. The filter was then air dried by pushing air through the plunger forcefully and quickly $2-3$ times. The outlet nozzle of the precipitator was dried using a Kim wipe to prevent ethanol carryover. The precipitator was then attached to a $5 \mathrm{~mL}$ syringe and placed over a microcentrifuge tube. A $1 \mathrm{~mL}$ volume of buffer TE (elution buffer) was added to the syringe and the DNA eluted into the tube. The eluate was transferred back into the syringe and filtered a second time through the same precipitator into the same collection tube.

To determine the concentration and purity of the DNA, a Nanodrop ND-1000 spectrophotometer (BioLab, Auckland, NZ) was used. The Nanodrop was blanked using 1 $\mu \mathrm{L}$ of buffer TE. Then, $1 \mu \mathrm{L}$ of plasmid DNA was placed onto the silver tip sensor and the amount of DNA present measured. Yields of above $500 \mathrm{ng} / \mu \mathrm{L}$ with an $A^{260 / 280}$ ratio of 1.7 to 1.9 were considered acceptable and used for transfections. When yields of high concentration were obtained, $500 \mu \mathrm{L}$ of the $2 \mathrm{~mL}$ bacterial culture was added to $500 \mu \mathrm{L}$ of $80 \%$ glycerol in a microcentrifuge tube and frozen at $-80^{\circ} \mathrm{C}$ for long-term storage. A scraping of this frozen stock could be streaked directly onto LB agar plates containing ampicillin to obtain single colonies containing the plasmid of interest. 


\begin{tabular}{|c|c|c|c|}
\hline Plasmid DNA & Vector & Resistance & Generous gifts from \\
\hline myc-rKOPr & pcDNA3 & Ampicillin $(100 \mu \mathrm{g} / \mathrm{mL})$ & L. Devi (Jordan and Devi, 1999) \\
\hline YFP-hDAT & pciHyg & Ampicillin $(100 \mu \mathrm{g} / \mathrm{mL})$ & J. Javitch (Daws et al., 2002) \\
\hline
\end{tabular}

Table 2.2: Plasmid DNA vector and resistance information

myc-rKOPr, myc-tagged rat kappa opioid receptor; YFP-hDAT, yellow fluorescent proteintagged human dopamine transporter

\subsubsection{Cell growth and passaging}

Frozen HEK-293 cell stocks were thawed in a $37^{\circ} \mathrm{C}$ waterbath and immediately added to $10 \mathrm{~mL}$ of DMEM supplemented with $10 \%$ FCS, $200 \mu \mathrm{M} \mathrm{L-glutamine,} \mathrm{and} \mathrm{1 \%} \mathrm{penstrep.} \mathrm{Cells}$ were centrifuged at $300 \mathrm{~g}$ for $5 \mathrm{~min}$, supernatant discarded, and the cell pellet resuspended in $5 \mathrm{~mL}$ of growth medium. Cells were allowed to grow overnight in a $25 \mathrm{~cm}^{2}$ flask with a vented cap. They were fed the following day by removing the old culture medium and replacing it with fresh growth medium prewarmed to $37^{\circ} \mathrm{C}$. Once the culture reached $70-80 \%$ confluency, cells were either plated for use or passaged into new 75 $\mathrm{cm}^{2}$ flasks.

To passage cells, old culture medium was removed and $2 \mathrm{~mL}$ of TrypLE ${ }^{\mathrm{TM}}$ Express Enzyme (Life Technologies, Auckland, NZ) was added to detach the cells. After $5 \mathrm{~min}, 5 \mathrm{~mL}$ of growth medium was added to the cells and the bottom of the flask rinsed several times to ensure detachment of cells. The suspension was transferred into a $15 \mathrm{~mL}$ centrifuge tube and centrifuged at $300 \mathrm{~g}$ for $5 \mathrm{~min}$. The resulting supernatant was removed and the cell pellet resuspended in $5 \mathrm{~mL}$ of growth medium. One $\mathrm{mL}$ of this suspension was seeded into a new $75 \mathrm{~cm}^{2}$ flask containing $10 \mathrm{~mL}$ of culture medium to continue the cell line.

Cells were plated at different densities for different experiments. For biotinylation and Western blot experiments, $1 \times 10^{6}$ cells $/ \mathrm{mL}$ were plated onto $35 \mathrm{~mm}$ plastic bottom petri dishes, whereas $0.5 \times 10^{6}$ cells $/ \mathrm{mL}$ were plated onto $35 \mathrm{~mm}$ glass bottom petri dishes for confocal microscopy experiments. All $35 \mathrm{~mm}$ petri dishes contained $1 \mathrm{~mL}$ of growth medium. 


\subsubsection{Cell transfection}

HEK-293 cells were transfected $24 \mathrm{~h}$ after plating. Cells in $1 \mathrm{~mL}$ petri dishes were transfected using $3 \mu \mathrm{L}$ of Lipofectamine 2000 reagent made up to $50 \mu \mathrm{L}$ with Opti-MEM. This mixture was incubated at room temperature for $5 \mathrm{~min}$. During incubation, the desired plasmid DNA was added to another tube and made up to $50 \mu \mathrm{L}$ with Opti-MEM. The myctagged rat KOPr plasmid DNA (myc-rKOPr, from L. Devi) was made up to a concentration of $0.7 \mu \mathrm{g} / \mathrm{mL}$, whereas the yellow fluorescent protein-tagged human DAT plasmid DNA (YFP-hDAT, from J. Javitch) was made up to $0.4 \mu \mathrm{g} / \mathrm{mL}$. The two tubes were subsequently mixed together and incubated at room temperature for $30 \mathrm{~min}$. The cells' growth medium was replaced with antibiotic free growth medium (DMEM, 10\% FCS, $200 \mu \mathrm{M}$ L-glutamine) and $100 \mu \mathrm{L}$ of the transfection mixture was added to each petri dish. Cells were ready for use $48 \mathrm{~h}$ after transfection.

\subsection{6 trans-4-[4-(dimethylamino)styryl]-1-methylpyridinium (ASP') uptake}

Uptake of trans-4-[4-(dimethylamino)styryl]-1-methylpyridinium $\left(\mathrm{ASP}^{+}\right)$was carried out using methods previously described in Schwartz et al. (2003) and Bolan et al. (2007). The fluorescent monoamine transporter substrate, $\mathrm{ASP}^{+}$(Tocris Biosciences supplied by Pharmaco NZ Ltd, Auckland, NZ) was made into a $10 \mathrm{mM}$ stock solution in sterile KREBS buffer $\left(130 \mathrm{mM} \mathrm{NaCl}, 1.3 \mathrm{mM} \mathrm{KCl}, 2.2 \mathrm{mM} \mathrm{CaCl}, 1.2 \mathrm{mM} \mathrm{MgSO}_{4} .6 \mathrm{H}_{2} \mathrm{O}, 1.2 \mathrm{mM} \mathrm{KH}_{2} \mathrm{PO}_{4}\right.$, $10 \mathrm{mM}$ HEPES, $10 \mathrm{mM}$ D-glucose, $\mathrm{pH}$ 7.4; Appendix 1). This $\mathrm{ASP}^{+}$stock was diluted to required working concentrations with sterile KREBS buffer and maintained at $37^{\circ} \mathrm{C}$ in a water bath. A confocal laser scanning microscope (Olympus Fluoview ${ }^{\mathrm{TM}}$ FV1000, NZ) was used to monitor $\mathrm{ASP}^{+}$uptake in cells.

After $48 \mathrm{~h}$ post-transfection, growth medium was removed from the $35 \mathrm{~mm}$ glass bottom petri dish by aspiration and cells were washed twice with sterile KREBS buffer at $37^{\circ} \mathrm{C}$. The petri dishes containing cultured cells were then placed in an insulated, double-layer heating chamber (\#INU-21LCS-F1, Tokai Hit Co. Ltd, Japan) mounted on the confocal microscope stage to keep the temperature of the cells constant $\left(37^{\circ} \mathrm{C}\right)$ throughout the experiment. The microscope was adjusted to focus on a single layer of cells using a $40 \mathrm{X}$ water immersion lens $(N A=0.9)$. Once an appropriate layer of cells was located, the KREBS buffer was removed and a time course scan started every $5 \mathrm{~s}$ for $10 \mathrm{~min}$. Immediately 
after the first image, $1 \mathrm{~mL}$ of $\mathrm{ASP}^{+}$at $37^{\circ} \mathrm{C}$ was carefully added to the petri dish. $\mathrm{ASP}^{+}$ fluorescence was measured using a $559 \mathrm{~nm}$ laser (ASP filter: $559 \mathrm{~nm}$ excitation; $600 \mathrm{~nm}$ emission), whereas the YFP fluorescence was measured using a $473 \mathrm{~nm}$ laser (eYFP filter: $473 \mathrm{~nm}$ excitation; $527 \mathrm{~nm}$ emission). Images were also captured using differential interference contrast (DIC) in order to visualise the cells. All images were acquired and analysed for fluorescence intensity using the Olympus FV1000-ASW software.

\subsubsection{Cell surface biotinylation}

Cell surface biotinylation was carried out according to methods previously described in Bolan et al. (2007) and Zapata et al. (2007). Myc-rKOPr and YFP-hDAT co-transfected cells were washed twice with ice-cold phosphate buffered saline (PBS, $140 \mathrm{mM} \mathrm{NaCl}, 2.68 \mathrm{mM}$ $\mathrm{KCl}, 8.1 \mathrm{mM} \mathrm{Na}_{2} \mathrm{HPO}_{4}, 1.47 \mathrm{mM} \mathrm{KH}_{2} \mathrm{PO}_{4}$; Appendix 1) containing calcium and magnesium (PBS/Ca-Mg, PBS containing $0.1 \mathrm{mM} \mathrm{CaCl}_{2}, 1 \mathrm{mM} \mathrm{MgCl}$, pH 8.0; Appendix 1) before being incubated with $1 \mathrm{~mL}$ of $1 \mathrm{mg} / \mathrm{mL}$ EZ Link ${ }^{\mathrm{TM}}$ NHS-Sulfo-SS-Biotin (\#21331, Pierce Biotechnology, IL, USA) in PBS/Ca-Mg for $60 \mathrm{~min}$ at $4^{\circ} \mathrm{C}$. Cells were then washed twice with ice-cold PBS/Ca-Mg containing $100 \mathrm{mM}$ glycine and incubated at gentle shaking conditions with $\mathrm{PBS} / \mathrm{Ca}-\mathrm{Mg}$ for $20 \mathrm{~min}$ at $4^{\circ} \mathrm{C}$ to quench unreacted biotin. After $20 \mathrm{~min}$, cells were lysed in $500 \mu \mathrm{L}$ of radio-immunoprecipitation assay (RIPA) buffer (10 mM Tris$\mathrm{HCl}, 150 \mathrm{mM} \mathrm{NaCl}, 1 \mathrm{mM}$ EDTA, 1\% Triton-X-100, 0.1\% SDS, 1\% sodium deoxycholate, pH 7.5; Appendix 1) containing $10 \mu \mathrm{L} / \mathrm{mL}$ protease inhibitor cocktail (P8340, Sigma-Aldrich) for $60 \mathrm{~min}$ at $4^{\circ} \mathrm{C}$ with gentle shaking. Lysates were transferred into a microcentrifuge tube and centrifuged at $16,000 \mathrm{~g}$ for $30 \mathrm{~min}$ at $4^{\circ} \mathrm{C}$. A $50 \mu \mathrm{L}$ aliquot of the resultant supernatant (total fraction) was removed and stored on ice. A $400 \mu \mathrm{L}$ aliquot of supernatant was loaded into plugged spin Zeba desalt columns (Pierce Biotechnology) containing $100 \mu \mathrm{L}$ streptavidin beads (Immobilised NeutrAvidin Protein, \#29200, Pierce Biotechnology). This mixture was incubated for $60 \mathrm{~min}$ at room temperature with gentle mixing using a mini-labroller (Total Lab Solutions Ltd, Auckland, NZ).

After 60 min, plugs were removed from the columns and columns were centrifuged at 600 $\mathrm{g}$ for $1 \mathrm{~min}$. A $50 \mu \mathrm{L}$ volume of the lysate (internal fraction) was removed and stored on ice. The streptavidin beads were then washed 4 times with $400 \mu \mathrm{L}$ of RIPA buffer followed by centrifuging at $16,000 \mathrm{~g}$ for $1 \mathrm{~min}$ following each wash. The columns were plugged after 
the final wash and bound proteins eluted with $40 \mu \mathrm{L}$ of $5 \mathrm{X}$ reducing buffer $(62.5 \mathrm{mM}$ Tris$\mathrm{HCl} \mathrm{pH} \mathrm{6.8,2 \%} \mathrm{SDS,} 20 \%$ glycerol, $1 \%$ bromophenol blue; Appendix 1) containing $10 \% \beta$ mercaptoethanol for $30 \mathrm{~min}$ at room temperature. The plugs were removed and the columns centrifuged at $500 \mathrm{~g}$ for $1 \mathrm{~min}$ at $4^{\circ} \mathrm{C}$ to collect the cell surface fraction. Fifteen $\mu \mathrm{L}$ of $5 \mathrm{X}$ reducing buffer was also added to the total fraction, whereas $12 \mu \mathrm{L}$ of $5 \mathrm{X}$ reducing buffer was added to the internal fraction. All three fractions were then loaded onto gels for electrophoresis, after which gels were scanned for YFP-hDAT fluorescence and subsequent Western blotting studies carried out (section 2.2.9). Membranes were then immunoblotted for glyceraldehyde 3-phosphate dehydrogenase (GAPDH, details in Table 2.3) to ensure that cells had not lysed prior to biotin addition.

\subsubsection{Protein concentration assay}

Cell and brain tissue lysates were examined for their total protein concentration using the Bradford protein assay method according to the Bio-Rad protocol. All standards and samples were analysed in duplicate. Briefly, standards were prepared by diluting bovine serum albumin (Invitrogen) in RIPA buffer to concentrations of $0,0.25,0.5,0.75,1.0,1.5$, and $2.0 \mathrm{mg} / \mathrm{mL}$. Lysates were diluted (1:5 and 1:10) in RIPA buffer containing $10 \mu \mathrm{L} / \mathrm{mL}$ protease inhibitor to a total volume of $10 \mu \mathrm{L}$.

Five $\mu \mathrm{L}$ of the standards and samples were added to wells of a 96-well microplate (Millipore, France) and $250 \mu \mathrm{L}$ of the $1 \mathrm{X}$ Bradford dye reagent was pipetted into each well. Samples were then incubated at room temperature for at least $5 \mathrm{~min}$ before being read on a VERSAmax microplate reader (Molecular Devices Group, USA) at $595 \mathrm{~nm}$. The absorbance of the standards and samples were analysed using SOFT max PRO version 3.0 software (Molecular Devices Group, 1999, USA) and a standard concentration curve plotted in Microsoft Excel 2013. Duplicate readings were averaged and the standard concentration curved used to determine the concentration of protein in each sample. Samples were diluted to $2.5 \mathrm{mg} / \mathrm{mL}$ for SDS-PAGE and Western blotting (refer to Appendix 2.1 for determination of loading concentration). 


\subsubsection{SDS polyacrylamide gel electrophoresis and Western blotting}

Tissue or cell lysates were run on 15-lane SDS-PAGE gels (4\% stacking gel and $10 \%$ separating gel; Appendix 1) made prior to the experimental day and stored in soaked paper towels at $4^{\circ} \mathrm{C}$. The protein content in samples was quantified using the Bradford protein assay (section 2.2 .8 ) and $50 \mu \mathrm{g}$ protein was mixed with $5 \mathrm{X}$ reducing buffer. A 20 $\mu \mathrm{L}$ volume of the mixture was loaded into the wells and the gel run for $80 \mathrm{~min}$ at $140 \mathrm{~V}$. After electrophoresis, separated proteins on the gel were transferred onto an Immobilon PVDF membrane (0.2 $\mu \mathrm{M}$ pore size). The PVDF membrane was soaked in methanol for 1 - 2 min and subsequently transfer buffer (25 mM Tris, 190 mM glycine, 20\% methanol; Appendix 1) for 5 min prior to use. The transfer cassette was assembled in transfer buffer and inserted into the Bio-Rad transfer tank containing transfer buffer and an icepack. This transfer was run at $20 \mathrm{~V}$ for $16 \mathrm{~h}$ at $4^{\circ} \mathrm{C}$.

After $16 \mathrm{~h}$, the transfer cassette was disassembled and the membrane containing electrophoresed proteins washed for $5 \mathrm{~min}$ in 1X Tris buffered saline (TBS; $150 \mathrm{mM} \mathrm{NaCl}$, $50 \mathrm{mM}$ Tris, $\mathrm{pH}$ 7.5; Appendix 1). The membrane was blocked with the respective blocking solution (Table 2.3 ) for $1 \mathrm{~h}$ at room temperature on a shaker and then probed with primary antibody diluted in blocking buffer (Table 2.3) overnight at $4^{\circ} \mathrm{C}$ on a shaker. The next day, the membrane was washed 3 times with TBS supplemented with 0.1\% Tween-20 (T-TBS) before being probed with secondary antibody diluted in TBS (Table 2.3) at room temperature on the shaker for $1 \mathrm{~h}$. The membrane was subsequently washed 3 times with TBS and scanned using a FUJIFILM FLA-5000 scanner (Fujifilm, Tokyo, Japan).

In order to detect both phosphorylated and total proteins on the same PVDF membrane, membranes were first probed and scanned for the phosphorylated protein. Following that, membranes were stripped with stripping buffer (0.05 M Tris- $\mathrm{HCl}, 2 \% \mathrm{SDS}, 3.75 \% \beta$ mercaptoethanol, $\mathrm{pH}$ 6.8; Appendix 1) for $40 \mathrm{~min}$ at room temperature on a shaker and washed thoroughly $5-7$ times with T-TBS. The blocking solution was then applied to membranes for $1 \mathrm{~h}$ at room temperature, followed by the primary antibody of the total protein (Table 2.3). Membranes were incubated overnight at $4^{\circ} \mathrm{C}$ on a shaker. The next day, membranes were washed 3 times with T-TBS and probed with the appropriate 
secondary antibody for $1 \mathrm{~h}$. Membranes were then washed 3 times with TBS before being scanned as above.

All western blots were analysed using the ImageJ software (NIH). All band densities were background corrected and corrected for protein loading differences by normalising the density of phosphorylated protein bands to the corresponding total protein band. The data was then normalised to vehicle-treated controls to enable comparison between membranes. The size of visual protein bands were quantified using their linear migration properties (refer to Appendix 2.2) to enable identification of proteins.

\subsection{Statistical analysis}

All statistical analyses and graphs were generated using the GraphPad 5.01 software (GraphPad Software Inc., La Jolla, CA, USA), with the exception of sphericity calculations which were conducted using the IBM SPSS Statistics software (Version 22, IBM ${ }^{\odot}$ Corporation). All numbers given in bar graphs represent sample numbers. Data was verified for normality prior to analysis (wherever possible) using the Kolmogorov-Smirnov test. Unless otherwise stated, sphericity could be and was assumed for all repeated measures ANOVA tests. In the event that subject numbers were insufficient for sphericity measurements, the Greenhouse Geisser correction was utilised to verify significance of results. Results that revealed no difference in statistical significance were reported without the Greenhouse Geisser correction. 


\begin{tabular}{|c|c|c|c|c|c|c|c|c|c|c|c|c|}
\hline \multirow[b]{2}{*}{ Protein } & \multirow{2}{*}{$\begin{array}{l}\text { Size } \\
\text { (kDa) }\end{array}$} & \multicolumn{3}{|c|}{ Blocking } & \multicolumn{4}{|c|}{ Primary antibody } & \multicolumn{4}{|c|}{ Secondary antibody } \\
\hline & & Solution & Time & Conditions & Solution & $\begin{array}{c}\text { Dilution } \\
\text { factor }\end{array}$ & Time & Conditions & Solution & $\begin{array}{c}\text { Dilution } \\
\text { factor }\end{array}$ & Time & Conditions \\
\hline $\begin{array}{c}\text { pERK } \\
1 / 2\end{array}$ & $\begin{array}{l}42 \\
44\end{array}$ & $\begin{array}{c}5 \% \text { BSA } \\
\text { in T-TBS }\end{array}$ & $1 \mathrm{~h}$ & RT & $\begin{array}{c}\text { Mouse monoclonal } \\
\text { p-ERK } 1 / 2 \\
\text { (Santa Cruz supplied } \\
\text { by Thermo Fisher, } \\
\text { Auckland, NZ) }\end{array}$ & $1: 500$ & ON & $4^{\circ} \mathrm{C}$ & $\begin{array}{c}\text { Goat anti- } \\
\text { mouse Cy5 } \\
\text { (GE } \\
\text { Healthcare, } \\
\text { Auckland, } \\
\text { NZ) }\end{array}$ & $1: 5000$ & $1 \mathrm{~h}$ & RT, dark \\
\hline $\begin{array}{l}\text { ERK } \\
1 / 2\end{array}$ & $\begin{array}{l}42 \\
44\end{array}$ & $\begin{array}{l}5 \% \text { BSA } \\
\text { in T-TBS }\end{array}$ & $1 \mathrm{~h}$ & RT & $\begin{array}{c}\text { Rabbit monoclonal } \\
\text { ERK } 1 / 2 \\
\text { (Cell Signalling } \\
\text { supplied by Thermo } \\
\text { Fisher) }\end{array}$ & $1: 1000$ & ON & $4^{\circ} \mathrm{C}$ & $\begin{array}{l}\text { Goat anti- } \\
\text { rabbit Cy5 } \\
\text { (GE } \\
\text { Healthcare) }\end{array}$ & $1: 5000$ & $1 \mathrm{~h}$ & $\mathrm{RT}$, dark \\
\hline p-p38 & 43 & $\begin{array}{l}5 \% \text { BSA } \\
\text { in T-TBS }\end{array}$ & $1 \mathrm{~h}$ & RT & $\begin{array}{l}\text { Rabbit monoclonal } \\
\text { p-p38 } \\
\text { (Cell Signalling) }\end{array}$ & 1:1000 & ON & $4^{\circ} \mathrm{C}$ & $\begin{array}{l}\text { Goat anti- } \\
\text { rabbit Cy5 } \\
\text { (GE } \\
\text { Healthcare) }\end{array}$ & $1: 5000$ & $1 \mathrm{~h}$ & $\mathrm{RT}$, dark \\
\hline p38 & 43 & $\begin{array}{l}5 \% \text { BSA } \\
\text { in T-TBS }\end{array}$ & $1 \mathrm{~h}$ & RT & $\begin{array}{l}\text { Rabbit monoclonal } \\
\text { p38 } \\
\text { (Cell Signalling) }\end{array}$ & $1: 1000$ & ON & $4^{\circ} \mathrm{C}$ & $\begin{array}{l}\text { Goat anti- } \\
\text { rabbit Cy5 } \\
\text { (GE } \\
\text { Healthcare) }\end{array}$ & $1: 5000$ & $1 \mathrm{~h}$ & $\mathrm{RT}$, dark \\
\hline GAPDH & 40.2 & $\begin{array}{l}5 \% \text { BSA } \\
\text { in T-TBS }\end{array}$ & $1 \mathrm{~h}$ & RT & $\begin{array}{l}\text { Mouse monoclonal } \\
\text { anti-GAPDH (Abcam, } \\
\text { supplied by } \\
\text { Sapphire Bioscience, } \\
\text { Hamilton NZ) }\end{array}$ & $1: 5000$ & $1 \mathrm{~h}$ & RT & $\begin{array}{l}\text { Goat anti- } \\
\text { mouse Cy5 } \\
\text { (GE } \\
\text { Healthcare) }\end{array}$ & $1: 5000$ & $1 \mathrm{~h}$ & $\mathrm{RT}$, dark \\
\hline
\end{tabular}

Key: RT, room temperature; ON, overnight, BSA, bovine serum albumin

\section{Table 2.3: Antibodies used for Western blotting and their conditions}




\section{Chapter 3: Investigating the preclinical anti- cocaine effects of novel Sal A analogues}

\subsection{Introduction}

\subsubsection{Modelling craving and relapse behaviours: Reinstatement of drug seeking}

Drug self-administration studies have been widely practiced since it was discovered that animals will self-administer many different drugs of abuse. In 1962, Weeks described the use of an automated system that delivers i.v. infusions of morphine to unrestrained rats upon performance of a task (Weeks, 1962). The discovery that psychoactive substances have vastly similar abuse potential in both rats and non-human primates increased the popularity of the rat self-administration paradigm (Collins et al., 1983). The selfadministration model is today considered the gold standard for studying drug abuse preclinically because of the multiple similarities between animal and human drug use (Katz and Higgins, 2003; Carter and Griffiths, 2009). Thus, self-administration studies review a high predictive validity for human drug use, prompting its use as a tool to study potential pharmacotherapies preclinically (Haney and Spealman, 2008; O'Connor et al., 2011).

The drug self-administration paradigm is highly valued due to its voluntary nature by use of a 'Skinner box', an operant conditioning chamber (Skinner, 1953). In a drug selfadministration model, animals are implanted with an intrajugular catheter that is attached to a pump that delivers a drug infusion upon completion of physical tasks (Weeks, 1962; de Wit and Stewart, 1981). The drug acts as a positive reinforcer, prompting repetition of the tasks (Weeks, 1962). This association is rapidly learned and stably maintained in the continual presence of a rewarding drug. Some drugs, such as cocaine, are stronger inducers of self-administration than others, such as MDMA or nicotine. The operant self-administration paradigm reveals an animal's voluntary intake and temporal distribution of responses in the absence of human intervention (Sanchis-Segura and Spanagel, 2006), increasing the face value and predictive validity of studies. 
Due to the long term stability of self-administration behaviour, various manipulations such as introduction of an extinction and reinstatement phase can be conducted to test for changes in operant responses. In the extinction phase, absence of the reinforcer decreases task responses. The behaviour can be re-established by application of triggers such as exposure to a drug, drug-related cues, or stress (de Wit and Stewart, 1981; Erb et al., 1996). As all three are also main triggers for drug craving and subsequently relapse in humans (de Wit, 1996; Sinha et al., 1999), the reinstatement procedure has been used as an approximation of human relapse to drug abuse or as an assessment of drug seeking (Shalev et al., 2002; Katz and Higgins, 2003).

There are three main types of reinstatement models: the between-session, withinsession, and between-within session models. Each phase in the between-session procedure is conducted over consecutive daily sessions (Stretch et al., 1971), whereas the within-session procedure consists of all three phases conducted within the same day (de Wit and Stewart, 1981). Each session consists of $1-2 \mathrm{~h}$ of self-administration, followed by a $3-4 \mathrm{~h}$ extinction period before the reinstatement test (Shaham et al., 2003). The between-within procedure takes place over several days, in which the self-administration phase is conducted over different days, and the extinction and reinstatement phases conducted on the same day (Tran-Nguyen et al., 1998). Of the three, the between-session procedure has an advantage because it mimics human relapse more closely in terms of its temporal dimensions (Shalev et al., 2002). The between-session procedure was therefore used in this study to determine the actions of novel KOPr agonists on cocaine-induced reinstatement of drug seeking, according to procedures previously established in our laboratory (Prevatt-Smith et al., 2011; Riley et al., 2014).

It has been shown that traditional KOPr agonists such as U69,593 (0.3 or $0.32 \mathrm{mg} / \mathrm{kg}$, s.c.), $\mathrm{U} 50,488 \mathrm{H}(30 \mathrm{mg} / \mathrm{kg}$, i.p.), and spiradoline $(1.0 \mathrm{mg} / \mathrm{kg}$, i.p.) attenuate cocaine primeinduced reinstatement of cocaine seeking in rats (Schenk et al., 1999; Schenk et al., 2000; Morani et al., 2009). Enadoline (0.01 mg/kg, i.v.) and spiradoline $(0.3 \mathrm{mg} / \mathrm{kg}$, i.v.) also produced a similar decrease in squirrel monkeys (Rüedi-Bettschen et al., 2010). Further investigation of novel KOPr agonists showed that Sal A $(0.3$ and $1.0 \mathrm{mg} / \mathrm{kg}$, i.p.) (Morani et al., 2009), MOM Sal B (0.3 mg/kg, i.p.) (Morani et al., 2013), and Mesyl Sal B (0.3 and 
$1.0 \mathrm{mg} / \mathrm{kg}$, i.p.) (Simonson et al., 2015) also attenuated cocaine-induced drug seeking in male Sprague-Dawley rats. Therefore, the present study was designed to examine whether other Sal A analogues, specifically EOM Sal B and 16-brSal A, also reduce reinstatement of drug seeking behaviour in the same manner.

\subsubsection{Modelling cocaine-induced hyperactivity}

Acute cocaine administration produces drug-induced behavioural changes, of which an increase in locomotor activity is one of the most widely reported (Houdi et al., 1989; Cabib et al., 1991; O'Neill et al., 1999; Thorn et al., 2014). Motor enhancement provides an easy and quick method for evaluating the anti-cocaine effects of KOPr agonists. In support of this, U69,593 (0.16 - $0.32 \mathrm{mg} / \mathrm{kg}$, s.c.) (Heidbreder et al., 1993; Vanderschuren et al., 2000; Collins et al., 2001b)), U50,488 (10 mg/kg, i.p.) (Vanderschuren et al., 2000), and bremazocine ( $0.1 \mathrm{mg} / \mathrm{kg}, 0.32 \mathrm{mg} / \mathrm{kg}$, s.c.) (Vanderschuren et al., 2000; Collins et al., 2001b) have been shown to antagonise cocaine-induced hyperactivity. Sal A also produced a dose-dependent inhibitory effect on cocaine-induced hyperactivity, with a low dose $(0.3 \mathrm{mg} / \mathrm{kg}$, i.p.) showing no effect (Morani et al., 2009) and a higher dose $(2.0 \mathrm{mg} / \mathrm{kg}$, i.p.) attenuating the behaviour (Chartoff et al., 2008). However, the lack of information on the effects of the Sal A analogues, 16-brSal A or MOM Sal B, on cocaine-induced hyperactivity prompted the present study.

\subsection{Aims and hypotheses}

The present study aimed to investigate the anti-cocaine effects of an acute administration of the novel KOPr agonists, EOM Sal B, MOM Sal B, or 16-brSal A, in rats using the cocaineprimed reinstatement of drug seeking and cocaine-induced hyperactivity models. It is hypothesised that KOPr agonists would attenuate cocaine-induced behaviours, similar to Sal A. Some of the work in this chapter was published alongside work carried out by $\mathrm{Dr}$ Aashish Morani during his doctoral research (Morani et al., 2013) and Mr David Young during his Master's degree (Riley et al., 2014). The effects of MOM Sal B on cocaineprimed reinstatement of drug seeking and EOM Sal B on cocaine-induced hyperactivity are not presented as it was conducted by Dr Aashish Morani (Morani et al., 2013) and Dr Peter Bosch (Kivell Lab, unpublished data) respectively. 


\subsection{Methods}

\subsubsection{Materials}

For the tail flick test, KOPr agonists were dissolved in propylene glycol: DMSO in a ratio of $8: 2$ to make a $2 \mathrm{mg} / \mathrm{mL}$ stock solution. This stock was then diluted with PBS to make up a $0.1 \mathrm{mg} / \mathrm{mL}$ solution. KOPr agonists were dissolved in 75\% DMSO for reinstatement and cocaine-induced hyperactivity. Injections in mice were given at a volume of $10 \mathrm{~mL} / \mathrm{kg}$, whereas rats were given injection volumes of $1 \mathrm{~mL} / \mathrm{kg}$. All cocaine and KOPr agonist injections were administered i.p. either 5 min (Sal A, EOM Sal B, MOM Sal B) or $10 \mathrm{~min}$ (16-brSal A) before testing. The KOPr antagonist nor-BNI was dissolved in physiological saline and injected s.c. $24 \mathrm{~h}$ prior to testing.

\subsubsection{Hot water tail flick test}

In order to determine the pre-treatment time and duration of action of 16 -brSal A, the hot water tail flick test was conducted according to Simonin et al. (1998) and Simonson et al. (2015) . Mice were restrained in a transparent plexiglass tube (internal diameter of 24 $\mathrm{mm}$ ) and allowed to acclimatise for $15 \mathrm{~min}$. During this period, their tail length was measured and a third of the tail marked. The distal portion of the tail was then immersed in a water bath $\left(50 \pm 0.5^{\circ} \mathrm{C}\right)$, and the latency of tail flick measured three times and averaged to give the control latency. Mice were then given an i.p. injection of either vehicle, Sal A $(1.0 \mathrm{mg} / \mathrm{kg})$ or $16-\mathrm{brSal} \mathrm{A}(1.0 \mathrm{mg} / \mathrm{kg})$ and the latency of tail flick re-measured at $1,5,10,15,30,45,60,90,120$, and 150 min post-injection. The maximum possible effect of analgesia was calculated using the following formula:

\section{$\%$ Maximum possible effect $=100 \times[($ Test Latency - Control Latency $) /(10-$ Control Latency)]}

The test was aborted and subjects disqualified if mice displayed a latency of $10 \mathrm{~s}$ or more, to prevent overexposure to high temperatures and subsequent tail tissue damage (Simonin et al., 1998; Valjent et al., 2002). 


\subsubsection{Cocaine prime-induced reinstatement of drug seeking}

Male Sprague-Dawley rats underwent surgery and were trained to self-administer cocaine as described in sections 2.1 .3 and 2.1.4. Rats were allowed to stably self-administer cocaine $(0.5 \mathrm{mg} / \mathrm{kg})$ for at least 10 days before reinstatement testing commenced. Reinstatement of cocaine seeking was carried out in 3 phases (Figure 3.1). Phase 1 consisted of at least 2 days of cocaine self-administration for $2 \mathrm{~h}$ on an FR5 schedule of reinforcement. Rats progressed to phase 2 when their responses were within $20 \%$ of the baseline responses. In phase 2 (extinction), the light cue was removed and the cocainefilled syringe was replaced with a syringe containing heparinised saline. Rats were kept on this schedule until their lever presses dropped to less than 20 active lever responses in a single $2 \mathrm{~h}$ session (about $3-4$ days). Once extinction criteria were reached, rats were subjected to reinstatement (phase 3 ), in which they were injected with either vehicle $(75 \%$ DMSO), 16 -brSal A (0.3 or $1.0 \mathrm{mg} / \mathrm{kg}$ ), EOM Sal B (0.1 or $0.3 \mathrm{mg} / \mathrm{kg})$, or Sal A (0.3 mg/kg) before a priming injection of cocaine $(20 \mathrm{mg} / \mathrm{kg})$. Rats were then placed into the chamber and the light cue restored. The number of active lever presses reinforced with heparinised saline throughout this session was recorded. To determine if a recorded decrease in number of active lever presses was KOPr-mediated, rats were pre-treated with nor-BNI $24 \mathrm{~h}$ prior to a reinstatement session.

\subsubsection{Cocaine-induced locomotor hyperactivity}

Male Sprague-Dawley rats weighing between $300-350 \mathrm{~g}$ were habituated in activity chambers (Med Associates, ENV-520) for 30 min. Naïve rats were then given either an injection of vehicle or KOPr agonist (1.0 mg/kg of $16-\mathrm{brSal} A$ or $0.3 \mathrm{mg} / \mathrm{kg}$ of MOM Sal B) before an injection of cocaine $(20 \mathrm{mg} / \mathrm{kg})$. They were returned immediately into the activity chambers, where their horizontal movements were measured by two 16 beam infrared arrays for $60 \mathrm{~min}$. The breaks in the infrared beam were recorded by the Med Associates Activity Monitor software (Med Associates, SOF-811) as ambulatory counts. All experiments were carried out in the dark between 09:00 and 17:00 in the presence of white noise. 


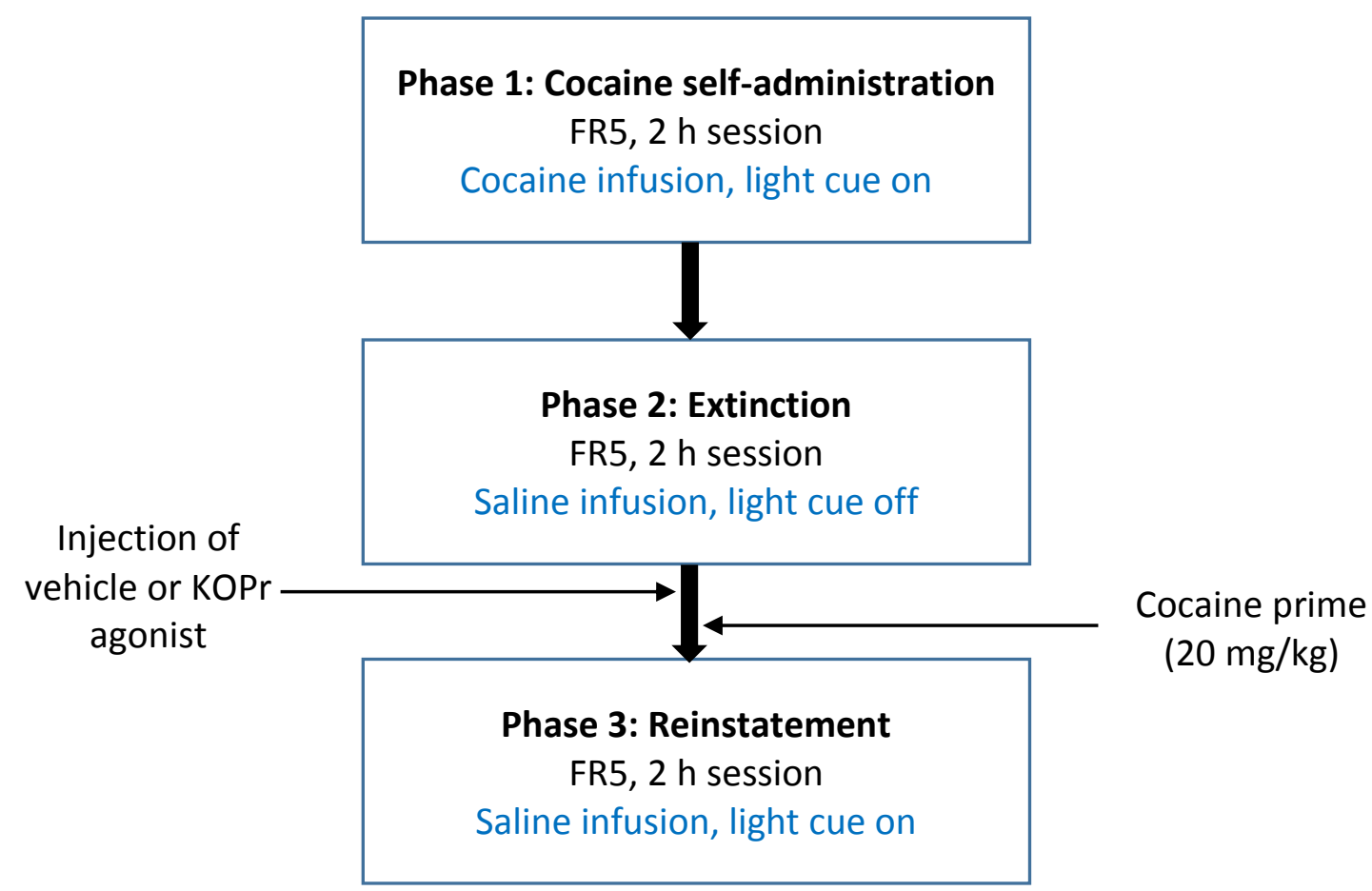

\section{Figure 3.1: Outline for cocaine-induced reinstatement of drug seeking}

In phase 1 , rats received an infusion of cocaine and a light cue upon completion of the fixed ratio. Once the number of active lever presses during two consecutive sessions varied less than $20 \%$ of the baseline, rats progressed to the extinction phase (phase 2). In phase 2, the light cue was removed and cocaine was replaced with heparinised saline. Once extinction criteria were achieved, phase 3 commenced. Rats were injected with either vehicle or KOPr agonist before a priming injection of cocaine $(20 \mathrm{mg} / \mathrm{kg}$, i.p.). They were placed into the chamber immediately after the cocaine injection and the number of active lever responses during this session recorded.

\subsubsection{Statistics}

For the tail flick and cocaine-induced hyperactivity tests, a one-way analysis of variance (ANOVA) with repeated measures was conducted. Paired comparisons with an applied Bonferroni correction was then used to compare the effects of the treatment to vehicle controls. In the reinstatement study, a repeated measures ANOVA was performed with the Bonferroni multiple comparison test to determine significance between treatment groups, whereas the time course data was analysed using a one-way ANOVA with repeated measures. Unless otherwise stated, all values are presented as mean \pm SEM. Data were considered statistically significant when $p \leq 0.05$. 


\subsection{Results}

\subsubsection{Onset and duration of action of 16-brSal A in vivo}

The hot water tail flick assay was used to determine the in vivo duration of action of KOPr agonists. Since Mauchley's test of sphericity indicated that the assumption of sphericity had been violated $\left[\chi^{2}(54)=124.637, p<0.0001\right]$, the Greenhouse Geisser correction was applied and a significant interaction was found $[F(9.052,117.679)=2.68, p=0.007]$ (oneway ANOVA with repeated measures, Figure 3.2). The treatments were then compared to each other and the vehicle at each time point using planned comparisons with the Bonferroni correction. This Sal A-produced anti-nociception was significant at the 5, 10 and 15 min time points. At $5 \mathrm{~min}(\mathrm{p}<0.05)$, a $14 \pm 3 \%$ increase in maximum possible effect was observed compared to control (Figure 3.2). The latency increased to $27 \pm 7 \%$ at 10 $\min (p<0.001)$ and $20 \pm 4 \%$ at $15 \mathrm{~min}(p<0.001)$. No significant differences were noted at later time points (Figure 3.2).

Similar to its parent compound, 16-brSal A also produced a significant increase in antinociception compared to vehicle controls at $10 \mathrm{~min}(\mathrm{p}<0.05)$, whereby 16 -brSal A-treated rats displayed a $15 \pm 4 \%$ increase in anti-nociceptive effects compared to vehicle-treated rats (Figure 3.2). This elevated analgesia was also seen at $15 \mathrm{~min}(15 \pm 5 \%, \mathrm{p}<0.05)$ and 30 $\min (15 \pm 3 \%, p<0.05)$. 16-brSal A produced the highest percentage of anti-nociceptive effect at $45 \mathrm{~min}$, with $21 \pm 6 \%$ of the maximum possible effect $(p<0.01)$. The effects of 16 brSal A started to decline after $45 \mathrm{~min}$, with a significant effect still observed at $60 \mathrm{~min}$ (19 $\pm 6 \%, p<0.001$ ) but not at any further time points (Figure 3.2). Although 16-brSal A showed a delayed onset and longer duration of analgesic action compared to Sal A, planned comparisons between Sal A and 16-brSal A presented no significant differences at any time points (Figure 3.2). 


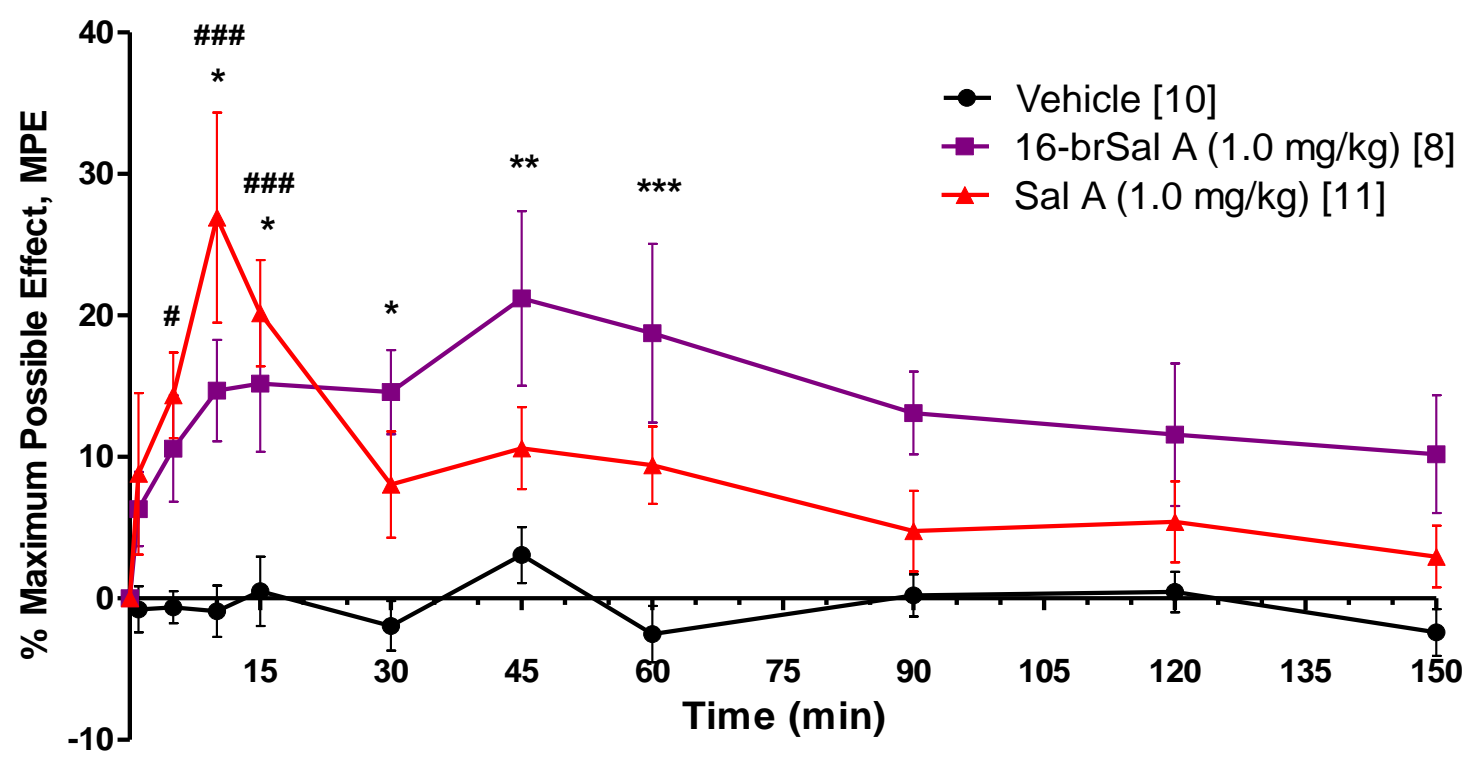

\section{Figure 3.2: 16-brSal A has a longer duration of action in vivo than Sal A}

Tail flick latencies in mice were measured before and after treatment injections at 1, 5, 10, 15, 30, 45, 60, 90, 120, and 150 min. 16-brSal A showed a significant increase in tail flick latency at 10, 15, 30,45 , and 60 min compared to vehicle controls, whereas Sal A showed a significant increase only at 5,10 , and 15 min. 16-brSal A displayed a slower onset and longer duration of action compared to Sal $A$, although no significant differences were found between the two. One-way ANOVA with repeated measures followed by planned comparisons with the Bonferroni correction, 16-brSal A: ${ }^{*} \mathrm{p}<0.05,{ }^{* *} \mathrm{p}<0.01,{ }^{* * *} \mathrm{p}<0.001$ compared to the vehicle; Sal $\mathrm{A}:{ }^{\# \#} \mathrm{p}<0.01,{ }^{\# \#} \mathrm{p}<0.001$ compared to the vehicle. Values presented as mean \pm SEM, $n=8-11$, number in square brackets represent $n$. 


\subsubsection{Effect of 16-brSal A and EOM Sal B on cocaine-induced drug seeking}

Rats were initially trained to self-administer cocaine on a FR1 schedule of reinforcement. The rate of self-administration acquisition behaviour of male Sprague-Dawley rats is shown in Figure 3.3A. Rats rapidly learnt the behaviour, with a mean acquisition time of 4 \pm 1 days (Figure 8.9 in Appendix 6.2). All rats achieved acquisition criteria by day 7 (Figure 3.3A). Rats then progressed to FR2, and subsequently the FR5 schedule of reinforcement. Rats stably self-administering cocaine on FR5 consistently displayed preference for the active lever, with a daily average of $189 \pm 3$ lever presses (Figure 3.3B). In contrast, responses on the inactive lever was almost non-existent, with mean daily levels of only $0.3 \pm 0.1$ lever responses (Figure 3.3B).

Prior to testing with KOPr agonists, rats were subjected to extinction and a cocaine primeonly reinstatement to verify establishment of cocaine-induced reinstatement behaviour. Figure $3.3 \mathrm{C}$ shows a comparison of the number of active lever responses during the baseline, extinction, and reinstatement phases. Comparison between treatments were carried out using the paired Student's $t$-test with the Bonferroni correction. Removal of the cocaine reinforcer significantly extinguished lever pressing to less than $10 \%$ of the baseline, producing $12 \pm 1$ active lever presses vs. baseline values of $210 \pm 11$ active lever responses ( $p<0.001$, Figure $3.3 C$ ). In contrast, exposure to a cocaine prime significantly reestablished lever pressing behaviour to $191 \pm 20$ active lever responses $(p<0.001$, Figure 3.3C). There was no significant difference between the number of baseline responses and reinstatement responses (Figure 3.3C). Rats were returned to a self-administration baseline between each reinstatement test. The baseline and extinction responses were consistently stable throughout the whole study (Figure 3.3D), indicating that changes in active lever responses when KOPr agonists were administered could be attributed to addition of the KOPr agonist. 
A)
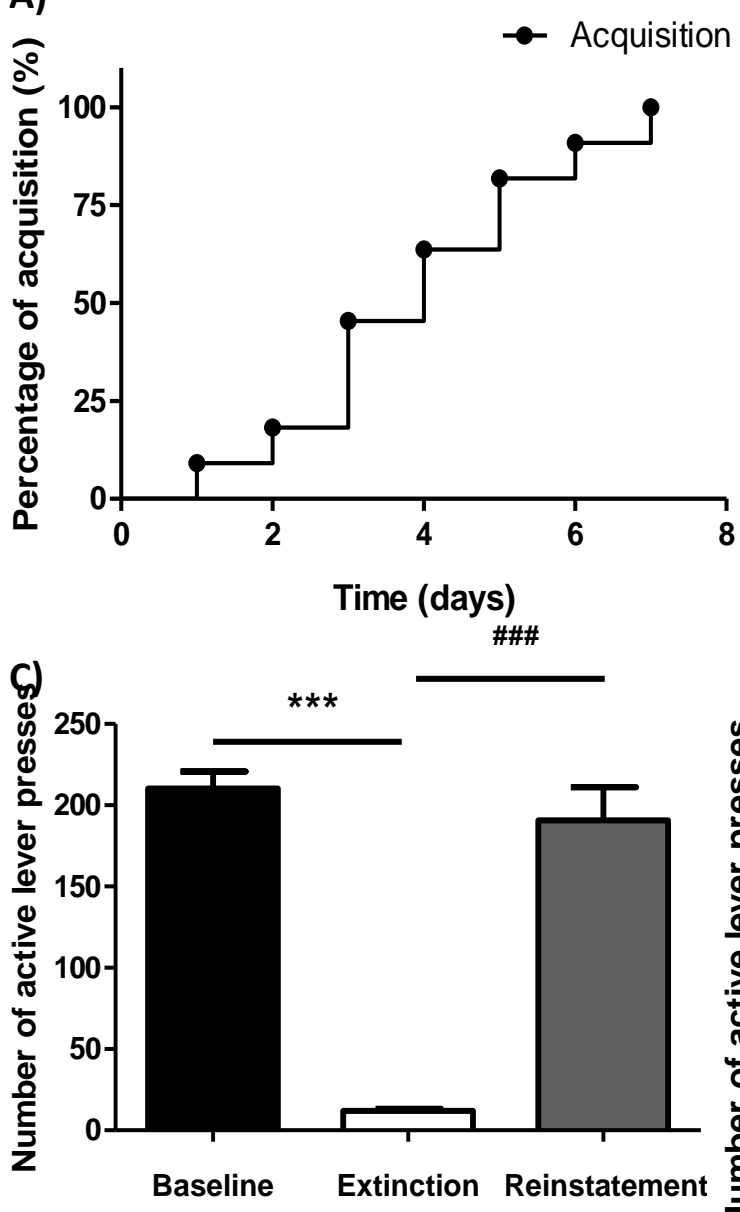

B)

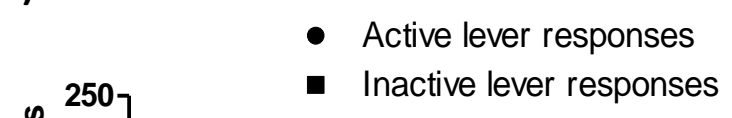

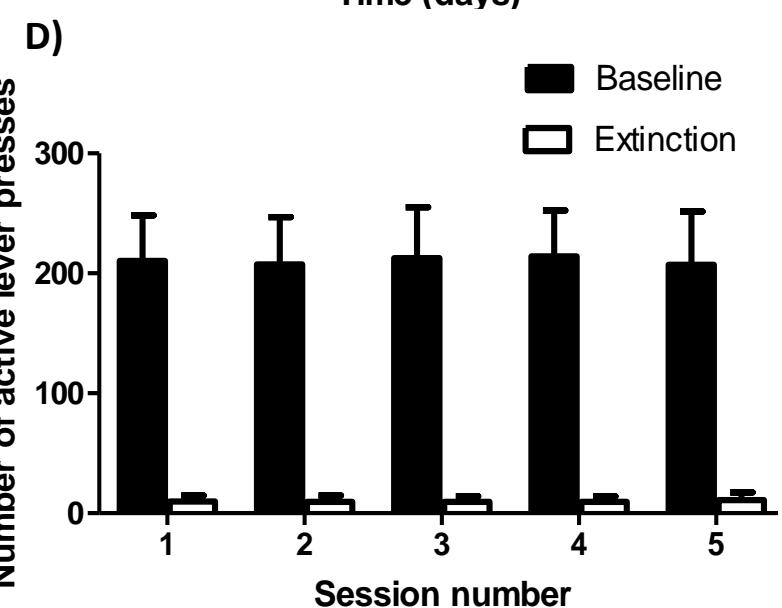

Figure 3.3: Self-administration training

A) The earliest rat acquired self-administration behaviour on day 1, whereas all rats achieved acquisition by day 7 . B) The number of active and inactive lever presses remained stable throughout self-administration training. C) Number of active lever responses in the extinction phase were significantly decreased compared to the baseline, whereas administration of a cocaine prime (20 $\mathrm{mg} / \mathrm{kg}$ ) significantly increased active lever presses in the reinstatement phase (both $p<0.001$, paired Student's $t$-test with the Bonferroni correction). D) Analysis of number of active lever presses during baseline and extinction phases prior to each reinstatement session revealed no significant changes throughout the study (a total of 6 reinstatement sessions). ${ }^{* *} p<0.001$ compared to baseline, $\# \#<<0.001$ compared to extinction. Values presented as mean $\pm S E M, n=11$. 
Following self-administration training, rats were divided into 2 groups. One group received 16 -brSal $A(0,0.3$, and $1.0 \mathrm{mg} / \mathrm{kg}, \mathrm{n}=6)$, whereas the other received EOM Sal $B$ $(0,0.1$, and $0.3 \mathrm{mg} / \mathrm{kg})$ prior to a reinstatement session $(\mathrm{n}=5)$. All KOPr agonists were delivered using a Latin square design (a counter-balanced manner in which each rat received treatments in a different order) to prevent treatment bias (Appendix 3.1), except for the selective KOPr antagonist, nor-BNI, which had to be delivered last due to longlasting effects of the compound (Endoh et al., 1992; Horan et al., 1992; Kishioka et al., 2013). A significant effect of treatment was found $[F(3,15)=8.349, p=0.0017]$ ( repeated measures ANOVA, Figure 3.4A), with 16-brSal A significantly reducing active lever presses in rats (paired Student's t-test with the Bonferroni correction). The lower dose of 16-brSal A $(0.3 \mathrm{mg} / \mathrm{kg})$ produced no significant effect compared to vehicle controls, resulting in 187 \pm 54 active lever responses vs. vehicle responding of $292 \pm 77$ active lever presses. On the other hand, the higher dose of $16-\mathrm{brSal} A(1.0 \mathrm{mg} / \mathrm{kg})$ reduced active lever responding by almost $60 \%$ compared to the vehicle, producing $126 \pm 35$ lever presses $(p<0.01$, Figure 3.4A). This 16-brSal A-modulated decrease was dependent on actions at the KOPr, since prior administration of nor-BNI $(10 \mathrm{mg} / \mathrm{kg})$ significantly inhibited the attenuation, resulting in $215 \pm 27$ active lever presses ( $p<0.05$, paired Student's $t$-test). However, further analysis on the cumulative responding in 5 min intervals revealed no significant interaction between time and treatments $[F(23,115)=1.030, p=0.4349]$ (one-way ANOVA with repeated measures, Figure $3.4 \mathrm{C})$. Sal $A(0.3 \mathrm{mg} / \mathrm{kg})$ also reduced lever pressing by almost $60 \%$ to $123 \pm 29$ active lever responses ( $p<0.01$, paired Student's t-test with the Bonferroni correction, Figure 3.4A). This finding is consistent with a previously published report, in which Sal A dose-dependently reduced cocaine-primed drug seeking in rats (Morani et al., 2009). 

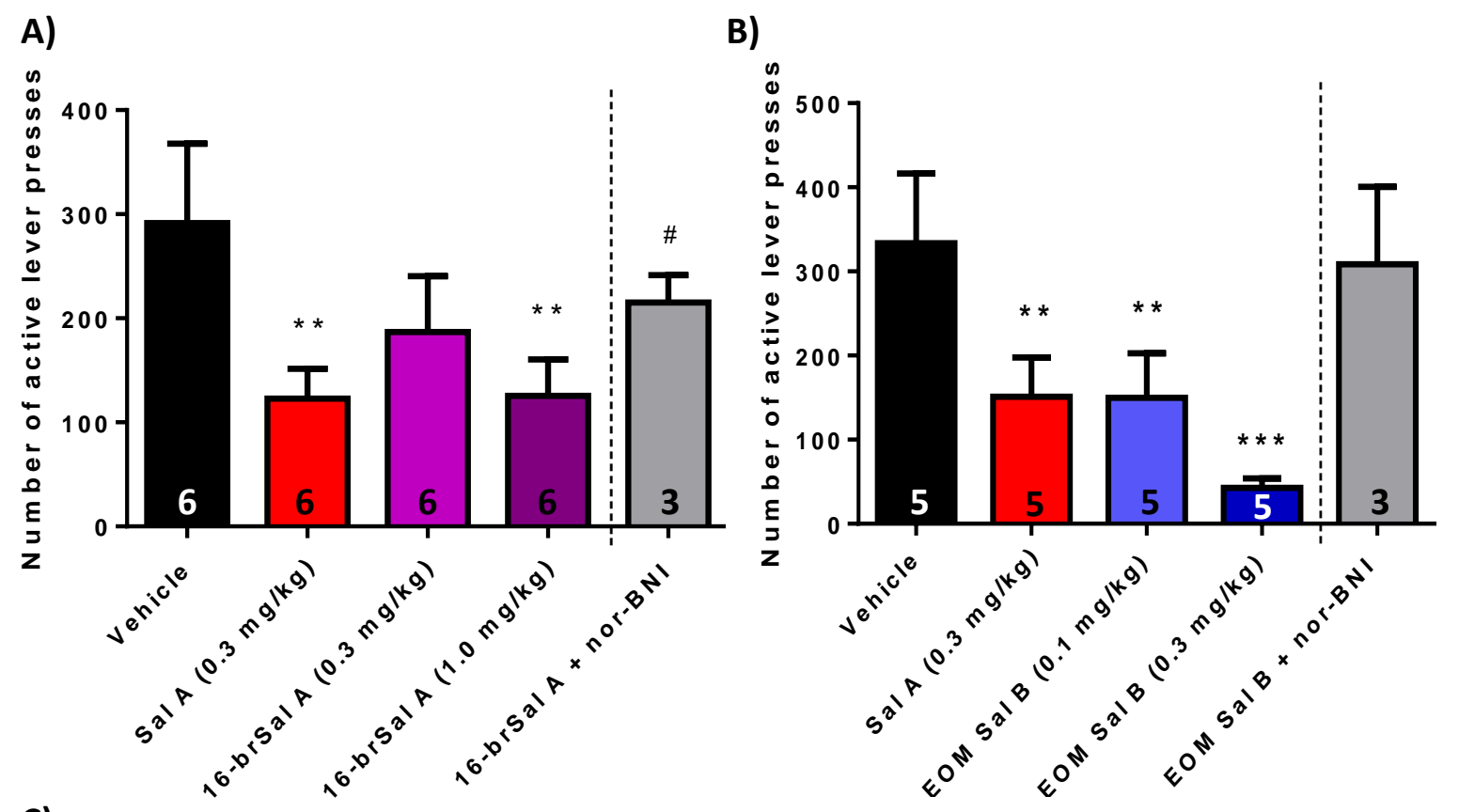

C)

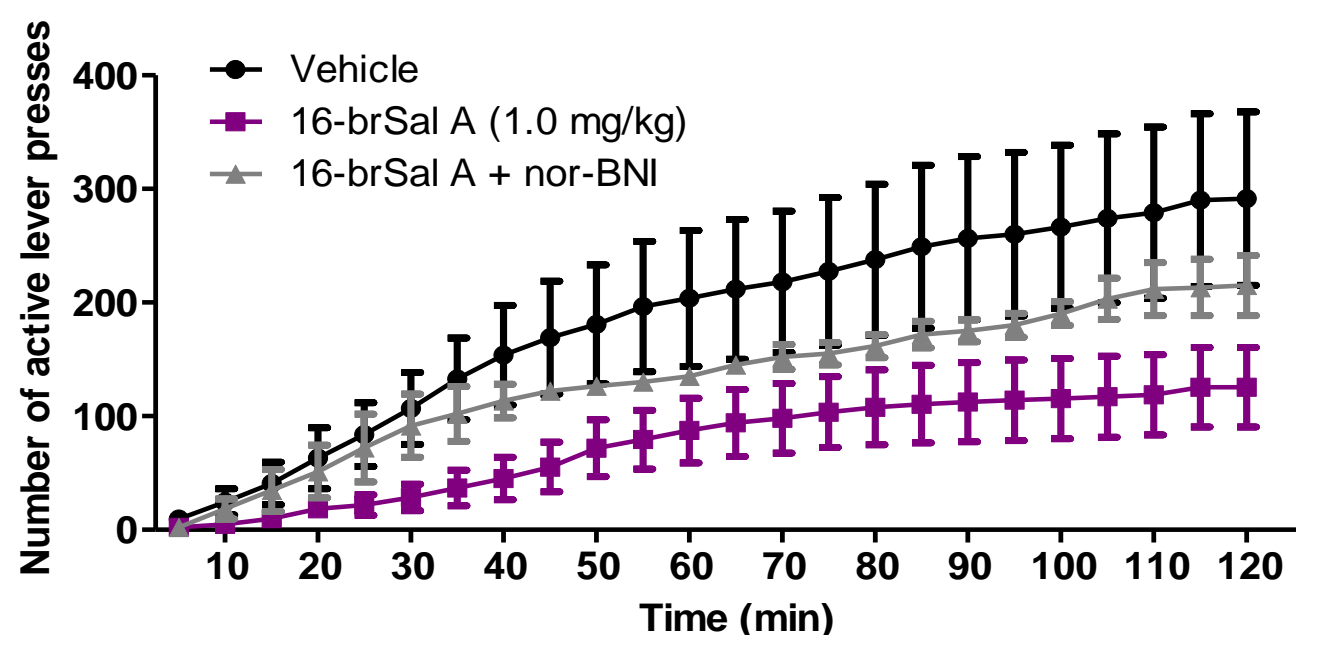

D)

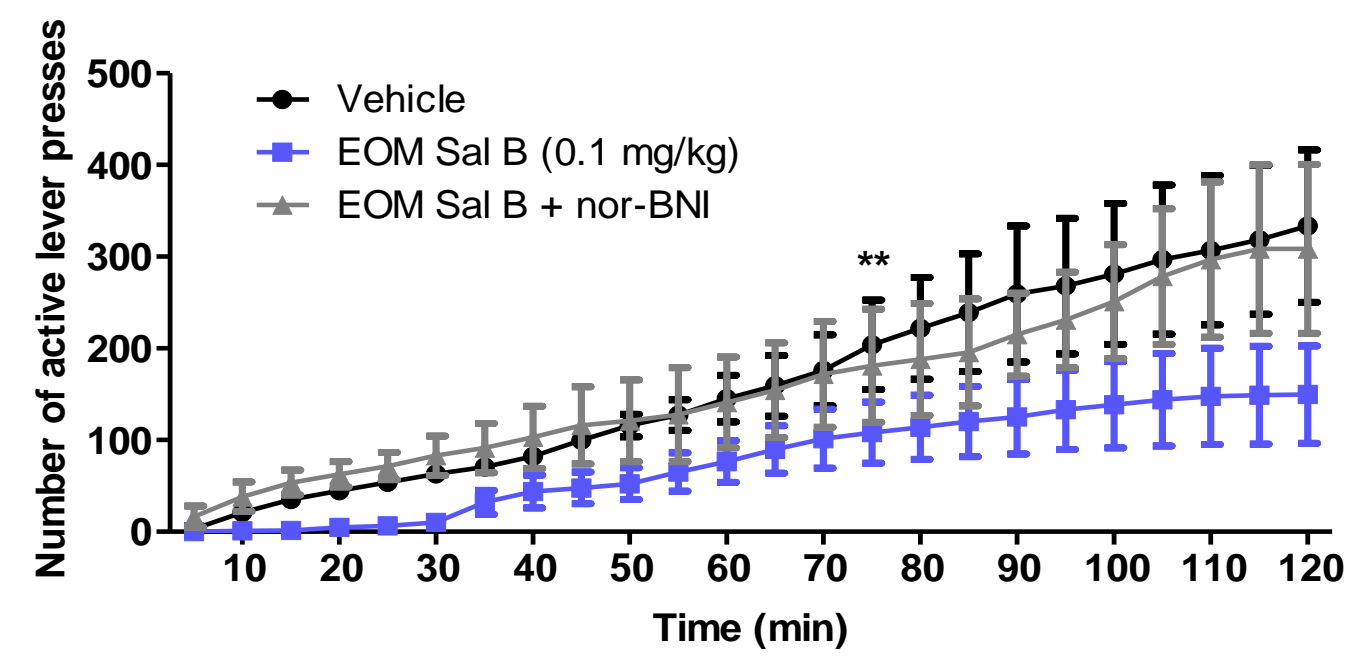




\section{Figure 3.4: 16-brSal A and EOM Sal B decrease cocaine-primed reinstatement of cocaine}

\section{seeking in rats}

A \& B) Rats received vehicle (75\% DMSO), 16-brSal A, EOM Sal B, or Sal A prior to cocaine (20 $\mathrm{mg} / \mathrm{kg}$, i.p.), and reinstatement was tested in a $2 \mathrm{~h}$ session. 16 -brSal $\mathrm{A}$, EOM Sal $B$, and Sal $A$ significantly attenuated cocaine-induced reinstatement of drug seeking compared to vehicle controls (repeated measures ANOVA followed by paired Student's t-tests with the Bonferroni correction, $n=5-6)$. Pre-treatment of rats with nor-BNI $(10 \mathrm{mg} / \mathrm{kg})$ significantly prevented the anticocaine effects of the higher dose of 16-brSal A, but no significant inhibition was seen of the EOM Sal B response ( $p=0.08$, paired Student's $t$-test, $n=3-6)$. C \& D) Analysis of the cumulative reinstatement responding in 5 min intervals revealed no significant interaction between time and treatment for 16-brSal A, but a significant interaction for EOM Sal B (one way ANOVA with repeated measures, $n=3-6)$. ${ }^{* *} p<0.01,{ }^{* * *} p<0.001$ compared to vehicle, ${ }^{\#} p<0.05$ compared to 16 -brSal $A$ $(1.0 \mathrm{mg} / \mathrm{kg})$. Values presented as mean \pm SEM. 
Rats in the EOM Sal B group also displayed a significant difference in mean number of active lever presses between vehicle and treatments $[F(3,12)=5.829, p=0.0107]$ (repeated measures ANOVA, Figure 3.4B). Multiple comparisons conducted with the Bonferroni correction showed that the lower dose of EOM Sal B $(0.1 \mathrm{mg} / \mathrm{kg})$ significantly decreased lever pressing behaviour to $150 \pm 53$ from vehicle values of $333 \pm 83$ active lever responses $(p<0.01)$. On the other hand, the higher dose of EOM Sal B $(0.3 \mathrm{mg} / \mathrm{kg})$ produced a $87 \%$ reduction in responding compared to vehicle-treated rats, resulting in 43 \pm 11 active lever presses ( $p<0.001$, Figure 3.4B). The parent compound, Sal A $(0.3 \mathrm{mg} / \mathrm{kg})$ attenuated active lever responding to a similar magnitude to the low dose of EOM Sal B, resulting in $151 \pm 47$ active lever responses ( $p<0.01$, Figure 3.4B). Pre-treatment of rats with nor-BNI increased active lever presses to $309 \pm 92$, but the difference was not significant $(p=0.08$, paired Student's $t$-test, Figure 3.4B). However, the distinct trend towards significance suggests that the anti-cocaine effects of EOM Sal B are likely to also be mediated by the KOPr. Further analysis of the cumulative reinstatement responding revealed a significant interaction between treatment and time $[F(23,92)=1.692, p=$ 0.0415] (one-way ANOVA with repeated measures, Figure 3.4D). A subsequent planned comparison with the Bonferroni correction between treatments at each time point revealed a significant effect of EOM Sal B compared to the vehicle at $75 \min (p<0.01$, Figure 3.4D).

Thus, both 16-brSal A and EOM Sal B dose-dependently decreased cocaine-induced reinstatement of drug seeking. 


\subsubsection{Effect of 16-brSal A and MOM Sal B on cocaine-induced hyperactivity}

To further evaluate the anti-cocaine effects of 16-brSal A and MOM Sal B, their effect on cocaine-induced motor activity were measured. Rats treated with 16-brSal A $(1.0 \mathrm{mg} / \mathrm{kg})$ displayed an activity count of $3789 \pm 1514$, a significant decrease compared to vehicle controls, which showed an average activity count of $9490 \pm 982$ ( $p<0.05$, Student's $t$-test, Figure $3.5 \mathrm{C}$ ). When analysed in segments of $5 \mathrm{~min}$, there was a significant interaction between treatment and time $[F(34,187)=2.50, p<0.0001]$ (one-way ANOVA with repeated measures, Figure 3.5A). Subsequent planned comparisons with the Bonferroni correction between treatments at each time point revealed that rats treated with 16-brSal A significantly differed from vehicle-treated rats in the first 20 min post-treatment. Pretreatment of rats with nor-BNI $(10 \mathrm{mg} / \mathrm{kg})$ significantly tripled the ambulatory counts of rats compared to $16-\mathrm{brSal} \mathrm{A}$ on its own, producing $12474 \pm 4454$ ambulatory counts $(p<0.05$, Student's $t$-test, Figure $3.5 \mathrm{C})$. Nor-BNI also significantly prevented the anticocaine effects of 16 -brSal $A$ at the 5 min time point ( $p<0.001$, Figure 3.5A). No significant difference in ambulatory counts was observed between the three groups of rats throughout habituation (Figure 3.5B); thus, the changes seen were due to addition of test compounds.

MOM Sal B-treated rats $(0.3 \mathrm{mg} / \mathrm{kg})$ produced $5159 \pm 1063$ ambulatory counts, a nonsignificant decrease in cocaine-induced hyperactivity compared to the vehicle $(75 \%$ DMSO, $7247 \pm 1678$ ambulatory counts, Figure 3.6C). There was no significant interaction observed $[F(17,170)=1.04, p=0.4204]$ and no main effect of treatment $[F(1,170)=1.43$, $p=0.2599$ ] when data were studied in intervals of $5 \mathrm{~min}$ (one-way ANOVA with repeated measures, Figure 3.6A). Both vehicle- and MOM Sal B-treated rats displayed no significant differences in locomotor counts during habituation, suggesting a similar basal ambulatory level prior to treatment with the KOPr agonist and cocaine (Figure 3.6B).

Thus, 16-brSal A, but not MOM Sal B, significantly decreased cocaine-induced hyperactivity in male Sprague-Dawley rats. 
A)
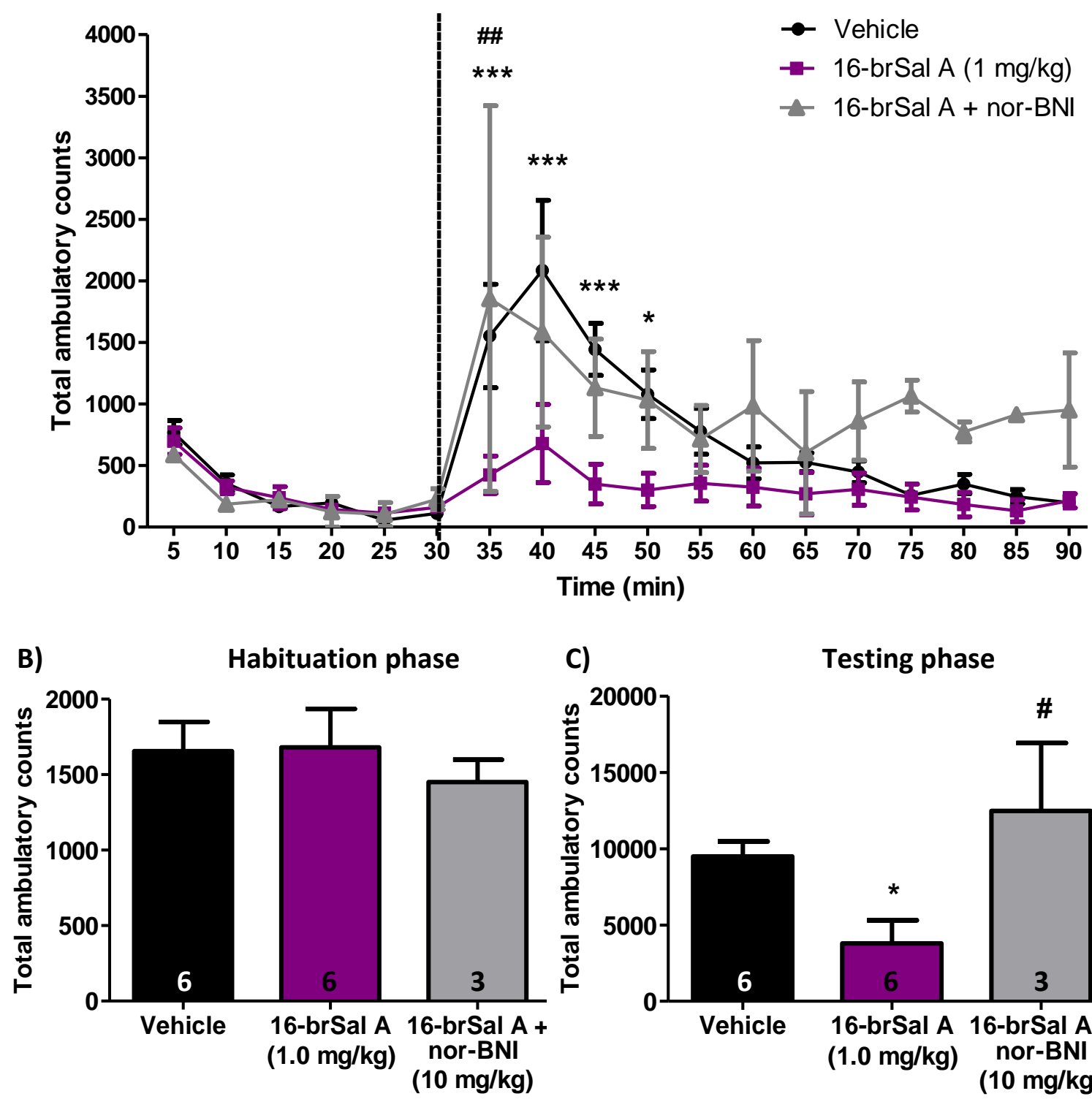

C)

Testing phase

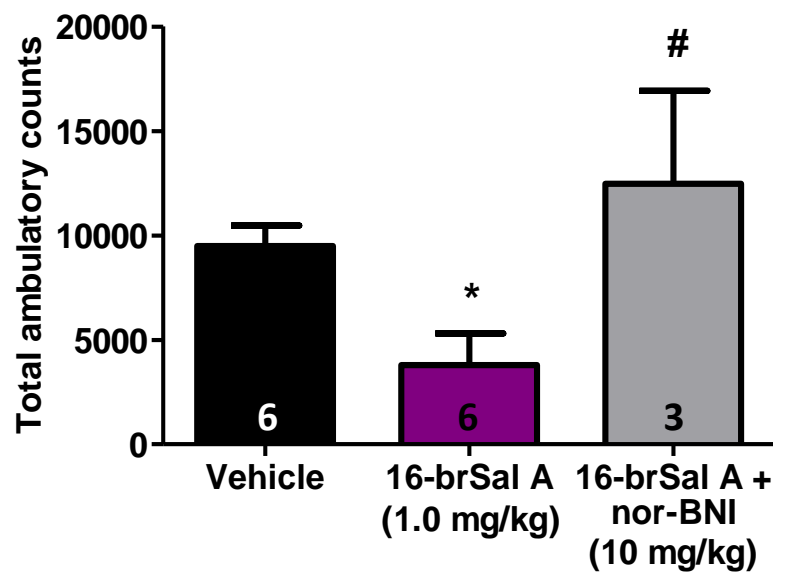

Figure 3.5: 16-brSal A reduces cocaine-induced locomotor hyperactivity

A) Rats treated with 16-brSal A followed by cocaine $(20 \mathrm{mg} / \mathrm{kg}$, i.p.) displayed a significant decrease in cocaine-induced hyperactivity at $5,10,15$, and $20 \mathrm{~min}$; an effect prevented at 5 min with prior administration of nor-BNI (one-way ANOVA with repeated measures followed by planned comparisons with the Bonferroni correction). The vertical line represents 16-brSal A and cocaine administration. B) There was no significant difference in activity between rats in the 3 different treatment groups during habituation. C) 16-brSal A produced a significant attenuation of cocaineinduced hyperactivity compared to the vehicle, an effect inhibited by nor-BNI (Student's $t$-test with the Bonferroni correction). ${ }^{*} p<0.05,{ }^{* * *} p<0.001$ compared to the vehicle, ${ }^{\#} p<0.05,{ }^{\#} p<0.01$ compared to 16-brSal A. Values presented as mean \pm SEM, $n=3-6$. 
A)

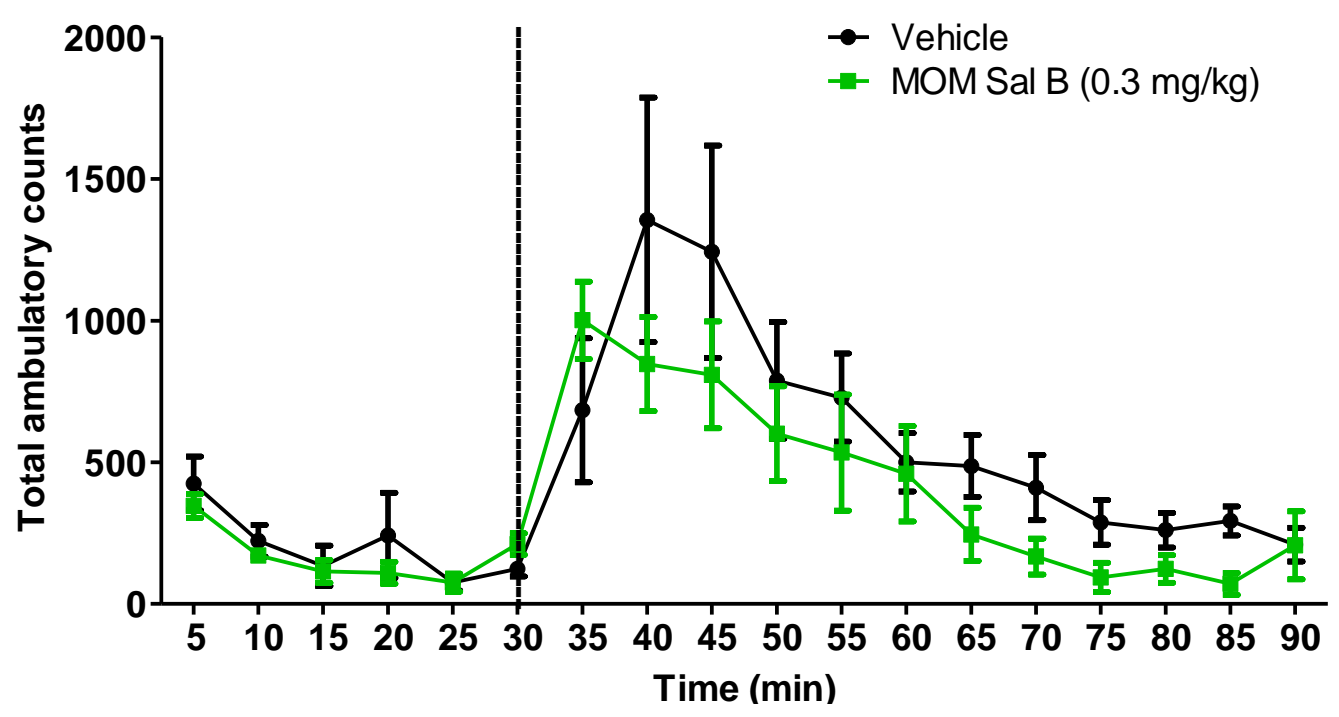

B)

Habituation phase

C)

Testing phase
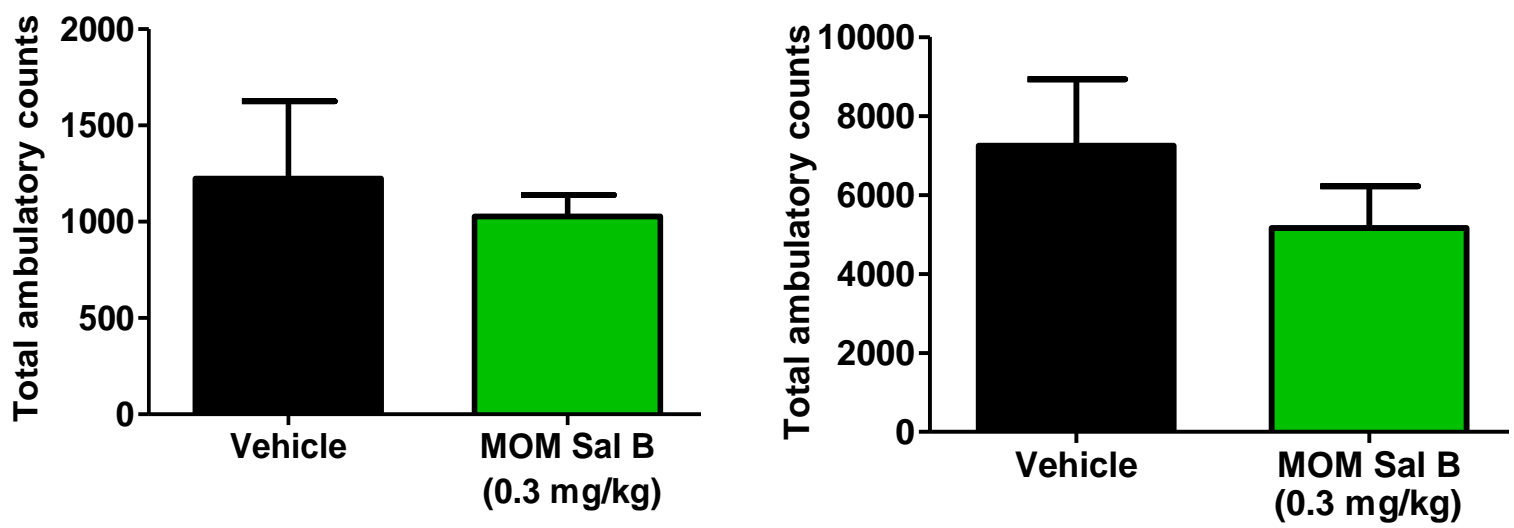

Figure 3.6: MOM Sal B does not alter cocaine-induced locomotor hyperactivity

A) Analysis of temporal distribution of ambulation in rats treated with MOM Sal B or vehicle $(75 \%$ DMSO) showed that MOM Sal B did not alter cocaine-enhanced motor function (one-way ANOVA with repeated measures). Administration of MOM Sal B and subsequently, cocaine, is indicated by the vertical line. B) Rats in both treatment groups displayed no significant difference in ambulatory counts during habituation (Student's $t$-test). C) Rats treated with MOM Sal B produced a slight but non-significant decrease in cocaine-induced hyperactivity compared to the vehicle (Student's $t$ test). Values presented as mean $\pm S E M, n=6$. 


\subsection{Discussion}

It has been shown that novel KOPr agonists have anti-cocaine properties, with analogues of Sal A such as MOM Sal B (Morani et al., 2013), Mesyl Sal B (Simonson et al., 2015), $\beta$ THP Sal B (Prevatt-Smith et al., 2011), and Ethy Sal A (Riley et al., 2014) attenuating cocaine-induced drug seeking in rats. Mesyl Sal B (Dr Aashish Morani, PhD thesis) and EOM Sal B (Kivell Lab, unpublished data) also prevented cocaine-induced hyperactivity at doses that decreased cocaine seeking, whereas Sal A inhibited cocaine-induced behavioural sensitisation (Morani et al., 2012). This study was therefore designed to explore the anti-cocaine effects of novel Sal A analogues on cocaine-induced drug seeking (16-brSal A, EOM Sal B) and cocaine-induced hyperactivity (16-brSal A, MOM Sal B).

\subsubsection{Onset and duration of action of 16-brSal A}

This study is the first to evaluate the in vivo pharmacokinetics of 16-brSal A, a C-16 Sal A analogue synthesised by Professor Thomas Prisinzano. Since KOPr agonists possess antinociceptive properties (Kivell and Prisinzano, 2010), a tail flick assay was used to determine the onset and duration of action of 16-brSal A. It was found that the effects of Sal A were consistent with the literature. A study in 2006 showed an increase in withdrawal latency with Sal A $(1.0 \mathrm{mg} / \mathrm{kg}$, i.p.) at $10 \mathrm{~min}$, but not 20 or $30 \mathrm{~min}$ postinjection (McCurdy et al., 2006). Another study showed that an intrathecal administration of Sal A (23.1 nmol) had an anti-nociceptive action in mice at 5 to 15 min post-injection but not at later times (John et al., 2006), indicating that Sal A has a significant but short duration of action regardless of study design or route of administration.

Interestingly, the present study found that 16-brSal A showed a slower onset (10 $\mathrm{min}$ ) but more prolonged duration of action (up to $60 \mathrm{~min}$ ) than Sal A (Figure 3.2). Although no significant differences were observed between the two treatments, this longer duration of action in vivo indicates that 16-brSal A may possess a better pharmacodynamic profile compared to Sal A. It is suggested that the improved pharmacodynamics displayed is due to a combination of metabolic stability and prolonged availability of free 16 -brSal $A$ in the blood. Previous positron emission tomography studies have shown that although the C-2 analogue of Sal A, EOM Sal B (i.p.), displays similar pharmacodynamics to Sal A in the rat 
brain, it showed a lower metabolism rate and decreased plasma protein affinity compared to Sal A. The authors suggested that the longer duration of action of EOM Sal B could be attributed to its improved metabolic stability and higher fraction of unbound compound compared to Sal A (Hooker et al., 2009a). It is also interesting to note that other Sal A analogues, MOM Sal B and Mesyl Sal B, for example, have also shown an increased duration of action when injected i.p., presumably because the drugs are gradually released from the injection site (Wang et al., 2008; Simonson et al., 2015). Taken together, these studies suggest that 16-brSal A showed a longer duration of in vivo action due to a combination of the injection route as well as a lower metabolism rate. Considering the short half-life of Sal A is its biggest shortcoming in terms of therapeutic potential, the increase in anti-nociceptive duration indicates that 16-brSal A may be a better candidate for clinical use.

\subsubsection{Cocaine self-administration and cocaine-induced reinstatement}

All rats trained to self-administer cocaine in daily $2 \mathrm{~h}$ sessions achieved acquisition criteria within 7 days (Figure 3.3A), consistent with a previous study in which $100 \%$ of the rats acquired cocaine $(0.5 \mathrm{mg} / \mathrm{kg} /$ infusion) self-administration behaviour within 13 training days (Schenk et al., 2007). Upon acquisition, self-administration behaviour was reliable and consistent throughout the course of the experiment, attesting to the reinforcing strength of cocaine (Aigner and Balster, 1978). Results show no escalation of cocaine (0.5 $\mathrm{mg} / \mathrm{kg} /$ infusion) intake in $2 \mathrm{~h}$ sessions (Figure 3.3B), unlike the expected increase in extended (6 h) access self-administration protocols (Chapter 6) (Ahmed and Koob, 1998). Following reliable self-administration on FR5 (Figure 3.3B), rats were subjected to extinction sessions (cocaine replaced with saline infusions and the light cue removed) until active lever presses were $<20$ in a $2 \mathrm{~h}$ session. At the beginning of the study, rats responded persistently for the active lever, requiring $6 \pm 1$ days to reach extinction criteria. However, fewer days were required as the experiment progressed, with extinction criteria reached within $2.3 \pm 0.1$ days by the $6^{\text {th }}$ reinstatement cycle (Appendix 3.2 ), indicating that rats were also quick to learn extinction behaviour.

Both 16-brSal A and EOM Sal B dose-dependently reduced active lever presses for cocaine, similar to that seen with other traditional and Sal A-derived KOPr agonists (Table 3.1). 
However, a higher dose of 16-brSal A was required to attenuate drug seeking behaviour despite in vitro studies showing the similar efficacies of 16-brSal A and Sal A at the KOPr (16-brSal A EC $50=0.040 \mathrm{nM}$ vs. Sal A EC $50=0.030 \mathrm{nM}$ ) (Riley et al., 2014). It is also interesting to note that EOM Sal B, which displays a greater efficacy compared to Sal A in discrimination studies (Peet and Baker, 2011) and in the $\left[{ }^{35} \mathrm{~S}\right] \mathrm{GTP}-\gamma-\mathrm{S}$ functional assay ( $\mathrm{EC}_{50}$ of $\mathrm{EOM}$ Sal $\mathrm{B}=0.65 \mathrm{nM}$ vs. $\mathrm{EC}_{50}$ of Sal $\mathrm{A}=40 \mathrm{nM}$ ) (Prevatt-Smith et al., 2011) significantly decreased reinstatement of cocaine seeking at a lower dose compared to Sal A (Figure 3.4B). However, although EOM Sal B is at least 10 times more potent than Sal A in vitro (Hooker et al., 2009a), the in vivo dose that produced similar attenuation of drug seeking as Sal A was only three times lower (0.1 mg/kg EOM Sal B vs. $0.3 \mathrm{mg} / \mathrm{kg}$ Sal A). A previous study involving MOM Sal B and U50,488 also showed similar discrepancies. Despite U50,488 being six times less potent than MOM Sal B $\left(U 50,488 \mathrm{EC}_{50}=3.4 \mathrm{nM}\right.$ vs. MOM Sal B EC $50=0.6 \mathrm{nM}$ ), U50,488 produced similar anti-nociceptive effects to MOM Sal $B(1 \mathrm{mg} / \mathrm{kg})$ in a rat hot plate assay only when a ten-fold dose of U50,488 was used (10 $\mathrm{mg} / \mathrm{kg}$ ) (Wang et al., 2008). Taken together, these studies suggest that although in vitro efficacy at KOPr is a good indication of pharmacological properties of an agonist, they do not always directly correlate to in vivo efficiency, affirming the need for direct measurements of behaviour in anti-cocaine studies.

The anti-cocaine effect of Sal A $(0.3 \mathrm{mg} / \mathrm{kg})$ reported in the present study is consistent with the literature (Morani et al., 2009), although this study reports a higher number of active lever presses during reinstatement compared to the former study. This difference is likely due to application of different reinstatement durations ( $2 \mathrm{~h}$ in this study vs. $1 \mathrm{~h}$ in Morani et al. (2009)) and experimental design (between-session in the present study vs. within-session in Morani et al. (2009)). Nonetheless, results from the present study are consistent with the ability of traditional and novel KOPr agonists to attenuate cocaineinduced reinstatement of drug seeking (Table 3.1) (Morani et al., 2009; Sun et al., 2010). Three rats in the EOM Sal B group and two rats in the 16-brSal A group received only one or two doses of KOPr agonist treatments due to catheter patency failure and were excluded from the study. Although the effect of 16-brSal A was inhibited with nor-BNI pretreatment (Figure 3.4A), this prevention was not significant with EOM Sal B ( $p=0.08$, Figure $3.4 B$ ) possibly due to low subject numbers $(n=3)$, a result of catheter patency loss 
before completion of the experiment. Nonetheless, temporal analysis showed that norBNI significantly attenuated EOM Sal B-mediated effects (Figure 3.4D), indicating that effect of EOM Sal B was KOPr-dependent. In support of this, cocaine-induced hyperactivity studies conducted in our laboratory with EOM Sal B showed that EOM Sal B significantly attenuated cocaine-induced hyperactivity in a nor-BNI-reversible manner (Kivell Lab, unpublished data).

\subsubsection{Cocaine-induced hyperactivity}

In light of the ability of both 16-brSal A and MOM Sal B to attenuate drug seeking, it was surprising to observe that only 16-brSal A, but not MOM Sal B, significantly decreased cocaine-induced hyperactivity in rats (Figure $3.5 \mathrm{C}$ and $3.6 \mathrm{C}$ ). It is important to note that there were no significant differences between vehicle- and MOM Sal B-treated rats in the habituation phase (Figure 3.6B), indicating that animals displayed similar levels of basal activity prior to cocaine or KOPr agonist treatment. The lack of a MOM Sal B $(0.3 \mathrm{mg} / \mathrm{kg})$ effect may be due to the lower dose used, since $1.0 \mathrm{mg} / \mathrm{kg}$ of 16 -brSal A was required to decrease cocaine-induced hyperactivity (Figure 3.5C). In support of this, the parent compound Sal A also produced no change in hyperactivity at $0.3 \mathrm{mg} / \mathrm{kg}$ (Morani et al., 2009), but attenuated cocaine-induced hyperactivity when a higher dose $(2.0 \mathrm{mg} / \mathrm{kg}$ ) was used (Chartoff et al., 2008). However, higher doses of Sal A (1-3.2 mg/kg) have been reported to produce adverse effects such as aversion, sedation, and a decrease in sucrose intake (Zhang et al., 2005; Ebner et al., 2010), indicating the possible existence of a threshold dose in which Sal A produces anti-addiction effects with minimal side effects. Taken together, these studies indicate that although higher doses of MOM Sal B may reduce cocaine-induced hyperactivity, it is highly likely that these doses will also elicit undesirable negative effects.

It is important to note that similar discrepancies in anti-cocaine properties of novel KOPr agonists have also been observed by others. As previously mentioned, Sal A produced no attenuation of hyperactivity at $0.3 \mathrm{mg} / \mathrm{kg}$, an effect mirrored by another Sal A analogue, Ethy Sal A (Kivell Lab, unpublished study). In contrast, EOM Sal B and Mesyl Sal B reduced cocaine-induced hyperactivity at doses that also attenuate cocaine seeking (Kivell Lab, unpublished data). Although such differences can possibly be attributed to the dose 
administered, differences in anti-cocaine effects may also be due to the distinct brain circuits underlying cocaine-induced reinstatement and cocaine-induced hyperactivity. A study conducted by McFarland and Kalivas (2001) showed that microinfusion of the D1/D2 DA receptor antagonist fluphenazine $(10 \mathrm{nmol} / \mathrm{side})$ into the dorsal PFC, but not the NAC core or ventral pallidum, blocked cocaine-induced reinstatement in rats, whereas administration of fluphenazine into the NAc core and ventral pallidum, but not the dorsal PFC decreased cocaine-induced hyperactivity. In addition, administration of baclofen and muscimol (GABA agonists) into the VTA, dorsal PFC, NAc core, and ventral pallidum prevented cocaine-induced reinstatement, but not spontaneous activity or cocaineinduced locomotion (McFarland and Kalivas, 2001), indicating that the brain circuitry mediating drug-primed reinstatement is distinct from that of locomotion. Since an ideal anti-cocaine pharmacotherapy should have a broad range of anti-cocaine effects, it is anticipated that 16-brSal A will be a more suitable candidate than MOM Sal B.

\subsubsection{Limitations and future directions}

Although the reinstatement model is widely used in the addiction field, this model is not an exact mirror of human craving or relapse because of its reliance on experimenteradministered triggers to elicit craving in subjects (Katz and Higgins, 2003). It is also important to note that laboratory animals are re-exposed to the drug of abuse prior to reinstatement, in contrast to human drug re-exposure where drug intake is a consequence of relapse (Katz and Higgins, 2003). Another limitation of this model is the availability of the reinforcer, whereby responses in the reinstatement phase are not reinforced, whereas drug seeking in humans results in drug intake (Shaham et al., 2003). As a result, some argue that the reinstatement model has weak construct validity (Katz and Higgins, 2003; Epstein et al., 2006). Therefore, successful drug treatments in the reinstatement model should not be taken as a universal solution to relapse, but instead be assimilated with other interventions such as counselling and social incentives (Higgins et al., 2002). Methodical assimilation of these interventions in the laboratory setting (i.e. availability of sucrose pellets or sexual partners) would increase the validity of reinstatement procedures as a model of relapse. 
Other models such as the CPP (Mueller and Stewart, 2000) and ICSS paradigms (Negus and Miller, 2014) can also be used to observe cocaine-induced effects, i.e. the rewarding properties of cocaine. The CPP paradigm consists of a conditioning period, in which animals are taught to associate distinct environments to different drugs, and a testing period in which rewarding effects of drugs are determined based on the animals' environmental preference. The CPP model requires a shorter training time and no surgery compared to drug self-administration, but relies on experimenter administered injections as opposed to voluntary drug intake (Prus et al., 2009). In addition, the CPP model requires administration of drug prior to the contextual learning phase, whereas animals in the selfadministration paradigm learn the task and are rewarded with administration of the drug; giving the self-administration model a better face validity to human drug taking behaviour than the CPP paradigm (reviewed in Prus et al. (2009)). The CPP model can also be used in parallel to the reinstatement of drug seeking paradigm by introduction of an extinction phase (whereby rats are placed in the cocaine-reinforced chamber without exposure to cocaine) and a reinstatement phase (in which rats would be injected with cocaine and allowed free access to chamber to determine their preferred chamber). On the other hand, ICSS is an operant responding model in which animals self-administer electrical pulses into brain regions associated with rewarding effects. In this paradigm, a decrease in ICSS threshold compared to basal levels indicates rewarding effects of the administered drug, and vice versa. The ICSS model has reported similar results to drug selfadministration, but the reduced likelihood that ICSS will produce evidence of a drug's abuse potential tip the scales in favour of the self-administration paradigm (reviewed in Negus and Miller (2014)). Nevertheless, it is noteworthy that traditional KOPr agonists have presented similar outcomes in cocaine-induced CPP and ICSS. Both U50,488 (5 $\mathrm{mg} / \mathrm{kg})$ and $U 69,593(0.04-0.32 \mathrm{mg} / \mathrm{kg})$ prevented sensitisation to the conditioned rewarding effects of cocaine in the rat CPP (Shippenberg et al., 1996; Shippenberg et al., 1998), whereas an acute administration of U50,488 (5 mg/ $\mathrm{kg}$, i.p.) $15 \mathrm{~min}$ prior to cocaine significantly suppressed cocaine-induced CPP in mice (McLaughlin et al., 2006). Both Sal A (2 mg/kg, i.p.) (Potter et al., 2011) and U69,593 (0.063-0.5 mg/kg, i.p.) (Tomasiewicz et al., 2008) also reduced the ability of cocaine to decrease ICSS thresholds. Therefore, effects seen in the present study could be complemented by using CPP and ICSS models. 
Lastly, the cocaine-induced behavioural sensitisation paradigm can also serve as a method to study additional anti-cocaine effects of Sal $A$ and its analogues. It has been found that repeated administration of cocaine produces behavioural sensitisation (Heidbreder et al., 1995), which is prevented by administration of $U 69593$ and U50,488 (Heidbreder et al., 1993; Heidbreder et al., 1995; Shippenberg et al., 1996; Shippenberg et al., 1998; Chefer et al., 1999) (Table 3.1). Although to the best of our knowledge, none of the Sal A analogues tested in the present study have been screened for their ability to modulate cocaine-induced behavioural sensitisation, a single administration of Sal A ( $0.3 \mathrm{mg} / \mathrm{kg}$, i.p.) (Morani et al., 2012) and Mesyl Sal B (0.3 mg/kg, i.p., Kivell Lab, unpublished study) prior to a cocaine prime $(20 \mathrm{mg} / \mathrm{kg}$, i.p.) on testing day prevented cocaine-induced behavioural sensitisation; indicating that Sal A-derived compounds activating the KOPr should also inhibit cocaine-produced sensitisation of locomotor effects.

\subsection{Conclusions}

Both 16-brSal and EOM Sal B successfully reduced cocaine-induced reinstatement of cocaine seeking to a similar extent as the parent compound Sal A, but produced differential effects on cocaine-induced hyperactivity. This discrepancy may possibly be due to a combination of different doses administered and the different circuitries underlying the two cocaine-evoked behaviours. 


\begin{tabular}{|c|c|c|c|c|c|c|c|c|c|c|c|}
\hline \multirow{2}{*}{$\begin{array}{c}\text { Anti-cocaine } \\
\text { behavioural } \\
\text { model }\end{array}$} & \multicolumn{10}{|c|}{ KOPr agonist } & \multirow[t]{2}{*}{ Animal } \\
\hline & U69,593 & U50,488 & Sal A & MOM Sal B & $\begin{array}{l}\text { EOM } \\
\text { Sal B }\end{array}$ & $\begin{array}{l}\text { Mesyl } \\
\text { Sal B }\end{array}$ & $\begin{array}{l}\beta \text {-THP } \\
\text { Sal B }\end{array}$ & $\begin{array}{c}16- \\
\text { brSal A }\end{array}$ & $\begin{array}{c}\text { Me Sal } \\
\text { A }\end{array}$ & $\begin{array}{l}\text { Ethy } \\
\text { Sal A }\end{array}$ & \\
\hline $\begin{array}{l}\text { Cocaine- } \\
\text { primed } \\
\text { reinstatement } \\
\text { of cocaine } \\
\text { seeking }\end{array}$ & $\begin{array}{c}\downarrow^{(1,2)} \\
0.32, \text { s.c. }\end{array}$ & $\begin{array}{c}\downarrow^{(2)} \\
30, \text { i.p. }\end{array}$ & $\begin{array}{c}\downarrow^{(2)} \\
0.3 \text {, i.p. }\end{array}$ & $\begin{array}{c}\downarrow^{(3)} \\
0.3, \text { i.p. }\end{array}$ & $\begin{array}{c}\downarrow^{*} \\
0.1, \text { i.p. }\end{array}$ & $\begin{array}{c}\downarrow^{(4)} \\
0.3, \text { i.p. }\end{array}$ & $\begin{array}{c}\downarrow^{(5)} \\
1.0, \text { i.p. }\end{array}$ & $\begin{array}{c}\downarrow^{*(6)} \\
1.0, \text { i.p. }\end{array}$ & $\begin{array}{c}\text { NE }{ }^{(6)} \\
1.0, \text { i.p. }\end{array}$ & $\begin{array}{c}\downarrow^{(6)} \\
0.1, \text { i.p. }\end{array}$ & Rats \\
\hline \multirow{2}{*}{$\begin{array}{c}\text { Self- } \\
\text { administration }\end{array}$} & $\begin{array}{c}\downarrow^{(1)} \\
0.32, \text { s.c. }\end{array}$ & $\begin{array}{c}\downarrow^{(8)} \\
10 \text {, i.p. }\end{array}$ & $\begin{array}{c}\text { NE* } \\
\text { 0.3, 2.0, i.p. } \\
\text { (Chapter 6) }\end{array}$ & - & - & - & - & - & - & - & Rats \\
\hline & - & $\begin{array}{c}\downarrow^{(17)} \\
0.032-0.1 \text { per } h, \\
\text { i.v. }\end{array}$ & $\begin{array}{c}\downarrow^{(9)} \\
\text { Variable } \\
\text { doses, i.v. }\end{array}$ & - & - & - & - & - & - & - & $\begin{array}{l}\text { Rhesus } \\
\text { monkeys }\end{array}$ \\
\hline \multirow{2}{*}{$\begin{array}{l}\text { Conditioned } \\
\text { place } \\
\text { preference }\end{array}$} & - & $\begin{array}{l}\downarrow^{(12)} \\
5, \text { s.c. }\end{array}$ & - & - & - & - & - & - & - & - & Rats \\
\hline & - & $\begin{array}{c}\downarrow \text { and } \uparrow(10,11) \\
\text { 5, i.p. }\end{array}$ & - & - & - & - & - & - & - & - & Mice \\
\hline Hyperactivity & $\begin{array}{c}\downarrow^{(15,16)} \\
0.32, \text { s.c. } \\
0.3, \text { s.c. }\end{array}$ & $\begin{array}{l}\downarrow^{(12,16)} \\
5 \text {, s.c. } \\
10, \text { i.p. }\end{array}$ & $\begin{array}{l}\downarrow^{(14)} \\
2, \text { i.p. } \\
\text { NE }{ }^{(2)} \\
0.3, \text { i.p. }\end{array}$ & $\begin{array}{l}\text { NE*(3) } \\
0.3, \text { i.p. }\end{array}$ & $\begin{array}{c}\downarrow^{(18)} \\
0.1 \text {, i.p. }\end{array}$ & $\begin{array}{c}\downarrow^{(18)} \\
0.3 \text {, i.p. }\end{array}$ & - & $\begin{array}{c}\downarrow^{*} \\
1.0, \text { i.p. }\end{array}$ & - & $\begin{array}{l}\text { NE } \\
0.1, \text { i.p. }\end{array}$ & Rats \\
\hline $\begin{array}{l}\text { Behavioural } \\
\text { sensitisation }\end{array}$ & $\begin{array}{l}\text { NE }{ }^{(16)} \\
0.3, \text { s.c. }\end{array}$ & $\begin{array}{l}\text { NE }{ }^{(16)} \\
10, \text { i.p. }\end{array}$ & $\begin{array}{c}\downarrow^{(13)} \\
0.3, \text { i.p. }\end{array}$ & - & - & $\begin{array}{c}\downarrow^{(18)} \\
0.3, \text { i.p. }\end{array}$ & - & - & - & - & Rats \\
\hline
\end{tabular}

${ }^{*}$ denotes this study, $\downarrow$ = attenuation, $\uparrow=$ potentiation, NE = no effect, - = study not found. Unless otherwise stated, doses are given in $\mathrm{mg} / \mathrm{kg}$.

\section{Table 3.1: Acute effects of U69,593, U50,488, Sal A, and Sal A analogues on anti-cocaine behavioural models}

1) Schenk et al. (1999)

2) Morani et al. (2009)

3) Morani et al. (2013)

4) Simonson et al. (2015)

$\mathscr{6}$ 5) Prevatt-Smith et al. (2011)
6) Riley et al. (2014)

7) Rüedi-Bettschen et al. (2010)

8) Glick et al. (1995)

9) Freeman et al. (2014)

10) Ehrich et al. (2014)
11) McLaughlin et al. (2006)

12) Crawford et al. (1995)

13) Morani et al. (2012)

14) Chartoff et al. (2008)

15) Collins et al. (2001b)
16) Vanderschuren et al. (2000)

17) Negus et al. (1997)

18) Kivell Lab, unpublished data 


\section{Chapter 4: Investigating the side effects of novel}

\section{Sal A analogues in preclinical models}

\subsection{Introduction}

Acute administration of traditional KOPr agonists produces anti-cocaine effects but can also cause an array of side effects. Activation of the KOPr has been reported to cause depression (Todtenkopf et al., 2004), sedation (Negus et al., 1997; Mello and Negus, 2000), emesis (Negus et al., 1997; Mello and Negus, 2000), decreased responding for food, and aversion in laboratory animals (Negus et al., 1997; Mello and Negus, 2000). In a similar manner, KOPr agonists are clinically associated with adverse effects such as dysphoria (Glick et al., 1998), insomnia (Glick et al., 1998; Kumagai et al., 2009; Kumagai et al., 2011), sedation (Walsh et al., 2001), irritability (Glick et al., 1998), confusion (Walsh et al., 2001), increased urinary output (Walsh et al., 2001), and psychotomimetic effects (Pfeiffer et al., 1986), preventing their development as therapies. Therefore, discovery of the structurally novel KOPr agonist, Sal A, which possesses similar anti-addiction effects (Morani et al., 2009) but fewer side effects in preclinical models (Morani et al., 2009; Morani et al., 2012) than traditional agonists has regenerated interest in the therapeutic use of KOPr agonists. This study will highlight use of different behavioural models to screen longer acting novel Sal A-derived agonists for adverse effects in vivo.

\subsubsection{Effect of KOPr agonists on sedation}

Previous studies have shown that traditional KOPr agonists such as ethylketocyclazocine, bremazocine, enadoline, Mr2033, and U50,488 produce sedative effects in rhesus monkeys (Mello and Negus, 2000). Although U50,488 (30 mg/kg, i.p.) reduced cocaineinduced drug seeking in rats (Morani et al., 2009), doses of U50,488 ranging from 2.5 to $40 \mathrm{mg} / \mathrm{kg}$ (s.c.) also showed sedative effects in rats (Gallantine and Meert, 2008). In addition, U50,488 (1-5 mg/0.5 mL, intrathecal) produced motor dysfunction in rabbits (Borgbjerg and Frigast, 1997), whereas ketocyclazocine (5 mg/kg, s.c.) produced sedation and ataxia in rats (Iwamoto, 1981). Cerebellar microinfusions $(2.5-10 \mathrm{nmol} / 100 \mathrm{~nL}$ artificial cerebrospinal fluid) of U50,488, spiradoline, and bremazocine enhanced 
ethanol-induced motor impairment, effects that were abolished with the intracerebellar administration of nor-BNI $(2 \mathrm{nmol})$, thus indicating that the impairment was a result of KOPr activation (Dar, 1998). Presence of these effects suggest that the attenuation of cocaine-induced effects by traditional KOPr agonists may not be specific to anti-cocaine behaviour but instead be due to sedation in general. However, recent studies using Sal A have revealed that Sal A $(0.3 \mathrm{mg} / \mathrm{kg}$, i.p.) does not affect spontaneous locomotion in the rat, a measure of sedation, at the minimal dose that attenuates drug seeking (Morani et al., 2009; Morani et al., 2012). There is therefore a need to study new analogues of Sal A such as 16-brSal A and MOM Sal B, to determine if they produce sedation, and if their cocaine antagonising effects observed in Chapter 3 are specific to anti-cocaine properties.

\subsubsection{Effect of KOPr agonists on depression}

Activation of the KOPr by dynorphin mediates stress, aversion, and depressive effects (Shirayama et al., 2004; Knoll and Carlezon Jr, 2010; Chartoff et al., 2012). Although depression is a subjective mood disorder that can be difficult to observe in the laboratory setting, pro- or anti-depressive properties of KOPr agonists can be examined and quantified preclinically using several behavioural models. One of the models frequently utilised is ICSS. In addition to its usefulness in determining the rewarding effects of drugs (section 3.5.4), ICSS can also be used to screen for depression; whereby elevated ICSS thresholds signify a depressive state (refer to Vlachou and Markou (2011); Negus and Miller (2014) for reviews). Other simple models practiced in the laboratory are the rodent tail suspension test (in which mice are suspended by their tails) and FST, studies based on an animal's instinct to escape and the inclination to give up upon learning that escape is impossible (Porsolt et al., 2001). In both models, the time rodents spend immobile is taken as an indication of a depressive-like state (Porsolt et al., 2001). All three models have been used extensively in the literature to examine the effects of KOPr agonists on depression. For example, U69,593 dose-dependently increased ICSS thresholds in rats (0.063 - $0.5 \mathrm{mg} / \mathrm{kg}$, i.p. in Tomasiewicz et al. (2008); $0.25-0.5 \mathrm{mg} / \mathrm{kg}$, i.p. in Bonano et al. (2014) and Todtenkopf et al. (2004)) and occurrences of immobility in the rat FST (0.3 - $10 \mathrm{mg} / \mathrm{kg}$, i.p.) (Mague et al., 2003). The other classic agonist, U50,488, also increased ICSS thresholds in mice (3.0, $5.5 \mathrm{mg} / \mathrm{kg}$, i.p.) (DiNieri et al., 2009) and occurrences of 
immobility in the mouse FST (Zhang et al., 2015). In contrast, administration of selective $\mathrm{KOPr}$ antagonists such as nor-BNI, 5'-guanidinonaltrindole, 5'acetamidinoethylnaltrindole, and JDTic decreased immobility occurrences in the rat FST (Mague et al., 2003; Beardsley et al., 2005; Carr et al., 2009), further supporting the role of KOPr in mediating depression. The known ability of KOPr agonists to induce depression and potentiate stress-induced relapse to drug seeking has been a major limitation of KOPr-based therapies.

Interestingly, discovery of Sal A represented a major step in the development of KOPrbased compounds with reduced depressive effects. At doses that display anti-cocaine properties, Sal A produced both pro- and anti-depressive effects in preclinical models. Although Sal A $(0.5-2 \mathrm{mg} / \mathrm{kg}$, i.p.) dose-dependently elevated ICSS thresholds in rats (Carlezon et al., 2006) and produced increased immobility time in the rat FST $(0.25-2$ $\mathrm{mg} / \mathrm{kg}$, i.p.) (Carlezon et al., 2006; Morani et al., 2012), Sal A (1 mg/kg, i.p.) also produced anti-depressive effects in rats subjected to chronic mild stress (Harden et al., 2012). Taken together, these studies show that Sal A has inconsistent effects on mood, suggesting that synthesis of Sal A analogues that lack the depressive effects of traditional KOPr agonists may be possible.

\subsubsection{Effect of KOPr agonists on modulation of natural reward}

The sucrose self-administration model is a simple method used to measure consumption of a natural reward. Similar to drug self-administration, animals are required to complete physical tasks, i.e. lever presses, in order to receive a sucrose reinforcer. This model is important in the study of KOPr agonists because the KOPr system plays a varied role in modulation of appetitive natural rewards such as sucrose. Although it is agreed, based on a large body of evidence, that KOPr agonists produce effects opposite to that of reward (Zimmer et al., 2001; McLaughlin and Chavkin, 2002; Liu-Chen, 2004; McLaughlin et al., 2006; Shippenberg et al., 2007; Carr and Mague, 2008; Tomasiewicz et al., 2008; Ebner et al., 2010; Knoll and Carlezon Jr, 2010; Wee and Koob, 2010) by decreasing DA neurotransmission (Di Chiara and Imperato, 1988b; Ebner et al., 2010), KOPr agonists have also been shown to stimulate appetite, food consumption, and sucrose intake 
(Cooper et al., 1985; Lynch and Burns, 1990; Ookuma et al., 1997; Ruegg et al., 1997; Badiani et al., 2001; Sipols et al., 2002). This contradiction to the classic anti-reward actions of the KOPr system can be observed with U50,488, whereby this compound (4 $\mathrm{mg} / \mathrm{kg}$, i.p.) both increased and decreased sucrose intake in rats (Badiani et al., 2001). In addition, the action of $U 50,488$ in reducing sucrose intake and sucrose seeking at doses lower than the dose required to attenuate cocaine seeking (doses that decrease sucrose intake $=5$ and $10 \mathrm{mg} / \mathrm{kg}$ vs. dose that attenuates drug seeking $=30 \mathrm{mg} / \mathrm{kg}$, i.p.) (Morani et al., 2009; Henderson-Redmond and Czachowski, 2014) suggests that U50,488 may attenuate cocaine intake by reducing intake of all rewarding stimuli. In support of this, U50,488 also decreased morphine self-administration in rats $(2.5-10.0 \mathrm{mg} / \mathrm{kg}$, i.p.) (Glick et al., 1995) and voluntary intake of a $10 \%$ ethanol solution (Lindholm et al., 2001). In contrast, Sal A $(0.3 \mathrm{mg} / \mathrm{kg}$, i.p.) showed no significant changes in intake of a $10 \%$ sucrose solution, indicating that Sal A neither stimulates nor inhibits intake of natural reward at the effective dose that attenuates cocaine seeking (Morani et al., 2009).

\subsubsection{Effect of KOPr agonists on anxiety}

It is generally agreed that activation of the KOPr system produces stress and anxietyrelated behaviours (refer to Van't Veer and Carlezon Jr (2013) for a recent review). Clinically, KOPr agonists such as MR2033 (3.5 $\mu \mathrm{g} / \mathrm{kg}$, i.v.) increased anxiety in healthy male volunteers (assessed by psychopathological evaluation scales) (Pfeiffer et al., 1986). In the rat (Bruchas et al., 2009; Valdez and Harshberger, 2012; Gillett et al., 2013) and mouse (Smith et al., 2012) models, U50,488 (5 and $10 \mathrm{mg} / \mathrm{kg}$, i.p.), produced a significant reduction in time spent on the open arm of an elevated plus maze (EPM). The EPM is a widely accepted procedure that examines an animal's conflict between its innate exploratory behaviour and the instinct to avoid open, brightly lit, elevated spaces (File et al., 2005). While anxiolytic drugs encourage exploratory behaviour, anxiogenic compounds such as KOPr agonists increase time spent in dark, covered spaces (File et al., 2005). Other tests used to determine anxiety-like effects are the light dark (LD) test, which uses a similar exploratory conflict as the EPM, and the defensive burying test, in which rodents displace bedding material towards sources of anxiogenic stimuli (refer to 
de Boer and Koolhaas (2003); File et al. (2005); Walf and Frye (2007); Ramos (2008) for reviews).

Consistent with the established role of KOPr in mediating anxiety-related behaviours, administration of KOPr antagonists such as nor-BNI $(3.0-30 \mathrm{mg} / \mathrm{kg}$, i.p.) or JDTic (1.0 $10 \mathrm{mg} / \mathrm{kg}$, i.p.) dose-dependently increased open arm time as well as open arm entries in the EPM (Knoll et al., 2007). Another KOPr antagonist, DIPPA (1 - $5 \mathrm{mg} / \mathrm{kg}$, s.c.) also dosedependently decreased defensive burying time in both male Wistar-Kyoto and SpragueDawley rats (Carr and Lucki, 2010), indicating the clear role of KOPr in modulating anxiety. It is therefore interesting to note that Sal A produces different effects on anxiety compared to traditional KOPr agonists. Clinically, inhalation of Sal A $(0.375-21 \mu \mathrm{g} / \mathrm{kg})$ produced low reports of anxiety (assessed using the State subscale of the State-Trait Anxiety Inventory) in healthy hallucinogen-using adults (MacLean et al., 2013). In addition, s.c. administration of Sal A $(0.1-160 \mu \mathrm{g} / \mathrm{kg})$ produced anxiolytic effects in mice, by increasing open arm time and entries in the EPM (Braida et al., 2009). These results suggest that Sal A may possess less stress-related anxiogenic effects than traditional KOPr agonists. However, to the best of our knowledge, there have been no studies evaluating anxiogenic properties of Sal A analogues at doses that show anti-cocaine properties, indicating a need to screen these compounds.

\subsubsection{Effect of KOPr agonists on aversion}

It is well established that KOPr activation mediates stress-related aversive properties (Shippenberg et al., 1993; Zhang et al., 2005; McLaughlin et al., 2006; Bruchas et al., 2007a; Land et al., 2008; Land et al., 2009; Bruchas and Chavkin, 2010; Wee and Koob, 2010; Chefer et al., 2013; Tejeda et al., 2013; Sufka et al., 2014; White et al., 2015). The most popular model to study aversive effects of a drug or stimulus in animals is the conditioned place aversion (CPA) paradigm, due to its low cost, non-invasive approach, and rapid data collection (Tzschentke, 2007; Prus et al., 2009). Similar to the CPP, it involves a conditioning phase that encourages the animal to create an association between the effects of a drug and the environment that the animal is placed in (i.e. the colour, texture, and smell of the chamber). The reduced amount of time spent by an 
animal voluntarily in the chamber after the conditioning period is an indication of the aversive effects of the drug.

Aversive effects in the CPA have been shown with U50,488 (1 mg/kg, s.c.) (Goktalay et al., 2006) and U69,593 (0.32 mg/kg, s.c.) (Tejeda et al., 2013) in male Sprague-Dawley rats. The aversive effects of $\mathrm{U} 69,593$ were KOPr-dependent, as prior administration of nor-BNI prevented U69,593-mediated aversion (Tejeda et al., 2013). However, just like its pro-/anti-depressive properties, Sal A produces inconsistent effects on aversion. Although Sal A produced place aversion in rats (0.3 and $1 \mathrm{mg} / \mathrm{kg}$, i.p.) (Sufka et al., 2014) and mice (1 and $3.2 \mathrm{mg} / \mathrm{kg}$, i.p.) (Zhang et al., 2005), another study examining Sal A at the dose that attenuated drug seeking $(0.3 \mathrm{mg} / \mathrm{kg}$, i.p.) displayed no significant changes when paired with a novel-tasting saccharin solution in the conditioned taste aversion (CTA) paradigm (Morani et al., 2009). These studies suggest that Sal A may exert fewer aversive effects compared to traditional agonists, and that Sal A-derived analogues may possibly be designed that lack the aversion responses but retain the anti-addictive effects.

\subsection{Aims and hypotheses}

The present study aimed to investigate the adverse effects of novel KOPr agonists including MOM Sal B, EOM Sal B, $\beta$-THP Sal B, and 16-brSal A with the objective of identifying an analogue with reduced severity of KOPr-mediated adverse effects. As typical side effects include sedation, modulation of natural reward, depression, anxiety, and aversion, novel Sal A-derived agonists will be screened for these effects at the minimal doses that attenuated drug seeking. Based on previous studies, it is hypothesised that Sal A analogues will produce fewer side effects compared to Sal $A$, as well as traditional KOPr agonists. Some of the work in this chapter was published along with work carried out by Dr Aashish Morani during his doctoral research (Morani et al., 2013) and Mr David Young during his Master's degree (Riley et al., 2014). 


\subsection{Methods}

\subsubsection{Materials}

KOPr agonists were dissolved in 75\% DMSO for spontaneous locomotor activity and FST. For all other tests, agonists were dissolved in DMSO:Tween-80:MilliQ water in a ratio of 2:1:7. Injections were given at a volume of $1 \mathrm{~mL} / \mathrm{kg}$. U69,593 was administered s.c. 15 min before testing, whereas all other agonists were administered i.p. either 5 min (Sal A, EOM Sal B, MOM Sal B) or 10 min (U50,488, 16-brSal A, $\beta$-THP Sal B) prior to testing, according to their previously determined onset of action (Figure 3.2 and Morani et al. (2009); Prevatt-Smith et al. (2011); Morani et al. (2013)).

\subsubsection{Spontaneous locomotor activity}

Male Sprague-Dawley rats weighing between 300 and $350 \mathrm{~g}$ were habituated in activity chambers (Med Associates, ENV-520) for 30 min. Rats were then given either vehicle or KOPr agonist (16-brSal A, $1.0 \mathrm{mg} / \mathrm{kg}$ or MOM Sal B, $0.3 \mathrm{mg} / \mathrm{kg}$ ) and returned to the activity chambers for a further $60 \mathrm{~min}$, as previously described (Morani et al., 2012). The number of ambulatory counts during habituation and testing sessions were recorded. All sessions were carried out in the dark between 09:00 and 17:00 in the presence of white noise.

\subsubsection{Forced swim test (FST)}

The FST was conducted according to methods modified from Porsolt (1979) and took place over 2 days. On both days, rats weighing between $350-400$ g were habituated to conditions in the experimental room 60 min prior to testing. A cylindrical swim chamber measuring $44 \mathrm{~cm}$ in height and $20 \mathrm{~cm}$ in diameter was filled with water (maintained at a temperature of $25 \pm 1^{\circ} \mathrm{C}$ ) to a depth of $35 \mathrm{~cm}$. On the first day, rats were habituated to forced swimming conditions for $15 \mathrm{~min}$. The next day, rats were injected with either vehicle or KOPr agonist (16-brSal A, $1.0 \mathrm{mg} / \mathrm{kg}$; MOM Sal B, $0.3 \mathrm{mg} / \mathrm{kg}$; EOM Sal B, 0.1 $\mathrm{mg} / \mathrm{kg}$; or $\beta$-THP Sal B, $1.0 \mathrm{mg} / \mathrm{kg}$ ) before a $5 \mathrm{~min}$ testing session. After each session, rats were removed from the swim chamber and dried using towels and a blow dryer. All test sessions were recorded using a Sony HDR-SR5E digital camera recorder, and videos were analysed in $5 \mathrm{~s}$ intervals by an observer blinded to experimental treatments. 


\subsubsection{Sucrose self-administration}

The sucrose reinforcement test was used to determine effects of experimental manipulations on a naturally rewarding, palatable stimuli. Sucrose is readily selfadministered by rats (Ferragud et al., 2009; Morani et al., 2009; Ebner et al., 2010; Harden et al., 2012; Morani et al., 2013), and is considered a relatively stable trait (Tonissaar et al., 2006). Rats weighing $260-300 \mathrm{~g}$ were maintained at $85 \%$ of their free-feeding weight and trained to self-administer sucrose pellets (Dustless Precision Pellet, $45 \mathrm{mg}$ sucrose: Able Scientific, Perth, Australia) in standard operant chambers (Med Associates, ENV001). The chambers contained 2 levers: one connected to a pellet dispenser (active lever) and one which was not connected for delivery of reward (inactive lever). Rats were initially trained on a FR1 schedule of reinforcement, in which a single active lever press was required for delivery of a single sucrose pellet accompanied by a light stimulus. Responses on the inactive lever produced no programmed consequences. Once rats acquired self-administration behaviour (at least 20 pellets during the 45 min session with an active:inactive lever ratio of more than 2:1), rats progressed to the FR5 schedule of reinforcement. Rats were subjected to sucrose self-administration for at least 5 days before testing to determine baseline responding levels. Once stable responding was achieved, rats were injected with either vehicle, U50,488 $(10 \mathrm{mg} / \mathrm{kg})$, U69,593 $(0.3$ $\mathrm{mg} / \mathrm{kg}), 16$-brSal A (1.0 mg/kg), EOM Sal B $(0.1 \mathrm{mg} / \mathrm{kg}), \beta$-THP Sal B $(1.0 \mathrm{mg} / \mathrm{kg})$, or Sal A $(0.3 \mathrm{mg} / \mathrm{kg}$ ) prior to testing sessions in a counterbalanced manner (Appendix 4.1). Active and inactive lever responses were recorded using Med Associates software (MED-PC IV, version 4.2). Rats were run in daily sessions from 1300 to 1345,6 days a week.

\subsubsection{Light dark (LD) test}

The LD test was conducted using a large white chamber ( $30 \times 30 \times 34 \mathrm{~cm})$ and a small black chamber $(15 \times 30 \times 34 \mathrm{~cm})$ connected by a small grey corridor $(8 \times 10 \times 34 \mathrm{~cm})$. All sessions took place in a dark room with three LED lamps (one for the white box, one for the corridor and one directed at the ceiling for recording). The intensity of light in each box was as follows: 100 lux in the white chamber, 10 lux in the black chamber, and 70 lux in the grey corridor. Rats weighing between 300-400 g were given either vehicle or KOPr agonist (16-brSal A, $1.0 \mathrm{mg} / \mathrm{kg}$ or Sal A, 0.3 or $1.0 \mathrm{mg} / \mathrm{kg}$ ) before being placed in the black 
chamber and given free access to the apparatus for $15 \mathrm{~min}$. Test sessions took place between 09:00 and 17:00 and were accompanied by white noise. All activity was tracked using SMART 3.0 software (PanLab, Harvard Apparatus, USA).

\subsubsection{Elevated plus maze (EPM)}

The EPM apparatus consisted of four arms (each arm $50 \mathrm{~cm}$ long and $10 \mathrm{~cm}$ wide) elevated $55 \mathrm{~cm}$ above the ground. Two of the arms had a small parapet measuring 2.5 $\mathrm{cm}$ in height surrounding them (open arms) and the other two arms were enclosed by high $40 \mathrm{~cm}$ black walls (closed arms). Animals were habituated to conditions in the testing room 60 min prior to the study. Rats weighing $300-400 \mathrm{~g}$ were given either vehicle or treatment (16-brSal A, $1.0 \mathrm{mg} / \mathrm{kg}$ or Sal A, 0.3 or $1.0 \mathrm{mg} / \mathrm{kg}$ ) before being placed at the centre of the apparatus facing an open arm. All activity was filmed for 5 min using a Sony HDR-SR5E digital camera recorder. The time spent on each arm was calculated by an observer blinded to experimental treatment. Open arm time was calculated only when rats had all four paws on the open arm (Walf \& Frye, 2007). Animals that fell off the maze during testing were excluded from analysis.

\subsubsection{Conditioned place aversion (CPA)}

CPA studies were conducted over 9 days using a biased procedure modified from Tejeda et al. (2013). Two large chambers $(30 \times 30 \times 34 \mathrm{~cm})$ were connected by a small corridor $(8 \times 10 \times 34 \mathrm{~cm})$, with removable sliding doors enabling isolation of each chamber. One of the large chambers had a textured black floor with black dotted patterns on its walls (henceforth known as the black chamber), whereas the other had a smooth white floor with black striped patterns on its walls (henceforth known as the white chamber). The corridor was a neutral zone with grey walls and floor. All sessions were conducted in the dark with two dim free-standing LED lamps, one aimed at the ceiling for recording purposes and the other directed into the corridor. The average light intensity in both conditioning chambers was 20 lux, whereas the corridor was illuminated at an intensity of 70 lux. White noise was present throughout all sessions to mask background noise. 
On habituation day, rats weighing $300-400 \mathrm{~g}$ were allowed free access to all chambers for $15 \mathrm{~min}$. On the second (pre-test) day, rats were again allowed free access to all chambers for $15 \mathrm{~min}$. Animals that showed more than $80 \%$ preference for a particular chamber or more than $40 \%$ preference for the corridor were excluded from testing. Rats were then subjected to $45 \mathrm{~min}$ of conditioning for six consecutive days, in which rats were injected with either vehicle or KOPr agonist (16-brSal A, $1.0 \mathrm{mg} / \mathrm{kg}$ or Sal A, $1.0 \mathrm{mg} / \mathrm{kg}$ ) and confined to respective chambers for $45 \mathrm{~min}$. Conditioning was conducted according to each animal's pre-test preference. KOPr agonist injections were followed by confinement in their preferred chamber and vehicle injections were followed by confinement in their less preferred chamber. The vehicle and $\mathrm{KOPr}$ agonists were administered on alternate days in a counterbalanced order. On the final (post-test) day, animals were placed in the corridor and allowed free access to both chambers for $15 \mathrm{~min}$. At the end of the testing period, the time spent in each chamber on pre- and post-test days were compared to determine changes in preference. Activity and total time spent in each chamber on pre- and post-test days were recorded and calculated using SMART 3.0.

\subsubsection{Statistics}

For the locomotor activity test, a one-way ANOVA with repeated measures was used to compare the time course data, whereas the total number of ambulatory counts was analysed using a Student's $t$-test. In sucrose self-administration, a within-subjects Latin square design was used to minimise treatment sequence bias, and the repeated measures one-way ANOVA was performed. This was followed by a post-hoc analysis made up of paired $t$-tests with the Bonferroni correction applied to determine significance. The Student's $t$-test was performed on FST data, whereas a paired Student's $t$-test was used to analyse the pre- and post- conditioning time in CPA experiments. A one-way ANOVA followed by paired $t$-tests with the Bonferroni correction applied was performed for data obtained in the LD and EPM tests. Data were considered statistically significant when $\mathrm{p} \leq 0.05$. 


\subsection{Results}

\subsubsection{Effect of 16-brSal A and MOM Sal B on sedation}

Since KOPr agonists are known to cause sedation, the Sal A derivatives, 16-brSal A and MOM Sal B were evaluated for their sedative properties. Neither 16-brSal A $(1.0 \mathrm{mg} / \mathrm{kg}$, Figure 4.1E) nor MOM Sal B $(0.3 \mathrm{mg} / \mathrm{kg}$, Figure 4.1F) treatment altered spontaneous locomotor activity in rats. 16-brSal A produced an average of $793 \pm 122$ ambulatory counts during the 60 min testing phase, whereas vehicle-treated rats (75\% DMSO) produced a mean of $778 \pm 123$ ambulatory counts (Figure 4.1E). Similarly, ambulatory counts of MOM Sal B (806 \pm 171$)$ were slightly, but not significantly higher than vehicle counts of $500 \pm 149(p=0.20$, Student's $t$-test, Figure $4.1 \mathrm{~F})$. Analysis of the $30 \mathrm{~min}$ habituation period also showed that rats in the vehicle group had no significant difference in ambulatory counts compared to rats in the 16-brSal A group (Figure 4.1C, Student's $t$ test) or the MOM Sal B group (Figure 4.1D, Student's $t$-test).

Further time course analysis in segments of $5 \mathrm{~min}$ for 16 -brSal $\mathrm{A}$ also revealed no significant interaction between treatment and time $[F(17,180)=0.55, p=0.9238]$ (oneway ANOVA with repeated measures, Figure 4.1A). There was also no significant effect of treatment $[F(1,180)=0.45, p=0.5046]$. The time course for MOM Sal B also displayed no significant interaction between treatment and time $[F(17,204)=0.64, p=0.8590]$ (oneway ANOVA with repeated measures, Figure 4.1B). However, rats treated with MOM Sal B showed a trend towards increase in locomotor activity compared to vehicle-treated rats $[F(1,204)=3.37, p=0.09]$. Thus, both 16 -brSal A and MOM Sal B showed no sedative properties in the locomotor activity test. 
A)

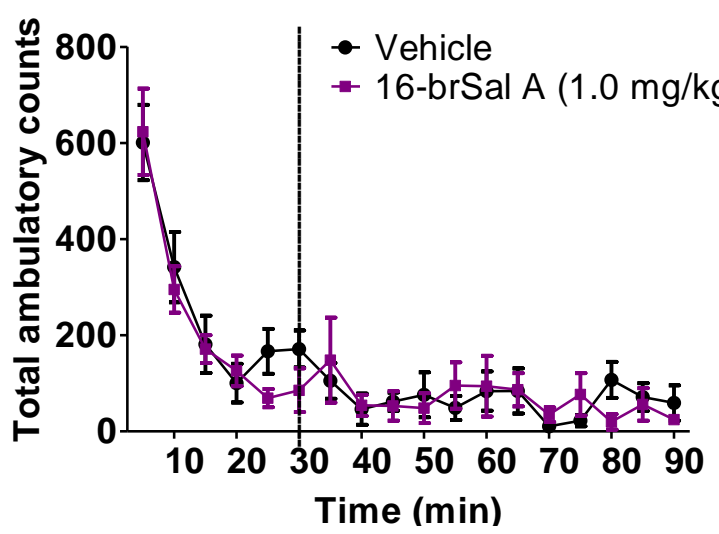

C)

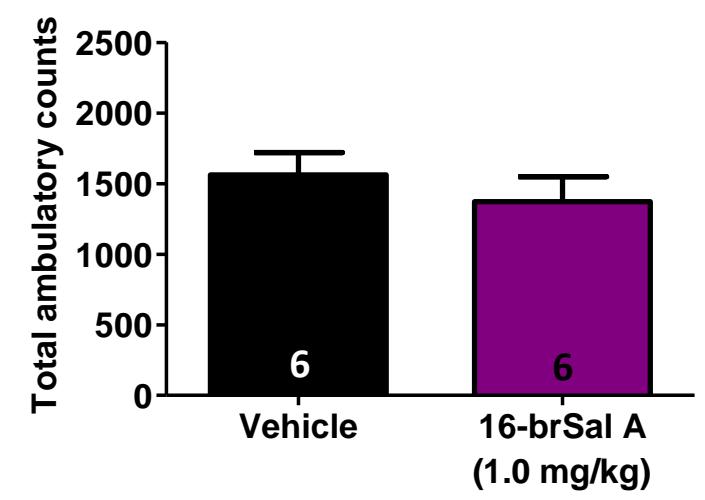

E)

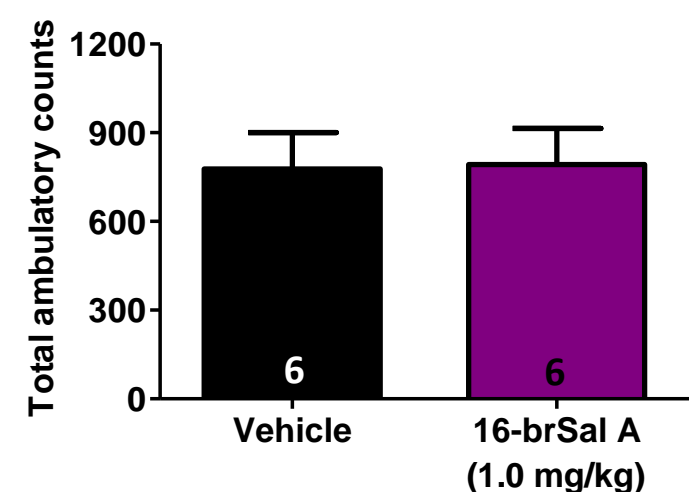

B)

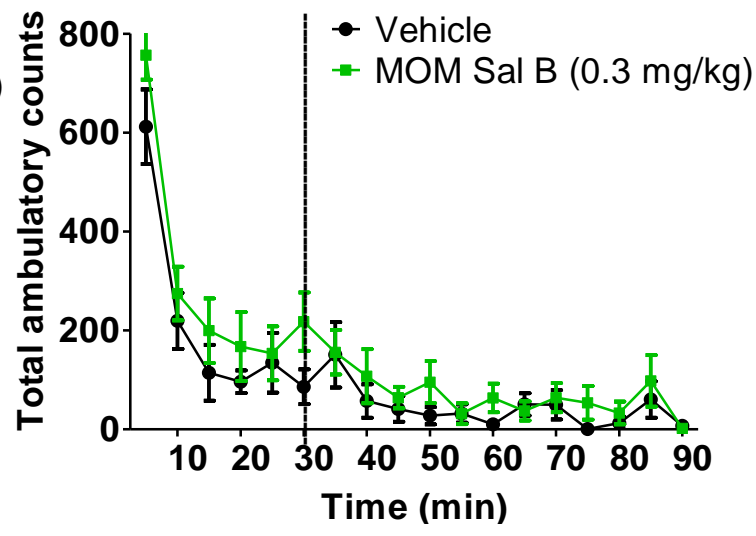

D) Habituation phase

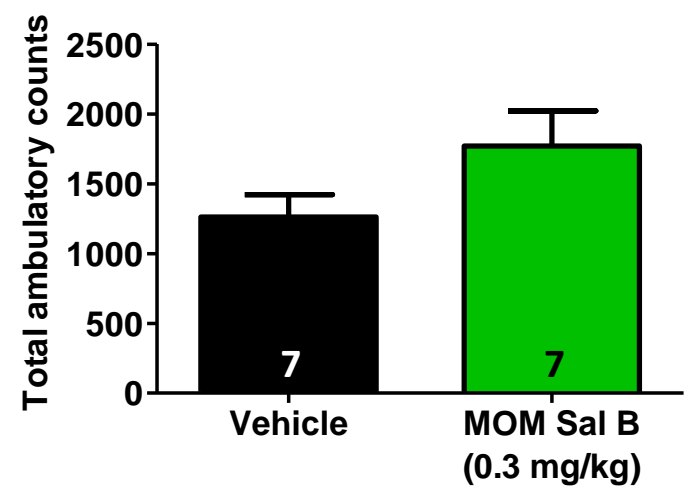

F) Testing phase

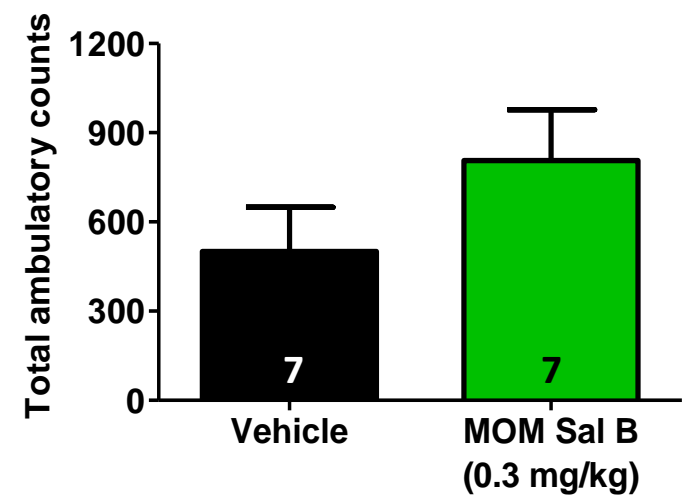


Figure 4.1: 16-brSal A and MOM Sal B do not alter spontaneous locomotor activity in

\section{$\underline{\text { rats }}$}

Rats were habituated in activity chambers for 30 min prior to vehicle (75\% DMSO), 16 -brSal A, or MOM Sal B injections, followed by 60 min of testing (vertical line represents administration of treatment). A) 16-brSal A- and B) MOM Sal B-treated rats show no significant changes in ambulatory counts compared to vehicle controls (one-way ANOVA with repeated measures). Total ambulatory counts recorded during the $30 \mathrm{~min}$ habituation period showed no significant change in ambulatory counts between vehicle and C) 16-brSal A or D) MOM Sal B groups (Student's $t$-test). Total ambulatory counts from the 60 min testing period showed no significant differences between vehicle controls and E) 16-brSal A or F) MOM Sal B (Student's $t$-test). Values presented as mean \pm SEM, $n=6-7$. 


\subsubsection{Effect of 16-brSal A, EOM Sal B, MOM Sal B, and $\beta$-THP Sal B on depression}

Administration of KOPr agonists have been reported to produce depressive-like behaviours in laboratory animals (Todtenkopf et al., 2004; Carlezon et al., 2006; Ebner et al., 2010), compelling the investigation of four novel Sal A-derived analogues (MOM Sal B, EOM Sal B, 16-brSal A, and $\beta$-THP Sal B) that have shown reduction in cocaine-primed drug seeking behaviour but no sedative properties. Consistent with the literature, drug naïve rats treated with MOM Sal B $(0.3 \mathrm{mg} / \mathrm{kg})$, showed a significant increase in immobility time (112 \pm 21 s compared to $73 \pm 16$ s for vehicle controls) ( $p<0.05$, Student's $t$-test, Figure $4.2 \mathrm{C}$ ). This increase in immobility was accompanied by a decrease in swimming time (MOM Sal B = 74 $\pm 26 \mathrm{~s}$ vs. vehicle $=171 \pm 9 \mathrm{~s})(\mathrm{p}<0.01$, Student's $t$-test, Figure $4.2 \mathrm{C})$. In contrast, $16-\mathrm{brSal} \mathrm{A}(1.0 \mathrm{mg} / \mathrm{kg})$ produced an average immobility time of $115 \pm 19 \mathrm{~s}$, which was not significantly different to vehicle-treated controls (85 $\pm 20 \mathrm{~s}$, Student's $t$-test, Figure 4.2A). $\beta$-THP Sal B $(1.0 \mathrm{mg} / \mathrm{kg})$ also showed no significant change in immobility time compared to controls, with $\beta$-THP Sal B-treated rats showing an average immobility time of $84 \pm 9$ s compared to vehicle values of $87 \pm 21 \mathrm{~s}$ (Figure 4.2D). On the other hand, although EOM Sal B-treated rats $(0.1 \mathrm{mg} / \mathrm{kg})$ did not display a significant increase in immobility time in the FST (EOM Sal B $=94 \pm 26$ vs. vehicle $=85 \pm$ 20 s), EOM Sal B significantly decreased swimming behaviour by $50 \%$, from vehicle values of $144 \pm 15 \mathrm{~s}$ to $74 \pm 20 \mathrm{~s}$ ( $p<0.05$, Student's $t$-test, Figure 4.2B). This decrease was complemented by a significant increase in the time spent climbing, from vehicle values of $71 \pm 15 \mathrm{~s}$ to $131 \pm 18 \mathrm{~s}$ ( $p<0.05$, Student's $t$-test, Figure 4.2B). In summary, only MOM Sal B (but not 16-brSal A, EOM Sal B, $\beta$-THP Sal B) showed depressive-like effects in the FST. 
A)

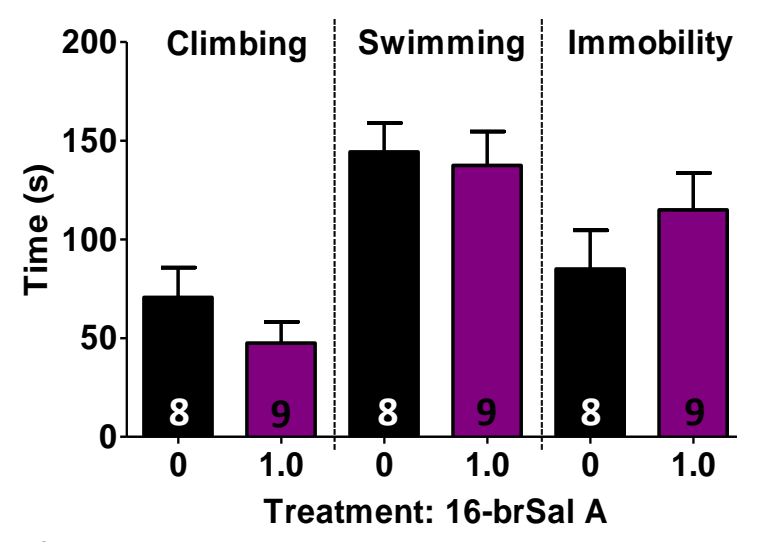

C)

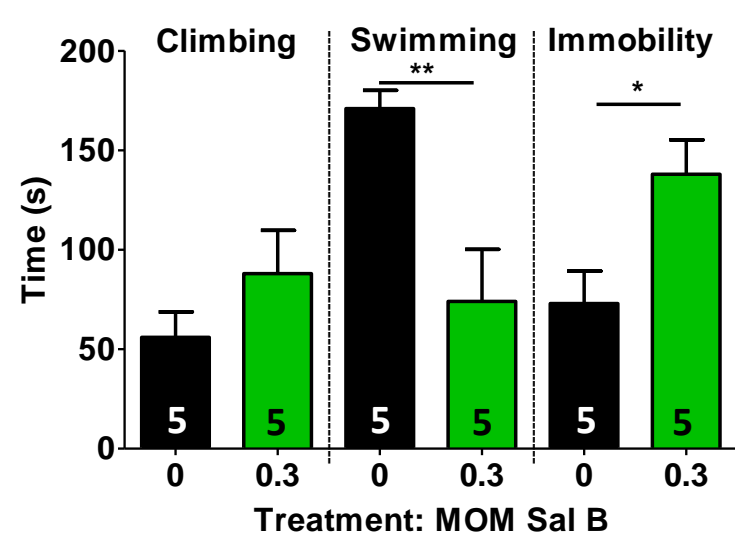

B)

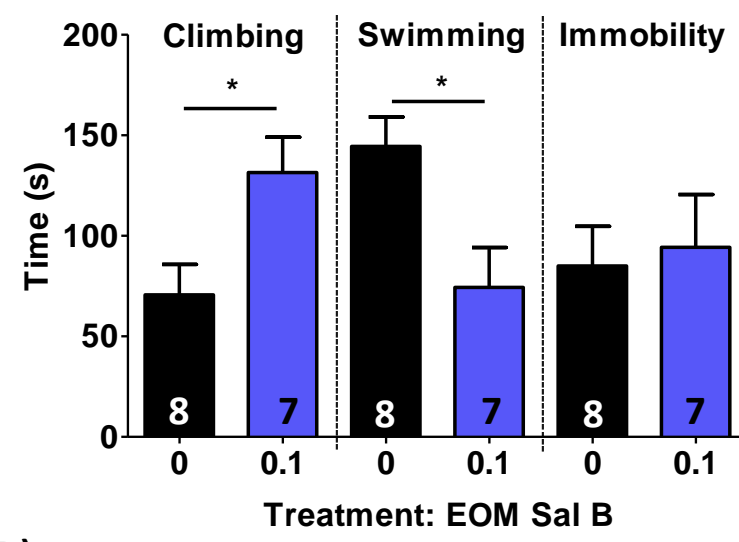

D)

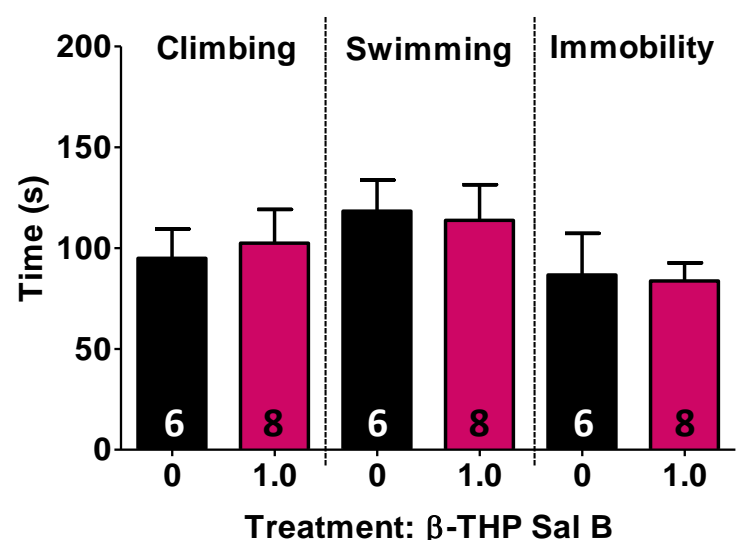

Figure 4.2: MOM Sal B increases occurrences of immobility in the rat FST

Climbing, swimming, and immobility time in the FST with A) 16-brSal A (purple bars), B) EOM Sal B (blue bars), C) MOM Sal B (green bars), or D) $\beta$-THP Sal B (pink bars) treatment. Black bars represent vehicle (75\% DMSO) controls. A) Rats treated with 16-brSal A displayed no significant change in climbing, swimming or immobility time. B) EOM Sal B-treated rats also displayed no change in occurrences of immobility, although a significant increase in climbing time accompanied by a significant decrease in swimming time was observed. C) Rats given MOM Sal B exhibited a significant increase in occurrences of immobility accompanied by a decrease in swimming time. D) $\beta$-THP Sal B-treated rats produced no change in climbing, swimming, or immobility time. All KOPr treatments were compared to vehicle controls. Student's $t$-test, ${ }^{*} p<0.05,{ }^{*} p<0.01$ compared to vehicle controls. Values presented as mean \pm SEM, $n=5-9$. 


\subsubsection{Effect of 16-brSal A, EOM Sal B, and $\beta$-THP Sal B on sucrose self-administration}

Sal A analogues that produced no significant depressive-like effects (16-brSal A, EOM Sal $B$, and $\beta$-THP Sal B) were subjected to further testing for their effects on sucrose reward, since traditional KOPr agonists have been shown to reduce sucrose self-administration. There was a significant effect of treatment on the number of active lever presses $[F(7,42)$ $=42.6, p<0.0001$ ] (repeated measures one-way ANOVA, Figure 4.3A). Planned comparisons conducted with the Bonferroni correction showed that administration of the vehicle alone (DMSO:Tween-80:MilliQ water in a ratio of 2:1:7) produced $497 \pm 54$ active lever presses, a response that was not significantly different from baseline $(493 \pm$ 37 lever responses). However, U50,488 (10 mg/kg) significantly decreased sucrose selfadministration to less than $5 \%$ of control, from $497 \pm 54$ to $21 \pm 11$ active lever presses $(p<0.001$, Figure $4.3 A)$. In contrast, $U 69,593(0.3 \mathrm{mg} / \mathrm{kg})$ produced a trend towards an increase in responding for sucrose, resulting in $618 \pm 31$ active lever responses $(p=0.051$, Figure 4.3A). Sal A (0.3 mg/kg, $600 \pm 46$ active lever responses) and 16-brSal A (1.0 mg/kg, $529 \pm 55$ active lever presses) produced no significant difference in sucrose selfadministration compared to the vehicle. On the other hand, EOM Sal B $(0.1 \mathrm{mg} / \mathrm{kg})$ and $\beta$-THP Sal $B(1.0 \mathrm{mg} / \mathrm{kg}, p<0.05)$ both increased responding for sucrose, producing $616 \pm$ 33 and $626 \pm 54$ active lever presses respectively; although statistical analysis revealed that the EOM Sal B-mediated increase fell just short of statistical significance $(p=0.056$, paired $t$-tests with the Bonferroni correction, Figure 4.3A).

The temporal analysis of responding using a one-way ANOVA with repeated measures revealed a significant interaction between treatment and time $[F(8.758,61.303)=2.887$, $p=0.007$ ] (Greenhouse Geisser correction applied as Mauchly's test of sphericity indicated that sphericity could not be assumed $\left[\chi^{2}(2)=18.959, p<0.0001\right]$, Figure 4.3B). Planned comparisons with the Bonferroni correction showed that EOM Sal B significantly increased sucrose intake at $15-30 \mathrm{~min}(p<0.05)$. $\beta$-THP Sal B also produced a significant increase at $30-45$ min compared to vehicle-treated controls (from $9 \pm 1$ to $14 \pm 2$ active lever responses) ( $p<0.001$, Figure 4.3B). U69,593 significantly increased active lever responding at $15-30 \mathrm{~min}$ and $30-45 \mathrm{~min}$ ( $p<0.05$ and $p<0.01$ respectively), whereas U50,488 almost completely abolished responding for sucrose $(p<0.001$, Figure 4.3B). Thus, the only Sal A analogue that did not alter responding for sucrose was 16-brSal A. 

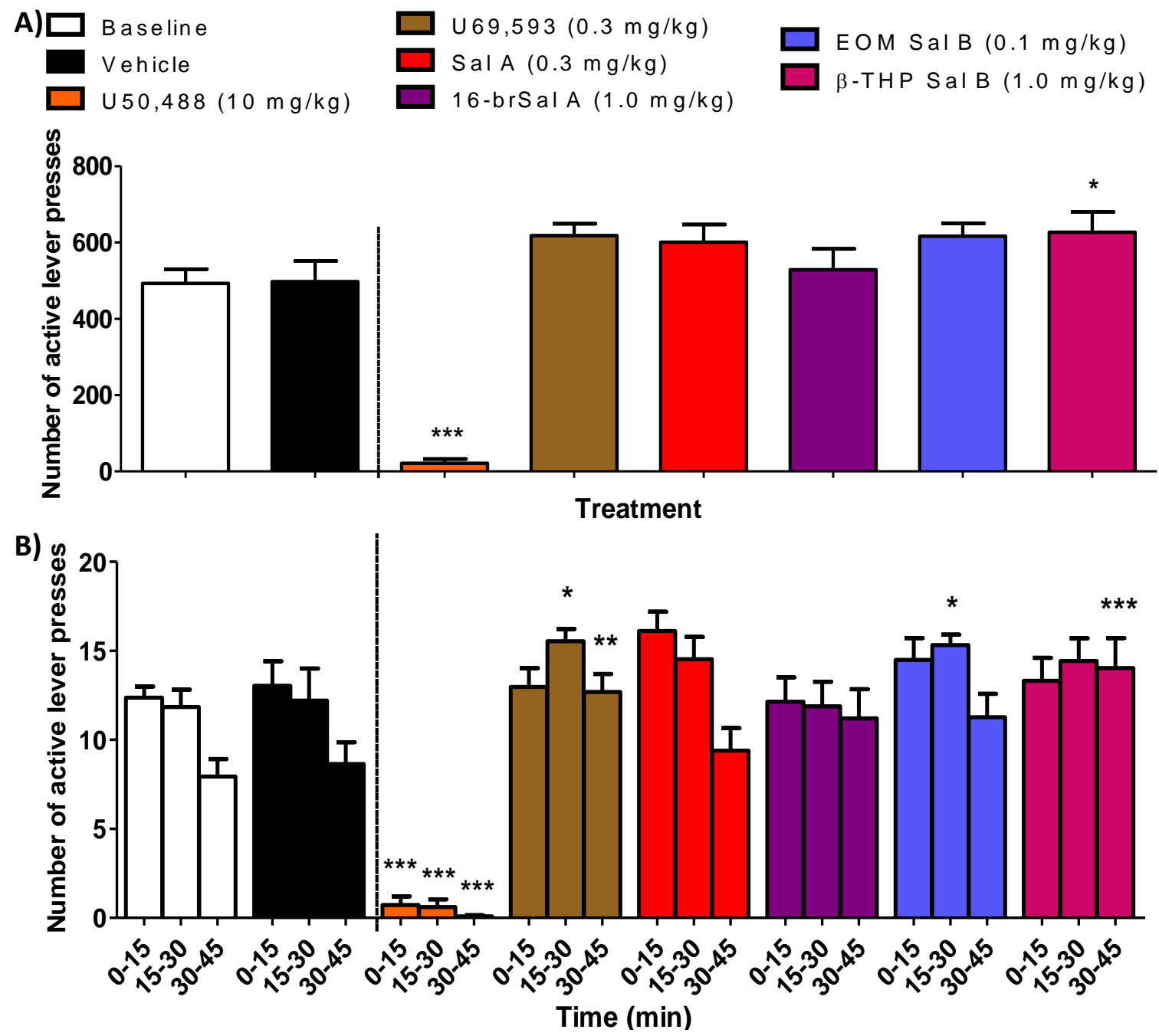

Figure 4.3: U50,488, EOM Sal B, and $\beta$-THP Sal B significantly alters responding for

\section{sucrose in rats}

A) U69,593, Sal A, 16-brSal A, and EOM Sal B did not significantly alter total responding for sucrose. U50,488 significantly decreased responding compared to the vehicle, whereas $\beta$-THP Sal B-treated rats increased responding for sucrose (one-way ANOVA with repeated measures followed by paired $t$-tests with the Bonferroni correction). B) Analysis of active lever presses in 15 min bins showed that U69,593 significantly increased lever pressing for sucrose at $15-45 \mathrm{~min}$. In contrast, U50,488 eliminated responding for sucrose throughout the session. EOM Sal B significantly increased responding for sucrose at $15-30 \mathrm{~min}$, whereas $\beta$-THP Sal B increased responding from 30 - 45 min (one-way ANOVA with repeated measures followed by planned comparisons with the Bonferroni correction applied). Sal A and 16-brSal A did not significantly alter sucrose self-administration. ${ }^{*} p<0.05,{ }^{* *} p<0.01,{ }^{* * *} p<0.001$ compared to time-matched controls. Values presented as mean \pm SEM, $n=7$. 


\subsubsection{Effect of 16-brSal A on anxiety}

Because KOPr agonism has been shown to cause anxiety, LD and EPM tests were used to see whether 16-brSal A - the only compound that did not show significant pro-depression or modulation of natural reward - was anxiogenic. In the LD test, a significant effect of treatment was observed $[F(3,55)=5.964, p=0.0014]$ (one-way ANOVA, Figure 4.4A). Subsequent planned comparisons with the Bonferroni correction showed that Sal A (1.0 $\mathrm{mg} / \mathrm{kg}$ ) produced a significant three-fold reduction in the light box time compared to vehicle controls, from $154 \pm 20 \mathrm{~s}$ to $45 \pm 12 \mathrm{~s}(\mathrm{p}<0.001)$. However, rats treated with the lower dose of Sal A $(0.3 \mathrm{mg} / \mathrm{kg})$ produced no change in the amount of time spent in the light box (123 $\pm 17 \mathrm{~s})$ compared to vehicle controls (Figure 4.4A). The Sal A analogue 16brSal A (108 \pm 17 s) also did not significantly alter light box time compared to controls (Figure 4.4A). There were no significant differences observed between the total distance travelled by rats in all four treatment groups throughout testing (inset in Figure 4.4A), indicating that the lack of anxiety seen was not due to sedation.

To further explore the anxiogenic effects of 16 -brSal A, rats were also tested using the EPM. Two rats were excluded from analysis because they fell off the maze during testing. A significant effect of treatment was observed $[F(3,75)=3.460, p=0.0205]$ (one-way ANOVA, Figure 4.4B).Consistent with the LD test, Sal A $(1.0 \mathrm{mg} / \mathrm{kg})$ produced a $36 \%$ decrease in time spent on the open arm, from vehicle values of $102 \pm 12 \mathrm{~s}$ to $65 \pm 11 \mathrm{~s}$ $(p<0.05$, Student's $t$-test with the Bonferroni correction applied, Figure $4.4 \mathrm{~B})$. It is noteworthy that the lower dose of Sal A $(0.3 \mathrm{mg} / \mathrm{kg})$ also significantly decreased open arm time, with an open arm time of $59 \pm 12 \mathrm{~s}$. In contrast, rats treated with 16-brSal A showed no significant changes in open arm time ( $82 \pm 21 \mathrm{~s})$ compared to vehicle controls (Figure 4.4B), in agreement with results obtained in the LD test. Thus, there was no evidence of anxiogenic side effects for 16-brSal A, despite Sal A having an anxiogenic effect in rats. 
A)

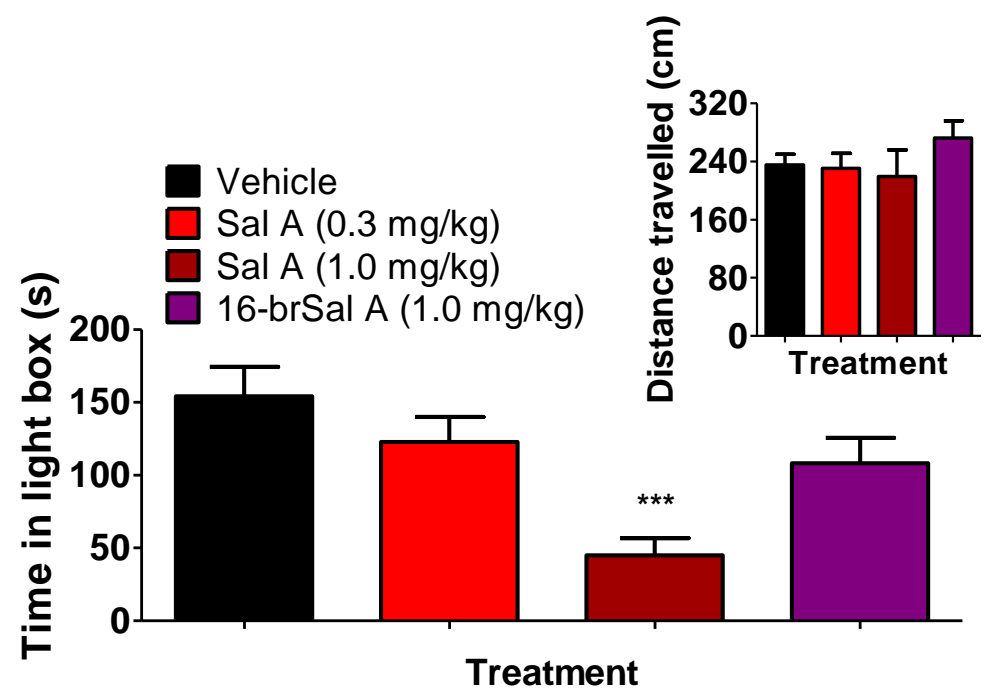

B)

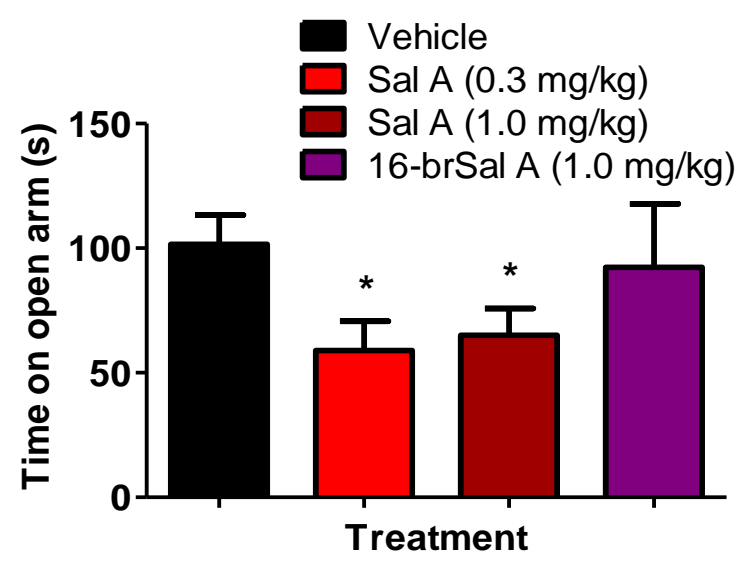

Figure 4.4: 16-brSal A produces no significant anxiogenic effects in rat anxiety models

A) Rats treated with 16-brSal A displayed no significant change in light box time compared to vehicle controls (DMSO:Tween-80:MilliQ water in a 2:1:7 ratio). In comparison, Sal A-treated rats $(1.0 \mathrm{mg} / \mathrm{kg})$ significantly showed decreased light box time. Inset: None of the treatments produced significant changes in distance travelled throughout the testing period. B) 16-brSal A did not significantly alter open arm time compared to vehicle-treated rats. In contrast, Sal A significantly decreased time spent on the open arm. One-way ANOVA followed by a post-hoc of Student's $t$ tests with the Bonferroni correction, ${ }^{*} p<0.05,{ }^{* * *} p<0.001$ compared to vehicle controls. Values presented as mean \pm SEM, $n=13-24$. 


\subsubsection{Effect of 16-brSal A on aversion}

Since previous studies have shown that Sal A produces aversion in rats (Sufka et al., 2014), 16-brSal A was also tested for aversive effects using the CPA model. Two rats were excluded from further testing as they showed extreme preference for a particular chamber in the pre-test. A further 11 rats were excluded from the study because they showed more than $40 \%$ preference for the corridor. Consistent with previous findings, Figure 4.5 shows that Sal A $(0.3 \mathrm{mg} / \mathrm{kg})$ significantly reduced the time rats spent in the paired chamber by $19 \%$ (pre-test of $57 \pm 5 \%$ vs. post-test of $38 \pm 7 \%$ ) ( $p<0.05$, paired Student's $t$-test). 16 -brSalA $(1.0 \mathrm{mg} / \mathrm{kg}$ ) also significantly reduced the amount of time rats spent in the paired chamber by $18 \%$ (from $56 \pm 4 \%$ before conditioning to $38 \pm 4 \%$ after conditioning) ( $p<0.05$, paired Student's $t$-test, Figure 4.5). In contrast, vehicle-treated rats (DMSO:Tween-80:MilliQ water in a 2:1:7 ratio) did not produce any significant changes in percentage of time spent in the paired chamber, with only a $8 \%$ decrease observed ( 44 $\pm 4 \%$ before conditioning vs. $36 \pm 4 \%$ after conditioning). Thus, 16 -brSal A produced aversive effects in rats in a similar manner to Sal A.

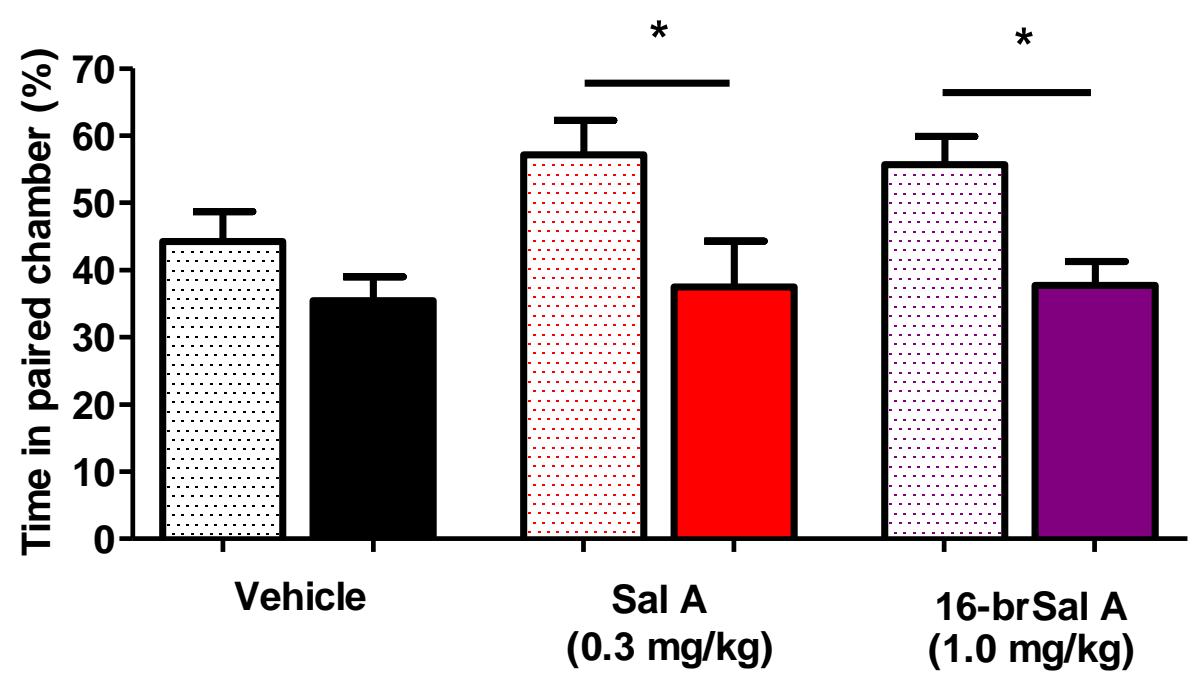

Treatment

Figure 4.5: Sal A and 16-brSal A produce conditioned place aversion in rats

Rats treated with Sal A and 16-brSal A displayed a significant decrease in time spent in the paired chamber compared to vehicle-treated rats (DMSO:Tween-80:MilliQ water at 2:1:7) in the CPA test. Stippled bars represent the pre-test period, solid filled bars represent the post-test period. Paired Student's $t$-test, ${ }^{*} \mathrm{p}<0.05$ compared to the pre-conditioning phase. Values presented as mean \pm SEM, $\mathrm{n}=8-9$. 


\subsection{Discussion}

Unlike traditional KOPr agonists that consistently produce KOPr-mediated adverse effects such as dysphoria, depression, hallucinations, sedation, emesis, and aversion, Sal A displays an inconsistent profile in several behavioural studies such as depression and aversion. Although Sal $A$ has shown negative effects at higher doses, it is important to note that anti-cocaine properties of Sal $\mathrm{A}$ are present at a dose that produces minimal side effects (0.3 mg/kg) (Morani et al., 2009; Morani et al., 2012). This notable difference between Sal A and traditional KOPr agonists may be due to a difference in KOPr binding or targets of the respective drugs. In support of this, a study conducted by Fantegrossi et al. (2005) found that administration of nor-BNI (30 mg/kg, i.p.) $24 \mathrm{~h}$ prior to testing inhibited motor incoordination induced by Sal A ( $2 \mathrm{mg} / \mathrm{kg}$ ) but not U69,593 (1 mg/kg). The authors also found that effects of U69,593 were eliminated 5 days following nor-BNI administration, and suggested that the differences in antagonism observed were due to dissimilar binding interactions (Fantegrossi et al., 2005). In support of this, it was found that Sal A binds to the KOPr in the same binding pocket as U69,593, but via different residues (Yan et al., 2005). Since it is highly probable that Sal A analogues will retain the improved side effect profile and binding properties of Sal A, this study investigated the adverse effects of several Sal A derivatives with known anti-cocaine effects.

\subsubsection{Sedative effects}

Previous studies have shown that Sal A can produce sedation in rats and rhesus monkeys. A single administration of Sal A (2 mg/kg, i.p.) significantly decreased locomotor activity in rats during the first $15 \mathrm{~min}$ of testing (Chartoff et al., 2008), whereas $0.032-0.1 \mathrm{mg} / \mathrm{kg}$ of Sal A (i.v.) produced dose-dependent sedation in rhesus monkeys (Butelman et al., 2009). It is, however, important to note that the doses used in those studies were much higher than the minimal anti-cocaine doses for those animals. The study conducted by Chartoff et al. (2008) utilised a seven-fold higher dose of Sal A (2 mg/kg vs. $0.3 \mathrm{mg} / \mathrm{kg}$ required for attenuation of drug seeking in Morani et al. (2009)), whereas Butelman et al. (2009) administered doses that were at least 10 times higher than that required for punishing cocaine self-administration $(0.003-0.01 \mathrm{mg} / \mathrm{kg}$, i.v.) (Freeman et al., 2014). As these studies suggest that sedative properties of Sal A and its derivatives may be dose- 
dependent, it is noteworthy that the minimal dose of Sal A $(0.3 \mathrm{mg} / \mathrm{kg}$, i.p. $)$ that attenuated drug-induced reinstatement does not alter spontaneous locomotion in rats (Morani et al., 2012). In addition, Morani et al. (2009) also showed that Sal A did not attenuate responses for a natural reward ( $10 \%$ sucrose) at $0.3 \mathrm{mg} / \mathrm{kg}$, indicating that the reduction in drug seeking responses by Sal A were specific to anti-cocaine properties, and not due to sedation or disruption of motor control. Similar to Sal A, this study found that both 16-brSal A- and MOM Sal B-treated rats showed no significant sedation at doses that attenuate drug seeking (Figure 4.1E and 4.1F). It is therefore important to note that the attenuation of drug seeking behaviour observed in Morani et al. (2013) and Figure 3.4A cannot be attributed to sedation. In addition, it can also be deduced that the 16-brSal Amediated decreases in cocaine-induced hyperactivity seen in Figure 3.5C was not caused by sedation.

It is, however, important to note that MOM Sal B produced a non-significant increase in ambulatory counts (Figure 4.1F, $p=0.20$ ). Although it can be argued that this increase may be due to the low ambulatory counts produced by vehicle-treated rats in the MOM Sal B study, further analysis of all rats in both the 16-brSal A and MOM Sal B study found no significant differences between vehicle-treated rats during habituation (Figure 8.5A in Appendix 4.2) or testing (Figure 8.5B in Appendix 4.2). Interestingly, a previous study conducted by Wang et al. (2008) found that MOM Sal B (1 - $5 \mathrm{mg} / \mathrm{kg}$, s.c.) increased ambulation and exploration on the Y-maze in rats (Wang et al., 2008), suggesting that MOM Sal B may actually increase locomotor activity. Although the different dose (1 - 5 $\mathrm{mg} / \mathrm{kg}$ vs. $0.3 \mathrm{mg} / \mathrm{kg}$ ) and route of administration (s.c. vs. i.p.) used in Wang et al. (2008) may be the reason results in that study differs from the present one, it may be possible that this study's non-significant increase in activity was a real increase that is responsible for the lack of attenuation in cocaine-induced hyperactivity studies by MOM Sal B (Figure 3.6C). Nonetheless, these studies collectively show that MOM Sal B did not produce significant sedation in rats, indicating that the anti-cocaine effects at its minimal effective dose are specific for cocaine.

In support of these observations, work using Sal A analogues such as Mesyl Sal B (0.3 $\mathrm{mg} / \mathrm{kg}$, i.p.) (Simonson et al., 2015), $\beta$-THP Sal B (1.0 mg/kg, i.p.) (Kivell Lab, unpublished 
data), EOM Sal B (0.1 mg/kg, i.p.) (Kivell Lab, unpublished data), and Ethy Sal A $(0.1 \mathrm{mg} / \mathrm{kg}$ ) (Riley et al., 2014) have also shown that these derivatives do not produce sedation at doses that attenuate cocaine seeking. In addition, another study investigating a G-protein biased Sal A analogue, 22-thiocyanatosalvinorin A (RB-64, 3mg/ $\mathrm{kg}$ ), found that this analogue did not decrease locomotion at the dose that produced analgesic effects (White et al., 2015). Taken together, these studies provide evidence that novel Sal A-derived analogues do not produce sedation that is characteristic of classic KOPr agonists.

\subsubsection{Depressive effects}

Sal A (0.25 to $2 \mathrm{mg} / \mathrm{kg}$, i.p.) has reported depressive effects in rats (Carlezon et al., 2006; Morani et al., 2012), effects that are attributed to KOPr-mediated decreases in DA neurotransmission in the NAc (Carlezon et al., 2006; Ebner et al., 2010). In contrast to previous findings, a recent study conducted by Harden et al. (2012) showed that $1 \mathrm{mg} / \mathrm{kg}$ Sal A reverses anhedonia in the chronic mild stress model in rats, a model the authors claim to be superior to the FST because the stressor and testing sessions are conducted at different times (Harden et al., 2012). Lower doses of Sal A also decreased immobility time in the FST in rats $(10-1000 \mu \mathrm{g} / \mathrm{kg}$, s.c.) and in the tail suspension test in mice $(0.001$ - $1.0 \mu \mathrm{g} / \mathrm{kg}$, s.c.) (Braida et al., 2009), suggesting that Sal A can produce anti-depressive effects. Sal A (0.1 and $0.2 \mu \mathrm{g} / \mathrm{kg}$, i.m.) also increased swimming behaviour in zebra fish (Braida et al., 2007), whereas the plant from which Sal A is extracted, Salvia divinorum, produced anti-depressive effects in a woman with a history of depression (Hanes, 2001). These results indicate that the anti-depressive effects of Sal A are present irrespective of species. This variable effect of Sal A on depression raises the possibility that synthetic derivatives of Sal A that would produce fewer depressive effects may be designed.

Of all the KOPr agonists investigated, MOM Sal B was the only compound to produce a significant increase in immobility in the FST (Figure 4.2C). This is consistent with a study which found that MOM Sal B (1 - $5 \mathrm{mg} / \mathrm{kg}$, s.c.) decreased vertical rearing and grooming in rats, subtle behaviour which the authors interpreted to indicate depression (Wang et al., 2008). On the other hand, EOM Sal B, $\beta$-THP Sal B, and 16-brSal A produced no significant changes in immobility time, implying no significant depressive-like effects. 
However, it is interesting to note that EOM Sal B-treated rats displayed a significant decrease in swimming time accompanied by a significant increase in climbing time (Figure 4.2B). Although this effect was complementary and did not produce a net decrease in mobility time, the differential mobility actions can potentially be attributed to modulation of 5-HT (swimming) and NE (climbing) systems. A study conducted by Detke et al. (1995) found that selective NE uptake inhibitors (desipramine and maprotiline) increased climbing behaviour, whereas selective 5-HT reuptake inhibitors (fluoxetine, sertraline, and paroxetine) increased occurrences of swimming in the FST (Detke et al., 1995). Investigation of EOM Sal B-mediated actions on these two systems is beyond the scope of this study, but further studies are required in order to determine the EOM Sal B-mediated mechanism in modulating mood.

Although 16-brSal A and $\beta$-THP Sal B produced no significant change in occurrences of swimming, climbing, or immobility in the rat FST (Figure 4.2A and 4.2D), it is important to note that control rats in the $\beta$-THP Sal B study exhibited elevated climbing time and decreased swimming time compared to other vehicle-treated rats (Figure 4.2D). Therefore, to confirm that the lack of $\beta$-THP Sal B-mediated depression was not due to this discrepancy, the swimming, climbing, and immobility time of all vehicle-treated rats were compared (Appendix 4.3). As this analysis revealed no significant differences, the lack of $\beta$-THP Sal B-mediated depression was not due to different climbing and swimming time of vehicle-treated rats in this study (one-way ANOVA with the Bonferroni multiple comparison test). It is also important to note that interpretation of FST data is based solely on immobility time, whereby an increase in immobility indicates depressive-like effects and vice versa (Porsolt, 1979; Porsolt et al., 2001; Carlezon et al., 2006). Since no significant differences in immobility time can be observed between control rats in the $\beta$ THP Sal B study compared to the other studies (Appendix 4.3), it can be inferred that the non-significant discrepancies observed in behaviour between control rats may be due to temporal differences and variation between batches of animals.

Taken together, these results show that MOM Sal B produced significant depressive effects at the minimal dose that attenuated drug seeking, in a similar manner as another Sal A analogue, Mesyl Sal B (Simonson et al., 2015). On the other hand, EOM Sal B, $\beta$-THP 
Sal B, and 16-brSal A all produced no significant depressive-like effects, encouraging further side effect screening of these compounds.

\subsubsection{Modulation of natural reward}

The action of KOPr agonists on sucrose self-administration is complicated due to the contrasting anti-reward and pro-feeding properties of KOPr agonists. Acute activation of the KOPr has been implicated in feeding and satiety (reviewed in Cooper et al. (1985) and Glass et al. (1999)). In support of this, intracerebroventricular (i.c.v.) administration of U50,488 (26 $\mu \mathrm{g}$ ) $15 \mathrm{~min}$ prior to testing stimulated sucrose feeding (45 mg pellets), whereas nor-BNI (50 $\mu$ g, i.c.v.) significantly decreased sucrose feeding in rats (Sipols et al., 2002). However, it is important to note that presentation of anti-reward or profeeding properties of KOPr agonists depends on sucrose concentration and dose of KOPr agonist. A dose-response study showed that lower doses of U50,488 (4 mg/kg, i.p.) increased sucrose intake in rats (Badiani et al., 2001), whereas rats given higher doses of U50,488 (5 - $10 \mathrm{mg} / \mathrm{kg}$, i.p.) decreased sucrose intake (Henderson-Redmond and Czachowski, 2014). Interestingly, Badiani et al. (2001) also discovered that the dose of U50,488 that increased sucrose intake (4 mg/kg, i.p.) only did so when sucrose concentrations were high ( $32 \%$ and $40 \%)$, whereas decreased intake was seen when sucrose concentrations were low (1\%). The authors suggested that this phenomenon is due to anti-dipsogenic properties of KOPr agonists, in which activation of KOPr by U50,488 stimulates feeding while supressing water intake (Badiani and Stewart, 1992; Badiani and Stewart, 1993). Therefore, the U50,488-mediated decrease in intake of a $1 \%$ sucrose solution may because this solution did not markedly differ from water in terms of solute content (Badiani et al., 2001). Thus, in an effort to prevent KOPr-mediated adipsia from interfering with the outcome of the present study, effects of KOPr agonists on natural reward were studied using self-administration of $45 \mathrm{mg}$ sucrose pellets.

Application of doses identical to or higher than the minimal dose that decreases cocaine seeking establishes the side effect profile of a KOPr agonist at its minimal effective anticocaine dose. Therefore, preclinical studies on the effects of KOPr agonists on natural reward have been conducted using these doses. Previous studies have found that 
although Sal A did not alter sucrose intake (Morani et al., 2009), the C-2 analogue of Sal A, MOM Sal B, attenuated self-administration of a $10 \%$ sucrose solution in rats at 0.3 $\mathrm{mg} / \mathrm{kg}$ (Morani et al., 2013). These studies suggest that despite their similarities in producing anti-cocaine effects, Sal A and its analogues can evoke different responses in modulating natural reward. In support of this, studies conducted using Mesyl Sal B (0.3 and $1.0 \mathrm{mg} / \mathrm{kg}$, i.p.), Ethy Sal A (0.3 mg/kg, i.p.), and Me Sal A (0.3 mg/kg, i.p.) showed no significant changes in sucrose self-administration (Kivell Lab, unpublished data).

The present study found that U50,488 inhibited sucrose self-administration (Figure 4.3A), consistent with data published by Henderson-Redmond and Czachowski (2014). On the other hand, U69,593 produced a significant increase in sucrose self-administration from $15-45$ min (Figure 4.3B), evidence that classic KOPr agonists can present both antireward and pro-feeding properties. Interestingly, although $\beta$-THP Sal B and EOM Sal B produced significant increases in sucrose intake, both Sal A and 16-brSal A showed no changes in sucrose self-administration (Figure 4.3B). Results in this study is consistent with work which showed that a threshold dose of Sal A $(0.25 \mathrm{mg} / \mathrm{kg}$, i.p.) did not significantly alter sucrose intake in a PR model of self-administration (Ebner et al., 2010). The magnitude and rate of sucrose self-administration responding observed in this study can also be used to support locomotor activity data, in which none of the Sal A analogues tested attenuated active lever responding (Figure 4.3A) or produced sedation (Figure 4.1E, Kivell Lab, unpublished study, Morani et al. (2012). Interestingly, rats treated with Sal A or 16-brSal A (compounds that did not alter sucrose intake) displayed a consistent decrease in responding for sucrose with the progression of time, similar to the trend observed in baseline and vehicle controls (Figure 4.3B). In contrast, U69,593-, EOM Sal B, and $\beta$-THP Sal B-treated rats (which showed an increase in sucrose intake) displayed an inverted U-shaped trend, in which the highest intake of sucrose was seen during the middle of the self-administration session (Figure 4.3B). These responding patterns suggest that EOM Sal B and $\beta$-THP Sal B may act to increase sucrose intake in a similar manner to U69,593, whereas Sal A- and 16-brSal A-treated rats act in a similar manner to vehicle controls. Although one can argue that different onset and/or pharmacokinetics of these compounds may play a role in the differential effects observed, it is important to note that Sal A has been reported to decrease sucrose intake as early as 10 min post- 
injection, effects that last until at least 40 min post-injection; sufficient for this study's test duration of 45 min (Ebner et al., 2010). EOM Sal B (Peet and Baker, 2011), 16-brSal A (Figure 3.2), and $\beta$-THP Sal B (Prevatt-Smith et al., 2011) have also reported similar onset times and longer duration of action compared to Sal A, indicating that the different effects of these compounds cannot be attributed to differences in pharmacokinetics alone.

The role of KOPr in stimulating appetite may be due to the presence of orexin, a neuropeptide that facilitates rewarding effects of food and drugs of abuse. The hypothalamic KOPr system has been implicated to modulate orexigenic effects (RomeroPico et al., 2013), with KOPr and orexin co-localised within synaptic vesicles in the hypothalamus (Muschamp et al., 2014). The heterodimerisation (Chen et al., 2015) and co-transmission of both peptides may be a mechanism by which KOPr regulates feeding (Muschamp et al., 2014). Although discussion of the relationship between KOPr and orexin is beyond the scope of this study, the extent by which KOPr and orexin cooperatively regulate sucrose self-administration warrants further investigation. Nonetheless, it is important to note that this study aimed to evaluate actions of novel Sal A derivatives on modulating natural reward, whereby deviation from normal behaviour (either an increase or decrease) should be regarded as a side effect. It is important to remember that although an increase in modulation of natural reward is not viewed as a negative effect, it has the potential to promote excessive intake of naturally rewarding food, causing weight gain. Thus, since results have shown that EOM Sal B and $\beta$-THP Sal B significantly increase sucrose self-administration, the only Sal A analogue that did not alter sucrose intake (or produce depressive-like effects), 16-brSal A, represents the most suitable KOPr agonist that can be further developed as an effective KOPr-based therapy. Therefore, further screening tests and discussion will only include 16-brSal A.

\subsubsection{Anxiogenic and aversive properties}

Activation of the KOPr increases anxiety in humans (Pfeiffer et al., 1986), mice (Smith et al., 2012), and rats (Bruchas et al., 2009; Valdez and Harshberger, 2012; Gillett et al., 2013), whereas blockade of KOPr by GNTI ( $3 \mathrm{nmol}$ in $3 \mu \mathrm{L}$, intracisternally) or nor-BNI (10 
$\mathrm{mg} / \mathrm{kg}$, i.p.) significantly increases open arm entries and time in a similar manner to prodynorphin knock-out mice (Wittmann et al., 2009), supporting a role of KOPr in mediating anxiety. KOPr-mediated aversion has also been demonstrated in rats (Goktalay et al., 2006; Tejeda et al., 2013) and mice (Skoubis et al., 2001; Chefer et al., 2013) (refer to Table 4.1 for a detailed summary). However, in a manner similar to its effects on depression and natural reward, Sal A deviates from normal KOPr-mediated behaviour in regulation of anxiety and aversion. Sal A produced little to no anxiety in humans with histories of hallucinogen use (MacLean et al., 2013). In fact, work conducted using rats showed that Sal A (0.1-160 $\mu \mathrm{g} / \mathrm{kg}$, s.c.) increased exploratory behaviour by increasing open arm time in the EPM, an indication of anxiolytic effects (Braida et al., 2009). The non-typical actions of Sal A extends to its aversive properties, whereby Sal A produces both aversion (0.3, 1.0, and $3.2 \mathrm{mg} / \mathrm{kg}$, i.p.) (Zhang et al., 2005; Sufka et al., 2014) as well as preference (0.1 and $40 \mu \mathrm{g} / \mathrm{kg}$, s.c.) (Braida et al., 2007; Braida et al., 2008) in laboratory animals.

In the present study, Sal A $(0.3 \mathrm{mg} / \mathrm{kg})$ produced significant anxiety in the EPM but not the LD behavioural test (Figure 4.4A and 4.4B), a discrepancy that may be due to the ability of EPM and LD models to assess different aspects of anxiety. Since anxiety is a multidimensional emotion (Ramos, 2008), similar discrepancies have been observed in other studies. A previous investigation using the neurokinin 1 receptor antagonist, NKP608 $(0.003-0.3 \mathrm{mg} / \mathrm{kg})$, showed anxiolytic effects only when tested using the rat open field, but not the EPM test. In addition, NKP608 only exhibited anxiolytic effects in male, but not female Lewis rats. (Vendruscolo et al., 2003). It is also important to note that Wistar Kyoto rats exhibit an increased level of basal anxiety compared to SpragueDawley rats (Carr and Lucki, 2010). Taken together, these studies that the outcome of anxiety tests depends on the experimental model, sex, strain, genetic background, as well as environmental conditions of test subjects (Vendruscolo et al., 2003; Vendruscolo et al., 2006; Wahlsten et al., 2006). Since the present study utilised subjects from the same environmental background, strain, sex, and age, it can be concluded that the discrepancies obtained in the LD and EPM study are most likely due to the different test models themselves. It is therefore not surprising that Sal A produced differential results in the LD and EPM, although the higher dose of Sal A $(1.0 \mathrm{mg} / \mathrm{kg})$ showed significant 
anxiogenic effects in both models. Since the total distance travelled by Sal A- and vehicletreated rats in the LD test did not significantly differ (inset in Figure 4.4A), it is inferred that the Sal A-produced decrease in light box time is specific for anxiogenic effects and not a result of sedation. In contrast to Sal A, rats treated with 16-brSal A did not exhibit significant anxiogenic effects in the LD test, although a non-significant decrease in light box time was observed ( $p=0.37$, Figure $4.4 A$ ). To confirm that this non-significant effect was indeed due to a lack of anxiety, further testing of 16-brSal A was conducted using the EPM. Since 16-brSal A also showed no significant anxiogenic effects in the EPM test (Figure 4.4B), the non-significant decrease observed in the LD may be due to the different groups of rats used and different experimental days, i.e. biological variation in responses.

To further determine the adverse effect profile of 16 -brSal A, the aversive properties of 16-brSal A were examined using a 3-chambered CPA paradigm. Previous studies have shown that $U 69,593$ produces CPA in wild-type mice but not in conditional knock-out mice that do not possess KOPr on DA neurons (Chefer et al., 2013), indicating the role of KOPr in mediating aversion. However, Sal A has shown contrasting results, with aversion seen in Sprague-Dawley rats $(0.3-1.0 \mathrm{mg} / \mathrm{kg}$, i.p.) (Sufka et al., 2014) and mice (1.0 - 3.2 $\mathrm{mg} / \mathrm{kg}$, i.p.) (Zhang et al., 2005), but preference observed in Wistar rats $(0.1-40 \mu \mathrm{g} / \mathrm{kg}$, s.c.) (Braida et al., 2008) and zebra fish (0.2 and $0.5 \mu \mathrm{g} / \mathrm{kg}$, i.m.) (Braida et al., 2007). In this study, 16-brSal A-treated rats decreased the amount of time spent in the paired chamber, indicating pro-aversive properties. Similarly, rats given Sal A also showed CPA (Figure 4.5). However, it is interesting to note that this same dose of Sal A $(0.3 \mathrm{mg} / \mathrm{kg}) \mathrm{did}$ not produce aversion in the CTA (Morani et al., 2012). In fact, Morani et al. (2012) found that an acute administration of Sal $\mathrm{A}$ at this dose produced a trend towards preference (Morani et al., 2012). It is important to note that traditional KOPr agonists such as U50,488 (0.90, $1.60 \mathrm{mg} / \mathrm{kg}$ s.c.) have shown CTA in rats (Davis et al., 2009), indicating that this model can be used to evaluate aversive properties. However, a recent study comparing CTA and CPA models found that they are not directly comparable due to the contrast in temporal presentation of conditioned cues and drug injection (taste cues presented prior to drug injection in the CTA vs. place cues presented after drug injection in the CPA) (Gore-Langton et al., 2015). Although the authors suggested that CTA may be more sensitive than CPA, the emetic lithium chloride produced a CTA but not CPA (Gore- 
Langton et al., 2015), indicating that the pharmacodynamics of a compound impacts the outcome of CTA. In addition, rewarding drugs such as cocaine (Isaac et al., 1989) and morphine (Simpson and Riley, 2005) produced place preference but taste aversion in rats despite the same dose and route of administration used. It is also important to remember that the CTA procedure incorporates a water deprivation step that can cause stress to animals, resulting in a confounding factor (Anderson et al., 2014). Since KOPr agonists are also known to be diuretic (Dykstra et al., 1987), use of the CTA model with a palatable liquid may be inappropriate due to the availability of fluids during the test. Therefore, the CPA model is more appropriate for testing compounds associated with the stress response such as the KOPr agonists used in this study.

One proposed mechanism that may help explain the contrasting behavioural side effects of novel vs. traditional KOPr agonists on anxiety and aversion is the concept of functional selectivity (section 1.6). It has been hypothesised that certain KOPr ligands are capable of selectively activating the G-protein pathway (suggested to produce physiological KOPrmediated effects), while only weakly recruiting $\beta$-arrestin (suggested to be responsible for KOPr-mediated adverse effects) (Phillips et al., 2013; Zhou et al., 2013; Shonberg et al., 2014; Violin et al., 2014). In theory, G-protein biased, functionally selective KOPr ligands would show desirable therapeutic anti-addiction properties with few KOPrmediated side effects. This hypothesis was supported by a recent study conducted using a biased Sal A analogue, RB-64, that produced analgesic effects without causing sedation or anhedonia. Surprisingly, despite its fewer KOPr-mediated side effects, RB-64 (3 mg/kg, s.c.) still caused aversion in mice (White et al., 2015). In fact, White et al. (2015) found that U69,593 (1 mg/kg), Sal A (3 mg/kg), and RB-64 (3 mg/kg) produced CPA even in $\beta$ arrestin-knock-out mice, indicating that aversion may be in fact dependent on signalling at the G-protein pathway (White et al., 2015). However, further studies are warranted in order to thoroughly determine the side effect profile of 16-brSal A.

\subsubsection{Limitations and future directions}

The fewer KOPr-mediated effects displayed by Sal A and its novel analogues in this study indicate that Sal A-derived agonists, especially 16-brSal A, may potentially be developed 
as therapeutic agents. However, one major limitation in laboratory side effect screening of KOPr agonists is that the behavioural paradigms used generally produce the adverse effect themselves. For example, the FST is used to produce depressive-like effects and screen for anti-depressants (Porsolt, 1979), whereas the EPM elicits anxiety and is used to screen for anxiolytic compounds in rats (Braida et al., 2009). Therefore, a compound that does not produce a difference compared to vehicle controls may still be depressive or aversive, although subtle differences may not be detected using these tests. To that effect, a combination of behavioural models can be used to supplement observations obtained from a single test. Behavioural studies such as ICSS (whereby an elevated threshold signifies pro-depression) and chronic mild stress model can be conducted to complement FST findings, whereas anxiogenic properties can be evaluated using the open field test (to identify time spent in the centre vs. periphery of the apparatus). Integration of anxiety models (including open field, EPM, and LD) are also more effective when screening for anxiety (Ramos, 2008). The aversive properties of KOPr agonists can also be investigated using the CTA paradigm to eliminate any discrepancy produced by differences in test sensitivity. Nonetheless, although the EPM, LD, FST, and CPA screening models cannot be taken as conclusive, stand-alone tests, these paradigms are widely accepted and have been widely used as valid methods for screening adverse effects (Leri and Franklin, 2000; Pliakas et al., 2001; Skoubis et al., 2001; Zhang et al., 2005; Carlezon et al., 2006; Goktalay et al., 2006; Morani et al., 2012; Chefer et al., 2013; Morani et al., 2013; Tejeda et al., 2013; Sufka et al., 2014).

In order to further investigate effects of KOPr agonists, dose-response studies can be conducted to further support results observed in this investigation. For example, varying doses of 16-brSal A can be used in studies involving modulation of natural reward to determine the threshold dose above which it affects intake of a natural reward. KOPr agonists also produce differential effects according to gender, with only female mice forming CPA with $2.5 \mathrm{mg} / \mathrm{kg}$ of U50,488 (i.p.) but male mice requiring a $10 \mathrm{mg} / \mathrm{kg}$ dose (i.p.) to cause CPA (Robles et al., 2014), suggesting that gender differences can play an important role in modulating the differential effects of KOPr agonists. Therefore, further studies determining the effects of novel KOPr agonists on male and female rats would help elucidate these differences. 
Other structurally novel, non Sal A-derived KOPr agonists have also showcased the possibility of new therapeutic compounds with fewer side effects. For example, (+)- 3acetyl-6-chloro-2-[2-(3-(N-(2-ethoxyethyl)-N-isopropylamino) propoxy)-5methoxyphenyl]benzothiazoline 0,0'-diacetyl-L-tartarate (SA14867) produced analgesia in rodents, as well as anti-pruritic effects in monkeys with minimal sedation (TsukaharaOhsumi et al., 2010; Tsukahara-Ohsumi et al., 2011). Another novel agonist, nalfurafine, currently prescribed in Japan for pruritus (Kumagai et al., 2009; Kumagai et al., 2011) also supresses rewarding effects of cocaine in rats without producing sedation or aversion (reviewed in Fujii et al. (2012)). In addition, clinical trial of CR845 (i.v.), a peripheral acting $\mathrm{KOPr}$ agonist, reduced post-operative pain with minimal nausea/vomiting (CARA Therapeutics, 2015). However, none of these agonists have been trialled clinically as anticocaine therapeutics to-date, indicating the need for preclinical and clinical investigation to determine the safety and efficacy of these agonists as anti-addiction treatments.

\subsection{Conclusions}

MOM Sal B and 16-brSal A produced no sedaton in rats, but MOM Sal B increased FST immobility time, indicating depressive-like effects. EOM Sal B, $\beta$-THP Sal B, and 16-brSal A displayed no significant depressive-like effects in the rat FST. However, $\beta$-THP Sal B and EOM Sal B significantly increased temporal sucrose self-administration, indicating that $\beta$ THP Sal B and EOM Sal B modulate intake of natural reward. In contrast, 16-brSal A did not significantly alter sucrose intake in rats. Taken together, these studies show 16-brSal A to be a KOPr agonist with the least side effects, making it the compound of choice for further studies. 16-brSal A produced no significant anxiogenic effects but caused significant aversion in the CPA. The minimal side effects of 16-brSal A makes it tempting to suggest that 16-brSal A may be a functionally selective agonist at the KOPr. However, further studies investigating the cellular actions of $16-\mathrm{brSal} A$ at the KOPr are required to establish its mechanism of action in more detail (Chapter 5 ). 


\begin{tabular}{|c|c|c|c|c|c|c|c|c|c|c|}
\hline \multirow[b]{2}{*}{ Side effect test } & \multicolumn{10}{|c|}{ KOPr agonist } \\
\hline & U69,593 & U50,488 & Sal A & $\begin{array}{l}\text { MOM } \\
\text { Sal B }\end{array}$ & $\begin{array}{c}\text { EOM Sal } \\
\text { B }\end{array}$ & $\begin{array}{l}\text { Mesyl } \\
\text { Sal B }\end{array}$ & $\begin{array}{c}\beta \text {-THP } \\
\text { Sal B }\end{array}$ & $\begin{array}{c}16-b r S a l \\
\text { A }\end{array}$ & $\begin{array}{c}\text { Me Sal } \\
\text { A }\end{array}$ & $\begin{array}{c}\text { Ethy Sal } \\
\text { A }\end{array}$ \\
\hline $\begin{array}{c}\text { Sedation } \\
\text { (Locomotor } \\
\text { activity/Rotarod in } \\
\text { rats) }\end{array}$ & - & $\begin{array}{c}\uparrow^{(6)} \\
2.5-40, \text { s.c. }\end{array}$ & $\begin{array}{c}\text { NE }(7,18) \\
0.125-2.0, \\
\text { i.p. }\end{array}$ & $\begin{array}{l}\text { NE*(14) } \\
0.3, \text { i.p. }\end{array}$ & $\begin{array}{c}\text { NE (9) } \\
0.1, \text { i.p. }\end{array}$ & $\begin{array}{c}\text { NE (8) } \\
0.3 \text {, i.p. }\end{array}$ & $\begin{array}{l}\text { NE }{ }^{(9)} \\
1.0, \text { i.p. }\end{array}$ & $\begin{array}{c}\text { NE* }^{*} \\
1.0, \text { i.p. }\end{array}$ & $\begin{array}{l}\text { NE }{ }^{(10)} \\
1.0, \text { i.p. }\end{array}$ & $\begin{array}{l}\text { NE }{ }^{(10)} \\
0.3 \text {, i.p. }\end{array}$ \\
\hline $\begin{array}{c}\text { Sedation } \\
\text { (Unresponsive to } \\
\text { stimuli and postural } \\
\text { relaxation in rhesus } \\
\text { monkeys) }\end{array}$ & $\begin{array}{c}\uparrow(1,3) \\
0.01-0.056, \text { i.v. } \\
0.01-0.032, \text { s.c. }\end{array}$ & $\begin{array}{c}\uparrow^{(5)} \\
0.10 \text { per h, i.v. }\end{array}$ & $\begin{array}{c}\text { 个 (1) } \\
0.032-0.1 \\
\text { i.v. }\end{array}$ & - & - & - & - & - & - & - \\
\hline $\begin{array}{c}\text { Sedation } \\
\text { (Inverted screen } \\
\text { performance / } \\
\text { Locomotor activity } \\
\text { in mice) }\end{array}$ & $\begin{array}{c}\uparrow^{(2)} \\
1.0, \text { i.p. }\end{array}$ & $\begin{array}{c}\uparrow^{(4)} \\
1.0,10, \text { i.p. }\end{array}$ & $\begin{array}{c}\uparrow^{(2)} \\
0.5-2.0, \text { i.p. } \\
\mathrm{NE}^{(19)} \\
0.001-1 \\
\mu \mathrm{g} / \mathrm{kg}, \text { s.c. }\end{array}$ & - & - & - & - & - & - & - \\
\hline $\begin{array}{l}\text { Modulation of } \\
\text { sucrose self- } \\
\text { administration in } \\
\text { rats }\end{array}$ & $\begin{array}{c}\mathbf{N E}^{*} \\
0.3, \text { s.c. }\end{array}$ & $\begin{array}{c}\uparrow^{(11)} \\
26 \mu g \text {, i.c.v. } \\
\uparrow \text { and } \downarrow \text { (12) } \\
4 \text {, i.p. } \\
\downarrow \text { (13) } \\
2.5-10 \text {, i.p. }\end{array}$ & $\begin{array}{c}\text { NE }{ }^{(7)} \\
0.3,1.0, \text { i.p. } \\
\downarrow^{(15)} \\
2.0 \text {, i.p. }\end{array}$ & $\begin{array}{c}\downarrow^{(14)} \\
0.3, \text { i.p. }\end{array}$ & $\begin{array}{c}\text { NE* }^{*} \\
0.1 \text {, i.p. }\end{array}$ & $\begin{array}{l}\text { NE }{ }^{(9)} \\
0.3,1.0 \text {, } \\
\text { i.p. }\end{array}$ & $\begin{array}{c}\text { NE* }^{*} \\
1.0, \text { i.p. }\end{array}$ & $\begin{array}{c}\mathrm{NE}^{*} \\
1.0, \text { i.p. }\end{array}$ & $\begin{array}{c}\text { NE }{ }^{(9)} \\
0.3 \text {, i.p. }\end{array}$ & $\begin{array}{c}\text { NE }{ }^{(9)} \\
0.3 \text {, i.p. }\end{array}$ \\
\hline $\begin{array}{l}\text { Depression (Forced } \\
\text { swim test in rats) }\end{array}$ & $\begin{array}{c}\uparrow^{(37)} \\
0.3-10, \text { i.p. }\end{array}$ & - & $\begin{array}{c}\uparrow^{(7,18)} \\
0.25-2.0, \text { i.p. } \\
\downarrow^{(19)} \\
10-1000 \\
\mu g / k g, \text { s.c. }\end{array}$ & $\begin{array}{l}\text { 个*(14) } \\
0.3, \text { i.p. }\end{array}$ & $\begin{array}{c}\text { NE* } \\
0.1, \text { i.p. }\end{array}$ & $\begin{array}{c}\uparrow(9) \\
0.3 \text {, i.p. }\end{array}$ & $\begin{array}{c}\text { NE* }^{*} \\
1.0, \text { i.p. }\end{array}$ & $\begin{array}{c}\text { NE* }^{*} \\
1.0, \text { i.p. }\end{array}$ & - & - \\
\hline $\begin{array}{c}\text { Depression } \\
\text { (Tail suspension } \\
\text { test in mice) }\end{array}$ & - & - & $\begin{array}{c}\downarrow^{(19)} \\
0.001-1.0 \\
\mu \mathrm{g} / \mathrm{kg}, \text { s.c. }\end{array}$ & - & - & - & - & - & - & - \\
\hline
\end{tabular}




\begin{tabular}{|c|c|c|c|c|c|c|c|c|c|c|}
\hline \multirow[b]{2}{*}{ Side effect test } & \multicolumn{10}{|c|}{ KOPr agonist } \\
\hline & U69,593 & U50,488 & Sal A & $\begin{array}{l}\text { MOM } \\
\text { Sal B }\end{array}$ & $\begin{array}{l}\text { EOM } \\
\text { Sal B }\end{array}$ & $\begin{array}{l}\text { Mesyl } \\
\text { Sal B }\end{array}$ & $\begin{array}{l}\beta-\mathrm{THP} \\
\text { Sal B }\end{array}$ & $\begin{array}{c}\text { 16-brSal } \\
\mathrm{A}\end{array}$ & $\begin{array}{c}\text { Me Sal } \\
\text { A }\end{array}$ & $\begin{array}{l}\text { Ethy } \\
\text { Sal A }\end{array}$ \\
\hline $\begin{array}{l}\text { Depression (Intracranial } \\
\text { self-stimulation in mice) }\end{array}$ & - & $\begin{array}{c}\uparrow^{(20)} \\
3.0,5.5 \text {, i.p. }\end{array}$ & - & & - & - & - & - & - & - \\
\hline $\begin{array}{c}\text { Depression } \\
\text { (Chronic mild stress in rats) }\end{array}$ & - & - & $\begin{array}{r}\downarrow^{(26)} \\
1.0, \text { i.p. }\end{array}$ & & - & - & - & - & - & - \\
\hline $\begin{array}{l}\text { Depression (Intracranial } \\
\text { self-stimulation in rats) }\end{array}$ & $\begin{array}{c}\uparrow(21-23,38) \\
0.18-0.56 \\
\text { i.p. }\end{array}$ & - & $\begin{array}{c}\text { 个 and } \downarrow{ }^{(15,18,24,25)} \\
1.0-4.0, \text { i.p. } \\
\text { NE }{ }^{(15)} \\
0.25 \text {, i.p. }\end{array}$ & - & - & - & - & - & - & - \\
\hline $\begin{array}{c}\text { Anxiety } \\
\text { (Light dark test in rats) }\end{array}$ & - & - & $\begin{array}{c}\uparrow^{*} \\
1.0, \text { i.p. }\end{array}$ & - & - & - & $\begin{array}{c}\uparrow^{(9)} \\
1.0, \text { i.p. }\end{array}$ & $\begin{array}{l}\text { NE* }^{*} \\
1.0 \text {, i.p. }\end{array}$ & - & - \\
\hline $\begin{array}{c}\text { Anxiety } \\
\text { (Elevated plus maze in rats) }\end{array}$ & - & $\begin{array}{l}\uparrow^{(17,27,28)} \\
5,10 \text {, i.p. }\end{array}$ & $\begin{array}{c}\downarrow^{(19)} \\
0.1-160 \mu \mathrm{g} / \mathrm{kg} \text {, s.c. } \\
\uparrow^{*} \\
0.3,1.0, \text { i.p. }\end{array}$ & - & - & - & - & $\begin{array}{l}\text { NE* }^{*} \\
1.0, \text { i.p. }\end{array}$ & - & - \\
\hline $\begin{array}{c}\text { Anxiety } \\
\text { (Elevated plus maze mice) }\end{array}$ & - & $\begin{array}{l}\uparrow^{(29)} \\
5, \text { i.p. }\end{array}$ & a & - & - & - & - & - & - & - \\
\hline $\begin{array}{l}\text { Conditioned place aversion } \\
\text { (a } \downarrow \text { indicates (PP) in rats }\end{array}$ & $\begin{array}{c}\uparrow^{(31)} \\
0.32, \text { s.c. }\end{array}$ & $\begin{array}{c}\text { }^{(33,34)} \\
1, \text { s.c. } \\
\uparrow(9) \\
10 \text {, i.p. }\end{array}$ & $\begin{array}{c}\uparrow^{*(35)} \\
0.3-1.0 \text {, i.p. } \\
\downarrow^{(16)} \\
0.1-40 \mu \mathrm{g} / \mathrm{kg}, \text { s.c. }\end{array}$ & - & - & - & $\begin{array}{c}\uparrow^{(9)} \\
1.0, \text { i.p. }\end{array}$ & $\begin{array}{c}\uparrow^{*} \\
1.0, \text { i.p. }\end{array}$ & - & - \\
\hline $\begin{array}{l}\text { Conditioned place aversion } \\
\text { in mice }\end{array}$ & $\begin{array}{c}\uparrow^{(30)} \\
0.32 \text {, s.c. }\end{array}$ & $\begin{array}{l}\uparrow^{(32)} \\
2, \text { s.c. }\end{array}$ & $\begin{array}{c}\uparrow^{(36)} \\
1.0-3.2, \text { i.p. }\end{array}$ & - & - & - & - & - & - & - \\
\hline
\end{tabular}

*denotes this study, $\downarrow=$ attenuation, $\uparrow=$ presence of effect/potentiation, $\mathrm{NE}=$ no effect, - = study not found. Unless otherwise stated, doses are given in $\mathrm{mg} / \mathrm{kg}$.

\section{Table 4.1: Acute effects of U69,593, U50,488, Sal A, and Sal A analogues on KOPr-mediated adverse effects}


1) Butelman et al. (2009)

2) Fantegrossi et al. (2005)

3) Butelman and Kreek (2001)

4) Paris et al. (2011)

5) Negus et al. (1997)

6) Gallantine and Meert (2008)

7) Morani et al. (2009)

8) Simonson et al. (2015)

9) Kivell Lab, unpublished data

10) Riley et al. (2014)

11) Sipols et al. (2002)

12) Badiani et al. (2001)

13) Henderson-Redmond and Czachowski (2014)
14) Morani et al. (2013)

15) Ebner et al. (2010)

16) Braida et al. (2008)

17) Bruchas et al. (2009)

18) Carlezon et al. (2006)

19) Braida et al. (2009)

20) DiNieri et al. (2009)

21) Bonano et al. (2014)

22) Tomasiewicz et al. (2008)

23) Do Carmo et al. (2009)

24) Potter et al. (2011)

25) Beguin et al. (2008)

26) Harden et al. (2012)
27) Gillett et al. (2013)

28) Valdez and Harshberger (2012)

29) Smith et al. (2012)

30) Chefer et al. (2013)

31) Tejeda et al. (2013)

32) Skoubis et al. (2001)

33) Leri and Franklin (2000)

34) Goktalay et al. (2006)

35) Sufka et al. (2014)

36) Zhang et al. (2005)

37) Mague et al. (2003)

38) Todtenkopf et al. (2004) 


\section{Chapter 5: Investigating the cellular}

\section{mechanisms of 16-brSal $A$ in rat brain tissue and}

\section{HEK-293 cells}

\subsection{Introduction}

Although the anti-cocaine and adverse effects of KOPr agonists are generally agreed upon, the cellular mechanisms by which KOPr agonists exert their behavioural actions are not yet fully known. Since a better understanding of KOPr-mediated cellular mechanisms is crucial to the development of novel KOPr agonists as therapeutic agents, this study will focus on the acute actions of 16-brSal A on DAT function and expression, as well as the activation of downstream MAPK signalling transduction pathways such as ERK1/2 and p38.

\subsubsection{KOPr modulation of DAT function and expression}

Activation of the KOPr antagonises the rewarding effects of cocaine, suggesting that KOPr agonists and cocaine exert opposite cellular effects on DA neurotransmission. Cocaine has a high affinity for DAT (Chefer and Shippenberg, 2002) and prevents DA uptake by binding to DAT (Huang et al., 2009), whereas acute KOPr activation modulates DA neurotransmission by decreasing DA release (Di Chiara and Imperato, 1988b; Shippenberg et al., 1993; Margolis et al., 2003; Ebner et al., 2010) and increasing DA uptake via DAT (Thompson et al., 2000; Kivell et al., 2014; Simonson et al., 2015). Although an earlier study showed that injection of $U 69,593(0.32 \mathrm{mg} / \mathrm{kg}$, s.c.) for three days had no effect on DA uptake in the rat dStr and NAc (Izenwasser et al., 1998), a later study showed that acute injection of U69,593 (0.32 mg/ $\mathrm{kg}$, i.p.) led to significant increases in DAT function after $2 \mathrm{~h}$, an effect inhibited by pre-treatment with nor-BNI to block the $\mathrm{KOPr}(10 \mathrm{mg} / \mathrm{kg}$, s.c.) (Thompson et al., 2000). Acute administration of U50,488 (500 nM) also produced a KOPr-mediated, MEK1/2-dependent increase in DA uptake in the NAc, dStr, and PFC of male rats (Simonson et al., 2015), whereas repeated treatment with U69,593 (0.32 mg/kg, s.c.) for 5 days caused a significant decrease in DAT function in the NAc (Thompson et al., 2000) that was accompanied by a decrease in DAT density in the 
dStr (Collins et al., 2001a; Collins et al., 2001b). Differences between these studies suggest that repeated administration of KOPr agonists exerts opposite effects on DAT compared to acute treatments.

The acute effects of KOPr agonists on DAT are also seen in cell models, with YFP-hDAT and myc-rKOPr expressing HEK-293 cells exhibiting a significant increase in DAT function when treated with U50,488, Sal A (5 and $10 \mu \mathrm{M})$, or the Sal A derivative, Mesyl Sal B (10 $\mu \mathrm{M})$ (Simonson et al., 2015). These effects were inhibited by pre-incubation with nor-BNI $(1 \mu \mathrm{M})$. However, it is interesting to note that the increase in DAT function following Mesyl Sal B treatment was not accompanied by an increase in DAT cell surface expression, unlike that seen with Sal A and U69,593 (Simonson et al., 2015). The DAT cell surface response to Mesyl Sal B suggests that although all KOPr agonists modulate DAT function, novel Sal A analogues may regulate DAT in a different manner. This differential regulation may account for different anti-cocaine profiles of the different Sal A derivatives in comparison to classic agonists - for example, Mesyl Sal B $(0.3 \mathrm{mg} / \mathrm{kg}$, i.p.) attenuated behavioural sensitisation to repeated cocaine, whereas U69,593 $(0.3 \mathrm{mg} / \mathrm{kg}$, s.c.) and $\mathrm{U} 50,488$ (10 mg/kg, i.p.) did not (for a summary of anti-cocaine effects, refer to Table 3.1) (Vanderschuren et al., 2000; Morani et al., 2012). For this reason, investigation of 16brSal A-modulated DAT function and expression is crucial to determine the mechanism by which it mediates anti-cocaine behavioural responses.

\subsubsection{KOPr-mediated ERK1/2 and p38 MAPK activation}

KOPr activation is linked to several MAPK signalling networks, with the ERK1/2 pathway being the most well-studied (Pearson et al., 2001; Belcheva et al., 2005; Potter et al., 2011; Yoshizawa et al., 2011; Kivell et al., 2014) (refer to Bruchas and Chavkin (2010) for a full review). KOPr-mediated activation of ERK1/2 increases DAT function and DAT cell surface expression (Moron et al., 2003; Bolan et al., 2007; Zapata et al., 2007), indicating that KOPr-evoked upregulation of DAT function probably occurs via ERK1/2-dependent signalling pathways. Both traditional (U50,488 and U69,593) (Belcheva et al., 2005; McLennan et al., 2008; Simonson et al., 2015) and Sal A-derived agonists (Sal A, MOM Sal B, and Mesyl Sal B) activate ERK1/2 leading to phosphorylated ERK1/2 (McLennan et al., 
2008; Kivell et al., 2014; Simonson et al., 2015), although the extent of ERK1/2 phosphorylation differs according to the agonist (refer to Table 5.4 at the end of this chapter for a full summary). ERK1/2 activation occurs in two phases: an early, $\beta$-arrestinindependent phase (suggested to produce the physiological anti-pain and anti-cocaine properties of KOPr agonist) and a late, $\beta$-arrestin-dependent phase (implicated in the negative behavioural properties of KOPr agonists) (McLennan et al., 2008; Al-Hasani and Bruchas, 2011; Phillips et al., 2013; Zhou et al., 2013). It is therefore interesting to note that although U69,593 (1 $\mu \mathrm{M})$ generated both rapid (5 min) and sustained (120 min) phosphorylation of ERK1/2 in immortalised astrocyte cultures (McLennan et al., 2008), Sal A activated ERK1/2 only in the early phase (5 min) (McLennan et al., 2008). This pattern of ERK activation is mirrored in the rat NAc, in which Potter et al. (2011) showed that acute administration Sal A rapidly activated ERK1/2 at the 15 min time point (Potter et al., 2011).

The unique activation displayed by Sal $A$ is matched by its synthetic derivatives. MOM Sal $B$, which has a seven-fold greater potency for the KOPr in GTP- $\gamma$ S binding assays $\left(E_{50}\right.$ : MOM Sal B = 0.6 nM; Sal A = 4.5 nM) and a longer half-life in vitro (Lee et al., 2005) showed a lack of late phase ERK1/2 phosphorylation in immortalised astrocyte cultures (McLennan et al., 2008). Another Sal A derivative, Mesyl Sal B, which has similar binding affinity to Sal $\mathrm{A}$ (Ki of $2.3 \mathrm{nM}$ compared to Sal A Ki of $1.9 \mathrm{nM}$ ) and potency as Sal A (EC 50 measured using the GTP- $\gamma$ S binding assay: Mesyl Sal B $=30 \mathrm{nM}$, Sal $A=40$ ) also produced only a rapid, $\beta$-arrestin-independent but not the sustained, $\beta$-arrestin-dependent activation of ERK1/2 in KOPr-expressing HEK-293 cells and rat dStr, NAc, and PFC (Simonson et al., 2015). This differential ERK1/2 signalling of novel KOPr agonists supports the idea of functional selectivity, whereby different KOPr compounds can selectively activate particular pathways rather than the full complement of signalling pathways.

The concept of functional selectivity also extends to p38, another molecule downstream of $\beta$-arrestin recruitment. However, p38 activation contrasts with that of KOPr-mediated ERK1/2 activation in that p38 is GRK3/ $\beta$-arrestin dependent (Bruchas et al., 2006) whereas ERK1/2 has both $\beta$-arrestin-dependent and -independent phases (McLennan et al., 2008). Due to the fact that $\beta$-arrestin recruitment mediates KOPr-induced stressful, 
dysphoric, and aversive behavioural effects (Bruchas et al., 2006; Bruchas et al., 2007a; Carr and Mague, 2008; Whitmarsh, 2010), therapeutically desirable, functionally selective/biased KOPr ligands should in theory not activate p38 and only produce phosphorylation of the $\beta$-arrestin-independent phase of ERK1/2. In support of this, recent studies have identified $6^{\prime}$-GNTI as a G-protein biased KOPr ligand that can produce prolonged anti-nociception in a rat model of prostaglandin $E_{2}$-stimulated thermal allodynia (Berg et al., 2012) without recruiting $\beta$-arrestin (Rives et al., 2012). As there is great continual support for research into identification of functionally selective therapeutic agents, it is crucial to determine whether 16 -brSal A recruits $\beta$-arrestin by investigating downstream ERK1/2 and p38 MAPK activation. This would provide the possibility of correlating specific cell signalling pathways activated to the side effect profiles previously identified in Chapter 4.

\subsection{Aims and hypotheses}

The aim of this study was to determine whether 16-brSal A modulates DA neurotransmission via regulation of DAT in in vitro (HEK-293 cells expressing KOPr and DAT) and ex vivo (rat dStr and NAc) models, as has been demonstrated by the parent compound Sal A. The ability of KOPr agonists to modulate DAT in an ERK1/2-dependent manner is believed to be a key mechanism in the regulation of cocaine reward. However, traditional and Sal A-derived KOPr agonists have shown differences in ERK1/2-regulation of DAT, with traditional agonists producing both $\beta$-arrestin-dependent and -independent ERK1/2 activation, whereas Sal $A$ and its analogues produced only $\beta$-arrestin-independent ERK1/2 phosphorylation. Therefore, this study was directed at determining whether the improved side effect profile of 16-brSal A could be correlated to an ability to only weakly recruit $\beta$-arrestin, by investigating 16-brSal A-mediated activation of ERK1/2 and the $\beta$ arrestin-dependent p38 MAPK pathway. The hypothesis of the present study is that 16brSal A will upregulate DAT function without altering DAT cell surface expression. In addition, it is hypothesised that 16-brSal A will activate only the early, $\beta$-arrestinindependent phase of ERK1/2 but not the sustained, late phase of ERK1/2 or p38 MAPK. 


\subsection{Methods}

\subsubsection{Materials}

Protease inhibitor (P8340), phosphatase inhibitor (P5726), and U0126 were purchased from Sigma-Aldrich. The KOPr specific anti-myc antibody (Ab18185, Mouse monoclonal Ab to Myc) was purchased from Abcam and the Alexa Zenon ${ }^{\circledR} 647$ labelling kit (Molecular Probes $^{\mathrm{TM}}{ }^{\text {) }}$ was obtained from Life Technologies. For all in vitro and ex vivo testing, $\mathrm{KOPr}$ agonists were dissolved in $100 \%$ DMSO to prepare a $50 \mathrm{mM}$ stock concentration, whereas nor-BNI and U0126 were dissolved in 100\% DMSO to prepare a $10 \mathrm{mM}$ stock concentration. These stock solutions were diluted with KREBS buffer to prepare working concentrations for respective experiments. All concentrations given refer to either working concentrations (vehicle) or final concentrations (Sal A, 16-brSal A, nor-BNI, U0126) unless otherwise stated. For ex vivo experiments, 16-brSal A was dissolved in $75 \%$ DMSO. All injections were administered i.p. at a volume of $1 \mathrm{~mL} / \mathrm{kg}$.

\subsubsection{Immuno-labelling of myc-rKOPr for visualisation}

HEK-293 cells transiently expressing myc-rKOPr and YFP-hDAT were visualised to determine KOPr expression $48 \mathrm{~h}$ after transfection, using the anti-myc antibody conjugated to Zenon ${ }^{\circledR}$ Alexa 647 according to the manufacturer's protocol. Briefly, 472 $\mu \mathrm{L}$ of serum-free Dulbecco's Modified Eagle's Medium (DMEM, Life Technologies) was pre-warmed to $37^{\circ} \mathrm{C}$ in a microcentrifuge tube, before addition of $4 \mu \mathrm{L}$ of anti-myc antibody (Abcam, 1:125 dilution) and $12 \mu \mathrm{L}$ of Zenon ${ }^{\circledR} 647$ reagent (1:3 dilution). The mixture was incubated for $5 \mathrm{~min}$ at room temperature before addition of $12 \mu \mathrm{L}$ of Zenon ${ }^{\circledR}$ blocking reagent, and incubated for a further $5 \mathrm{~min}$ at room temperature. The mixture was added to $35 \mathrm{~mm}$ petri dishes containing transfected cells and incubated for $30 \mathrm{~min}$ in the dark at $37^{\circ} \mathrm{C}$. After incubation, the mixture was removed and cells were washed three times with KREBS buffer. One $\mathrm{mL}$ of KREBS buffer was then added to the cells, and the dishes were mounted on the confocal microscope in an insulated, double-layered $37^{\circ} \mathrm{C}$ heating chamber (\#INU-21LCS-F1, Tokai Hit Co. Ltd, Japan) for visualisation. The myc-rKOPr fluorescence was measured using the $635 \mathrm{~nm}$ laser (Alexa Fluor 647 filter: 635 $\mathrm{nm}$ excitation; $668 \mathrm{~nm}$ emission) while YFP-DAT fluorescence was visualised with the 473 $\mathrm{nm}$ laser according to section 2.2.6. 


\subsection{3 trans-4-[4-(dimethylamino)styryl]-1-methylpyridinium (ASP+) uptake}

All experiments were carried out in an insulated, stage-mounted incubator at $37^{\circ} \mathrm{C}$. To validate the $\mathrm{ASP}^{+}$uptake technique, cells co-expressing myc-rKOPr and YFP-hDAT were visualised in the $35 \mathrm{~mm}$ glass bottomed petri dish and KREBS buffer removed. A reference image was captured $(t=0)$, followed immediately by addition of $\operatorname{ASP}^{+}(1 \mathrm{~mL}, 10 \mu \mathrm{M})$ in KREBS buffer. Images were captured every $5 \mathrm{~s}$ for $10 \mathrm{~min}$. Cells expressing YFP-hDAT were manually outlined and the intensity of the YFP fluorescence in these regions of interest on the initial scan recorded using Olympus Fluoview FV1000 software. This initial intensity of YFP fluorescence is taken as a quantification of DAT cell surface expression. The intensity of $\mathrm{ASP}^{+}$accumulation $250 \mathrm{~s}$ after $\mathrm{ASP}^{+}$addition was determined, corrected for background autofluorescence (by subtracting the average ASP ${ }^{+}$intensity seen in nontransfected cells at $250 \mathrm{~s}$ ), and graphed in relation to their initial YFP-hDAT expression (intensity of YFP-hDAT fluorescence at $t=0$ ).

$\mathrm{ASP}^{+}$uptake tests were conducted as described in section 2.2.6. Briefly, KREBS buffer was removed from the dishes and a reference image of YFP-hDAT expression captured prior to addition of $1 \mathrm{~mL}$ of $\mathrm{ASP}^{+}(10 \mu \mathrm{M})$ in KREBS buffer to cells. Images were continually captured every $5 \mathrm{~s}$ for 10 min, with either a vehicle (0.02\% DMSO) or KOPr agonist $(5,10$ $\mu \mathrm{M}$ of $16-\mathrm{brSal} \mathrm{A}$ or $10 \mu \mathrm{M}$ of Sal A) added at $5 \mathrm{~min}$. To determine the KOPr-mediated action of 16 -brSal A, cells were pre-incubated with either vehicle (0.01\% DMSO) or norBNI $(1 \mu \mathrm{M})$ for 30 min prior to testing. On the other hand, the ERK1/2-depedent effect of 16-brSal A was verified by pre-incubating cells with either vehicle $(0.2 \% \mathrm{DMSO})$ or the MEK1/2 inhibitor, U0126 $(20 \mu \mathrm{M})$, for $30 \mathrm{~min}$ before testing. Since MEK directly phosphorylates ERK1/2, the inhibition of MEK prevents the activation of ERK1/2. ASP uptake data were analysed by manually outlining cells expressing YFP-hDAT to allow for appropriate selection of cells. A linear trendline of $\mathrm{ASP}^{+}$accumulation was generated over a period of $60 \mathrm{~s}$ prior to addition of the KOPr agonist and at the time of maximal change in uptake. Cells that did not have linear uptake were discarded as it indicated cell drift. The percentage change in rate of $\mathrm{ASP}^{+}$uptake was then calculated by comparing the change in slope of $\mathrm{ASP}^{+}$accumulation before and after KOPr agonist treatment [(uptake rate after drug - uptake rate before drug)/uptake rate before drug $\times 100]$. 
For $\mathrm{ASP}^{+}$uptake kinetics, HEK-293 cells co-expressing YFP-hDAT and myc-rKOPr were incubated with either vehicle (0.02\% DMSO) or 16-brSal A (10 $\mu \mathrm{M})$ in $1 \mathrm{~mL}$ of KREBS buffer for 2 min prior to removal of KREBS and imaging of the reference photo. Varying concentrations of $\operatorname{ASP}^{+}(1,2,3,4,5,6,7,8,9,12$, and $16 \mu \mathrm{M})$ containing vehicle or 16brSal A were added to different petri dishes and the linear slope of $\mathrm{ASP}^{+}$uptake for selected cells generated over a period of $60 \mathrm{~s}$ at the time of maximal effect. The background autofluorescence in a dish of cells was determined by manually outlining non-transfected cells using the Fluoview FV1000 software and averaging the rate of increase in $\mathrm{ASP}^{+}$fluorescence of these cells. All $\mathrm{ASP}^{+}$uptake kinetics data generated were corrected for background autofluorescence (by subtracting the average rate of $\mathrm{ASP}^{+}$ uptake seen in non-transfected cells) and normalised to YFP-hDAT expression. The normalised data were then entered into GraphPad Prism and uptake with 16-brSal A pretreatment compared to vehicle controls. All data generated for both $\mathrm{ASP}^{+}$uptake and $\mathrm{ASP}^{+}$kinetic studies in KOPr and DAT expressing cells were collected from a minimal of 3 separate transfections.

\subsubsection{Rotating disk electrode voltammetry (RDEV)}

The experiment was carried out and data analysed as described in section 2.1.7. Briefly, rats weighing between $300-400$ g were sacrificed via $\mathrm{CO}_{2}$ asphyxiation and decapitated. The brain regions of interest (dStr and NAc) were rapidly dissected on ice within $5 \mathrm{~min}$, suspended in $294 \mu \mathrm{L}$ of aerated KREBS buffer, and placed in the RDEV chamber. To determine the kinetics of DA uptake using the low to infinite trans model, tissue suspensions were treated with either vehicle (0.001\% DMSO) or 16-brSal A (500 nM) 4 min prior to sequential addition of DA. To determine the KOPr-mediated effect of 16 brSal A using the zero trans model, nor-BNI $(1 \mu \mathrm{M})$ was added to tissue suspensions 30 min prior to 16-brSal A treatment.

\subsubsection{Cell surface biotinylation}

Cells were treated with either the vehicle $(0.02 \%$ DMSO) or 16 -brSal A $(10 \mu \mathrm{M})$ for 2 min at $37^{\circ} \mathrm{C}$ before the start of the experiment. The cells were then washed with ice cold PBS/Ca-Mg and processed according to section 2.2.7. YFP-hDAT expression was 
measured by quantifying the intensity of the YFP tag using ImageJ software. Background readings were subtracted from the sample bands to yield background corrected values. The background corrected internal and cell surface fraction bands were normalised to total protein, generating arbitrary values. These values were then normalised to vehicle controls within the same gel to enable comparison between different gels.

\subsubsection{ERK1/2 and p38 MAPK activation}

For in vivo treatments, drug naïve rats weighing between 300 and 400 g were injected i.p. with either vehicle (75\% DMSO) or $16-\mathrm{brSal} A(1.0 \mathrm{mg} / \mathrm{kg})$ at selected times $(0,10-15$, 30 , or $120 \mathrm{~min}$ ) and sacrificed following $\mathrm{CO}_{2}$ asphyxiation. The dStr, NAc, and PFC was then removed within 5 min according to section 2.1.6. The brain tissue was homogenised in $100 \mu \mathrm{L}$ (NAc) or $200 \mu \mathrm{L}$ (dStr and PFC) of ice-cold RIPA buffer containing both protease and phosphatase inhibitors. Homogenates were then centrifuged at 16,000 g for $15 \mathrm{~min}$ at $4^{\circ} \mathrm{C}$ and the DNA pellet removed.

For in vitro treatments, HEK-293 cells transiently expressing myc-rKOPr were serum starved in serum-free DMEM for 30 min prior to testing. Cells were then incubated with 16-brSal A $(10 \mu \mathrm{M})$ for $0,5,10,15,30,60,120$, or $180 \mathrm{~min}$ at $37^{\circ} \mathrm{C}$. To determine the KOPr- or ERK1/2-mediated action of 16-brSal A, cells were pre-treated with vehicle (0.01\% DMSO for U0126 or 0.2\% DMSO for nor-BNI), KOPr antagonist nor-BNI (1 $\mu \mathrm{M})$ or MEK1/2 inhibitor U0126 (20 $\mu \mathrm{M}) 30$ min prior to a 10 min incubation with 16-brSal A (the amount of time that caused a largest increase in ERK1/2 activation according to the time course experiments). At the end of the incubation period, cells were washed twice with ice-cold sterile PBS and lysed with $200 \mu \mathrm{L}$ RIPA buffer containing protease and phosphatase inhibitors at $4^{\circ} \mathrm{C}$. Lysates were transferred into a microcentrifuge tube and centrifuged at $16,000 \mathrm{~g}$ for $30 \mathrm{~min}$ at $4^{\circ} \mathrm{C}$.

Both tissue and cell lysates were removed after centrifugation to be used for the Bradford protein assay and Western blotting studies. Resultant membranes were probed for pERK/ERK or p-p38/p38, and band densities quantified using ImageJ according to section 2.2.9. 


\subsubsection{Statistics}

For pilot $\mathrm{ASP}^{+}$uptake experiments, a linear regression was generated to determine the relationship between $\mathrm{ASP}^{+}$accumulation and YFP-hDAT cell-surface expression. $\mathrm{ASP}^{+}$ accumulation studies and DA uptake measured by single addition of DA using RDEV techniques were analysed using a one-way ANOVA followed by the Student's $t$-test with the Bonferroni correction applied. All $\mathrm{ASP}^{+}$and DA uptake kinetics data were entered into the GraphPad 5 software and a Michaelis-Menten curve fitted. The Vmax and Km values were obtained for each repeat and the mean and SEM calculated. An unpaired Student's $t$-test was performed on Vmax and $\mathrm{Km}$ values to determine significant changes between treatment groups. The quantitative analysis of DAT cell surface expression, ERK1/2 phosphorylation, and p38 activation were also conducted using an unpaired Student's $t$ test. Data were considered statistically significant when $p \leq 0.05$. 


\subsection{Results}

\subsubsection{Validation of YFP-hDAT and myc-rKOPr co-transfection in HEK-293 cells}

HEK-293 cells transiently co-transfected with YFP-hDAT and myc-rKOPr were immunolabelled with anti-myc antibody conjugated to Zenon ${ }^{\circledR}$ Alexa 647 and viewed under a confocal microscope to determine validity of co-transfection. The expression of YFP-hDAT is shown in Figure 5.1B, whereas expression of myc-rKOPr is shown in Figure 5.1C. The co-expression of both proteins is displayed in Figure 5.1D, thus indicating successful cotransfection. The yellow arrows point to a co-transfected cell whereas the white arrows point to a non-transfected cell. 
A)

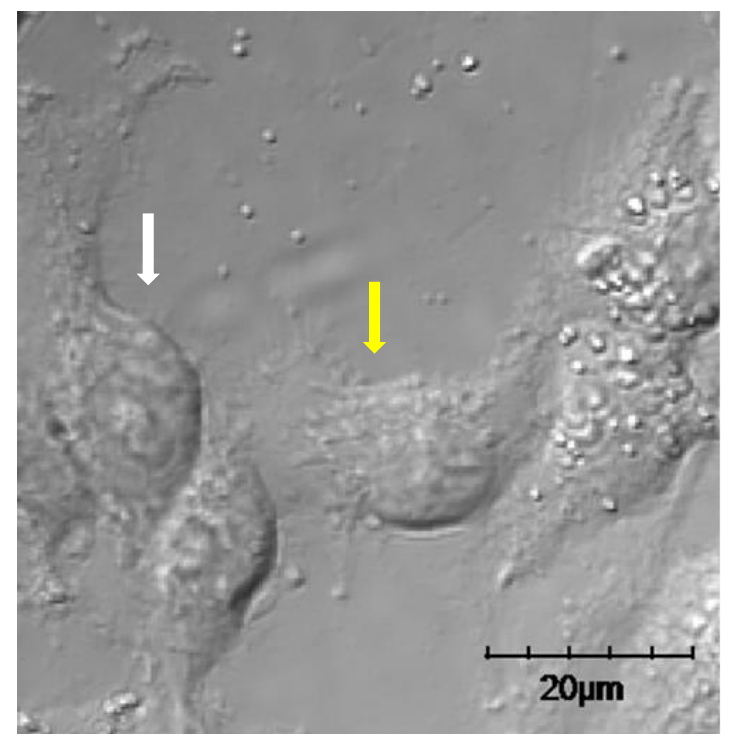

C)

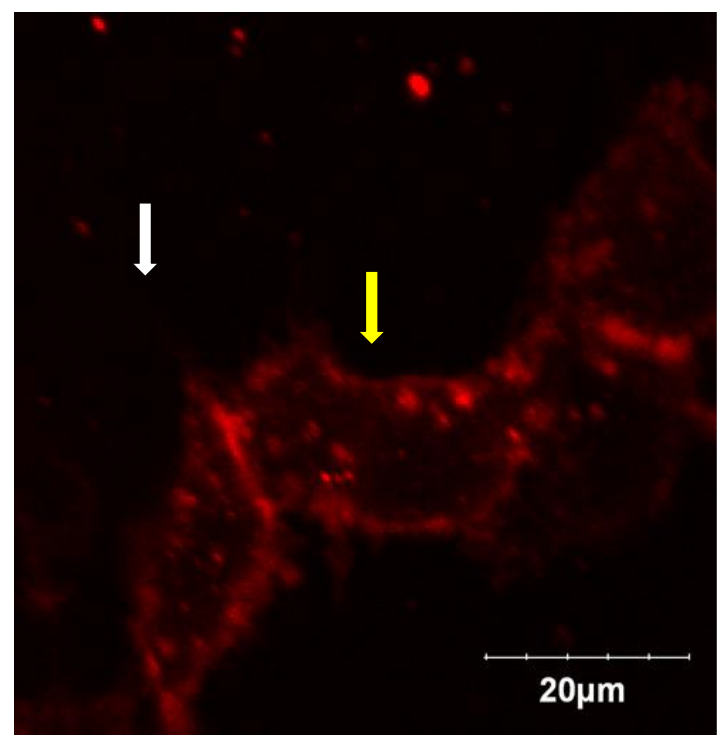

B)

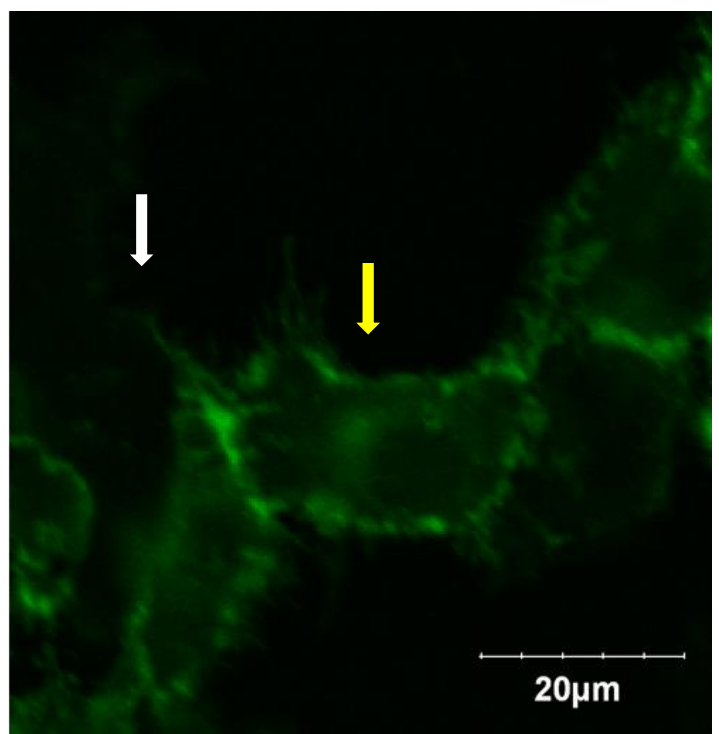

D)

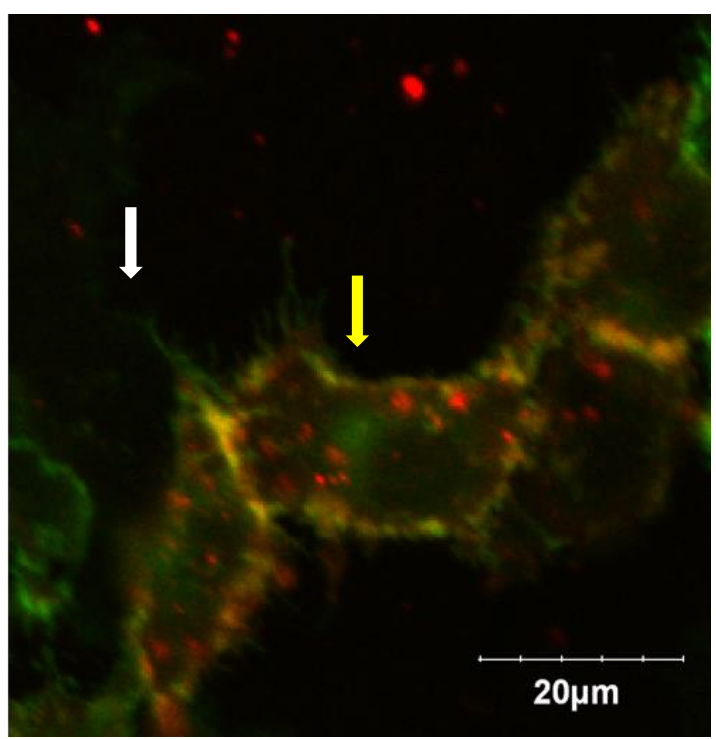

\section{Figure 5.1: Validation of YFP-hDAT and myc-rKOPr co-expression in HEK-293 cells}

HEK-293 cells co-expressing YFP-hDAT and myc-rKOPr were immuno-labelled for myc-rKOPr and imaged. Cells were visualised using A) differential interference contrast (DIC) and imaged for B) YFP-hDAT expression (green), C) myc-rKOPr expression (red), and D) an overlay of YFP-hDAT and myc-rKOPr expression in HEK-293 cells. Membrane colocalisation of DAT and KOPr is shown by yellow immunofluorescence. White arrows, non-transfected cell; yellow arrows, YFP-hDAT and myc-rKOPr co-transfected cell. 


\subsubsection{Validation of $\mathrm{ASP}^{+}$uptake in KOPr and DAT co-expressing HEK-293 cells}

Pilot experiments were conducted with HEK-293 cells co-expressing YFP-hDAT and mycrKOPr to validate the $\mathrm{ASP}^{+}$technique. KOPr and DAT expressing cells displayed a rapid, initial binding phase, followed by a gradual linear accumulation of $\mathrm{ASP}^{+}$over time for at least $10 \mathrm{~min}$ after $\mathrm{ASP}^{+}$addition (Figure 5.2A). Quantification of $\mathrm{ASP}^{+}$fluorescence intensity at $250 \mathrm{~s}$ after $\mathrm{ASP}^{+}$addition revealed a linear correlation between $\mathrm{ASP}^{+}$ accumulation and YFP-hDAT expression, showing that $\mathrm{ASP}^{+}$uptake directly correlates to surface expression of DAT ( $p<0.0001$ compared to $r^{2}=0$, Figure 5.2B). However, to minimise variability and prevent signal saturation, cells that displayed YFP-hDAT expression outside the $40-150$ AFU range were not used for experimental analyses. The gradual accumulation of $\mathrm{ASP}^{+}$over time is displayed in Figure 5.3, in which KOPr and DAT co-expressing cells displayed a large accumulation of $\mathrm{ASP}^{+}$, whereas non-transfected cells showed little to no $\mathrm{ASP}^{+}$uptake. 
A)

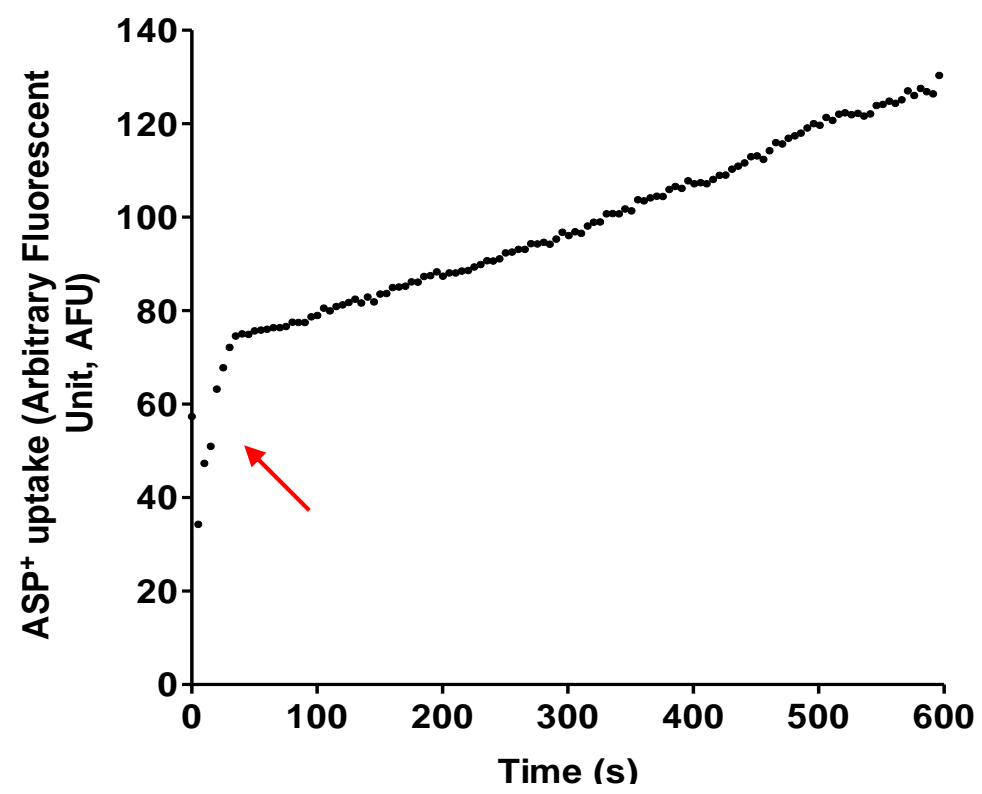

B)

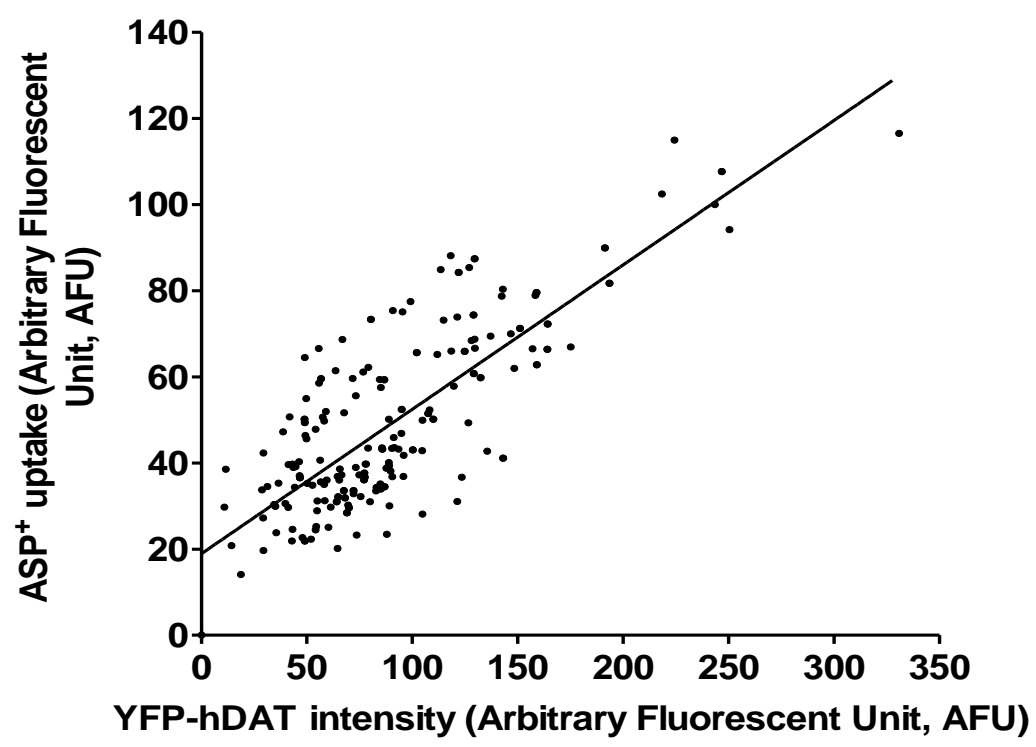

Figure 5.2: Validation of $\mathrm{ASP}^{+}$uptake study

A) ASP+ uptake in HEK-293 cells co-expressing YFP-hDAT and myc-rKOPr over 10 min. After an initial rapid binding phase (red arrow), $\mathrm{ASP}^{+}$uptake occurs in a linear fashion for at least $10 \mathrm{~min}$. B) Intensity of ASP+ fluorescence at $250 \mathrm{~s}$ after addition to KOPr and DAT expressing HEK-293 cells. Accumulation of ASP+ is dependent on expression of YFP-hDAT, as cells with higher expression of YFP-hDAT display increased $\mathrm{ASP}^{+}$accumulation compared to cells with lower YFPhDAT expression $\left(p<0.0001\right.$ compared to $\left.r^{2}=0\right)$. 
宫 A)

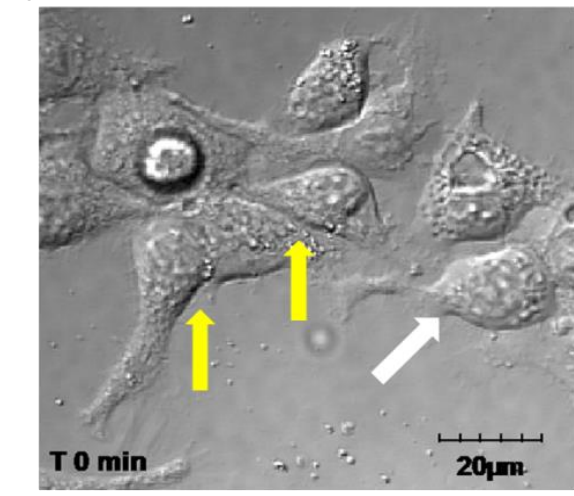

E)

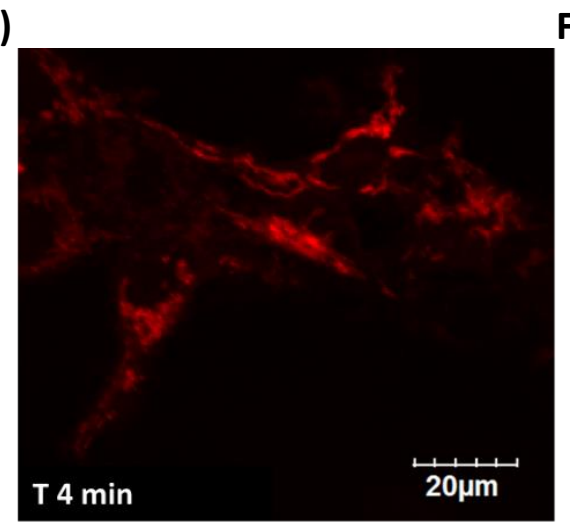

B)

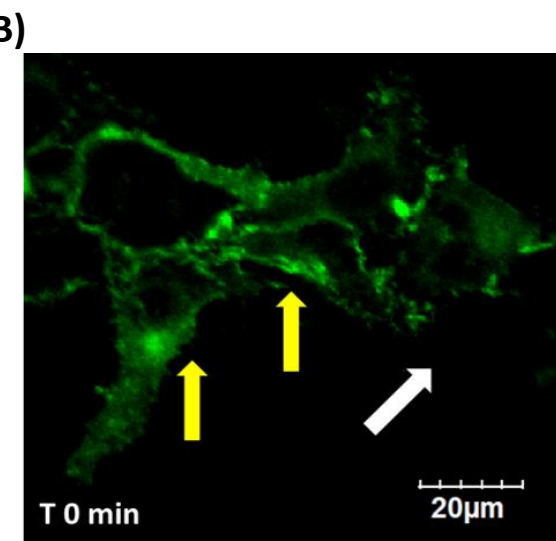

C)

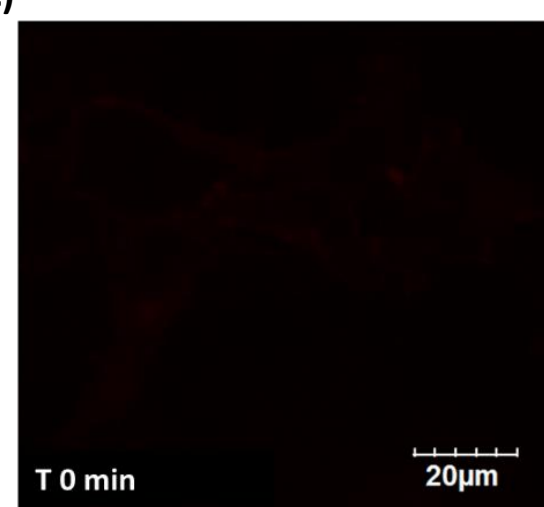

D)
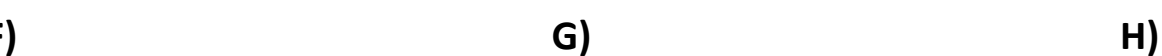

$\mathbf{T} 2 \mathrm{~min}$ $\frac{1111 \text {-1 }}{20 \mu \mathrm{m}}$

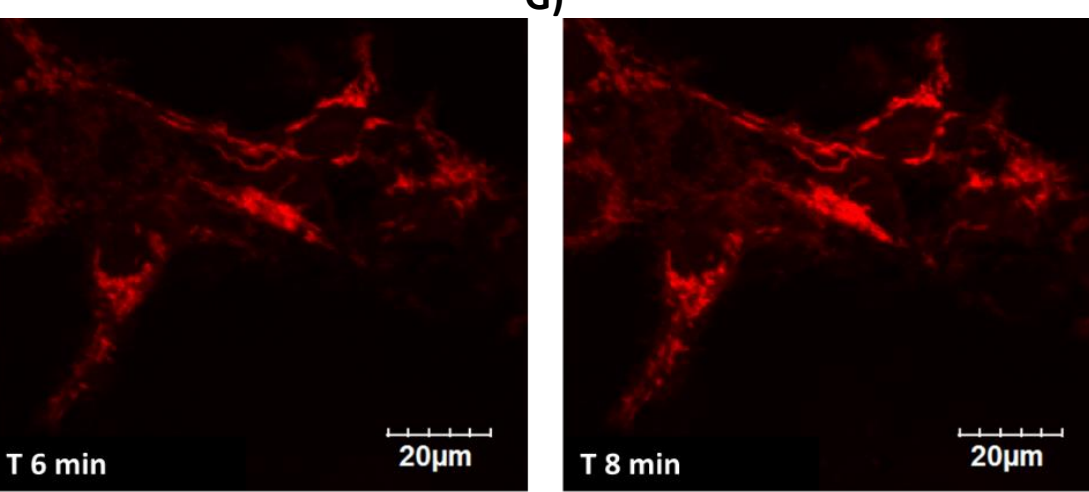
)

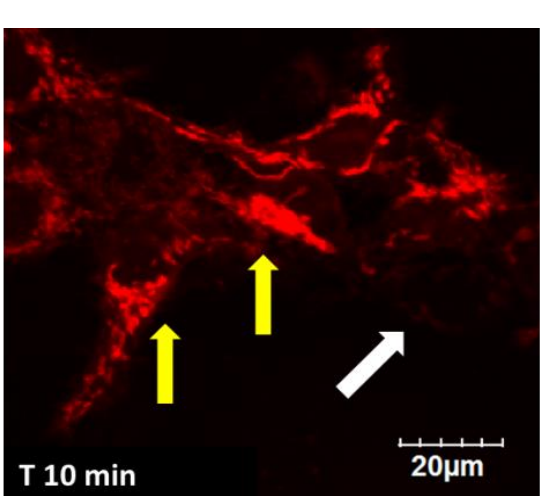

Figure 5.3: Time course of ASP ${ }^{+}$uptake in YFP-hDAT and myc-rKOPr co-transfected HEK-293 cells

$\mathrm{ASP}^{+}$accumulation in HEK-293 cells was imaged over a period of 10 min. Cells were visualised using A) differential interference contrast (DIC) and imaged for B) YFP-hDAT expression (green), C) and ASP+ accumulation (red). Cells co-expressing YFP-hDAT and myc-rKOPr (yellow arrows) displayed an increase in $\mathrm{ASP}^{+}$accumulation with time, whereas the non-transfected cell (white arrows) accumulated little to no $\mathrm{ASP}^{+}$. Increase in $\mathrm{ASP}^{+}$fluorescence at $\left.\mathrm{D}\right) 2$ min, $\mathrm{E}$ ) $4 \mathrm{~min}, \mathrm{~F}) 6 \mathrm{~min}, \mathrm{G}) 8 \mathrm{~min}$, and H) $10 \mathrm{~min}$ is shown. 


\subsubsection{Modulation of DAT in HEK-293 cells following acute administration with 16-brSal}

\section{A}

To determine the effect of 16-brSal A on DAT function in HEK-293 cells expressing KOPr and DAT, cells were subjected to the $\mathrm{ASP}^{+}$uptake test. Analysis of the data showed a significant difference between treatments $[F(3,229)=12.59, p<0.0001]$ (one-way ANOVA, Figure 5.4A). Subsequent Student's $t$-tests with the Bonferroni correction applied revealed that 16-brSal A produced a concentration-dependent increase in the rate of $\mathrm{ASP}^{+}$uptake (Fig. 5.4A). Five $\mu \mathrm{M}$ of 16 -brSal $\mathrm{A}$ led to a $5 \pm 3 \%$ increase in $\mathrm{ASP}^{+}$uptake, whereas $10 \mu \mathrm{M}$ 16-brSal A caused a $20 \pm 8 \%$ increase in $\mathrm{ASP}^{+}$uptake compared to the control ( $-5 \pm 3 \%$ change in uptake). Sal $\mathrm{A}(10 \mu \mathrm{M})$ led to a larger increase in $\mathrm{ASP}^{+}$uptake compared to the control, with a $48 \pm 11 \%$ change $(p<0.001)$. The Sal A-induced increase also exceeded that caused by $10 \mu \mathrm{M} 16-\mathrm{brSal} A$ ( $p<0.05$ ). To determine whether the 16 brSal A-mediated increase in $\mathrm{ASP}^{+}$uptake was KOPr-dependent, cells were pre-treated with nor-BNI $(1 \mu \mathrm{M}) 30$ min prior to addition of 16-brSal A $(10 \mu \mathrm{M})$. A significant effect of treatment was observed $[F(3,197)=3.878, p=0.0101]$ (one-way ANOVA, Figure 5.4B). Pre-treatment of cells with nor-BNI alone produced no significant effect on control ASP $^{+}$ uptake (-6 $\pm 9 \%$ change in $\mathrm{ASP}^{+}$uptake compared to the control of $-5 \pm 3 \%$, Figure $\left.5.4 \mathrm{~B}\right)$. However, nor-BNI completely inhibited the 16 -brSal A-mediated increase in rate of $\mathrm{ASP}^{+}$ accumulation, returning it to control-only values (from $23 \pm 8 \%$ to $-5 \pm 10 \%, p<0.05$ ) (Figure 5.4B).

The $\mathrm{ASP}^{+}$uptake experiment was also conducted with varying concentrations of $\mathrm{ASP}^{+}$ (from 1 to $16 \mu \mathrm{M}$ ) in order to determine changes in the affinity of $\mathrm{ASP}^{+}$for DAT $(\mathrm{Km})$ and the maximum uptake velocity of $\mathrm{ASP}^{+}$by DAT (Vmax) (Figure 5.5A). 16-brSal A (10 $\left.\mu \mathrm{M}\right)$ significantly increased both Vmax (Figure 5.5B) and $\mathrm{Km}$ (Figure 5.5C) of $\mathrm{ASP}^{+}$uptake compared to vehicle ( $p<0.0001$, unpaired Student's $t$-test). Table 5.1 shows a summary of vehicle and 16-brSal A Vmax and $\mathrm{Km}$ values for rate of $\mathrm{ASP}^{+}$uptake.

Thus, 16-brSal A increased DAT-mediated $\mathrm{ASP}^{+}$uptake in KOPr and DAT co-expressing HEK-293 cells. 
A)

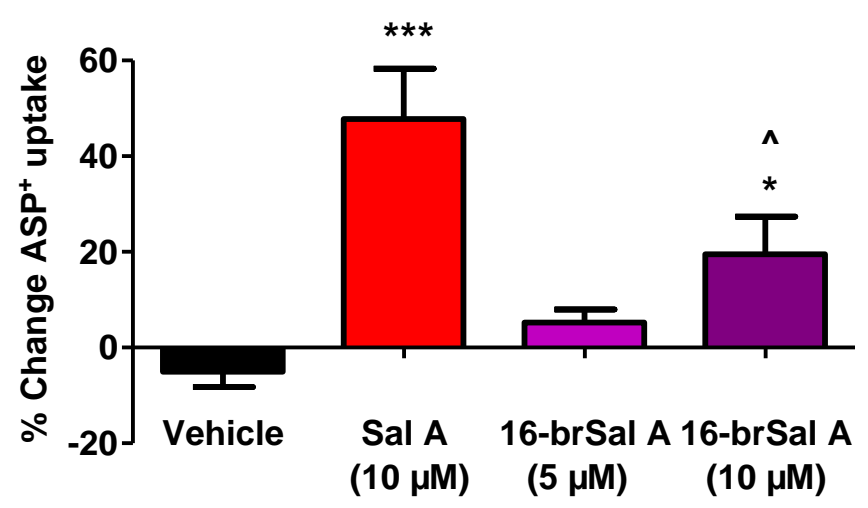

B)

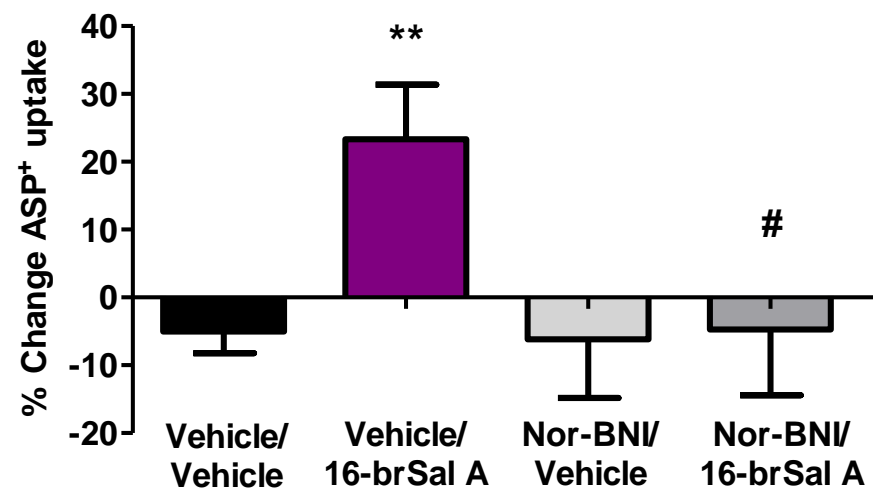

Figure 5.4: 16-brSal A increases ASP ${ }^{+}$uptake in YFP-hDAT and myc-rKOPr co-expressing HEK-293 cells in a KOPr-dependent manner

A) KOPr and DAT co-expressing cells treated 16-brSal A showed a dose-dependent increase in rate of $\mathrm{ASP}^{+}$uptake compared to vehicle-treated controls (0.02\% DMSO). B) Pre-treatment of cells with nor-BNI $(1 \mu \mathrm{M})$ or the nor-BNI vehicle $(0.01 \%$ DMSO) for 30 min prior to $10 \mu \mathrm{M}$ of 16 -brSal A shows that nor-BNI prevented 16-brSal A-evoked increases in the rate of ASP+ uptake. Addition of nor-BNI alone did not alter $\mathrm{ASP}+$ uptake compared to controls. Results were obtained from a minimum of 3 separate transfections. One-way ANOVA followed by Student's $t$-tests with the Bonferroni correction applied, ${ }^{*} p<0.05,{ }^{* *} p<0.01,{ }^{* * *} p<0.001$ compared to vehicle controls; ${ }^{\wedge} p<0.05$ compared to Sal A, ${ }^{p} p<0.05$ compared to vehicle/16-brSal A. Values presented as mean $\pm S E M, n$ $=48-65$ cells. 
A)

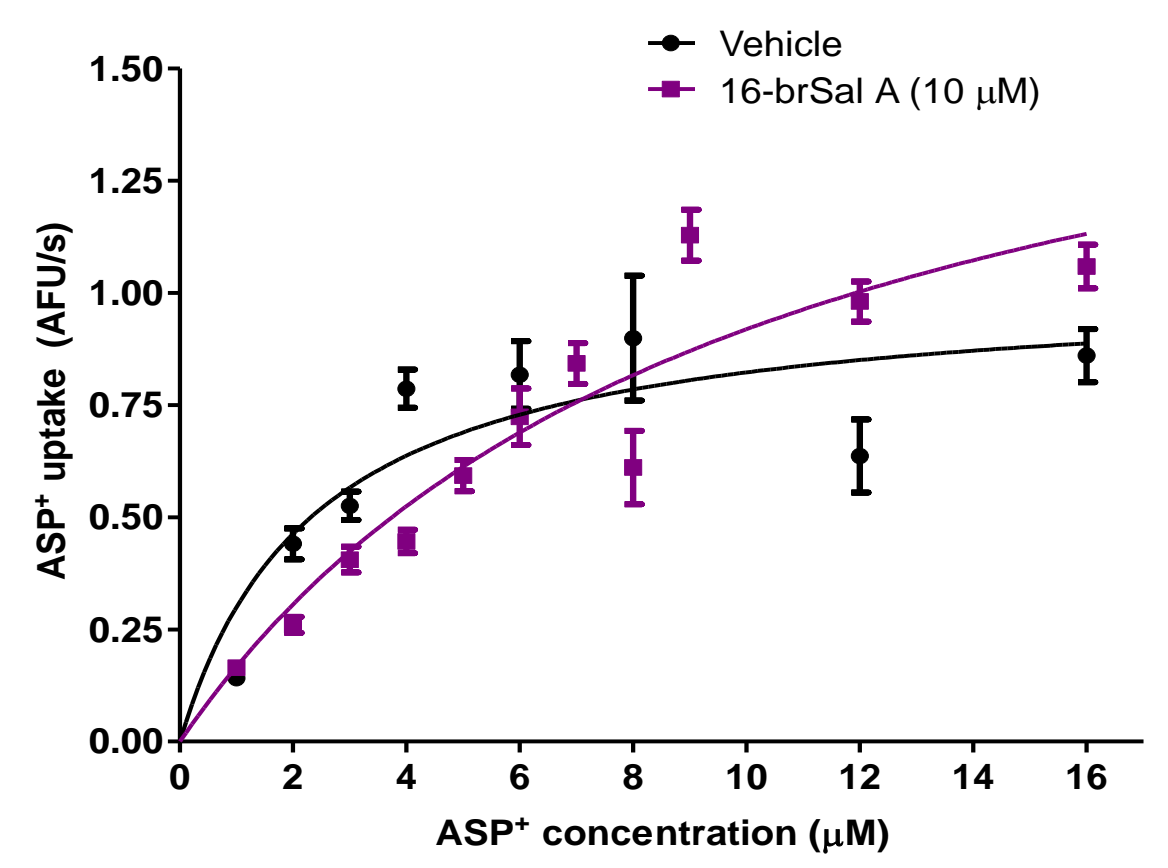

B)

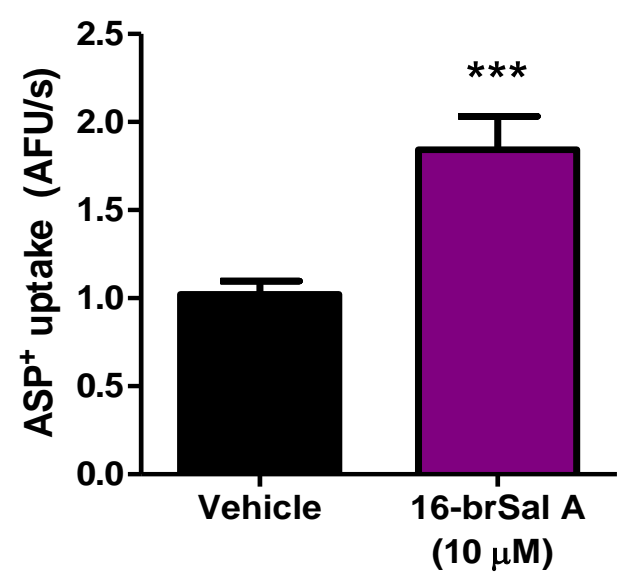

C)

$\mathrm{Km}$

Figure 5.5: 16-brSal A increases the Vmax and $\mathrm{Km}$ of ASP ${ }^{+}$uptake by DAT in YFP-hDAT and myc-rKOPr expressing HEK-293 cells

A) Calculation of net ASP+ accumulation showed that DAT and KOPr co-expressing HEK-293 cells treated with 16-brSal A produced an increase in B) Vmax and C) $\mathrm{Km}$ of ASP+ uptake compared to vehicle-treated $\left(0.02 \%\right.$ DMSO) cells. Unpaired Student's $t$-test, ${ }^{* * *} p<0.0001$ compared to vehicle. Values presented as mean \pm SEM, $n=238-262$ cells with replicates from at least 3 separate transfections. 


\begin{tabular}{|l|c|c|}
\hline \multicolumn{1}{|c|}{ Treatment } & Vmax (AFU/s) & $\mathrm{Km}(\mu \mathrm{M})$ \\
\hline Vehicle $(0.02 \% \mathrm{DMSO})$ & $1.0 \pm 0.1$ & $2.4 \pm 0.5$ \\
\hline 16 -brSal A $(10 \mu \mathrm{M})$ & $1.8 \pm 0.2^{* * *}$ & $10.1 \pm 1.9^{* * *}$ \\
\hline
\end{tabular}

Student's $t$-test. ${ }^{* * *} \mathrm{p}<0.0001$ compared to the Vmax or $\mathrm{Km}$ of the control. Values presented as mean \pm SEM, $n=238-262$ cells.

Table 5.1: 16-brSal A increases Vmax and Km of ASP' ${ }^{+}$uptake in YFP-hDAT and myc-rKOPr co-expressing HEK-293 cells 


\subsubsection{Modulation of DAT in the rat dStr and NAc following acute treatment with 16-brSal}

\section{A}

The KOPr is strategically located to modulate DA uptake via regulation of DAT, a mechanism suggested to be responsible for the anti-cocaine behaviours of KOPr agonists. To determine if the 16-brSal A regulation of DAT seen in KOPr and DAT co-expressing HEK293 cells extends to native models, the dStr and NAc of drug naïve rats were treated with 16-brSal A ( $500 \mathrm{nM}$ ) before sequential addition of DA. The uptake of DA was measured using the RDEV low to infinite trans model. There was a significant difference in DA uptake between 16-brSal A- and vehicle-treated (0.001\% DMSO) dStr suspensions $[F(1,100)=$ $32.78, p<0.0001$ ] (one-way ANOVA with repeated measures, Figure 5.6A). A similar significant effect was also noted in the $\operatorname{NAc}[F(1,103)=39.13, p<0.0001]$ (one-way ANOVA with repeated measures, Figure 5.7A). Further analysis found that 16-brSal A produced a significant 39\% increase in Vmax of DA uptake in the dStr $(p<0.05$, Student's $t$-test, Figure 5.6B). This increase was greater in the NAc, with $16-\mathrm{brSal} A$ producing a $45 \%$ increase in Vmax of DA uptake compared to the vehicle ( $<<0.05$, Student's $t$-test, Figure 5.7B). There were no significant changes in Km values following 16-brSal A treatment in the dStr or NAc (Figure 5.6C and 5.7C). Table 5.2 presents a detailed summary of vehicle- and 16brSal A-induced Vmax and Km values in the rat dStr and NAc using RDEV.

The effect of the KOPr antagonist, nor-BNI on the changes produced by 16-brSal A were investigated using a single addition of DA $(2 \mu \mathrm{M})$ in the RDEV zero trans model. There was a significant effect of treatment in both the rat $d \operatorname{Str}[F(2,21)=6.054, p=0.0084]$ and NAc $[F(2,24)=8.623, p=0.0015]$ (one way ANOVA, Figure 5.8). Pre-treatment of both the rat dStr and NAc with nor-BNI (1 $\mu \mathrm{M})$ significantly inhibited the 16-brSal A-modulated increase of DA uptake ( $p<0.05, p<0.01$, Student's $t$-test with the Bonferroni correction, Figure 5.8). Using the single addition model, 16-brSal A produced a $49 \%$ increase in DA uptake in the dStr, which was inhibited by prior addition of nor-BNI. In a similar manner, 16-brSal A produced a significant $50 \%$ increase in DA uptake in the NAc, an effect not seen when nor-BNI was used (Figure 5.8 and Table 5.3).

Thus, 16-brSal A increased DA uptake in the rat dStr and NAc in a KOPr-dependent manner using the RDEV model. 
A)

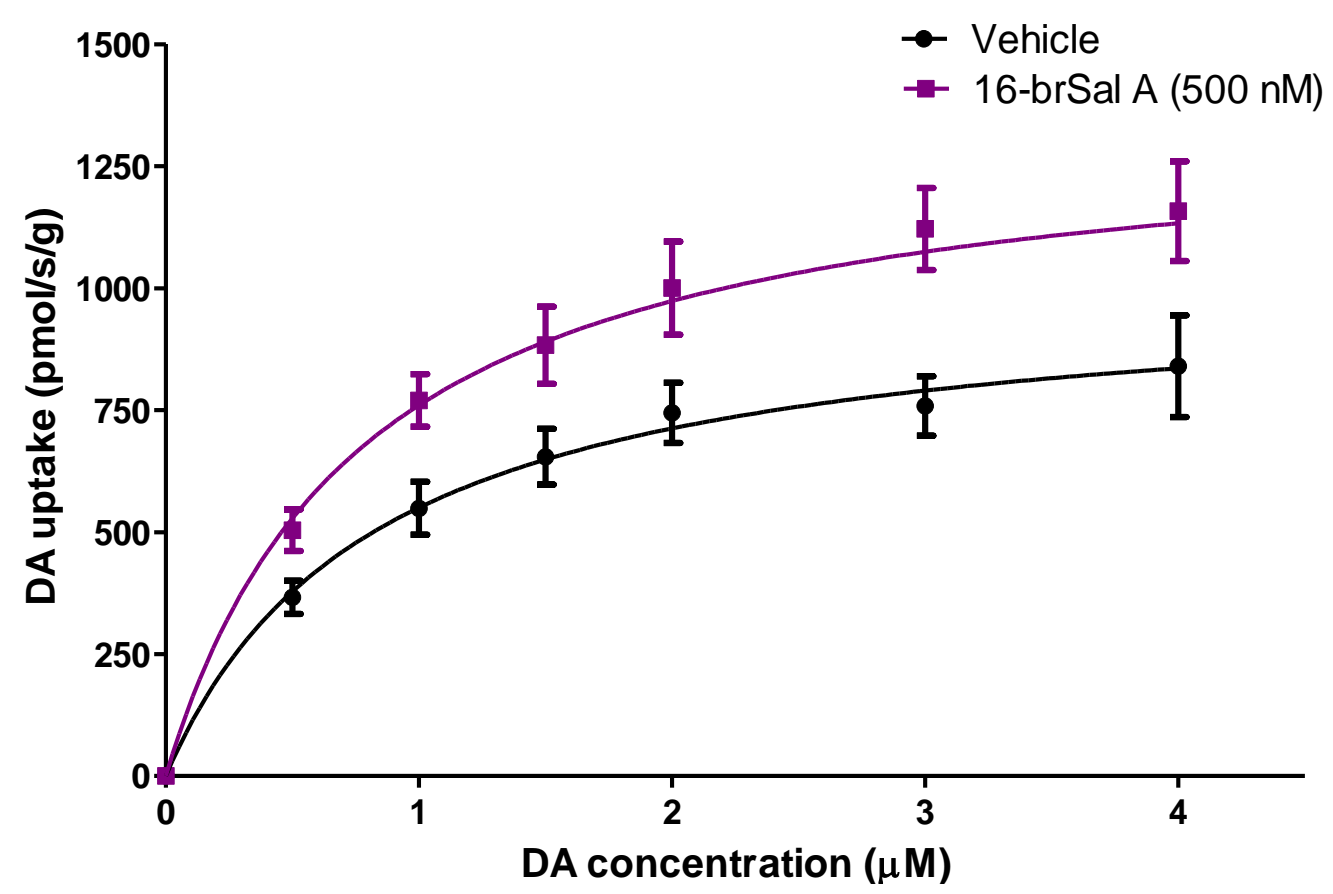

B)

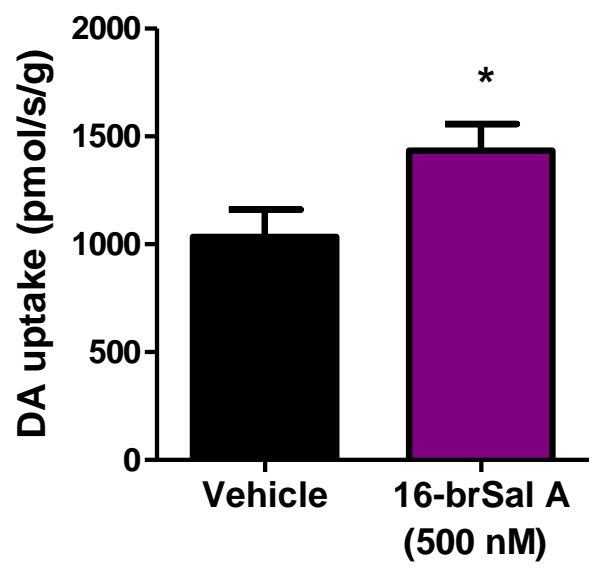

C)

$\mathrm{Km}$

\section{Figure 5.6: 16-brSal A increases DA uptake in the rat dStr}

The dStr of drug naïve rats was isolated and the minced tissue incubated with either vehicle $(0.001 \%$ DMSO) or 16 -brSal A 4 min prior to sequential DA addition. DA uptake was measured using the low to infinite trans model. 16-brSal A produced a significant increase in DA uptake compared to vehicle-treated controls (one-way ANOVA with repeated measures) with an increase in B) Vmax but no change in C) $\mathrm{Km}$ (Student's $t$-test). ${ }^{*} \mathrm{p}<0.05$ compared to vehicle. Values presented as mean \pm SEM, $n=9$. 
A)

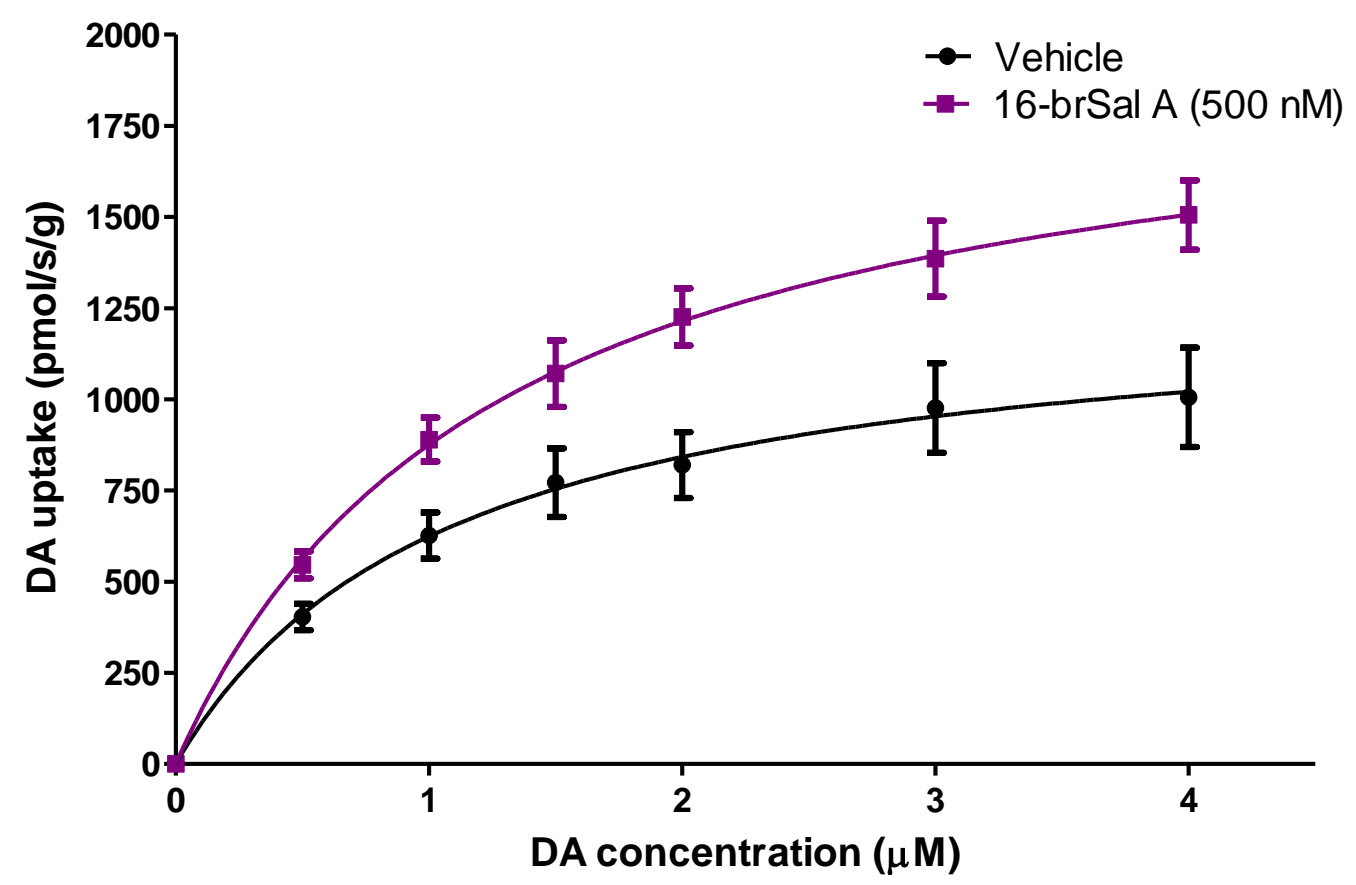

B)

Vmax

C)

$\mathrm{Km}$
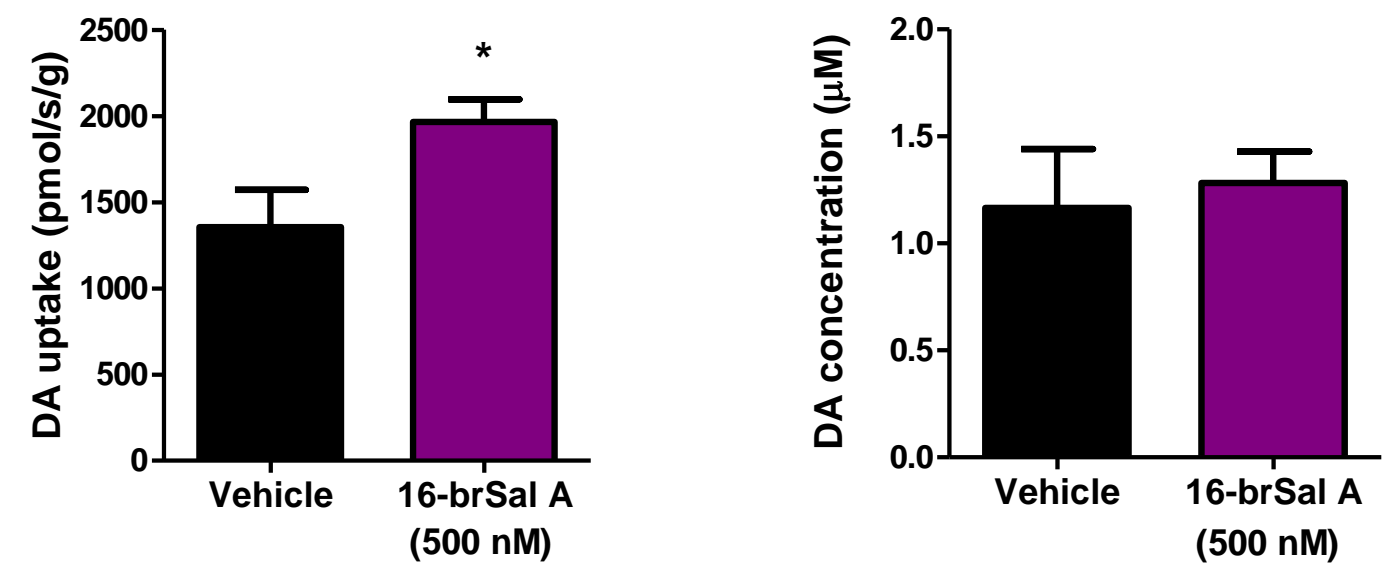

\section{Figure 5.7: 16-brSal A increases DA uptake in the rat NAC}

Kinetics of DA uptake in the rat NAc measured by RDEV (low to infinite trans model) revealed that 16-brSal A produced a significant increase in DA uptake compared to the vehicle (0.001\% DMSO) (one-way ANOVA with repeated measures). 16-brSal A increased the B) Vmax but not the C) Km of DA uptake compared to vehicle controls (Student's $t$-test). ${ }^{*} \mathrm{p}<0.05$ compared to the vehicle. Values presented as mean $\pm S E M, n=9$. 


\begin{tabular}{|c|c|c|c|c|}
\hline \multirow[b]{2}{*}{ Brain region } & \multicolumn{2}{|c|}{ Vmax (pmol/s/g) } & \multicolumn{2}{|c|}{$\mathrm{Km}(\mu \mathrm{M})$} \\
\hline & $\begin{array}{l}\text { Vehicle } \\
(0.001 \% \\
\text { DMSO) } \\
\end{array}$ & $\begin{array}{c}\text { 16-brSal A } \\
\text { (500 nM) }\end{array}$ & $\begin{array}{l}\text { Vehicle } \\
(0.001 \% \\
\text { DMSO) } \\
\end{array}$ & $\begin{array}{c}\text { 16-brSal A } \\
\text { (500 nM) }\end{array}$ \\
\hline $\begin{array}{l}\text { Dorsal } \\
\text { Striatum } \\
\text { (dStr) }\end{array}$ & $1034 \pm 126$ & $1433 \pm 124^{*}$ & $0.90 \pm 0.18$ & $0.94 \pm 0.14$ \\
\hline $\begin{array}{c}\text { Nucleus } \\
\text { Accumbens } \\
\text { (NAc) }\end{array}$ & $1356 \pm 215$ & $1965 \pm 132 *$ & $1.17 \pm 0.28$ & $1.28 \pm 0.15$ \\
\hline
\end{tabular}

Student's $t$-test. * $\mathrm{p}<0.05$ compared to the Vmax of vehicle. Values presented as mean $\pm \mathrm{SEM}, \mathrm{n}=$ 9

Table 5.2: 16-brSal A increases Vmax but not Km of DA uptake in the rat dStr and NAC 


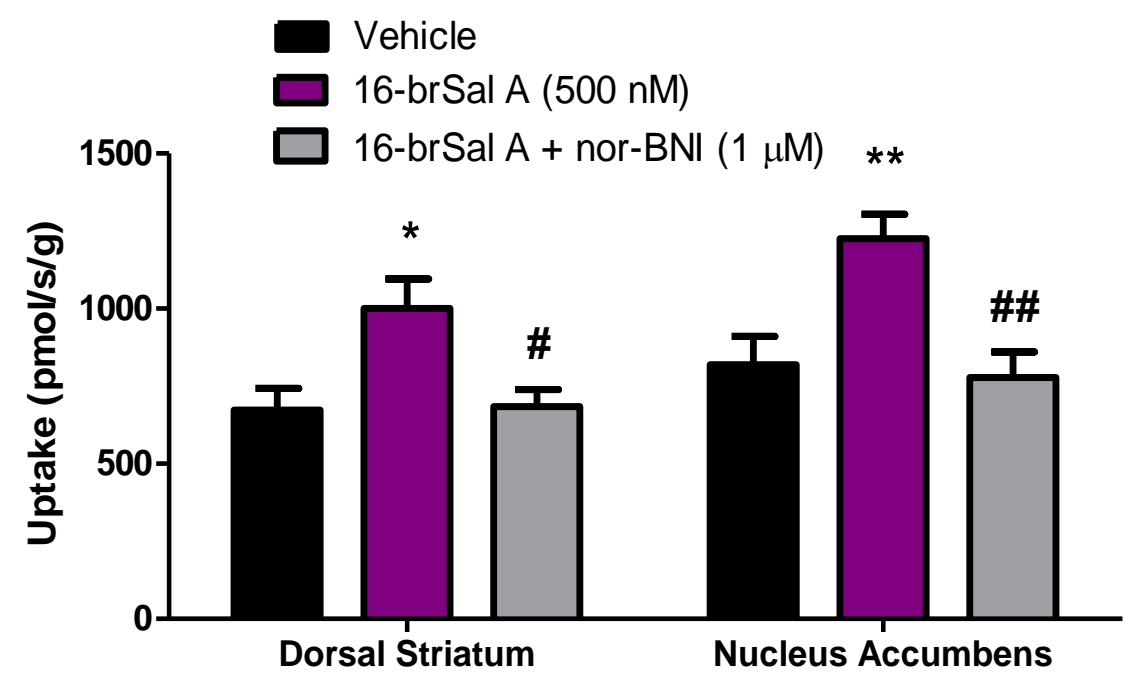

Figure 5.8: The selective KOPr antagonist nor-BNI inhibits 16-brSal A-mediated increase of DA uptake in the dStr and NAC

16-brSal $A$ increased DA uptake in the rat dStr and NAc when tested using a single $2 \mu \mathrm{M}$ addition of DA (RDEV zero trans model). Pre-treatment of tissue suspensions with nor-BNI significantly inhibited the 16-brSal A-induced increase in DA uptake. One-way ANOVA followed by Student's $t$ tests with the Bonferroni correction, ${ }^{*} p<0.05,{ }^{* *} p<0.01$ compared to the vehicle; ${ }^{\#} p<0.05,{ }^{\# \#} p<0.01$ compared to 16-brSal A. Values presented as mean \pm SEM, $n=8-9$.

\begin{tabular}{|c|c|c|c|}
\hline \multirow{2}{*}{ Brain region } & \multicolumn{3}{|c|}{ DA uptake using a single DA (2 $\mu \mathrm{M})$ addition (pmol/s/g) } \\
\cline { 2 - 4 } & $\begin{array}{c}\text { Vehicle } \\
\text { (0.001\% DMSO) }\end{array}$ & $\begin{array}{c}\text { 16-brSal A } \\
\text { (500 } \mathbf{~ n M ) ~}\end{array}$ & $\begin{array}{c}\mathbf{1 6} \text { brSal A + nor-BNI } \\
\text { (500 nM/1 } \boldsymbol{\mu M} \text { ) }\end{array}$ \\
\hline $\begin{array}{c}\text { Dorsal Striatum } \\
\text { (dStr) }\end{array}$ & $673.3 \pm 70.0$ & $1000 \pm 95.3^{*}$ & $683.8 \pm 55.9^{\#}$ \\
\hline $\begin{array}{c}\text { Nucleus } \\
\text { Accumbens (NAc) }\end{array}$ & $820.0 \pm 90.6$ & $1226 \pm 78.7^{* *}$ & $777.7 \pm 83.3^{\# \#}$ \\
\hline
\end{tabular}

One-way ANOVA followed by Student's $t$-tests with the Bonferroni correction. ${ }^{*} \mathrm{p}<0.05$, ${ }^{* *} \mathrm{p}<0.01$

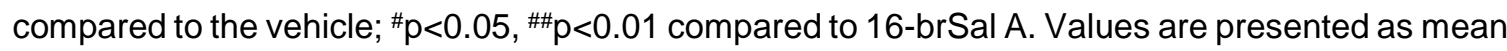
\pm SEM in pmol/s/g, $n=8-9$.

Table 5.3: The 16-brSal A-evoked increase of DA uptake in the rat dStr and NAc is inhibited with prior addition of nor-BNI 


\subsubsection{Effect of ERK1/2 inhibition on the 16-brSal A-evoked increase in DAT function}

It has previously been shown that KOPr-mediated DAT regulation is ERK1/2-dependent, whereby KOPr agonists activate ERK1/2 (Belcheva et al., 2005), and ERK1/2 activation increases DAT function and expression (Moron et al., 2003). In order to confirm whether 16-brSal A also modulates DAT via an ERK1/2-dependent pathway, HEK-293 cells transiently expressing YFP-hDAT and myc-rKOPr were pre-treated with the MEK1/2 inhibitor, U0126 (20 $\mu \mathrm{M})$ for 30 min prior to measuring $\mathrm{ASP}^{+}$uptake. A significant effect of treatment was found $[F(3,206)=5.813, p=0.0008]$ (one-way ANOVA, Figure 5.9A). Planned comparisons using Student's t-test with the Bonferroni correction showed that 16-brSal A (10 $\mu \mathrm{M})$ again significantly increased $\mathrm{ASP}^{+}$uptake compared to the vehicle (0.02\% DMSO) as in Figure 5.4, with the change in $\mathrm{ASP}^{+}$uptake increasing from $-5 \pm 3 \%$ to $23 \pm 8 \%(p<0.01$, Figure 5.9A). Although addition of $\mathrm{U} 0126$ alone had no significant effect on the change in rate of $\mathrm{ASP}^{+}$uptake, it prevented the 16-brSal A-evoked increase in rate of $\mathrm{ASP}^{+}$uptake, bringing it back to vehicle-level values of $-8 \pm 4 \%(p<0.01$, Figure $5.9 \mathrm{~A})$.

As the ERK1/2-dependent upregulation of DAT observed could have been due to either an increase in transport activity of DAT or an increase in DAT cell surface expression or both, DAT and KOPr expressing HEK-293 cells were treated with either vehicle $0.02 \%$ DMSO) or 16-brSal A (10 $\mu \mathrm{M})$ to determine changes in the cell surface fractions of DAT. Cell surface biotinylation studies revealed that 16-brSal A did not increase cell surface expression of YFP-hDAT (1.02 \pm 0.03 fold compared to control). 16-brSal A also had a nonsignificant effect on the internal fraction ( $7 \pm 8 \%$ increase compared to the vehicle) (Figure 5.9B, Student's $t$-test).

In summary, 16-brSal A increased DAT function, but not DAT cell surface expression, in an ERK1/2-dependent manner. 
A)

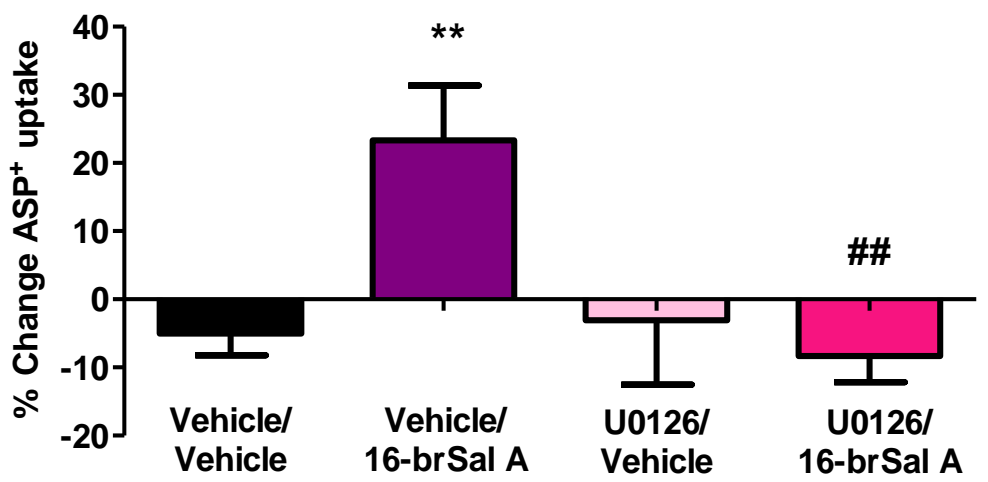

B)

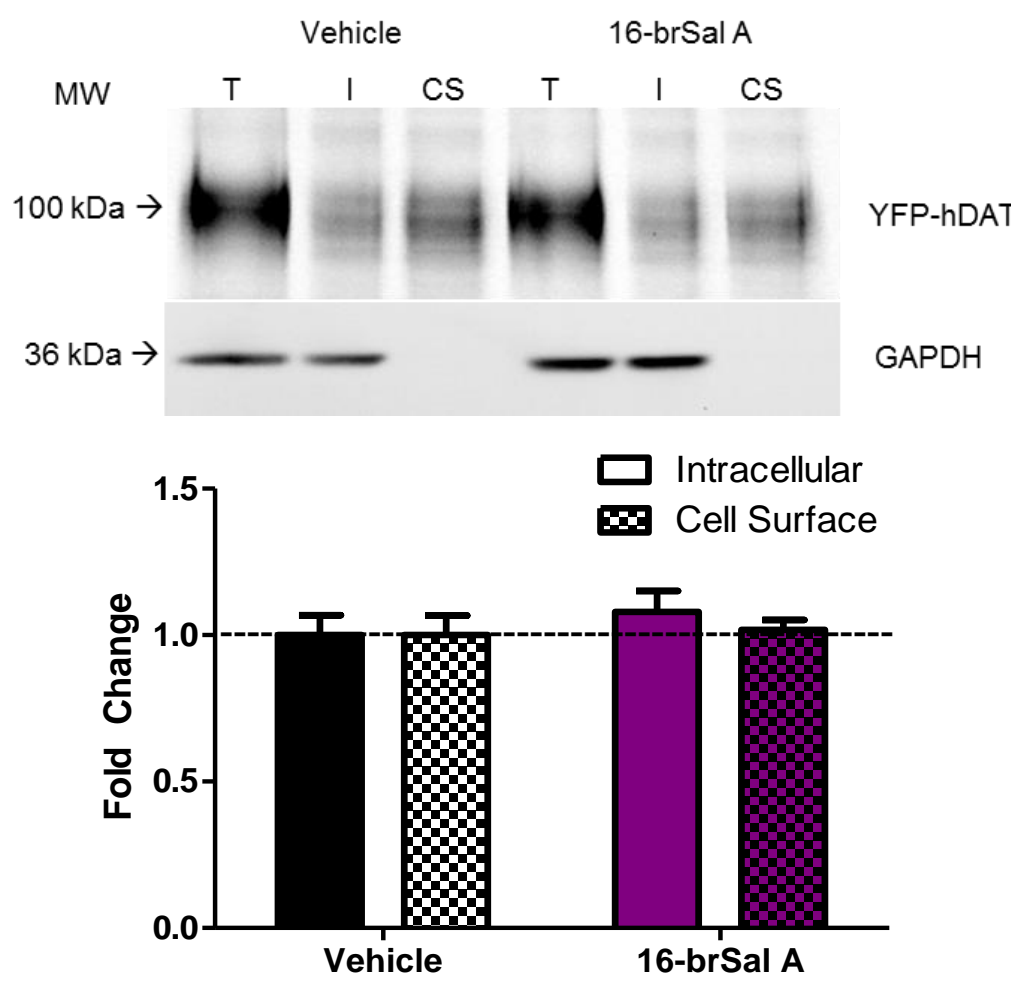

Figure 5.9: 16-brSal A increases DAT function in an ERK1/2-dependent manner but does not alter DAT cell surface expression

A) 16-brSal A $(10 \mu \mathrm{M})$ produced a significant increase in ASP+ uptake in HEK-293 cells coexpressing YFP-hDAT and myc-rKOPr, an effect that was prevented by prior incubation with the MEK1/2 inhibitor U0126 (20 $\mu \mathrm{M}$ ) (Student's $t$-test with the Bonferroni correction applied, $\mathrm{n}=53-65$ cells from at least 3 separate transfections). B) Cell surface biotinylation studies showed that the 16-brSal A-upregulation of DAT was not due to an increase in DAT cell surface expression (Student's $t$-test, $\mathrm{n}=7-8$ petri dishes containing cells from at least 3 separate transfections). ${ }^{* *} \mathrm{p}<0.01$ compared to the vehicle/vehicle group, ${ }^{*} \mathrm{p}<0.01$ compared to compared to vehicle/16brSal A. Values presented as mean \pm SEM. $T$, total fraction; I, intracellular fraction; $C S$, cell surface fraction 


\subsubsection{Effect of 16-brSal A on ERK1/2 activation}

To determine the phases of ERK1/2 activation by which 16-brSal A regulates DAT function, cells expressing KOPr, but not DAT, were incubated with 16-brSal A (10 $\mu \mathrm{M})$ for $0,5,10$, $15,30,60,120$, and $180 \mathrm{~min} .16-\mathrm{brSal}$ A produced a significant $50 \pm 20 \%$ increase in ERK1 phosphorylation $(p<0.05)$ and $50 \pm 10 \%$ increase in ERK2 activation at $5 \mathrm{~min}(p<0.01)$. A similar increase at 10 min was noted, with 16-brSal A producing an increase in ERK1 (1.5 \pm 0.1 fold, $p<0.05)$ and ERK2 phosphorylation $(1.8 \pm 0.2$ fold, $p<0.01$, Student's $t$-test, Figure 5.10A). Although ERK2 was also significantly activated 30 min post-treatment with a $50 \pm 20 \%$ increase in phosphorylation $(p<0.05), 16$-brSal A produced no changes in ERK1/2 activation at any of the other time points (Figure 5.10A). To determine whether the modulation of ERK1/2 by 16-brSal A was KOPr-mediated, nor-BNI ( $1 \mu \mathrm{M})$ was added to the KOPr-expressing cells. Addition of nor-BNI alone did not alter ERK1/2 activation compared to the vehicle, but completely inhibited the 16-brSal A-mediated activation of early ER1/2 ( $p<0.05$, Student's $t$-test, Figure 5.10B). In contrast, prior treatment of cells with U0126 (20 $\mu \mathrm{M})$ completely abolished the ERK1/2 phosphorylation compared to vehicle controls ( $p<0.0001$, Student's $t$-test, Figure 5.10B).

Figure 5.10: 16-brSal A selectively activates the early but not late phase of ERK1/2 in HEK-293 cells transiently expressing YFP-hDAT and myc-rKOPr in a KOPr-dependent manner

A) 16-brSal A $(10 \mu \mathrm{M})$ significantly increased ERK1/2 at 5 and $10 \mathrm{~min}$, as well as ERK2 at $30 \mathrm{~min}$ post-treatment in KOPr and DAT expressing HEK-293 cells. No significant change in ERK1/2 phosphorylation was noted at other time points. B) 16-brSal A significantly increased ERK1/2 activation compared to the control, an effect that was inhibited by prior incubation of cells with the KOPr antagonist nor-BNI $(1 \mu \mathrm{M})$. Addition of nor-BNI alone did not alter phosphorylation of ERK1/2 compared to the vehicle. Pre-treatment of cells with the MEK1/2 inhibitor U0126 (20 $\mu \mathrm{M})$ eliminated ERK1/2 phosphorylation compared to the control. Student's $t$-test, ${ }^{*} \mathrm{p}<0.05,{ }^{* *} \mathrm{p}<0.01$ compared to time 0 in $(A) ;{ }^{*} p<0.05,{ }^{* * * *} p<0.0001$ compared to the vehicle/vehicle group, ${ }^{*} p<0.05$ compared to vehicle/16-brSal $A$ in (B). Values presented as mean \pm SEM, $n=7-8$ dishes from at least 3 separate transfections. 
A)
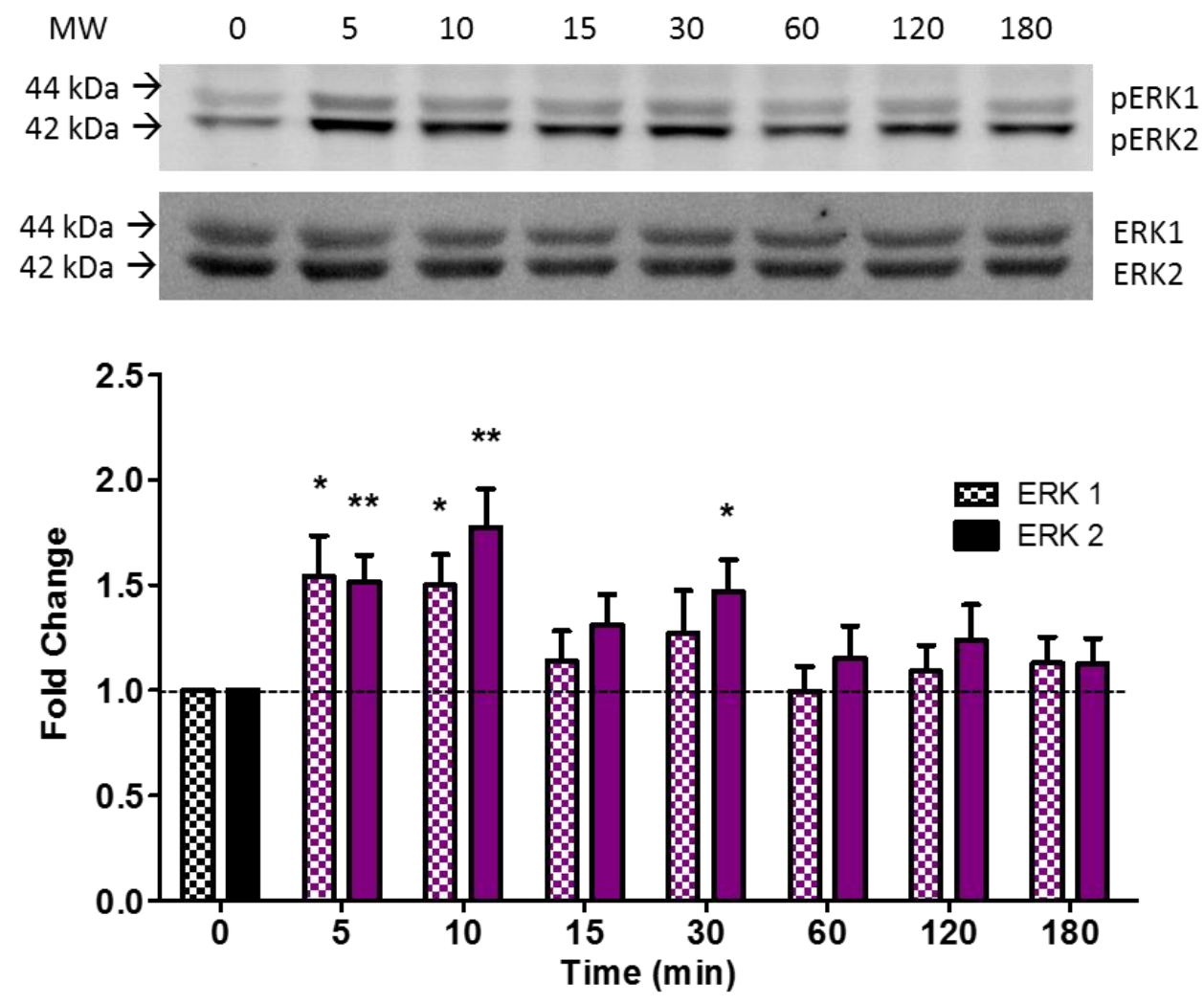

B)

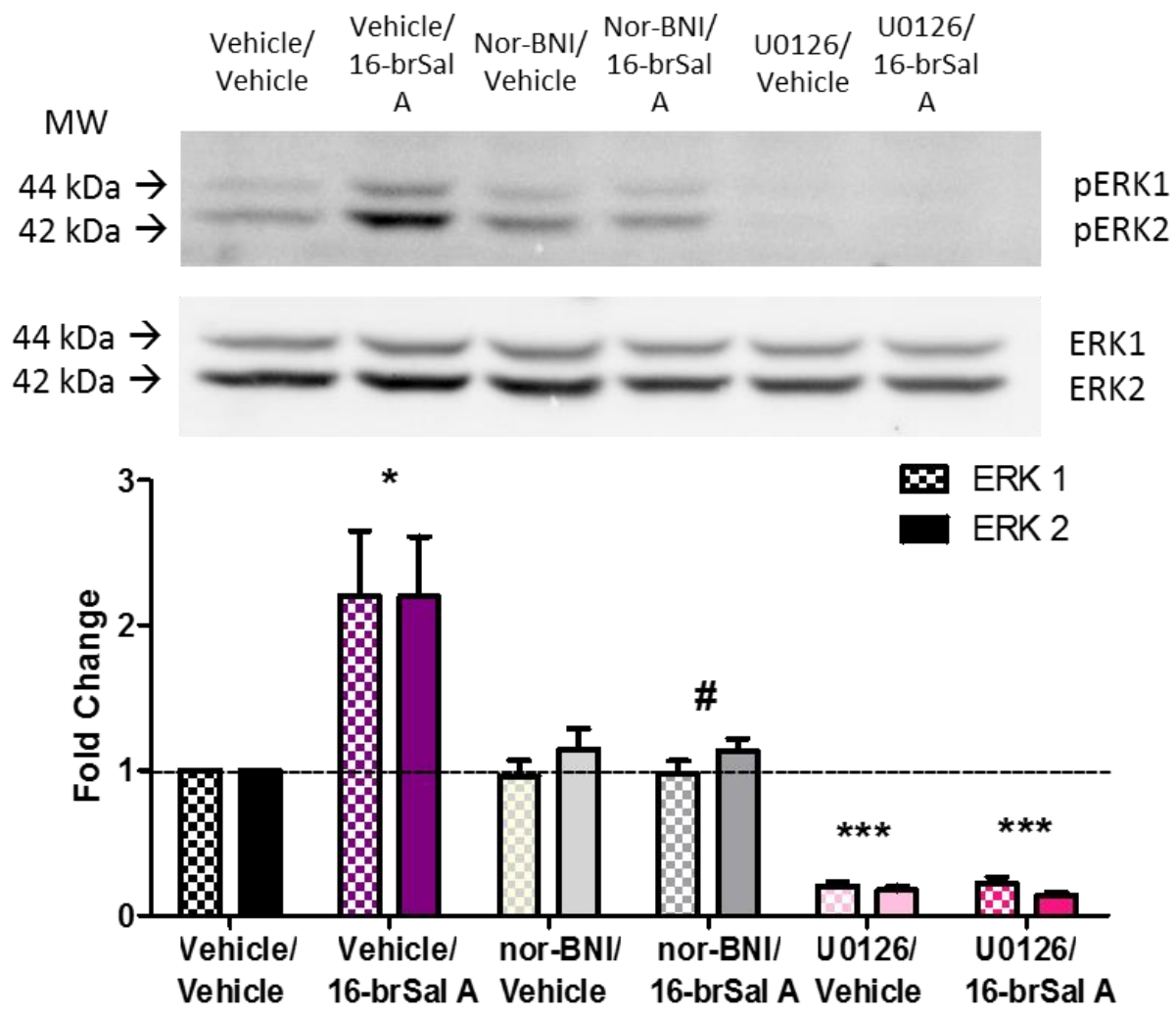


Activation of ERK1/2 in the adult rat dStr, NAc, and PFC was also investigated to determine whether the increase in ERK1/2 phosphorylation seen in vitro extends to in vivo systems. In Western blot studies, 16-brSal A (1.0 mg/ $\mathrm{kg}$ ) showed a significant $22 \pm$ $10 \%$ increase in ERK2 activation at $10-15 \mathrm{~min}$ in the dStr and a significant $24 \pm 10 \%$ increase in ERK2 phosphorylation in the PFC ( $p<0.05$, Student's $t$-test, Figure 5.11A and 5.11C). In the NAc, 16-brSal A significantly increased phosphorylation of ERK1 by $13 \pm 6 \%$ at $10-15 \mathrm{~min}$, whereas a $23 \pm 10 \%$ increase in ERK2 was noted the same time point ( $p<0.05$, Student's $t$-test, Figure 5.11B). However, no significant activation of ERK1/2 was noted at 30 or $120 \mathrm{~min}$ in all the brain regions investigated (Figure 5.11). 
A)

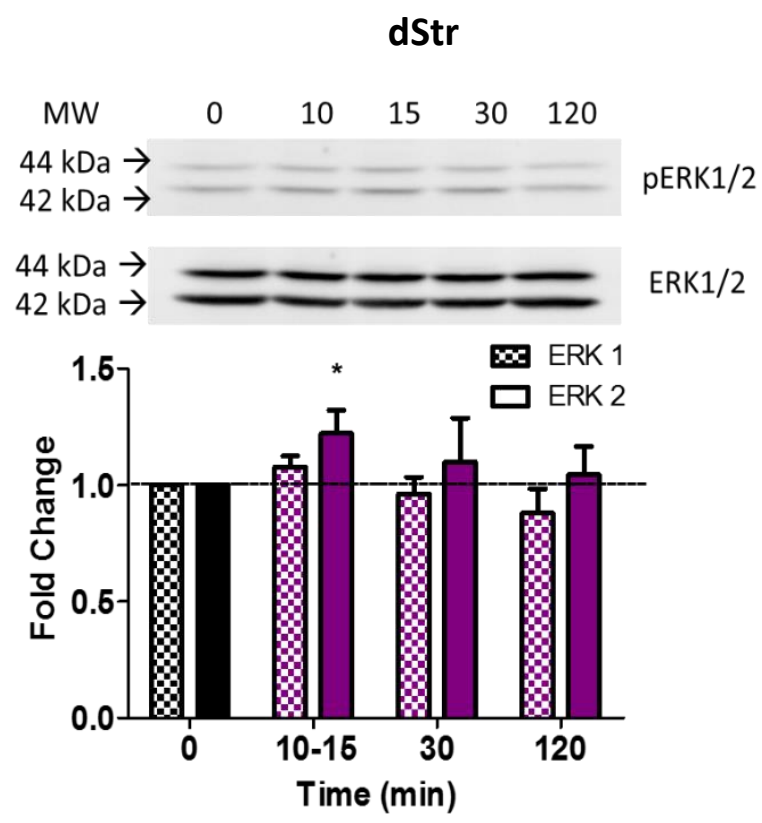

B)

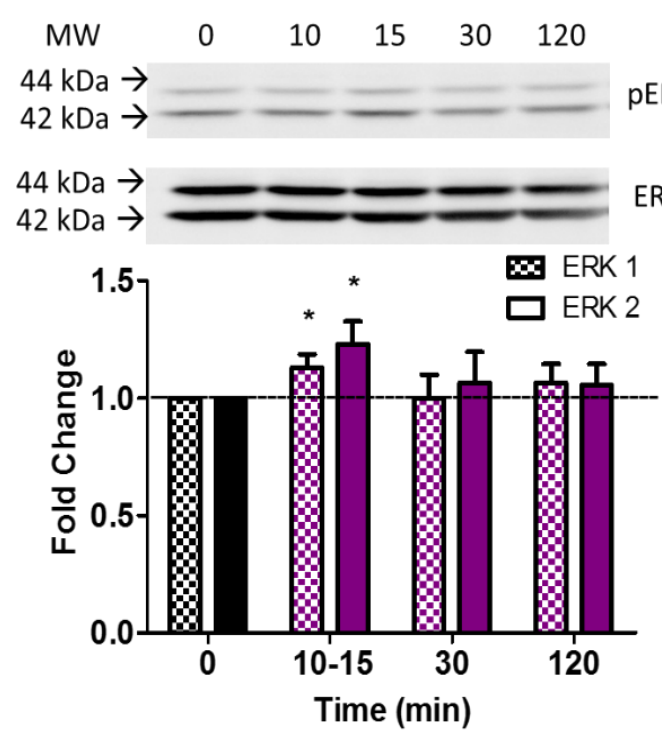

C)

PFC

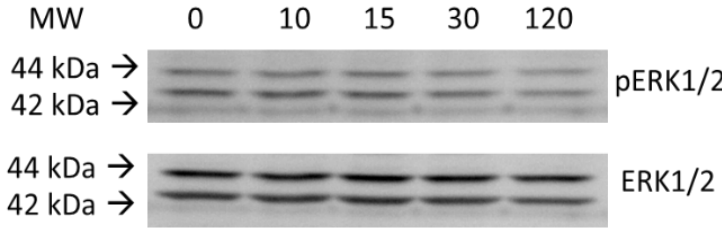

Figure 5.11: 16-brSal A modulates ERK1/2 phosphorylation in the rat dStr, NAc, and PFC 16-brSal A (1.0 mg/kg, i.p.) produced significant increases in A) ERK2 activation in the dStr at 10 - 15 min, B) ERK1 and ERK2 activation in the NAc 10 - 15 min post-injection, and C) ERK2 activation at $10-15 \mathrm{~min}$ in the PFC. Student's $t$-test, ${ }^{*} \mathrm{p}<0.05$ compared to time 0 . Values presented as mean \pm SEM, $n=6$ (except for timepoint $10-15$, whereby $n=12$ ). 


\subsubsection{Effect of 16-brSal A treatment on p38 MAPK activation}

Figure $5.12 \mathrm{~A}$ shows that an acute injection of $16-\mathrm{brSal} \mathrm{A}(1.0 \mathrm{mg} / \mathrm{kg})$ in an adult rat led to a non-significant trend towards an increase in p38 activation at $10-15 \mathrm{~min}$ in the dStr (17 $\pm 12 \%, p=0.18$ ) but no change at 30 or 120 min. A significant $12 \pm 5 \%$ increase in $p 38$ activation was seen in the NAc at $10-15$ min following 16-brSal A administration $(p<0.05$, Student's $t$-test, Figure 5.12B). No significant changes in p38 activation was observed in the PFC at any time points (Figure 5.12C). 
A)
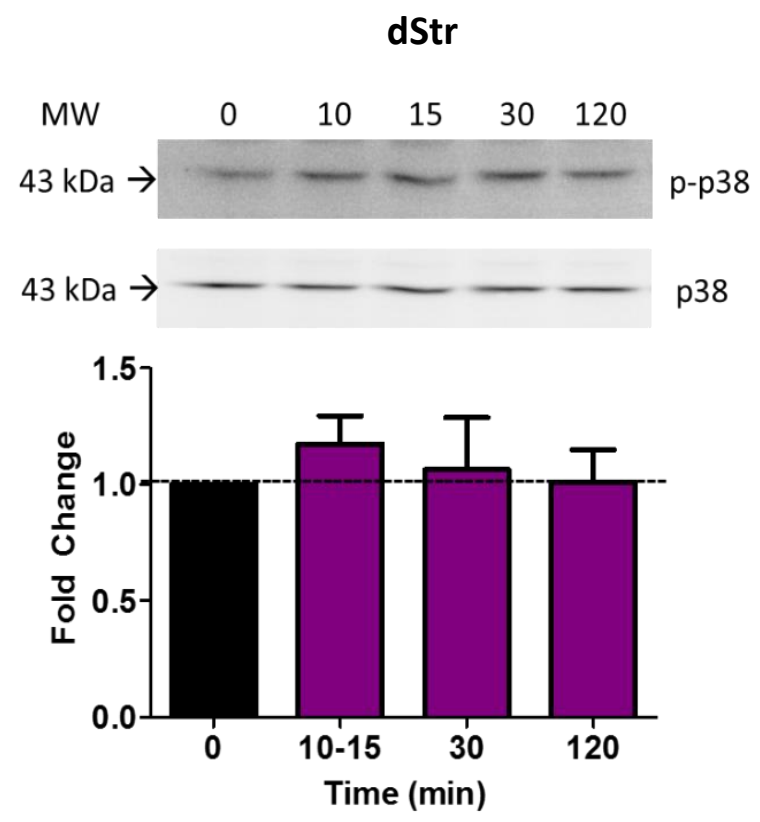

B)

NAC

C)

PFC

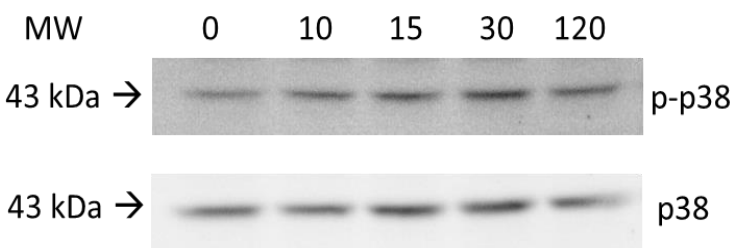

$\begin{array}{llllll}\text { MW } & 0 & 10 & 15 & 30 & 120\end{array}$

$43 \mathrm{kDa} \rightarrow-\mathrm{p}-\mathrm{p} 38$
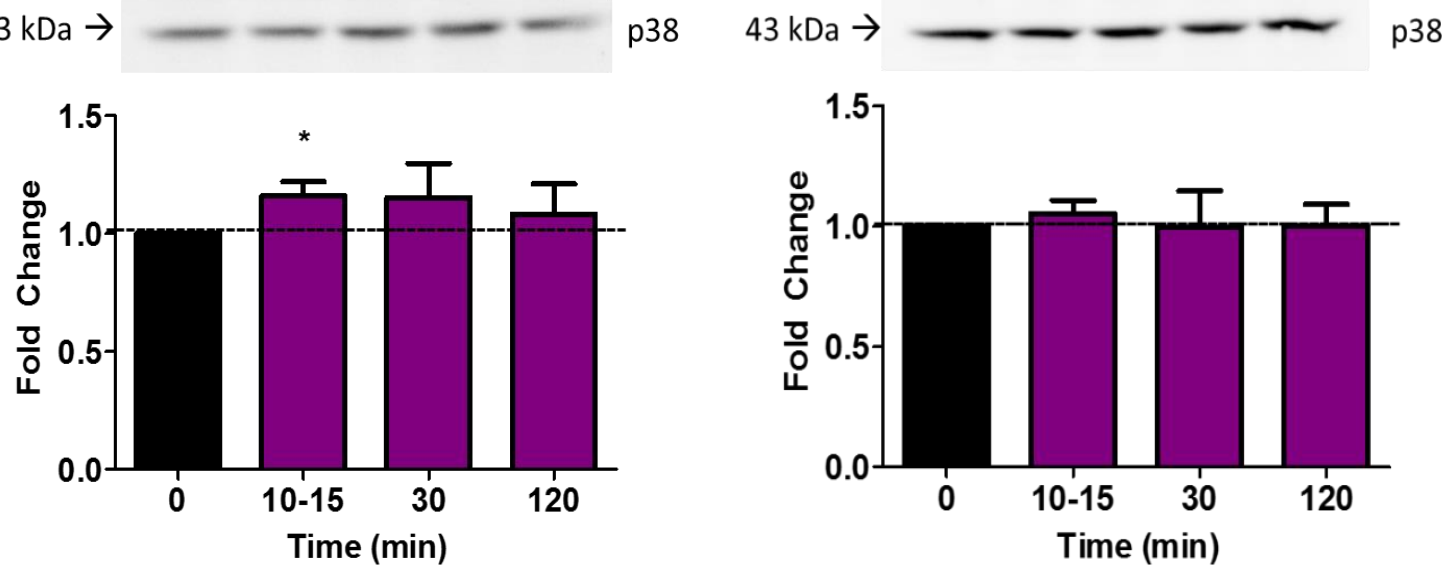

\section{Figure 5.12: 16-brSal A modulates p38 phosphorylation the rat NAC}

16-brSal A (1.0 mg/kg, i.p.) produced A) no significant change in p38 MAPK activation in the dStr, but B) increased p38 phosphorylation at $10-15 \mathrm{~min}$ in the NAc. C) 16-brSal A did not alter p38 activation in the PFC. Student's $t$-test, ${ }^{*} p<0.05$ compared to time 0 . Values presented as mean \pm SEM, $n=6$ (except for timepoint 10-15, whereby $n=12$ ). 


\subsection{Discussion}

Recently, there has been growing interest in KOPr signalling pathways, following the discovery that KOPr agonists with biased signalling for the G-protein pathway have therapeutic properties with fewer KOPr-mediated adverse effects. With this in mind, this study investigated the cellular effects of $16-\mathrm{brSal} \mathrm{A}$, a KOPr agonist shown to have desirable anti-cocaine effects (Chapter 3 ) and minimal side effects (Chapter 4).

\subsubsection{Validation of YFP-hDAT and myc-rKOPr expression in HEK-293 cells for ASP ${ }^{+}$uptake testing}

This study utilised HEK-293 cells transiently transfected with myc-rKOPr and YFP-hDAT to conduct $\mathrm{ASP}^{+}$uptake testing. HEK-293 cells are easy to grow, manipulate, and transfect. They also have very low basal levels of endogenous solute carrier transporters (a group of transporters which include DAT) (Ahlin et al., 2009) and KOPr (Blake et al., 1997; Wu and Wong, 2005), indicating that expression of KOPr and DAT in the present study was due to transfection. In addition, HEK-293 cells display neuronal characteristics in that they express the major neurofilament subunit NF-M, a protein normally found only in neuronal cells (Shaw et al., 2002). The resemblance of HEK-293 cells to neuronal cells make them desirable as in vitro models in this study, as the effects of 16-brSal A on these cells could be corroborated to the in vivo 16-brSal A-mediated actions on neuronal cells.

$\mathrm{ASP}^{+}$is an analogue of the neurotoxic compound, 1-methyl-4-phenylpyridinium (MPP+), which is used to induce Parkinson's disease-like features in laboratory animals (Schwartz et al., 2003). $\mathrm{ASP}^{+}$is a fluorescent substrate that can be used to monitor monoamine transporter function (Schwartz et al., 2003). Previous studies have used $\mathrm{ASP}^{+}$uptake to measure DAT (Bolan et al., 2007; Zapata et al., 2007; Oz et al., 2010a; Kivell et al., 2014), SERT (Oz et al., 2010b), and NET (Schwartz et al., 2003; Schwartz et al., 2005) function in cells. The use of $\mathrm{ASP}^{+}$uptake in this study was valuable as it allowed determination of real-time DAT function in single cells. Validation of $\mathrm{ASP}^{+}$uptake experiments showed that the $\mathrm{ASP}^{+}$method in this study showed the same pattern of uptake in accordance with previously published work (Bolan et al., 2007; Zapata et al., 2007; Kivell et al., 2014), whereby a rapid binding phase preceded a linear uptake phase that was maintained for 
at least $10 \mathrm{~min}$ (Figure 5.2A). The linear uptake phase of $\mathrm{ASP}^{+}$was extremely useful as it allowed evaluation of acute 16-brSal A in a within-cell design. In addition, the present study found that co-transfection of cells with both KOPr and DAT did not compromise the trend of $\mathrm{ASP}^{+}$uptake by DAT (Figure 5.2A), as this linear pattern is similar to that seen in cells expressing only YFP-hDAT (Dr Bridget Simonson, PhD thesis 2011). The present study finding that $\mathrm{ASP}^{+}$uptake correlates to expression of cell surface DAT (Figure 5.2B) supports results from a previous study that show $\mathrm{ASP}^{+}$uptake to be proportional to hSERT, a monoamine transporter that can also transport $\mathrm{ASP}^{+}$(Oz et al., 2010b). However, when used in a between-cell design, since $\mathrm{ASP}^{+}$uptake correlates to the expression of cell surface DAT (Figure 5.2B), the increase in rate of $\mathrm{ASP}^{+}$uptake observed could be an indirect measure due to variable DAT expression. Therefore, all between-cell ASP ${ }^{+}$uptake data were normalised to initial DAT cell surface expression. These data were also background corrected to eliminate non-DAT-mediated uptake, since HEK-293 cells possess endogenous mechanisms such as organic cation transport systems for $\mathrm{ASP}^{+}$ accumulation (Stachon et al., 1996; Schwartz et al., 2003). In addition, cells that displayed YFP-hDAT expression outside the 40-150 AFU range were not analysed due to their poor rate of $\mathrm{ASP}^{+}$uptake in correlation to DAT cell surface expression. Cells below this AFU range exhibited $\mathrm{ASP}^{+}$accumulation similar to that of non-transfected cells, whereas cells above this AFU range displayed signal saturation before the end of the 10 min testing period.

\subsubsection{6-brSal A modulates DAT function in a KOPr-dependent manner without altering DAT cell surface expression}

16-brSal A (5 $\mu \mathrm{M}, 10 \mu \mathrm{M})$ produced a concentration-dependent increase in the rate of $\mathrm{ASP}^{+}$uptake (Figure 5.4A). A dose-dependent increase of rate of $\mathrm{ASP}^{+}$uptake has also been observed in other studies using the parent compound, Sal $A$ at 3, 5, and $10 \mu \mathrm{M}$ in HEK-293 (Simonson et al., 2015) and EM4 cells (modified HEK-293 cells stably expressing the macrophage scavenger receptor with increased adherence to tissue culture plastic (Robbins and Horlick, 1998)) (Kivell et al., 2014) co-expressing KOPr and DAT. This increase in DAT function was also seen with $5 \mu \mathrm{M}$ and $10 \mu \mathrm{M}$ of U50,488 in DAT and KOPr co-expressing HEK-293 cells (Simonson et al., 2015). In the present study, 16-brSal A 
showed a lower increase in rate of $\mathrm{ASP}^{+}$uptake compared to the parent compound Sal $\mathrm{A}$ (Figure 5.4A), a result that cannot be attributed to differences in potency since 16-brSal $A$ and Sal A show similar efficacies at $\operatorname{KOPr}\left(\right.$ Sal $A E C_{50}=0.030 \mathrm{nM}$ vs. $16-b r S a l ~ A E C_{50}=$ $0.040 \mathrm{nM}$ ) (Riley et al., 2014). However, previous studies have found similar differential effects, in that although Sal A has a similar to greater efficacy for KOPr than U69,593 and U50,488 (Table 1.1), EM4 cells co-expressing DAT and KOPr exhibited over a 60\% increase in rate of $\mathrm{ASP}^{+}$accumulation when treated with Sal $\mathrm{A}(10 \mu \mathrm{M})$, but only about $40 \%$ and 20\% respectively when U69,593 (10 $\mu \mathrm{M})$ and U50,488 (10 $\mu \mathrm{M})$ were used (Kivell et al., 2014). In addition, Mesyl Sal B (10 $\mu \mathrm{M})$, which has similar efficacy at KOPr to Sal A (Sal A $E C_{50}=40 \mathrm{nM}$ vs. Mesyl Sal B EC $50=30 \mathrm{nM}$ ) showed only a $20 \%$ increase in rate of $\mathrm{ASP}^{+}$ uptake compared to over a 40\% increase for Sal A in HEK-293 cells (Simonson et al., 2015). Taken together, these studies and the present study indicate that the efficacy of KOPr agonists at KOPr cannot be taken as a direct correlation to the potency of these compounds in vitro, similar to the dose-related effects observed in vivo (discussed in section 3.5.2). Nonetheless, it is interesting to note that the decreased magnitude of the $\mathrm{ASP}^{+}$uptake rate change by $10 \mu \mathrm{M} 16$-brSal $\mathrm{A}$ in the present study is also reflected in the higher in vivo dose of 16 -brSal A needed to decrease reinstatement of drug seeking behaviour than Sal A (Figure 3.4A). From these findings, the reduced ability of 16-brSal A to increase DAT activity compared to Sal A matched the in vivo doses required for each compound. This suggests that KOPr modulation of DAT is an important regulating mechanism in modulating drug-seeking behaviour following a cocaine prime. It was also shown that regulation of DAT by 16-brSal A occurred in a KOPr-dependent manner (Figure 5.4B), similar to previous work using U50,488, U69,593, Sal A, and Mesyl Sal B (Kivell et al., 2014; Simonson et al., 2015).

To further evaluate the 16-brSal A-mediated increase of DAT function, kinetic studies were conducted using both $\mathrm{ASP}^{+}$uptake and RDEV. Kinetic studies are useful because they reveal the mechanisms behind the increase in transporter function. Changes in Vmax with no change in $\mathrm{Km}$ would indicate alterations in DAT cell surface expression, whereby an increase in Vmax indicates either an increase in transporter activity, an increase in cell surface expression, or both. On the other hand, changes in $\mathrm{Km}$ indicate a change in the affinity of a substrate for the transporter, with an increase in $\mathrm{Km}$ indicating a decrease in 160 
binding affinity and vice versa. RDEV experiments were conducted using the dStr and NAC because both these striatal regions contain terminals of the nigrostriatal and mesolimbic DA neurons, and express high levels of DAT and KOPr (Boja and Kuhar, 1989; Marshall et al., 1990; Richfield, 1991; Meshul and McGinty, 2000; Svingos et al., 2001; Gulley and Zahniser, 2003). In addition, it has previously been reported that DA uptake in the dStr and NAc is primarily dependent on DAT (Moron et al., 2002), and that the KOPr is apposed to DAT in striatal axons and varicosities (Svingos et al., 2001). Taken together, these studies indicate that the KOPr is strategically located in the striatum to modulate DA uptake via regulation of DAT.

Although both $\mathrm{ASP}^{+}$uptake and RDEV kinetic studies revealed that 16-brSal A increased Vmax, it was surprising to find that 16 -brSal $\mathrm{A}$ increased $\mathrm{Km}$ in the $\mathrm{ASP}^{+}$uptake model (Table 5.1) but not in the RDEV study (Table 5.2). However, this difference could be attributed to the extent of full brain circuitry and signalling mechanisms present in the two different environments (native vs. heterologous), a possible different regulatory system of DAT by the KOPr in both systems, varying levels of KOPr and DAT co-expression, or different effects of different uptake substrates (DA in the rat model versus ASP ${ }^{+}$in the cell model). Another possible reason for this discrepancy is that the $\mathrm{ASP}^{+}$uptake study used a between-sample design, which potentially increases data variability, whereas the RDEV kinetics study utilised sequential DA additions to the same sample. However, it is interesting to note that a previous study also showed that Sal $\mathrm{A}(10 \mu \mathrm{M})$ produced no significant change in the $\mathrm{Km}$ of $\mathrm{ASP}^{+}$uptake in striatal synaptosomes but significantly increased the Km when KOPr and DAT co-expressing EM4 cells were used (Kivell et al., 2014), proving that the kinetics of DAT in native systems can differ from that of cellular models. Nonetheless, the increased $\mathrm{Vmax}$ in both studies indicate that 16-brSal A increased DA uptake by increasing either DAT function or DAT expression, or a combination of the two. Further studies using cell surface biotinylation showed that $16-$ brSal A did not alter cell surface expression of DAT in YFP-hDAT and myc-rKOPr coexpressing HEK-293 cells (Figure 5.9B), evidence that 16-brSal A regulates DAT via an increase in DAT function. One possible reason for this increase may be the elevation in DAT-mediated DA uptake turnover rate (Meiergerd and Schenk, 1994). 
It is important to note that Thompson et al. (2000) has previously used microdialysis techniques to show that modulation of DA uptake by U69,593 was opposite to that of cocaine, a mechanism suggested to be responsible for its anti-cocaine effects (Thompson et al., 2000). In support of this, the authors showed that acute KOPr activation by U69,593 at a dose that attenuated drug seeking behaviour $(0.32 \mathrm{mg} / \mathrm{kg}, \mathrm{s.c})$ produced an increase in DA uptake by increasing DAT function in the NAc of rats $2 \mathrm{~h}$ after agonist treatment (Thompson et al., 2000). Therefore, it can be argued that the similar increase in DAT function produced by 16-brSal A may account for the cocaine-antagonistic action of 16brSal $A$ in the reinstatement of drug seeking and hyperactivity models observed in Chapter 3. It is also intriguing to note that a simple use of the KOPr antagonist, nor-BNI, inhibited both the behavioural anti-cocaine effects (Chapter 3 ) as well as the increase in DAT function (Figure $5.4 \mathrm{~B}$ and 5.8 ) produced by 16 -brSal $A$, further supporting the suggestion that 16-brSal A antagonises the effects of cocaine by increasing DAT function in a KOPr-dependent manner.

Despite the similar effects of KOPr agonists in increasing DA uptake, differences in DAT regulation mechanisms between agonists have been noted. It has been shown that Mesyl Sal B regulates DAT in a similar manner to 16-brSal A, since Mesyl Sal B increased the Vmax of DA uptake in the RDEV model without increasing DAT cell surface expression in KOPr and DAT expressing HEK-293 cells (Simonson et al., 2015). In direct contrast, both U69,593 (10 $\mu \mathrm{M})$ and Sal A (10 $\mu \mathrm{M})$ upregulated DA uptake by producing a significant increase in DAT cell surface expression (Simonson et al., 2015). Further evidence of this differential regulation comes from an earlier study in which Sal $\mathrm{A}(10 \mu \mathrm{M})$ increased DAT cell surface expression in KOPr and DAT co-expressing EM4 cells, as well as striatal synaptosomes (Kivell et al., 2014). Taken together, these findings indicate that 16-brSal A regulates DAT function and expression in a different manner to Sal A and U69,593. In view of the fact that 16 -brSal A produces fewer KOPr-mediated side effects than Sal A and U69,593 (Table 4.1), it may be possible that the differential mechanisms involved in regulation of DAT may correlate to the KOPr-mediated adverse effects observed. However, further studies involving KOPr signal transductions are required to determine if such correlation exists. Therefore, 16-brSal A was also studied for several different KOPr signalling mechanisms (discussed in the following section). 


\subsubsection{6-brSal A regulates DAT by activating the early, but not late phase of ERK1/2}

One factor influencing DAT regulation is the ERK1/2 signalling pathway, whereby modulation of DAT is ERK1/2-dependent. A study examining the relationship between DAT and ERK1/2 revealed that inhibition of ERK1/2 by the MEK1/2 inhibitor U0126 (50 $\mu \mathrm{M})$ or the ERK1/2 inhibitor PD98059 $(50 \mu \mathrm{M})$ led to a decrease in DAT function and expression in striatal synaptosomes as well as HEK-293 cells expressing DAT (Moron et al., 2003). However, a later study by Kivell et al. (2014) showed that although inhibition of ERK1/2 kinase with PD98059 (10 $\mu \mathrm{M})$ prevented the Sal A-mediated increases in ASP ${ }^{+}$ uptake, PD98058 did not alter $\mathrm{ASP}^{+}$uptake on its own (Kivell et al., 2014). Similarly, PD98059 also inhibited Sal A-mediated increase of DA uptake in striatal tissue (Kivell et al., 2014), whereas U0126 prevented the Mesyl Sal B-induced increase of DAT function in the rat striatum (Simonson et al., 2015). It was therefore not surprising to find that U0126 $(20 \mu \mathrm{M})$ did not alter $\mathrm{ASP}^{+}$uptake but significantly prevented the 16-brSal A-mediated increase in $\mathrm{ASP}^{+}$accumulation (Figure 5.9A), indicating that 16 -brSal A modulates DAT function in an ERK1/2-dependent manner. However, subsequent time course investigation using Western blotting in KOPr expressing HEK-293 cells revealed that 16brSal $\mathrm{A}(10 \mu \mathrm{M})$ rapidly phosphorylated ERK1/2 only at $5-10 \mathrm{~min}$, but not at later time points (120 or $180 \mathrm{~min}$, Figure $5.10 \mathrm{~A}$ ). Interestingly, this pattern of ERK1/2 activation supports the hypothesis that 16-brSal A produces fewer KOPr-mediated side effects due to its differences in KOPr signalling, because the traditional agonist U69,593 (1 $\mu \mathrm{M})$ has shown ERK1/2 activation in two distinct phases: a $\beta$-arrestin-independent, initial activation occurring at $5 \mathrm{~min}$ and a $\beta$-arrestin-dependent, late phase occurring at $2 \mathrm{~h}$ posttreatment in immortalised astrocytes (McLennan et al., 2008). In contrast, a study using Sal A - which shows fewer side effects than traditional KOPr agonists - showed that Sal A stimulated only early (5 $\mathrm{min}$ ) but not late phase (120 $\mathrm{min}$ ) ERK1/2 in immortalised rat cortical astrocytes (McLennan et al., 2008). The potent C-2 derivative of Sal A, MOM Sal B $(1 \mu \mathrm{M})$ also produced only $\beta$-arrestin-independent, rapid ERK1/2 activation in immortalised astrocytes (McLennan et al., 2008), suggesting that novel KOPr agonists with fewer behavioural adverse effects phosphorylate ERK1/2 in a different manner compared to traditional KOPr agonists. It has been recently hypothesised, based on the concept of functional selectivity, that $\beta$-arrestin recruitment is responsible for $\mathrm{KOPr}$ mediated adverse effects (Phillips et al., 2013; Zhou et al., 2013). In support of this, a 
ligand preferentially activating G-protein signalling, RB-64, showed no sedation, motor incoordination, or anhedonia-like effects (White et al., 2015).

Further extension of the ERK1/2 investigation found that the 16-brSal A effects on ERK1/2 activation extends to in vivo systems, in which $16-\mathrm{brSal} \mathrm{A}(1.0 \mathrm{mg} / \mathrm{kg})$ displayed the same pattern of $\beta$-arrestin-independent only ERK1/2 activation in the dStr, NAc, and PFC of rats (Figure 5.11). This pattern of ERK1/2 activation has also previously been demonstrated by Mesyl Sal B $(2.0 \mathrm{mg} / \mathrm{kg})$ in the dStr, NAc, and PFC of male Sprague-Dawley rats (Simonson et al., 2015). This in vivo finding further strengthens the suggestion that the ERK1/2 phosphorylation produced by $16-$ brSal A correlates to its improved side effect profile (Table 4.1, refer to Kivell et al. (2013) for a recent review). Although this study did not directly measure the inability of 16 -brSal $A$ to recruit $\beta$-arrestin, it is possible that 16 brSal A may act as a functionally selective or biased ligand at the KOPr. Since development of functionally selective KOPr agonists are of major therapeutic interest, the present study also investigated the effects of 16 -brSal $A$ on a second $\beta$-arrestin-dependent signalling pathway, p38 kinase.

\subsubsection{6-brSal A activates p38 MAPK in the rat NAc}

This study utilised only the in vivo rat model to determine the effect of 16-brSal A (1.0 $\mathrm{mg} / \mathrm{kg}$, i.p.) on p38 activation because Sal A $(10 \mu \mathrm{M})$ did not increase p38 phosphorylation in KOPr and DAT co-expressing EM4 cells (Kivell et al., 2014). However, Sal A caused KOPrmediated p38 activation with similar efficacy and potency as $\mathrm{U} 50,488$ in striatal astrocytes (U50,488 EC50 $=501 \pm 348 \mathrm{nM}$; Sal A EC $50=316 \pm 304 \mathrm{nM}$ ) (Bruchas et al., 2006), an effect that can possibly be attributed to use of the in vitro cellular model in Kivell et al. (2014) and native system in in Bruchas et al. (2006) (also discussed in section 5.5.2). In support of this, $U 69,593$ and $U 50,488$ showed no activation of p38 MAPK in EM4 cells (Kivell et al., 2014), although KOPr activation by U50,488 (10 mg/kg) has been linked to p38 phosphorylation in vivo (Robles et al., 2014). The present study found that 16brSal A significantly activated p38 MAPK only in the NAc of rats (Figure 5.12B). However, although this increase in p38 MAPK was significant $(p=0.0403)$, it is important to note that the magnitude of this increase was minimal (only a $12 \%$ increase) and observed due 
to elevated levels of phosphorylated p38 in 5 out of 11 rats. Thus, activation of p38 MAPK in the NAc may not be biologically relevant and may be a result of variation between rats, day-to-day variability, or the variability caused by experimenter-administered injections. In addition, it is also noteworthy that no significant p38 phosphorylation was observed in the dStr (Figure 5.12A) or PFC (Figure 5.12C), unlike ERK1/2 which was significantly activated in all three regions (Figure 5.11). Although it can be argued that this may be due to the fact that p38 MAPK is not required for modulation of DAT activity, unlike ERK1/2 (Moron et al., 2003; Kivell et al., 2014), it is suggested that this lack of significant p38 MAPK activation in areas other than the NAc may be because of its ability to only weakly recruit $\beta$-arrestin.

Previous reports have shown that activation of p38 MAPK requires phosphorylation of serine-369 in the C-terminal domain of the KOPr by GRK3 and subsequent $\beta$-arrestin recruitment (Bruchas et al., 2006; Bruchas et al., 2007a). P38 MAPK is reported to play a key component in KOPr-mediated aversion, dysphoria, and swim stress immobility (Bruchas et al., 2006; Bruchas et al., 2007a; Violin and Lefkowitz, 2007; Land et al., 2008; Bruchas and Chavkin, 2010; Violin et al., 2014). Since the present study has showed that 16-brSal A (1.0 mg/kg) produced no significant depressive-like effects in male SpragueDawley rats (Figure 4.2A), it is suggested that this reduced adverse effect profile may be partly due to its reduced or absent signalling of the GRK3/ $\beta$-arrestin-dependent $p 38$ MAPK pathway. However, one problem remains with this argument, in that 16 -brSal A showed aversive effects in the CPA paradigm (Figure 4.5), an effect that could potentially be correlated to its ability to activate p38 MAPK in the NAc. Incidentally, the time point of p38 MAPK activation seen in the NAc $(10-15 \mathrm{~min})$ also corresponds to the in vivo onset time of the 16-brSal A response in rats (10 $\mathrm{min})$. In addition, previous studies have shown that U50,488 (2.5 mg/kg, i.p.) produced KOPr-modulated CPA in wild-type but not KOPr knock-out mice (Land et al., 2009). This aversion was not restored upon injection of a lentiviral-KOPr construct encoding a mutant receptor unable to activate p38 MAPK into the dorsal raphe nucleus, indicating that activation of p38 is required to produce KOPrmediated aversion (Land et al., 2009). U50,488 (20 mg/kg, i.p.) also significantly increased p38 phosphorylation in mouse striatal tissue, but mice pre-treated with a selective p38 inhibitor, SB203580 (0.5 nmol, i.c.v.), showed no U50,488-mediated CPA (Bruchas et al., 
2007a). Taken together, these studies suggest that the increase in p38 activation (and consequently, aversive effects) seen in the present study was indeed due to 16-brSal A. However, a recent study conducted by White et al. (2015) showed astonishing results that contradict the long-standing and popular belief that CPA behaviour requires recruitment of $\beta$-arrestin. In this study, the authors found that an s.c. injection of U69,593 (1 mg/kg), Sal A (3 mg/kg), or the G-protein biased KOPr agonist, RB-64 (3 mg/kg) still produced CPA in $\beta$-arrestin knock-out mice (White et al., 2015), indicating that KOPrproduced aversion may in fact be mediated by the G-protein pathway. Although further studies are warranted to determine whether 16-brSal A is indeed a functionally selective agonist at the KOPr, the ability of 16-brSal A to activate certain aspects of KOPr signalling pathways as shown by the selective ERK1/2 and p38 MAPK signalling indicates that it may be functionally selective at the KOPr, which may in turn explain its improved side effect profile discussed in Chapter 4.

\subsubsection{Limitations and future directions}

The main limitation in this study was the use of differential cellular (HEK-293 cells) and non-human (adult Sprague-Dawley rats) experimental models. A study previously conducted by McLennan et al. (2008) showed that although MOM Sal B activated only early phase ERK1/2 in immortalised astrocytes, both early and late phase ERK1/2 phosphorylation was seen in primary astrocytes, suggesting that different experimental models may produce contrasting results (McLennan et al., 2008). The activated KOPr signalling pathways may also depend on species. Schattauer and colleagues (2012) found that although human and rodent KOPr share a $94 \%$ overall sequence homology, the amino acid residues involved in GRK3/ $\beta$-arrestin signalling pathway are not conserved. For example, the residue at position 358 is an asparagine (not a GRK substrate) in rodent KOPr, but a serine in human KOPr (Schattauer et al., 2012). Although U50,488 produced no significant differences in p38 and ERK1/2 activation in both rodent and human KOPr, the non-selective partial KOPr agonist, pentazocine was more potent at activating p38 in human KOPr than rodent KOPr (Schattauer et al., 2012). This indicates that different experimental models can produce different outcomes. However, it is important to note that although evaluation of 16 -brSal A-mediated effects in native systems are preferable, 
the in vivo dose of 16-brSal A required for collection of a single tissue sample $(1 \mathrm{mg} / \mathrm{kg})$ was seven-fold greater than that required for an in vitro study (based on a $350 \mathrm{~g}$ rat and use of $10 \mu \mathrm{M}$ for in vitro treatments). Since Sal A analogues are in limited supply and costly to synthesise, the use of HEK-293 cells was deemed to be a suitable alternative screening tool. The use of cellular models was also preferred to encourage a reduction in number of animal subjects required.

It is important to note that the present study did not investigate the effect of 16 -brSal A on DA release. Previous studies have shown that KOPr agonists decrease basal DA release (Ebner et al., 2010) as well as inhibit cocaine-induced release of DA (Zhang et al., 2004b; Zhang et al., 2004a). Therefore, further studies investigating the effect of 16-brSal A on both basal and cocaine-induced DA levels could be evaluated using in vivo microdialysis. To conduct this study, rats can be implanted with a guide cannula and a microdialysis probe inserted into the dStr and NAc. The dialysate samples collected before and after 16-brSal A administration could be compared to determine changes in DA release. To determine whether 16-brSal A will inhibit changes in cocaine-induced increase in DA levels, 16-brSal A can be administered 10 min prior to a cocaine injection.

Further experiments that can be carried out to extend the present findings include verification of the results obtained. For example, total internal reflection microscopy (TIRFM) can be used to support cell surface biotinylation studies, which revealed a lack of 16-brSal A-mediated modulation on DAT expression. TIRFM is a microscopy technique in which a laser beam is directed to the sample at an angle larger than the critical angle, so that the beam is internally reflected rather than refracted through the sample. This technique allows visualisation and analysis of events at the cell surface due to its shallow penetration (within $100 \mathrm{~nm}$ ) (Axelrod, 2003), without having to account for the inability of biotin to attach to proteins within cell membrane invaginations (Furman et al., 2009). However, TIRFM can also provide higher cell surface fraction readings by measuring DAT molecules that are close to the cell membrane, suggesting that multiple complementary techniques are required to confirm the effects of 16 -brSal A on DAT cell surface expression. The use of rat striatal synaptosomes instead of KOPr and DAT expressing HEK- 
293 cells can also aid in determining if the absence of 16 -brSal A-modulation on DAT cell surface expression extend to the native system.

Other additional studies that can be carried out include high throughput $\mathrm{ASP}^{+}$uptake studies - conducted with 96-well plates and a fluorescence plate reader - which will greatly increase the speed and magnitude of data collection. High throughput studies will also enable rapid investigation of multiple novel KOPr agonists simultaneously. The investigation of chronic effects of 16-brSal A is beyond the scope of this study; however, Acri et al. (2001) showed that repeated administration of U69,593 (0.32 mg/kg, s.c.) for 3 days did not affect DA neurotransmission in the dStr of rats (Acri et al., 2001). This is in contrast to a study by Thompson et al. (2000) which showed that repeated treatment of rats with U69,593 (0.32 mg/kg, s.c.) for 5 consecutive days decreased DA uptake by downregulating DAT in the NAc (Thompson et al., 2000). On the other hand, repeated use of the novel KOPr agonist Sal A (1 mg/kg, i.p.) did not alter DA release or uptake in the dStr (Gehrke et al., 2008) but produced an increase in ERK1/2 and CREB activation $(2.0 \mathrm{mg} / \mathrm{kg}$, i.p.) in the NAc of rats (Potter et al., 2011). Taken together, these results suggest that KOPr-mediated regulation of DAT and activation of signalling pathways are ligand and region specific, necessitating the investigation of repeated 16-brSal A administration in order to determine its longer term mechanism of action. It is also noteworthy that CREB activation is associated with KOPr-mediated stress and depressivelike effects (Pliakas et al., 2001; Mague et al., 2003; Kreibich and Blendy, 2004) suggesting that since 16-brSal A produced fewer side effects compared to Sal A, it should produce minimal $\beta$-arrestin-dependent CREB activation (Manson et al., 2011). Finally, since this study did not directly quantify $\beta$-arrestin recruitment but instead used inferred observations based on $\beta$-arrestin-dependent signalling pathways, investigations involving biased signalling studies such as the $\beta$-arrestin recruitment assays are needed in order to evaluate whether 16-brSal A is indeed a functionally selective KOPr agonist.

\subsection{Conclusions}

16-brSal A significantly increased DAT function in a KOPr- and ERK1/2-dependent manner, but did not induce a change in binding affinity or cell surface expression of DAT. Although 
KOPr and DAT expressing HEK-293 cells caused a decrease in DAT binding affinity to ASP ${ }^{+}$, this effect could be attributed to a difference in experimental models. Results in this study suggest that the increase in 16-brSal A-mediated DAT function may contribute to the antiaddiction properties of 16 -brSal A. Investigation of MAPK signalling pathways also indicated that 16-brSal A selectively activated the early, but not late, phase of ERK1/2 while only marginally activating p38 MAPK, suggesting that the differential activation pattern of 16-brSal A may account for its improved side effect profile. As p38 and late phase ERK1/2 signalling are $\beta$-arrestin-dependent pathways, it is possible that 16 -brSal A may be a biased agonist at the KOPr. However, future $\beta$-arrestin recruitment studies of 16-brSal A are required to determine if 16-brSal A is a G-protein biased KOPr agonist. 


\begin{tabular}{|c|c|c|c|c|c|c|c|c|c|}
\hline \multirow{2}{*}{$\begin{array}{l}\text { Signalling } \\
\text { pathway }\end{array}$} & \multicolumn{8}{|c|}{ KOPr agonist } & \multirow{2}{*}{ Model } \\
\hline & U69,593 & U50,488 & Sal A & MOM Sal B & EOM Sal B & Mesyl Sal B & $\beta$-THP Sal B & 16-brSal A & \\
\hline $\begin{array}{l}\text { ERK1/2 } \\
\text { MAPK }\end{array}$ & $\begin{array}{c}\uparrow \mathrm{P} 12^{(4)} \\
1 \mu \mathrm{M} \\
\text { (immortalised, } \\
\text { primary } \\
\text { astrocytes) } \\
\uparrow{ }^{(9)} \\
0.1 \mu \mathrm{M} \\
\text { (immortalised } \\
\text { astrocytes) }\end{array}$ & - & $\begin{array}{c}\uparrow P 1^{(4)} \\
\text { (immortalised } \\
\text { astrocytes) }\end{array}$ & $\begin{array}{c}\uparrow \mathrm{P} 1^{(4)} \\
1 \mu \mathrm{M} \\
\text { (immortalised } \\
\text { astrocytes) } \\
\uparrow \mathrm{P} 12^{(4)} \\
1 \mu \mathrm{M} \\
\text { (primary } \\
\text { astrocytes) }\end{array}$ & - & $\begin{array}{c}\text { 个 P1 (2) } \\
10 \mu \mathrm{M} \\
\text { (HEK-293) }\end{array}$ & - & $\begin{array}{c}\uparrow \text { P1* }^{*} \\
10 \mu \mathrm{M} \\
\text { (HEK-293) }\end{array}$ & Cellular \\
\hline \multirow[b]{2}{*}{ p38 MAPK } & - & $\begin{array}{c}\uparrow^{(5)} \\
20 \mathrm{mg} / \mathrm{kg} \text {, i.p. } \\
\text { (Str - mice) }\end{array}$ & - & - & - & - & - & $\begin{array}{c}\uparrow^{*} \\
1 \mathrm{mg} / \mathrm{kg} \text {, i.p. } \\
\text { (NAc - rat) }\end{array}$ & Animal \\
\hline & $\begin{array}{l}\text { NE }{ }^{(3)} \\
10 \mu M \\
(E M 4)\end{array}$ & $\begin{array}{c}\uparrow^{(6)} \\
10 \mu \mathrm{M} \\
(\text { AtT-20) } \\
\mathbf{N E}^{(3)} \\
10 \mu \mathrm{M} \\
(\mathrm{EM} 4)\end{array}$ & $\begin{array}{c}\uparrow^{(6)} \\
\text { (primary } \\
\text { astrocytes) } \\
\mathbf{N E}^{(3)} \\
10 \mu \mathrm{M} \\
\text { (EM4) }\end{array}$ & - & - & - & - & - & Cellular \\
\hline
\end{tabular}

*denotes this study, $\downarrow=$ attenuation, $\uparrow=$ presence of effect/potentiation, NE $=$ no effect, $\mathrm{P} 1=$ early phase ERK1/2, P12 = early and late phase ERK1/2, \#\# = studies currently ongoing in our laboratory, - = study not found. EM4 $=$ HEK-293 cells stably expressing macrophage scavenger receptor, HEK-293 $=$ human embryonic kidney cells, AtT-20 = mouse pituitary tumour cells

\section{Table 5.4: Acute effects of U69,593, U50,488, Sal A, and Sal A analogues on the ERK1/2 and p38 MAPK signalling pathways}




\begin{tabular}{|c|c|c|c|c|c|c|c|c|c|}
\hline \multirow{2}{*}{$\begin{array}{c}\text { KOPr- } \\
\text { mediated } \\
\text { modulation } \\
\text { of DAT }\end{array}$} & \multicolumn{8}{|c|}{ KOPr agonist } & \multirow{2}{*}{ Model } \\
\hline & U69,593 & U50,488 & Sal A & MOM Sal B & EOM Sal B & Mesyl Sal B & $\beta$-THP Sal B & 16-brSal A & \\
\hline \multirow{2}{*}{$\begin{array}{c}\text { DAT } \\
\text { function }\end{array}$} & $\begin{array}{c}\uparrow^{(1)} \\
0.32 \mathrm{mg} / \mathrm{kg}, \\
\text { s.c. } \\
\text { (NAc- rat) }\end{array}$ & $\begin{array}{c}\uparrow^{(2)} \\
500 \mathrm{nM} \text { (NAc, } \\
\mathrm{PFC}, \mathrm{dStr}-\text { rat) } \\
\uparrow^{(3)} \\
10 \mu \mathrm{M} \\
\text { (Str - rat) } \\
\end{array}$ & $\begin{array}{c}\uparrow^{(3)} \\
10 \mu \mathrm{M} \\
\text { (Str - rat) }\end{array}$ & $\begin{array}{c}\uparrow(7) \\
500 \mathrm{nM} \\
\text { (NAc, PFC, } \\
\text { dStr - rat) }\end{array}$ & \#\# & $\begin{array}{c}\uparrow^{(2)} \\
500 \mathrm{nM} \\
\text { (NAc, PFC, dStr - rat) }\end{array}$ & \#\# & $\begin{array}{c}\uparrow^{*} \\
500 \mathrm{nM} \\
\text { (NAc, dStr - } \\
\text { rat) }\end{array}$ & Animal \\
\hline & $\begin{array}{c}\uparrow^{(3)} \\
10 \mu \mathrm{M} \\
(\mathrm{EM} 4)\end{array}$ & $\begin{array}{c}\uparrow^{(2)} \\
5 \text { and } 10 \mu \mathrm{M}, \\
\text { (HEK-293) } \\
\uparrow^{(3)} \\
10 \mu \mathrm{M}(\mathrm{EM} 4) \\
\end{array}$ & $\begin{array}{c}\text { 个 }(2,3) \\
3,5,10 \mu M \\
(\text { HEK-293, } \\
\text { EM4) }\end{array}$ & $\begin{array}{c}\uparrow(7) \\
10 \mu \mathrm{M} \\
(\mathrm{HEK}-293 \\
\text { COS7, N2A) }\end{array}$ & - & $\begin{array}{c}\uparrow^{(2)} \\
10 \mu \mathrm{M} \\
\text { (HEK-293, COS7, } \\
\text { N2A) }\end{array}$ & - & $\begin{array}{c}\text { 个* } \\
10 \mu \mathrm{M} \\
\text { (HEK-293) }\end{array}$ & Cellular \\
\hline \multirow[b]{2}{*}{$\begin{array}{c}\text { DAT } \\
\text { expression }\end{array}$} & - & 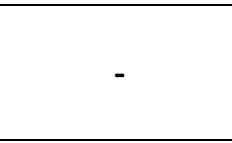 & $\begin{array}{c}\uparrow^{(3)} \\
10 \mu \mathrm{M} \\
\text { (Str- rat) }\end{array}$ & - & - & - & - & - & Animal \\
\hline & $\begin{array}{c}\uparrow^{(2)} \\
10 \mu \mathrm{M} \\
(\mathrm{HEK}-293)\end{array}$ & - & $\begin{array}{c}\text { 个 }(2,3) \\
10 \mu M \\
(H E K-293, \\
\text { EM4) }\end{array}$ & $\begin{array}{c}\uparrow^{(7)} \\
10 \mu \mathrm{M} \\
(\text { HEK-293) }\end{array}$ & - & $\begin{array}{c}\text { NE (2) } \\
10 \mu \mathrm{M} \\
(\mathrm{HEK}-293)\end{array}$ & - & $\begin{array}{c}\text { NE* }^{*} \\
10 \mu \mathrm{M} \\
(\mathrm{HEK}-293)\end{array}$ & Cellular \\
\hline
\end{tabular}

*denotes this study, $\downarrow=$ attenuation, $\uparrow=$ presence of effect/potentiation, NE = no effect, \#\# = studies currently ongoing in our laboratory, - = study not found. EM4 = HEK-293 cells stably expressing macrophage scavenger receptor, HEK-293 = human embryonic kidney cells, N2A = neuro-2A cells, COS7 = fibroblast cell line derived from African green monkey

\section{Table 5.5: Acute effects of U69,593, U50,488, Sal A, and Sal A analogues on DAT modulation}
1) Thompson et al. (2000)
5) Bruchas et al. (2007a)
6) Bruchas et al. (2006)
2) Simonson et al. (2015)
7) Kivell Lab, unpublished data
8) Potter et al. (2011)

9) Belcheva et al. (2005) 


\section{Chapter 6: Investigating the preclinical effects}

\section{of novel kappa opioid receptor agonists using}

\section{long access and progressive ratio self- administration paradigms}

\subsection{Introduction}

While no single preclinical behavioural model accurately models the complex nature of addition, a combination of several animal models can provide valuable information on different aspects of drug use. As outlined in Chapter 3, drug self-administration paradigms are considered the gold standard in preclinical addition studies due to their strong resemblance to voluntary drug intake in humans (Carter and Griffiths, 2009). Although simple drug self-administration models can only investigate the abuse liability of drugs (Koob and Le Moal, 2005), manipulations of these models allow investigation of different aspects of addiction, i.e., introducing an extinction phase and a subsequent drug prime. This drug primed reinstatement model mimics human craving and allows quantification of the compulsivity to seek a drug (used in Chapter 3). Other manipulations such as introduction of a chained schedule have also been developed, in which the conditioned behaviour is maintained by presentation of one or a few links in the chain of events or stimuli that precede presentation of the drug. For example, animals may be required to complete physical task requirements for presentation of a tone, and subsequent responses for the tone result in drug delivery (Ferster, 1957). However, chained schedules are rarely studied due to the difficulty in maintaining reinforced behaviour, particularly with studies made up of several links (Schindler et al., 2002). On the other hand, second order reinforcement schedules are a combination of two simple schedules, in which completion of physical tasks for the first schedule requirement results in presentation of a conditioned stimulus (such as a light cue or tone), whereas completion of the schedule requirements after an experimenter-determined time interval results in a drug delivery accompanied by the conditioned stimulus (Schindler et al., 2002). Second order manipulations are useful for studying the reinforcing effects of a 
drug without interference from the drug itself, as conditioned behaviours can usually be maintained for an extended amount of time prior to drug delivery. As a result, both drug seeking and drug taking behaviours can be investigated, with drug seeking behaviour evaluated prior to the first drug delivery and drug taking behaviour evaluated for the rest of the test session (reviewed in Schindler et al. (2002)). Another model of selfadministration is the discrete trial procedure, whereby a self-administration session is split into multiple distinct trial sessions (completion of a schedule requirement results in drug delivery) and inter-trial-intervals (completion of a schedule requirement results in no programmed consequences) (Dobrin and Roberts, 2012). Discrete trial procedures are useful because they can be used over $24 \mathrm{~h}$ periods without causing drug overdose, enabling investigation of self-administration diurnal rhythm (Dobrin and Roberts, 2012). Although discussion of the many different manipulations possible with drug selfadministration are beyond the scope of this study (refer to Markou et al. (1993) for a review of models of drug craving; Epstein et al. (2006) for a review of the reinstatement model; Schindler et al. (2002) for second order schedules; Dobrin and Roberts (2012) for

discrete trial procedures; and Panlilio and Goldberg (2007) for preclinical selfadministration models in general), it is important to determine if the anti-cocaine effects of novel KOPr agonists observed in Chapter 3 extend to other self-administration paradigms. To that end, the present study utilised two different self-administration paradigms: the long/extended access ( $\operatorname{LgA})$ and progressive ratio (PR) models.

\subsubsection{Extended or long access model}

Early studies using drug self-administration permitted unlimited access to the drug, a practice that most closely mimics the human condition. Unfortunately, unlimited access to drugs was reported to cause overdose and death. Unrestricted access to cocaine $(0.25$ and $1.0 \mathrm{mg} / \mathrm{kg}$, i.v.), a combination of cocaine $(1.0 \mathrm{mg} / \mathrm{kg}, \mathrm{i} . \mathrm{v})$ and morphine $(2.5 \mathrm{mg} / \mathrm{kg}$, i.v.), or codeine $(0.5$ and $2.5 \mathrm{mg} / \mathrm{kg}$, i.v.) caused convulsions and overdose in most monkeys within two to six weeks (Deneau et al., 1969). Another study showed that rhesus monkeys self-administrating i.v. infusions of cocaine $(0.2 \mathrm{mg} / \mathrm{kg}), \mathrm{d}$-amphetamine $(0.05$ $\mathrm{mg} / \mathrm{kg})$, or d-methamphetamine $(0.025 \mathrm{mg} / \mathrm{kg})$ in an unlimited access paradigm also died, with the monkeys self-administering cocaine dying in less than 5 days (Johanson et al., 
1976). Unlimited access to cocaine is more toxic than other drugs, with rats given unlimited access to cocaine $(1 \mathrm{mg} / \mathrm{kg}$, i.v.) reporting a $54 \%$ higher mortality rate than that of heroin $(100 \mu \mathrm{g} / \mathrm{kg}$, i.v.) (Bozarth and Wise, 1985). As a result of these high mortality rates, the short access (ShA) self-administration model ( 1 to $3 \mathrm{~h}$ duration) was introduced. The present project has previously used the ShA model to establish stable selfadministration behaviour in rats (Chapter 3). However, although ShA studies are useful for studying drug taking behaviour due to the resultant stable levels of responding (Ahmed and Koob, 1998), ShA models are ineffective at providing a complete portrayal of the human condition. Human drug addicts typically undergo a vicious cycle of drug binge and abstinence, as well as increase the amount and/or frequency of drug intake over time (Wee et al., 2007). This increase of intake, observed particularly during the development of drug dependence, is not portrayed by animals undergoing ShA selfadministration sessions. Therefore, in an effort to duplicate this compulsivity and loss of control in a laboratory setting, the LgA model (6 h) was introduced (Ahmed and Koob, 1998).

Rats given extended access to drugs typically show a gradual increase in drug taking behaviour known as escalation. Male Sprague-Dawley rats given LgA ( $7 \mathrm{~h})$ to cocaine $(0.5$ or $2.0 \mathrm{mg} / \mathrm{kg}$, i.v.) demonstrated a progressive increase in intake that ShA (3 h) rats did not $(0.5,1.0$, or $2.0 \mathrm{mg} / \mathrm{kg}$, i.v.) (Mantsch et al., 2004). Similarly, male Wistar rats given LgA $(6 \mathrm{~h})$ to cocaine $(0.4 \mathrm{mg} / \mathrm{kg})$ displayed a significant escalation in cocaine intake compared to ShA (1 h) rats (Ferrario et al., 2005). This escalation in cocaine intake could not be attributed to a higher efficacy of cocaine at increasing DA levels, since a single administration of cocaine $(0.125-1 \mathrm{mg} /$ injection) produced a similar magnitude of DA increase in the NAc of ShA and LgA rats (Ahmed et al., 2003). The escalation behaviour seen is therefore believed to correlate to the extended availability of cocaine and elevated cocaine intake. As a result, LgA self-administration sessions are said to more closely model uncontrollable drug taking in humans (Ahmed and Koob, 1998; Mantsch et al., 2001; Ahmed et al., 2003; Paterson and Markou, 2003; Ben-Shahar et al., 2004; Ferrario et al., 2005), suggesting that the LgA paradigm may be a more suitable preclinical model than the ShA paradigm. 
To the best of our knowledge, there have been no reports on the effects of KOPr agonists, including Sal A and its analogues, on animals given LgA to cocaine. However, studies utilising different models apart from the ShA paradigm have shown that KOPr agonists decrease cocaine self-administration. For example, Mr2033 (0.01 - $0.032 \mathrm{mg} / \mathrm{kg} / \mathrm{h}$, i.v.), bremazocine $(0.0032 \mathrm{mg} / \mathrm{kg} / \mathrm{h}$, i.v.), and enadoline $(0.001-0.0032 \mathrm{mg} / \mathrm{kg} / \mathrm{h}$, i.v.) significantly decreased cocaine $(0.01 \mathrm{mg} / \mathrm{kg})$ self-administration on a second order schedule of reinforcement in monkeys, in which cocaine infusions were conditioned to a green light. This dose-dependent, KOPr-mediated attenuation of cocaine selfadministration was seen throughout 10 days of treatment (Mello and Negus, 1998). In addition, Freeman et al. (2014) utilised a discrete trial choice procedure to show that rhesus monkeys given a choice between cocaine $(0.1 \mathrm{mg} / \mathrm{kg})$ or cocaine mixed with Sal A $(0.003-0.01 \mathrm{mg} / \mathrm{kg}$, i.v.) displayed a decrease in response for the paired drug option (Freeman et al., 2014). Taken together, these studies indicate that the anti-cocaine properties of KOPr agonists are consistent regardless of self-administration paradigms. Therefore, it is suggested that Sal A, as well as its synthetic derivatives should also present similar anti-cocaine effects in rats given extended access to cocaine.

\subsubsection{Progressive ratio model}

A common manipulation of the standard FR schedule of self-administration is the PR paradigm. While FR schedules require a fixed number of responses for delivery of the reinforcer, the PR schedule utilises an arithmetic or exponential series to increase the number of active responses required for each subsequent reinforcer. The increasing ratio requirements makes the PR paradigm a more effective tool to evaluate the reinforcing strength of drugs compared to FR schedules (Richardson and Roberts, 1996). Eventually, responding by subjects will reach a point where it ceases. The final reinforced ratio completed by subjects is known as the breakpoint (Cain and Bardo, 2010). This dependent variable is defined as the maximum effort subjects are willing to expend for a single delivery of the reinforcer. It is also used as a measure of a subject's motivation for the reinforcer (reviewed in Richardson and Roberts (1996)). Despite popularity of the PR schedule, there has been to our knowledge, no previous investigation of the effects of either traditional or novel KOPr agonists on cocaine self-administration using a PR 
schedule. However, a study conducted by Ebner et al. (2010) showed that $2.0 \mathrm{mg} / \mathrm{kg}$ of Sal A reduced the breakpoint in a PR sucrose self-administration paradigm (Ebner et al., 2010). Another study showed that addition of Sal A to oxycodone infusions $(0.006-0.1$ $\mathrm{mg} / \mathrm{kg}$, i.v.) decreased the breakpoint in responding for oxycodone in rhesus monkeys (Naylor et al., 2015). These studies emphasise that the KOPr system plays a role in modulating motivation for reward, making it likely that studying the effect of $\mathrm{KOPr}$ agonists on cocaine using a PR paradigm would produce interesting results.

\subsection{Aims and hypotheses}

Since KOPr agonists decrease cocaine self-administration by producing anti-cocaine effects in ShA paradigms, the present study aims to determine how KOPr agonists modulate different aspects of reward and addiction by using other self-administration paradigms. The LgA schedule was therefore utilised to evaluate effects of Sal A on extended access cocaine self-administration, whereas the PR model was used to determine the effects of Sal A and 16-brSal A on the motivation to self-administer cocaine. It is hypothesised that these KOPr agonists will attenuate drug taking in the LgA model and decrease motivation to seek out cocaine by reducing the breakpoint in the PR paradigm. 


\subsection{Methods}

\subsubsection{Materials}

Sal A was dissolved in 75\% DMSO for the LgA self-administration study. U69,593, U50,488, Sal A, and 16-brSal A was dissolved in DMSO:Tween-80:MilliQ water in a ratio of 2:1:7 for the PR study. Unless otherwise stated, all injections were administered i.p. either 5 (Sal A) or $10 \mathrm{~min}(\mathrm{U} 50,488,16$-brSal A) before testing at a volume of $1 \mathrm{~mL} / \mathrm{kg}$. U69,593 was administered s.c. 15 min before testing.

\subsubsection{Long access cocaine self-administration}

Rats underwent surgery and were trained to self-administer cocaine $(0.5 \mathrm{mg} / \mathrm{kg})$ as described in section 2.1.3 and 2.1.4. Once rats completed cocaine self-administration on FR5 for 4 weeks in $6 \mathrm{~h}$ sessions, they were administered either vehicle or Sal A 0.3 or 2.0 $\mathrm{mg} / \mathrm{kg}$ ) before commencement of a testing session. To determine the effects of repeated Sal A treatments on cocaine self-administration, vehicle or Sal A injections were given to rats self-administering cocaine for 3 consecutive days in a counterbalanced manner (Appendix 6.1).

\subsubsection{Progressive ratio self-administration}

Due to limited access to rats and utilising the $3 R$ principle of animal testing, two groups of rats were used for the PR paradigm. One group of rats $(n=6)$ was pre-exposed to a single injection of KOPr agonist and subjected to EPM testing a week prior to surgery. These rats were given either Sal A (1.0 mg/kg, $n=2)$, Mesyl Sal B $(0.3 \mathrm{mg} / \mathrm{kg}, \mathrm{n}=2)$, or $\mathrm{U} 50,488(10 \mathrm{mg} / \mathrm{kg}, \mathrm{n}=2)$. The other group of rats was drug naïve $(\mathrm{n}=8)$. All rats underwent surgery and were trained to self-administer cocaine in $2 \mathrm{~h}$ sessions on FR5 before progressing to PR sessions as described in section 2.1.3 and 2.1.4. Prior to testing, rats were subjected to at least 3 days of PR responding to establish a stable breakpoint (not more than \pm 2 infusions for 3 consecutive days). On testing day, rats were injected with either vehicle, 16-brSal A (1.0 or $2.0 \mathrm{mg} / \mathrm{kg}$ ), Sal A $(0.3$ or $2.0 \mathrm{mg} / \mathrm{kg}$ ), U50,488 (10 $\mathrm{mg} / \mathrm{kg})$, or $\mathrm{U} 69,593(1.0 \mathrm{mg} / \mathrm{kg})$ prior to commencement of a PR self-administration session. Each treatment was administered following a Latin square design (Appendix 6.1). 


\subsubsection{Statistics}

A paired Student's $t$-test was used to determine the significance of the number of active lever presses on day $1-2$ compared to day $20-21$ of the LgA study. To determine significance for total active lever presses in the LgA study, a repeated measures ANOVA was performed. In the PR study, a one-way ANOVA with repeated measures was used to analyse the time course self-administration training data, whereas a repeated measures ANOVA followed by paired $t$-tests with the Bonferroni correction was performed to determine significance of the number of infusions received by rats. Data were considered statistically significant when $p \leq 0.05$. 


\subsection{Results}

\subsubsection{Validation of the extended access cocaine self-administration paradigm}

Prior to testing with KOPr agonists, the stability of self-administration behaviour was examined in order to validate the LgA paradigm. Figure 6.1A shows the acquisition for cocaine self-administration on FR1, whereby acquisition was achieved when at least 20 active lever presses were performed within a $2 \mathrm{~h}$ session (with an active:inactive lever ratio of more than 2:1). Male Sprague-Dawley rats had a mean acquisition time of $5 \pm 1$ days (Figure 8.9 in Appendix 6.2), with all rats acquiring self-administration behaviour by day 10 (Figure 6.1A). After acquisition, rats consistently showed preference for the active lever over the inactive lever on FR5 (Figure 6.1B). In the $6 \mathrm{~h}$ training sessions, a progressive increase in active lever responses was observed over 21 days. However, analysis of data showed no significant escalation $[F(20,120)=1.58, p=0.069]$ (repeated measures ANOVA, Figure 6.1B). Since rats undergoing LgA models have been shown to escalate and the results of the ANOVA showed a trend towards significance, a comparison between the initial and final stages of self-administration training was also conducted. A paired Student's $t$-test showed a non-significant trend in escalation, with responses increasing by $37 \%$ from $554 \pm 20$ active lever responses on day $1-2$ to $760 \pm 112$ active lever presses on day $20-21$ ( $p=0.08$, Figure $6.1 C$ ). Since previous studies using LgA models have found that escalation is most notable during the first hour of selfadministration (Ahmed and Koob, 2004; Ahmed and Cador, 2006), the first hour of active lever responses in the present study was also analysed (Figure 6.1C). Responding during the first hour alone also showed a non-significant trend towards escalation $(p=0.06$, paired Student's $t$-test). On days $1-2$, rats produced $104 \pm 6$ lever presses in the first hour. This responding increased by $30 \%$ to $135 \pm 16$ lever presses by day $20-21$ (Figure 6.1C). Despite the escalating trend, the number of active lever responses stabilised by day 21 (Figure 6.1B). Comparison of baseline self-administration responding prior to each testing session revealed that responding for the active lever was consistent throughout all three testing sessions, with no major increases or decreases throughout the testing period (a total of approximately 6 weeks, Figure 6.1D). 
A)

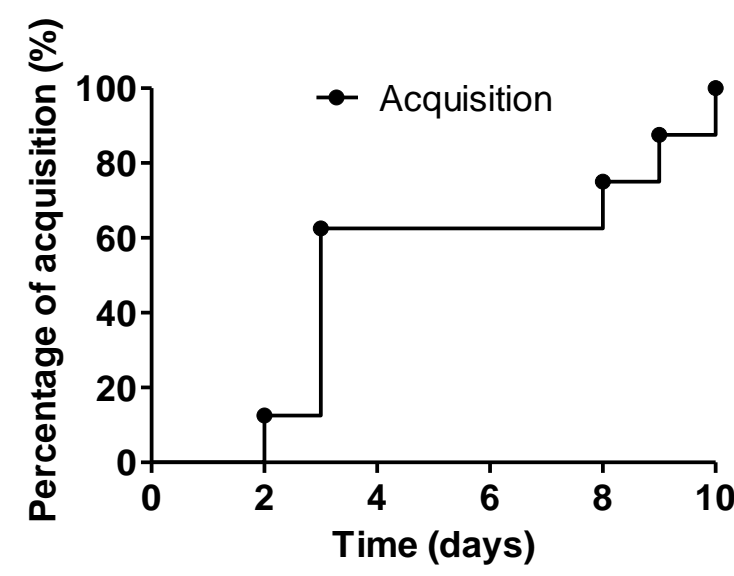

C)

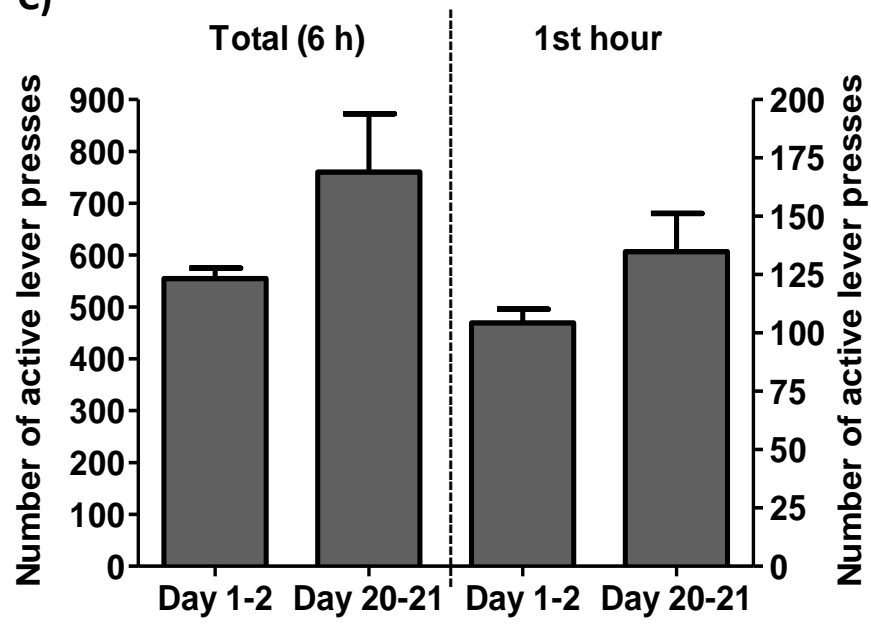

B)

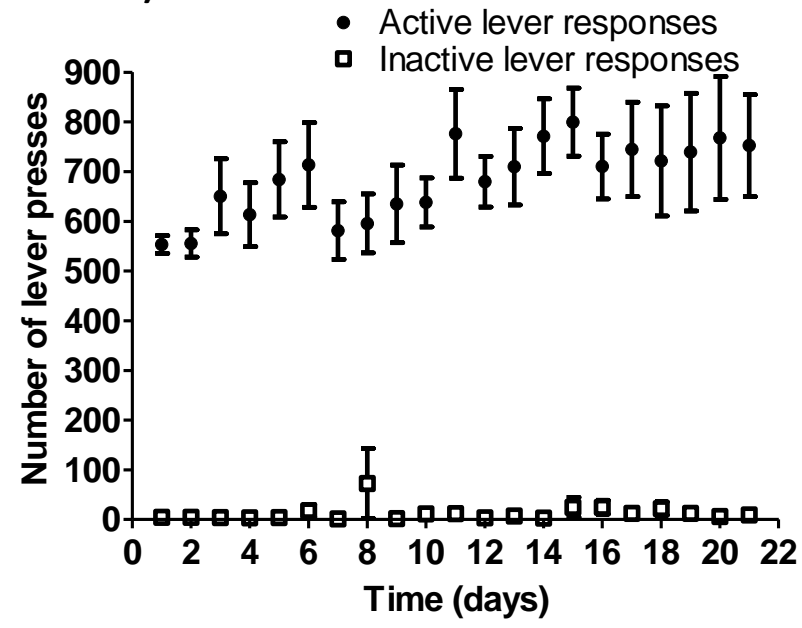

D)

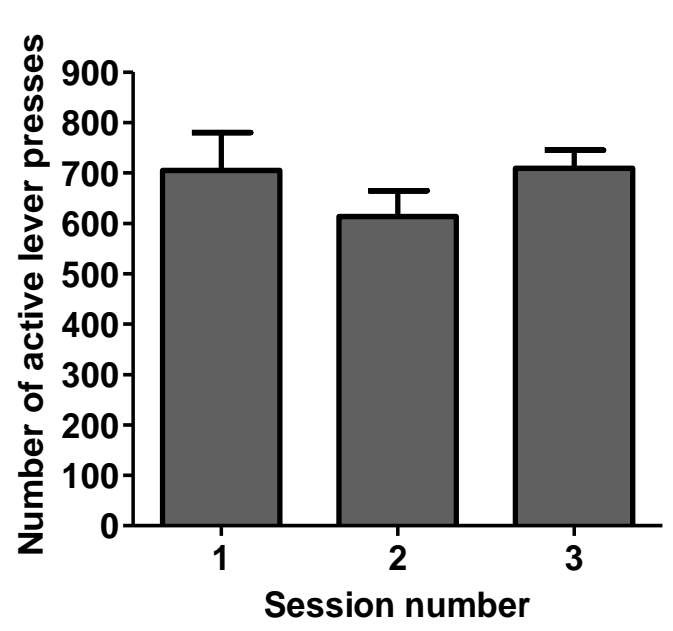

\section{Figure 6.1: Self-administration training}

A) Drug naïve rats began acquiring self-administration behaviour (FR1) on day 2, with all rats achieving acquisition by day 10. B) Number of active and inactive lever presses during $\operatorname{LgA}(6 \mathrm{~h})$ training sessions on the FR5 schedule prior to commencement of testing. No significant escalation was observed ( $p=0.069$, repeated measures ANOVA). C) Comparison of the number of active lever presses during the first and last two days of self-administration showed a trend towards an increase in the number of active lever responses ( $p=0.08$, paired Student's $t$-test). The number of responses in the first hour also showed a non-significant trend towards escalation $(p=0.06$, paired Student's $t$-test). D) Analysis of number of active lever presses throughout the baseline phases prior to commencement of each testing session revealed no significant changes in active lever responding throughout the experiment. Values presented as mean $\pm S E M, n=7$. 


\subsubsection{Effect of Sal A on extended access cocaine self-administration in rats}

Since previous studies have shown that Sal A produces anti-cocaine effects, the effect of Sal A on cocaine self-administration was also tested using the LgA paradigm. Rats were treated daily with vehicle (75\% DMSO) or Sal A ( 0.3 or $2.0 \mathrm{mg} / \mathrm{kg}$ ) over 3 continuous days. No significant effect of treatment was found $[F(8,40)=0.6840, p=0.7029]$ (repeated measures ANOVA, Figure 6.2A). Sal A-treated rats $(0.3 \mathrm{mg} / \mathrm{kg})$ produced a total of $721 \pm$ 70 active lever presses on day 1 , a response that was not significantly different than vehicle-treated controls (a mean response of $701 \pm 66$ active lever responses). Repeated administration of Sal A $(0.3 \mathrm{mg} / \mathrm{kg})$ for a further 2 days also produced no changes in active lever responses compared to vehicle controls, with Sal A-treated rats producing $698 \pm 78$ active lever responses on day 2 and $687 \pm 84$ active lever presses on day 3 (Figure 6.2A). On the other hand, rats given the vehicle produced $717 \pm 43$ active lever presses on day 2 and $714 \pm 63$ active lever responses on day 3 (Figure 6.2A). In addition to the low dose of Sal A $(0.3 \mathrm{mg} / \mathrm{kg})$, a higher dose of Sal A $(2.0 \mathrm{mg} / \mathrm{kg})$ was also tested. A single administration Sal A ( $2.0 \mathrm{mg} / \mathrm{kg}, 705 \pm 75$ lever presses) had no significant effect on selfadministration in rats given LgA to cocaine on day 1 (Figure 6.2A). Administration of Sal A for another two days also had no significant changes in the number of active lever responses compared to vehicle controls, with rats producing $588 \pm 79$ active lever presses on day 2 and $665 \pm 68$ active lever responses on day 3 .

Due to the short half-life of Sal A, the number of active lever responses in only the first hour of testing was also analysed. However, no significant changes in responding were noted between groups $[F(8,40)=0.3977, p=0.9152]$ (repeated measures ANOVA, Figure 6.2B). In the first hour, the lower dose $(0.3 \mathrm{mg} / \mathrm{kg})$ of Sal A produced an average of $141 \pm$ 16 active lever presses on day 1 , followed by $147 \pm 15$ active lever responses on day 2 , and $152 \pm 20$ active lever presses on day 3 . This Sal A-mediated responding was not significantly different from vehicle controls, which resulted in $131 \pm 16$ active lever presses on day $1,141 \pm 19$ on day 2 , and $133 \pm 15$ on day 3 . The higher dose of Sal A (2.0 $\mathrm{mg} / \mathrm{kg}$ ) also produced no significant alteration in active lever responding compared to vehicle-treated rats in the first hour of testing, with $139 \pm 15$ active lever responses on day 1 . A mean of $132 \pm 14$ active lever presses was observed on day 2 , whereas the third 
day of Sal A treatment yielded $133 \pm 23$ active lever responses in the first hour of testing (Figure 6.2B).

Thus, Sal A ( 0.3 or $2.0 \mathrm{mg} / \mathrm{kg}$ ) produced no significant changes in responding for cocaine utilising a LgA self-administration paradigm. 
A)

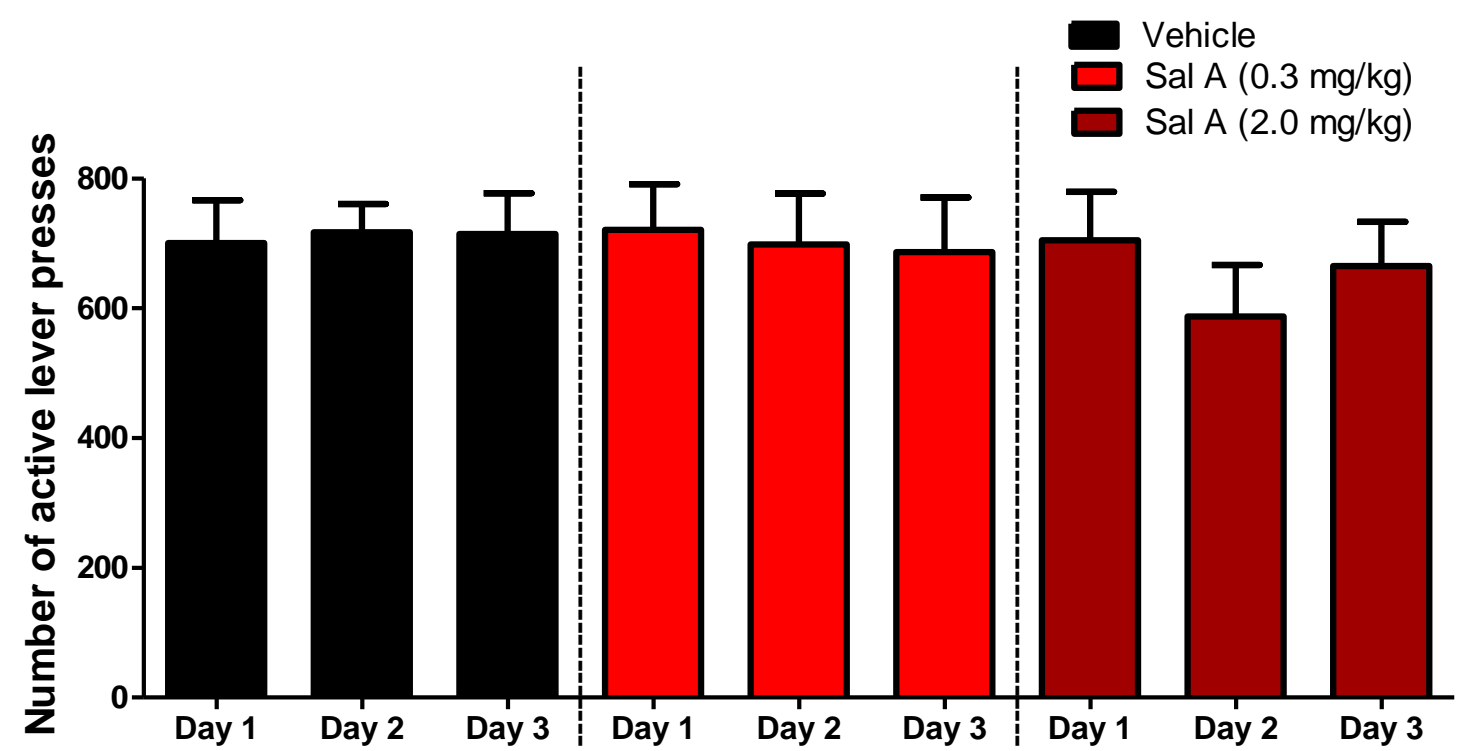

B)

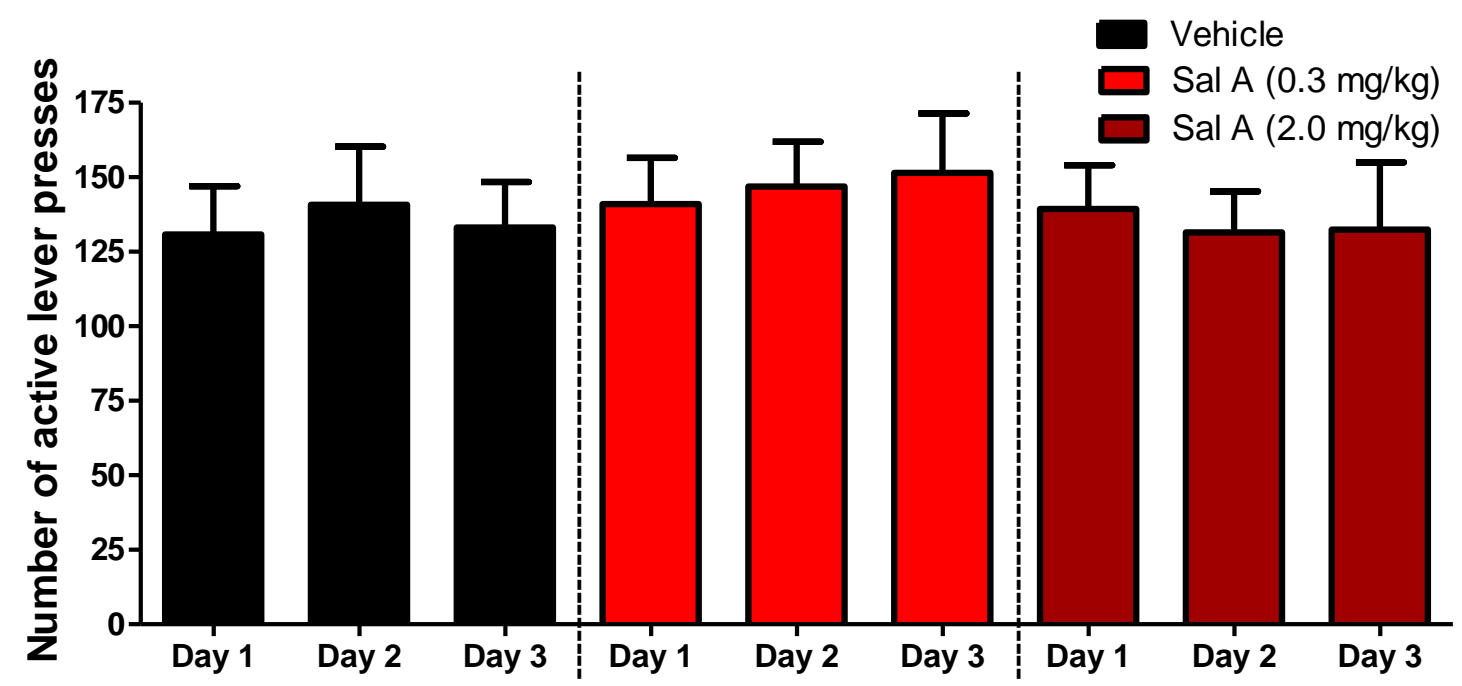

Figure 6.2: Sal A does not alter responding for cocaine in an extended access model of self-administration

Rats treated with Sal A displayed no significant changes in A) total active lever presses or B) active lever responding in the first hour of testing for all three days of treatment compared to time-matched vehicle (75\% DMSO) controls. Repeated measures ANOVA. Values presented as mean \pm SEM, $n$ $=6$. 


\subsubsection{Determination of differences between drug naïve and KOPr agonist pre-treated}

rats

The PR paradigm was used to determine the effect of KOPr agonists on the motivation of rats to self-administer cocaine. Since two subsets of rats were used for PR experiments, pilot studies were conducted to determine whether differences exist between the two groups. A Kaplan-Meier survival analysis was conducted to compare the different groups. Rats pre-treated with KOPr agonists had a median acquisition time of 11 days, longer than non-treated rats, which had a median acquisition time of 4 days (Figure 6.3A). A log-rank (Mantel-Cox) test conducted to determine differences in the survival distributions found that the two curves were statistically significantly different, $\chi^{2}(1)=9.661, p=0.0019$. Further analysis of the mean of acquisition times also showed that rats pre-treated with KOPr agonists were significantly slower in acquiring self-administration behaviour than drug naïve rats ( $p<0.01$, Student's $t$-test, Figure $6.3 C$ ). When analysed according to their respective pre-treatments, the Kaplan-Meier survival analysis also showed that rats pretreated with Sal A $(1.0 \mathrm{mg} / \mathrm{kg}$, median of 6.5 days), U50,488 $(10 \mathrm{mg} / \mathrm{kg}$, median time of 15 days) and Mesyl Sal B (0.3 mg/kg, median time of 12.5 days) were slower in acquiring self-administration behaviour compared to drug naïve rats (Figure 6.3B). A log-rank (Mantel-Cox) test conducted revealed statistically significant differences, $\chi^{2}(3)=11.97, p$ $=0.0075$. Analysis of the mean of acquisition times showed that rats pre-treated with U50,488 $(p<0.01)$ and Mesyl Sal B $(p<0.01)$ had significantly increased acquisition time compared to drug naïve rats, although no significant effect was observed in rats given Sal A $(1.0 \mathrm{mg} / \mathrm{kg}$ ) (one-way ANOVA followed by planned comparisons with the Bonferroni correction applied, Figure 6.3D).

The number of lever presses for the 16 training days prior to PR training is shown in Figure 6.4. Despite acquisition differences, there was no significant difference in active lever responses between drug naïve rats and rats pre-treated with $\operatorname{KOPr}$ agonists $[F(1,180)=$ $0.00, p=0.96$ ] (one-way ANOVA with repeated measures, Figure 6.4A). Further analysis according to their respective pre-treatments revealed no significant difference in active lever responses between drug naïve rats and rats pre-treated with U50,488, Mesyl Sal B, and Sal $A[F(3,150)=1.22, p=0.35]$ (one-way ANOVA with repeated measures, Figure 6.4B). There was also no significant difference in inactive lever responses between drug 
naïve rats and KOPr pre-treated rats on any day $[F(3,150)=1.65, p=0.24]$ (one-way ANOVA with repeated measures, Figure 6.4B). Due to the lack of significant differences between groups of rats, data from all rats were pooled and analysed together for the rest of the study.

A)

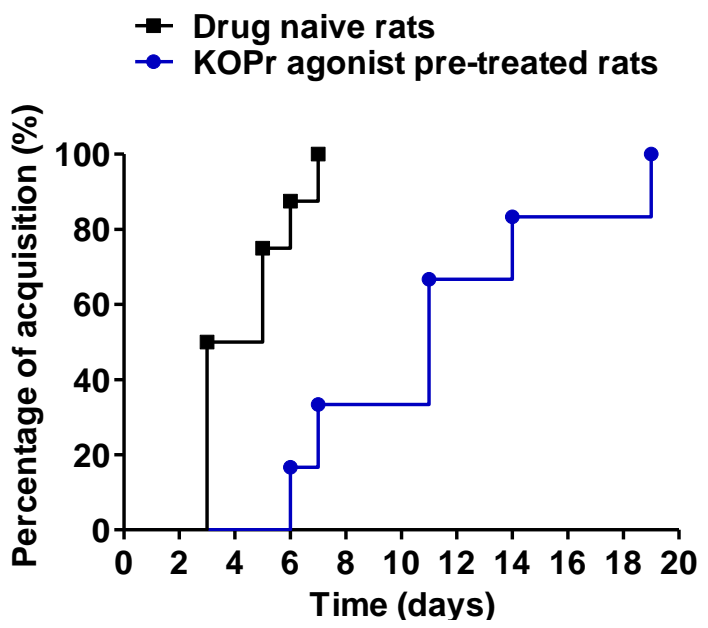

C)

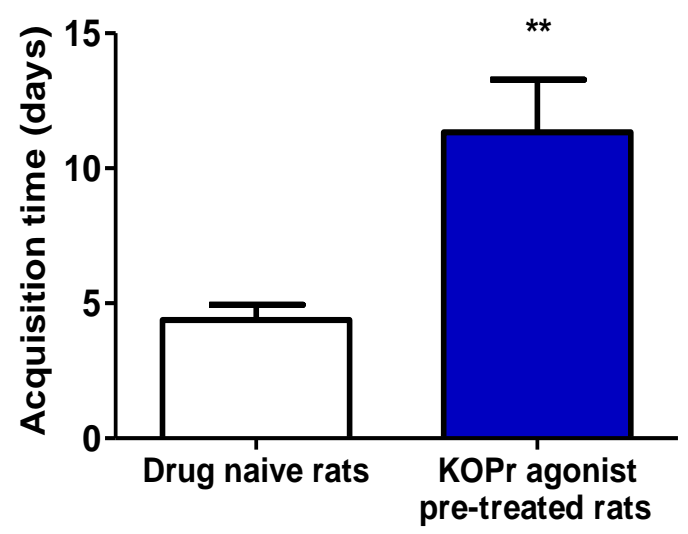

B)

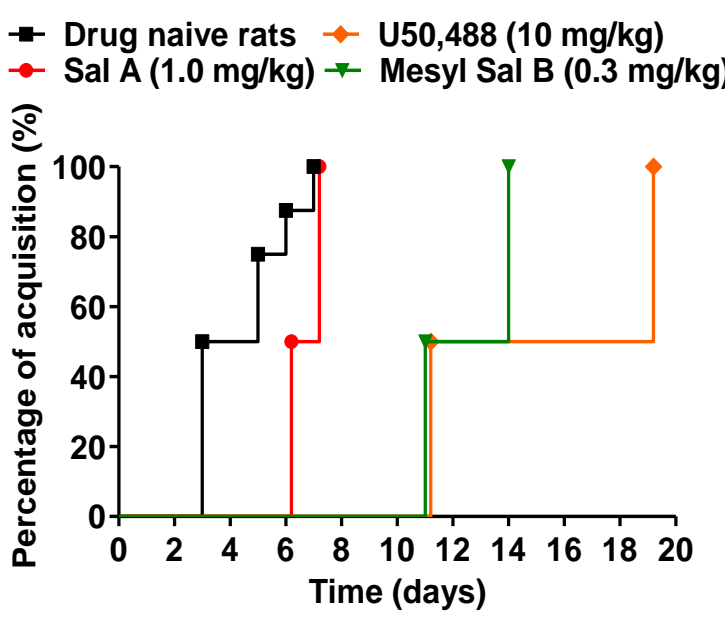

D)

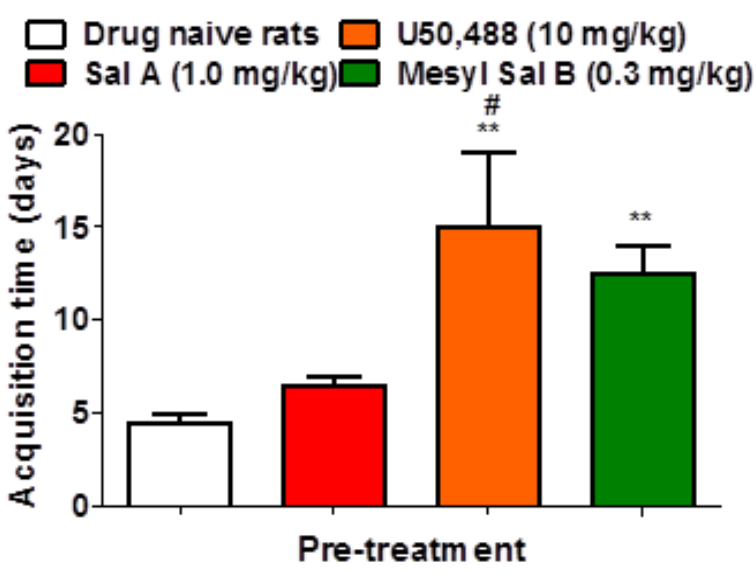

Figure 6.3: Mesyl Sal B and U50,488 pre-treatment increases cocaine self-administration acquisition time in rats

A) Drug naïve rats began acquiring self-administration behaviour on day 3 and achieved acquisition by day 7. Rats pre-treated with KOPr agonists a week before surgery began acquiring selfadministration behaviour on day 6 and achieved acquisition by day 20. B) Rats pre-treated with U50,488 and Mesyl Sal B showed larger delays in acquisition than Sal A. C) Rats pre-exposed to $\mathrm{KOPr}$ agonists showed a significant increase in acquisition time compared to drug naïve rats (Student's t-test). D) Rats pre-treated with U50,488 and Mesyl Sal B, but not Sal A, showed a significant increase in acquisition time compared to drug naïve rats (one-way ANOVA followed by planned comparisons). ${ }^{* *} \mathrm{p}<0.01$ compared to drug naïve rats, ${ }^{*} \mathrm{p}<0.05$ compared to rats pretreated with Sal $A$. Values presented as mean \pm SEM, $n=2-8$. 
A)

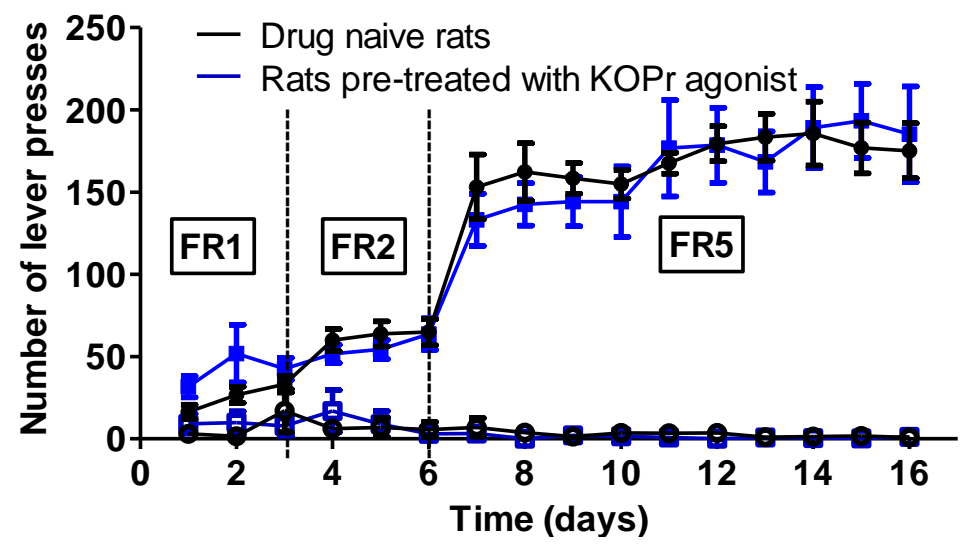

B)

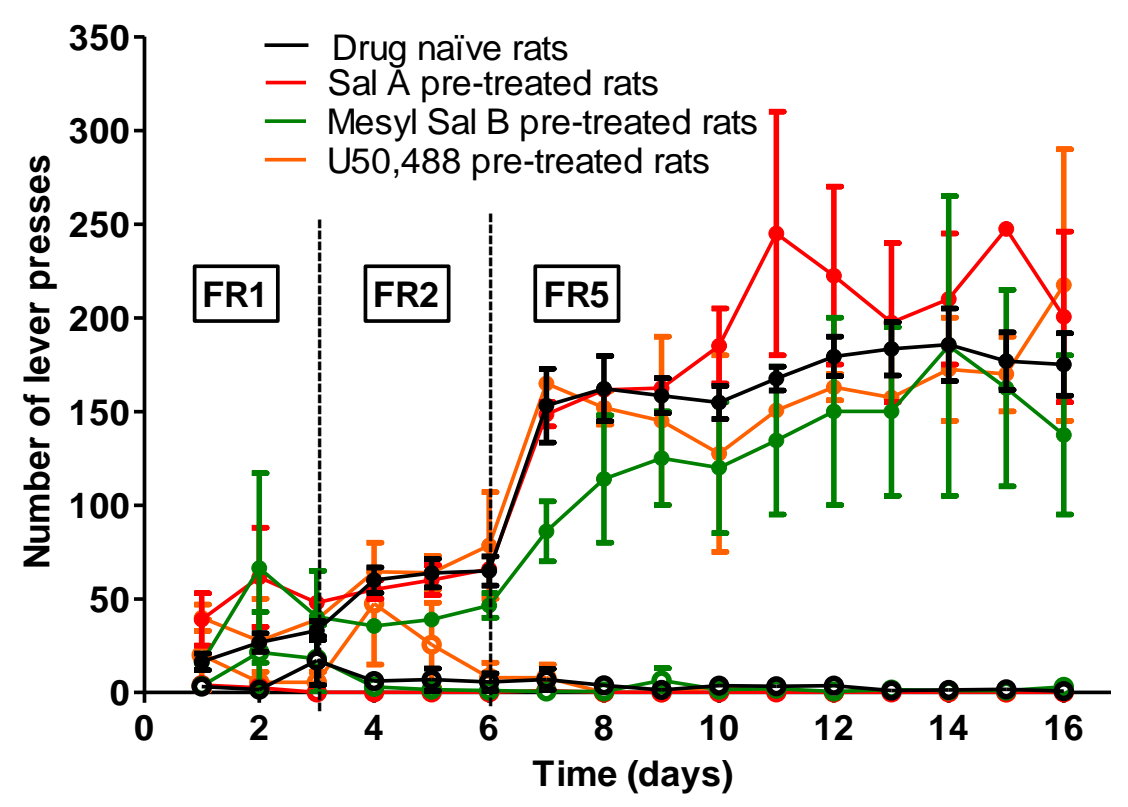

Figure 6.4: Pre-treatment with KOPr agonist does not alter the rate of active or inactive

\section{lever responding in rats}

A) Drug naïve rats and rats pre-treated with KOPr agonists (U50488, Sal A, and Mesyl Sal B) displayed a similar number of active (filled circles) and inactive lever responses (unfilled circles) throughout all training phases prior to commencement of the PR paradigm. B) There was no significant difference in active and inactive lever responding throughout all training phases in drug naïve rats or rats pre-treated with U50,488, Sal A, or Mesyl Sal B. One-way ANOVA with repeated measures. Values presented as mean \pm SEM, $n=2-8$. 


\subsubsection{Effect of novel and traditional KOPr agonists on cocaine self-administration using a progressive ratio schedule of reinforcement}

KOPr agonists that have previously shown anti-cocaine effects were tested for their effects on motivation to self-administer cocaine in the PR model. Analysis of the data showed that a significant effect of treatment was found $[F(6,78)=4.878, p=0.0003]$ (repeated measures ANOVA, Figure 6.5). Further post-hoc testing revealed that rats subjected to $U 50,488$, Sal A, or 16 -brSal A showed no significant difference in number of received infusions compared to vehicle controls (DMSO:Tween-80:MilliQ water in a ratio of 2:1:7) (paired $t$-tests with the Bonferroni correction applied, Figure 6.5). U50,488treated rats $(10 \mathrm{mg} / \mathrm{kg})$ showed a non-significant $14 \%$ decrease in number of received infusions compared to rats treated with vehicle, from $12.6 \pm 0.8$ infusions to $10.8 \pm 0.9$ infusions (Figure 6.5). Rats treated with $0.3 \mathrm{mg} / \mathrm{kg}$ Sal A self-administered $12.4 \pm 0.8$ infusions, similar to vehicle-treated rats (Figure 6.5). The higher dose of Sal A (2.0 mg/kg) also showed similar responding to the controls, with $12.9 \pm 0.6$ infusions (Figure 6.5). Rats given $1.0 \mathrm{mg} / \mathrm{kg}$ of 16 -brSal A prior to a PR test session self-administered $13.1 \pm 0.4$ infusions, similar to a $2.0 \mathrm{mg} / \mathrm{kg}$ dose of 16-brSal A (13.1 \pm 0.7 infusions) (Figure 6.5). On the other hand, rats treated with $U 69,593(1.0 \mathrm{mg} / \mathrm{kg})$ displayed a significant decrease in cocaine infusions, with $10.1 \pm 0.7$ infusions $(p<0.05$, paired $t$-test with the Bonferroni correction applied) (Figure 6.5). In summary, only rats treated with U69,593 showed a decrease in cocaine intake utilising a PR self-administration paradigm. 

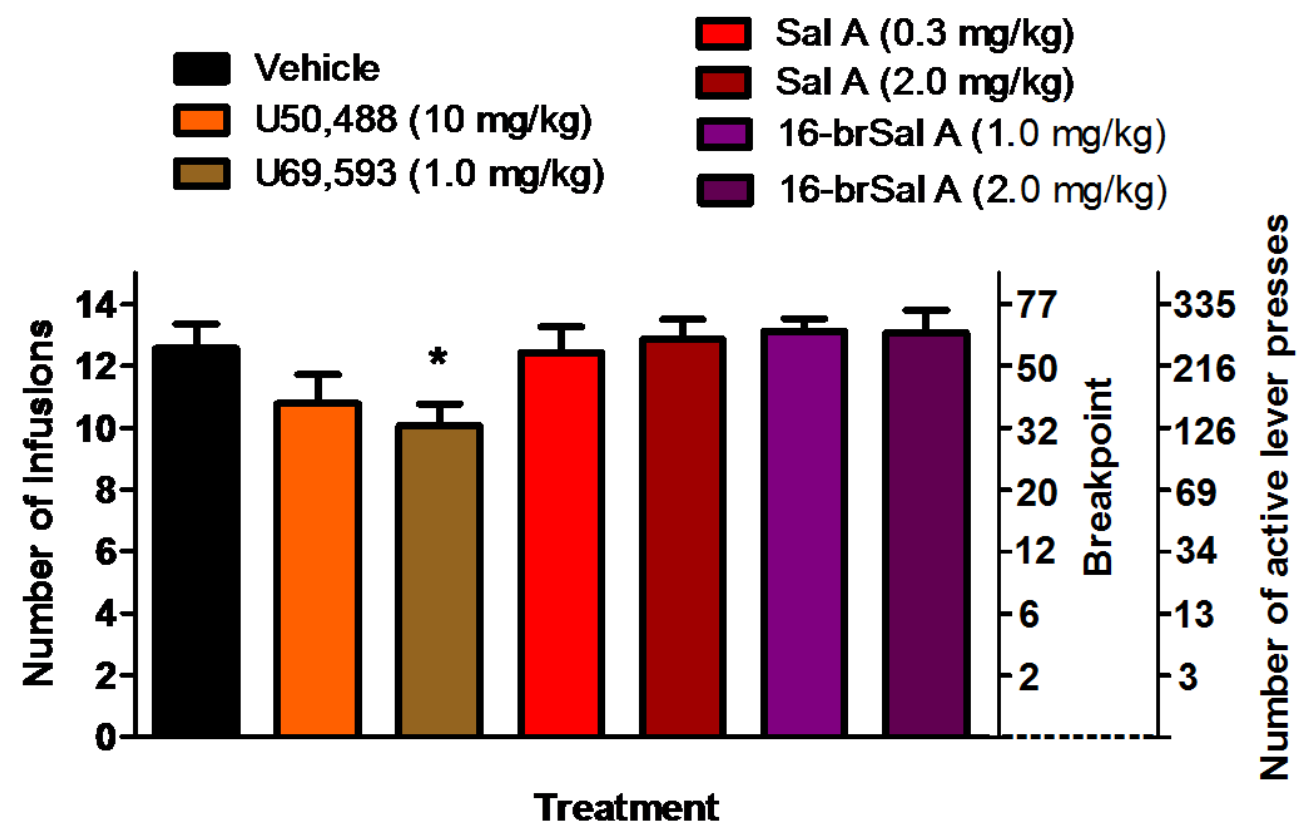

Figure 6.5: U69,593 decreases motivation to self-administer cocaine in rats using a PR

\section{schedule of reinforcement}

The number of infusions, breakpoint, and active lever responses made by all rats during PR testing sessions are presented. U69,593 significantly decreased the number of infusions compared to the vehicle controls. No significant changes were observed in rats administered U50,488, Sal A, or 16brSal A prior to a test session. Repeated measures ANOVA followed by paired $t$-tests with the Bonferroni correction, ${ }^{*} \mathrm{p}<0.05$ compared to vehicle. Values presented as mean $\pm \mathrm{SEM}, \mathrm{n}=14$. 


\subsection{Discussion}

Although many studies have investigated the effects of acute KOPr agonists on drug seeking and drug taking using ShA sessions, few studies have investigated the actions of Sal A and its analogues on cocaine self-administration using the LgA and PR paradigms. Therefore, this study was designed to investigate whether Sal A and 16-brSal A display anti-cocaine properties by reducing cocaine self-administration in rats given extended access to cocaine (LgA model) as well as the motivation to self-administer cocaine (PR schedule of reinforcement).

\subsubsection{Extended access cocaine self-administration}

Previous studies have shown that rats self-administering cocaine $(0.25 \mathrm{mg} /$ infusion $)$ displayed an escalation in responding for cocaine in the first hour of self-administration when progressing from ShA (1 h) to LgA (6 h) training sessions, whereas rats trained only on the ShA and LgA sessions did not (Knackstedt and Kalivas, 2007). Despite using a similar training paradigm to Knackstedt and Kalivas (2007), the present study showed a nonsignificant trend towards escalation in the first hour $(p=0.06)$ and throughout the session $(p=0.08$, Figure $6.1 C)$. This lack of significance may be attributed to the number of subjects used in this study $(n=7)$. However, it is important to note that significant escalation has been observed in as few as six rats (Ahmed and Koob, 1998). Escalation behaviour has also previously been shown five to eight days after commencement of LgA sessions (Ahmed and Koob, 1998; Ben-Shahar et al., 2004), indicating that the number of training sessions in the present study should have been sufficient to produce significant escalation. The lack of significant escalation observed in this study may also be due to the strain of rats used. Male Sprague-Dawley rats show minimal escalation when lower doses of cocaine $(0.5 \mathrm{mg} / \mathrm{kg})$ are used, but more pronounced escalation when higher doses of cocaine $(2.0 \mathrm{mg} / \mathrm{kg}$ ) are used (Mantsch et al., 2004). In support of this, a study by Kippin et al. (2006) showed that Wistar, but not Sprague-Dawley rats, displayed significant escalation when given extended access to cocaine $(0.4 \mathrm{mg} / \mathrm{kg})$. Calculation of cocaine intake in both rat strains revealed that in the first hour, Wistar rats that had undergone escalation self-administered the same amount of cocaine $(14 \mathrm{mg} / \mathrm{kg})$ as Sprague-Dawley rats on a ShA session (Kippin et al., 2006). This indicates that Sprague-Dawley rats have a 
higher basal level of cocaine intake than Wistar rats, and require higher doses of cocaine to show the same magnitude of escalation as Wistar rats (Kippin et al., 2006). In addition, rhesus monkeys given extended access $(4 \mathrm{~h})$ to cocaine $(0.1 \mathrm{mg} / \mathrm{kg})$ showed an increase in cocaine intake compared to monkeys given ShA ( $1 \mathrm{~h})$ to cocaine, but no escalation behaviour (Henry et al., 2009). Taken together, these studies suggest that the presence of escalation may depend on species, and only displays a trend towards significance in this study due to a combination of the use of male Sprague-Dawley rats and choice of cocaine dose $(0.5 \mathrm{mg} / \mathrm{kg})$.

In view of the fact that the threshold dose of Sal A $(0.3 \mathrm{mg} / \mathrm{kg})$ produces variable effects on anti-cocaine behaviour - i.e., $0.3 \mathrm{mg} / \mathrm{kg}$ of Sal A did not attenuate cocaine-induced hyperactivity (Morani et al., 2009) - a higher dose of Sal A $(2.0 \mathrm{mg} / \mathrm{kg}$ ) was also tested in the LgA paradigm. Although previous studies have shown that repeated administration of U69,593 produced anti-cocaine effects by attenuating cocaine-induced locomotion and sensitisation (Heidbreder et al., 1995; Shippenberg et al., 1996; Heidbreder et al., 1998; Collins et al., 2001b), it was not completely surprising to find that Sal A produced no significant changes in responses for cocaine. This is because repeated Sal A treatments $(2 \mathrm{mg} / \mathrm{kg})$ did not alter cocaine-induced locomotion when Sal A was administered in home cages (Chartoff et al., 2008). Repeated administration of a higher Sal A dose $(3.2 \mathrm{mg} / \mathrm{kg}$ ) also showed different effects to U69,593, with no changes in cocaine-induced hyperactivity observed in the rat (Gehrke et al., 2008). The lack of anti-cocaine effects observed may be due to a combination of factors: the rapid pharmacokinetics of Sal A, cocaine dose used, and LgA sessions.

It has been established that Sal A has a rapid onset and duration of action, with a recent study using positron emission tomography to show that Sal A $(0.6 \mathrm{mg} / \mathrm{kg}$, i.v. $)$ has a rapid uptake (maximum activity seen at approximately $13 \mathrm{~s}$ ) and clearance from the rat brain (half-life of $3.3 \mathrm{~min}$ ) (Placzek et al., 2015). When given i.p., Sal A consistently produced maximum effect between 20 and 40 min in rodents (Hooker et al., 2009b), indicating that the lack of active lever response attenuation may be due to the short half-life of Sal A. However, as analysis of only first hour responding also yielded no significant difference between Sal $A$ and vehicle treatments, the absence of a decrease in self-administration 
cannot be due to the short pharmacodynamics of Sal A alone. It is therefore suggested that the cocaine dose used in this study $(0.5 \mathrm{mg} / \mathrm{kg})$ may account for the lack of a KOPrmediated decrease in cocaine self-administration (Kuzmin et al., 1997; Schenk et al., 1999). It has been shown that U50,488 (1.2, $2.5 \mathrm{mg} / \mathrm{kg}$, i.p.) decreases self-administration of a 30 and $60 \mu \mathrm{g} /$ infusion of cocaine (Kuzmin et al., 1997), whereas U69,593 (0.32 mg/kg, s.c.) only reduces self-administration when cocaine doses are at least four-fold lower $(0.03-0.125 \mathrm{mg} / \mathrm{kg})$ than the one used in the present study $(0.5 \mathrm{mg} / \mathrm{kg})$ (Schenk et al., 1999). However, a later study by Schenk et al. (2001) showed that U69,593 $(0.32 \mathrm{mg} / \mathrm{kg}$, s.c.) attenuated self-administration of $0.5 \mathrm{mg} / \mathrm{kg}$ cocaine for up to $10 \mathrm{~h}$ (Schenk et al., 2001), indicating that the absence of attenuation seen in this study cannot be attributed to the dose of cocaine alone. Thus, it is suggested that use of the LgA paradigm may also be a contributing factor, as rats in the Schenk et al. (2001) study were only trained in $2 \mathrm{~h}$ sessions (Schenk et al., 2001), whereas rats in this study were trained in $6 \mathrm{~h}$ sessions for 21 days. Although LgA rats in the present study did not show significant escalation, it cannot be denied that LgA rats show elevated cocaine intake compared to ShA subjects due to the longer duration of cocaine access. In the present study, LgA rats had an average cocaine intake of $115 \mathrm{mg}$ throughout a $6 \mathrm{~h}$ session compared to ShA rats in Chapter 3, which only had an average cocaine intake of $31 \mathrm{mg}$ throughout a $2 \mathrm{~h}$ session. Since administration of non-contingent cocaine to cocaine self-administering rats maintains responses for the active lever (Norman and Tsibulsky, 2006), it is suggested that the continual presence and increased cocaine availability throughout the session sustains self-administration behaviour. It is also important to note that although administration of a KOPr agonist produced anti-cocaine effects by attenuating cocaineinduced changes in DA levels (Thompson et al., 2000), increased intake of cocaine produces increased elevation of DA levels. In support of this, LgA rats had a greater increase in elevation of DA levels in the NAc compared to ShA rats (DA levels in LgA rats $=750 \%$ of baseline vs. DA levels in ShA rats $=400 \%$ of baseline) (Ahmed et al., 2003) Therefore, it stands to reason that higher doses of KOPr agonists would be required to attenuate the elevated cocaine-induced increase of DA levels. Taken together, these studies indicate that the higher levels of circulating cocaine in $\operatorname{Lg} A$ rats maintains selfadministration behaviour, accounting for the lack of Sal A-mediated anti-cocaine effects at the doses used. 


\subsubsection{Progressive ratio schedule of reinforcement}

This study is the first to investigate the effect of a single, delayed ( 2 week) injection of KOPr agonist on acquisition of cocaine self-administration. A previous study using repeated U69,593 (0.32 mg/ $\mathrm{kg}$, s.c.) found that this compound produced no change in the acquisition latency for cocaine (approximately 7 days for both vehicle- and U69,593treated rats) (Schenk et al., 2001). However, three differences exist between this study and Schenk et al. (2001), the first being that Schenk et al. (2001) utilised repeated treatments of U69,593 for 5 consecutive days, whereas the present study used a single administration of KOPr agonists. While Schenk et al. (2001) examined the effects of U69,593 3 days prior to self-administration training, the KOPr agonists in this study were administered two weeks before commencement of self-administration. The present study also used a combination of KOPr agonists (none of which was U69,593), with differences noted between U50,488, Sal A, and Mesyl Sal B (Figure 6.3B and 6.3D). To further determine whether the increase in acquisition latency is comparable to the other self-administration studies conducted in the present project, further analysis was performed to compare the acquisition latency of rats trained for the reinstatement study in Chapter 3, rats trained for LgA self-administration testing, as well as rats used in the PR paradigm (Appendix 6.2). No significant difference in acquisition time was noted between all drug naïve rats, whereas pre-treatment of rats with KOPr agonists significantly increased acquisition latency (Figure 8.9 in Appendix 6.2), indicating that pre-treatment of rats with KOPr agonists increases the latency of rats to self-administer cocaine.

The increased acquisition latency observed may be due to an increase in basal hedonic states due to delayed effects of KOPr agonists. A recent report by Chartoff et al. (2015) showed that Sal A $(2.0 \mathrm{mg} / \mathrm{kg}$, i.p.) administered $24 \mathrm{~h}$ prior to testing produced a decrease in basal ICSS thresholds, an indication of rewarding properties. Sal A also attenuated cocaine-induced alterations of ICSS thresholds and cocaine-induced increases in DA levels in the NAc core, suggesting that Sal A decreases the sensitivity to cocaine reward (Chartoff et al., 2015). The authors therefore attributed the increase in hedonic state produced by delayed Sal A (indicated by the decrease in basal ICSS thresholds) to neuroplasticity of dopaminergic circuits and upregulation of DA synthesis (Chartoff et al., 2015). This elevated basal hedonic level would explain reluctance of rats to acquire self- 
administration behaviour in the present study. However, Sal A produced no alteration in acquisition latency in the present study, possibly due to its rapid duration of action. In support of this, effects of delayed Sal A were not observed by $48 \mathrm{~h}$ post-injection (Chartoff et al., 2015). Therefore, it can be argued that the increased acquisition latency seen with Mesyl Sal B and U50,488 may be due to their increased duration of action. Taken together, these studies support the use of KOPr agonists as preventative agents by decreasing the sensitivity to the rewarding effects of cocaine and increasing the latency to self-administer cocaine.

This work also demonstrates for the first time effects of novel KOPr agonists on cocaine self-administration using a PR paradigm. The progressive ratio sequence in this study $\left(5 \mathrm{e}^{(0.2 \times \text { Infusion\# })}-5\right.$; rounded to the nearest integer) was taken from Richardson and Roberts (1996). The ratio requirements generated were deemed suitable for the reinforcing effects of cocaine and ensured that PR sessions would end within 5 hours (Richardson and Roberts, 1996). It is important to note that data generated for the PR paradigm were analysed based on the number of infusions delivered, due to a lack of variance homogeneity in breakpoints. Since the number of lever requirements for each subsequent infusion did not increase linearly, use of a parametric ANOVA analysis was not possible (Richardson and Roberts, 1996). As each ratio requirement is considered a 'step', graphing the ordinal value of the final ratio (equivalent to the number of infusions) was more accurate for statistical analysis. Although ratio requirements in a PR experiment can be increased in a within- or between-session manner (the within-session model practices an increase in required responses during the same session or day, whereas the between-session model escalates ratio requirements at the beginning of each session) the within-session approach was used in this study because it allowed a more accurate evaluation of KOPr agonists as possible therapeutic agents (Richardson and Roberts, 1996).

None of the KOPr agonists studied, except U69,593, produced a decrease in PR breakpoint (Figure 6.5). A possible reason for this is the ability of cocaine to sustain selfadministration behaviour, as discussed previously (section 6.5.1). It is important to note that the breakpoint in within-session PR paradigms is established in the presence of high 
systemic cocaine levels, due to the low response requirements at the beginning of each session (Richardson and Roberts, 1996). In support of this, within-session PR paradigms produced larger breakpoints compared to between-session paradigms because of increasing blood cocaine levels (Gancarz et al., 2012). It is also important to note that the same argument used in section 6.5.1, in which a higher dose of KOPr agonist would be required to attenuate elevated cocaine-induced changes in DA levels, is also valid for the PR self-administration model. In addition, it was found that only high doses ( $1-3.2$ $\mathrm{mg} / \mathrm{kg}$, i.p.) but not the threshold dose $(0.25 \mathrm{mg} / \mathrm{kg}$, i.p.) of Sal A reduced DA levels in the NAc (Ebner et al., 2010) and dStr (Gehrke et al., 2008). Since rats undergoing PR sessions in the present study (Chapter 6 ) received three times the amount of cocaine compared to rats undergoing the cocaine-prime induced reinstatement test in Chapter 3 (PR $=21$ $\mathrm{mg}$ of cocaine by the $10^{\text {th }}$ infusion vs. reinstatement $=8 \mathrm{mg}$ of cocaine during the cocaine prime), it is suggested that doses of $U 50,488$, Sal A, and 16-brSal A higher than the minimum dose that decreased drug seeking would be required to decrease the motivation to self-administer cocaine. In support of this, U69,593 was only effective at $1.0 \mathrm{mg} / \mathrm{kg}$ (Figure 6.5), three times the dose required to attenuate drug seeking $(0.32$ mg/kg) (Schenk et al., 1999; Schenk et al., 2000; Schenk et al., 2001; Morani et al., 2009). It was therefore not surprising that U50,488 did not produce a decrease in PR breakpoint at $10 \mathrm{mg} / \mathrm{kg}$, since this dose is three-fold lower than the dose required to attenuate drug seeking (30 mg/kg) (Morani et al., 2009). However, as the single rat given $30 \mathrm{mg} / \mathrm{kg}$ of U50,488 in our study showed a complete absence of responsiveness due to severe sedation, further investigation of U50,488 was conducted using $10 \mathrm{mg} / \mathrm{kg}$ to prevent the non-specific effects of U50,488.

Further investigation with higher doses of Sal A and 16-brSal A $(2.0 \mathrm{mg} / \mathrm{kg})$ were also unsuccessful at lowering the breakpoint for cocaine. This lack of effect seen with Sal A may be due to its short half-life - also discussed in section 6.5.1 - and subsequently, a mismatch between peak effects of Sal A and cocaine (Richardson and Roberts, 1996). However, Ebner et al. (2010) showed that Sal A (2 mg/kg) produces a decrease in breakpoint for sucrose, suggesting that Sal A can be effective when used in a PR model (Ebner et al., 2010). Nonetheless, work previously conducted with sucrose selfadministration in our laboratory has shown that that KOPr agonists may exert differential 
effects on cocaine and sucrose, i.e. U69,593 $(0.32 \mathrm{mg} / \mathrm{kg}$ ) reduced cocaine (Schenk et al., 1999) but not sucrose self-administration (Chapter 4). Thus, the discrepancy with Sal A between our study and Ebner et al. (2010) may be due to the role of Sal A in mediating feeding and satiety (refer to Chapter 4 for more details). On the other hand, further efforts to investigate higher doses of 16 -brSal A were thwarted due to its inability to dissolve in the vehicle used (DMSO:Tween-80:MilliQ water in a ratio of 2:1:7). It is also important to note that another Sal A analogue, Ethy Sal A, successfully decreased the breakpoint for cocaine, but only at a 20-fold higher concentration than the minimal dose that attenuates drug seeking $(2.0 \mathrm{mg} / \mathrm{kg}$ in PR studies vs. $0.1 \mathrm{mg} / \mathrm{kg}$ for attenuation of drug seeking in Riley et al. (2014)) (Kivell Lab, unpublished study). Taken together, these studies indicate that novel KOPr agonists may be capable of reducing the motivation to self-administer cocaine, albeit only at doses higher than that required to attenuate cocaine-induced reinstatement of drug seeking.

\subsubsection{Limitations and future directions}

The continuous availability of cocaine throughout testing sessions served as the main limitation of both LgA and PR paradigms. Although experimenter observation and previous studies have noted that the likelihood of drug related behaviours such as hyperactivity or lever-related stereotypy from drug intake are low (Fowler et al., 2007), the rate of responding is likely to be influenced (to a certain point) by the presence of cocaine in the bloodstream (Norman and Tsibulsky, 2006). It is also important to note that although the within-session PR model can be used to determine if a pharmacological treatment disrupts cocaine self-administration, it is unable to determine the effect of the treatment on motivation to respond for the first drug infusion/relapse (Roberts and Andrews, 1997). To that end, other schedules such as the second order selfadministration paradigm (described in section 6.1) can be used to further evaluate the anti-cocaine effects of novel KOPr agonists in the absence, or at least, at low concentrations of cocaine.

Results obtained in the present study suggests that anti-cocaine effects of KOPr agonists in LgA and PR paradigms would be manifested at higher doses. Since current Sal A 
analogues have solubility issues at higher concentrations, possible use of a different vehicle such as 10\% Kolliphor ${ }^{\circledR}$ HS 15 (42966, Sigma Aldrich) may permit higher doses to be used. Synthesis of water soluble analogues are also highly desirable (to enable oral administration) and are currently in progress (Professor Thomas Prisinzano, personal communication). However, it is important to remember that higher doses of Sal A have been associated with adverse effects such as sedation (Fantegrossi et al., 2005), modulation of natural reward (Ebner et al., 2010), and depression (Carlezon et al., 2006). Thus, it stands to reason that higher doses of Sal A analogues will also present side effects such as sedation that may non-specifically decrease responding for cocaine.

Further studies such as usage of longer acting Sal A analogues in the LgA study should be conducted to determine the anti-cocaine potential of these compounds. Although this experiment was planned in the present study, it could not be completed due catheter patency failure in 4 out of 7 rats. Other studies using simultaneous administration of novel KOPr agonists and cocaine should also be studied as an alternative to the current pre-treatment method. As mentioned in section 6.1, mixtures of Sal A and reinforcers such as oxycodone or cocaine decrease self-administration and breakpoints under a PR schedule in rhesus monkeys (Freeman et al., 2014; Naylor et al., 2015). Since the i.v. route of administration requires lower doses of KOPr agonists than i.p. injections, it stands to reason that concurrent infusion of KOPr agonists and cocaine may be more effective at reducing self-administration in the LgA or PR paradigm. Alternatively, rats could be implanted with a programmable microinfusion pump to allow for temporally-controlled infusions of KOPr agonists, to ensure that the peak effects of the agonist coincides with cocaine infusions.

The potential for an acute administration of KOPr agonist to prevent acquisition of cocaine self-administration also necessitates further investigation, i.e. by increasing subject numbers and injecting different groups of rats with different KOPr agonists. These additional studies will help determine whether traditional and longer acting Sal A-derived agonists have similar inhibitory effects on cocaine self-administration. To further evaluate this, studies investigating the effect of KOPr agonists on learning and memory also need to be conducted. These studies would reveal whether the delayed acquisition observed 
in rats pre-treated with KOPr agonists is due to a specific anti-reward protective effect, or a possible learning deficit. Previous studies have shown that low doses of Sal A (80$640 \mu \mathrm{g} / \mathrm{kg}$, s.c.) impairs spatial long-term memory evaluated using a food pelletmotivated, eight arm radial maze test (Braida et al., 2011), whereas high doses of Sal A (2.0 and $4.0 \mathrm{mg} / \mathrm{kg}$, i.p.) disrupts the 5 -choice serial reaction time task, a food-motivated test used to measure attention (Nemeth et al., 2010).

\subsection{Conclusions}

Sal A (0.3 and $2.0 \mathrm{mg} / \mathrm{kg})$ did not decrease cocaine self-administration in rats given LgA (6 h) to cocaine. Sal A and 16-brSal A (1.0 and $2.0 \mathrm{mg} / \mathrm{kg}$ ) also did not decrease the breakpoint for cocaine in rats tested using the PR paradigm. However, U69,593 decreased the breakpoint for cocaine in the PR schedule of reinforcement, an effect that may be attributed to the high dose administered $(1.0 \mathrm{mg} / \mathrm{kg})$. Further investigation of higher doses of KOPr agonists or simultaneous infusion of a KOPr agonist and cocaine is crucial to elucidate the potential pharmacotherapeutic potential of KOPr agonists. 


\section{Chapter 7: Final Discussion}

\subsection{Significance of study}

Psychostimulant addiction is a major social and economic issue worldwide, with no current FDA approved pharmacotherapy to treat this disorder. Individuals suffering from substance use disorders have a high risk of relapse even after long abstinence periods (Nestler, 2001). Therefore, the development of therapeutic compounds to treat addiction is required. The aim of this PhD project was to identify novel KOPr agonists derived from Sal A as potential anti-cocaine therapies, with the hypothesis that analogues of Sal A will possess anti-cocaine effects with fewer side effects compared to traditional KOPr compounds. In order to evaluate the mechanisms underlying the behavioural effects, the cellular actions and cellular signalling pathways activated by the lead KOPr agonist, 16brSal A, were also studied. In theory, functionally selective KOPr agonists may activate specific cell signalling pathways, yielding the desired therapeutic actions without side effects. Since behavioural and cellular studies show that KOPr agonists derived from the structurally novel KOPr agonist, Sal A, can produce anti-cocaine effects with fewer typical KOPr-mediated adverse effects, these models suggest that Sal A-derived compounds have the potential to yield pharmacotherapies which are functionally selective at the KOPr. It is important to note that despite the many studies reporting on the chemistry and pharmacology (Rives et al., 2012; Zhou et al., 2013; Lovell et al., 2015; Zhou et al., 2015) as well as analgesic effects (Zhou et al., 2013; White et al., 2015) of functionally selective KOPr ligands, none have examined the anti-cocaine properties of these compounds. Therefore, the present study is an essential addition that will aid in the effort of developing functionally selective KOPr agonists as anti-cocaine therapies.

\subsection{Key findings}

The key findings of this study are summarised in Tables 7.1 and 7.2. 
Table 7.1: Summary of behavioural anti-cocaine and side effects of Sal A derivatives

\begin{tabular}{|c|c|c|c|c|c|}
\hline Test & Sal A & 16-brSal A & EOM Sal B & MOM Sal B & $\beta$-THP Sal B \\
\hline $\begin{array}{l}\text { Reinstatement of } \\
\text { drug seeking }\end{array}$ & Attenuated & Attenuated & Attenuated & - & - \\
\hline $\begin{array}{l}\text { Cocaine-induced } \\
\text { hyperactivity }\end{array}$ & - & Attenuated & - & No change & - \\
\hline Locomotor activity & - & No change & - & No change & - \\
\hline $\begin{array}{l}\text { Forced swim test } \\
\text { (FST) }\end{array}$ & - & No change & $\begin{array}{c}\text { Decreased } \\
\text { swimming, } \\
\text { increased } \\
\text { climbing }\end{array}$ & $\begin{array}{l}\text { Increased } \\
\text { immobility }\end{array}$ & No change \\
\hline $\begin{array}{l}\text { Sucrose self- } \\
\text { administration }\end{array}$ & No change & No change & Increased & - & Increased \\
\hline Light dark (LD) & $\begin{array}{l}\text { Decreased } \\
\text { light box } \\
\text { time }\end{array}$ & No change & - & - & - \\
\hline $\begin{array}{l}\text { Elevated plus maze } \\
\text { (EPM) }\end{array}$ & $\begin{array}{c}\text { Decreased } \\
\text { open arm } \\
\text { time }\end{array}$ & No change & - & - & - \\
\hline $\begin{array}{l}\text { Conditioned place } \\
\text { aversion (CPA) }\end{array}$ & $\begin{array}{l}\text { Produced } \\
\text { aversion }\end{array}$ & $\begin{array}{l}\text { Produced } \\
\text { aversion }\end{array}$ & - & - & - \\
\hline $\begin{array}{l}\text { Extended access } \\
\text { (LgA) self- } \\
\text { administration }\end{array}$ & No change & - & - & - & - \\
\hline $\begin{array}{l}\text { Progressive ratio } \\
\text { (PR) cocaine self- } \\
\text { administration }\end{array}$ & No change & No change & - & - & - \\
\hline
\end{tabular}

Table 7.2: Summary of cellular effects produced by 16-brSal A

\begin{tabular}{|l|l|}
\hline \multicolumn{1}{|c|}{ Test } & \multicolumn{1}{c|}{ 16-brSal A } \\
\hline ASP $^{+}$uptake & Increased uptake \\
\hline ASP $^{+}$kinetics Vmax and $\mathrm{Km}$ & Increased Vmax and Km \\
\hline DA uptake in tissue & Increased uptake in dStr and NAc \\
\hline Vmax and Km of DA uptake in tissue & Increased Vmax, no change in Km \\
\hline DAT cell surface expression & No change \\
\hline ERK1/2 phosphorylation in HEK-293 cells & Increased early phase ERK1/2 activation \\
\hline ERK1/2 phosphorylation in tissue & $\begin{array}{l}\text { Increased early phase ERK1/2 activation } \\
\text { in dStr, NAc, and PFC }\end{array}$ \\
\hline p38 MAPK phosphorylation in tissue & Increased p38 MAPK activation in NAc \\
\hline
\end{tabular}




\subsubsection{6-brSal A has a longer duration of action in vivo and similar anti-cocaine profile}

as Sal A

Since the advancement of Sal A into clinical settings has been perturbed by its short halflife, the discovery that 16-brSal A possesses a longer duration of anti-nociceptive action posits it as an agonist that may be more therapeutically viable than its parent compound. However, it was surprising to note that although 16-brSal A attenuated both cocaineinduced drug seeking and hyperactivity, the dose of 16-brSal A required to produce a similar magnitude of anti-cocaine behaviour was three-fold higher than Sal A $(1.0 \mathrm{mg} / \mathrm{kg}$ for 16-brSal A vs. $0.3 \mathrm{mg} / \mathrm{kg}$ for Sal A) despite its similar efficacy to Sal A at the KOPr (Table 1.3). A recent study conducted in non-human primates using 16-brSal $A$ found that it was also 3 times less potent than Sal $A$ at inducing release of prolactin, a translational neuroendocrine biomarker assay of KOPr agonist effects (Butelman et al., 2015). These studies suggest that the decrease in in vivo effectiveness of 16-brSal $A$ is similar irrespective of species, suggesting that a three-fold higher dose (compared to the effective dose of Sal A) will be required when evaluating behavioural effects of 16-brSal A in other animals or humans. On the other hand, discovery that the anti-cocaine effects of MOM Sal B (Morani et al., 2013), did not extend to hyperactivity studies suggests that differences in activation of the individual pathways involved in drug seeking and locomotion are involved (discussed in section 3.5.3). It is also important to note that although MOM-Sal B, U50,488H, and Sal A showed similar binding affinities to the human $\mathrm{KOPr}$ and similar G-protein activating potencies, MOM-Sal $\mathrm{B}\left(\mathrm{EC}_{50}=7.4 \mathrm{nM}\right)$ was approximately 13- and 70-fold more potent than $\mathrm{U} 50,488 \mathrm{H}\left(\mathrm{EC}_{50}=97.1 \mathrm{nM}\right)$ and Sal $\mathrm{A}$ $\left(E_{50}=522 \mathrm{nM}\right)$ respectively at internalising the human KOPr (Wang et al., 2008). This indicates that differences in MOM Sal B- and traditional KOPr-activated signalling pathways may also account for behavioural inconsistencies.

\subsubsection{6-brSal A has fewer side effects compared to traditional KOPr agonists, Sal A, or} other Sal A analogues

This portion of the study aimed to determine the adverse effect profile of Sal A analogues that showed attenuation of drug seeking behaviour. Side effect screening of KOPr agonists allowed elimination of less therapeutically desirable compounds from further studies, 
allowing for conservation of resources such as animal subjects and chemicals (required for synthesis of analogues from Sal A). It is important to note that the sedation results for 16-brSal A in this study are mirrored in primates. Butelman et al. (2015) showed that 16brSal A (0.0032 - $0.1 \mathrm{mg} / \mathrm{kg}$, i.v.) has no sedative effects in primates despite using a threefold higher dose (Butelman et al., 2015). In addition, screening of depression and modulation of natural reward indicated EOM Sal B, MOM Sal B, and $\beta$-THP Sal B were less desirable therapeutic compounds and highlighted 16-brSal A as the Sal A-derived KOPr agonist with the best therapeutic potential. Interestingly, although previous studies suggest that aversive properties are mediated by the $\beta$-arrestin-dependent p38 MAPK pathway (Bruchas et al., 2007a), a more recent study has shown that RB-64, a G-protein biased KOPr agonist, produced aversion in mice (White et al., 2015). This suggests that aversion may be regulated via the G-protein pathway, and not the $\beta$-arrestin pathway. Consequently, the promise of 16 -brSal $A$ as a possible therapeutic agent should not be disregarded despite the presence of 16-brSal A-mediated CPA. It is also important to remember that although most clinically available therapeutic interventions can be unpleasant and aversive, the presence of aversion is not entirely prohibitive due to the human ability to make a well informed decision between an unpleasant treatment and chronic illness. Therefore, it stands to reason that despite its aversive properties, 16-brSal A may possibly be a functionally selective ligand at the KOPr, although further studies are required to determine its actual intracellular signalling transduction pathway.

\subsubsection{6-brSal A modulates DAT function in a different manner than traditional KOPr agonists}

16-brSal A-modulated upregulation of DAT function in in vitro (HEK-293 cells coexpressing KOPr and DAT) and ex vivo (minced rat brain tissue) models decreases synaptic DA levels. This suggests that 16-brSal A exerts its anti-cocaine properties by antagonising the actions of cocaine in dopaminergic systems. Although it has been previously mentioned that inherent differences exist between cell models and native systems, it is important to note that use of cellular models allow for greater manipulation compared to animal models. In addition, the present study found that the in vitro and ex vivo models produced similar results - the only exception being the increase in $\mathrm{Km}$ values seen in the 
kinetics of $\mathrm{ASP}^{+}$uptake but not the RDEV study. Thus, although cellular models are unable to completely replace animal subjects, they are a suitable alternative that can aid in reduction of animal numbers. Differences in DAT regulation by 16 -brSal A compared to traditional KOPr agonists were also seen, and these may contribute to the reduced side effects observed. The three main differences in KOPr-mediated mechanisms observed with 16-brSal A were: 1) lack of elevated cell surface DAT expression (compared to U69,593 which showed an increase in DAT expression (Simonson et al., 2015)), 2) lack of late phase ERK1/2 phosphorylation (compared to U69,593 which showed both early and late phase ERK1/2 activation (McLennan et al., 2008)), and 3) minimal p38 MAPK activation (vs. robust p38 MAPK activation by U50,488 (Bruchas et al., 2007a)). As discussed previously, these cellular studies are consistent with the superior side effect profile of 16-brSal A, and strengthen the suggestion that 16-brSal A may be a biased agonist at the KOPr. However, it is important to note that the functionally selective properties of 16 -brSal A can only be established using direct $\beta$-arrestin recruitment methods, which were not undertaken in the present study.

\subsubsection{Sal A and 16-brSal A do not decrease LgA self-administration or motivation to self- administer cocaine}

The main goal of preclinical laboratory studies is to translate results from research into the clinical setting. Therefore, the present study used a range of self-administration models to screen for different anti-cocaine behaviours. In order to more closely represent human drug taking behaviour, the LgA study investigated the effect of Sal A on subjects with extended access to cocaine. On the other hand, the PR study investigated the effect of Sal A and 16-brSal A on the motivation to self-administer cocaine. Although Sal A and 16-brSal A had no significant changes in either paradigms, there is preliminary evidence that higher doses or concurrent infusions of these agonists alongside cocaine may yield anti-cocaine effects.

\subsection{Limitations and future directions}

The largest limitation in developing KOPr agonists as clinical therapies is the negative behavioural impact of KOPr activation on stress and mood. There has been a long-standing 
divide in the scientific community with regards to development of KOPr agonists as treatments for drug addiction. On one hand, studies such as the present one show that acute activation of the KOPr produces anti-reward effects. On the other hand, it cannot be denied that activation of the KOPr also produces negative behavioural effects similar to withdrawal-like effects that can promote drug seeking or taking (refer to section 1.4.1). This suggests instead that use of KOPr antagonists are more suitable for combating drug use (reviewed in Carroll and Carlezon (2013)). An example of these contrasting phenomena is a study by Beardsley et al. (2005) showing that the KOPr antagonist JDTic attenuates foot shock stress-induced reinstatement but potentiates cocaine-induced reinstatement of cocaine seeking (Beardsley et al., 2005).

Therefore, it is suggested that both KOPr agonists and antagonists can act as antiaddiction pharmacotherapies, albeit at diferent stages of the addiction cycle. The present study has shown that KOPr agonists can produce anti-cocaine effects, effectively reducing the hedonic effects of cocaine and therefore reduce cocaine use. This advocates the therapeutic application of KOPr agonists at the early stages of drug abuse (refer to Shippenberg (2009); Wee and Koob (2010); Kivell et al. (2013); Picetti et al. (2013) for more details) in an effort to halt the progress of the addiction cycle. In support of this, a study in which co-administration of KOPr agonists with a drug of abuse (cocaine or remifentanil) reduced use of the drug in non-human primates (Freeman et al., 2014) represents a good indication of the potential use of KOPr agonists in clinical settings. On the other hand, KOPr antagonists are more suitably applied in a later stage of the addiction cycle (the withdrawal stage) as they have been known to counteract the negative KOPr-mediated effects of withdrawal such as depression, stress, aversion, and anxiety (Chavkin, 2011; Picetti et al., 2013). Preclinical studies have shown that KOPr antagonists successfully reduced drug seeking behaviour by alleviating withdrawal symptoms such as stress and depression (Beardsley et al., 2005; Chartoff et al., 2012; Schank et al., 2012). In addition, since the emergence of a negative emotional state is a key to the development of dependence (and subsequently addiction) and is associated with compulsive drug taking (Koob 2008; Koob and Le Moal 1997), KOPr antagonists are also capable of attenuating escalation behaviour and reducing the motivation for drug intake (Wee et al., 2009; Schlosburg et al., 2013). Application of either KOPr agonists or 
KOPr antagonists in a clinical setting would therefore depend on the behavioural/emotional state as well as the phase of addiction that an individual is in.

Another intriguing fact to note is that activation of the KOPr is a dynamic process that is dependent on timing of the drug pairing. For example, U50,488 produced a significant attenuation of cocaine-induced place preference when administered $15 \mathrm{~min}$ prior to cocaine, but potentiated CPP when given 60 min prior to cocaine (McLaughlin et al., 2006; Ehrich et al., 2014). A recent study also showed that administration of Sal A 15 or $60 \mathrm{~min}$ prior to cocaine potentiated cocaine-induced decreases in ICSS thresholds. However, administration of Sal A $24 \mathrm{~h}$ before cocaine attenuated the cocaine-induced decreases in ICSS thresholds (Chartoff et al., 2015). This study by Chartoff et al. (2015) also reported a different timing effect compared to previous work, in that Sal A potentiated cocaineinduced effects when administered 15 min prior to cocaine, whereas U50,488 attenuated cocaine-induced effects when administered $15 \mathrm{~min}$ prior to cocaine. It is, however, important to remember that due to its rapid onset of action, $15 \mathrm{~min}$ is three times the normal onset time required for Sal A to produce anti-cocaine effects. Previous work has shown that the anti-cocaine effects of Sal A in rats - such as attenuation of cocaineinduced drug seeking and cocaine-induced sensitisation - occur when Sal $A$ is administered 5 min prior to cocaine (Morani et al., 2009; Morani et al., 2012)). In fact, an earlier study, also conducted by Chartoff et al. (2008), found that Sal A ( $2 \mathrm{mg} / \mathrm{kg}$, i.p.) inhibited cocaine-induced hyperactivity in rats when administered 5 min before cocaine (Chartoff et al., 2008). Therefore, it follows that the potentiation of cocaine-induced effects observed by Chartoff et al. (2015) is due to the delay after Sal A administration before cocaine was delivered.

Taken together, the work discussed above suggests the possibility of a three-phase KOPr agonist action. KOPr agonists first exert 1) an immediate anti-cocaine effect (i.e. when Sal A was administered 5 min prior to cocaine as in the Chartoff et al. (2008); Morani et al. (2009) and Morani et al. (2012) studies), followed by 2) negative, withdrawal-like effects that potentiates cocaine-induced actions (i.e. when Sal A was administered $15-60$ min prior to cocaine as in Chartoff et al. (2015) and when U50,488 was administered 60 min prior to cocaine as in McLaughlin et al. (2006) and Ehrich et al. (2014)), and lastly 3) an 
attenuation of cocaine-induced effects (i.e. when Sal A was administered $24 \mathrm{~h}$ prior to cocaine as in Chartoff et al. (2015)). The latter attenuation observed may be due to neuroplasticity within the mesolimbic circuits of reward, or suppression of KOPr signalling (Chartoff et al., 2015). Thus, it is important to note that these studies indicate that application of KOPr agonists should take place either immediately prior to cocaine intake or an extended period of time prior to cocaine in order to be able to attenuate the effects of cocaine. Therefore, further work should include a time course study to determine the appropriate therapeutic period of these compounds. A time course investigation using identified G-protein biased KOPr agonists will also establish differences in temporal effects between the classic and functionally selective agonists.

The present study has shown that novel Sal A-derived agonists have the potential to produce fewer negative side effects on mood than classical KOPr agonists. If the cocainepotentiating effects and stress-induced drug taking/seeking effects of KOPr agonists are solely due to the negative regulation of KOPr agonists on mood, it might be possible to eliminate the temporal limitations of KOPr agonists. In support of this, studies of a functionally selective G-protein biased KOPr agonist, RB-64, have demonstrated minimal KOPr-mediated negative effects (White et al., 2015). Thus, it can be hypothesised that the use of functionally selective KOPr agonists that produce no negative mood regulation will not only prevent the withdrawal-like encouragement of drug taking/seeking, but also inhibit the potentiation of drug-induced effects. The identification of functionally selective compounds may hold the key to developing such therapies. Therefore, quantification of $\beta$-arrestin signalling following administration of novel KOPr agonists are essential to determine the functional selectivity of these agonists (also mentioned in section 5.5.5). In addition, future studies involving behavioural screening should only be conducted after identification of biased agonism to ensure that all KOPr agonists studied will produce minimal KOPr-mediated side effects.

It is important to note that the present study involved substantial behavioural work that was conducted in a limited number of animals (as low as $n=5$ ). Experiments which involved pre-treating animals with nor-BNI involved even lower subject numbers $(n=3)$. These low numbers were due to the effort of reducing animal usage, as well as the the 
high cost and dose of nor-BNI required. However, use of the same animals (with aid of the Latin square design) allowed for within-subjects statistical analysis which provided increased statistical power and a reduction in error associated with individual differences. Another limitation of the present study was the use of a single dose of KOPr agonists in most studies. Since Sal A and its derivatives have shown contradicting effects at low vs. high doses (refer to Table 4.1 and Section 4.5 for a detailed discussion), dose-response studies should be conducted in order to investigate the threshold at which these compounds exert their therapeutic ability without producing adverse effects. Although these experiments were not conducted due to limited supply of KOPr agonists, use of each agonist at the 'minimal effective dose that attenuates cocaine-induced drug seeking' enabled this study to identify a single agonist with the best therapeutic potential (16-brSal A). Therefore, further behavioural experimentation should incorporate dose-response studies using 16-brSal A to determine its optimal therapeutic dose range.

There are a number of additional behavioural studies that could be performed to extend the findings of this study. One of the models crucial to this study that shoud be complemented with another test is the FST, due to the impact of KOPr agonists on depressive-like behaviour. It is important to note that the FST was originally designed for assessing anti-depressive effects (Porsolt, 1979; Porsolt et al., 2001) as the test involves forced swimming, which in itself is used to generate stress and depressive-like behaviours (McLaughlin et al., 2006). As a result, the FST has been said to be a poor model for assessing depression. Therefore, newer approaches such as the sweet drive test should be carried out in tandem with the FST in order to confirm the results of this study (MateusPinheiro et al., 2014). The sweet drive test measures a rodent's preference for sweet pellets in a non-aversive environment and integrates it with ultrasonic vocalisations to determine anhedonic behaviour. The sweet drive test has an advantage over the classic sucrose consumption model (also used to assess depressive-like behaviour) due to the lack of a water deprivation period, which eliminates the interference of thirst on the test outcome (Mateus-Pinheiro et al., 2014). In addition, the sweet drive test has been found to effectively measure anhedonia in different rat strains as well as both male and female rats (Mateus-Pinheiro et al., 2014), indicating the robustness of this model. 
The self-administration models used in the present study were chosen in an effort to replicate human drug taking and seeking behaviour. Since it has been suggested that the LgA self-administration model more closely models human drug use (refer to section 6.1.1), further studies should include the cocaine-induced reinstatement study in rats given extended access to cocaine. This could be achieved by first training rats to selfadminister cocaine in $2 \mathrm{~h}$ sessions, followed by $6 \mathrm{~h}$ training sessions in an effort to promote escalation behaviour. As rats given extended access to cocaine display enhanced reinstatement, in that LgA rats showed reinstatement at more cocaine doses than ShA rats (Knackstedt and Kalivas, 2007), it would be interesting to determine if an acute administration of novel KOPr agonists can attenuate drug seeking behaviour in LgA rats in a similar manner to that seen in the present study.

In an effort to determine the repeated or chronic effects of KOPr agonists on their anticocaine actions, a cocaine-induced hyperactivity study could also be conducted following repeated treatments with novel Sal A-derived agonists. This could be achieved by administering novel KOPr agonists to rats for five days prior to testing. Since repeated administration of KOPr agonists has been reported to produce tolerance (Suzuki et al., 2004), it would be interesting to determine the extent to which novel KOPr agonists can be given before exhibition of tolerance. This study could be conducted by separately injecting different groups of rats with novel KOPr agonists for a range of treatment durations (from a single day to 10 continuous days) prior to testing in a cocaine-induced hyperactivity paradigm. This study can also be extended by using identified G-protein biased KOPr agonists to determine whether biased agonism does indeed reduce or eliminate exhibition of tolerance.

The investigation of 16-brSal A-mediated cellular effects in this study was limited by use of a single cell line (HEK-293). Therefore, further investigation of KOPr-mediated effects should be extended to include other neuronal cell lines such as N2A or SH-SY5Y cells. It would also be interesting to investigate the effects of 16-brSal A and other novel KOPr agonists on cocaine-induced changes in DA uptake. To that end, future studies could be conducted using both $\mathrm{ASP}^{+}$and RDEV, whereby DAT function and expression of cells treated with cocaine can be compared to cells pre-treated with a KOPr agonist prior to 
administration of cocaine. Results obtained from this study would provide detailed mechanistic information on the anti-cocaine effects of novel Sal A-derived KOPr agonists.

\subsection{Final conclusions}

The combination of a behavioural and cellular studies utilised in the present study provides important information on the anti-cocaine effects of novel Sal A-derived agonists, as well as the mechanism by which their inhibition occurs. Identification of suitable compounds by preclinical testing will lead to better development of clinically viable therapeutics. This is the first study to show the anti-cocaine effects, minimal side effects, and regulation of DAT function by 16-brSal A. The minimal KOPr-mediated adverse effects of 16-brSal A, together with its activation of only early phase ERK1/2 and little activation of p38 MAPK demonstrates differences between 16-brSal A and traditional KOPr agonists. Thus, novel analogues of Sal A such as 16-brSal A may represent a new class of anti-cocaine therapeutic agents that produce specific anti-cocaine effects. Since development of anti-cocaine pharmacotherapies is one of the most important approach to combatting cocaine addiction, studies such as the present one will help in identifying new therapeutics and aid in further developing existing therapies. 


\section{Chapter 8: Supplementary information}

\section{Appendix 1: Solutions}

10X Phosphate Buffered Saline (PBS) pH 7.4

$\begin{array}{lll} & \text { Concentration } & \mathrm{g} / \mathrm{L} \\ \mathrm{NaCl} & 1.4 \mathrm{M} & 80 \\ \mathrm{KCl} & 26.8 \mathrm{mM} & 2.0 \\ \mathrm{Na}_{2} \mathrm{HPO}_{4} & 81.0 \mathrm{mM} & 26.8 \\ \mathrm{KH}_{2} \mathrm{PO}_{4} & 14.7 \mathrm{mM} & 2.4\end{array}$

KREBS Buffer $\mathrm{pH} 7.4$

$\begin{array}{lll} & \text { Concentration } & \mathrm{g} / \mathrm{L} \\ \mathrm{NaCl} & 130 \mathrm{mM} & 7.6 \\ \mathrm{KCl} & 1.3 \mathrm{mM} & 0.10 \\ \mathrm{CaCl}_{2} & 2.2 \mathrm{mM} & 0.24 \\ \mathrm{MgSO}_{4} \cdot 6 \mathrm{H}_{2} \mathrm{O} & 1.2 \mathrm{mM} & 0.27 \\ \mathrm{KH}_{2} \mathrm{PO}_{4} & 1.2 \mathrm{mM} & 0.16 \\ \mathrm{HEPES} & 10 \mathrm{mM} & 2.6 \\ \text { D-Glucose } & 10 \mathrm{mM} & 1.8\end{array}$

RIPA buffer $\mathrm{pH} 7.5$

$\begin{array}{lll} & \text { Concentration } & \mathrm{g} / \mathrm{L} \\ \text { Tris- } \mathrm{HCl} & 10 \mathrm{mM} & 1.2 \\ \mathrm{NaCl} & 150 \mathrm{mM} & 8.766 \\ \text { EDTA } & 1 \mathrm{mM} & 0.37 \\ \text { Triton-X-100 } & 1 \% & 10 \mathrm{~mL} \\ \text { SDS } & 0.1 \% & 1.0 \\ \text { Sodium deoxycholate } & 1 \% & 10\end{array}$


$\underline{5 X \text { Reducing Buffer }}$

\section{Concentration}

Tris- $\mathrm{HCl}, \mathrm{pH} 6.8$

$62.5 \mathrm{mM}$

SDS

$2 \%$

Glycerol

$20 \%$

Bromophenol Blue

$1 \%$

Gels (Enough for 2 gels)

$\begin{array}{ll}\text { 10\% Separating Gel } & \text { Volume } \\ \text { dH2O } & 8 \mathrm{~mL} \\ 1.5 \text { M Tris pH } 8.8 & 5 \mathrm{~mL} \\ 10 \% \text { SDS } & 200 \mu \mathrm{L} \\ \text { Acrylamide } & 6.66 \mathrm{~mL} \\ 10 \% \text { APS } & 100 \mu \mathrm{L} \\ \text { TEMED } & 10 \mu \mathrm{L}\end{array}$

Gel was layered with $100 \%$ isopropanol while setting. Isopropanol was poured off prior to addition of the stacking gel.

4\% Stacking Gel

$\mathrm{dH} 2 \mathrm{O}$

$0.5 \mathrm{M}$ Tris $\mathrm{pH} 6.8$

$10 \%$ SDS

Acrylamide

$10 \%$ APS

TEMED

10X Running Buffer

SDS

Tris- $\mathrm{HCl}$

Glycine
Concentration

$35 \mathrm{mM}$

$250 \mathrm{mM}$

$1.9 \mathrm{M}$
Volume

$6.1 \mathrm{~mL}$

$2.5 \mathrm{~mL}$

$100 \mu \mathrm{L}$

$1.33 \mathrm{~mL}$

$50 \mu \mathrm{L}$

$10 \mu \mathrm{L}$

$\mathrm{g} / \mathrm{L}$

10

30.3

144.1 
Western Transfer Buffer

$\begin{array}{lll} & \text { Concentration } & \mathrm{g} / \mathrm{L} \\ \text { Glycine } & 190 \mathrm{mM} & 14.4 \\ \text { Tris- } \mathrm{HCl} & 25 \mathrm{mM} & 3.03 \\ \text { Methanol } & 20 \% & 200 \mathrm{~mL}\end{array}$

$\underline{10 X \text { TBS pH } 7.5}$

$\begin{array}{lll} & \text { Concentration } & \mathrm{g} / \mathrm{L} \\ \text { Tris } \mathrm{HCl} & 500 \mathrm{mM} & 60.5 \\ \mathrm{NaCl} & 1.5 \mathrm{M} & 87.6\end{array}$

$\underline{\text { T-TBS }}$

1X TBS containing $0.1 \%$ Tween-20

$\underline{\text { Stripping Buffer }}$

SDS

Concentration

g/L

$69 \mathrm{mM}$

20

$0.05 \mathrm{M} \mathrm{TrisHCl}, \mathrm{pH} 6.8$

$992.5 \mathrm{~mL}$

$\beta$-mercaptoethanol

$7.5 \mathrm{~mL}$ 


\section{Appendix 2.1: Determination of ideal protein loading concentration}

Prior to analysis of cell and tissue lysates, the amount of protein loaded on SDS-gels gel were optimised to ensure that protein concentrations fell within the linear detection range and did not produce saturation. Figure 8.1 shows representative ERK (Figure 8.1A) and p38 MAPK (Figure 8.1B) Western blots showing a linear relationship between the amount of protein loaded and density of bands seen. All protein concentrations were determined using the Bradford protein assay.

A)

$50 \mathrm{kDa}$

$37 \mathrm{kDa}$
ERK

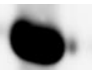

\section{(}

B)

$50 \mathrm{kDa}$

$37 \mathrm{kDa}$

\section{$0.5 \quad 1.25 \quad 2.5 \quad 4.0$}

\section{$\begin{array}{llll}0.5 & 1.25 & 2.5 & 4.0\end{array}$}

\section{Protein concentration $(\mathrm{mg} / \mathrm{mL})$}

C)

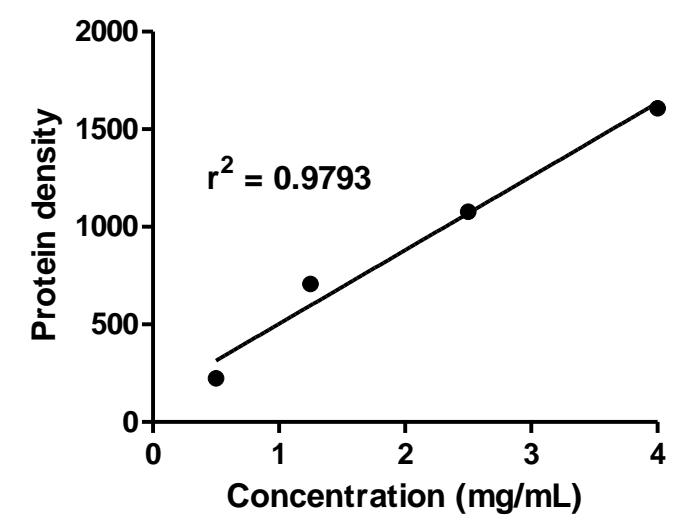

D)

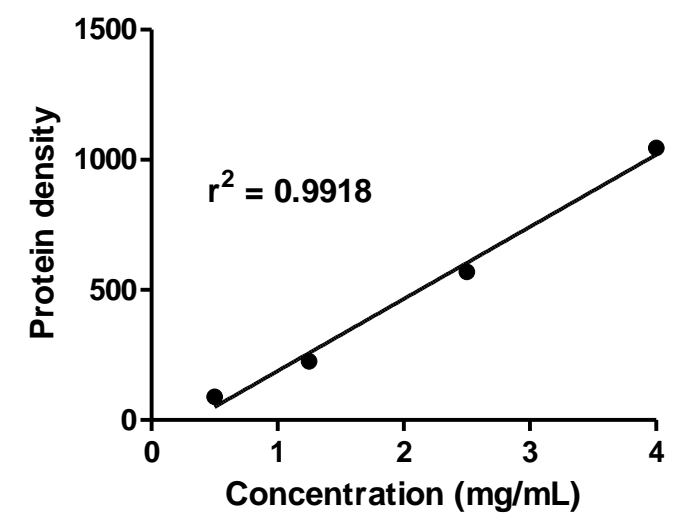

\section{Figure 8.1: The density of protein bands is dependent on protein concentration}

Western blotting studies conducted showed that for both A) ERK and B) P38 MAPK, the density of bands increased in correlation to protein concentration. The $2.5 \mathrm{mg} / \mathrm{mL}$ concentration was chosen as the ideal loading concentration as it was within the linear loading range. 


\section{Appendix 2.2: Protein band size quantification}

To quantify the size of protein bands, the distance migrated by the 6 standard bands of the molecular weight marker (Precision Plus Protein ${ }^{\mathrm{TM}}$ Dual Colour Standards, \#1610374, purchased from Bio-Rad) were measured from the top of the membrane in $\mathrm{mm}$. These measurements were plotted against the molecular weight of respective bands to produce a linear regression. The distance travelled by protein bands in this study, i.e. ERK1/2 and p38, were calculated using the standard linear regression.

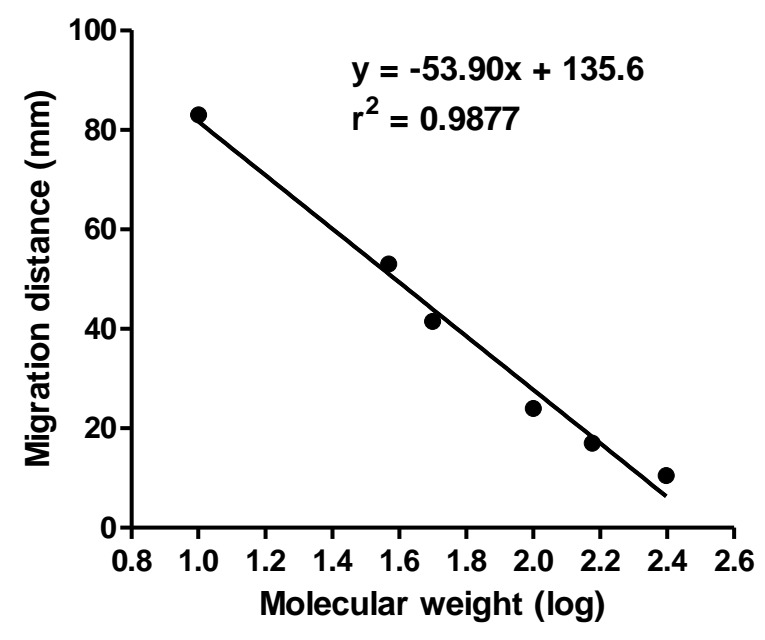

\begin{tabular}{|c|c|c|c|}
\hline Protein & Distance $(\mathbf{m m})$ & $\begin{array}{c}\text { Calculated molecular weight } \\
\mathbf{( k D a )}\end{array}$ & $\begin{array}{c}\text { Molecular weight } \\
\text { (kDa) }\end{array}$ \\
\hline ERK1 & 48.0 & 42.2 & 42 \\
\hline ERK2 & 46.5 & 45.0 & 44 \\
\hline P38 & 48.5 & 41.3 & 43 \\
\hline
\end{tabular}

\section{Figure 8.2: Determination of protein band size}

The known molecular weight of standard bands and their migration distance was used to calculate the molecular weight of protein bands produced in this study. The migration distance of protein bands correlate linearly to the log of their molecular weight. The migration distance of protein bands were measured, substituted into the equation, and a reverse log taken to determine band size. The expected molecular weights $(\mathrm{kDa})$ shown in the table were obtained from the manufacturer's (Cell Signalling) data sheet. 


\section{Appendix 3.1: Counterbalanced treatment order for the reinstatement}

\section{study}

A within-subjects design was used for reinstatement studies, meaning that all rats received each treatment group (except for the nor-BNI pre-treatment). Both experiments were designed in a counterbalanced order using the Latin square design to control for any effect of treatment order.

A)

\begin{tabular}{|c|c|c|c|c|c|}
\hline Rat & Session 1 & Session 2 & Session 3 & Session 4 & Session 5 \\
\hline 1 & Vehicle & $\begin{array}{c}0.316-\mathrm{brSal} \\
\mathrm{A}\end{array}$ & 1.0 16-brSal A & 0.3 Sal A & - \\
\hline 2 & $\begin{array}{c}0.316-\mathrm{brSal} \\
\mathrm{A}\end{array}$ & $\begin{array}{c}1.016-\text { brSal } \\
\text { A }\end{array}$ & 0.3 Sal A & Vehicle & - \\
\hline 3 & $\begin{array}{c}1.016-\text { brSal } \\
\text { A }\end{array}$ & $0.3 \mathrm{Sal}$ A & Vehicle & 0.3 16-brSal A & $\begin{array}{l}1.016 \text {-brSal A } \\
+10 \text { nor-BNI } \\
\end{array}$ \\
\hline 4 & 0.3 Sal A & Vehicle & 0.3 16-brSal A & $1.016-\mathrm{brSal} \mathrm{A}$ & - \\
\hline 5 & Vehicle & $0.3 \mathrm{Sal}$ A & 1.0 16-brSal A & 0.3 16-brSal A & $\begin{array}{l}1.016-\mathrm{brSal} \text { A } \\
+10 \text { nor-BNI }\end{array}$ \\
\hline 6 & 0.3 Sal A & $\begin{array}{c}1.016-\mathrm{brSal} \\
\mathrm{A}\end{array}$ & $0.316-\mathrm{brSal} A$ & Vehicle & $\begin{array}{c}1.016-\mathrm{brSal} A \\
+10 \text { nor-BNI }\end{array}$ \\
\hline
\end{tabular}

B)

\begin{tabular}{|c|c|c|c|c|c|}
\hline Rat & Session 1 & Session 2 & Session 3 & Session 4 & Session 5 \\
\hline 1 & Vehicle & $\begin{array}{c}0.1 \text { EOM Sal } \\
\text { B }\end{array}$ & 0.3 EOM Sal B & 0.3 Sal A & $\begin{array}{c}1.016-\mathrm{brSal} A \\
+10 \text { nor-BNI }\end{array}$ \\
\hline 2 & $\begin{array}{c}0.1 \text { EOM Sal } \\
\text { B }\end{array}$ & $\begin{array}{c}0.3 \text { EOM Sal } \\
\text { B }\end{array}$ & 0.3 Sal A & Vehicle & - \\
\hline 3 & $\begin{array}{c}0.3 \text { EOM Sal } \\
\text { B }\end{array}$ & 0.3 Sal A & Vehicle & 0.1 EOM Sal B & - \\
\hline 4 & 0.3 Sal A & $\begin{array}{c}\text { Vehicle } \\
5\end{array}$ & 0.1 EOM Sal B & 0.3 EOM Sal B & $\begin{array}{c}1.016-\mathrm{brSal} \text { A } \\
+10 \text { nor-BNI }\end{array}$ \\
\hline Vehicle & $\begin{array}{c}0.3 \text { EOM Sal } \\
\text { B }\end{array}$ & 0.1 EOM Sal B & 0.3 Sal A & $\begin{array}{c}1.016-\mathrm{brSal} A \\
+10 \text { nor-BNI }\end{array}$ \\
\hline
\end{tabular}

Table 8.1: Treatment design used in cocaine-induced reinstatement of drug seeking

Treatment order for rats in the A) 16-brSal A $(n=6)$ and B) EOM Sal B $(n=5)$ group. Numbers represent doses used, i.e. $0.3=0.3 \mathrm{mg} / \mathrm{kg}$. Vehicle $=75 \%$ DMSO. All vehicle and $\mathrm{KOPr}$ agonists were given i.p., whereas nor-BNI was given s.c. 


\section{Appendix 3.2: Number of days required to reach extinction criteria}

Rats stably self-administering cocaine on a FR5 schedule of reinforcement were subjected to extinction sessions prior to reinstatement. During extinction, cocaine infusions were replaced with saline and the light cue was removed. Extinction sessions continued until active lever presses were $<20$ in a $2 \mathrm{~h}$ session. Due to the within-subjects study design, rats were subjected to a total of six extinction and reinstatement phases. In the first extinction phase, rats responded persistently for the active lever, requiring a mean of 6.0 \pm 0.7 days to achieve extinction. However, rats quickly learned the behaviour, with rats achieving extinction in an average of $2.3 \pm 0.1$ days by the 6th extinction phase.

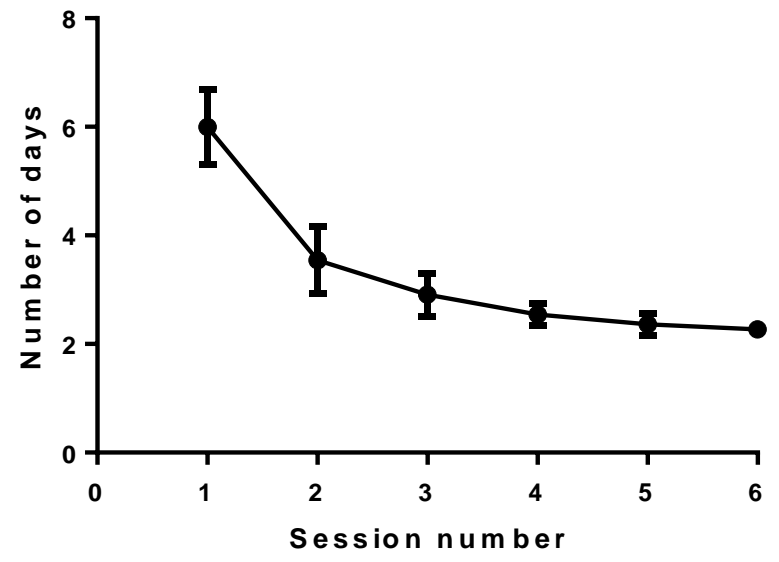

Figure 8.3: Number of days required for extinction decreased with progression of the study

Rats required fewer number of days to achieve extinction criteria as the study progressed. 


\section{Appendix 3.3: Comparison between vehicle groups in the cocaine-induced hyperactivity study}

In the cocaine-induced hyperactivity study, all rats were subjected to $30 \mathrm{~min}$ of habituation in the activity chambers 30 min prior to vehicle (75\% DMSO) or KOPr agonist (16-brSal A, $1.0 \mathrm{mg} / \mathrm{kg}$ or MOM Sal B, $0.3 \mathrm{mg} / \mathrm{kg}$ ) treatment. Rats were then given an injection of cocaine $(20 \mathrm{mg} / \mathrm{kg}$ ) and subjected to a $60 \mathrm{~min}$ testing period. To ensure that the data obtained was not due to a difference in vehicle-evoked responding, the number of ambulatory counts produced by rats treated with vehicle in the habituation (Figure 8.4A) and testing (Figure 8.4B) phases were compared between the two experiments. Control rats did not significantly differ in number of ambulatory counts produced in the habituation ( $p=0.36$, Student's $t$-test) or testing ( $p=0.28$, Student's $t$-test) phase, indicating that all rats treated with vehicle showed no significant difference in basal or cocaine-induced locomotor activity.

A)

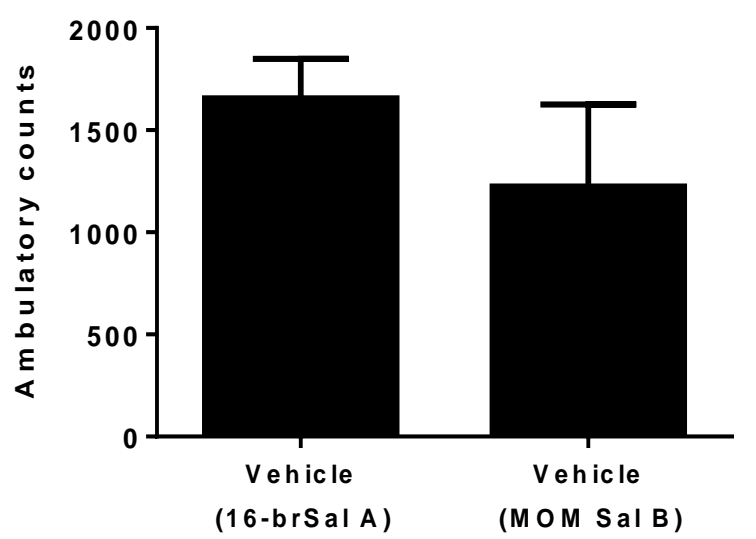

B)

Testing phase

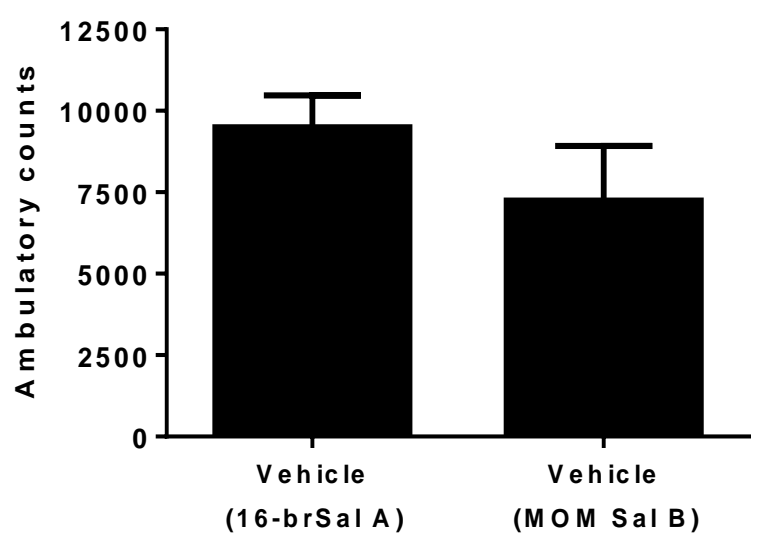

Figure 8.4: Number of total ambulatory counts produced by vehicle-treated rats in the

\section{hyperlocomotion study}

Rats in both the 16-brSal A and MOM Sal B study groups showed no significant difference in the number of ambulatory counts in the A) habituation or B) testing phase of the cocaine-induced hyperactivity experiment. Student's $t$-test. Values presented as mean $\pm S E M, n=6$. 
Appendix 4.1: Counterbalanced treatment order for the sucrose selfadministration study

A within-subjects design, in which all rats received each treatment, was used for the sucrose self-administration study. The study was designed in a counterbalanced order using the Latin square design to control for any effect of treatment order.

\begin{tabular}{|c|c|c|c|c|c|c|c|}
\hline Rat & Session 1 & Session 2 & Session 3 & Session 4 & Session 5 & Session 6 & Session 7 \\
\hline 1 & Vehicle & $\begin{array}{c}10 \\
\text { U50,488 }\end{array}$ & $\begin{array}{c}1.0 \\
\text { U69,593 }\end{array}$ & $\begin{array}{c}0.1 \\
\text { EOM Sal } \\
\text { B }\end{array}$ & $\begin{array}{c}0.3 \\
\text { Sal A }\end{array}$ & $\begin{array}{c}1.0 \\
16-b r S a l \\
\text { A }\end{array}$ & $\begin{array}{c}1.0 \beta- \\
\text { THP Sal B }\end{array}$ \\
\hline 2 & $\begin{array}{c}10 \\
\text { U50,488 }\end{array}$ & $\begin{array}{c}1.0 \\
\text { U69,593 }\end{array}$ & $\begin{array}{c}0.1 \\
\text { EOM Sal } \\
\text { B }\end{array}$ & $\begin{array}{c}0.3 \\
\text { Sal A }\end{array}$ & $\begin{array}{c}1.0 \\
16-b r S a l \\
A\end{array}$ & $\begin{array}{c}1.0 \beta- \\
\text { THP Sal B }\end{array}$ & Vehicle \\
\hline 3 & $\begin{array}{c}1.0 \\
\text { U69,593 }\end{array}$ & $\begin{array}{c}0.1 \\
\text { EOM Sal } \\
\text { B }\end{array}$ & $\begin{array}{c}0.3 \\
\text { Sal A }\end{array}$ & $\begin{array}{c}1.0 \\
16-b r S a l \\
A\end{array}$ & $\begin{array}{c}1.0 \beta- \\
\text { THP Sal B }\end{array}$ & Vehicle & $\begin{array}{c}10 \\
\text { U50,488 }\end{array}$ \\
\hline 4 & $\begin{array}{c}0.1 \\
\text { EOM Sal } \\
\text { B }\end{array}$ & $\begin{array}{c}0.3 \\
\text { Sal A }\end{array}$ & $\begin{array}{c}1.0 \\
16-b r S a l \\
A\end{array}$ & $\begin{array}{c}1.0 \beta- \\
\text { THP Sal B }\end{array}$ & Vehicle & $\begin{array}{c}10 \\
\text { U50,488 }\end{array}$ & $\begin{array}{c}1.0 \\
\text { U69,593 }\end{array}$ \\
\hline 5 & $\begin{array}{c}0.3 \\
\text { Sal A }\end{array}$ & $\begin{array}{c}1.0 \\
16-b r S a l \\
\text { A }\end{array}$ & $\begin{array}{c}1.0 \beta- \\
\text { THP Sal B }\end{array}$ & Vehicle & $\begin{array}{c}10 \\
\text { U50,488 }\end{array}$ & $\begin{array}{c}1.0 \\
\text { U69,593 }\end{array}$ & $\begin{array}{c}0.1 \\
\text { EOM Sal } \\
\text { B }\end{array}$ \\
\hline 6 & $\begin{array}{c}1.0 \\
16-b r S a l \\
\text { A }\end{array}$ & $\begin{array}{c}1.0 \beta- \\
\text { THP Sal B }\end{array}$ & Vehicle & $\begin{array}{c}10 \\
\text { U50,488 }\end{array}$ & $\begin{array}{c}1.0 \\
\text { U69,593 }\end{array}$ & $\begin{array}{c}0.1 \\
\text { EOM Sal } \\
\text { B }\end{array}$ & $\begin{array}{c}0.3 \\
\text { Sal A }\end{array}$ \\
\hline 7 & $\begin{array}{c}1.0 \beta- \\
\text { THP Sal B }\end{array}$ & Vehicle & $\begin{array}{c}10 \\
\text { U50,488 }\end{array}$ & $\begin{array}{c}1.0 \\
\text { U69,593 }\end{array}$ & $\begin{array}{c}0.1 \\
\text { EOM Sal } \\
\text { B }\end{array}$ & $\begin{array}{c}0.3 \\
\text { Sal A }\end{array}$ & $\begin{array}{c}1.0 \\
16-b r S a l \\
\text { A }\end{array}$ \\
\hline
\end{tabular}

Table 8.2: Treatment order for rats in the sucrose reinforcement study

Rats were given KOPr agonist treatment in a Latin square design to prevent treatment order bias $(\mathrm{n}=7)$. Numbers represent doses used, i.e. $0.3=0.3 \mathrm{mg} / \mathrm{kg}$. Vehicle = DMSO:Tween-80:MilliQ water in a ratio of 2:1:7. All vehicle and KOPr agonists (except for U69,593) were given i.p. U69,593 was given s.c. 


\section{Appendix 4.2: Comparison of vehicle groups in the locomotor activity}

\section{study}

In the locomotor activity study, all rats were subjected to $30 \mathrm{~min}$ of habituation in the activity chambers for 30 min prior to vehicle (75\% DMSO) or KOPr agonist (16-brSal A, 1.0 $\mathrm{mg} / \mathrm{kg}$ or MOM Sal B, $0.3 \mathrm{mg} / \mathrm{kg}$ ) treatment. Rats were then returned to the activity chamber for a further $60 \mathrm{~min}$. To ensure that the data obtained was not due to a difference in vehicle-evoked responding, the number of ambulatory counts produced by rats treated with vehicle in the habituation (Figure 8.5A) and testing (Figure 8.5B) phases were compared between the two experiments. Control rats did not significantly differ in number of ambulatory counts produced in the habituation ( $p=0.21$, Student's $t$-test) or testing ( $p=0.19$, Student's $t$-test) phase, indicating that all vehicle-treated rats showed no significant difference in locomotor activity.

A)

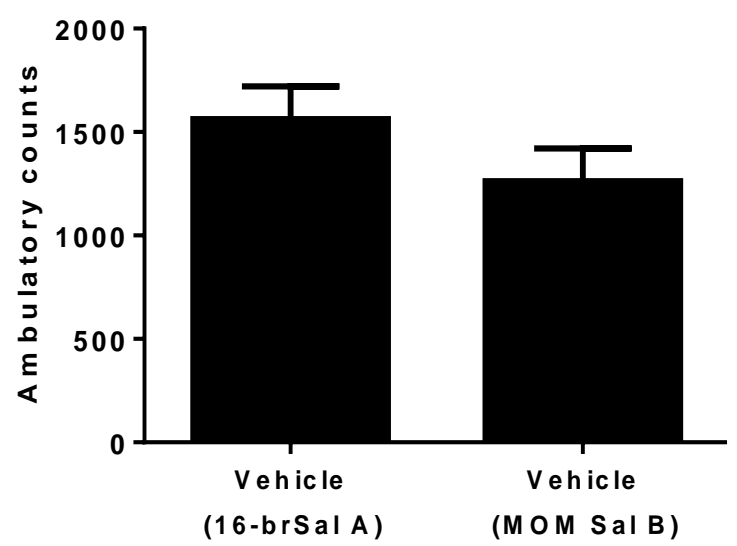

B) Testing phase

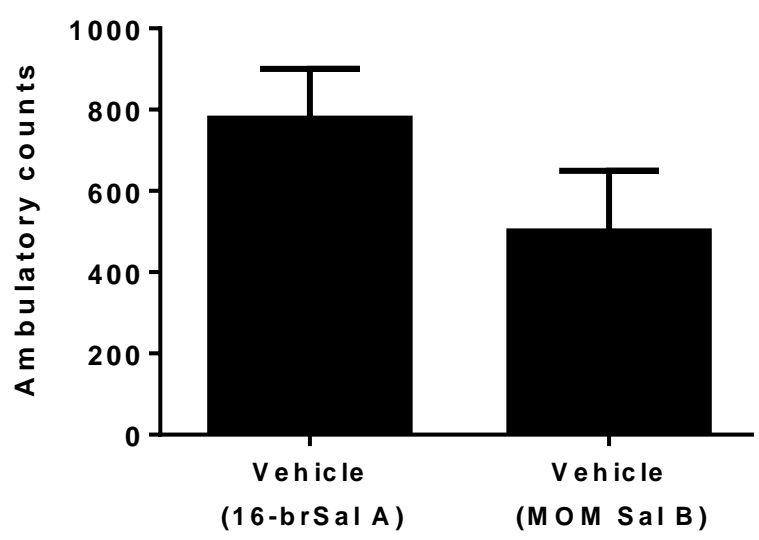

Figure 8.5: Number of total ambulatory counts produced by vehicle-treated rats in the open field locomotor activity study

Rats in both the 16-brSal A and MOM Sal B study showed no significant difference in the number of ambulatory counts in the A) habituation and B) testing phase of the locomotor activity study. Student's $t$-test. Values presented as mean \pm SEM, $n=6-7$. 


\section{Appendix 4.3: Comparison of vehicle-treated rats of different groups in the FST study}

The FST comprised of four different studies, with each study comparing a single KOPr agonist treatment (either 16-brSal A, EOM Sal B, MOM Sal B, or $\beta$-THP Sal B) to vehicletreated controls (75\% DMSO). To ensure that the significant data obtained were not due to discrepancies between climbing, swimming, and immobility time in rats treated with the vehicle, the occurrences of swimming, climbing, and immobility in all control rats were analysed. Figure 8.6 shows the responses of vehicle-treated rats in their respective study groups. Control rats did not show any significant differences in the climbing $[F(3,23)=$ $0.9618, p=0.4275]$, swimming $[F(3,23)=1.772, p=0.1805]$ or immobility time $[F(3,23)=$ $0.08060, p=0.9699$ ] (one-way ANOVA), indicating administration of the vehicle evoked the same response in rats despite the temporal difference of separate experiments.

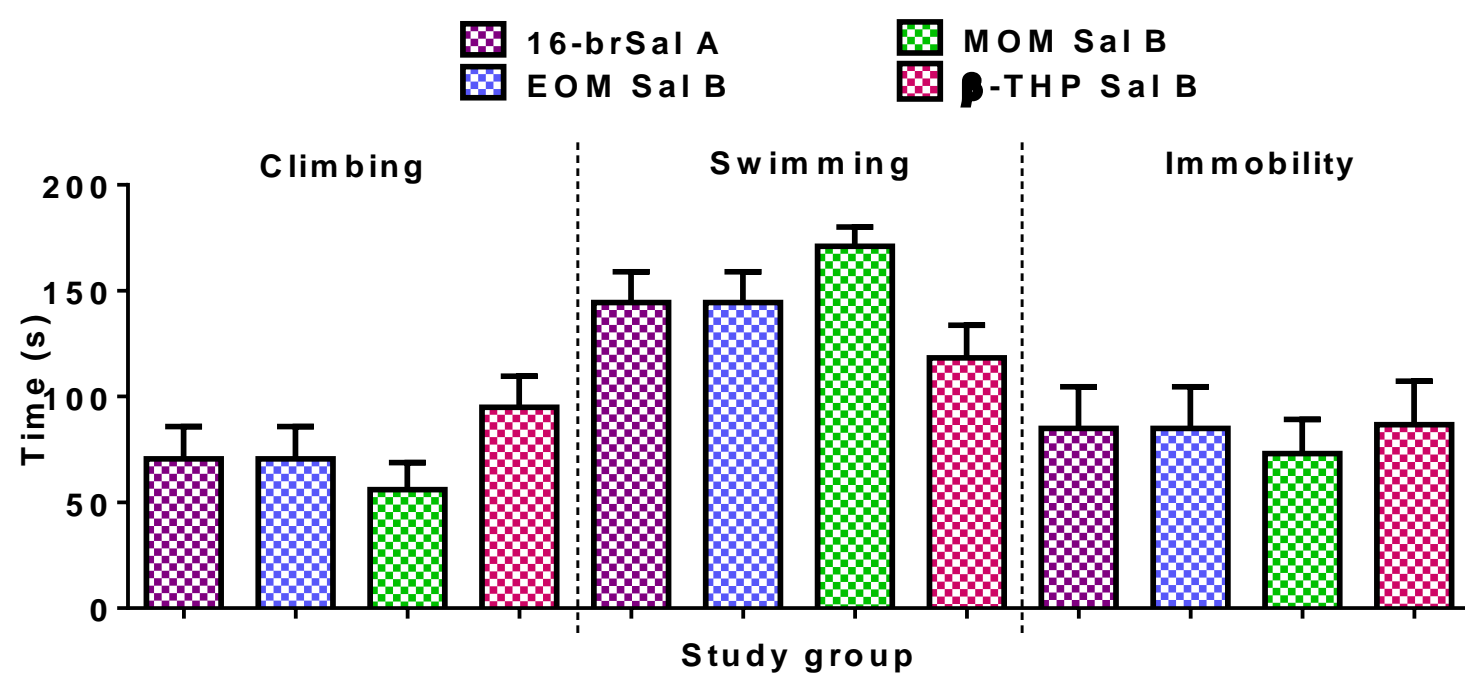

Figure 8.6: Time spent climbing, swimming, and immobile for vehicle-treated rats in all study groups

Rats treated with the vehicle (75\% DMSO) showed no significant difference in climbing, swimming, or immobility time. One-way ANOVA. Values presented as mean \pm SEM, $n=5-9$. 


\section{Appendix 5.1: Non-specific uptake in $\mathrm{ASP}^{+}$uptake kinetics by non- transfected cells}

In a between-cell design $\mathrm{ASP}^{+}$uptake study, all data had to be background corrected to eliminate non-DAT-mediated uptake, i.e. uptake via endogenous transport systems found in HEK-293 cells (Stachon et al., 1996; Schwartz et al., 2003). Figure 8.7A shows the nonDAT-mediated accumulation of $\mathrm{ASP}^{+}$accumulation, in which non-transfected HEK-293 cells display a linear pattern of $\mathrm{ASP}^{+}$uptake. Figure 8.7B shows the $\mathrm{ASP}^{+}$accumulation in HEK-293 cells co-expressing YFP-hDAT and myc-rKOPr prior to non-specific background fluorescence subtraction, whereas Figure 8.7C shows the $\mathrm{ASP}^{+}$accumulation in HEK-293 cells co-expressing YFP-hDAT and myc-rKOPr after background correction. 
A)

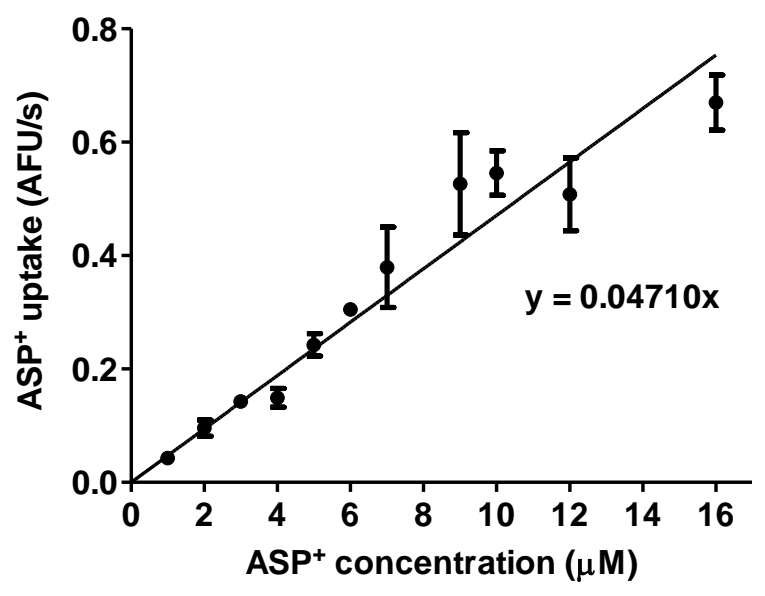

B)

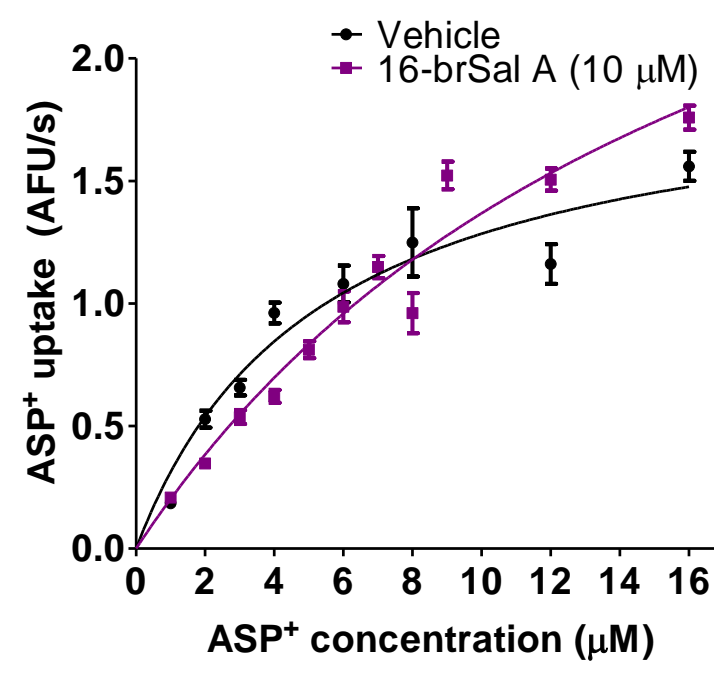

C)

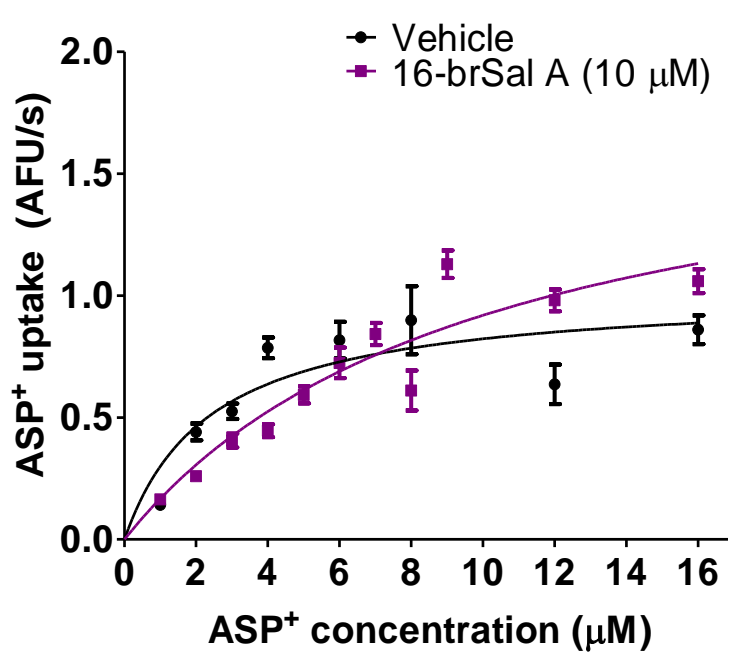

\section{Figure 8.7: Increase of background autofluorescence in $\mathrm{ASP}^{+}$uptake kinetics}

A) The rate of background autofluorescence increase was determined by evaluating the increase in ASP+ fluorescence intensity in non-transfected cells. Non-transfected HEK-293 cells produced a linear increase in background autofluorescence. The values of $\mathrm{ASP}^{+}$uptake kinetics conducted in KOPr and DAT co-expressing HEK-293 cells shown B) before and C) after background autofluorescence subtraction. Values presented as mean \pm SEM, $n=238-262$ cells from at least 3 separate transfections. 


\section{Appendix 5.2: Representative Western blot membranes}

Western blots were performed using specific antibodies for pERK1/2 (Figure 8.8A), ERK1/2 (Figure 8.8B), p-p38 (Figure 8.8C), and p38 MAPK (Figure 8.8D). Control blots in which samples were probed with only the secondary antibody was also performed to check for non-specific binding (Figure 8.8E and 8.8F).

A)

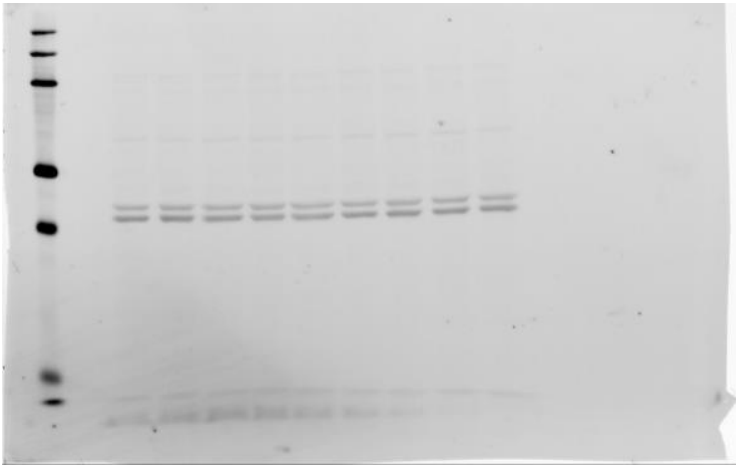

C)

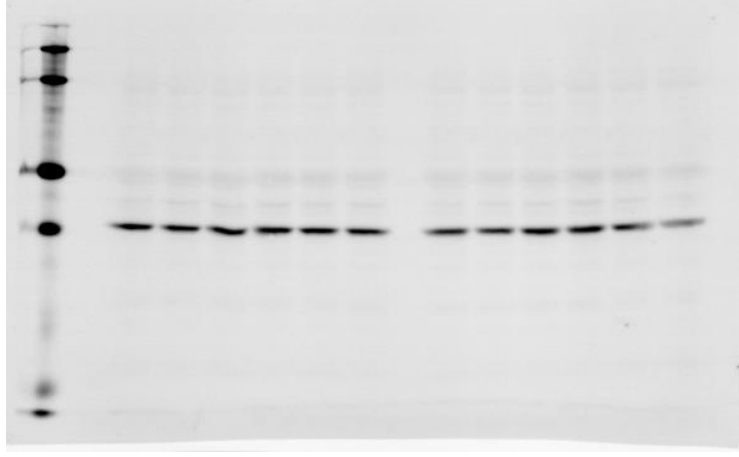

E)

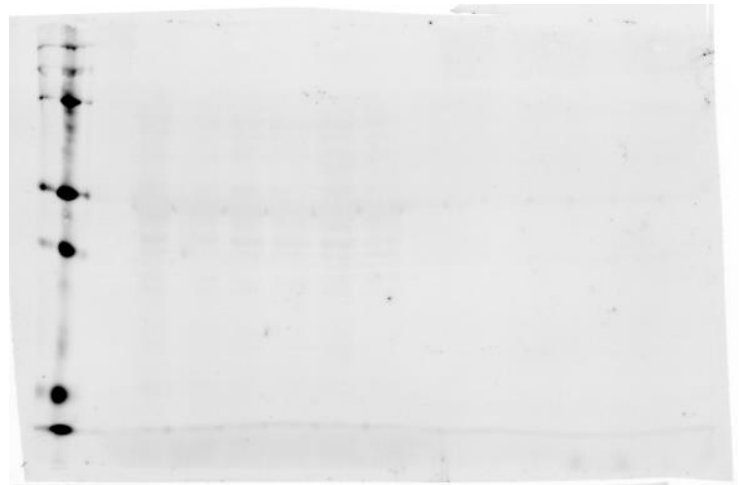

B)

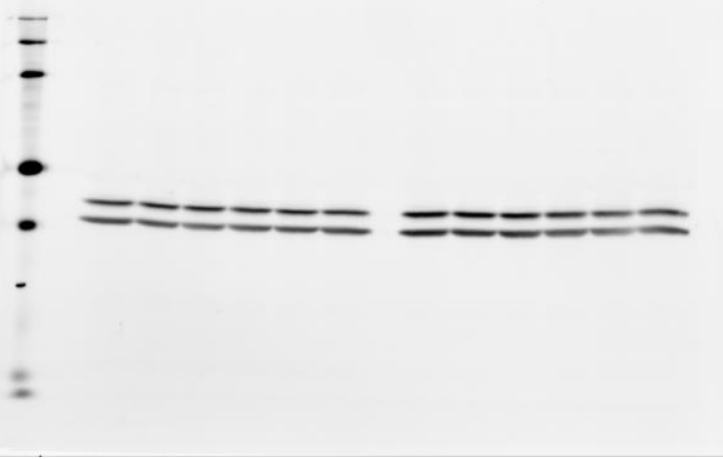

D)

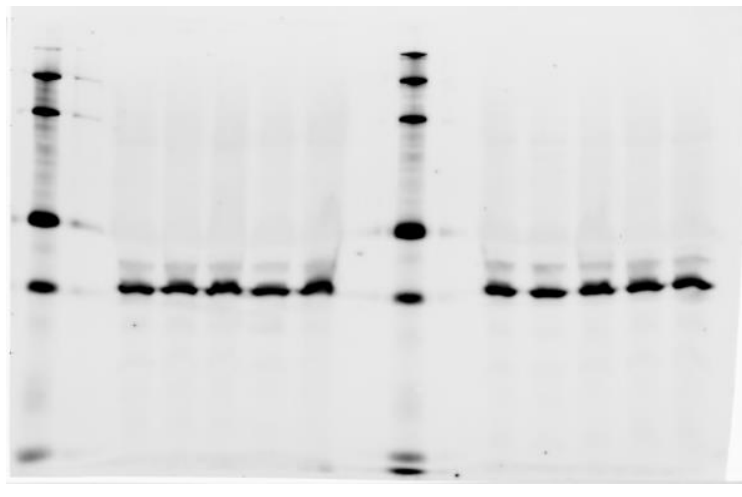

F)

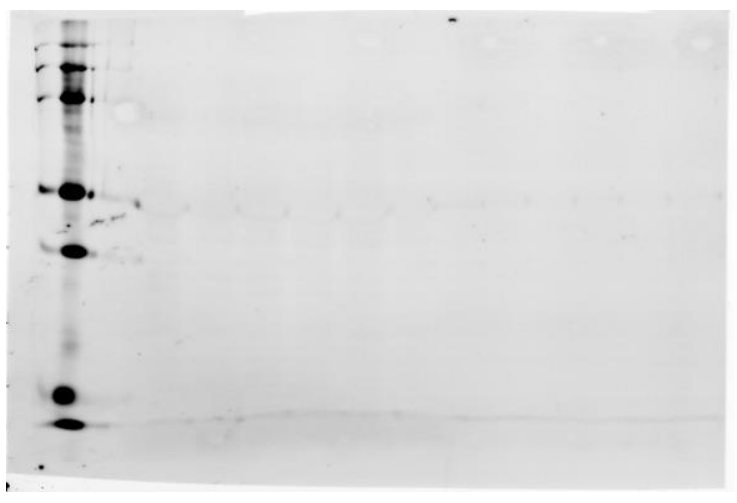

Figure 8.8: Representative Western blot membranes

Representative membranes shown for A) pERK1/2, B) ERK1/2, C) p-p38 MAPK, D) p38 MAPK, E) non-specific binding of anti-mouse Cy5, and F) non-specific binding of anti-rabbit Cy5. 
Appendix 6.1: Counterbalanced treatment order for the LgA and PR selfadministration study

A within-subjects design, in which all rats received each treatment, was used for both the LgA (Table 8.3) and PR (Table 8.4) studies. All treatments in both experiments were administered according to a Latin square design to control for any effect of treatment order.

\begin{tabular}{|c|c|c|c|}
\hline Rat & Session 1 & Session 2 & Session 3 \\
\hline $\mathbf{1}$ & Vehicle & 0.3 Sal A & 2.0 Sal A \\
\hline $\mathbf{2}$ & 0.3 Sal A & 2.0 Sal A & Vehicle \\
\hline $\mathbf{3}$ & 2.0 Sal A & Vehicle & 0.3 Sal A \\
\hline $\mathbf{4}$ & Vehicle & 2.0 Sal A & 0.3 Sal A \\
\hline $\mathbf{5}$ & 2.0 Sal A & 0.3 Sal A & Vehicle \\
\hline $\mathbf{6}$ & 0.3 Sal A & Vehicle & 2.0 Sal A \\
\hline
\end{tabular}

\section{Table 8.3: Treatment order for rats in the LgA self-administration study}

Rats were given vehicle or Sal A treatment in a counterbalanced manner to prevent treatment order bias $(n=6)$. Numbers represent doses used, i.e. $0.3=0.3 \mathrm{mg} / \mathrm{kg}$. Vehicle $=75 \%$ DMSO. All treatments were given i.p. 


\begin{tabular}{|c|c|c|c|c|c|c|c|c|}
\hline Rat & Pre-treatment & Session 1 & Session 2 & Session 3 & Session 4 & Session 5 & Session 6 & Session 7 \\
\hline 1 & 0.3 Mesyl Sal B & Vehicle & 10 U50,488 & 1.0 U69,593 & 0.3 Sal A & $2.0 \mathrm{Sal} \mathrm{A}$ & $1.016-\mathrm{brSal}$ A & $2.016-\mathrm{brSal} A$ \\
\hline 2 & 0.3 Mesyl Sal B & $10 \cup 50,488$ & $1.0 \cup 69,593$ & $0.3 \mathrm{Sal} A$ & $2.0 \mathrm{Sal}$ A & 1.0 16-brSal A & $2.016-\mathrm{brSal}$ A & Vehicle \\
\hline 3 & 1.0 Sal A & $1.0 \cup 69,593$ & 0.3 Sal A & $2.0 \mathrm{Sal} \mathrm{A}$ & $1.016-\mathrm{brSal} A$ & 2.0 16-brSal A & Vehicle & $10 \cup 50,488$ \\
\hline 4 & 1.0 Sal A & $0.3 \mathrm{Sal}$ A & 2.0 Sal A & $1.016-\mathrm{brSal}$ A & $2.016-\mathrm{brSal} \mathrm{A}$ & Vehicle & $10 \cup 50,488$ & $1.0 \cup 69,593$ \\
\hline 5 & $10 \cup 50,488$ & $2.0 \mathrm{Sal} \mathrm{A}$ & $1.016-\mathrm{brSal} \mathrm{A}$ & $2.016-\mathrm{brSal}$ A & Vehicle & $10 \cup 50,488$ & $1.0 \cup 69,593$ & $0.3 \mathrm{Sal} \mathrm{A}$ \\
\hline 6 & $10 \cup 50,488$ & $1.016-\mathrm{brSal} A$ & $2.016-\mathrm{brSal}$ A & Vehicle & $10 \cup 50,488$ & $1.0 \cup 69,593$ & 0.3 Sal A & $2.0 \mathrm{Sal} \mathrm{A}$ \\
\hline 7 & None & $2.016-\mathrm{brSal}$ A & Vehicle & $10 \cup 50,488$ & $1.0 \cup 69,593$ & 0.3 Sal A & $2.0 \mathrm{Sal} \mathrm{A}$ & $1.016-\mathrm{brSal} A$ \\
\hline 8 & None & Vehicle & $2.016-\mathrm{brSal} \mathrm{A}$ & $1.016-\mathrm{brSal}$ A & $2.0 \mathrm{Sal} \mathrm{A}$ & 0.3 Sal A & $1.0 \cup 69,593$ & 10 U50,488 \\
\hline 9 & None & $2.016-\mathrm{brSal} \mathrm{A}$ & $1.016-\mathrm{brSal} A$ & $2.0 \mathrm{Sal} \mathrm{A}$ & 0.3 Sal A & 1.0 U69,593 & $10 \cup 50,488$ & Vehicle \\
\hline 10 & None & $1.016-\mathrm{brSal} \mathrm{A}$ & 2.0 Sal A & 0.3 Sal A & $1.0 \cup 69,593$ & $10 \cup 50,488$ & Vehicle & $2.016-\mathrm{brSal} A$ \\
\hline 11 & None & $2.0 \mathrm{Sal}$ A & 0.3 Sal A & $1.0 \cup 69,593$ & $10 \cup 50,488$ & Vehicle & $2.016-\mathrm{brSal} A$ & $1.016-\mathrm{brSal} A$ \\
\hline 12 & None & $0.3 \mathrm{Sal}$ A & $1.0 \cup 69,593$ & $10 \cup 50,488$ & Vehicle & 2.0 16-brSal A & $1.016-\mathrm{brSal} A$ & 2.0 Sal A \\
\hline 13 & None & $1.0 \cup 69,593$ & $10 \cup 50,488$ & Vehicle & $2.016-\mathrm{brSal}$ A & 1.0 16-brSal A & 2.0 Sal A & 0.3 Sal A \\
\hline 14 & None & $10 \cup 50,488$ & Vehicle & $2.016-\mathrm{brSal}$ A & $1.016-\mathrm{brSal} A$ & $2.0 \mathrm{Sal}$ A & 0.3 Sal A & $1.0 \cup 69,593$ \\
\hline
\end{tabular}

\section{Table 8.4: Treatment order for rats in the PR study}

Rats were given vehicle or KOPr agonists in a Latin square design to prevent treatment order bias $(n=14)$. Numbers represent doses used, i.e. $0.3=0.3$ $\mathrm{mg} / \mathrm{kg}$. Vehicle = DMSO:Tween-80:MilliQ water in a ratio of 2:1:7. All vehicle and KOPr agonists (except for U69,593) were given i.p. U69,593 was given s.c. 


\section{Appendix 6.2: Comparison of acquisition periods in all self-administration}

studies

In this PhD project, three cocaine self-administration paradigms were used: the ShA, $\operatorname{LgA}$, and PR models. Despite the different self-administration model utilised, all rats were trained in the same fashion, in which rats were initially trained on the FR1 schedule of reinforcement. Comparison of drug naïve rats (checkered bars) in all three selfadministration paradigms showed no significant differences in mean acquisition time (Figure 8.9), indicating that all rats acquired self-administration behaviour in a similar duration despite all three experiments being carried out at different times. In contrast, rats pre-treated with KOPr agonist (striped bar) in the PR study showed a significantly increased acquisition period compared to drug naïve rats in all three experimental models (one-way ANOVA with the Bonferroni post-test, Figure 8.9).

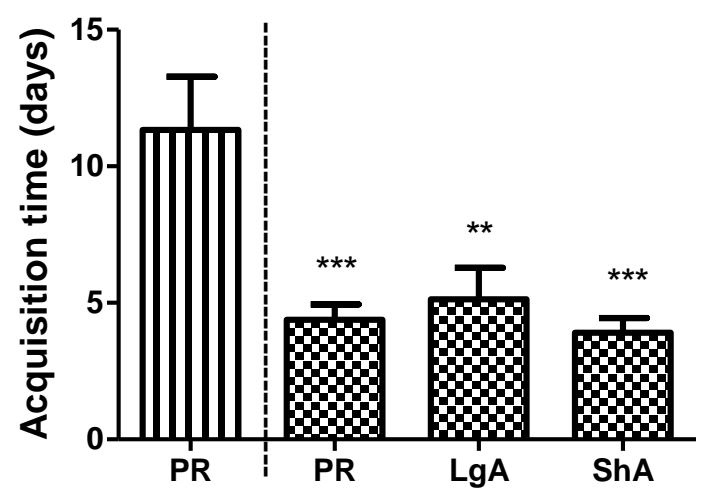

Self-administration model

Figure 8.9: Self-administration acquisition period of drug naïve and KOPr pre-treated $\underline{\text { rats }}$

Rats pre-treated with KOPr agonists (striped bar) showed a significantly longer acquisition period compared to drug naïve rats (checkered bars). One-way ANOVA with the Bonferroni post-test, ${ }^{* *} p<0.01,{ }^{* * *} p<0.001$ compared to rats pre-treated with KOPr agonist. Values presented as mean \pm SEM, $n=6-11$. 


\section{Appendix 6.3: Comparison of baselines throughout the PR study}

The number of cocaine infusions prior to each KOPr agonist administration was compared to ensure that these baselines infusions did not drastically change throughout the PR study. The number of infusions was consistently stable throughout the whole study (Figure 8.10), indicating that changes in number of infusions when KOPr agonists were administered in testing sessions could be attributed to addition of the KOPr agonist.

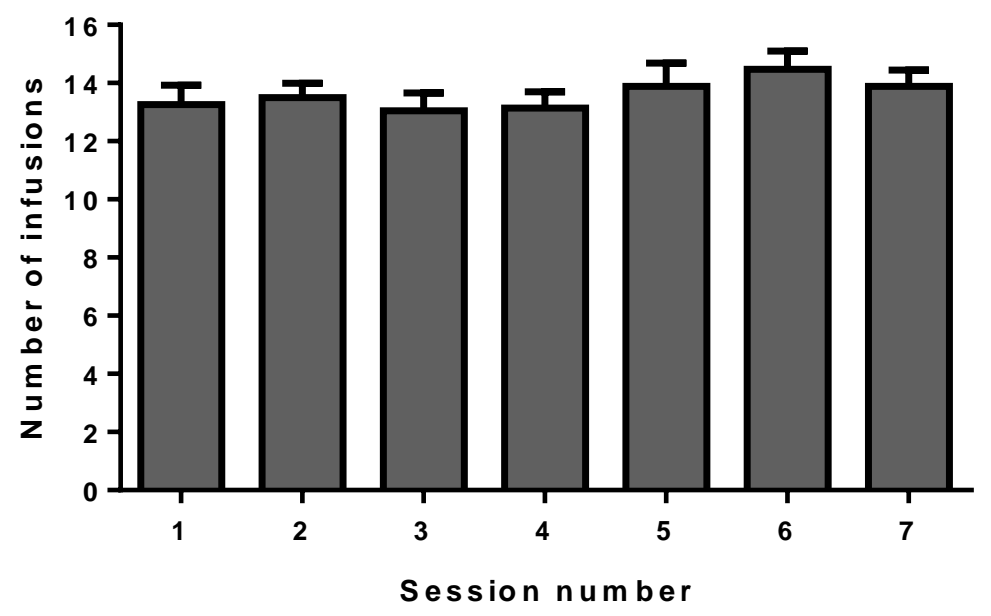

Figure 8.10: Baseline number of infusions received by rats prior to PR test sessions

Comparison of the baseline cocaine self-administration prior to each test session revealed that number of infusions received was consistent throughout all testing sessions, with no major increases or decreases throughout the experiment (a total of 10 weeks). Values presented as mean \pm SEM, $n=14$. 


\section{References}

Acri, J. B., Thompson, A. C. \& Shippenberg, T. (2001). Modulation of pre- and postsynaptic dopamine D2 receptor function by the selective kappa-opioid receptor agonist U69593. Synapse, 39, 343-350.

Addy, P. H. (2012). Acute and post-acute behavioral and psychological effects of salvinorin A in humans. Psychopharmacology, 220, 195-204.

Addy, P. H., Garcia-Romeu, A., Metzger, M. \& Wade, J. (2015). The subjective experience of acute, experimentally-induced Salvia divinorum inebriation. Psychopharmacology, 29, 426-435.

Ahlin, G., Hilgendorf, C., Karlsson, J., Szigyarto, C. A.-K., Uhlén, M. \& Artursson, P. (2009). Endogenous gene and protein expression of drug-transporting proteins in cell lines routinely used in drug discovery programs. Drug Metabolism and Disposition, 37, 2275-2283.

Ahmed, S. \& Koob, G. (1998). Transition from moderate to excessive drug intake: change in hedonic set point. Science, 282, 298-300.

Ahmed, S. H. \& Cador, M. (2006). Dissociation of psychomotor sensitization from compulsive cocaine consumption. Neuropsychopharmacology, 31, 563-571.

Ahmed, S. H. \& Koob, G. F. (2004). Changes in response to a dopamine receptor antagonist in rats with escalating cocaine intake. Psychopharmacology, 172, 450-4.

Ahmed, S. H., Lin, D., Koob, G. F. \& Parsons, L. H. (2003). Escalation of cocaine selfadministration does not depend on altered cocaine-induced nucleus accumbens dopamine levels. Journal of Neurochemistry, 86, 102-13.

Aigner, T. G. \& Balster, R. L. (1978). Choice behavior in rhesus monkeys: Cocaine versus food. Science, 201, 534-535.

Al-Hasani, R. \& Bruchas, M. R. (2011). Molecular mechanisms of opioid receptordependent signaling and behavior. Anesthesiology, 115, 1363-81.

Alper, K. R. (2001). Ibogaine: A review. The Alkaloids: Chemistry and Biology, 56, 1-38.

American Psychiatric Association, U. S. A. (2013). Diagnostic and statistical manual of mental disorders. Arlington, VA.

Anderson, A. L., Reid, M. S., Li, S. H., Holmes, T., Shemanski, L., Slee, A., Smith, E. V., Kahn, R., Chiang, N., Vocci, F., Ciraulo, D., Dackis, C., Roache, J. D., Salloum, I. M., Somoza, E., Urschel, H. C., 3rd \& Elkashef, A. M. (2009). Modafinil for the treatment of cocaine dependence. Drug and Alcohol Dependence, 104, 133-9.

Anderson, R. I., Morales, M., Spear, L. P. \& Varlinskaya, E. I. (2014). Pharmacological activation of kappa opioid receptors: Aversive effects in adolescent and adult male rats. Psychopharmacology (Berl), 231, 1687-93.

Ardila, A., Rosselli, M. \& Strumwasser, S. (1991). Neuropsychological deficits in chronic cocaine abusers. International Journal of Neuroscience, 57, 73-9.

Avidorreiss, T., Zippel, R., Levy, R., Saya, D., Ezra, V., Barg, J., Matusleibovitch, N. \& Vogel, Z. (1995). Kappa-opioid receptor-transfected cell-lines - modulation of adenylylcyclase activity following acute and chronic opioid treatments. FEBS Letters, 361, 70-74.

Axelrod, D. (2003). Total internal reflection fluorescence microscopy in cell biology. Methods in Enzymology, 361, 1-33. 
Badiani, A., Rajabi, H., Nencini, P. \& Stewart, J. (2001). Modulation of food intake by the $\mathrm{k}$ opioid U-50,488H: Evidence for an effect on satiation. Behavioural Brain Research, 118, 179-186.

Badiani, A. \& Stewart, J. (1992). The kappa-opioid U-50,488H suppresses the initiation of nocturnal spontaneous drinking in normally hydrated rats. Psychopharmacology, 106, 463-473.

Badiani, A. \& Stewart, J. (1993). Enhancement of the prophagic but not of the antidipsogenic effect of $\mathrm{U}-50,488 \mathrm{H}$ after chronic amphetamine. Pharmacology Biochemistry and Behavior, 44, 77-86.

Bailey, C. P. \& Husbands, S. M. (2014). Novel approaches for the treatment of psychostimulant and opioid abuse - Focus on opioid receptor-based therapies. Expert Opinion on Drug Discovery, 9, 1333-44.

Baker, J., Jatlow, P., Pade, P., Ramakrishnan, V. \& McCance-Katz, E. F. (2007). Acute cocaine responses following cocaethylene infusion. The American Journal of Drug and Alcohol Abuse, 33.

Baker, L. E., Panos, J. J., Killinger, B. A., Peet, M. M., Bell, L. M., Haliw, L. A. \& Walker, S. L. (2009). Comparison of the discriminative stimulus effects of salvinorin $A$ and its derivatives to U69,593 and U50,488 in rats. Psychopharmacology, 203, 203-211.

Beardsley, P., Howard, J., Shelton, K. \& Carroll, F. I. (2005). Differential effects of the novel kappa opioid receptor antagonist, JDTic, on reinstatement of cocaine-seeking induced by footshock stressors vs cocaine primes and its antidepressant-like effects in rats. Psychopharmacology, 183, 118-126.

Beardsley, P. M. \& Hauser, K. F. (2014). Glial modulators as potential treatments of psychostimulant abuse. Advances in Pharmacology, 69, 1-69.

Beaulieu, J.-M. \& Gainetdinov, R. R. (2011). The physiology, signaling, and pharmacology of dopamine receptors. Pharmacological Reviews, 63, 182-217.

Béguin, C., Duncan, K. K., Munro, T. A., Ho, D. M., Xu, W., Liu-Chen, L.-Y., Carlezon, W. A. \& Cohen, B. M. (2009). Modification of the furan ring of salvinorin A: Identification of a selective partial agonist at the kappa opioid receptor. Bioorganic and Medicinal Chemistry, 17, 1370-1380.

Beguin, C., Potter, D. N., DiNieri, J. A., Munro, T. A., Richards, M. R., Paine, T. A., Berry, L., Zhao, Z., Roth, B. L., Xu, W., Liu-Chen, L.-Y., Carlezon, W. A., Jr. \& Cohen, B. M. (2008). N-methylacetamide analog of salvinorin A: A highly potent and selective kappa-opioid receptor agonist with oral efficacy. Journal of Pharmacology and Experimental Therapeutics, 324, 188-195.

Belcheva, M. M., Clark, A. L., Haas, P. D., Serna, J. S., Hahn, J. W., Kiss, A. \& Coscia, C. J. (2005). Mu and kappa opioid receptors activate ERK/MAPK via different protein kinase $\mathrm{C}$ isoforms and secondary messengers in astrocytes. Journal of Biological Chemistry, 280, 27662-27669.

Ben-Shahar, O., Ahmed, S. H., Koob, G. F. \& Ettenberg, A. (2004). The transition from controlled to compulsive drug use is associated with a loss of sensitization. Brain Research, 995, 46-54.

Berg, K. A., Rowan, M. P., Gupta, A., Sanchez, T. A., Silva, M., Gomes, I., McGuire, B. A., Portoghese, P. S., Hargreaves, K. M., Devi, L. A. \& Clarke, W. P. (2012). Allosteric interactions between $\delta$ and $\mathrm{K}$ opioid receptors in peripheral sensory neurons. Molecular Pharmacology, 81, 264-272. 
Berridge, K. C. (1996). Food reward: Brain substrates of wanting and liking. Neuroscience and Biobehavioral Reviews, 20, 1-25.

Berridge, K. C. \& Robinson, T. E. (1995). The mind of an addicted brain: Neural sensitization of wanting versus liking. Current Directions in Psychological Science, 71-76.

Berridge, K. C. \& Robinson, T. E. (1998). What is the role of dopamine in reward: hedonic impact, reward learning, or incentive salience? Brain Research Reviews, 28, 309369.

Blake, A. D., Bot, G., Li, S., Freeman, J. C. \& Reisine, T. (1997). Differential agonist regulation of the human k-opioid receptor. Journal of Neurochemistry, 68, 18461852.

Bode, A. M. \& Dong, Z. (2007). The functional contrariety of JNK. Molecular Carcinogenesis, 46, 591-8.

Boja, J. W. \& Kuhar, M. J. (1989). $\left[{ }^{3} \mathrm{H}\right]$ cocaine binding and inhibition of $\left[{ }^{3} \mathrm{H}\right]$ dopamine uptake is similar in both the rat striatum and nucleus accumbens. European Journal of Pharmacology, 173, 215-217.

Bolan, E. A., Kivell, B., Jaligam, V., Oz, M., Jayanthi, L. D., Han, Y., Sen, N., Urizar, E., Gomes, I., Devi, L. A., Ramamoorthy, S., Javitch, J. A., Zapata, A. \& Shippenberg, T. S. (2007). D-2 receptors regulate dopamine transporter function via an extracellular signalregulated kinases 1 and 2-dependent and phosphoinositide 3 kinase-independent mechanism. Molecular Pharmacology, 71, 1222-1232.

Bonano, J. S., Runyon, S. P., Hassler, C., Glennon, R. A. \& Stevens Negus, S. (2014). Effects of the neuropeptide $S$ receptor antagonist RTI-118 on abuse-related facilitation of intracranial self-stimulation produced by cocaine and methylenedioxypyrovalerone (MDPV) in rats. European Journal of Pharmacology, 743, 98-105.

Borgbjerg, F. M. \& Frigast, C. (1997). Segmental effects on motor function following different intrathecal receptor agonists and antagonists in rabbits. Acta Anaesthesiologica Scandinavica, 41, 586-594.

Bozarth, M. A. \& Wise, R. A. (1985). Toxicity associated with long-term intravenous heroin and cocaine self-administration in the rat. Journal of the American Medical Association, 254, 81-83.

Braida, D., Capurro, V., Zani, A., Rubino, T., Vigano, D., Parolaro, D. \& Sala, M. (2009). Potential anxiolytic- and antidepressant-like effects of salvinorin $A$, the main active ingredient of Salvia divinorum, in rodents. British Journal of Pharmacology, 157, 844-853.

Braida, D., Donzelli, A., Martucci, R., Capurro, V. \& Sala, M. (2011). Learning and memory impairment induced by salvinorin $A$, the principal ingredient of Salvia divinorum, in Wistar rats. International Journal of Toxicology, 30, 650-661.

Braida, D., Limonta, V., Capurro, V., Fadda, P., Rubino, T., Mascia, P., Zani, A., Gori, E., Fratta, W., Parolaro, D. \& Sala, M. (2008). Involvement of kappa-opioid and endocannabinoid system on salvinorin A-induced reward. Biological Psychiatry, 63, 286-292.

Braida, D., Limonta, V., Pegorini, S., Zani, A., Guerini-Rocco, C., Gori, E. \& Sala, M. (2007). Hallucinatory and rewarding effect of salvinorin A in zebrafish: kappa-opioid and CB1-cannabinoid receptor involvement. Psychopharmacology, 190, 441-448. 
Brecht, M.-L. \& Herbeck, D. (2014). Time to relapse following treatment for methamphetamine use: A long-term perspective on patterns and predictors. Drug and Alcohol Dependence, 139, 18-25.

Brensilver, M., Heinzerling, K. G. \& Shoptaw, S. (2013). Pharmacotherapy of amphetamine-type stimulant dependence: An update. Drug and Alcohol Review, 32, 449-460.

Bruchas, M., Land, B. \& Chavkin, C. (2010). The dynorphin/kappa opioid system as a modulator of stress-induced and pro-addictive behaviors. Brain Research, 1314, 44-55.

Bruchas, M. R. \& Chavkin, C. (2010). Kinase cascades and ligand-directed signaling at the kappa opioid receptor. Psychopharmacology, 210, 137-147.

Bruchas, M. R., Land, B. B., Aita, M., Xu, M., Barot, S. K., Li, S. \& Chavkin, C. (2007a). Stressinduced p38 mitogen-activated protein kinase activation mediates kappa-opioiddependent dysphoria. Journal of Neuroscience, 27, 11614-11623.

Bruchas, M. R., Land, B. B., Lemos, J. C. \& Chavkin, C. (2009). CRF1-R activation of the dynorphin/kappa opioid system in the mouse basolateral amygdala mediates anxiety-like behavior. PLOS One, 4.

Bruchas, M. R., Macey, T. A., Lowe, J. D. \& Chavkin, C. (2006). Kappa opioid receptor activation of p38 MAPK is GRK3- and arrestin-dependent in neurons and astrocytes. The Journal of Biological Chemistry, 281, 18081-18089.

Bruchas, M. R., Xu, M. \& Chavkin, C. (2008). Repeated swim stress induces kappa opioidmediated activation of extracellular signal-regulated kinase 1/2. Neuroreport, 19, 1417-1422.

Bruchas, M. R., Yang, T., Schreiber, S., Defino, M., Kwan, S. C., Li, S. \& Chavkin, C. (2007b). Long-acting kappa opioid antagonists disrupt receptor signaling and produce noncompetitive effects by activating c-Jun N-terminal kinase. Journal of Biological Chemistry, 282, 29803-11.

Bulling, S., Schicker, K., Zhang, Y. W., Steinkellner, T., Stockner, T., Gruber, C. W., Boehm, S., Freissmuth, M., Rudnick, G., Sitte, H. H. \& Sandtner, W. (2012). The mechanistic basis for noncompetitive ibogaine inhibition of serotonin and dopamine transporters. Journal of Biological Chemistry, 287, 18524-34.

Burnette, W. B., Bailey, M. D., Kukoyi, S., Blakely, R. D., Trowbridge, C. G. \& Justice, J. B. (1996). Human norepinephrine transporter kinetics using rotating disk electrode voltammetry. Analytical Chemistry, 68, 2932-2938.

Butelman, E. R., Harris, T. J. \& Kreek, M. J. (2004). The plant-derived hallucinogen, salvinorin A, produces kappa-opioid agonist-like discriminative effects in rhesus monkeys. Psychopharmacology, 172, 220-4.

Butelman, E. R. \& Kreek, M. J. (2001). kappa-Opioid receptor agonist-induced prolactin release in primates is blocked by dopamine $\mathrm{D}(2)$-like receptor agonists. European Journal of Pharmacology, 423, 243-9.

Butelman, E. R., Prisinzano, T. E., Deng, H., Rus, S. \& Kreek, M. J. (2009). Unconditioned behavioral effects of the powerful kappa-opioid hallucinogen salvinorin $A$ in nonhuman primates: Fast onset and entry into cerebrospinal fluid. Journal of Pharmacology and Experimental Therapeutics, 328, 588-597.

Butelman, E. R., Riley, A. P., Prisinzano, T. E. \& Kreek, M. J. (Year). Comparative effects of a semi-synthetic salvinorin $A$ analog, 16- $B r$-salvinorin $A$ in a translational 
neuroendocrine biomarker assay, and on sedation scores. In: International Narcotics Research Conference 2015 Phoenix, Arizona, USA.

Butelman, E. R., Yuferov, V. \& Kreek, M. J. (2012). Kappa-opioid receptor/dynorphin system: Genetic and pharmacotherapeutic implications for addiction. Trends in Neurosciences, 35, 587-596.

Cabib, S., Castellano, C., Cestari, V., Filibeck, U. \& Puglisi-Allegra, S. (1991). D1 and D2 receptor antagonists differently affect cocaine-induced locomotor hyperactivity in the mouse. Psychopharmacology, 105, 335-339.

Cain, M. \& Bardo, M. (2010). Progressive-ratio schedule. In: Stolerman, I. (ed.) Encyclopedia of Psychopharmacology. Springer Berlin Heidelberg.

CARA Therapeutics, U. (2015). Pipeline and technologies. URL: http://www.caratherapeutics.com/pipeline-technologies.shtml.

Carboni, E., Tanda, G. L., Frau, R. \& Chiara, G. D. (1990). Blockade of the noradrenaline carrier increases extracellular dopamine concentrations in the prefrontal cortex: Evidence that dopamine is taken up in vivo by noradrenergic terminals. Journal of Neurochemistry, 55, 1067-1070.

Carlezon, W. A., Beguin, C., DiNieri, J. A., Baumann, M. H., Richards, M. R., Todtenkopf, M. S., Rothman, R. B., Ma, Z. Z., Lee, D. Y. W. \& Cohen, B. M. (2006). Depressive-like effects of the kappa-opioid receptor agonist salvinorin $A$ on behavior and neurochemistry in rats. Journal of Pharmacology and Experimental Therapeutics, 316, 440-447.

Carlezon, W. A., Duman, R. S. \& Nestler, E. J. (2005). The many faces of CREB. Trends in Neurosciences, 28, 436-445.

Carr, G. V., Bangasser, D. A., Bethea, T., Young, M., Valentino, R. J. \& Lucki, I. (2009). Antidepressant-like effects of kappa-opioid receptor antagonists in Wistar Kyoto rats. Neuropsychopharmacology, 35, 752-763.

Carr, G. V. \& Lucki, I. (2010). Comparison of the kappa-opioid receptor antagonist DIPPA in tests of anxiety-like behavior between Wistar Kyoto and Sprague Dawley rats. Psychopharmacology, 210, 295-302.

Carr, G. V. \& Mague, S. D. (2008). P38: The link between the k-opioid receptor and dysphoria. The Journal of Neuroscience, 28, 2299-2300.

Carroll, F. I. \& Carlezon, W. A. (2013). Development of kappa opioid receptor antagonists. Journal of Medicinal Chemistry, 56, 2178-2195.

Carter, L. P. \& Griffiths, R. R. (2009). Principles of laboratory assessment of drug abuse liability and implications for clinical development. Drug and Alcohol Dependence, 105, S14-S25.

Chartoff, E., Sawyer, A., Rachlin, A., Potter, D., Pliakas, A. \& Carlezon, W. A. (2012). Blockade of kappa opioid receptors attenuates the development of depressive-like behaviors induced by cocaine withdrawal in rats. Neuropharmacology, 62, 167-76.

Chartoff, E. H., Ebner, S. R., Sparrow, A., Potter, D., Baker, P. M., Ragozzino, M. E. \& Roitman, M. F. (2015). Relative timing between kappa opioid receptor activation and cocaine determines the impact on reward and dopamine release. Neuropsychopharmacology.

Chartoff, E. H., Potter, D., Damez-Werno, D., Cohen, B. M. \& Carlezon, W. A. (2008). Exposure to the selective kappa-opioid receptor agonist salvinorin A modulates the behavioral and molecular effects of cocaine in rats. Neuropsychopharmacology, 33, 2676-2687. 
Chavkin, C. (2011). The therapeutic potential of kappa-opioids for treatment of pain and addiction. Neuropsychopharmacology, 36, 369-70.

Chavkin, C., James, I. F. \& Goldstein, A. (1982). Dynorphin is a specific endogenous ligand of the kappa-opioid receptor. Science, 215, 413-415.

Chavkin, C., Sud, S., Jin, W. Z., Stewart, J., Zjawiony, J. K., Siebert, D. J., Toth, B. A., Hufeisen, S. J. \& Roth, B. L. (2004). Salvinorin A, an active component of the hallucinogenic sage Salvia divinorum is a highly efficacious kappa-opioid receptor agonist: Structural and functional considerations. Journal of Pharmacology and Experimental Therapeutics, 308, 1197-1203.

Chefer, V., Thompson, A. C. \& Shippenberg, T. S. (1999). Modulation of cocaine-induced sensitization by k-opioid receptor agonists: Role of the nucleus accumbens and medial prefrontal cortex. Annals of the New York Academy of Sciences, 877, 803806.

Chefer, V. I., Backman, C. M., Gigante, E. D. \& Shippenberg, T. S. (2013). Kappa opioid receptors on dopaminergic neurons are necessary for kappa-mediated place aversion. Neuropsychopharmacology, 38, 2623-2631.

Chefer, V. I., Czyzyk, T., Bolan, E. A., Moron, J., Pintar, J. E. \& Shippenberg, T. S. (2005). Endogenous kappa-opioid receptor systems regulate mesoaccumbal dopamine dynamics and vulnerability to cocaine. Journal of Neuroscience, 25, 5029-5037.

Chefer, V. I., Moron, J. A., Hope, B., Rea, W. \& Shippenberg, T. S. (2000). Kappa-opioid receptor activation prevents alterations in mesocortical dopamine neurotransmission that occur during abstinence from cocaine. Neuroscience, 101, 619-627.

Chefer, V. I. \& Shippenberg, T. S. (2002). Changes in basal and cocaine-evoked extracellular dopamine uptake and release in the rat nucleus accumbens during early abstinence from cocaine: Quantitative determination under transient conditions. Neuroscience, 112, 907-919.

Chen, J., Zhang, R., Chen, X., Wang, C., Cai, X., Liu, H., Jiang, Y., Liu, C. \& Bai, B. (2015). Heterodimerization of human orexin receptor 1 and kappa opioid receptor promotes protein kinase A/cAMP-response element binding protein signaling via a Galphas-mediated mechanism. Cell Signalling, 27, 1426-1438.

Chen, R., Furman, C. A. \& Gnegy, M. E. (2010). Dopamine transporter trafficking: Rapid response on demand. Future Neurology, 5, 123-134.

Chen, Y. L., Law, P.-Y. \& Loh, H. H. (2006). Nuclear factor KB signaling in opioid functions and receptor gene expression. Journal of Neuroimmune Pharmacology, 1, 270279.

Ciliax, B. J., Drash, G. W., Staley, J. K., Haber, S., Mobley, C. J., Miller, G. W., Mufson, E. J., Mash, D. C. \& Levey, A. I. (1999). Immunocytochemical localization of the dopamine transporter in human brain. Journal of Comparative Neurology, 409, 3856.

Ciliax, B. J., Heilman, C., Demchyshyn, L. L., Pristupa, Z. B., Ince, E., Hersch, S. M., Niznik, H. B. \& Levey, A. I. (1995). The dopamine transporter: Immunochemical characterization and localization in brain. Journal of Neuroscience, 15, 1714-23.

Clayton, C. C., Xu, M. \& Chavkin, C. (2009). Tyrosine phosphorylation of Kir3 following Kopioid receptor activation of p38 MAPK causes heterologous desensitization. Journal of Biological Chemistry, 284, 31872-31881. 
Codd, E. E. (1995). High affinity ibogaine binding to a mu opioid agonist site. Life Sciences, 57, $\mathrm{Pl} 315-20$.

Collins, R. J., Weeks, J. R., Cooper, M. M., Good, P. I. \& Russell, R. R. (1983). Prediction of abuse liability of drugs using IV self-administration by rats. Psychopharmacology, 82, 6-13.

Collins, S. L., D'Addario, C. \& Izenwasser, S. (2001a). Effects of kappa-opioid receptor agonists on long-term cocaine use and dopamine neurotransmission. European Journal of Pharmacology, 426, 25-34.

Collins, S. L., Gerdes, R. M., D'Addario, C. \& Izenwasser, S. (2001b). Kappa opioid agonists alter dopamine markers and cocaine-stimulated locomotor activity. Behavioural Pharmacology, 12, 237-45.

Collins, S. L., Kunko, P. M., Ladenheim, B., Cadet, J. L., Carroll, F. I. \& Izenwasser, S. (2002). Chronic cocaine increases kappa-opioid receptor density: Lack of effect by selective dopamine uptake inhibitors. Synapse, 45, 153-158.

Cooper, S. J., Jackson, A. \& Kirkham, T. C. (1985). Endorphins and food intake: Kappa opioid receptor agonists and hyperphagia. Pharmacology, Biochemistry and Behavior, 23, 889-901.

Crawford, C. A., McDougall, S. A., Bolanos, C. A., Hall, S. \& Berger, S. P. (1995). The effects of the kappa agonist $U-50,488$ on cocaine-induced conditioned and unconditioned behaviors and Fos immunoreactivity. Psychopharmacology, 120, 392-9.

Cunha, T. M., Souza, G. R., Domingues, A. C., Carreira, E. U., Lotufo, C. M., Funez, M. I., Verri, W. A., Jr., Cunha, F. Q. \& Ferreira, S. H. (2012). Stimulation of peripheral kappa opioid receptors inhibits inflammatory hyperalgesia via activation of the $\mathrm{PI3Kgamma/AKT/nNOS/NO} \mathrm{signaling} \mathrm{pathway.} \mathrm{Molecular} \mathrm{Pain,} \mathrm{8,} 10$.

Cunningham, C. W., Rothman, R. B. \& Prisinzano, T. E. (2011). Neuropharmacology of the naturally occurring k-opioid hallucinogen salvinorin A. Pharmacological Reviews, 63, 316-347.

Dackis, C. A., Lynch, K. G., Yu, E., Samaha, F. F., Kampman, K. M., Cornish, J. W., Rowan, A., Poole, S., White, L. \& O'Brien, C. P. (2003). Modafinil and cocaine: A doubleblind, placebo-controlled drug interaction study. Drug and Alcohol Dependence, 70, 29-37.

Dar, M. S. (1998). Involvement of K-opioids in the mouse cerebellar adenosinergic modulation of ethanol-induced motor incoordination. Alcoholism: Clinical and Experimental Research, 22, 444-454.

Davis, C. M., Rice, K. C. \& Riley, A. L. (2009). Opiate-agonist induced taste aversion learning in the Fischer 344 and Lewis inbred rat strains: Evidence for differential mu opioid receptor activation. Pharmacology, Biochemistry and Behavior, 93, 397-405.

Daws, L. C., Callaghan, P. D., Morón, J. A., Kahlig, K. M., Shippenberg, T. S., Javitch, J. A. \& Galli, A. (2002). Cocaine increases dopamine uptake and cell surface expression of dopamine transporters. Biochemical and Biophysical Research Communications, 290, 1545-1550.

de Boer, S. F. \& Koolhaas, J. M. (2003). Defensive burying in rodents: Ethology, neurobiology and psychopharmacology. European Journal of Pharmacology, 463, 145-61.

de Wit, H. (1996). Priming effects with drugs and other reinforcers. Experimental and Clinical Psychopharmacology, 4, 5. 
de Wit, H. \& Stewart, J. (1981). Reinstatement of cocaine-reinforced responding in the rat. Psychopharmacology, 75, 134-143.

Degenhardt, L. \& Hall, W. (2012). Extent of illicit drug use and dependence, and their contribution to the global burden of disease. Lancet, 379, 55-70.

Deneau, G., Yanagita, T. \& Seevers, M. (1969). Self-administration of psychoactive substances by the monkey. Psychopharmacologia, 16, 30-48.

Detke, M. J., Rickels, M. \& Lucki, I. (1995). Active behaviors in the rat forced swimming test differentially produced by serotonergic and noradrenergic antidepressants. Psychopharmacology, 121, 66-72.

Di Chiara, G. \& Imperato, A. (1988a). Drugs abused by humans preferentially increase synaptic dopamine concentrations in the mesolimbic system of freely moving rats. Proceedings of the National Academy of Sciences, 85, 5274-5278.

Di Chiara, G. \& Imperato, A. (1988b). Opposite effects of mu-opiate and kappa-opiate agonists on dopamine release in the nucleus accumbens and in the dorsal caudate of freely moving rats. Journal of Pharmacology and Experimental Therapeutics, 244, 1067-1080.

DiMattio, K. M., Ehlert, F. J. \& Liu-Chen, L.-Y. (2015). Intrinsic relative activities of $\mathrm{k}$ opioid agonists in activating $\mathrm{G} \alpha$ proteins and internalizing receptor: Differences between human and mouse receptors. European Journal of Pharmacology, 761, 235-244.

DiNieri, J. A., Nemeth, C., Parsegian, A., Carle, T., Gurevich, V. V., Gurevich, E., Neve, R. L., Nestler, E. J. \& Carlezon, W. A. (2009). Altered sensitivity to rewarding and aversive drugs in mice with inducible disruption of CREB function within the nucleus accumbens. Journal of Neuroscience, 29, 1855-1859.

Do Carmo, G. P., Folk, J. E., Rice, K. C., Chartoff, E., Carlezon, W. A., Jr. \& Negus, S. S. (2009). The selective non-peptidic delta opioid agonist SNC80 does not facilitate intracranial self-stimulation in rats. European Journal of Pharmacology, 604, 5865.

Dobrin, C. V. \& Roberts, D. C. (2012). Cocaine self-administration in rats: Discrete trials procedures. Methods in Molecular Biology, 829, 291-302.

Dykstra, L., Gmerek, D. E., Winger, G. \& Woods, J. H. (1987). Kappa opioids in rhesus monkeys. I. Diuresis, sedation, analgesia and discriminative stimulus effects. Journal of Pharmacology and Experimental Therapeutics, 242, 413-420.

Earles, C. \& Schenk, J. O. (1998). Rotating disk electrode voltammetric measurements of dopamine transporter activity: An analytical evaluation. Analytical Biochemistry, 264, 191-198.

Ebner, S. R., Roitman, M. F., Potter, D. N., Rachlin, A. B. \& Chartoff, E. H. (2010). Depressive-like effects of the kappa opioid receptor agonist salvinorin $A$ are associated with decreased phasic dopamine release in the nucleus accumbens. Psychopharmacology, 210, 241-252.

Ehrich, J. M., Phillips, P. E. \& Chavkin, C. (2014). Kappa opioid receptor activation potentiates the cocaine-induced increase in evoked dopamine release recorded in vivo in the mouse nucleus accumbens. Neuropsychopharmacology, 39, 3036-48.

Elsworth, J. D. \& Roth, R. H. (1997). Dopamine synthesis, uptake, metabolism, and receptors: Relevance to gene therapy of Parkinson's disease. Experimental Neurology, 144, 4-9. 
Endoh, T., Matsuura, H., Tanaka, C. \& Nagase, H. (1992). Nor-binaltorphimine: A potent and selective kappa-opioid receptor antagonist with long-lasting activity in vivo. Archives Internationales de Pharmacodynamie et de Thérapie, 316, 30-42.

Epling, C. \& Jativa-M, C. D. (1962). A new species of Salvia S. divinorum sp. nov. (Labiatae) from Mexico. Botanical Museum Leaflets Harvard University, 20, 75-76.

Epstein, D. H., Preston, K. L., Stewart, J. \& Shaham, Y. (2006). Toward a model of drug relapse: An assessment of the validity of the reinstatement procedure. Psychopharmacology, 189, 1-16.

Erb, S., Shaham, Y. \& Stewart, J. (1996). Stress reinstates cocaine-seeking behavior after prolonged extinction and a drug-free period. Psychopharmacology, 128, 408-412.

Eriksen, J., Bjorn-Yoshimoto, W. E., Jorgensen, T. N., Newman, A. H. \& Gether, U. (2010a). Postendocytic sorting of constitutively internalized dopamine transporter in cell lines and dopaminergic neurons. Journal of Biological Chemistry, 285, 27289-301.

Eriksen, J., Jorgensen, T. N. \& Gether, U. (2010b). Regulation of dopamine transporter function by protein-protein interactions: new discoveries and methodological challenges. Journal of Neurochemistry, 113, 27-41.

Fantegrossi, W. E., Kugle, K. M., Valdes, L. J., Koreeda, M. \& Woods, J. H. (2005). Kappaopioid receptor-mediated effects of the plant-derived hallucinogen, salvinorin A, on inverted screen performance in the mouse. Behavioural Pharmacology, 16, 627-633.

Farooq, M. U., Bhatt, A. \& Patel, M. (2009). Neurotoxic and cardiotoxic effects of cocaine and ethanol. Journal of Medical Toxicology, 5, 134-8.

Ferragud, A., Velazquez-Sanchez, C., Hernandez-Rabaza, V., Nacher, A., Merino, V., Carda, M., Murga, J. \& Canales, J. J. (2009). A dopamine transport inhibitor with markedly low abuse liability suppresses cocaine self-administration in the rat. Psychopharmacology, 207, 281-289.

Ferrario, C. R., Gorny, G., Crombag, H. S., Li, Y., Kolb, B. \& Robinson, T. E. (2005). Neural and behavioral plasticity associated with the transition from controlled to escalated cocaine use. Biological Psychiatry 58, 751-9.

Ferster, C. B. (1957). Schedules of reinforcement. New York: New York, Appleton-CenturyCrofts.

File, S., Lippa, A., Beer, B. \& Lippa, M. (2005). Animal tests of anxiety. Current Protocols in Pharmacology, Unit 5.38-Unit 5.38.

Fowler, S. C., Covington, H. E., 3rd \& Miczek, K. A. (2007). Stereotyped and complex motor routines expressed during cocaine self-administration: results from a 24-h binge of unlimited cocaine access in rats. Psychopharmacology, 192, 465-78.

Freeman, K., Naylor, J., Prisinzano, T. \& Woolverton, W. (2014). Assessment of the kappa opioid agonist, salvinorin A, as a punisher of drug self-administration in monkeys. Psychopharmacology, 231, 2751-2758.

Fujii, H., Nagase, H. \& Hirayama, S. (2012). Opioid kappa receptor selective agonist TRK820 (nalfurafine hydrochloride). INTECH Open Access Publisher.

Furman, C. A., Chen, R., Guptaroy, B., Zhang, M., Holz, R. W. \& Gnegy, M. (2009). Dopamine and amphetamine rapidly increase dopamine transporter trafficking to the surface: Live-cell imaging using total internal reflection fluorescence microscopy. Journal of Neuroscience, 29, 3328-3336. 
Gallantine, E. L. \& Meert, T. F. (2008). Antinociceptive and adverse effects of $\mu$ - and kopioid receptor agonists: A comparison of morphine and U50488-H. Basic and Clinical Pharmacology and Toxicology, 103, 419-427.

Gancarz, A. M., Kausch, M. A., Lloyd, D. R. \& Richards, J. B. (2012). Between-session progressive ratio performance in rats responding for cocaine and water reinforcers. Psychopharmacology, 222, 215-223.

Gehrke, B. J., Chefer, V. I. \& Shippenberg, T. S. (2008). Effects of acute and repeated administration of salvinorin $A$ on dopamine function in the rat dorsal striatum. Psychopharmacology, 197, 509-517.

Gillett, K., Harshberger, E. \& Valdez, G. R. (2013). Protracted withdrawal from ethanol and enhanced responsiveness stress: Regulation via the dynorphin/kappa opioid receptor system. Alcohol, 47, 359-65.

Glass, M. J., Billington, C. J. \& Levine, A. S. (1999). Opioids and food intake: Distributed functional neural pathways? Neuropeptides, 33, 360-368.

Glick, S. D. \& Maisonneuve, I. M. (2000). Development of novel medications for drug addiction. The legacy of an African shrub. Annals of the New York Academy of Sciences, 909, 88-103.

Glick, S. D., Maisonneuve, I. M. \& Pearl, S. M. (1997). Evidence for roles of kappa-opioid and NMDA receptors in the mechanism of action of ibogaine. Brain Research, 749, 340-3.

Glick, S. D., Maisonneuve, I. M., Raucci, J. \& Archer, S. (1995). Kappa-opioid inhibition of morphine and cocaine self-administration in rats. Brain Research, 681, 147-152.

Glick, S. D., Visker, K. E. \& Maisonneuve, I. M. (1998). Effects of cyclazocine on cocaine self-administration in rats. European Journal of Pharmacology, 357, 9-14.

Global Drug Survey, G. (2014). Last 12 month prevalence of top 20 drugs In: Winstock, D. A. (ed.).

Glue, P., Lockhart, M., Lam, F., Hung, N., Hung, C.-T. \& Friedhoff, L. (2015). Ascending-dose study of noribogaine in healthy volunteers: Pharmacokinetics, pharmacodynamics, safety, and tolerability. The Journal of Clinical Pharmacology, 55, 189-194.

Goktalay, G., Cavun, S., Levendusky, M. C., Hamilton, J. R. \& Millington, W. R. (2006). Glycyl-glutamine inhibits nicotine conditioned place preference and withdrawal. European Journal of Pharmacology, 530, 95-102.

Gore-Langton, J. K., Flax, S. M., Pomfrey, R. L., Wetzell, B. B. \& Riley, A. L. (2015). Measures of the aversive effects of drugs: A comparison of conditioned taste and place aversions. Pharmacology Biochemistry and Behavior, 134, 99-105.

Grabowski, J., Roache, J. D., Schmitz, J. M., Rhoades, H., Creson, D. \& Korszun, A. (1997). Replacement medication for cocaine dependence: Methylphenidate. Journal of Clinical Psychopharmacology, 17, 485-488.

Graham, F. L., Smiley, J., Russell, W. C. \& Nairn, R. (1977). Characteristics of a human cell line transformed by DNA from human adenovirus type 5. Journal of General Virology, 36, 59-74.

Gramsch, C., Hollt, V., Pasi, A., Mehraein, P. \& Herz, A. (1982). Immunoreactive dynorphin in human-brain and pituitary. Brain Research, 233, 65-74.

Graves, S. M., Rafeyan, R., Watts, J. \& Napier, T. C. (2012). Mirtazapine, and mirtazapinelike compounds as possible pharmacotherapy for substance abuse disorders: 
Evidence from the bench and the bedside. Pharmacology and Therapeutics, 136, 343-53.

Groer, C. E., Tidgewell, K., Moyer, R. A., Harding, W. W., Rothman, R. B., Prisinzano, T. E. \& Bohn, L. M. (2007). An opioid agonist that does not induce mu-opioid receptorarrestin interactions or receptor internalization. Molecular Pharmacology, 71, 549-57.

Gulley, J. M. \& Zahniser, N. R. (2003). Rapid regulation of dopamine transporter function by substrates, blockers and presynaptic receptor ligands. European Journal of Pharmacology, 479, 139-152.

Hagan, C. E., Neumaier, J. F. \& Schenk, J. O. (2010). Rotating disk electrode voltammetric measurements of serotonin transporter kinetics in synaptosomes. Journal of Neuroscience Methods, 193, 29-38.

Hall, F. S., Li, X. F., Sora, I., Xu, F., Caron, M., Lesch, K. P., Murphy, D. L. \& Uhl, G. R. (2002). Cocaine mechanisms: Enhanced cocaine, fluoxetine and nisoxetine place preferences following monoamine transporter deletions. Neuroscience, 115, 153161.

Hall, F. S., Sora, I., Drgonova, J., Li, X. F., Goeb, M. \& Uhl, G. R. (2004). Molecular mechanisms underlying the rewarding effects of cocaine. Annals of the New York Academy of Sciences, 1025, 47-56.

Hanes, K. R. (2001). Antidepressant effects of the herb Salvia divinorum: A case report. Journal of Clinical Psychopharmacology, 21, 634-635.

Haney, M. \& Spealman, R. (2008). Controversies in translational research: Drug selfadministration. Psychopharmacology, 199, 403-419.

Harden, M. T., Smith, S. E., Niehoff, J. A., McCurdy, C. R. \& Taylor, G. T. (2012). Antidepressive effects of the kappa-opioid receptor agonist salvinorin $A$ in a rat model of anhedonia. Behavioural Pharmacology 23, 710-5.

Harding, W. W., Tidgewell, K., Byrd, N., Cobb, H., Dersch, C. M., Butelman, E. R., Rothman, R. B. \& Prisinzano, T. E. (2005). Neoclerodane diterpenes as a novel scaffold for mu opioid receptor ligands. Journal of Medicinal Chemistry, 48, 4765-4771.

Heal, D. J., Gosden, J. \& Smith, S. L. (2014). Dopamine reuptake transporter (DAT) "inverse agonism" - A novel hypothesis to explain the enigmatic pharmacology of cocaine. Neuropharmacology, 87, 19-40.

Heidbreder, C. A., Babovicvuksanovic, D., Shoaib, M. \& Shippenberg, T. S. (1995). Development of behavioral sensitization to cocaine - Influence of kappa-opioid receptor agonists. Journal of Pharmacology and Experimental Therapeutics, 275, 150-163.

Heidbreder, C. A., Goldberg, S. R. \& Shippenberg, T. S. (1993). The kappa-opioid receptor agonist U-69593 attenuates cocaine-induced behavioral sensitization in the rat. Brain Research, 616, 335-338.

Heidbreder, C. A., Schenk, S., Partridge, B. \& Shippenberg, T. S. (1998). Increased responsiveness of mesolimbic and mesostriatal dopamine neurons to cocaine following repeated administration of a selective kappa-opioid receptor agonist. Synapse, 30, 255-262.

Henderson-Redmond, A. \& Czachowski, C. (2014). Effects of systemic opioid receptor ligands on ethanol-and sucrose seeking and drinking in alcohol-preferring $(P)$ and Long Evans rats. Psychopharmacology, 231, 4309-4321. 
Henry, P. K., Davis, M. \& Howell, L. L. (2009). Effects of cocaine self-administration history under limited and extended access conditions on in vivo striatal dopamine neurochemistry and acoustic startle in rhesus monkeys. Psychopharmacology, 205, 237-247.

Higgins, S. T., Alessi, S. M. \& Dantona, R. L. (2002). Voucher-based incentives. A substance abuse treatment innovation. Addictive Behaviors, 27, 887-910.

Himmelsbach, C. (1941). The morphine abstinence syndrome, its nature and treatment. Annals of Internal Medicine, 15, 829-839.

Hobbs, M., Remington, B. \& Glautier, S. (2005). Dissociation of wanting and liking for alcohol in humans: $A$ test of the incentive-sensitisation theory. Psychopharmacology, 178, 493-499.

Hooker, J. M., Munro, T. A., Béguin, C., Alexoff, D., Shea, C., Xu, Y. \& Cohen, B. M. (2009a). Salvinorin $A$ and derivatives: Protection from metabolism does not prolong shortterm, whole-brain residence. Neuropharmacology, 57, 386-391.

Hooker, J. M., Patel, V., Kothari, S. \& Schiffer, W. K. (2009b). Metabolic changes in the rodent brain after acute administration of salvinorin A. Molecular Imaging and Biology, 11, 137-143.

Hooker, J. M., Xu, Y., Schiffer, W., Shea, C., Carter, P. \& Fowler, J. S. (2008). Pharmacokinetics of the potent hallucinogen, salvinorin $A$ in primates parallels the rapid onset and short duration of effects in humans. Neurolmage, 41, 1044-1050.

Horan, P., Taylor, J., Yamamura, H. I. \& Porreca, F. (1992). Extremely long-lasting antagonistic actions of nor-binaltorphimine (nor-BNI) in the mouse tail-flick test. Journal of Pharmacology and Experimental Therapeutics 260, 1237-43.

Houdi, A. A., Bardo, M. T. \& Van Loon, G. R. (1989). Opioid mediation of cocaine-induced hyperactivity and reinforcement. Brain Research, 497, 195-198.

Huang, X., Gu, H. H. \& Zhan, C.-G. (2009). Mechanism for cocaine blocking the transport of dopamine: Insights from molecular modeling and dynamics simulations. Journal of Physical Chemistry $B, 113$.

Hudmon, A., Choi, J.-S., Tyrrell, L., Black, J. A., Rush, A. M., Waxman, S. G. \& Dib-Hajj, S. D. (2008). Phosphorylation of sodium channel Nav1. 8 by p38 mitogen-activated protein kinase increases current density in dorsal root ganglion neurons. Journal of Neuroscience, 28, 3190-3201.

Hurd, Y. L. \& Herkenham, M. (1993). Molecular alterations in the neostriatum of human cocaine addicts. Synapse, 13, 357-369.

Hutsell, B. A., Cheng, K., Rice, K. C., Negus, S. S. \& Banks, M. L. (2015). Effects of the kappa opioid receptor antagonist nor-binaltorphimine (nor-BNI) on cocaine versus food choice and extended-access cocaine intake in rhesus monkeys. Addiction Biology, n/a-n/a.

Isaac, W. L., Nonneman, A. J., Neisewander, J., Landers, T. \& Bardo, M. T. (1989). Prefrontal cortex lesions differentially disrupt cocaine-reinforced conditioned place preference but not conditioned taste aversion. Behavioural Neuroscience, 103, 345-55.

Ismayilova, N. \& Shoaib, M. (2010). Alteration of intravenous nicotine self-administration by opioid receptor agonist and antagonists in rats. Psychopharmacology, 210, 211220.

Iwamoto, E. T. (1981). Locomotor activity and antinociception after putative mu, kappa and sigma opioid receptor agonists in the rat: Influence of dopaminergic agonists 
and antagonists. Journal of Pharmacology and Experimental Therapeutics, 217, 451-460.

Izenwasser, S., Acri, J. B., Kunko, P. M. \& Shippenberg, T. (1998). Repeated treatment with the selective kappa opioid agonist U-69593 produces a marked depletion of dopamine D2 receptors. Synapse, 30, 275-283.

Jacobsen, L. K., Giedd, J. N., Gottschalk, C., Kosten, T. R. \& Krystal, J. H. (2001). Quantitative morphology of the caudate and putamen in patients with cocaine dependence. American Journal of Psychiatry, 158, 486-489.

Johanson, C. E., Balster, R. L. \& Bonese, K. (1976). Self-administration of psychomotor stimulant drugs: The effects of unlimited access. Pharmacology Biochemistry and Behavior, 4, 45-51.

John, T. F., French, L. G. \& Erlichman, J. S. (2006). The antinociceptive effect of salvinorin A in mice. European Journal of Pharmacology, 545, 129-133.

Johnson, K. W., Matsuda, K. \& Iwaki, Y. (2014). Ibudilast for the treatment of drug addiction and other neurological conditions. Clinical Investigation, 4, 269-279.

Johnson, M. W., MacLean, K. A., Reissig, C. J., Prisinzano, T. E. \& Griffiths, R. R. (2011). Human psychopharmacology and dose-effects of salvinorin A, a kappa opioid agonist hallucinogen present in the plant Salvia divinorum. Drug and Alcohol Dependence, 115, 150-155.

Jones, K. T., Zhen, J. \& Reith, M. E. A. (2012). Importance of cholesterol in dopamine transporter function. Journal of Neurochemistry, 123, 700-715.

Jordan, B. A. \& Devi, L. A. (1999). G-protein-coupled receptor heterodimerization modulates receptor function. Nature, 399, 697-700.

Kam, A. Y., Chan, A. S. \& Wong, Y. H. (2004a). Kappa-opioid receptor signals through Src and focal adhesion kinase to stimulate $\mathrm{c}$-Jun $\mathrm{N}$-terminal kinases in transfected COS-7 cells and human monocytic THP-1 cells. Journal of Pharmacology and Experimental Therapeutics, 310, 301-10.

Kam, A. Y., Chan, A. S. \& Wong, Y. H. (2004b). Phosphatidylinositol-3 kinase is distinctively required for $\mathrm{mu}$-, but not kappa-opioid receptor-induced activation of c-Jun $\mathrm{N}$ terminal kinase. Journal of Neurochemistry, 89, 391-402.

Katz, J. L. \& Higgins, S. T. (2003). The validity of the reinstatement model of craving and relapse to drug use. Psychopharmacology, 168, 21-30.

Kenakin, T. (2007). Collateral efficacy in drug discovery: Taking advantage of the good (allosteric) nature of 7TM receptors. Trends in Pharmacological Sciences, 28, 407415.

Killinger, B. A., Peet, M. M. \& Baker, L. E. (2010). Salvinorin A fails to substitute for the discriminative stimulus effects of LSD or ketamine in Sprague-Dawley rats. Pharmacology Biochemistry and Behavior, 96, 260-5.

Kippin, T. E., Fuchs, R. A. \& See, R. E. (2006). Contributions of prolonged contingent and noncontingent cocaine exposure to enhanced reinstatement of cocaine seeking in rats. Psychopharmacology, 187, 60-67.

Kishioka, S., Kiguchi, N., Kobayashi, Y., Yamamoto, C., Saika, F., Wakida, N., Ko, M. C. \& Woods, J. H. (2013). Pharmacokinetic evidence for the long-lasting effect of norbinaltorphimine, a potent kappa opioid receptor antagonist, in mice. Neuroscience Letters, 552, 98-102. 
Kivell, B., Ewald, A. \& Prisinzano, T. (2013). Salvinorin A analogs and other kappa-opioid receptor compounds as treatments for cocaine abuse. Advances in Pharmacology, 69, 481-511.

Kivell, B., Kahlig, K., Galli, A., Javitch, J. A. \& Shippenberg, T. (2004). Regulation of dopamine transporter function and cell surface expression by kappa opioid receptors. Society for Neuroscience Abstract Viewer and Itinerary Planner, 280.12.

Kivell, B. \& Prisinzano, T. E. (2010). Kappa opioids and the modulation of pain. Psychopharmacology, 210, 109-119.

Kivell, B., Uzelac, Z., Sundaramurthy, S., Rajamanickam, J., Ewald, A., Chefer, V., Jaligam, V., Bolan, E., Simonson, B. \& Annamalai, B. (2014). Salvinorin A regulates dopamine transporter function via a kappa opioid receptor and ERK1/2-dependent mechanism. Neuropharmacology, 86, 228-240.

Kloner, R. A., Hale, S., Alker, K. \& Rezkalla, S. (1992). The effects of acute and chronic cocaine use on the heart. Circulation, 85, 407-419.

Knackstedt, L. A. \& Kalivas, P. W. (2007). Extended access to cocaine self-administration enhances drug-primed reinstatement but not behavioral sensitization. Journal of Pharmacology and Experimental Therapeutics 322, 1103-9.

Knoll, A. T. \& Carlezon Jr, W. A. (2010). Dynorphin, stress, and depression. Brain Research, 1314, 56-73.

Knoll, A. T., Meloni, E. G., Thomas, J. B., Carroll, F. I. \& Carlezon, W. A. (2007). Anxiolyticlike effects of kappa-opioid receptor antagonists in models of unlearned and learned fear in rats. Journal of Pharmacology and Experimental Therapeutics 323, 838-845.

Koenig, X. \& Hilber, K. (2015). The anti-addiction drug ibogaine and the heart: A delicate relation. Molecules, 20, 2208-28.

Koob, G. \& Kreek, M. J. (2007). Stress, dysregulation of drug reward pathways, and the transition to drug dependence. American Journal of Psychiatry, 164, 1149-1159.

Koob, G. F. (2008). A role for brain stress systems in addiction. Neuron, 59, 11-34.

Koob, G. F. (2015). The dark side of emotion: The addiction perspective. European Journal of Pharmacology, 753, 73-87.

Koob, G. F., Ahmed, S. H., Boutrel, B., Chen, S. A., Kenny, P. J., Markou, A., O'Dell, L. E., Parsons, L. H. \& Sanna, P. P. (2004). Neurobiological mechanisms in the transition from drug use to drug dependence. Neuroscience and Biobehavioral Reviews 27, 739-49.

Koob, G. F., Le, H. T. \& Creese, I. (1987). The D1 dopamine receptor antagonist SCH 23390 increases cocaine self-administration in the rat. Neuroscience Letters, 79, 315-320.

Koob, G. F. \& Le Moal, M. (1997). Drug abuse: Hedonic homeostatic dysregulation. Science, 278, 52-58.

Koob, G. F. \& Le Moal, M. (2001). Drug addiction, dysregulation of reward, and allostasis. Neuropsychopharmacology, 24, 97-129.

Koob, G. F. \& Le Moal, M. (2005). Plasticity of reward neurocircuitry and the 'dark side' of drug addiction. Nature Neuroscience, 8, 1442-4.

Koob, G. F. \& Le Moal, M. (2008). Addiction and the brain antireward system. Annual Review of Psychology, 59, 29-53.

Kreibich, A. S. \& Blendy, J. A. (2004). cAMP response element-binding protein is required for stress but not cocaine-induced reinstatement. Journal of Neuroscience, 24, 6686-92. 
Kuhar, M. J., Ritz, M. C. \& Boja, J. W. (1991). The dopamine hypothesis of the reinforcing properties of cocaine. Trends in Neurosciences, 14, 299-302.

Kumagai, H., Ebata, T., Takamori, K., Miyasato, K., Muramatsu, T., Nakamoto, H., Kurihara, M., Yanagita, T. \& Suzuki, H. (2011). Efficacy and safety of a novel k-agonist for managing intractable pruritus in dialysis patients. American Journal of Nephrology, 36, 175-183.

Kumagai, H., Ebata, T., Takamori, K., Muramatsu, T., Nakamoto, H. \& Suzuki, H. (2009). Effect of a novel kappa-receptor agonist, nalfurafine hydrochloride, on severe itch in 337 haemodialysis patients: A Phase III, randomized, double-blind, placebocontrolled study. Nephrology Dialysis Transplantation, gfp588.

Kuzmin, A. V., Gerrits, M. A. F. M. \& Van Ree, J. M. (1998). k-Opioid receptor blockade with nor-binaltorphimine modulates cocaine self-administration in drug-naive rats. European Journal of Pharmacology, 358, 197-202.

Kuzmin, A. V., Semenova, S., Gerrits, M., Zvartau, E. E. \& VanRee, J. M. (1997). Kappaopioid receptor agonist $\mathrm{U} 50,488 \mathrm{H}$ modulates cocaine and morphine selfadministration in drug-naive rats and mice. European Journal of Pharmacology, 321, 265-271.

Lamb, K., Tidgewell, K., Simpson, D. S., Bohn, L. M. \& Prisinzano, T. E. (2012). Antinociceptive effects of herkinorin, a MOP receptor agonist derived from salvinorin $A$ in the formalin test in rats: New concepts in mu opioid receptor pharmacology. Drug and Alcohol Dependence, 121, 181-188.

Land, B. B., Bruchas, M. R., Lemos, J. C., Xu, M., Melief, E. J. \& Chavkin, C. (2008). The dysphoric component of stress is encoded by activation of the dynorphin kappaopioid system. Journal of Neuroscience, 28, 407-414.

Land, B. B., Bruchas, M. R., Schattauer, S., Giardino, W. J., Aita, M., Messinger, D., Hnasko, T. S., Palmiter, R. D. \& Chavkin, C. (2009). Activation of the kappa opioid receptor in the dorsal raphe nucleus mediates the aversive effects of stress and reinstates drug seeking. Proceedings of the National Academy of Sciences of the United States of America, 106, 19168-73.

Larsen, M. B., Sonders, M. S., Mortensen, O. V., Larson, G. A., Zahniser, N. R. \& Amara, S. G. (2011). Dopamine transport by the serotonin transporter: A mechanistically distinct mode of substrate translocation. Journal of Neuroscience 31, 6605-15.

Leal, M. B., Michelin, K., Souza, D. O. \& Elisabetsky, E. (2003). Ibogaine attenuation of morphine withdrawal in mice: Role of glutamate $\mathrm{N}$-methyl-D-aspartate receptors. Progress in Neuro-Psychopharmacology and Biological Psychiatry, 27, 781-5.

Lee, D. Y., Karnati, V. V., He, M., Liu-Chen, L.-Y., Kondaveti, L., Ma, Z., Wang, Y., Chen, Y., Beguin, C. \& Carlezon, W. A. (2005). Synthesis and in vitro pharmacological studies of new C (2) modified salvinorin A analogues. Bioorganic and Medicinal Chemistry Letters, 15, 3744-3747.

Lefloch, R., Pouyssegur, J. \& Lenormand, P. (2008). Single and combined silencing of ERK1 and ERK2 reveals their positive contribution to growth signaling depending on their expression levels. Molecular and Cellular Biology, 28, 511-527.

Leppa, S. \& Bohmann, D. (1999). Diverse functions of JNK signaling and c-Jun in stress response and apoptosis. Oncogene, 18, 6158-62.

Leri, F. \& Franklin, K. B. (2000). Diazepam in the ventral striatum dissociates dopaminedependent and dopamine-independent place conditioning. Neuroreport, 11, 2553-7. 
Leshner, A. I. (1997). Addiction is a brain disease, and it matters. Science, 278, 45-47.

Letchworth, S. R., Nader, M. A., Smith, H. R., Friedman, D. P. \& Porrino, L. J. (2001). Progression of changes in dopamine transporter binding site density as a result of cocaine self-administration in rhesus monkeys. Journal of Neuroscience, 21, 27992807.

Levin, F. R., Evans, S. M., Brooks, D. J. \& Garawi, F. (2007). Treatment of cocaine dependent treatment seekers with adult ADHD: Double-blind comparison of methylphenidate and placebo. Drug and Alcohol Dependence, 87, 20-29.

Li, J.-G., Benovic, J. L. \& Liu-Chen, L.-Y. (2000). Mechanisms of agonist-induced downregulation of the human $\mathrm{K}$-opioid receptor: Internalization is required for downregulation. Molecular Pharmacology, 58, 795-801.

Li, J.-G., Luo, L.-Y., Krupnick, J. G., Benovic, J. L. \& Liu-Chen, L.-Y. (1999). U50,488H-induced internalization of the human $\mathrm{\kappa}$ opioid receptor involves a $\beta$-arrestin- and dynamindependent mechanism: $\mathrm{k}$ receptor internalization is not required for mitogenactivated protein kinase activation. Journal of Biological Chemistry, 274, 1208712094.

Li, L.-B., Chen, N., Ramamoorthy, S., Chi, L., Cui, X.-N., Wang, L. C. \& Reith, M. E. (2004). The role of $\mathrm{N}$-glycosylation in function and surface trafficking of the human dopamine transporter. Journal of Biological Chemistry, 279, 21012-21020.

Lindholm, S., Werme, M., Brené, S. \& Franck, J. (2001). The selective k-opioid receptor agonist $\mathrm{U} 50,488 \mathrm{H}$ attenuates voluntary ethanol intake in the rat. Behavioural Brain Research, 120, 137-146.

Listos, J., Merska, A. \& Fidecka, S. (2011). Pharmacological activity of salvinorin A, the major component of Salvia divinorum. Pharmacological Reports, 63, 1305-1309.

Liu-Chen, L. Y. (2004). Agonist-induced regulation and trafficking of kappa opioid receptors. Life Sciences, 75, 511-536.

Logrip, M. L., Janak, P. H. \& Ron, D. (2009). Blockade of ethanol reward by the kappa opioid receptor agonist U50,488H. Alcohol, 43, 359-365.

Lovell, K. M., Frankowski, K. J., Stahl, E. L., Slauson, S. R., Yoo, E., Prisinzano, T. E., Aube, J. \& Bohn, L. M. (2015). Structure-activity relationship studies of functionally selective kappa opioid receptor agonists that modulate ERK $1 / 2$ phosphorylation while preserving $\mathrm{G}$ protein over beta-arrestin2 signaling bias. ACS Chemical Neuroscience.

Lozama, A., Cunningham, C. W., Caspers, M. J., Douglas, J. T., Dersch, C. M., Rothman, R. B. \& Prisinzano, T. E. (2011). Opioid receptor probes derived from cycloaddition of the hallucinogen natural product salvinorin A. Journal of Natural Products, 74, 71826.

Lu, L., Grimm, J., Dempsey, J. \& Shaham, Y. (2004). Cocaine seeking over extended withdrawal periods in rats: Different time courses of responding induced by cocaine cues versus cocaine priming over the first 6 months. Psychopharmacology, 176, 101-108.

Lynch, W. C. \& Burns, G. (1990). Opioid effects on intake of sweet solutions depend both on prior drug experience and on prior ingestive experience. Appetite, 15, 23-32.

Mach, R. H., Smith, C. R. \& Childers, S. R. (1995). Ibogaine possesses a selective affinity for $\sigma 2$ receptors. Life Sciences, 57, PL57-PL62. 
MacLean, K. A., Johnson, M. W., Reissig, C. J., Prisinzano, T. E. \& Griffiths, R. R. (2013). Dose-related effects of salvinorin $A$ in humans: Dissociative, hallucinogenic, and memory effects. Psychopharmacology, 226, 381-92.

Mague, S. D., Pliakas, A. M., Todtenkopf, M. S., Tomasiewicz, H. C., Zhang, Y., Stevens, W. C., Jones, R. M., Portoghese, P. S. \& Carlezon, W. A. (2003). Antidepressant-like effects of kappa-opioid receptor antagonists in the forced swim test in rats. Journal of Pharmacology and Experimental Therapeutics, 305, 323-330.

Mah, S. J., Tang, Y., Liauw, P. E., Nagel, J. E. \& Schneider, A. S. (1998). Ibogaine acts at the nicotinic acetylcholine receptor to inhibit catecholamine release. Brain Research, 797, 173-80.

Maillet, E. L., Milon, N., Heghinian, M. D., Fishback, J., Schurer, S. C., Garamszegi, N. \& Mash, D. C. (2015). Noribogaine is a G-protein biased kappa-opioid receptor agonist. Neuropharmacology, 99, 675-88.

Maisonneuve, I. M., Archer, S. \& Glick, S. D. (1994). U50,488, a к opioid receptor agonist, attenuates cocaine-induced increases in extracellular dopamine in the nucleus accumbens of rats. Neuroscience Letters, 181, 57-60.

Manson, M. E., Corey, D. A., Rymut, S. M. \& Kelley, T. J. (2011). Beta-arrestin-2 regulation of the cAMP response element binding protein. Biochemistry, 50, 6022-9.

Mantsch, J., Ho, A., Schlussman, S. \& Kreek, M. (2001). Predictable individual differences in the initiation of cocaine self-administration by rats under extended-access conditions are dose-dependent. Psychopharmacology, 157, 31-39.

Mantsch, J. R., Yuferov, V., Mathieu-Kia, A.-M., Ho, A. \& Kreek, M. J. (2004). Effects of extended access to high versus low cocaine doses on self-administration, cocaineinduced reinstatement and brain mRNA levels in rats. Psychopharmacology, 175, 26-36.

Margolis, E. B., Hjelmstad, G. O., Bonci, A. \& Fields, H. L. (2003). Kappa-opioid agonists directly inhibit midbrain dopaminergic neurons. Journal of Neuroscience, 23, 99819986.

Margolis, E. B., Lock, H., Chefer, V. I., Shippenberg, T. S., Hjelmstad, G. O. \& Fields, H. L. (2006). Kappa opioids selectively control dopaminergic neurons projecting to the prefrontal cortex. Proceedings of the National Academy of Sciences of the United States of America, 103, 2938-2942.

Mariani, J. J. \& Levin, F. R. (2012). Psychostimulant treatment of cocaine dependence. Psychiatric Clinics of North America 35, 425-39.

Marinova, Z., Vukojevic, V., Surcheva, S., Yakovleva, T., Cebers, G., Pasikova, N., Usynin, I., Hugonin, L., Fang, W. J., Hallberg, M., Hirschberg, D., Bergman, T., Langel, U., Hauser, K. F., Pramanik, A., Aldrich, J. V., Terenius, L. \& Bakalkin, G. (2005). Translocation of dynorphin neuropeptides across the plasma membrane - A putative mechanism of signal transmission. Journal of Biological Chemistry, 280, 26360-26370.

Markou, A., Weiss, F., Gold, L., Caine, S. B., Schulteis, G. \& Koob, G. (1993). Animal models of drug craving. Psychopharmacology, 112, 163-182.

Marshall, J. F., O'Dell, S. J., Navarrete, R. \& Rosenstein, A. J. (1990). Dopamine high-affinity transport site topography in rat brain: Major differences between dorsal and ventral striatum. Neuroscience, 37, 11-21.

Martinez, D., Broft, A., Foltin, R. W., Slifstein, M., Hwang, D.-R., Huang, Y., Perez, A., Frankle, W. G., Cooper, T., Kleber, H. D., Fischman, M. W., Laruelle, M. \& Frankel, 
W. G. (2004). Cocaine dependence and D2 receptor availability in the functional subdivisions of the striatum: relationship with cocaine-seeking behavior. Neuropsychopharmacology, 29, 1190-1202.

Masahiko, F., Tsutomu, S., Minoru, N., Miwa, M. \& Hiroshi, N. (1993). Blockade of morphine reward through the activation of $\mathrm{k}$-opioid receptors in mice. Neuropharmacology, 32, 1315-1323.

Mash, D. C., Kovera, C. A., Buck, B. E., Norenberg, M. D., Shapshak, P., Hearn, W. L. \& Sanchez-Ramos, J. (1998). Medication development of ibogaine as a pharmacotherapy for drug dependence. Annals of the New York Academy of Sciences, 844, 274-92.

Mash, D. C., Kovera, C. A., Pablo, J., Tyndale, R. F., Ervin, F. D., Williams, I. C., Singleton, E. G. \& Mayor, M. (2000). Ibogaine: Complex pharmacokinetics, concerns for safety, and preliminary efficacy measures. Annals of the New York Academy of Sciences, 914, 394-401.

Mash, D. C., Pablo, J., Ouyang, Q., Hearn, W. L. \& Izenwasser, S. (2002). Dopamine transport function is elevated in cocaine users. Journal of Neurochemistry, 81, 292300.

Mash, D. C. \& Staley, J. K. (1999). D3 dopamine and kappa opioid receptor alterations in human brain of cocaine-overdose victims. Annals of the New York Academy of Sciences, 877, 507-522.

Mateus-Pinheiro, A., Patricio, P., Alves, N. D., Machado-Santos, A. R., Morais, M., Bessa, J. M., Sousa, N. \& Pinto, L. (2014). The Sweet Drive Test: Refining phenotypic characterization of anhedonic behavior in rodents. Frontiers in Behavioural Neuroscience, 8, 74.

McCurdy, C. R., Sufka, K. J., Smith, G. H., Warnick, J. E. \& Nieto, M. J. (2006). Antinociceptive profile of salvinorin $A$, a structurally unique kappa opioid receptor agonist. Pharmacology Biochemistry and Behavior, 83, 109-113.

McFarland, K. \& Kalivas, P. W. (2001). The circuitry mediating cocaine-induced reinstatement of drug-seeking behavior. Journal of Neuroscience, 21, 8655-8663.

McLaughlin, J. P. \& Chavkin, C. (2002). Endogenous kappa opioid systems mediate responses to forced swim stress and drug reward. Society for Neuroscience Abstract Viewer and Itinerary Planner, 2002, Abstract No. 9.8.

McLaughlin, J. P., Land, B. B., Li, S., Pintar, J. E. \& Chavkin, C. (2006). Prior activation of kappa opioid receptors by $\mathrm{U} 50,488$ mimics repeated forced swim stress to potentiate cocaine place preference conditioning. Neuropsychopharmacology, 31, 787-94.

McLaughlin, J. P., Marton-Popovici, M. \& Chavkin, C. (2003). Kappa opioid receptor antagonism and prodynorphin gene disruption block stress-induced behavioral responses. Journal of Neuroscience, 23, 5674-5683.

McLaughlin, J. P., Myers, L. C., Zarek, P. E., Caron, M. G., Lefkowitz, R. J., Czyzyk, T. A., Pintar, J. E. \& Chavkin, C. (2004). Prolonged kappa opioid receptor phosphorylation mediated by G-protein receptor kinase underlies sustained analgesic tolerance. Journal of Biological Chemistry, 279, 1810-8.

McLennan, G. P., Kiss, A., Miyatake, M., Belcheva, M. M., Chambers, K. T., Pozek, J. J., Mohabbat, Y., Moyer, R. A., Bohn, L. M. \& Coscia, C. J. (2008). Kappa opioids promote the proliferation of astrocytes via $\mathrm{G}$ beta gamma and beta-arrestin 2- 
dependent MAPK-mediated pathways. Journal of Neurochemistry, 107, 17531765.

Meiergerd, S. \& Schenk, J. (1995). Measurement of the time-resolved kinetics of biogenic amine release and transporter activity by rotating disk electrode voltammetry in vitro. In: Boulton, A., Baker, G. \& Adams, R. (eds.) Voltammetric Methods in Brain Systems. Humana Press.

Meiergerd, S. M. \& Schenk, J. O. (1994). Kinetic evaluation of the commonality between the site(s) of action of cocaine and some other structurally similar and dissimilar inhibitors of the striatal transporter for dopamine. Journal of Neurochemistry, 63, 1683-1692.

Mello, N. K. \& Negus, S. S. (1998). Effects of kappa opioid agonists on cocaine- and foodmaintained responding by rhesus monkeys. Journal of Pharmacology and Experimental Therapeutics, 286, 812-824.

Mello, N. K. \& Negus, S. S. (2000). Interactions between kappa opioid agonists and cocaine - Preclinical studies. In: Glick, S. D. \& Maisonneuve, I. M. (eds.) New Medications for Drug Abuse.

Mendelson, J. E., Coyle, J. R., Lopez, J. C., Baggott, M. J., Flower, K., Everhart, E. T., Munro, T. A., Galloway, G. P. \& Cohen, B. M. (2011). Lack of effect of sublingual salvinorin A, a naturally occurring kappa opioid, in humans: a placebo-controlled trial. Psychopharmacology, 214, 933-939.

Merg, F., Filliol, D., Usynin, I., Bazov, I., Bark, N., Hurd, Y. L., Yakovleva, T., Kieffer, B. L. \& Bakalkin, G. (2006). Big dynorphin as a putative endogenous ligand for the kappaopioid receptor. Journal of Neurochemistry, 97, 292-301.

Meshul, C. \& McGinty, J. (2000). Kappa opioid receptor immunoreactivity in the nucleus accumbens and caudate-putamen is primarily associated with synaptic vesicles in axons. Neuroscience, 96, 91-99.

Ministry of Health, N. (2010). Drug use in New Zealand: Key results of the 2007/08 New Zealand alcohol and drug use survey. Wellington: Ministry of Health.

Missale, C., Nash, S. R., Robinson, S. W., Jaber, M. \& Caron, M. G. (1998). Dopamine receptors: From structure to function.

Mitchell, J. M., Liang, M. T. \& Fields, H. L. (2005). A single injection of the kappa opioid antagonist norbinaltorphimine increases ethanol consumption in rats. Psychopharmacology, 182, 384-392.

Mittelstadt, P. R., Salvador, J. M., Fornace, J. A. J. \& Ashwell, J. D. (2005). Activating p38 MAPK: New tricks for an old kinase. Cell Cycle, 4, 1189-1192.

Mittenberg, W. \& Motta, S. (1993). Effects of chronic cocaine abuse on memory and learning. Archives of Clinical Neuropsychology, 8, 477-83.

Morani, A., Ewald, A., Prevatt-Smith, K., Prisinzano, T. E. \& Kivell, B. (2013). The 2-methoxy methyl analogue of salvinorin A attenuates cocaine-induced drug seeking and sucrose reinforcements in rats. European Journal of Pharmacology.

Morani, A. S., Kivell, B., Prisinzano, T. E. \& Schenk, S. (2009). Effect of kappa-opioid receptor agonists $\mathrm{U} 69593, \mathrm{U} 50488 \mathrm{H}$, spiradoline and salvinorin A on cocaineinduced drug-seeking in rats. Pharmacology Biochemistry and Behavior, 94, 244249.

Morani, A. S., Schenk, S., Prisinzano, T. E. \& Kivell, B. M. (2012). A single injection of a novel kappa opioid receptor agonist salvinorin $A$ attenuates the expression of cocaineinduced behavioral sensitization in rats. Behavioural Pharmacology, 23, 162-170. 
Mori, T., Yoshizawa, K., Ueno, T., Nishiwaki, M., Shimizu, N., Shibasaki, M., Narita, M. \& Suzuki, T. (2013). Involvement of dopamine D2 receptor signal transduction in the discriminative stimulus effects of the kappa-opioid receptor agonist $\mathrm{U}-50,488 \mathrm{H}$ in rats. Behavioural Pharmacology, 24, 275-81.

Moron, J. A., Brockington, A., Wise, R. A., Rocha, B. A. \& Hope, B. T. (2002). Dopamine uptake through the norepinephrine transporter in brain regions with low levels of the dopamine transporter: Evidence from knock-out mouse lines. Journal of Neuroscience, 22, 389-95.

Moron, J. A., Zakharova, I., Ferrer, J. V., Merrill, G. A., Hope, B., Lafer, E. M., Lin, Z. C., Wang, J. B., Javitch, J. A., Galli, A. \& Shippenberg, T. S. (2003). Mitogen-activated protein kinase regulates dopamine transporter surface expression and dopamine transport capacity. Journal of Neuroscience, 23, 8480-8488.

Mowry, M., Mosher, M. \& Briner, W. (2003). Acute physiologic and chronic histologic changes in rats and mice exposed to the unique hallucinogen salvinorin A. Journal of Psychoactive Drugs, 35, 379-382.

Mueller, D. \& Stewart, J. (2000). Cocaine-induced conditioned place preference: reinstatement by priming injections of cocaine after extinction. Behavioural Brain Research, 115, 39-47.

Muschamp, J. W., Hollander, J. A., Thompson, J. L., Voren, G., Hassinger, L. C., Onvani, S., Kamenecka, T. M., Borgland, S. L., Kenny, P. J. \& Carlezon, W. A. (2014). Hypocretin (orexin) facilitates reward by attenuating the antireward effects of its cotransmitter dynorphin in ventral tegmental area. Proceedings of the National Academy of Sciences of the United States of America, 111, E1648-E1655.

Nader, M. A., Morgan, D., Gage, H. D., Nader, S. H., Calhoun, T. L., Buchheimer, N., Ehrenkaufer, R. \& Mach, R. H. (2006). PET imaging of dopamine D2 receptors during chronic cocaine self-administration in monkeys. Nature Neuroscience 9, 1050-1056.

National Institute On Drug Abuse, U. S. A. (2008). Addiction science: From molecules to managed care. Retrieved from www. drugabuse.gov.

National Institute On Drug Abuse, U. S. A. (2012). Principles of drug addiction treatment A research based guide (Third Edition). Retrieved from www.drugabuse.gov.

Naylor, J., Prisinzano, T. \& Freeman, K. (2015). Self-administration of oxycodone alone or as a mixture with the kappa agonist, salvinorin $A$, by monkeys under a progressive ratio schedule of reinforcement. Drug and Alcohol Dependence, 146, e48-e49.

Negus, S. S., Mello, N. K., Portoghese, P. S. \& Lin, C. E. (1997). Effects of kappa opioids on cocaine self-administration by rhesus monkeys. Journal of Pharmacology and Experimental Therapeutics, 282, 44-55.

Negus, S. S. \& Miller, L. L. (2014). Intracranial self-stimulation to evaluate abuse potential of drugs. Pharmacological Reviews, 66, 869-917.

Nemeth, C. L., Paine, T. A., Rittiner, J. E., Beguin, C., Carroll, F. I., Roth, B. L., Cohen, B. M. \& Carlezon, W. A., Jr. (2010). Role of kappa-opioid receptors in the effects of salvinorin A and ketamine on attention in rats. Psychopharmacology, 210, 263274.

Nestler, E. J. (2001). Molecular basis of long-term plasticity underlying addiction. Nature Reviews Neuroscience, 2, 119-128.

Nirenberg, M. J., Chan, J., Pohorille, A., Vaughan, R. A., Uhl, G. R., Kuhar, M. J. \& Pickel, V. M. (1997a). The dopamine transporter: Comparative ultrastructure of 
dopaminergic axons in limbic and motor compartments of the nucleus accumbens. Journal of Neuroscience, 17, 6899-6907.

Nirenberg, M. J., Chan, J., Vaughan, R. A., Uhl, G. R., Kuhar, M. J. \& Pickel, V. M. (1997b). Immunogold localization of the dopamine transporter: an ultrastructural study of the rat ventral tegmental area. Journal of Neuroscience, 17, 5255-62.

Nirenberg, M. J., Vaughan, R. A., Uhl, G. R., Kuhar, M. J. \& Pickel, V. M. (1996). The dopamine transporter is localized to dendritic and axonal plasma membranes of nigrostriatal dopaminergic neurons. Journal of Neuroscience, 16, 436-47.

Noble, F., Lenoir, M. \& Marie, N. (2015). The opioid receptors as targets for drug abuse medication. British Journal of Pharmacology, 172, 3964-3979.

Norman, A. B. \& Tsibulsky, V. L. (2006). The compulsion zone: A pharmacological theory of acquired cocaine self-administration. Brain Research, 1116, 143-52.

Nyberg, F. \& Hallberg, M. (2007). Neuropeptides in hyperthermia. Progress in Brain Research, 162, 277-293.

O'Connor, E. C., Chapman, K., Butler, P. \& Mead, A. N. (2011). The predictive validity of the rat self-administration model for abuse liability. Neuroscience and Biobehavioral Reviews, 35, 912-938.

O'Neill, M. F., Heron-Maxwell, C. L. \& Shaw, G. (1999). 5-HT 2 receptor antagonism reduces hyperactivity induced by amphetamine, cocaine, and MK-801 but not D 1 agonist C-APB. Pharmacology Biochemistry and Behavior, 63, 237-243.

Ookuma, K., Barton, C., York, D. A. \& Bray, G. A. (1997). Effect of enterostatin and kappaopioids on macronutrient selection and consumption. Peptides, 18, 785-91.

Oz, M., Jaligam, V., Galadari, S., Petroianu, G., Shuba, Y. M. \& Shippenberg, T. S. (2010a). The endogenous cannabinoid, anandamide, inhibits dopamine transporter function by a receptor-independent mechanism. Journal of Neurochemistry, 112, 1454-1464.

Oz, M., Libby, T., Kivell, B., Jaligam, V., Ramamoorthy, S. \& Shippenberg, T. S. (2010b). Real-time, spatially resolved analysis of serotonin transporter activity and regulation using the fluorescent substrate, ASP+. Journal of Neurochemistry, 114, 1019-1029.

Panlilio, L. V. \& Goldberg, S. R. (2007). Self-administration of drugs in animals and humans as a model and an investigative tool. Addiction, 102, 1863-1870.

Paris, J. J., Reilley, K. J. \& McLaughlin, J. P. (2011). Kappa opioid receptor-mediated disruption of novel object recognition: Relevance for psychostimulant treatment. Journal of Addiction Research and Therapy, S4.

Passarella, D., Barilli, A., Efange, S. M., Elisabetsky, E., Leal, M. B., Lesma, G., Linck, V. M., Mash, D. C., Martinelli, M., Peretto, I., Silvani, A. \& Danieli, B. (2006). Natureinspired indolyl-2-azabicyclo[2.2.2]oct-7-ene derivatives as promising agents for the attenuation of withdrawal symptoms: synthesis of 20-desethyl-20hydroxymethyl-11-demethoxyibogaine. Natural Product Research, 20, 758-65.

Passarella, D., Favia, R., Giardini, A., Lesma, G., Martinelli, M., Silvani, A., Danieli, B., Efange, S. M. \& Mash, D. C. (2003). Ibogaine analogues. Synthesis and preliminary pharmacological evaluation of 7-heteroaryl-2-azabicyclo[2.2.2]oct-7-enes. Bioorganic \& Medicinal Chemistry, 11, 1007-14.

Paterson, N. E. \& Markou, A. (2003). Increased motivation for self-administered cocaine after escalated cocaine intake. NeuroReport, 14, 2229-2232. 
Paxinos, G. \& Watson, C. (2005). The rat brain in stereotaxic coordinates (4th and 5th eds.) Academic press. San Diego (1998 and 2005).

Pearson, G., Robinson, F., Beers Gibson, T., Xu, B. E., Karandikar, M., Berman, K. \& Cobb, M. H. (2001). Mitogen-activated protein (MAP) kinase pathways: Regulation and physiological functions. Endocrine Reviews, 22, 153-83.

Peet, M. M. \& Baker, L. E. (2011). Salvinorin B derivatives, EOM-Sal B and MOM-Sal B, produce stimulus generalization in male Sprague-Dawley rats trained to discriminate salvinorin A. Behavioural Pharmacology, 22, 450-457.

Perez-Reyes, M., Di Guiseppi, S., Ondrusek, G., Jeffcoat, A. R. \& Cook, C. E. (1982). Freebase cocaine smoking. Clinical Pharmacology and Therapeutics, 32, 459-65.

Pfeiffer, A., Brantl, V., Herz, A. \& Emrich, H. M. (1986). Psychotomimesis mediated by kappa-opiate receptors. Science, 233, 774-776.

Phillips, A., Zhou, L., Lovell, K., Frankowski, K., Slausen, S., Prisinzano, T. E., Aube, J. \& Bohn, L. M. (2013). Development of biased agonists at the kappa opioid receptor. The FASEB Journal, 27, 1171.3.

Picetti, R., Schlussman, S. D., Zhou, Y., Ray, B., Ducat, E., Yuferov, V. \& Kreek, M. J. (2013). Addictions and stress: Clues for cocaine pharmacotherapies. Current Pharmaceutical Design

Pierce, R. C., O'Brien, C. P., Kenny, P. J. \& Vanderschuren, L. J. (2012). Rational development of addiction pharmacotherapies: Successes, failures, and prospects. Cold Spring Harbor Perspectives in Medicine, 2, a012880.

Pineyro, G. (2009). Membrane signalling complexes: Implications for development of functionally selective ligands modulating heptahelical receptor signalling. Cell Signalling, 21, 179-85.

Placzek, M. S., Van de Bittner, G. C., Wey, H. Y., Lukas, S. E. \& Hooker, J. M. (2015). Immediate and persistent effects of salvinorin A on the kappa opioid receptor in rodents, monitored in vivo with pet. Neuropsychopharmacology.

Pliakas, A. M., Carlson, R. R., Neve, R. L., Konradi, C., Nestler, E. J. \& Carlezon, W. A., Jr. (2001). Altered responsiveness to cocaine and increased immobility in the forced swim test associated with elevated CAMP response element-binding protein expression in nucleus accumbens. Journal of Neuroscience, 21, 7397-403.

Porsolt, R. D. (1979). Animal-model of depression. Biomedicine, 30, 139-140.

Porsolt, R. D., Brossard, G., Hautbois, C. \& Roux, S. (2001). Rodent models of depression: Forced swimming and tail suspension behavioral despair tests in rats and mice. Current Protocols in Neuroscience, Chapter 8, Unit 8.10A.

Potter, D. N., Damez-Werno, D., Carlezon, W. A., Jr., Cohen, B. M. \& Chartoff, E. H. (2011). Repeated exposure to the kappa-opioid receptor agonist salvinorin $A$ modulates extracellular signal-regulated kinase and reward sensitivity. Biological Psychiatry, 70, 744-753.

Povlock, S. L. \& Schenk, J. O. (1997). A multisubstrate kinetic mechanism of dopamine transport in the nucleus accumbens and its inhibition by cocaine. Journal of Neurochemistry, 69, 1093-1105.

Prevatt-Smith, K. M., Lovell, K. M., Simpson, D. S., Day, V. W., Douglas, J. T., Bosch, P., Dersch, C. M., Rothman, R. B., Kivell, B. \& Prisinzano, T. E. (2011). Potential drug abuse therapeutics derived from the hallucinogenic natural product salvinorin $A$. Medicinal Chemistry Communications, 2, 1217-1222. 
Prisinzano, T. E. (2005). Psychopharmacology of the hallucinogenic sage Salvia divinorum. Life Sciences, 78, 527-531.

Prus, A. J., James, J. R. \& Rosecrans, J. A. (2009). Conditioned place preference. Methods of Behavior Analysis in Neuroscience, 2, 59-76.

Przekop, P. \& Lee, T. (2009). Persistent psychosis associated with Salvia divinorum use. American Journal of Psychiatry, 166, 832-832.

Raman, M., Chen, W. \& Cobb, M. H. (2007). Differential regulation and properties of MAPKs. Oncogene, 26, 3100-12.

Ramos, A. (2008). Animal models of anxiety: Do I need multiple tests? Trends in Pharmacological Sciences, 29, 493-498.

Redila, V. A. \& Chavkin, C. (2008). Stress-induced reinstatement of cocaine seeking is mediated by the kappa opioid system. Psychopharmacology, 200, 59-70.

Rees, D. \& Hunter, J. (1990). Opioid receptors. Comprehensive Medicinal Chemistry. New York: Pergamon Press.

Reid, M. S., Hsu, K., Jr., Souza, K. H., Broderick, P. A. \& Berger, S. P. (1996). Neuropharmacological characterization of local ibogaine effects on dopamine release. Journal of Neural Transmission, 103, 967-85.

Richardson, N. R. \& Roberts, D. C. (1996). Progressive ratio schedules in drug selfadministration studies in rats: A method to evaluate reinforcing efficacy. Journal of Neuroscience Methods, 66, 1-11.

Richfield, E. K. (1991). Quantitative autoradiography of the dopamine uptake complex in rat brain using [3H] GBR 12935: Binding characteristics. Brain Research, 540, 1-13.

Riley, A. P., Groer, C. E., Young, D., Ewald, A. W., Kivell, B. M. \& Prisinzano, T. E. (2014). Synthesis and K-opioid receptor activity of furan-substituted salvinorin A analogues. Journal of Medicinal Chemistry, 57, 10464-10475.

Rives, M.-L., Rossillo, M., Liu-Chen, L.-Y. \& Javitch, J. A. (2012). 6'-Guanidinonaltrindole (6'GNTI) is a G protein-biased K-opioid receptor agonist that inhibits arrestin recruitment. Journal of Biological Chemistry, 287, 27050-27054.

Rkieh, N., Cloke, J. M., Gallagher, N., Winters, B. D. \& Leri, F. (2014). Drugs of abuse as memory modulators: A study of cocaine in rats. Psychopharmacology, 231, 23392348.

Robbins, A. \& Horlick, R. (1998). Macrophage scavenger receptor confers an adherent phenotype to cells in culture. Biotechniques, 25, 240-244.

Roberts, D. \& Andrews, M. M. (1997). Baclofen suppression of cocaine self-administration: Demonstration using a discrete trials procedure. Psychopharmacology, 131, 271277.

Roberts, D. C. S., Corcoran, M. E. \& Fibiger, H. C. (1977). On the role of ascending catecholaminergic systems in intravenous self-administration of cocaine. Pharmacology Biochemistry and Behavior, 6, 615-620.

Robinson, M. J. F., Robinson, T. E. \& Berridge, K. C. (2013). Chapter 39 - Incentive Salience and the Transition to Addiction. In: Miller, P. M. (ed.) Biological Research on Addiction. San Diego: Academic Press.

Robinson, T. E. \& Berridge, K. C. (1993). The neural basis of drug craving - An incentivesensitization theory of addiction. Brain Research Reviews, 18, 247-291.

Robinson, T. E. \& Berridge, K. C. (2001). Incentive-sensitization and addiction. Addiction, 96, 103-114. 
Robles, C. F., McMackin, M. Z., Campi, K. L., Doig, I. E., Takahashi, E. Y., Pride, M. \& Trainor, B. C. (2014). Effects of kappa opioid receptors on conditioned place aversion and social interaction in males and females. Behavioural Brain Research, 262, 84-93.

Rocha, B. A. (2003). Stimulant and reinforcing effects of cocaine in monoamine transporter knockout mice. European Journal of Pharmacology, 479, 107-115.

Rocha, B. A., Fumagalli, F., Gainetdinov, R. R., Jones, S. R., Ator, R., Giros, B., Miller, G. W. \& Caron, M. G. (1998). Cocaine self-administration in dopamine-transporter knockout mice. Nature Neuroscience, 1, 132-137.

Romero-Pico, A., Vazquez, M. J., Gonzalez-Touceda, D., Folgueira, C., Skibicka, K. P., Alvarez-Crespo, M., Van Gestel, M. A., Velasquez, D. A., Schwarzer, C., Herzog, H., Lopez, M., Adan, R. A., Dickson, S. L., Dieguez, C. \& Nogueiras, R. (2013). Hypothalamic kappa-opioid receptor modulates the orexigenic effect of ghrelin. Neuropsychopharmacology, 38, 1296-307.

Roth, B. L., Baner, K., Westkaemper, R., Siebert, D., Rice, K. C., Steinberg, S., Ernsberger, P. \& Rothman, R. B. (2002). Salvinorin A: A potent naturally occurring nonnitrogenous kappa opioid selective agonist. Proceedings of the National Academy of Sciences of the United States of America, 99, 11934-11939.

Roux, P. P. \& Blenis, J. (2004). ERK and p38 MAPK-activated protein kinases: A family of protein kinases with diverse biological functions. Microbiology and Molecular Biology Reviews, 68, 320-344.

Rüedi-Bettschen, D., Rowlett, J. K., Spealman, R. D. \& Platt, D. M. (2010). Attenuation of cocaine-induced reinstatement of drug seeking in squirrel monkeys: Kappa opioid and serotonergic mechanisms. Psychopharmacology, 210, 169-177.

Ruegg, H., Yu, W.-Z. \& Bodnar, R. J. (1997). Opioid-receptor subtype agonist-induced enhancements of sucrose intake are dependent upon sucrose concentration. Physiology and Behavior, 62, 121-128.

Rush, C. R. \& Stoops, W. W. (2012). Agonist replacement therapy for cocaine dependence: A translational review. Future Medicinal Chemistry, 4, 245-265.

Rusin, K. I., Giovannucci, D. R., Stuenkel, E. L. \& Moises, H. C. (1997). Kappa-opioid receptor activation modulates $\mathrm{Ca}^{2+}$ currents and secretion in isolated neuroendocrine nerve terminals. Journal of Neuroscience, 17, 6565-74.

Sanchis-Segura, C. \& Spanagel, R. (2006). Behavioural assessment of drug reinforcement and addictive features in rodents: An overview. Addiction Biology, 11, 2-38.

Sandi, C., Borrell, J. \& Guaza, C. (1988). Involvement of kappa type opioids on ethanol drinking. Life Sciences, 42, 1067-1075.

Schank, J. R., Goldstein, A. L., Rowe, K. E., King, C. E., Marusich, J. A., Wiley, J. L., Carroll, F. I., Thorsell, A. \& Heilig, M. (2012). The kappa opioid receptor antagonist JDTic attenuates alcohol seeking and withdrawal anxiety. Addiction Biology, 17, 634647.

Schattauer, S. S., Miyatake, M., Shankar, H., Zietz, C., Levin, J. R., Liu-Chen, L. Y., Gurevich, V. V., Rieder, M. J. \& Chavkin, C. (2012). Ligand directed signaling differences between rodent and human kappa-opioid receptors. Journal of Biological Chemistry, 287, 41595-607.

Schenberg, E. E., de Castro Comis, M. A., Chaves, B. R. \& da Silveira, D. X. (2014). Treating drug dependence with the aid of ibogaine: A retrospective study. Journal of Psychopharmacology, 28, 993-1000. 
Schenk, J. O., Wright, C. \& Bjorklund, N. (2005). Unraveling neuronal dopamine transporter mechanisms with rotating disk electrode voltammetry. Journal of Neuroscience Methods, 143, 41-47.

Schenk, S., Hely, L., Lake, B., Daniela, E., Gittings, D. \& Mash, D. C. (2007). MDMA selfadministration in rats: Acquisition, progressive ratio responding and serotonin transporter binding. European Journal of Neuroscience, 26, 3229-3236.

Schenk, S., Partridge, B. \& Shippenberg, T. S. (1999). U69593, a kappa-opioid agonist, decreases cocaine self-administration and decreases cocaine-produced drugseeking. Psychopharmacology, 144, 339-346.

Schenk, S., Partridge, B. \& Shippenberg, T. S. (2000). Reinstatement of extinguished drugtaking behavior in rats: Effect of the kappa-opioid receptor agonist, U69593. Psychopharmacology, 151, 85-90.

Schenk, S., Partridge, B. \& Shippenberg, T. S. (2001). Effects of the kappa-opioid receptor agonist, U69593, on the development of sensitization and on the maintenance of cocaine self-administration. Neuropsychopharmacology, 24, 441-450.

Schindler, C. W., Panlilio, L. V. \& Goldberg, S. R. (2002). Second-order schedules of drug self-administration in animals. Psychopharmacology, 163, 327-344.

Schlosburg, J. E., Whitfield, T. W., Park, P. E., Crawford, E. F., George, O., Vendruscolo, L. F. \& Koob, G. F. (2013). Long-term antagonism of $\mathrm{k}$ opioid receptors prevents escalation of and increased motivation for heroin intake. Journal of Neuroscience, 33, 19384-19392.

Schmid, C. L., Streicher, J. M., Groer, C. E., Munro, T. A., Zhou, L. \& Bohn, L. M. (2013). Functional selectivity of $6^{\prime}$-guanidinonaltrindole $\left(6^{\prime}\right.$-GNTI) at k-opioid receptors in striatal neurons. Journal of Biological Chemistry, 288, 22387-22398.

Schmidt, M. D., Schmidt, M. S., Butelman, E. R., Harding, W. W., Tidgewell, K., Murry, D. J., Kreek, M. J. \& Prisinzano, T. E. (2005). Pharmacokinetics of the plant-derived kappa-opioid hallucinogen salvinorin A in nonhuman primates. Synapse, 58, 208210.

Schwartz, J. W., Blakely, R. D. \& DeFelice, L. J. (2003). Binding and transport in norepinephrine transporters - Real-time, spatially resolved analysis in single cells using a fluorescent substrate. Journal of Biological Chemistry, 278, 9768-9777.

Schwartz, J. W., Novarino, G., Piston, D. W. \& DeFelice, L. J. (2005). Substrate binding stoichiometry and kinetics of the norepinephrine transporter. Journal of Biological Chemistry, 280, 19177-19184.

Sershen, H., Hashim, A. \& Lajtha, A. (1997). Ibogaine and cocaine abuse: Pharmacological interactions at dopamine and serotonin receptors. Brain Research Bulletin, 42, 161-8.

Shaham, Y., Shalev, U., Lu, L., de Wit, H. \& Stewart, J. (2003). The reinstatement model of drug relapse: History, methodology and major findings. Psychopharmacology, 168, 3-20.

Shalev, U., Grimm, J. W. \& Shaham, Y. (2002). Neurobiology of relapse to heroin and cocaine seeking: A review. Pharmacological Reviews, 54, 1-42.

Shaw, G., Morse, S., Ararat, M. \& Graham, F. L. (2002). Preferential transformation of human neuronal cells by human adenoviruses and the origin of HEK 293 cells. The FASEB Journal, 16, 869-871.

Shearer, J., Sherman, J., Wodak, A. \& Beek, I. v. (2002). Substitution therapy for amphetamine users. Drug and Alcohol Review, 21, 179-185. 
Shippenberg, T., LeFevour, A. \& Heidbreder, C. (1996). Kappa-opioid receptor agonists prevent sensitization to the conditioned rewarding effects of cocaine. Journal of Pharmacology and Experimental Therapeutics, 276, 545-554.

Shippenberg, T. S. (2009). The dynorphin/kappa opioid receptor system: A new target for the treatment of addiction and affective disorders? Neuropsychopharmacology, 34, 247-247.

Shippenberg, T. S., Balskubik, R. \& Herz, A. (1993). Examination of the neurochemical substrates mediating the motivational effects of opioids - Role of the mesolimbic dopamine system and D-1 vs D-2 dopamine-receptors. Journal of Pharmacology and Experimental Therapeutics, 265, 53-59.

Shippenberg, T. S., Chefer, V. I., Zapata, A. \& Heidbreder, C. A. (2001). Modulation of the behavioral and neurochemical effects of psychostimulants by kappa-opioid receptor systems. In: QuinonesJenab, V. (ed.) Biological Basis of Cocaine Addiction.

Shippenberg, T. S., LeFevour, A. \& Thompson, A. C. (1998). Sensitization to the conditioned rewarding effects of morphine and cocaine: differential effects of the kappa-opioid receptor agonist U69593. European Journal of Pharmacology, 345, 27-34.

Shippenberg, T. S., Zapata, A. \& Chefer, V. I. (2007). Dynorphin and the pathophysiology of drug addiction. Pharmacology and Therapeutics, 116, 306-321.

Shirayama, Y., Ishida, H., Iwata, M., Hazama, G. I., Kawahara, R. \& Duman, R. S. (2004). Stress increases dynorphin immunoreactivity in limbic brain regions and dynorphin antagonism produces antidepressant-like effects. Journal of Neurochemistry, 90, 1258-68.

Shonberg, J., Lopez, L., Scammells, P. J., Christopoulos, A., Capuano, B. \& Lane, J. R. (2014). Biased agonism at $\mathrm{G}$ protein-coupled receptors: The promise and the challengesa medicinal chemistry perspective. Medicinal Research Reviews.

Shorter, D., Domingo, C. B. \& Kosten, T. R. (2015). Emerging drugs for the treatment of cocaine use disorder: A review of neurobiological targets and pharmacotherapy. Expert Opinion on Emerging Drugs, 20, 15-29.

Siebert, D. (2014). The legal status of Salvia divinorum.

Siebert, D. J. (1994). Salvia-divinorum and salvinorin-A - New pharmacological findings. Journal of Ethnopharmacology, 43, 53-56.

Simonin, F., Gaveriaux-Ruff, C., Befort, K., Matthes, H., Lannes, B., Micheletti, G., Mattei, M.-G., Charron, G., Bloch, B. \& Kieffer, B. (1995). Kappa-opioid receptor in humans: CDNA and genomic cloning, chromosomal assignment, functional expression, pharmacology, and expression pattern in the central nervous system. Proceedings of the National Academy of Sciences of the United States of America 92, 70067010.

Simonin, F., Valverde, O., Smadja, C., Slowe, S., Kitchen, I., Dierich, A., Le Meur, M., Roques, B. P., Maldonado, R. \& Kieffer, B. L. (1998). Disruption of the K-opioid receptor gene in mice enhances sensitivity to chemical visceral pain, impairs pharmacological actions of the selective $\mathrm{k}$-agonist $\mathrm{U}-50,488 \mathrm{H}$ and attenuates morphine withdrawal. The European Molecular Biology Organization Journal, 17, 886-897.

Simonson, B., Morani, A. S., Ewald, A. W. M., Walker, L., Kumar, N., Simpson, D., Miller, J. H., Prisinzano, T. E. \& Kivell, B. M. (2015). Pharmacology and anti-addiction effects of the novel $\mathrm{K}$ opioid receptor agonist Mesyl Sal B, a potent and long-acting analogue of salvinorin A. British Journal of Pharmacology, 172, 515-531. 
Simpson, D. S., Katavic, P. L., Lozama, A., Harding, W. W., Parrish, D., Deschamps, J. R., Dersch, C. M., Partilla, J. S., Rothman, R. B. \& Navarro, H. (2007). Synthetic studies of neoclerodane diterpenes from Salvia divinorum: Preparation and opioid receptor activity of salvinicin analogues. Journal of Medicinal Chemistry, 50, 35963603.

Simpson, G. R. \& Riley, A. L. (2005). Morphine preexposure facilitates morphine place preference and attenuates morphine taste aversion. Pharmacology Biochemistry and Behavior, 80, 471-9.

Sinha, R., Catapano, D. \& O'Malley, S. (1999). Stress-induced craving and stress response in cocaine dependent individuals. Psychopharmacology, 142, 343-351.

Sipols, A. J., Bayer, J., Bennett, R. \& Figlewicz, D. P. (2002). Intraventricular insulin decreases kappa opioid-mediated sucrose intake in rats. Peptides, 23, 2181-2187.

Skinner, B. F. (1953). Science and human behavior. Simon and Schuster.

Skoubis, P., Matthes, H., Walwyn, W., Kieffer, B. \& Maidment, N. (2001). Naloxone fails to produce conditioned place aversion in $\mu$-opioid receptor knock-out mice. Neuroscience, 106, 757-763.

Slack, A., Nana, G., Webster, M., Stokes, F. \& Wu, J. (2009). Costs of harmful alcohol and other drug use. Berl economics. Wellington, New Zealand: Business and Economic Research Limited.

Smith, J. S., Schindler, A. G., Martinelli, E., Gustin, R. M., Bruchas, M. R. \& Chavkin, C. (2012). Stress-induced activation of the dynorphin/kappa-opioid receptor system in the amygdala potentiates nicotine conditioned place preference. Journal of Neuroscience 32, 1488-95.

Solomon, R. L. \& Corbit, J. D. (1974). An opponent-process theory of motivation: I. Temporal dynamics of affect. Psychological Review, 81, 119.

Somoza, E. C., Winhusen, T. M., Bridge, T. P., Rotrosen, J. P., Vanderburg, D. G., Harrer, J. M., Mezinskis, J. P., Montgomery, M. A., Ciraulo, D. A., Wulsin, L. R. \& Barrett, J. A. (2004). An open-label pilot study of methylphenidate in the treatment of cocaine dependent patients with adult attention deficit/hyperactivity disorder. Journal of Addictive Diseases, 23, 77-92.

Sora, I., Hall, F. S., Andrews, A. M., Itokawa, M., Li, X.-F., Wei, H.-B., Wichems, C., Lesch, K.-P., Murphy, D. L. \& Uhl, G. R. (2001). Molecular mechanisms of cocaine reward: Combined dopamine and serotonin transporter knockouts eliminate cocaine place preference. Proceedings of the National Academy of Sciences of the United States of America, 98, 5300-5305.

Sora, I., Wichems, C., Takahashi, N., Li, X.-F., Zeng, Z., Revay, R., Lesch, K.-P., Murphy, D. L. \& Uhl, G. R. (1998). Cocaine reward models: Conditioned place preference can be established in dopamine-and in serotonin-transporter knockout mice. Proceedings of the National Academy of Sciences, 95, 7699-7704.

Spanagel, R., Herz, A. \& Shippenberg, T. S. (1992). Opposing tonically active endogenous opioid systems modulate the mesolimbic dopaminergic pathway. Proceedings of the National Academy of Sciences of the United States of America, 89, 2046-2050.

Stachon, A., Schlatter, E. \& Hohage, H. (1996). Dynamic monitoring of organic cation transport processes by fluorescence measurements in LLC-PK ${ }_{1}$ cells. Cellular Physiology and Biochemistry, 6, 72-81. 
Stretch, R., Gerber, G. J. \& Wood, S. M. (1971). Factors affecting behavior maintained by response-contingent intravenous infusions of amphetamine in squirrel monkeys. Canadian Journal of Physiology and Pharmacology, 49, 581-589.

Sufka, K. J., Loria, M. J., Lewellyn, K., Zjawiony, J. K., Ali, Z., Abe, N. \& Khan, I. A. (2014). The effect of Salvia divinorum and Mitragyna speciosa extracts, fraction and major constituents on place aversion and place preference in rats. Journal of Ethnopharmacology, 151, 361-364.

Sun, W. L., Xue, Y. Q., Huang, Z. F. \& Steketee, J. D. (2010). Regulation of cocainereinstated drug-seeking behavior by kappa-opioid receptors in the ventral tegmental area of rats. Psychopharmacology, 210, 179-188.

Suzuki, T., Izumimoto, N., Takezawa, Y., Fujimura, M., Togashi, Y., Nagase, H., Tanaka, T. \& Endoh, T. (2004). Effect of repeated administration of TRK-820, a kappa-opioid receptor agonist, on tolerance to its antinociceptive and sedative actions. Brain Research, 995, 167-75.

Svingos, A. L., Chavkin, C., Colago, E. E. \& Pickel, V. M. (2001). Major coexpression of kappa-opioid receptors and the dopamine transporter in nucleus accumbens axonal profiles. Synapse, 42, 185-92.

Taussig, R., Iniguez-Lluhi, J. A. \& Gilman, A. G. (1993). Inhibition of adenylyl cyclase by Gi alpha. Science, 261, 218-221.

Tejeda, H. A., Counotte, D. S., Oh, E., Ramamoorthy, S., Schultz-Kuszak, K. N., Bäckman, C. M., Chefer, V., O'Donnell, P. \& Shippenberg, T. S. (2013). Prefrontal cortical kappaopioid receptor modulation of local neurotransmission and conditioned place aversion. Neuropsychopharmacology, 38, 1770-1779.

Teksin, Z. S., Lee, I. J., Nemieboka, N. N., Othman, A. A., Upreti, V. V., Hassan, H. E., Syed, S. S., Prisinzano, T. E. \& Eddington, N. D. (2009). Evaluation of the transport, in vitro metabolism and pharmacokinetics of Salvinorin A, a potent hallucinogen. European Journal of Pharmaceutics and Biopharmaceutics, 72, 471-7.

Thomas, M. J., Beurrier, C., Bonci, A. \& Malenka, R. C. (2001). Long-term depression in the nucleus accumbens: A neural correlate of behavioral sensitization to cocaine. Nature Neuroscience, 4, 1217-23.

Thompson, A. C., Zapata, A., Justice, J. B., Vaughan, R. A., Sharpe, L. G. \& Shippenberg, T. S. (2000). Kappa-opioid receptor activation modifies dopamine uptake in the nucleus accumbens and opposes the effects of cocaine. Journal of Neuroscience, 20, 9333-9340.

Thomsen, M., Hall, F. S., Uhl, G. R. \& Caine, S. B. (2009). Dramatically decreased cocaine self-administration in dopamine but not serotonin transporter knock-out mice. Journal of Neuroscience, 29, 1087-1092.

Thorn, D. A., Zhang, C., Zhang, Y. \& Li, J.-X. (2014). The trace amine associated receptor 1 agonist RO5263397 attenuates the induction of cocaine behavioral sensitization in rats. Neuroscience Letters, 566, 67-71.

Todtenkopf, M. S., Marcus, J. F., Portoghese, P. S. \& Carlezon, W. A. (2004). Effects of kappa-opioid receptor ligands on intracranial self-stimulation in rats. Psychopharmacology, 172, 463-470.

Tomasiewicz, H. C., Todtenkopf, M. S., Chartoff, E. H., Cohen, B. M. \& Carlezon, W. A. (2008). The kappa-opioid agonist U69,593 blocks cocaine-induced enhancement of brain stimulation reward. Biological Psychiatry, 64, 982-988. 
Tonissaar, M., Herm, L., Rinken, A. \& Harro, J. (2006). Individual differences in sucrose intake and preference in the rat: Circadian variation and association with dopamine D2 receptor function in striatum and nucleus accumbens. Neuroscience Letters, 403, 119-24.

Torres, G. E. (2006). The dopamine transporter proteome. Journal of Neurochemistry, 97, 3-10.

Tran-Nguyen, L. T., Fuchs, R. A., Coffey, G. P., Baker, D. A., O'Dell, L. E. \& Neisewander, J. L. (1998). Time-dependent changes in cocaine-seeking behavior and extracellular dopamine levels in the amygdala during cocaine withdrawal. Neuropsychopharmacology, 19, 48-59.

Trifilieff, P. \& Martinez, D. (2013). Kappa-opioid receptor signaling in the striatum as a potential modulator of dopamine transmission in cocaine dependence. Frontiers in Psychiatry, 4.

Tsukahara-Ohsumi, Y., Tsuji, F., Niwa, M., Hata, T., Narita, M., Suzuki, T., Sasano, M. \& Aono, H. (2011). The kappa opioid receptor agonist SA14867 has antinociceptive and weak sedative effects in models of acute and chronic pain. European Journal of Pharmacology, 671, 53-60.

Tsukahara-Ohsumi, Y., Tsuji, F., Niwa, M., Nakamura, M., Mizutani, K., Inagaki, N., Sasano, M. \& Aono, H. (2010). SA14867, a newly synthesized kappa-opioid receptor agonist with antinociceptive and antipruritic effects. European Journal of Pharmacology, 647, 62-7.

Turchan, J., Przewłocka, B., Lasoń, W. \& Przewłocki, R. (1998). Effects of repeated psychostimulant administration on the prodynorphin system activity and kappa opioid receptor density in the rat brain. Neuroscience, 85, 1051-1059.

Tzschentke, T. M. (2007). Measuring reward with the conditioned place preference (CPP) paradigm: Update of the last decade. Addiction Biology, 12, 227-462.

Unterwald, E. M., Kreek, M. J. \& Cuntapay, M. (2001). The frequency of cocaine administration impacts cocaine-induced receptor alterations. Brain Research, 900, 103-109.

Unterwald, E. M., Rubenfeld, J. M. \& Kreek, M. J. (1994). Repeated cocaine administration upregulates kappa and mu, but not delta opioid receptors. Neuroreport, 5, 16131616.

Urban, J. D., Clarke, W. P., von Zastrow, M., Nichols, D. E., Kobilka, B., Weinstein, H., Javitch, J. A., Roth, B. L., Christopoulos, A., Sexton, P. M., Miller, K. J., Spedding, M. \& Mailman, R. B. (2007). Functional selectivity and classical concepts of quantitative pharmacology. Journal of Pharmacology and Experimental Therapeutics 320, 1-13.

US Department of Justice, U. (2011). Drugs of abuse. In: Administration, D. E. (ed.). US Department of Justice.

Valdés III, L. J., Díaz, J. \& Paul, A. G. (1983). Ethnopharmacology of ska María Pastora (Salvia divinorum, Epling AND Játiva-M.). Journal of Ethnopharmacology, 7, 287312.

Valdes, L. J. (1994). Salvia-divinorum and the unique diterpene hallucinogen, salvinorin (divinorin)-A. Journal of Psychoactive Drugs, 26, 277-283.

Valdez, G. R. \& Harshberger, E. (2012). Kappa opioid regulation of anxiety-like behavior during acute ethanol withdrawal. Pharmacology Biochemistry and Behavior, 102, 44-7. 
Valjent, E., Mitchell, J. M., Besson, M.-J., Caboche, J. \& Maldonado, R. (2002). Behavioural and biochemical evidence for interactions between $\Delta 9$-tetrahydrocannabinol and nicotine. British Journal of Pharmacology, 135, 564-578.

Van't Veer, A. \& Carlezon Jr, W. A. (2013). Role of kappa-opioid receptors in stress and anxiety-related behavior. Psychopharmacology, 1-18.

Vanderschuren, L. J., Schoffelmeer, A. N., Wardeh, G. \& De Vries, T. J. (2000). Dissociable effects of the kappa-opioid receptor agonists bremazocine, U69593, and U50488H on locomotor activity and long-term behavioral sensitization induced by amphetamine and cocaine. Psychopharmacology, 150, 35-44.

Vendruscolo, L. F., Takahashi, R. N., Brüske, G. R. \& Ramos, A. (2003). Evaluation of the anxiolytic-like effect of NKP608, a NK1-receptor antagonist, in two rat strains that differ in anxiety-related behaviors. Psychopharmacology, 170, 287-293.

Vendruscolo, L. F., Terenina-Rigaldie, E., Raba, F., Ramos, A., Takahashi, R. N. \& Mormède, P. (2006). Evidence for a female-specific effect of a chromosome 4 locus on anxiety-related behaviors and ethanol drinking in rats. Genes, Brain and Behavior, 5, 441-450.

Violin, J. D., Crombie, A. L., Soergel, D. G. \& Lark, M. W. (2014). Biased ligands at G-proteincoupled receptors: Promise and progress. Trends in Pharmacological Sciences, 35, 308-316.

Violin, J. D. \& Lefkowitz, R. J. (2007). Beta-arrestin-biased ligands at seven-transmembrane receptors. Trends in Pharmacological Sciences, 28, 416-22.

Vlachou, S. \& Markou, A. (2011). Intracranial self-stimulation. In: Olmstead, M. C. (ed.) Animal Models of Drug Addiction. Humana Press.

Volkow, N. D., Fowler, J. S. \& Wang, G.-J. (1999). Imaging studies on the role of dopamine in cocaine reinforcement and addiction in humans. Journal of Psychopharmacology, 13, 337-345.

Volkow, N. D., Fowler, J. S., Wang, G. J., Hitzemann, R., Logan, J., Schlyer, D. J., Dewey, S. L. \& Wolf, A. P. (1993). Decreased dopamine D2 receptor availability is associated with reduced frontal metabolism in cocaine abusers. Synapse, 14, 169-177.

Volkow, N. D., Hitzemann, R., Wang, G.-J., Fowler, J. S., Wolf, A. P., Dewey, S. L. \& Handlesman, L. (1992). Long-term frontal brain metabolic changes in cocaine abusers. Synapse, 11, 184-190.

Volkow, N. D., Mullani, N., Gould, K. L., Adler, S. \& Krajewski, K. (1988). Cerebral blood flow in chronic cocaine users: A study with positron emission tomography. The British Journal of Psychiatry, 152, 641-648.

Waetzig, V. \& Herdegen, T. (2005). Context-specific inhibition of JNKs: Overcoming the dilemma of protection and damage. Trends in Pharmacological Sciences, 26, 45561.

Wahlsten, D., Bachmanov, A., Finn, D. A. \& Crabbe, J. C. (2006). Stability of inbred mouse strain differences in behavior and brain size between laboratories and across decades. Proceedings of the National Academy of Sciences of the United States of America 103, 16364-16369.

Waldhoer, M., Fong, J., Jones, R. M., Lunzer, M. M., Sharma, S. K., Kostenis, E., Portoghese, P. S. \& Whistler, J. L. (2005). A heterodimer-selective agonist shows in vivo relevance of $\mathrm{G}$ protein-coupled receptor dimers. Proceedings of the National Academy of Sciences of the United States of America, 102, 9050-9055. 
Walf, A. A. \& Frye, C. A. (2007). The use of the elevated plus maze as an assay of anxietyrelated behavior in rodents. Nature Protocols, 2, 322-8.

Walsh, S. L., Strain, E. C., Abreu, M. E. \& Bigelow, G. E. (2001). Enadoline, a selective kappa opioid agonist: Comparison with butorphanol and hydromorphone in humans. Psychopharmacology, 157, 151-162.

Wang, Y., Chen, Y., Xu, W., Lee, D. Y. W., Ma, Z., Rawls, S. M., Cowan, A. \& Liu-Chen, L.-Y. (2008). 2-methoxymethyl-salvinorin $B$ is a potent kappa opioid receptor agonist with longer lasting action in vivo than salvinorin A. Journal of Pharmacology and Experimental Therapeutics, 324, 1073-1083.

Wang, Y., Tang, K., Inan, S., Siebert, D., Holzgrabe, U., Lee, D. Y. W., Huang, P., Li, J. G., Cowan, A. \& Liu-Chen, L. Y. (2005). Comparison of pharmacological activities of three distinct kappa ligands (salvinorin A, TRK-820 and 3FLB) on kappa opioid receptors in vitro and their antipruritic and antinociceptive activities in vivo. Journal of Pharmacology and Experimental Therapeutics, 312, 220-230.

Watson, S. J., Khachaturian, H., Coy, D., Taylor, L. \& Akil, H. (1982). Dynorphin is located throughout the CNS and is often co-localized with alpha-neo-endorphin. Life Sciences, 31, 1773-1776.

Wee, S. \& Koob, G. F. (2010). The role of the dynorphin-kappa opioid system in the reinforcing effects of drugs of abuse. Psychopharmacology, 210, 121-135.

Wee, S., Orio, L., Ghirmai, S., Cashman, J. R. \& Koob, G. F. (2009). Inhibition of kappa opioid receptors attenuated increased cocaine intake in rats with extended access to cocaine. Psychopharmacology, 205, 565-575.

Wee, S., Specio, S. E. \& Koob, G. F. (2007). Effects of dose and session duration on cocaine self-administration in rats. Journal of Pharmacology and Experimental Therapeutics, 320, 1134-1143.

Weeks, J. R. (1962). Experimental morphine addiction: Method for automatic intravenous injections in unrestrained rats. Science, 138, 143-144.

West, R. (2001). Theories of addiction. Addiction, 96, 3-13.

White, K. L., Robinson, J. E., Zhu, H., DiBerto, J. F., Polepally, P. R., Zjawiony, J. K., Nichols, D. E., Malanga, C. J. \& Roth, B. L. (2015). The G protein-biased kappa-opioid receptor agonist RB-64 is analgesic with a unique spectrum of activities in vivo. Journal of Pharmacology and Experimental Therapeutics, 352, 98-109.

Whitmarsh, A. J. (2010). A central role for p38 MAPK in the early transcriptional response to stress. BMC Biology, 8, 47.

Willmore-Fordham, C. B., Krall, D. M., McCurdy, C. R. \& Kinder, D. H. (2007). The hallucinogen derived from Salvia divinorum, salvinorin A, has kappa-opioid agonist discriminative stimulus effects in rats. Neuropharmacology, 53, 481-486.

Wise, R. A. (2009). Roles for nigrostriatal-not just mesocorticolimbic-dopamine in reward and addiction. Trends in Neurosciences, 32, 517-524.

Wittmann, W., Schunk, E., Rosskothen, I., Gaburro, S., Singewald, N., Herzog, H. \& Schwarzer, C. (2009). Prodynorphin-derived peptides are critical modulators of anxiety and regulate neurochemistry and corticosterone. Neuropsychopharmacology, 34, 775-785.

Wu, E. H. T. \& Wong, Y. H. (2005). Activation of $\delta-$, K-, and $\mu$-opioid receptors induces phosphorylation of tuberin in transfected HEK 293 cells and native cells. Biochemical and Biophysical Research Communications, 334, 838-844. 
Wu, Q., Reith, M. E., Kuhar, M. J., Carroll, F. I. \& Garris, P. A. (2001). Preferential increases in nucleus accumbens dopamine after systemic cocaine administration are caused by unique characteristics of dopamine neurotransmission. Journal of Neuroscience 21.

Xi, Z.-X. \& Gardner, E. L. (2008). Hypothesis-driven medication discovery for the treatment of psychostimulant addiction. Current Drug Abuse Reviews 1, 303.

Xi, Z. X., Fuller, S. A. \& Stein, E. A. (1998). Dopamine release in the nucleus accumbens during heroin self-administration is modulated by kappa opioid receptors: An in vivo fast-cyclic voltammetry study. Journal of Pharmacology and Experimental Therapeutics, 284, 151-161.

Xu, F., Gainetdinov, R. R., Wetsel, W. C., Jones, S. R., Bohn, L. M., Miller, G. W., Wang, Y.M. \& Caron, M. G. (2000). Mice lacking the norepinephrine transporter are supersensitive to psychostimulants. Nature Neuroscience, 3, 465-471.

Xu, M., Bruchas, M. R., Ippolito, D. L., Gendron, L. \& Chavkin, C. (2007). Sciatic nerve ligation-induced proliferation of spinal cord astrocytes is mediated by kappa opioid activation of p38 mitogen-activated protein kinase. Journal of Neuroscience, 27, 2570-81.

Yan, F., Mosier, P. D., Westkaemper, R. B., Stewart, J., Zjawiony, J. K., Vortherms, T. A., Sheffler, D. J. \& Roth, B. L. (2005). Identification of the molecular mechanisms by which the diterpenoid salvinorin A binds to kappa-opioid receptors. Biochemistry, 44, 8643-8651.

Yoshizawa, K., Narita, M., Saeki, M., Narita, M., Isotani, K., Horiuchi, H., Imai, S., Kuzumaki, N. \& Suzuki, T. (2011). Activation of extracellular signal-regulated kinase is critical for the discriminative stimulus effects induced by U-50,488H. Synapse, 65, 105261.

Zapata, A., Kivell, B., Han, Y., Javitch, J. A., Bolan, E. A., Kuraguntla, D., Jaligam, V., Oz, M., Jayanthi, L. D., Samuvel, D. J., Ramamoorthy, S. \& Shippenberg, T. S. (2007). Regulation of dopamine transporter function and cell surface expression by D3 dopamine receptors. Journal of Biological Chemistry, 282, 35842-35854.

Zhang, L.-S., Wang, J., Chen, J.-C., Tao, Y.-m., Wang, Y.-h., Xu, X.-j., Chen, J., Xu, Y.-g., Xi, T., Hu, X.-W., Wang, Y.-j. \& Liu, J.-g. (2015). Novel kappa-opioid receptor agonist MB$1 \mathrm{C}-\mathrm{OH}$ produces potent analgesia with less depression and sedation. Acta Pharmacologica Sinica.

Zhang, L., Elmer, L. W. \& Little, K. Y. (1998). Expression and regulation of the human dopamine transporter in a neuronal cell line. Molecular Brain Research, 59, 66-73.

Zhang, Y., Butelman, E. R., Schlussman, S. D., Ho, A. \& Kreek, M. J. (2004a). Effect of the endogenous kappa opioid agonist dynorphin $A(1-17)$ on cocaine-evoked increases in striatal dopamine levels and cocaine-induced place preference in C57BL/6J mice. Psychopharmacology, 172, 422-429.

Zhang, Y., Butelman, E. R., Schlussman, S. D., Ho, A. \& Kreek, M. J. (2004b). Effect of the kappa opioid agonist R-84760 on cocaine-induced increases in striatal dopamine levels and cocaine-induced place preference in $\mathrm{C57BL} / 6 \mathrm{~J}$ mice. Psychopharmacology, 173, 146-152.

Zhang, Y., Butelman, E. R., Schlussman, S. D., Ho, A. \& Kreek, M. J. (2005). Effects of the plant-derived hallucinogen salvinorin $A$ on basal dopamine levels in the caudate putamen and in a conditioned place aversion assay in mice: agonist actions at kappa opioid receptors. Psychopharmacology, 179, 551-558. 
Zhou, L., Lovell, K. M., Frankowski, K. J., Slauson, S. R., Phillips, A. M., Streicher, J. M., Stahl, E., Schmid, C. L., Hodder, P., Madoux, F., Cameron, M. D., Prisinzano, T. E., Aubé, J. \& Bohn, L. M. (2013). Development of functionally selective, small molecule agonists at kappa opioid receptors. Journal of Biological Chemistry, 288, 3670336716.

Zhou, L., Stahl, E. L., Lovell, K. M., Frankowski, K. J., Prisinzano, T. E., Aubé, J. \& Bohn, L. M. (2015). Characterization of kappa opioid receptor mediated, dynorphin-stimulated [35S]GTPYS binding in mouse striatum for the evaluation of selective KOR ligands in an endogenous setting. Neuropharmacology, 99, 131-141.

Zhu, J., Chen, C., Xue, J. C., Kunapuli, S., DeRiel, J. K. \& Liu-Chen, L. Y. (1995). Cloning of a human kappa opioid receptor from the brain. Life Sciences, 56, PI201-7.

Zimmer, A., Valjent, E., Konig, M., Zimmer, A. M., Robledo, P., Hahn, H., Valverde, O. \& Maldonado, R. (2001). Absence of delta -9-tetrahydrocannabinol dysphoric effects in dynorphin-deficient mice. Journal of Neuroscience, 21, 9499-505. 